Illinois State University

ISU ReD: Research and eData

Theses and Dissertations

$11-10-2013$

\title{
An Archaeological And Historical Search For German Ethnicity At The Janis-Ziegler Site (23sg272)
}

Lisa Marie Dretske

Illinois State University, Imdretske@gmail.com

Follow this and additional works at: https://ir.library.illinoisstate.edu/etd

Part of the History Commons, and the History of Art, Architecture, and Archaeology Commons

\section{Recommended Citation}

Dretske, Lisa Marie, "An Archaeological And Historical Search For German Ethnicity At The Janis-Ziegler Site (23sg272)" (2013). Theses and Dissertations. 68.

https://ir.library.illinoisstate.edu/etd/68

This Thesis is brought to you for free and open access by ISU ReD: Research and eData. It has been accepted for inclusion in Theses and Dissertations by an authorized administrator of ISU ReD: Research and eData. For more information, please contact ISUReD@ilstu.edu. 


\author{
AN ARCHAEOLOGICAL AND HISTORICAL SEARCH \\ FOR GERMAN ETHNICITY AT THE \\ JANIS-ZIEGLER SITE (23SG272)
}

Lisa M. Dretske

354 Pages

December 2013

Ste. Genevieve, Missouri is a small town south of St. Louis, settled by the French and home to the Janis-Ziegler site (23SG272). However, the German Ziegler family along with other German families resided in the town beginning in the early nineteenth century. The Ziegler family used the Janis-Ziegler site as a home and a tobacco shop. Beginning in 2006, archaeological investigations went underway on the Janis-Ziegler site. The purpose of this research is to figure out to what degree the Ziegler family showed their ethnicity while living at the Janis-Ziegler house. The second purpose of the research is to figure out whether the German ethnicity of the Ziegler family be uncovered through the material culture.

In this research, I reviewed the English and German newspapers from nineteenth-century Ste. Genevieve, to reveal any ethnic differences between the advertisements. I then compared the findings of the advertisements to the 
archaeological and Ziegler probate inventory data. By doing this, the Ziegler family ethnic preferences became more apparent. I also included research on the landscape at the Janis-Ziegler house and inheritance practices of the Ziegler family, since these areas may also reveal the Ziegler ethnic identity. 
AN ARCHAEOLOGICAL AND HISTORICAL SEARCH

FOR GERMAN ETHNICITY AT THE

JANIS-ZIEGLER SITE (23SG272)

\author{
LISA M. DRETSKE
}

A Thesis Submitted in Partial Fulfillment of the Requirements for the Degree of

MASTER OF ARTS

Department of Sociology and Anthropology

ILLINOIS STATE UNIVERSITY

2013 


\begin{abstract}
All rights reserved
INFORMATION TO ALL USERS

The quality of this reproduction is dependent upon the quality of the copy submitted.

In the unlikely event that the author did not send a complete manuscript and there are missing pages, these will be noted. Also, if material had to be removed, a note will indicate the deletion.
\end{abstract}

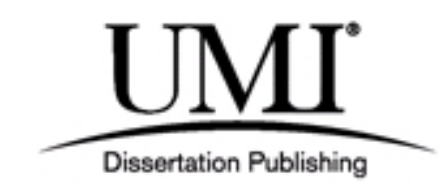

UMI 1552459

Published by ProQuest LLC (2014). Copyright in the Dissertation held by the Author.

Microform Edition (c) ProQuest LLC.

All rights reserved. This work is protected against unauthorized copying under Title 17, United States Code

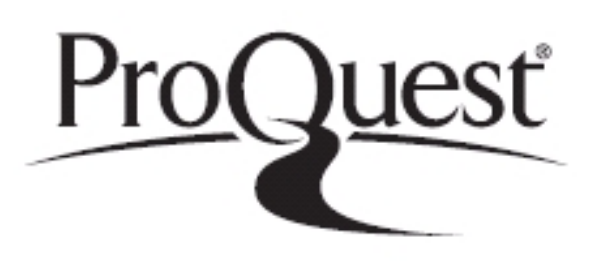

ProQuest LLC.

789 East Eisenhower Parkway

P.O. Box 1346

Ann Arbor, MI $48106-1346$ 
AN ARCHAEOLOGICAL AND HISTORICAL SEARCH

FOR GERMAN ETHNICITY AT THE

JANIS-ZIEGLER SITE (23SG272)

LISA M. DRETSKE

COMMITTEE MEMBERS:

Elizabeth Scott, Chair

Kathryn Sampeck 


\section{ACKNOWLEDGMENTS}

The author wishes to thank Dr. Elizabeth Scott and Dr. Kathryn Sampeck for their guidance throughout the research and writing process. Additionally, I wish to give a special thanks to Elizabeth Scott for her dedicated support and time she put into helping with this thesis. I also wish to thank my family and friends for their love and support throughout my graduate career. Thank you for pushing me to continue my research each and every day. Finally, I take full responsibility for any errors or omissions, both logic and fact, in this body of work. 


\section{CONTENTS}

Page

ACKNOWLEDGMENTS

CONTENTS

TABLES $\quad$ iv

FIGURES Vi vi

CHAPTER

I. INTRODUCTION 1

Research Questions 2

History of Ste. Genevieve $\quad 5$

German Immigration to America $\quad 6$

Janis-Ziegler House/Green Tree Tavern 14

Historical Archaeology of German-American Sites 16

Moravians in Wachovia 16

The Gibbs Farmstead $\quad 17$

Germans in the Southern Colonies 18

The Swiss-Appenzellers 18

CRM Work on German Sites:

Site 11MO779, Site 11M0792, Site 11MO770N 20

Other Sites in Ste. Genevieve 21

II. THEORETICAL FRAMEWORK 23

Archaeology of Identity $\quad 23$

Consumer Behavior $\quad 27$

Consumer Behavior and Newspapers 29

Landscape Archaeology $\quad 30$ 
III. METHODOLOGY 32

Newspaper Analysis 33

Additional Archival Analysis $\quad 36$

Artifact Analysis $\quad 37$

Landscape Analysis $\quad 42$

Summary $\quad 42$

IV. ANALYSIS OF DATA 44

Newspaper, Inventory, and Archaeological Analysis 44

Household Maintenance-Ceramics 44

Household Maintenance-Glass $\quad 52$

Household Maintenance-Other $\quad 54$

Architecture $\quad 56$

Household Furnishings $\quad 60$

Clothing 64

Personal Items $\quad 70$

Household Production Activity $\quad 74$

Household Production for Sale $\quad 83$

Pharmaceuticals $\quad 84$

Foodstuffs $\quad 90$

Alcohol 95

Inheritance Practices $\quad 99$

Landscape Analysis $\quad 105$

$\begin{array}{ll}\text { Summary } & 109\end{array}$

$\begin{array}{ll}\text { V. CONCLUSIONS } & 113\end{array}$

$\begin{array}{ll}\text { Potential for Further Research } & 116\end{array}$

$\begin{array}{ll}\text { REFERENCES } & 118\end{array}$

$\begin{array}{ll}\text { APPENDIX A: Newspaper Databases } & 127\end{array}$

APPENDIX B: Archaeological Databases $\quad 235$

APPENDIX C: Inventory Analysis 337 


\section{TABLES}

Table

Page

4.1. English Newspaper Household Maintenance-Ceramics Ware Types

4.2. German Newspapers Household Maintenance-Ceramics Ware Types

4.3. Ceramic MNV of Unit 150R0, Ziegler Occupation (1833-1900) and Ziegler and Late Occupation (1850-Present)

4.4. Ceramic MNV of Unit 110R30, Levels 1-6: Ziegler Occupation (1833-1900) and Ziegler and Late Occupation (1850-Present)

4.5. Vessel forms from Unit 110R30, Levels 1-6: Ziegler Occupation (1833-1900) and Ziegler and Late Occupation (1850-Present) and Unit 150R0, Levels 1-9: Ziegler Occupation (1833-1900) and Ziegler and Late Occupation (1850-Present)

4.6. English Newspaper Household Maintenance-Other 54

4.7. German-English Newspaper Household Maintenance-Other 55

4.8. English Newspapers Architecture 57

4.9. German Newspapers Architecture 58

4.10. English Newspapers Furnishings 60

4.11. German Newspapers Household Furnishings 61

4.12. English Newspapers Clothing Types 65

4.13. German Newspapers Clothing Types 66 
4.14. English Newspapers Personal Items 70

4.15. German Newspapers Personal Items 71

4.16. English Newspapers Household Production Activity 75

4.17. German Newspapers Household Production Activity 77

4.18. German-English Newspaper Pharmaceuticals 88

4.19. English Newspapers Foodstuffs 91

4.20. German Newspapers Foodstuffs 93

4.21. English Newspapers Alcohol 96

4.22. German Newspaper Alcohol 97 


\section{FIGURES}

Figure Page

1.1. Janis-Ziegler House and Surrounding Property in the 1880s (SMSM1880)

3.1. Map of the Janis-Ziegler/Green Tree Tavern Site (23SG272) Courtesy of Meredith Hawkins

4.1. Advertisement for Dish Forms in Herold December $21^{\text {st }}, 1895$

4.2. F. \& L.U. Store Advertisement for Glassware in Ste. Genevieve Herald, December $17^{\text {th }}, 1892$.

4.3. Oefen Advertisement Herold Dezember 17, 1892

4.4. Mrs. F. Leavenworth Milliner! from Fair Play 23, 1875

4.5. Hamm \& Co. Advertisement from Freie Presse July 22, 1876

4.6. Rudolph Gun Co. from Fair Play December 23, 1875

4.7. Dealsche Pfeife Advertisement from Herold Dezember 17, 1892

4.8. California Gold! Advertisement from Ste. Genevieve Democrat June 8,1850

4.9. Agricultural Warehouse Advertisement from Freie Presse July 22, 1876

4.10. Dr. Whittier Advertisement from Freie Presse July 22, 1876

86

4.11. Ayer's Cherry Pectoral Advertisement from Herald December 17,1892

4.12. Ste. Genevieve Bakery Advertisement from Herald December 17,1892 
4.13. Neuer Spezereiladen Advertisement from Freie Presse July 22,1876

4.14. Val Rottler's Beer Advertisement from Ste. Genevieve Herald, December $17^{\text {th }}, 1892$ 


\section{CHAPTER I \\ INTRODUCTION}

The Janis-Ziegler/Green Tree Tavern site (23G272) in Ste. Genevieve, Missouri, has been the focus of several archaeological theses since excavations began there in 2006. Meredith Hawkins (2007) excavated at the site, using the artifact assemblages to identify how the Janis and Ziegler families each used the property. She also included an analysis of both families' economic standing and ethnic association according to the archaeological record. Matt Cox (2009) looked at the consumption patterns of the Ziegler family. Based on past suggestions by Hawkins (2007), Cox (2009) analyzed the historical documentation and archaeological assemblage to identify the Ziegler's level of wealth as well as reveal whether or not they were "living beyond their means."

Like Hawkins's (2007) and Cox's (2009) theses, my thesis will also examine the material culture recovered from excavations at the JanisZiegler/Green Tree Tavern site (23G272). Along with the archaeological materials, I will also examine the historical documentation from various archives in order to identify how the Ziegler family may have expressed their German ethnic identity. Meredith Hawkins (2007) suggests that the material culture related to the Ziegler occupation period revealed that the family acculturated. In 
saying this, she suggests that the German Ziegler household implemented ideas and materials from another ethnic group into their own. This research will take a more in-depth look at ways to identify German ethnicity from the material culture and historical documents related to the Zieglers.

This study will investigate Germans living in America beginning in the 1820s, a topic rarely investigated in historical and archaeological writings. Historians have dealt mainly with German immigrants living in America in the 1850's and later. Few archaeologists have focused on German sites in the New World (South 1999; Idol and Trage 1995, Idol, Trage, and Kirchen 1996; Groover 2003), even fewer on Germans in French-dominated settlements (Wesler1999). Finally, this study will contribute to a more comprehensive history of Ste. Genevieve by shedding light on its German immigrant community.

\section{Research Questions}

There are two questions I propose to research. First, I wish to address to what degree did the German Ziegler family express their ethnicity while living at the Janis-Ziegler house? I will look at the historical and archaeological data: newspapers, probate records, wills, and artifacts. I hope to analyze how this changed between the ownership of Mathias and Barbara Ziegler (1833-1860) and Francis and Josephine Ziegler (1860-1900). Penner (1997) notes that ethnicity is not static, but constantly changing or adapting to the environment. He also suggests that in order to look at ethnicity in the archaeological record, archaeologists should look at the boundaries each group creates (Penner 1997: 
267). An ethnic group shows the important aspects that bind them together by expressing cultural symbols to produce boundaries between themselves and others (Penner 1997: 268). By investigating these boundaries, archaeologists can identify the cultural aspects most important to each ethnic group.

Along with these questions, I will also keep in mind the possibility that the Zieglers may have chosen not to identify themselves as German, at least in a public context. They may have preferred or found benefit in "fitting in" with the French in Ste. Genevieve. The Zieglers did not move into German communities in Missouri, such as the town of Hermann. Instead they moved into a predominately French town. Mathias Ziegler was a business partner with Felix Valle, and for the sake of the business, the Zieglers may have chosen not to express their German ethnicity. There is also a possibility that the Zieglers only emphasized their German ethnicity in private. In his study of the Seguí household in colonial Florida, James Cusick (2000) found that the material culture of this Minorcan family resembled that of the Spanish gentry (Cusick 2000:52). Through the probate records and archaeological data, Cusick (2000) looked at the architecture of the Seguí house. The front façade had "Georgian symmetry with Spanish features," which was in the local fashion of combining Minorcan, Spanish, and English traditions. The back of the house, where the family spent more of their time, was in the local Spanish tradition, which corresponds to the other material culture analyzed from the Seguí households (Cusick 2000:52). Similarly, the Ziegler family could have emphasized their ethnicity in private. 
In the second generation, that of Francis Ziegler, there is also the possibility of finding evidence of a household of mixed ethnicity. He married a French woman, Josephine Moreau, in 1856. This marriage likely involved the combining of French and German traditions, resulting in a mixed ethnic identity for the household that may appear in the archaeological record. I will also consider that perhaps the Zieglers put class distinction ahead of ethnicity, stressing their economic position more than their ethnic traditions.

The second question I wish to address is, is it possible to identify the German ethnicity of the Ziegler family from the material culture of the JanisZiegler site? In Hawkins' thesis (2007), she looked at the economic standings and ethnic markers from the archaeological remains. She wrote that her efforts to look at the German ethnicity of the Ziegler occupation were inconclusive, based on her focus on "markers" as proposed by Elliott and Elliott (2002). Hawkins (2007) felt that the results were inconclusive for two possible reasons. First, the Ziegler family seemed to have acculturated, taking on the culture of their French neighbors. Second, the occupational sequences could not be differentiated at the time between the Mathias and Barbara ownership (1833-1860) and the Francis and Josephine ownership (1860-1900). Because Hawkins (2007) based much of her analysis of German ethnicity on the article by Elliott and Elliott (2002), I wish to reexamine German ethnicity at the Janis-Ziegler site using several methods. Rita Folse Elliott and Daniel T. Elliott (2002) compress the eighteenth-century German colonial experience in the British colonies into the single explanation of acculturation without acknowledging other possibilities of 
interaction such as resistance and ethnogenesis. However, every German group immigrating to America may not have taken this approach. Also, Elliott and Elliott (2002) do not discuss any Germans moving into non-German settlements. For this reason, the Ziegler occupation of the Janis-Ziegler house deserves a closer examination of the archaeological assemblage and historical documentation looking for ways in which they may have shown their ethnicity.

\section{History of Ste. Genevieve}

Ste. Genevieve, Missouri is located just over 60 miles south of St. Louis, Missouri. The town was considered to be a part of the region called the Illinois Country (Ekberg 1996:4). In the eighteenth century, French Jesuit missionaries were traveling along the Mississippi River, setting up outposts. At first the French control along the river was sparse (Ekberg 1996:4), but by 1750, the area in and around Ste. Genevieve along the Mississippi was occupied by the French (Ekberg 1985:25). However, in 1763, because of the Treaty of Paris, the land west of the Mississippi was granted to the Spanish, while the English gained the territory to the east of the river (Ekberg 1985: 41). Though the Spanish gained possession of Ste. Genevieve, the French remained prominent in the region (Ekberg 1985:55). In 1803, the area transferred to US rule with the Louisiana Purchase.

The area around Ste. Genevieve was originally settled because of the siltenriched soils, excellent for agriculture. The lands east of the river, such as the towns of Kaskaskia and Prairie du Rocher, were losing their nutrients and were 
becoming scarce (Ekberg 1996:21). Along with agriculture, several other industries emerged, such as lead mining and salt extraction (Ekberg 1985:1011). Though there has been much disagreement on the exact year Ste. Genevieve was founded, the most probable time was in the 1750s (Ekberg 1996:12). Flooding was a major problem for the inhabitants of Ste. Genevieve throughout the eighteenth century. 1785 was "the year of the big waters," forcing the town members out of their homes. This flood proved to be the final straw, and the townspeople moved to new settlements on higher ground, establishing New Ste. Genevieve by 1790 (Ekberg 1985:421).

\section{German Immigration to America}

As mentioned previously, people began migrating Ste. Genevieve primarily for the rich agricultural lands. Germans were also starting to move into the region but for reasons of hardship in their homeland. During the early nineteenth century, the German kingdoms as a whole were experiencing both economic and political woes. Smaller landowners and farmers were pushed out by the larger estate owners. Also, the marketplaces were filled with products produced from outside each region, causing economic decline of village industries. Lastly, the kingdoms were dealing with threats of revolution and upheaval against the monarchy (Wilhelm 1988:211). In general, the German kingdoms were swarmed with individuals looking for a better life elsewhere, away from political and economic frustrations. 
Besides looking at the nation of Germany, it is also important to look at the historical situation on a smaller scale. The Ziegler family initially came from the region of Bavaria in Germany, and I will look at the history of this region, as well as explain why they and so many others chose to emigrate. What we now know as Germany was divided into many smaller kingdoms with their own rules. Once the Holy Roman Empire lost control over the German kingdoms in 1806, The Confederation of the Rhine formed a protective union of sixteen kingdoms, including Bavaria, under French protection (Werner 1977:11). Bavaria aligned strongly with the French in order to regain some of their lost lands. When Bavaria began to depend too much on the French alliance, they became fearful of falling with the decline in Napoleon's power. On April 25, 1813, they declared neutrality (Werner 1977:13).

In Vienna, in 1815, 38 German kingdoms joined to form a new confederation (Werner 1977:14). In essence, this confederation was a union against the fear of revolution (Werner 1977:20). In 1818, Bavaria added a third Edict to their constitution allowing for more freedom of the press (Werner 1977:16). This continued to be an issue within the confederation into the 1830 s, since the other German kingdoms had more conservative constitutions. Bavaria tried to form a strong union with the other southern German states in case Austria were to get involved in a war; however, Bavaria's continued interest in regaining lands from Baden and Württemberg made this union impossible (Werner 1977:48). 
In 1819, Bavaria and the other German kingdoms were dealing with economic instability. British goods were taking over the market, depleting the German competition (Werner 1977:50). This joint problem for all the German kingdoms opened the door for further discussion of a southern German state union. However, Bavaria's desire for remaining independent and its refusal to dismiss land claims against Baden ruined their efforts for a customs union of the southern German kingdoms (Werner 1977:54). At this point in the 1820s, the Ziegler family is believed to have left Bavaria and moved, eventually, to Ste. Genevieve. Perhaps one of the reasons for their relocation was due to the economic hardships in Bavaria at this time.

Another reason so many Bavarians emigrated was because of the political instability, in addition to the economic problems. When the revolutions broke out in France as well as Poland and Belgium in the 1830s, the German kingdoms began to fear revolutions in their own lands (Werner 1977:87). Though Ludwig I of Bavaria did not fear French insurgency on Bavarian lands as much as other German kingdoms, he did fear revolts of French-sympathetic regions of Bavaria, such as the Rhenish provinces (Werner 1977:89). In 1833, a group of students and other academic affiliates conspired to attack the Federal Assembly in Frankfurt. They hoped to set off a revolution throughout all the German kingdoms. Unfortunately for them, Frankfurt soldiers stopped their revolt, thus ending their plan for a grand revolution (Werner 1977:134-5). This attempt at a revolt set off alarms from not only Bavarian leaders, but other German kingdoms, the confederation, and other countries as well (Werner 1977:151). This was a 
moment of great uncertainty for the German kingdoms, convincing many German people in Bavaria to immigrate to the United States.

The term "German" can be confusing when looking at German-Americans. Because "Germans" emigrated to America before Germany became one unified country in 1871 , it can be difficult to define who these people really were. Should they be defined by the kingdom from which they originated? Or as "Germans"? For the purpose of this paper, most of the research concerns the region of Bavaria, from which the Zieglers originated. However, some other German generalities are made because even though German speaking people came from different regions and spoke different dialects, they did appear to continue to share their language and similar customs despite their differences (Fogleman 1996:86). For this reason, in this paper, the general term "German" is used pertaining to German speaking people.

Germans moved to Jamestown as early as 1611 (Davis-DuBois and Schweppe 1936:27). The numbers of Germans moving into America increased more by the mid-seventeenth century, many into Dutch and English territories. Many of the first Germans were of a religious affiliation, moving to America to settle in communities, such as the Mennonites and the Quakers. By the eighteenth century a "continuous stream" of Germans were emigrating to America (Davis-DuBois and Schweppe 1936:33). By 1750, sections of New York and Pennsylvania held communities of German immigrants, including Germantown of Pennsylvania. From the east coast, the immigrants moved through the Valley of Virginia and then further west (Wittke 1952:6). Many of the 
original German immigrants turned to farming, much like the Germans in the region of Ste. Genevieve (Wittke 1952:7). During the nineteenth century, "socioeconomical" reasons played a significant role in German families' decision to emigrate as well (Moltmann 1985:15).

The early emigrating Germans did not always find an easy trip to the New World. One popular method of paying for transportation was the Redemption System. A wealthier man would pay for the passage fees for the German emigrant, in exchange for a year or more of services or labor (Moltmann 1985:16). This system sometimes had negative results. Many were tricked or promised inexpensive travel by ship, only to find themselves stuck at sea with no money, starving, sick, and even sold into captivity (Davis-DuBois and Schweppe 1936:35). Even some better-off Germans had their luggage and belongings stolen at sea, forcing them into serfdom upon arrival in America (Faust 1927:69). However, by the 1820 s the Redemption system fell out of use. New shipping laws and guidelines provided safer passages for emigrants, but also took away the previous inexpensive method for German emigrants to move to America (Moltmann 1985:16).

Because of the new transportation guidelines, the type of German emigrants changed as well. Poor emigrants could now no longer afford to move to America, unless paid for by their families or the state (Moltmann 1985:17-18). Mostly it was Germans who could afford the payment for transport on their own or sold their property for the payment, who immigrated to America (Moltmann 1985:18). 
By the early eighteenth century, Germans, especially Catholic Germans, began moving into French Louisiana. Many of them came after reading the writings encouraging Germans to move into the area for the rich lands available (Davis-DuBois and Schweppe 1936:37). People labeled Neuländer (Newlanders) commonly distributed pamphlets and convinced German people to move to America. However, not every person was honest about the immigration experience and embellished their experiences in America to receive money from ship owners (Faust 1927: 61). Some governments even outlawed the Neuländer from working in their kingdoms (Faust 1927:62). Germans, compared to other ethnicities, were encouraged to relocate to lands to the west because of their great farming abilities and accomplishments observed in Pennsylvania and other areas of the New World.

In $1817,60,000$ people resided in the territory that would become Missouri. By 1821, when Missouri became a state, still no Germans were documented to have settled in the new state. But, in 1824, Gottfried Duden traveled to Missouri to start a home (Faust 1927:440). Beginning in 1824, while living on his farm in Warren County, Missouri (located just under 200 miles northwest of Ste.Genevieve), he wrote a novel describing his journey to Missouri and a romanticized description of the available land. His novel was titled, Berichte über eine Reise nach den westlichen Staaten Nordamerikas und einen mehrjährigen Aufenthalt am Missouri (Report on a Journey to the Western States of North America and a stay of Several Years Along the Missouri) (Faust 1927:441). Duden's book promoted the immigration of other educated Germans 
to the western territories in America, especially Missouri. He hoped that with the immigration of more of his fellow Germans that they could make a new "Germania" in Missouri, saying that:

If a small city were founded with the intention of serving the American Germans as a center of culture, one would soon see a rejuvenated Germania arise and the European Germans would then have a second country here, such as the British have (Duden 1980:179).

Though this was a highly "romanticized" book about Missouri, it enticed many different professions and types of Germans to the Midwest of America (DavisDuBois and Schweppe 1936:55). Gottfried Duden (1980:127) also encouraged Germans to move into Missouri because of the abundance of salt and other types of fuel besides wood, like coal.

Other sources promoted the emigration to the New World as well. Magazines or journals such as Allgemeine Auswanderungszeitung of Rudolfstadt and Der Deutsche Auswanderer of Frankfurt advertised the trip to America and where new emigrants could purchase land (Wyman 1984:59). Personal letters from friends and family who had already moved to America also enticed Germans to emigrate. In the early nineteeth century, many German leaders would confiscate any letters that they might come across that were convincing Germans to move to America (Wyman 1984:59).

Beginning in 1825, a wave of German immigrants moved into Missouri. According to the Missouri Republican, by 1837, 30,000 Germans had moved into Missouri, with 6,000 living in St. Louis (Rowan and Primm 1983:4). From 1830 to 1848, a wave of educated Germans immigrated to Missouri. They were coined 
the "Latin settlement" because they grew up learning Latin and Greek. The group consisted of "barons and counts, engineers, preachers, artists, and other representatives of the cultivated German classes" (van Ravenswaay 1977:31). Once these new immigrants moved to Missouri and other western states, they were forced to learn how to farm and other forms of labor (Faust 1927:442). By 1850, the German population in America was at 573,225 and quickly rose to 1,302,136 in 1860 (Wyman 1984:50). In Ste. Genevieve were settled predominately Catholic Germans from the region of Baden, in southwestern Germany (Kamphoefner 1988:186). By the middle of the nineteenth century, it is believed that more Germans lived in the area of Ste. Genevieve than French, because they were attracted to its exceptional agricultural potential (Franzwa 1998:103).

The Germans did not arrive into certain regions with open arms. They were put down and expected to assimilate to the culture and ways of living of the dominant group. Some German people, like Friedrich Kapp who immigrated in 1848, supported the full assimilation into American culture, and believed the isolated groups of German culture would eventually fail (Wittke 1952:179). However, many Germans were determined to preserve their cultural ways including their language, foodways, language, religion, and many other trait to "overcome any feeling of inferiority" (Wittke 1952:59). Karl Heinzen was said to have commented at a public lecture in Boston in 1866 that "one might hate Germany and leave it, [as he and other Forty-eighters had done], but one could 
never deny one's nationality, because it was deeply rooted in a man's blood, his education, and the history and traditions of his people" (Wittke 1952:180).

\section{Janis-Ziegler House/Green Tree Tavern}

Nicolas Janis, a wealthy older French gentleman, moved his family to New Ste. Genevieve in 1790 (Cox 2009:6). He quickly built what we know today as the Janis-Ziegler House in a French vernacular architectural style. François was given the property in 1796, and his father, Nicolas, died several years later in 1804 (Cox 2009:9; Hawkins 2007:32). The Janis-Ziegler house was the meeting place for the first Freemasons in Missouri; beginning in 1807, meetings were held in the Green Tree Tavern that operated in one end of the building (Cox 2009:9). In 1833, after the death of François, his heirs sold the house to Mathias Ziegler (Cox 2009:11).

Mathias and Barbara Ziegler originally came from the village of Weingarten located near Stadt Brutzel located on the Main River in the region of Bavaria (Petition by Mathias and Barbara Ziegler 1827). They moved to Ste. Genevieve in the 1820s (Hawkins 2007: 34; Federal Census 1830, 1860). As mentioned previously, the 1820 s were a time of economic and political instability in Bavaria and the rest of Germany. By 1827, Mathias had entered into business with Felix Valle, a prominent French merchant in town. Mathias Ziegler and his family moved into the Janis-Ziegler 


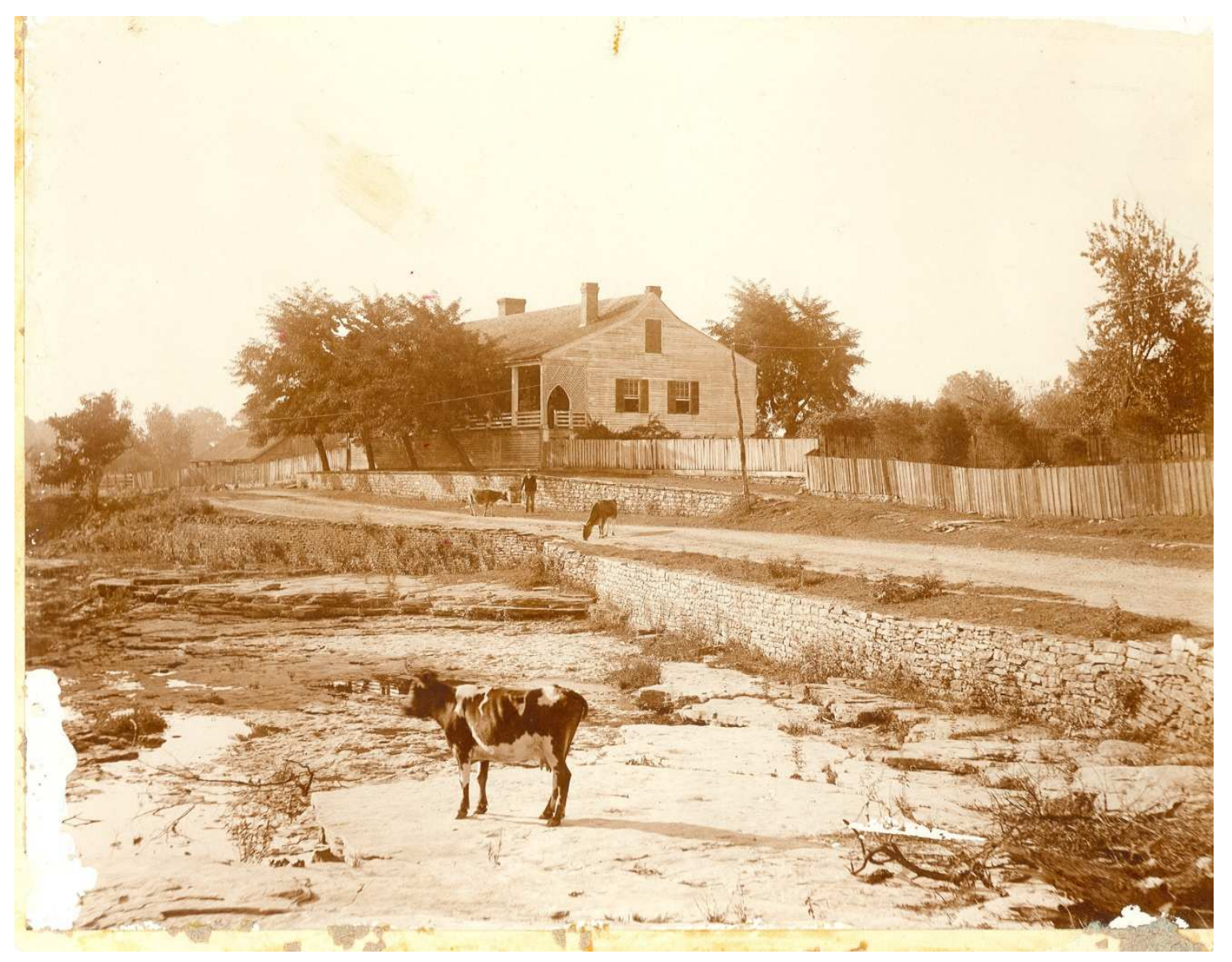

Figure 1.1. Janis-Ziegler House and Surrounding Property in the 1880s (SHSM 1880)

house in 1833, using it for a residence as well as the location of their wholesale tobacco business (Cox 2009:12). When Mathias died in 1835 at the age of 39, his wife, Barbara, took over the family business and the estate, and with their children operated the tobacco business until the early 1850s (Hawkins 2007; Cox 2009; Federal Census 1850). At his death, Mathias left six children-John Alexander, Francis J., Louis, Mary A., and Charles (Federal Census 1850). In 1860, Francis Ziegler purchased the house from his mother, Barbara. Francis 
had married Josephine Moreau, a daughter from a nearby French family, in 1856. Together they had four daughters and one son. While in their possession, Francis and Josephine remodeled the house in Victorian fashion (Scott 2009: personal communication). In 1883, Josephine Moreau Ziegler died; Francis J. Ziegler died seven years later, in 1900. The Ziegler home was willed to his four daughters and stayed in the Ziegler family until 1939, when Josephine and Helen Ziegler, grand-daughters of Francis, sold the property to the Moranville family (Cox 2009:14). After this, the building passed through several owners unrelated to the Ziegler family.

The Janis-Ziegler/Green Tree Tavern site (23G272) has gone through many changes throughout the centuries. The generational and family changes at this site may reveal some answers to the questions I have proposed concerning the extent that the Ziegler family showed their German ethnicity throughout the nineteenth century.

\section{Historical Archaeology of German-American Sites}

\section{Moravians in Wachovia}

One German-speaking group, for which there is some archaeological evidence, is the Moravians who settled in North Carolina in 1753. The Moravians first moved to America in 1736 as a small settlement in Georgia. Because of the pressures from their neighbors and military (they refused to fight in the war with the Spanish), by 1740 they had moved to Pennsylvania. From Pennsylvania, a small group of eleven Moravians moved to North Carolina, while the remainder 
continued to live in Pennsylvania. They first settled in the town of Bethabara. From there, they established Bethania and then Salem, the appointed central town in the region of Wachovia (Murtagh 1967: 6-7). Stanley South was the first to excavate the Moravian settlement in Wachovia, beginning in 1963 (South 1999). His primary focus during his first years of excavating was uncovering the pottery shop of master potter Gottfried Aust from 1756.

The Moravians were known for their isolation from other groups, and therefore, writings about the Moravians by other groups are not common. However, the Moravians kept their own records, which Dr. Adelaide Fries translated into eight volumes of the Records of the Moravians in North Carolina (1922). Because of the Moravian leaders' excessive control over their townspeople, their isolation has been a major point of research throughout the years. However, as historically documented, over time they began to interact more with the surrounding communities, their German identity less visible within archaeological data (Thomas 1994).

\section{The Gibbs Farmstead}

The Gibbs Farmstead was a German-American farmstead in Knox County, Tennessee, with a considerable amount of documentary and archaeological material available. The Nicholas Gibbs site was first excavated during the spring and summer of 1987 by Charles Faulkner (1988) and again in 1988. Mark D. Groover uses both the historical resources and archaeological material, in An Archaeological Study of Rural Capitalism and Material Life: The 
Gibbs Farmstead in Southern Appalachia, 1790-1920 (2003). He develops a detailed family chronology with the help of historical records and oral history, in order to better understand what was happening on the Gibbs farmstead during the nineteenth century on an economic level. He also touches on world systems theory and how it relates to his archaeological study of the Gibbs farmstead.

\section{Germans in the Southern Colonies}

Rita Folse Elliott and Daniel T. Elliott (2002) focus on eighteenth-century German immigrants in the southern colonies. The article focuses on both the history and archaeological materials of German ethnic groups in the southern colonies, to understand how they contributed to southern culture (Elliott and Elliott 2002:80). Germans are not typically researched in colonial archaeology or history, making this article one of the few. Identifying German ethnic traits proves to be difficult since the Germans moved into regions economically and politically controlled by other colonizing groups, such as the English (Elliott and Elliott 2002:88). Elliott and Elliott (2002) identify these ethnic traits when looking at the architecture, ceramics, and medicines found in these German settlements in the southern colonies. The authors state that through these three areas of material culture, German ethnicity may be identified archaeologically.

\section{The Swiss-Appenzellers}

Bruce Penner (1997) focuses on another German speaking group, the Swiss-Appenzellers. He follows the theories proposed by Eriksen (1992), 
explaining that when looking for ethnicity, archaeologists should not look at isolated groups, but look at the "boundaries" and interaction between the groups. Eriksen (1992:30) states that "the fundamental aspect of ethnicity is the very act of communicating and maintaining cultural differences." Eriksen identifies the importance of looking at "ethnic signs" at the point of interaction (1992:32). Bruce Penner (1997:266) agrees with looking at the boundaries in ethnicity research, and states that "Ethnic social identities are most visible in response to change, and so it is that during change ethnicity will be most visible in the archaeological record." In order to look at these boundaries and the ways the Swiss-Appenzellers changed once they immigrated to the southern colonies, he first describes the pre-immigration lifeways. Next he compares these past lifeways - foodways, architecture, livelihood, spatial organization, etc. - to the archaeological data found from their settlements in the southern colonies of America. Penner (1997) did this to better understand what aspects of SwissAppenzeller ethnicity they continued to incorporate and which aspects they chose to change.

Penner (1997) also discusses another practice for continuing ethnic identity in the New World, inheritance practices. He explains the difference between partible inheritance and impartible inheritance. Partible inheritance is the more equal form of inheritance for the heirs. In this practice, the female and male heirs may split the movable assets while male heirs split the land. In some cases, every child, regardless of gender, may receive equal assets. Impartible inheritance is when only one heir, usually the eldest son, receives all of the 
assets (Penner 1997: 300). Penner (1997) examines the pre-immigration inheritance practices and then compares them to those in the new settlements over time. In this way, Penner (1997) illustrates how inheritance practices can be considered when researching ethnicity in the colonial period.

CRM work on German Sites: Site 11MO779, Site 11MO792, Site 11MO77ON

Between 1990 and 1994, Thomas E. Emerson led an investigation through the Illinois Transportation Archaeological Research Program, conducting Phase I and Phase II archaeological investigations of a route between Columbia and Waterloo, Illinois, on the eastern side of the Mississippi, northeast of Ste. Genevieve (Gums 2009). Three main sites were investigated, 11MO779, $11 \mathrm{MO} 770 \mathrm{~N}$, and $11 \mathrm{MO} 792$. Two of these sites were inhabited by people of German ancestry during the mid- to late-nineteenth century (11MO779, 11M0792). The other site was inhabited by an American born family of German decent (11MO770N).

After Phase I and Phase II investigations, the research showed that these sites were farmsteads from the early to late nineteenth century (Gums 2009:1). German families began moving into this region in the 1830 s, as they did on the other side of the river in Ste. Genevieve (Gums 2009:6). Site $11 \mathrm{MO} 779$ is located two miles southeast of Columbia, Illinois (Gums 2009:19). This site was inhabited by Franz and Mary Boehme, a couple from Hanover, who bought the 
property in 1851 (Gums 2009:19). On the farmstead the Boehme family operated a grocery and later a tavern until 1871 (Gums 2009:21).

Several families lived on site $11 \mathrm{MO} 792$ throughout the nineteenth century. One family, the Gaertner family, lived on the farmstead from 1854 to 1873 . Four of the household members were recorded to have been born in Germany, while the children of the house were born in America (Gums 2009:121). These sites are another example of German families immigrating to the Midwest during the 1830s.

Other German Sites in Ste. Genevieve

The Delassus-Kern house was investigated in 1997 by the Middle Mississippi Survey project, conducted by Murray State University's Wickliffe Mounds Research Center and Southeast Missouri State University (Wesler 1999:67). The primary research question was who was the builder and original inhibitors of the Delassus-Kern house? The house was built in the French style originally affiliated with the earlier resident, Pierre Delassus DeLuziere in the late eighteenth century. However, the 1997 Middle Mississippi Survey project concluded that the house was most likely constructed in the 1830 s by Martin Sweek (Wesler 1999:86). The Delassus family passed down the residence through several generations, then sold the property to various other people not related to the family in the 1830 s. In 1855 , the German immigrant Kern family purchased the property, including the house and a working plantation, for $\$ 1000$ (Wesler 1999:70). The Kern family lived at this residence for over one hundred 
years. Throughout the Kern ownership, they made many improvements to the French style house, including a second story and an orchard next to the house (Wesler 1999:70).

After reviewing Hawkins (2006) and Cox's (2009) theses, I developed several research questions. First, to what degree did the Ziegler family express their ethnicity while living at the Janis-Ziegler house? Second, is it possible to reveal the Ziegler's German ethnicity from the material culture from the site? The Zieglers arrived in Ste. Genevieve in the early nineteenth century, just as many other Germans were immigrating to America. However, the Ziegler family chose to live in a French dominated town, unlike most other Germans who moved into areas amongst other German immigrants. Therefore, through these questions, my research may uncover the ethnic identity of the Ziegler family throughout the generations. 


\section{CHAPTER II}

\section{THEORETICAL FRAMEWORK}

In my research, I hoped to answer two questions: did the Ziegler family show their ethnicity in the archaeological and historical record, and to what degree and method did they show their ethnicity? In order to answer these two questions, three theoretical approaches were used: the archaeology of identity, consumer behavior, and landscape archaeology. This research looks at nineteenth-century newspapers, the archaeological record from the Janis-Ziegler house/Green Tree Tavern (23SG272), and the wills and probate inventories from the Ziegler family in order to identify whether or not the Zieglers showed any distinct ethnic identity while occupying the Janis-Ziegler site. These materials were analyzed using the theories of identity archaeology, consumer behavior and also the growing theory of landscape archaeology.

\section{Archaeology of Identity}

My research concerns the archaeology of identity, with a primary focus on ethnic identity. The archaeology of identity explores different social identities and the "relationships" between different groups (Meskell 2001:188). In archaeology it is important to look at different groups of people because, "archaeologists can illuminate the lives of anonymous social groups within increasingly complex, 
modern societies" (Orser 2004:239). Ethnicity is one of these groups. The word ethnicity has had many explanations throughout the years and amongst scholars. In historical archaeology an ethnic group can be best explained as "an assemblage of people who share enough physical and cultural characteristics to define themselves as 'us,' and to define everyone else as 'them'" (Orser 2004:251). Identifying different ethnic groups in the material culture has proven difficult in the past. For this reason, many historical archaeologists have focused on finding "ethnic markers," in order to identify an ethnic group in the material culture. Charles Orser, Jr. (2004:252) describes 'ethnic markers' as “individual artifacts or groups of artifacts that can undeniably indicate the presence of certain ethnic groups at archaeological sites." However, even ethnic markers are always changing and can be difficult to assign an ethnicity or group.

In historical archaeology of North America, scholars have focused on finding evidence of the ethnic identity of the French (e.g., Mann 2008; Nassaney 2008; Scott 2001a; Steen 2002), and increasingly of Africans (e.g., Babson 1990; Fennell 2000; Ferguson 1992; McDavid 2002). However, evidence of German ethnicity has been largely ignored by historical archaeologists except for a few articles and monographs (e.g., Groover 1994; Elliott and Elliott 2002; Penner 1997; South 1977).

Past studies of ethnicity in America have focused on architecture (Groover 1994, 2003; Elliott and Elliott 2002; Penner 1997; Mann 2008), foodways (Scott 2001b; Groover 1994; Dietler 2007), and ceramics (Elliott and Elliott 2002; Ferguson 1992). Charles van Ravenswaay (1977:13) wrote that the new 
German immigrants probably felt isolated to some extent from the other ethnic groups living around them, allowing them to keep some of their old German customs. For this reason, I hope to identify whether the Ziegler family showed their ethnicity while living at the Janis-Ziegler house/Green Tree Tavern.

The Zieglers purchased a house in the French style from the Janis family, and therefore, architecture may not be useful in analyzing ethnicity in this case. This was a common situation because the French and then the Anglo-Americans moved into the Mississippi Valley first, constructing buildings in their traditions before the Germans moved into the region (van Ravenswaay 1977:19). However, probate records may reveal how the Zieglers used their living space. Currently faunal analysis is being conducted on the materials excavated from the Janis/Ziegler house. Faunal remains may reveal the dietary habits of the Ziegler family, which could then be compared to those from other German households, such as the Gibbs farmstead (Groover 2003), and possibly compared to faunal remains from a contemporaneous French household. At other sites, ceramics have been useful in identifying consumer choice in relation to ethnicity (Elliott and Elliott 2002; Groover 2003; Mann 2008). By the 1830s, Ste. Genevieve's markets were dominated by British goods; therefore, the Zieglers were not purchasing German ceramics. However, the type and style of British ceramics they purchased may still reveal the German consumer choices of the Ziegler family in the nineteenth century, as will be explained further in the methodology. Inheritance practices may be another source for identifying the persistence of German ethnicity among the Ziegler family members. Bruce R. 
Penner (1997) investigated inheritance practices to examine ethnicity and change over time. Penner (1997) used the work by S. Gross (1996) to understand German inheritance practices. Gross (1996:196) investigated two townships in central Minnesota that were settled by German Catholics from various regions in what became Germany, including Bavaria. In his investigation, Gross (1996:211) found that the "values" of the German Catholics transferred from Germany to their new homes in Minnesota. Gross (1996:197) stated that German immigrants from Bavaria maintained impartible inheritance practices, which is the passing down of property to a single heir, usually the eldest son. Gross (1996:194) believed that German-American inheritance practices may be different from those of other ethnic groups. Other ethnic groups may be more "aggressive" economically, focusing on being "entrepreneurs," relying less on keeping farmland within their families and more on social growth in a community. For this reason, inheritance practices may reveal another method the Zieglers showed their ethnicity.

The Ziegler family was not like other German immigrant families in Missouri, and they decided to move into the French dominated town of Ste. Genevieve and not a German settlement. They also conducted business in partnership with a prominent French family in town, the Vallé family. For this reason, the German ethnicity of the Ziegler family in the archaeological and historical record may not be as visible or apparent as it would be for Germans living in the German towns elsewhere in Missouri. However, this search may shed some light on how Germans interacted with non-Germans in French- 
dominated regions, and how this was transcribed in the archaeological and historical records.

\section{Consumer Behavior}

Consumer behavior is best defined as "the behavior that consumers display in searching for, using, evaluating, and disposing of products, services, and ideas which they expect will satisfy their needs" (Schiffman and Kanuk 1987:6). Consumer behavior is an important area of study for historical archaeologists because archaeologists come into contact with consumer goods in their research regularly. Archaeologists can study the consumer by looking at the physical goods the consumer left behind. Though consumer behavior did not start as an anthropological theory, it is related to social behavior (Henry 1991:3). The main aspect of consumer behavior in which archaeologists can play a role is "post-use disposition" (Henry 1991:4). This could include re-use, disposal, or loss

of the item (Henry 1991:5). Historical archaeologists not only look at the archaeological record, but also look at probate records, wills, newspapers, inventory books, and other historical documents to study commodities and what they meant to different people (Orser 2004:103).

The "decision to consume" can be explained by two factors: external influences and internal influences (Henry 1991:4). The external influences are the aspects archaeologists research, especially in the area of the social and cultural importance. Ethnicity is one external influence which may affect the consumer behavior of a person. The internal influence is much more difficult for 
archaeologists to study, since it deals with the psychological aspect of the consumer (Henry 1991:9).

In the last two decades, historical archaeologists have focused on commodities and the consumer revolution (McCracken 1988; Orser 2004; Yentsch and Beaudry 2001; Pogue 2001; Shackel and Little 1992; Pendery 1992; Cook, Yamin, and McCarthy 1996; Henry 1991). Historical archaeologists have started to focus on looking at the meaning behind consumption and not just what was being consumed and disposed (Cook, Yamin, and McCarthy 1996:50). They have also begun to look at how people in the past used consumer goods to show their identity or status (Pogue 2001:51; Pendery 1992:66). Cook, Yamin, and McCarthy (1996:51) best state the use of historical archaeology for the study of consumption:

The class and ethnic differences between occupants of archaeological sites provided the opportunity to explore the material expression of class and ethnicity, a promise that has not yet been fully realized, but which constitutes, at this writing, an ongoing project within the discipline. For this reason, consumerism has been an increasing focus by historical archaeologists throughout the years.

Consumerism was not only what people were purchasing, but knowing how to use the products. Knowing how to properly use an item might separate you in a different social group (Martin 1996:76). For example, the elite would use consumer goods to separate themselves from other classes, making it more complex at mealtimes to show they were part of an elite group (Martin 1996:78). Therefore, consumer goods became "social and behavioral props" which archaeologists can analyze (Martin 1996:79). As stated by Ann Smart Martin (1996:82), "Material objects matter because they are complex symbolic bundles 
of social, cultural, and individual meaning fused into something we can see, touch, and own." These "material objects" become what archaeologists work with today.

However, not every person acts in the same manner, and consumer goods and their meanings are always changing. Consumerism is reflected differently from one region to the other as well as from one individual to another (Pogue 2001:53). In the same way, consumer goods may have different meanings to people throughout time (Shackel and Little 1992:8). This not only makes studying consumer goods more complex, but more complicated when drawing connections from one region or time to another.

\section{Consumer Behavior and Newspapers}

At the Gibbs farmstead in Knox County, Tennessee, Mark Groover (2005) looked at consumerism in Appalachia. He showed that printed advertisements reached their peak, being as prominent as they are in today's society, by the third quarter of the nineteenth century (2005:260). However, printed advertisements were infiltrating U.S. newspapers even earlier than the nineteenth century, and played a prominent role amongst newly immigrating Germans.

Newspapers were an important aspect of German life. At the end of the eighteenth century, after the Revolutionary Era, German newspapers began to be more popular, especially in Maryland and Pennsylvania (Fogleman 1996:149). Many intellectual German immigrants worked in the newspaper as a source of income when they first moved to America (Wittke 1952:262). This being so, the production of German newspapers doubled between the years 1848 to 1852 
(Wittke 1952:262). The height of German press in America was between the years 1850 to 1870 , after which German language newspapers started to go into decline (Wittke 1952:263). As early as 1845, already two German newspapers came out daily in St. Louis (Faust 1927:447-448). Early on, many German newspapers in America were only German translations of English newspapers. Also, many German newspapers focused on political stances (Wittke 1952:264). Many of the more popular German newspapers were eventually taken over by American publishers for a profit, which included English advertisements, but allowed for the continuation of a German newspaper in America (Wittke 1952:270). These advertisements made up the majority of the newspapers in the eighteenth and early nineteenth century (Jones 1993:25). They give insight into what goods the English and German households were purchasing and using during this time. In Ste. Genevieve, two German papers were produced in the nineteenth century, Freie Presse and Herold. These newspapers and the advertisements printed within them may reveal some typical consumer habits of Germans in Ste. Genevieve, and potentially, the Ziegler family.

\section{Landscape Archaeology}

Landscape analysis became a focus amongst archaeologists beginning in the 1950s when Gordon Willey (1953) published his work Prehistoric Settlement Patterns in the Viru Valley, Peru. He believed that social and cultural influences played a part in landscape formation (Shackel 2003:4) By the 1970s, archaeologists were focusing on the functional uses people associated with the 
different areas of their landscapes (Shackel 2003:4). Archaeologists revealed that people used their landscapes for many different purposes throughout history, e.g. cooking, production, and to show wealth or control (Zierden 1996:287). By the 1980s, landscape archaeology took on a whole new purpose: to preserve the landscapes and gardens of "great men" from America's past (Shackel 2003:4). The use of Geographic Information System technology has helped improve this purpose of landscape archaeology, making it easier to map out landscapes (Shackel 2003:6). By uncovering the environment people lived in, archaeologists can reveal aspects about a society in much the same way that other artifacts reveal aspects about a people. Therefore, landscape archaeology has a permanent place in historical archaeology and can contribute to understanding the Ziegler family at the Janis-Ziegler House/Green Tree Tavern.

Three theoretical approaches are used when researching the ethnicity of the Ziegler family at the Janis-Ziegler house: identity archaeology, consumer behavior, and landscape archaeology. With identity archaeology, the primary focus is on the ethnic identity of the family. Consumer behavior and the rise of consumerism and newspapers also play a role in my research. Lastly, landscape archaeology is used by analyzing the Ziegler use of space at the Janis-Ziegler house, and how this may reveal their ethnic identity. With these three approaches in mind, I researched the archaeological and historical data relevant to the Ziegler occupation of the Janis-Ziegler house. 


\section{CHAPTER III \\ METHODOLOGY}

Research was performed on nineteenth-century English and German newspapers from Ste. Genevieve, the Ziegler probate inventory, census records, the Ziegler wills, and the archaeological assemblage. The primary research was done with the English and German newspapers, focusing on the Fair Play (in English), the Ste. Genevieve Democrat (in English), Herald (in English and German), Freie Presse (in German), and Herold (in German). The English newspaper advertisements and the German newspaper advertisements were then examined and compared to find potential indicators of ethnic preferences. These observations were then considered when analyzing the archaeological record from the Ziegler Occupation (1833-1900) and the Ziegler and Late Occupation (1850-present) contexts and the Ziegler probate inventory. Together the newspaper, archaeological, and other historical data provide an answer to whether the Ziegler family showed their ethnicity despite living and working amongst their English-speaking neighbors. 


\section{Newspaper Analysis}

For the Ste. Genevieve newspaper analysis, I reviewed a sample from the Fair Play (in English), the Ste. Genevieve Democrat (in English), Herald (in English and German), Herold (in German), and Freie Press (in German). I looked at papers fromevery three years between 1850 and 1895 . The newspapers were held at the Missouri Historical Society Archives in St. Louis, the State Historical Society of Missouri in Rolla, and the Ste. Genevieve Branch Library. Obtaining copies of these newspapers was conducted through interlibrary loan as well as traveling to St. Louis and Ste. Genevieve. I photocopied and then scanned each newspaper issue, to better analyze each advertisement.

Whenever possible, I looked at the newspaper issue dated just prior to Christmas to reveal any specific consumer items the Germans may have traditionally used for the holiday. Germans were fond of festivals and other celebrations throughout the year, none more than Christmas. Albert Bernhardt Faust (1927:474) commented that Germans had shown Americans the "joy of living," putting much effort into their "merrymaking, festivals, and the Christmas celebration." Rachel Davis-DuBois and Emma Schweppe (1972:80) discussed that though German traditions may have deteriorated once the family transplanted to the New World, German holiday customs were continued amongst German-Americans. In the Fair Play from December 23, 1875, the newspaper published an article explaining "Christmas in Germany." The article begins by explaining how big a simple birthday celebration is for a small village, 
with the townspeople providing lush gifts and celebrating with song and dance throughout the day for a schoolmaster's birthday. By explaining such a grand festival for a birthday, the author helps you imagine how grand the Christmas holiday might have been for Germans as well.

Add to these domestic occasions the public festive days of the Church and the State, and you can imagine that German life has holidays enough. Christmas and similar days are occasions of incredible festivities throughout Germany. Santa Claus has no better dominion (Fair Play, 23 December 1875).

The holiday was so important to the Germans that in the 1860's, in areas settled by Germans in America, stores and businesses closed on Christmas Day (Wittke 1952:286). Germans even made the custom of decorating Christmas trees the grand tradition it is today amongst most Americans. Also, the tradition of sending Christmas cards to family and friends was first started by the German gentleman, Louis Prang, in the 1840's (Wittke 1952:286). Therefore, I focused the newspaper analysis around newspaper issues from the Christmas season, to find material item related to these ethnic holiday traditions.

For the Fair Play, I analyzed the newspapers from 1872-1880. For the Herald, I analyzed newspapers from 1883-1901. These dates were chosen because the Herold is only available beginning in 1882; however, I also looked at the Fair Play beginning in June 14,1872, since that one issue is available. I also looked at the Freie Press from July 22, 1876, the Democrat from June 8, 1850, because few of these issues were available within the time frame being researched. I would have liked to use earlier issues in my analysis, but few pre1870 Ste. Genevieve newspapers were preserved or available for research. 
To analyze the advertisements from the German and the English newspapers, I made an Access spreadsheet recording the specific products or items being advertised, separating them into specific categories: Household Maintenance-Ceramics, Household Maintenance-Glass, Household Maintenance-Other, Architecture, Household Furnishings, Clothing, Personal Items, Household Production Activity, Household Production Activity For Sale, Pharmaceuticals, Foodstuffs, and Alcohol. This is a variation of the categories originally used by Meredith Hawkins (2007) to search for ethnicity the Ziegler household. She used the categories: ceramics, glass, beads, metal, clinker/cinder, coal, construction material, "tumbled" materials, animal remains, and plant remains. These categories are also similar to those used by Stanley South (1978) in his search for patterns of cultural processes at domestic sites. However, I made my categories according to ethnic attributes in the newspapers. I limited my newspaper analysis to the advertisements defined as a paid announcement, or goods for sale, in the newspapers. Therefore, I did not include items/services done free of charge, notices to the public that are not selling goods or services, or job listings. I separated the German and the English newspapers and the German-English newspaper into three databases (the German-English newspaper is the Ste. Genevieve Herald once it became half in German and half in English), in order to better compare and contrast the advertisements between the different language groups. Using the conclusions drawn from the newspaper analysis, I analyzed other historical data and the archaeological record. 


\section{Additional Archival Analysis}

Along with the newspaper analysis, I analyzed the Ziegler probate inventory, Ste. Genevieve census records, and the Ziegler family wills. Meredith Hawkins and Dr. Elizabeth Scott previously gathered these documents from the Ste. Genevieve County Archives, the Missouri Historical Society in St. Louis, and the Western Manuscript Collection at the University of Missouri at Rolla. With the probate inventory, I categorized each entry into the same Access database template as the newspaper advertisements. With conclusions drawn from the newspaper analysis, I looked at the probate inventory categories to reveal whether the Zieglers showed either English or German ethnic preferences.

In the second stage of my analysis, I looked at the wills of Mathias Ziegler (1835), Francis Ziegler (1897), and Joseph C. Ziegler (1925), to identify the inheritance practices used by the Ziegler family throughout the generations. Other research has shown that inheritance practices can be sensitive to a family's ethnicity, especially German families (Groover 2003; Penner 1997;

Gross 1996). I also wanted to reveal whether these inheritance practices changed throughout the generations. I also compared the Ziegler inheritance practices to French practices in Ste. Genevieve. Inheritance practices may reveal another way in which the Ziegler family expressed their ethnicity or chose to acculturate after a few generations.

I also review the business ledgers for Valle and Ziegler currently held by the Missouri Historical Society, noting when the Mathias Ziegler business ledgers 
changed from recording business in German to recording it in English, and what this means about each generation of the Ziegler family.

\section{Artifact Analysis}

I looked at the artifacts from the 2006-2009 field seasons for the archaeological analysis. I took the artifact analysis from the Janis-Ziegler site (23SG272): units 110R30 and 150R0 (Figure 3.1). 


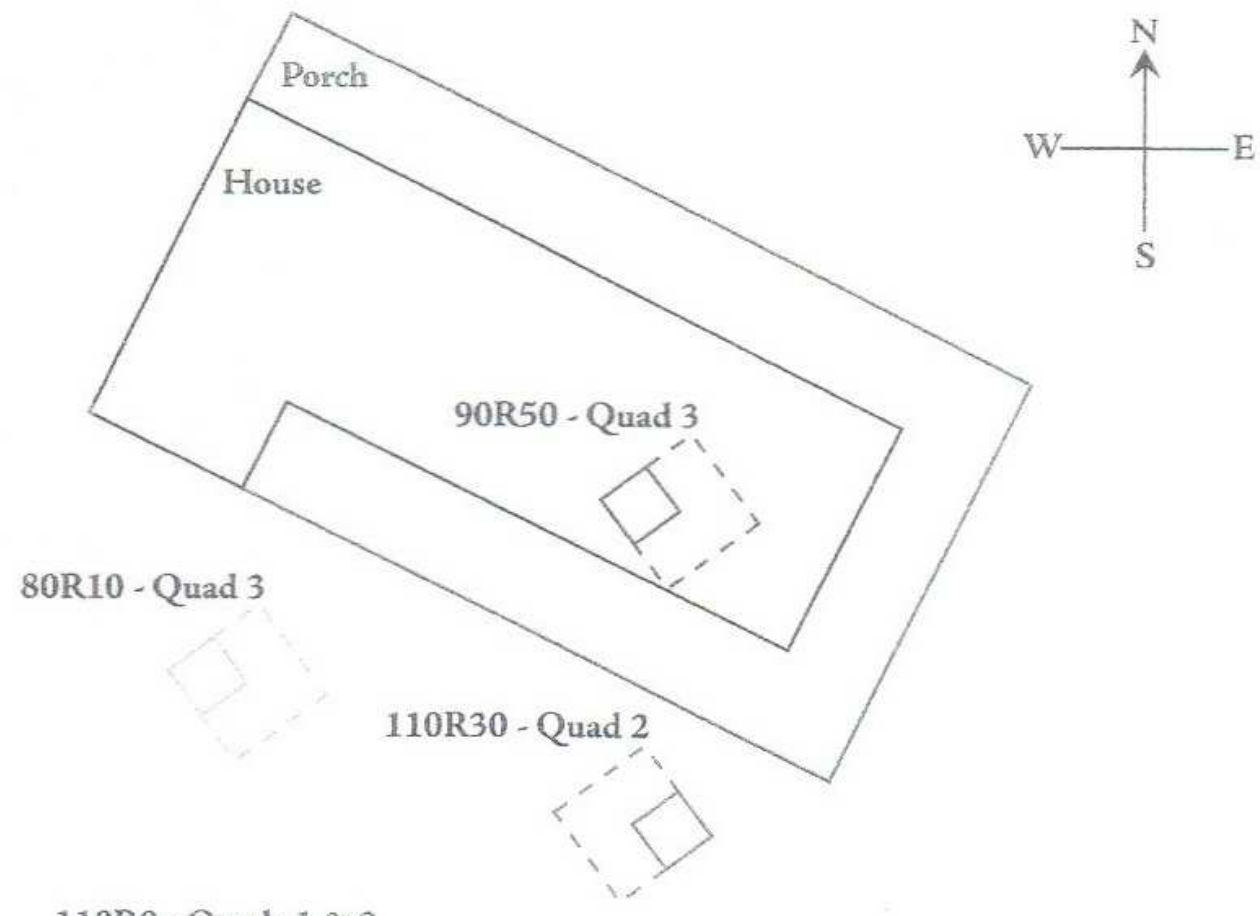

110R0 - Quads $1 \& 3$

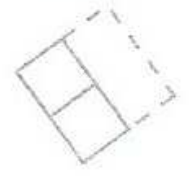

150R0 - Quad 1

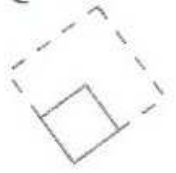

Key for Excatation Linit

$$
\begin{aligned}
& \text { Quadrant } \\
& \text { Unit - } \ldots \\
& \text { Scale } \theta_{m \rightarrow-m^{\prime}=10^{\prime}}^{\prime}
\end{aligned}
$$

Figure 3.1. Map of the Janis-Ziegler/Green Tree Tavern Site (23SG272) Courtesy of Meredith Hawkins 
Unit $150 R 0$ is located on the southeast portion of the property behind the JanisZiegler house. Unit 110R30 was located directly east of the house, just off the gallery or porch (Hawkins 2007:42).The levels associated with the Ziegler occupation were analyzed by Hawkins (2007). She used ceramics to identify which levels were part of the Ziegler occupation, because ceramics offered the most accurate dates (Hawkins 2007:47). Hawkins then provided a list of minimum number of vessels (MNV) for each unit, in order to figure out the mean ceramic date (MCD) for each level (Hawkins 2007:48). She also utilized other artifacts, like tarpaper/asphalt shingles, in order to differentiate between the Janis occupation and the Ziegler occupation levels (Hawkins 2007:53). She concluded that each unit (110R30 and 150R0) had three occupational periods (Hawkins 2007:54):

110R30: Janis Occupation (1790-1833)-Levels 7-13

Ziegler Occupation (1833-1900)-Levels 5 \& 6

Ziegler and Late Occupation (1850-present)-Levels 1-4

150R0: Janis Occupation (1790-1833)-Levels $10 \& 11$

Ziegler Occupation (1833-1900)-Levels 5-9

Ziegler and Late Occupation (1850-present)-Levels 1-4 
Therefore, I used the artifacts associated with the levels considered to be the Ziegler Occupation and the Ziegler and Late Occupation, according to Hawkins's research.

I put the archaeological findings into the same categories as the newspaper and probate inventory analysis. Then I compared the findings from the newspaper analysis to the archaeological record from the Ziegler house. If the Ziegler family was using German-targeted items, this would reveal one way the Zieglers were emphasizing their ethnicity.

Like Matt Cox (2009) and Meredith Hawkins (2007), I take another look to see if I can find "Germanness" but also potentially "non-Germanness" in the archaeological collection. Matt Cox analyzed the ceramics uncovered in the pre1900 Ziegler occupation (Cox 2009:80), and suggests that the ceramics reflected the French heritage of Josephine, who moved into the Ziegler house in 1856 when she married Francis (Cox 2009:81-82). I broadened Matt Cox's research by looking at different material culture categories (not just ceramics) to compare to the newspaper advertisement categories. I expanded the time period from Matt Cox's study by looking at the data from pre-1900 as well as post-1900, since the Ziegler occupation of the Janis/Ziegler house lasted until the late 1930s.

I reviewed the newspapers that coincide with popular German holidays, hoping to find artifacts that relate to traditional holiday customs. For example, winter celebrations included Fastnacht and Weihnachten (Christmas). Fastnacht was a winter holiday where usually the younger members of society dressed up 
and wore masks while roaming their neighborhoods asking for goodies. For these holidays, immigrants made traditional doughnuts and cookies. I also looked in the probate inventories and the artifacts to find evidence of the equipment necessary for making these desserts. For example, Springerle are cookies that Germans made during Weihnachten and other holidays. In order to make these cookies, the person who is baking used a Springerle Roller or a board with imprinted designs that were transferred onto the cookie dough before baking. Also, Germans were fond of baking Krapfen (doughnuts), especially during Fastnacht. In order to make these desserts, the baker used a deep frying to fry the doughnuts. Items like these would be German ethnic markers. Finding these specific cooking utensils and dishes would show that the Zieglers continued to act like their other German neighbors in Ste. Genevieve.

Germans were also known for their frugality and cleanliness (Long 1972: 2-3), sometimes holding onto possessions longer than they were in style in order to save money. This can be seen archaeologically by finding artifacts that predate others in the same level, showing the keeping of heirlooms. Mark Groover (2003:167) suggests that economic position may be revealed when looking at ceramics and comparing them to the newspaper advertisements and national trends during this time. However, the German ethnic trait of frugality may hinder this; they may not have put their money in material items, but other commodities such as land. 


\section{Landscape Analysis}

I also investigated further the traditional German use of space. Germans typically used their back or side doors more than front doors. For this reason, they would extend their walk-way from the driveway to the back door (Coggeshall 1986:194). Besides evidence of a walk way in this location, perhaps the number of artifacts found in the backyard units compared to the front units will show evidence of this preferred use of the back living space.

Cleanliness can also be seen by looking at the landscape. Brian D. Crane (2000) suggests that refuse disposal may vary from one cultural group to another. "Different groups of people may have responded differently to the evolving corpus of rules and common practice surrounding refuse disposal. In addition, different standards may have been applied to yards used as workspace compared to yards used for gardens or recreation space" (Crane 2000:29). Therefore, fewer artifacts found in main living spaces, or negative data, in comparison to the work areas of the property, could indicate a concern with cleanliness.

\section{Summary}

In summary, I compared the advertisements in the nineteenth-century English newspapers in Ste. Genevieve to the German newspapers, to find any German or English preferences as far as products being advertised. Then I compared these observations to find whether or not the Ziegler family showed 
their ethnic identity in their probate inventories and archaeological record. I also looked for any signs of "Germanness" or "non-Germanness" in the probate inventory and the archaeological record, as discussed previously. This includes analyzing the probate records and the wills to identify the form of inheritance practices each Ziegler generation used, which may be another method for expressing ethnicity. I also analyzed the landscape use by the Ziegler family to identify whether they may have shown their German ethnicity through their use of space. 


\section{CHAPTER IV}

\section{ANALYSIS OF DATA}

This analysis will uncover to what degree the German Ziegler family expressed their ethnicity while living at the Janis-Ziegler house. First, a review is conducted of each category from the newspaper analysis. By comparing and contrasting the advertisements between the German and English newspapers, this process may reveal possible ethnic trends. These observations are then compared to the Ziegler probate inventory and the archaeological data.

The second part of the analysis was a study of the landscape and the inheritance practices of the Ziegler family throughout the century they inhabited the Janis-Ziegler house. This study hopes to reveal whether or not it is possible to identify the German ethnicity of the Ziegler family by looking at their consumption patterns and from the material culture of the Janis-Ziegler site.

\section{Newspaper, Inventory, and Archaeological Analysis} Household Maintenance-Ceramics

The first category discussed in the newspaper analysis will be Household Maintenance-Ceramics. In the English newspapers reviewed, advertisements for ceramics made up 13 of the 187 total advertisements (7.0\%). In the German 
newspapers, ceramics made up 5 advertisements out of 173 (2.9\%). The German -English newspaper advertisements had 1 advertisement for ceramics out of $86(1.2 \%)$. The English newspapers listed several ware types, of which Queensware was advertised most, followed by china and stoneware (Table 4.1).

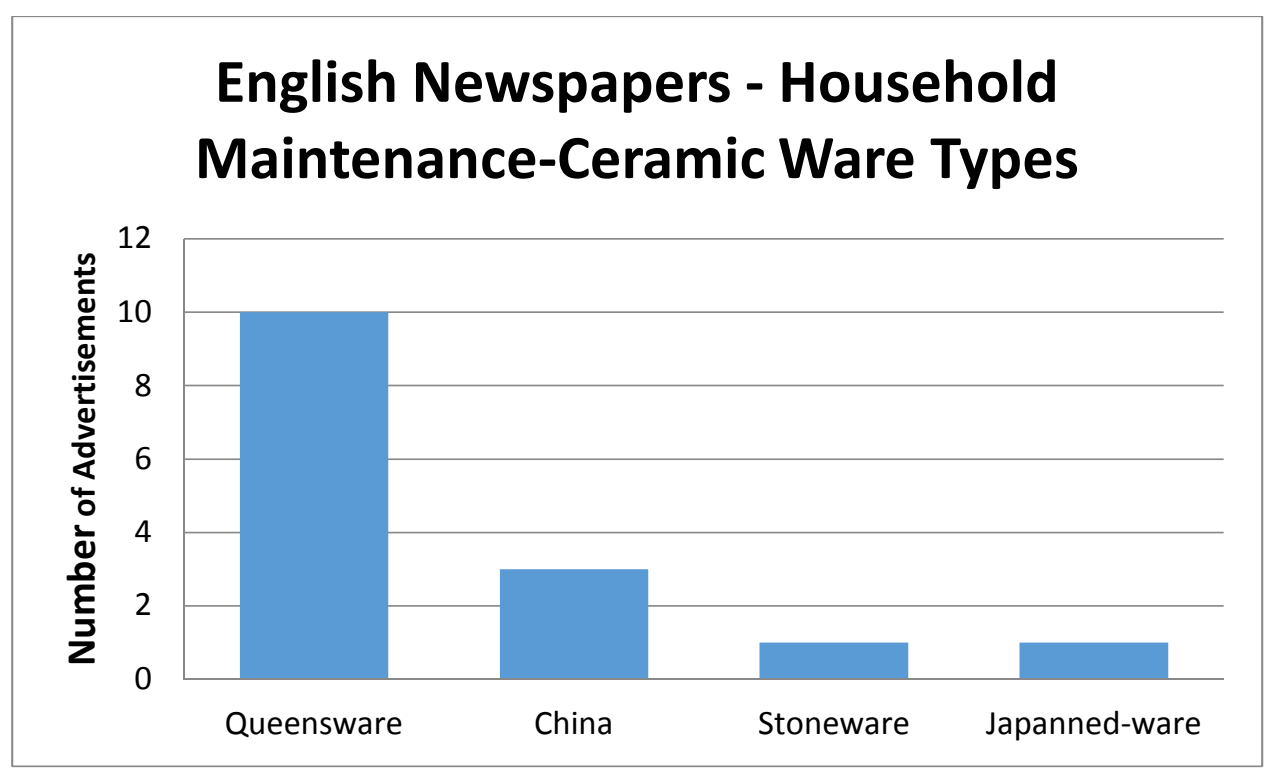

Table 4.1. English Newspaper Household Maintenance-Ceramics Ware Types

The English newspapers also advertised for specific dish forms such as water pitchers, butter dishes, and pickle dishes. The German newspapers had considerably fewer ceramic advertisements, most of which were for Porzeilan (porcelain), and then Steingut (stoneware) (Table 4.2). 


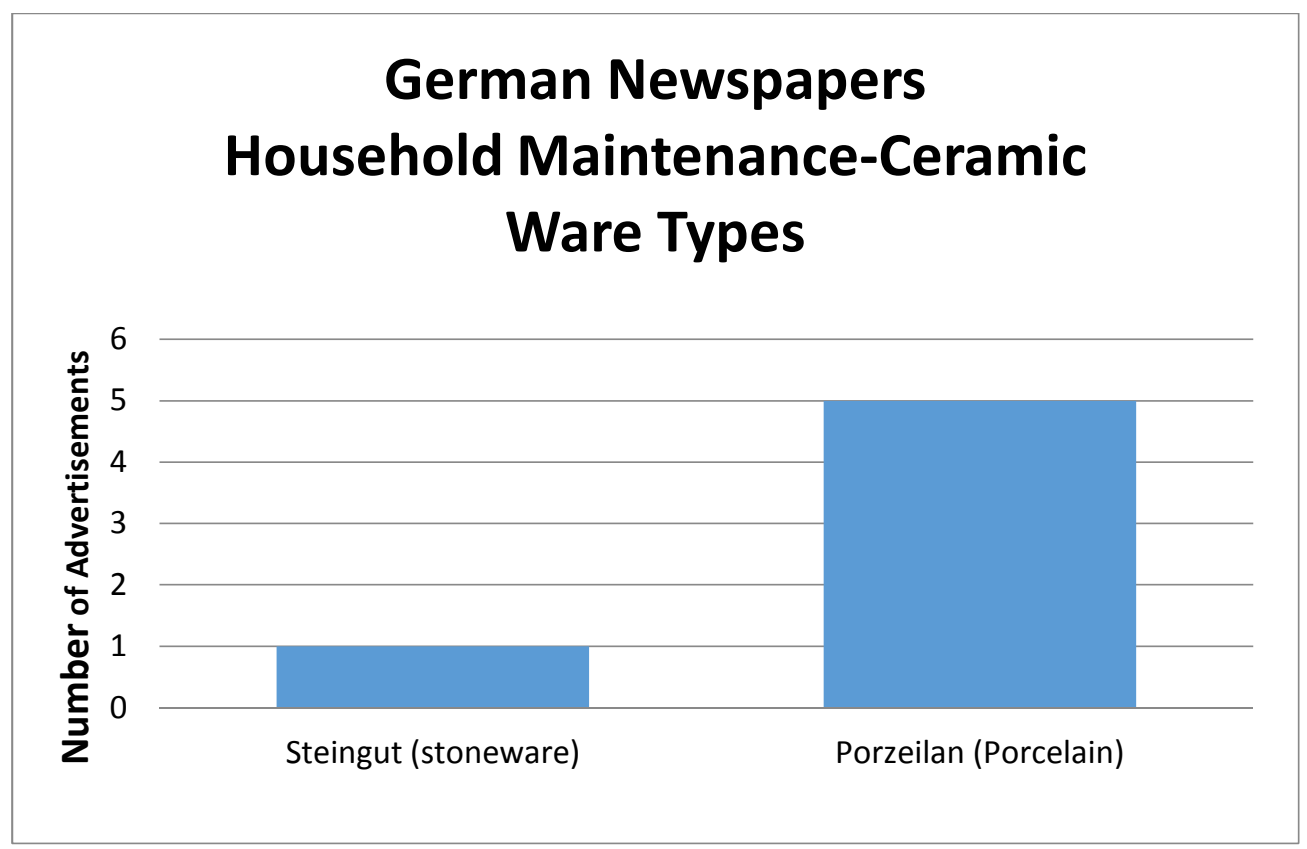

Table 4.2. German Newspapers Household Maintenance-Ceramics Ware Types

The German newspapers did not advertise for specific ceramic forms like the English newspapers. The German-English newspaper only had one listing and it did not include ware type; instead, the advertisement was for dish forms, such as fancy cups, saucers, tea sets, and chamber sets (Figure 4.1). 


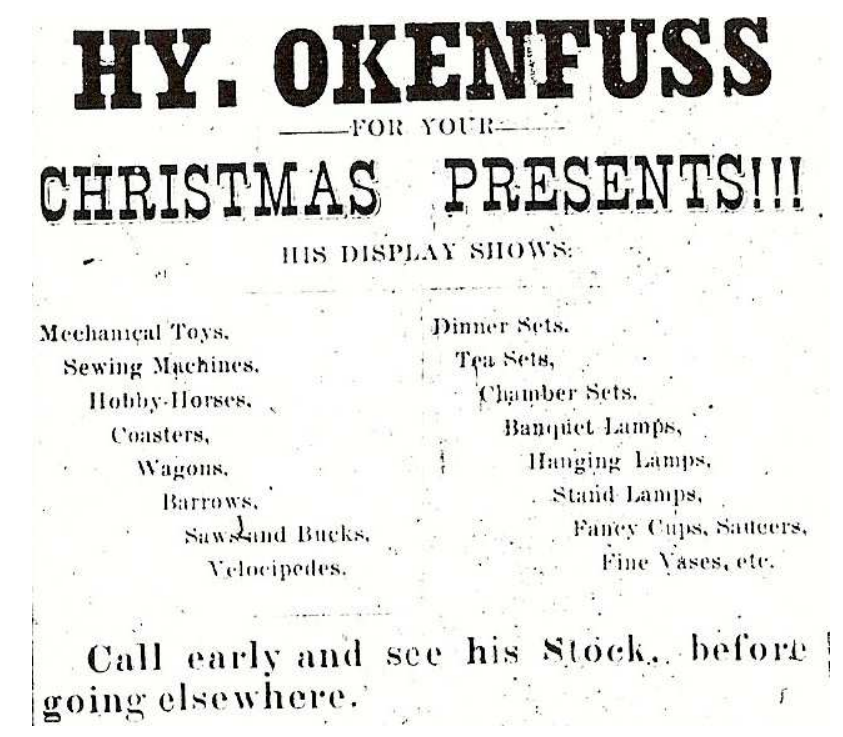

Figure 4.1. Advertisement for dish forms in Herold December $21^{\text {st }}, 1895$

The German newspapers had considerably fewer advertisements for ceramics than did the English newspapers. Perhaps the Germans tended to be frugal, and therefore, bought new ceramics less often making advertisements for ceramics in the German newspapers less common. All five of the five German newspaper advertisements for ceramics advertised for porcelain (Table 4.2). In the English newspapers, only three of the 13 ceramic advertisements listed china (Table 4.1). Queensware was listed in 10 of the 13 English ceramic advertisements. Queensware was not listed in any German advertisements in my sample. Christopher Fennell (2003:275) noted that "Queensware," and by that he meant the inexpensive cream-colored "Queensware that was made popular by Josiah Wedgwood," was mainly popular in the late eighteenth century until around 1820 in the major urban areas. However, the popularity of Queensware only appeared to grow in the first half of the nineteenth century in 
the rural areas that he studied in New England. However, this may be because in Philadelphia, potters began producing a similar inexpensive cream-ware, calling it "Queensware" (Fennell 2003:276). A similar circumstance in Ste. Genevieve may have occurred in the nineteenth century.

The Ziegler probate inventory only lists one specific ware type, Stoneware; however, it lists several specific vessel forms. The German newspapers did not specify vessel forms in the advertisements while the English and GermanEnglish newspapers did. It is not possible, then to associate specific vessel forms with either ethnic group in an absolute way.

Meredith Hawkins (2007) defined what ware types were present in the archaeological record and developed a minimum number of vessels (MNV) for the archaeological records for units $150 \mathrm{R} 0$ and $110 \mathrm{R} 30$. The MNV for the Ziegler Occupation (1833-1900) and the Ziegler and Late Occupation (1850present) levels were used in my analysis. The ware types were generalized in order to fit with the newspaper analysis. For example, Hawkins (2007) differentiated between creamware, pearlware, white ware, and refined white earthenware in her MNV. However, the newspapers generalized these under the same term, Queensware. For my archaeological analysis, the term 'whitecolored refined earthenwares' is used in reference to a combined count of creamware, pearlware, whiteware, and refined white earthenware vessels to better compare to the newspapers' use of the term "Queensware."

The ceramic assemblage from unit 150R0, located in the yard behind the Janis-Ziegler/Green Tree Tavern, contained a minimum of 40 vessels and was 
made up primarily of white-colored refined earthenwares, followed by course red earthenware, stoneware, and yellowware (Table 4.3). Other ceramic sherds were not large enough to be clearly differentiated. No porcelain was found in this unit for the Ziegler occupation.

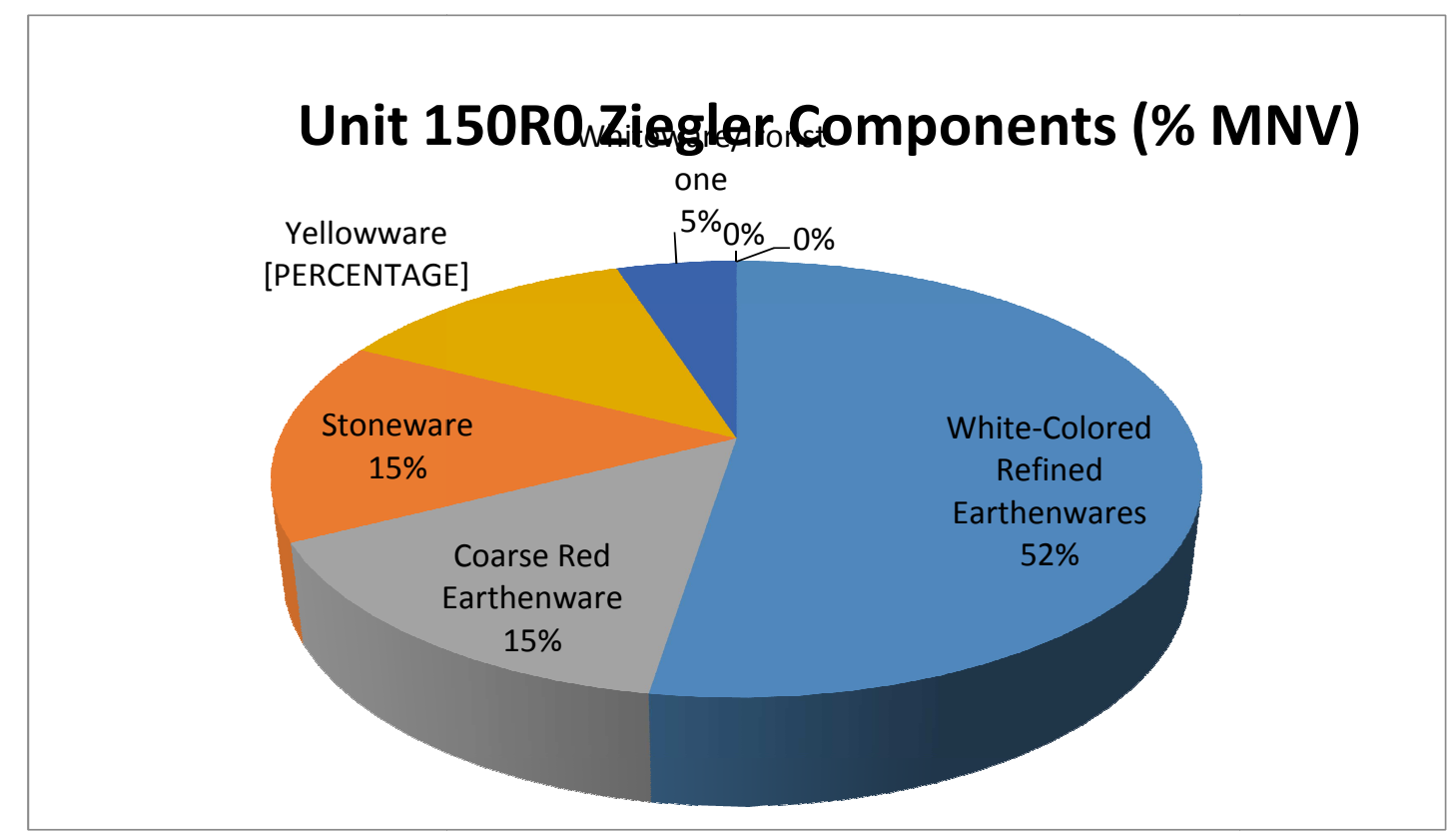

Table 4.3. Ceramic MNV of unit 150R0, Ziegler Occupation (1833-1900) and Ziegler and Late Occupation (1850-present)

The ceramic assemblage from unit 110R30, located just off the back porch of the Janis-Ziegler/Green Tree Tavern, contained a minimum of 198 vessels, including an even larger percentage of white-colored refined earthenwares (71\%). Other ware types were coarse red earthenware, stoneware, and a small percentage of yellowware, porcelain, and unidentifiable ceramic sherds (Table 4.4). This unit does contain porcelain, but, only one vessel. 


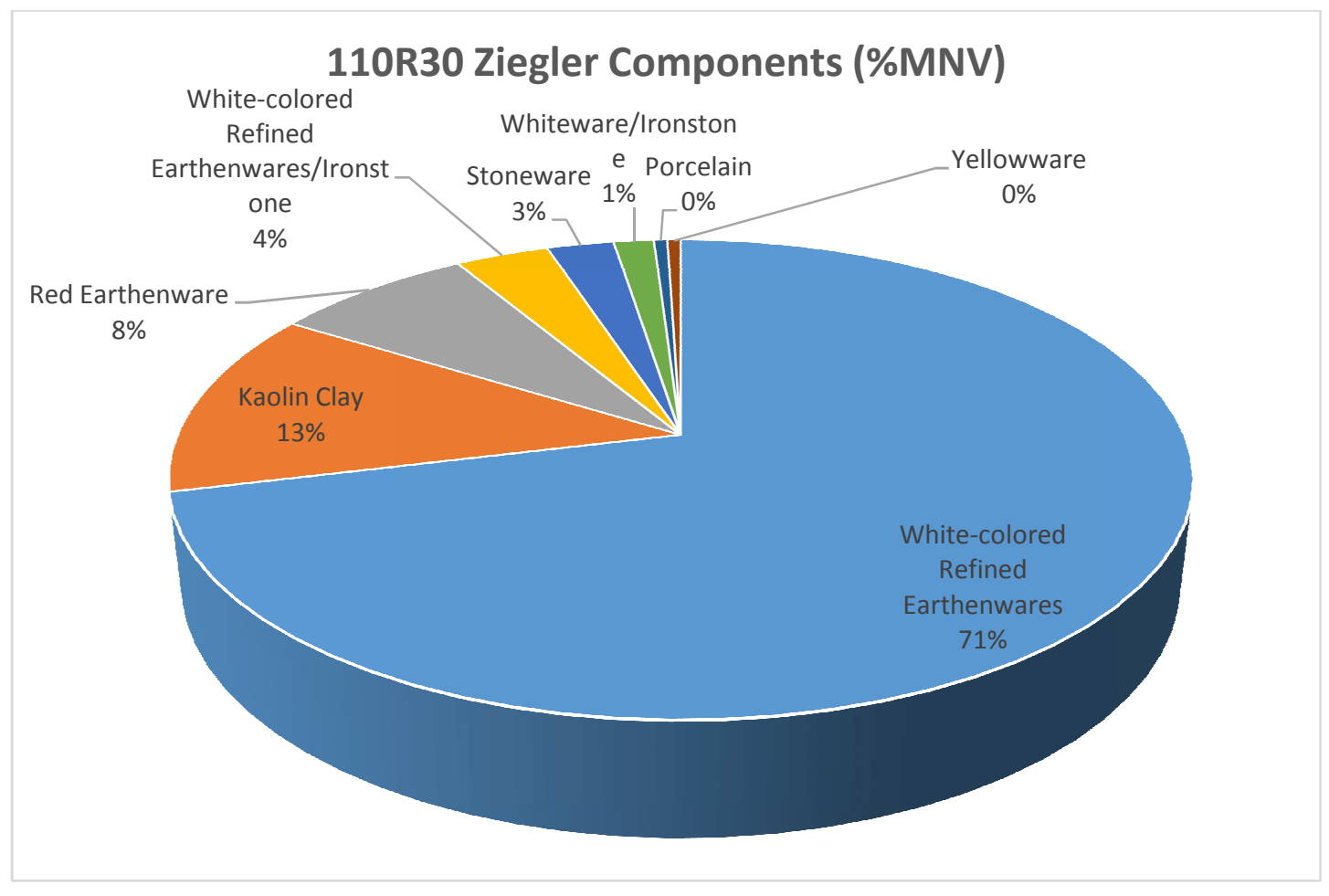

Table 4.4. Ceramic MNV of unit 110R30, levels 1-6: Ziegler Occupation (18331900) and Ziegler and Late Occupation (1850-present)

When the German and English newspapers are compared to the archaeological record, it appears as though the Ziegler family followed the tradition of the English speaking people in the community in terms of ceramic use. The English newspapers advertised primarily for Queensware, while the German newspapers advertised for primarily Porzeilan (porcelain). Both units show the use of white-colored refined earthenwares as their primary ware of choice; very little porcelain or china was found in these two units. However, with this observation must be stated that the archaeological data only allows for the comparison of ceramics left behind or discarded by the Ziegler family. This does not reveal any of the ware types that the Zieglers may have passed on 
throughout the years and took better care of while living in the JanisZiegler/Green Tree Tavern. This could be the reason so little porcelain was found in the archaeological record. Porcelain was one of the most expensive ware types in the nineteenth century (Miller 1980:32). Also, George Miller (1991:11) noticed during his research that porcelain rarely is found in archaeological assemblages in America prior to 1850. Unfortunately, the only ware type listed in the Ziegler probate inventory was stoneware; the rest of the ceramics on the inventory were listed by vessel form.

In Meredith Hawkins (2007) research she listed the identifiable vessel forms found in units $110 \mathrm{R} 30$ and $150 \mathrm{R} 0$ (Table 4.5).

\begin{tabular}{|l|l|}
\hline 110R30 & 150R0 \\
\hline Flowerpot & Flowerpot \\
\hline Bowl & Bowl \\
\hline Plate & Plate \\
\hline Mug & Medium bowl \\
\hline Teacup & Pitcher \\
\hline Cups & Large Crock \\
\hline Large bowl & Large Bowl \\
\hline Saucer & Saucer \\
\hline & Medium-large Bowl \\
\hline & Small-medium bowl \\
\hline & Serving dish \\
\hline & Small-medium cosmetic jar or canister \\
\hline
\end{tabular}

Table 4.5. Vessel Forms from Unit 110R30, Levels 1-6: Ziegler Occupation (1833-1900) and Ziegler and Late Occupation (1850-Present) and Unit 150R0, levels 1-9: Ziegler Occupation (1833-1900) and Ziegler and Late Occupation (1850-Present) 
Many of these vessel forms were also advertised in the English newspapers: water pitchers, dinner sets, tea sets. Other vessel forms were mentioned as well in the English newspaper advertisements but they were not identified in the Ziegler archaeological record: water pitcher, dinner set, butter dish, pickle dish. The Ziegler probate inventory also has some similarities to the English newspaper advertisements. The probate inventory lists dinner plates, smaller plates, dishes, soup tureen, bowls, cups and saucers, tea pot, sugar dish, and pitchers. By comparing the English newspaper advertisements to the Ziegler probate inventory and the archaeological analysis, it appears that the Zieglers were purchasing similar vessel forms as their English-speaking neighbors. Unfortunately, the German newspaper advertisements do not contain advertisements for vessel forms in comparison.

\section{Household Maintenance-Glass}

The occurrence of advertisements for Household Maintenance-Glass was fairly even between the English and German newspapers. The English newspapers advertised for glass in five of the 187 advertisements $(2.7 \%)$. The German newspapers advertised for glass in four of the 173 advertisements (2.3\%). In the German-English newspaper, household glass was not advertised at all. This category was not as widely advertised as other categories. The German newspapers did not advertise for glass after 1876. The English newspapers only had one advertisement for glass after the 1870s, found in the Ste. Genevieve Herald in 1892 (Figure 4.2) 


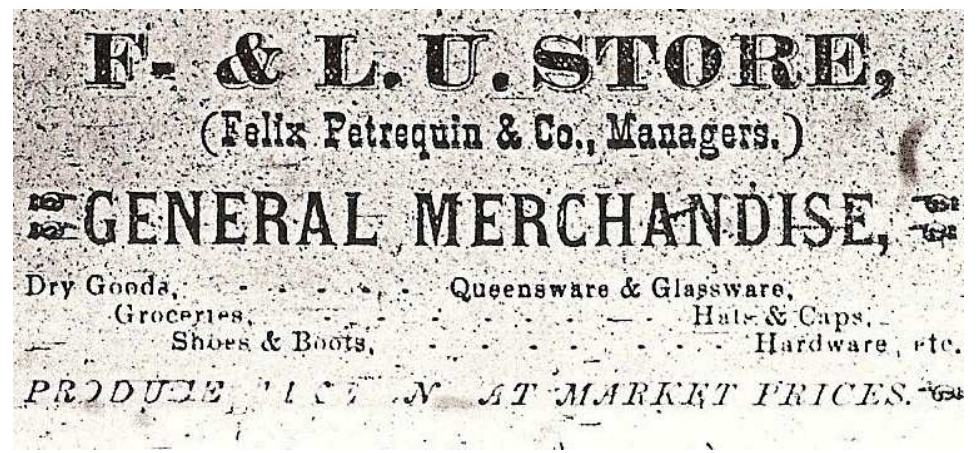

Figure 4.2. F. \& L.U. Store Advertisement for Glassware in Ste. Genevieve Herald, December $17^{\text {th }}, 1892$.

The glass advertisements appeared to decline over the years and did not appear in any of the later newspaper issues reviewed. This confirms what Jane Busch (1987:68) noted, that when bottle production increased by 1880 , the bottle prices declined. Therefore, advertisements for glassware and bottles were less profitable. Neither English nor German newspapers contained enough information about glass vessels nor glass ware types to reveal any ethnic differences.

The Ziegler probate inventory lists several forms of glass: tumblers, pitchers, and plates. The archaeological data includes fragments of different colored glass; however, the fragments are too small to discern, the vessel forms. Since the advertisements, probate inventory, and archaeological data do not provide comparable sets of information, it is not possible to detect any significant ethnic differences in the use of glass in the household. 
Household Maintenance-Other

The category Household Maintenance-Other includes any household maintenance objects in the advertisements that are not ceramics or glass. In the English newspapers, advertisements for Household Maintenance-Other made up five out of 187 advertisements (2.7\%). The German newspapers had two advertisements for this category out of $173(1.2 \%)$. The German-English newspaper had three advertisements for Household Maintenance-Other out of 86 (3.5\%). In the English newspapers, three out of the five advertisements in this category listed cutlery (Table 4.6).

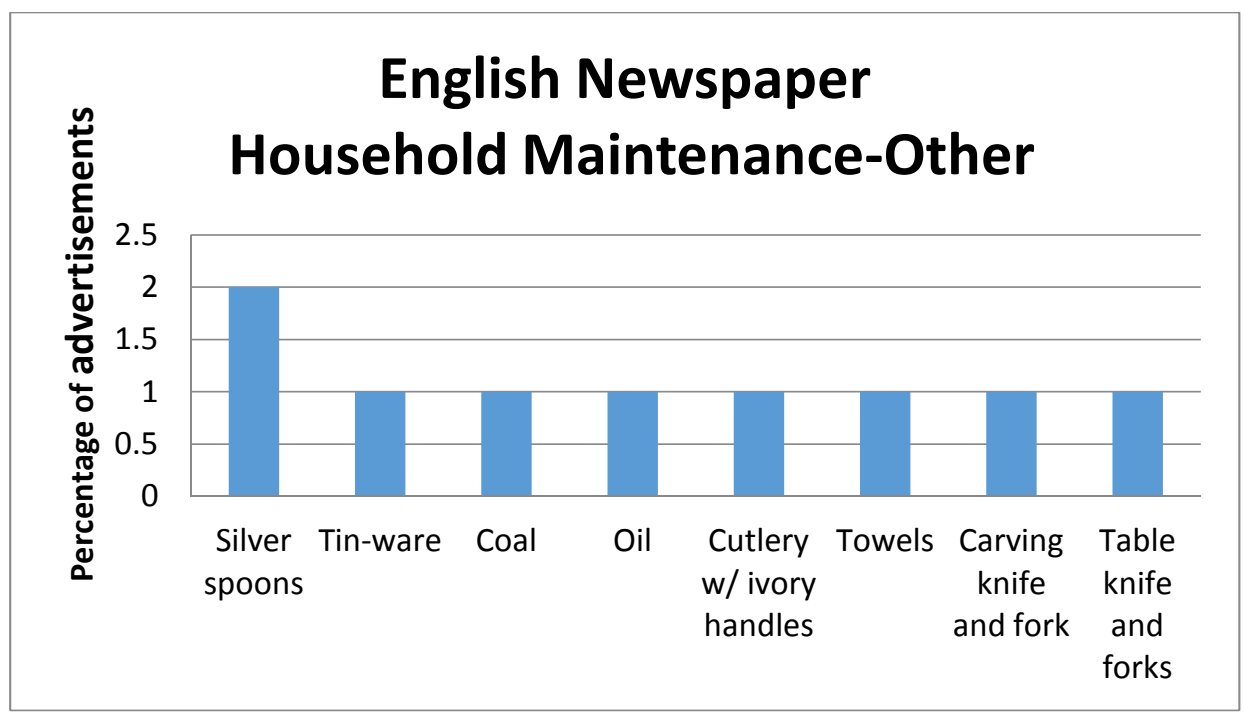

Table 4.6. English Newspaper Household Maintenance-Other

Some of the "other" items listed were towels, oil, coal, and tin-ware. The German newspapers only advertised for gold und silberwaaren (gold and silver goods). Silberwaaren is commonly translated to mean silverware, and therefore the 
advertisement was included in the Household Maintenance-Other category. The German-English newspaper did not advertise for any silverware. Instead the newspaper advertised for stove polish, coasters, and fine vases (Table 4.7).

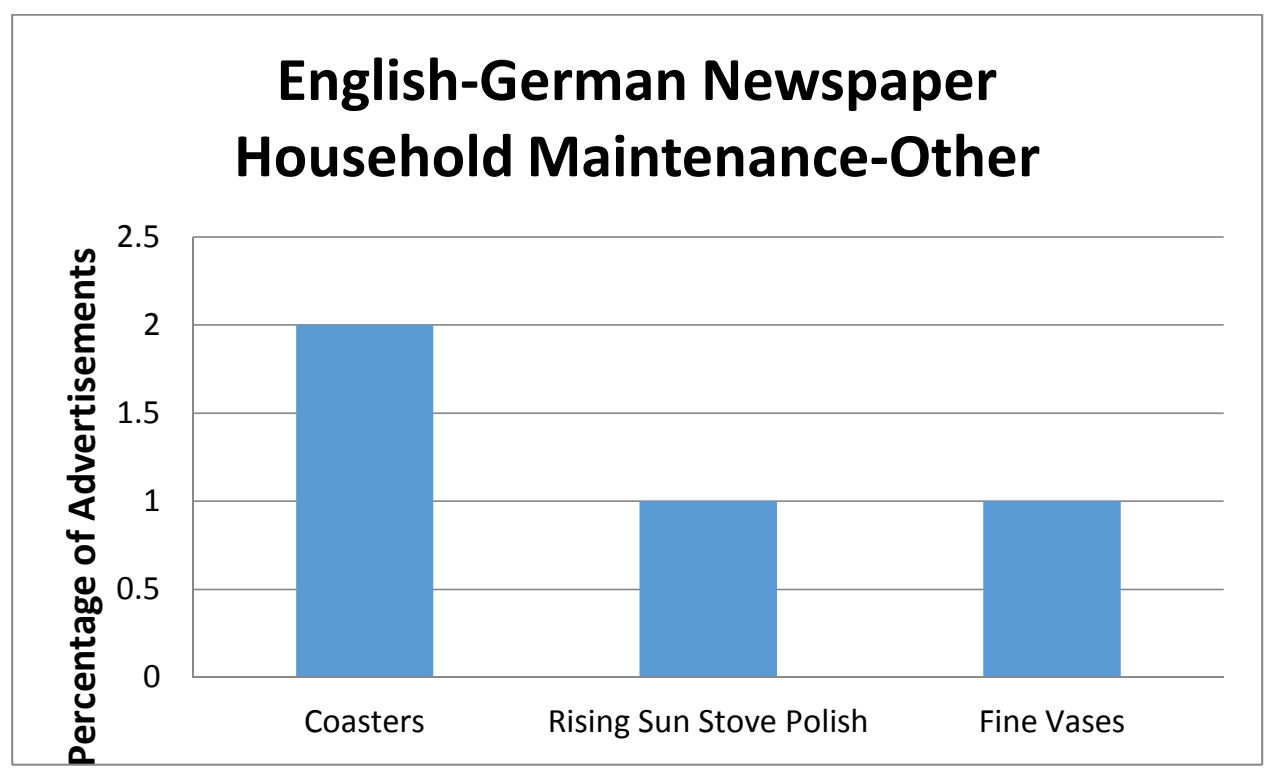

Table 4.7. German-English Newspaper Household Maintenance-Other

Nothing stands out as different between the English and the German newspaper advertisements in the Household Maintenance-Other category, besides the English newspapers having more advertisements in this category than the German newspapers.

The Ziegler probate inventory includes 50 listings that could be considered in the Household Maintenance-Other category. These items appear to have been used mainly in two different types of maintenance, kitchen and cleaning. Many of the items are different types of cutlery or cooking utensils, along with a churn, crocks and other cooking pots, tin ware, and candle sticks. The cleaning 
items are mainly large tubs and buckets. Though the Ziegler inventory is detailed, the newspaper analysis does not suggest any ethnic characteristics that can be identified in the inventory.

Similarly, the archaeological data does not suggest any ethnic traits. Most of artifacts in this category are metal scraps or fragments. One item that was identifiable was a handle fragment for a pewter spoon. Pewter cutlery was not listed specifically in any of the newspapers, so any association with one group or another is not known.

\section{Architecture}

The Architecture category contains materials and tools used in constructing and maintaining structures. The English newspapers advertised for architectural items in 15 out of 187 advertisements (8.0\%). In the German newspapers, 11 advertisements out of 173 advertised for architectural items (6.4\%). The German-English newspaper had five advertisements out of 86 for this category (5.8\%). The English newspapers mainly advertised for general architectural items; the 15 architectural advertisements used the general term "hardware" 10 times (Table 4.8). 


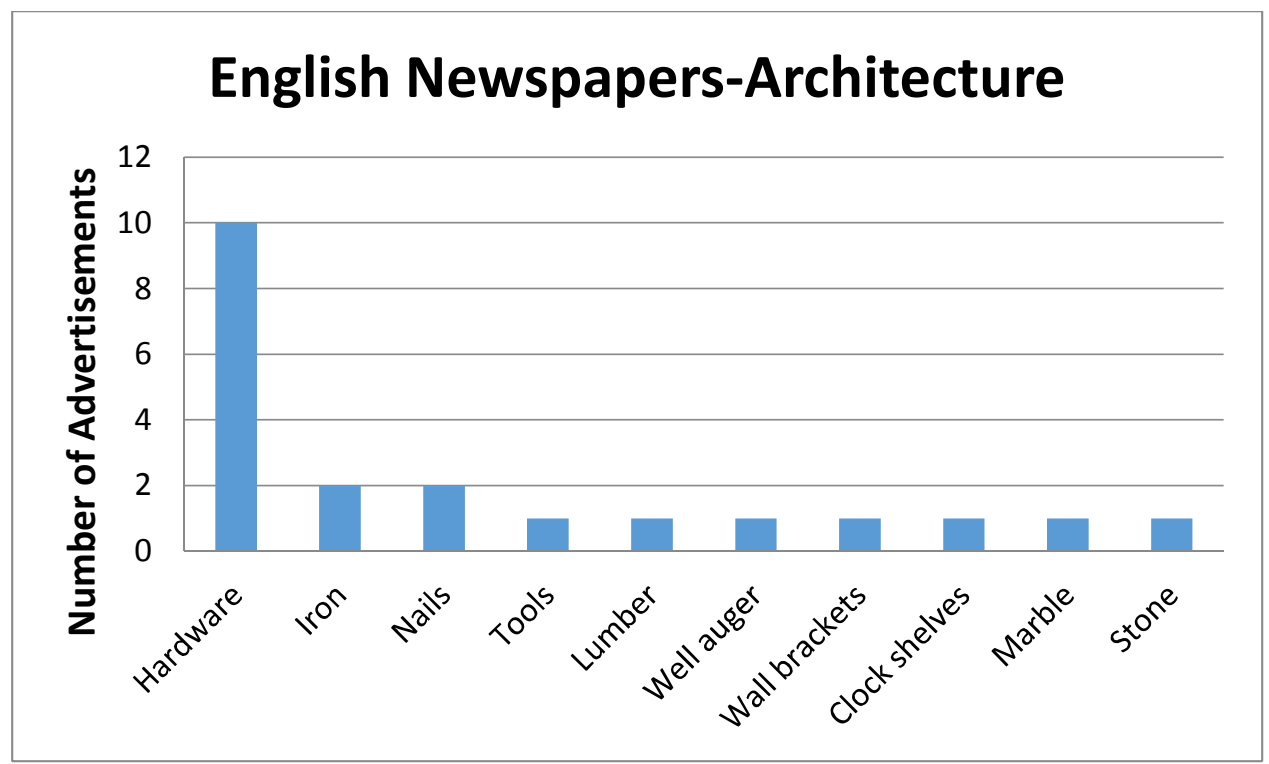

Table 4.8. English Newspapers Architecture

The other advertisements in English newspapers were for tools, iron, lumber, a well auger, wall bracket, shelves, nails, and marble/stone. Each of these items was only advertised for once or twice out of the 15 architectural advertisements.

In the German newspapers, only five of the 11 architecture advertisements were for general hardware (Table 4.9). 


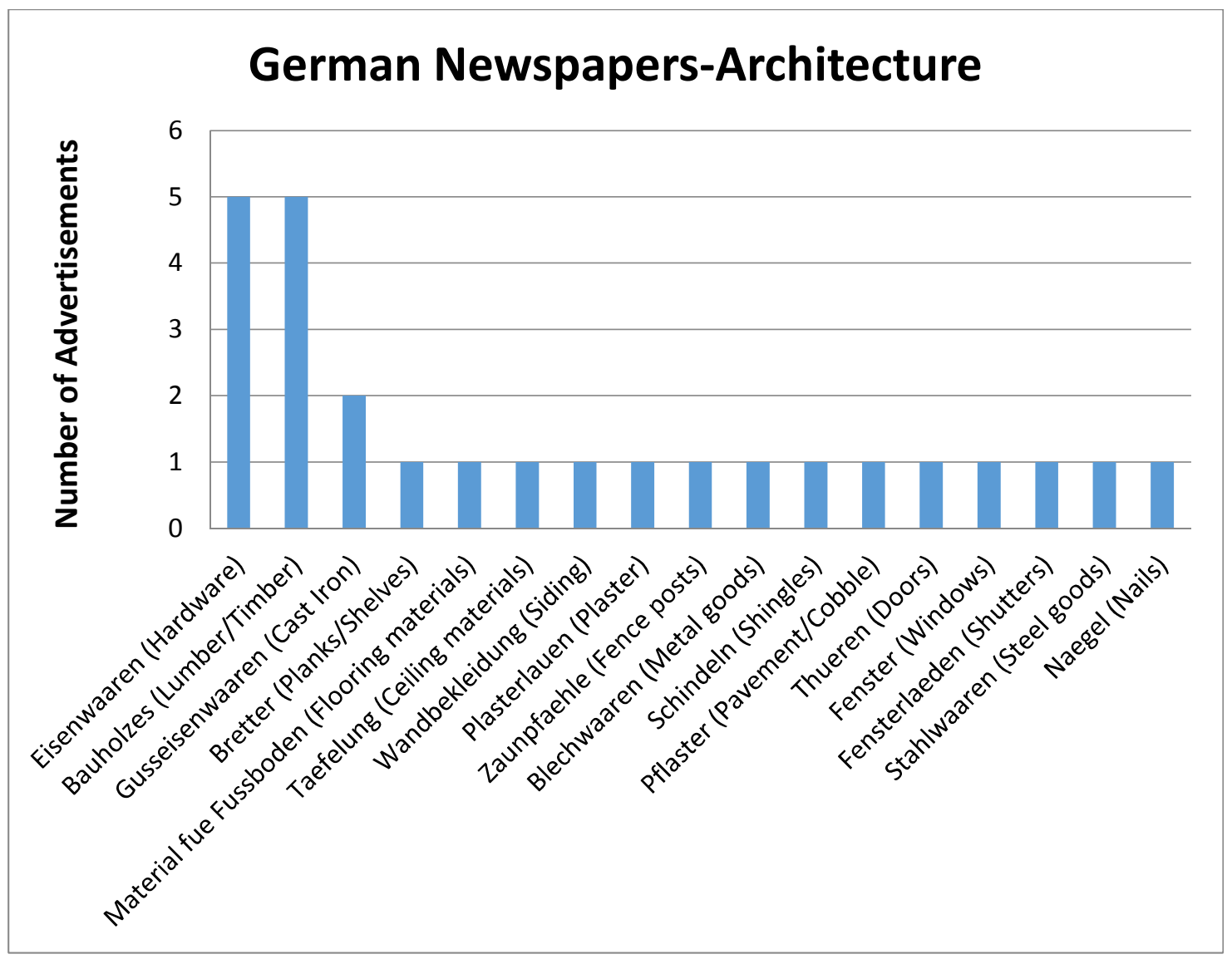

Table 4.9. German Newspapers Architecture

Also, five of the advertisements listed timber or lumber. The German newspapers advertised for many more specific architectural items than the English newspapers. These items were related to heavy household repairs or building projects, with advertisements for doors, windows, roofing materials, etc. In the English newspapers, the advertisements for architectural items were for light household repairs or improvements, such as nails, general tools, and shelves. 
The Ziegler probate inventory listed seven items that would be included in the architecture category: a wheel barrow, hand saw, hammers, pr. pinchers, chisel, square and sundry tools, and nails. The archaeological data in the architecture category mainly consists of lime plaster/mortar and window glass, neither of which can be considered German ethnic architectural traits. This may also be due to the fact that the Ziegler family purchased the already built home from the French Janis family and did not build their own home. However, one would think the Ziegler family might have conducted minor construction projects on their property while inhabiting the Janis-Ziegler house. It is possible that they borrowed tools from neighbors and friends to make any additions or alterations throughout the years, which would not have turned up in the inventory.

Despite the Ziegler inventory not providing much insight into this category, the newspapers themselves may reveal some information about the German community as a whole in Ste. Genevieve. Perhaps the reason the hardware stores listed more detailed advertisements in the German newspapers compared to the English newspapers is because by the 1870s, most of the carpenters in town were German immigrants. German carpenters were encouraged to immigrate to North America because of the high demand for their labor (van Ravenswaay 1977:303). Also, many German-speaking farmers manufactured their own furniture and other crafts (van Ravenswaay 1977:305). 


\section{Household Furnishings}

The next category analyzed was Household Furnishings. In the English newspapers, 12 advertisements out of 187 were for Household Furnishings (6.4\%). In the German newspapers, 13 out of 173 were for Household Furnishings (7.5\%). The German-English newspapers only had three advertisements for Household Furnishings (3.5\%). In the English newspapers, many kinds of furnishings were advertised, ranging from stoves to tables to hanging lamps, but none stood out in frequency (Table 4.10).

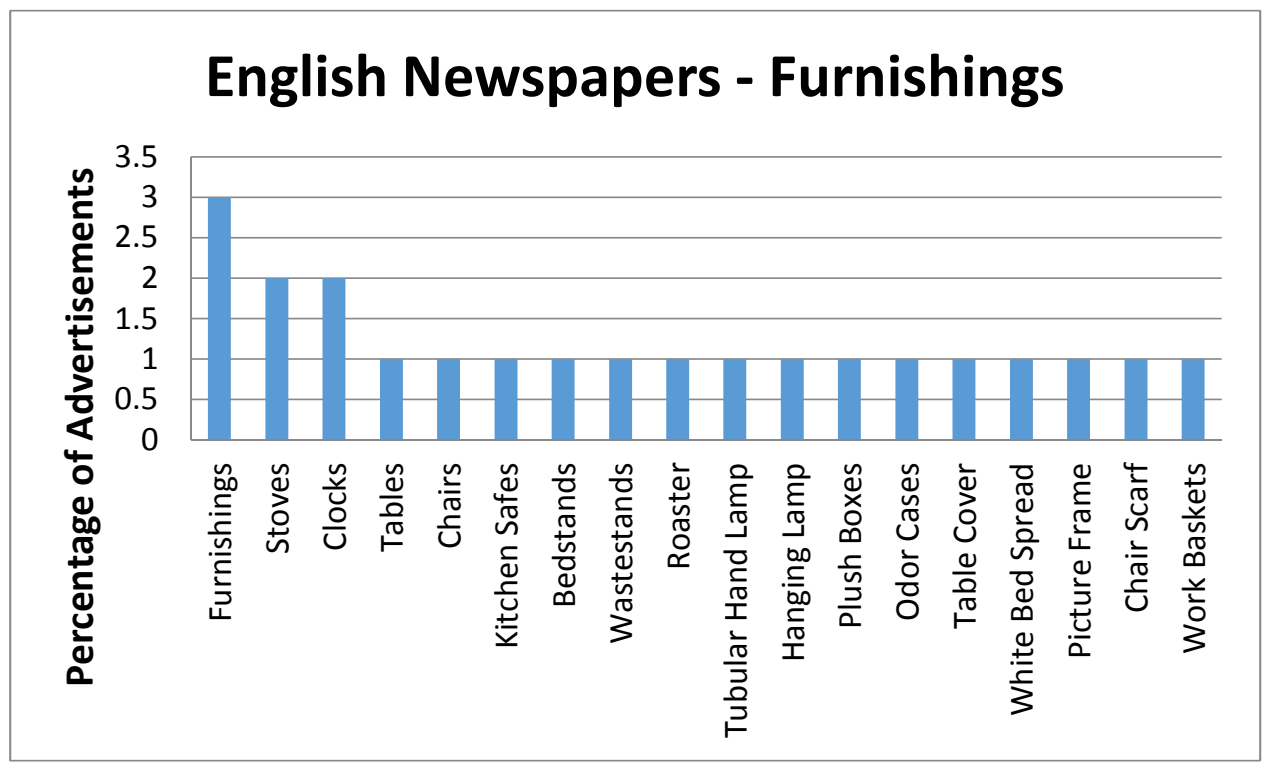

Table 4.10. English Newspapers Furnishings

The German newspapers, on the other hand, advertised for one item more than others in this category, the clock (Uhren). Clocks were advertised in seven out of the 13 advertisements in the Household Furnishings category (54\%) (Table 4.11). 


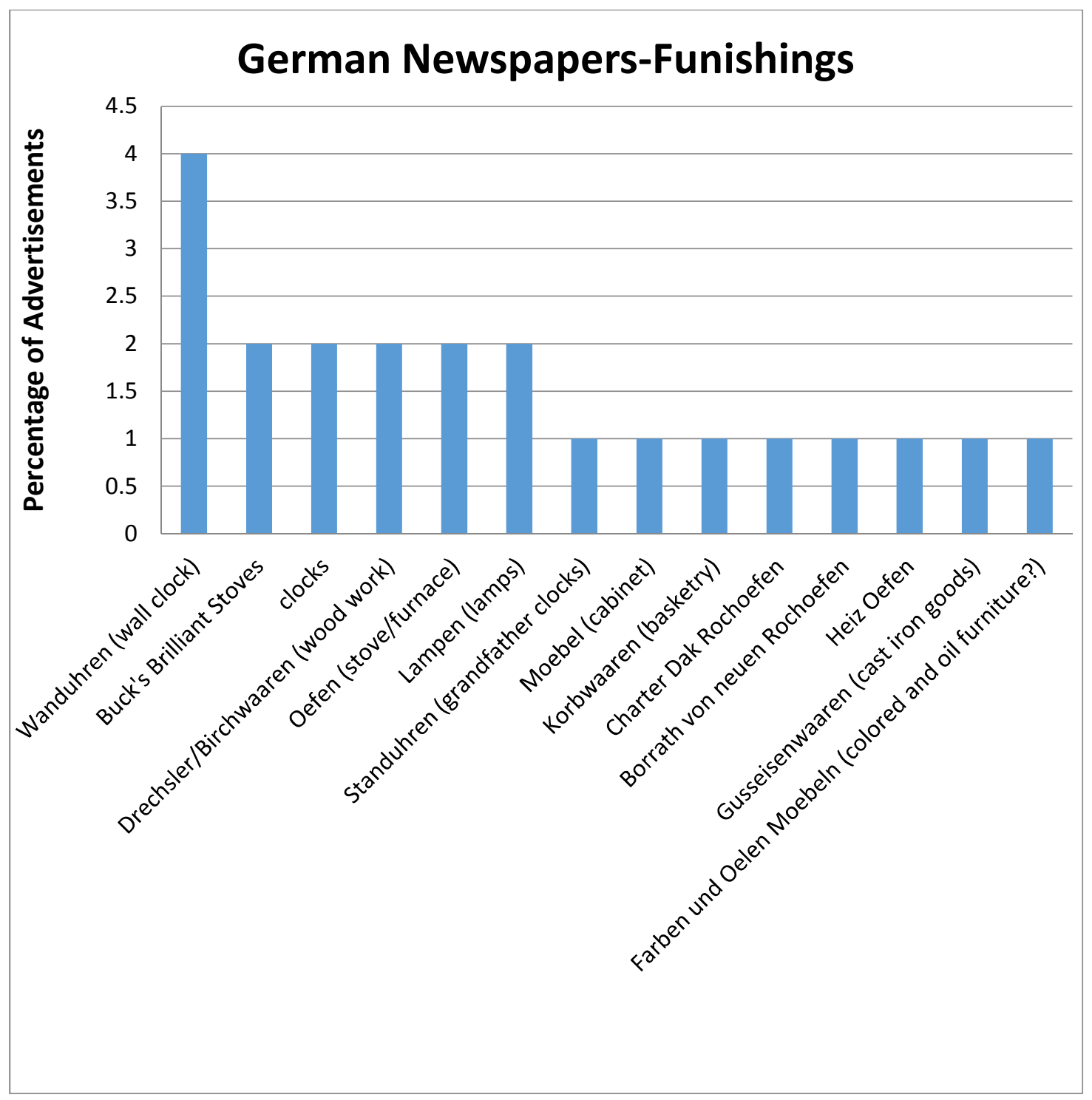

Table 4.11. German Newspapers Household Furnishings

The English newspapers only advertised for clocks in two out of 12 advertisements in this category (9\%) (Table 4.9). This substantial amount of advertising for clocks may be a sign of German ethnicity. 
Ovens and stoves were also advertised for significantly more in the German newspapers than the English newspapers. An example of a German newspaper advertisement for an Oven is shown below (Figure 4.3).

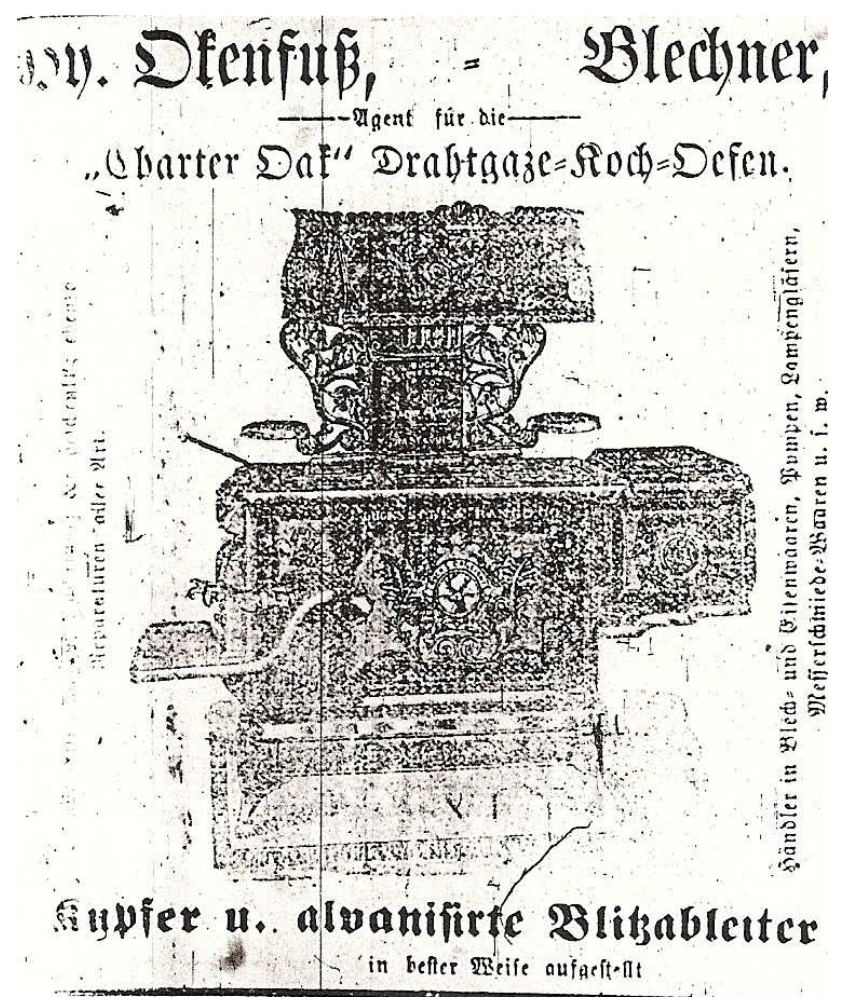

Figure 4.3. Oefen Advertisement Herold Dezember 17, 1892

In the German newspapers, four of the 13 Household Furnishing advertisements are for ovens or stoves (31\%). In the English newspapers, two advertisements listed stoves of the 12 advertisements for Household Furnishings (17\%). Fennell (2003:202) noted that Germans liked fuel efficient stoves because of the lack of wood available in Germany, even though wood was more available once they moved to America. Germans were also known for being economical, in favor of using stoves over open fireplaces in order to save wood (Faust 1927:29). 
Perhaps this explains why the German newspapers advertised for stoves or ovens more than the English newspapers in Ste. Genevieve. The German stove and oven advertisements did not discuss the efficiency of the equipment itself, but that varieties of stoves and ovens were available as well as prompt service.

In the German newspapers, three of the 13 Household Furnishing advertisements listed cabinets, wooden furniture, and/or wooden goods (23\%). According to the Ziegler probate inventory, the Zieglers owned several pieces of wooden furniture including: walnut cupboard, walnut tables, cupboard, set of Windsor chairs, com. chairs, rocking chair, cherry table, cherry bureau, cherry press, sewing cherry stand, and a pine writing desk. The Zieglers were similar to their German speaking neighbors in that they appreciated and owned many wooden furnishings for their home. As stated previously, German immigrants found lucrative jobs in America as craftsmen and woodworkers. The Zieglers could have bought these furnishings from German-speaking craftsmen, produced pieces themselves, or brought furnishings from Germany; however, this cannot be fully determined from the Ziegler probate inventory.

Another interesting thing about the German newspapers is that they did not include advertisements for chairs scarves, bed spreads, or table covers like the English newspapers did. This might be because German women may have been producing their own cloth goods, and therefore they were not carried in the stores. The Ziegler inventory does list table cloths, bed spreads, and other linens, however, these could very well have been made by members of the Ziegler household. This will also be discussed further in the next section about clothing. 
The Ziegler probate inventory shows a large array of Household Furnishings, but does not list any clocks. The inventory does list "Stove \& pipes," but this cannot necessarily be linked to a German ethnic preference because English speaking people also had stoves. Some other listings for Household Furnishings in the Ziegler probate inventory are for bedding, desks, beds, cupboards, chests, and other pieces of furniture, as well as the linen table clothes and bed spreads. The archaeological data did not include any material that can be identified as household furnishings.

\section{Clothing}

In the English newspapers reviewed, advertisements for clothing made up 34 of the 187 advertisements (18\%). For the German newspapers, clothing advertisements made up 20 out of the 173 German advertisements (12\%). The German-English newspaper had 6 advertisements for clothing out of 86 advertisements (7.0\%). Shown below are two Tables; Table 4.12 presents the clothing types that appeared in the English newspapers and Table 4.13, the clothing types in German newspapers. 


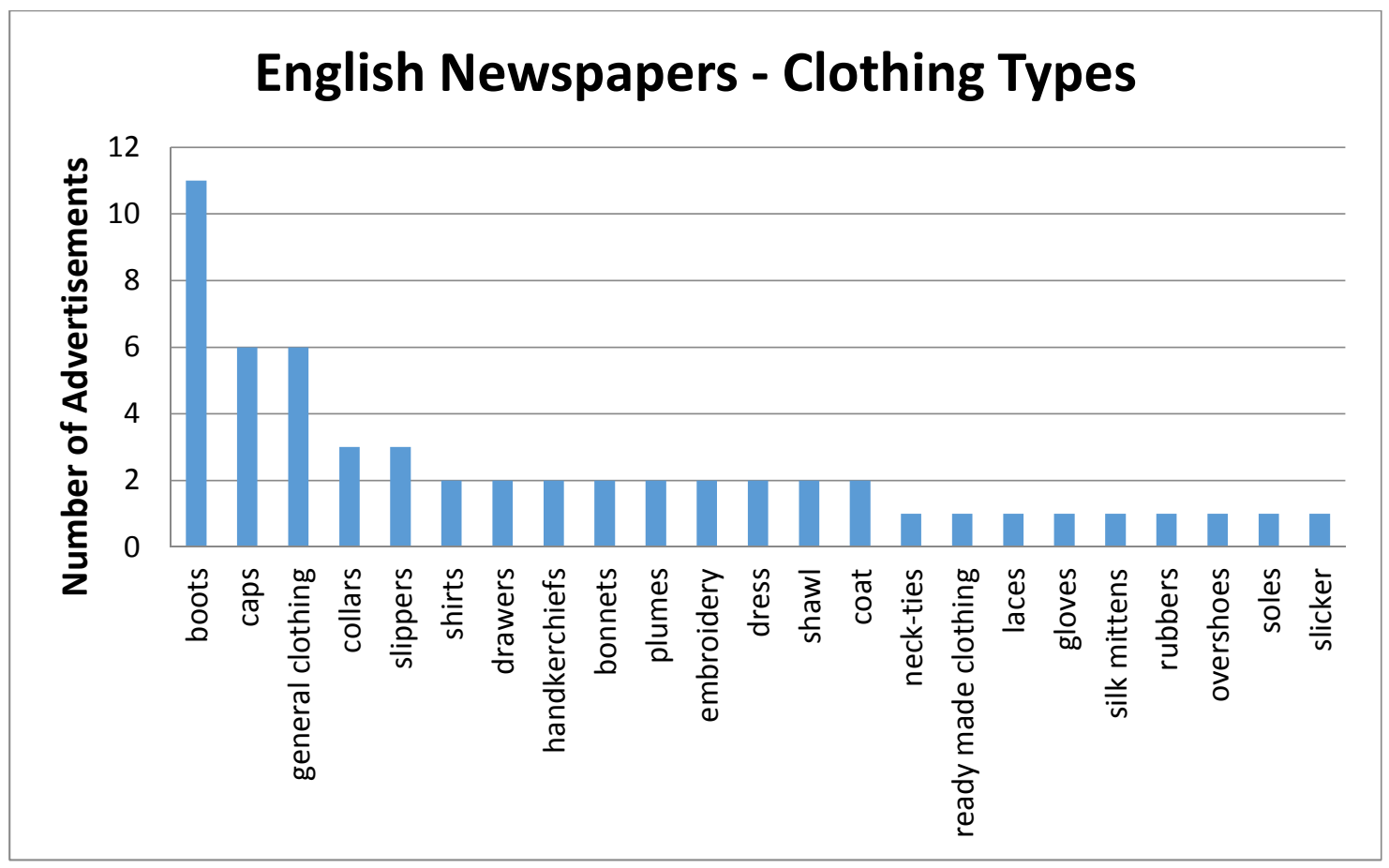

Table 4.12. English Newspapers Clothing Types 


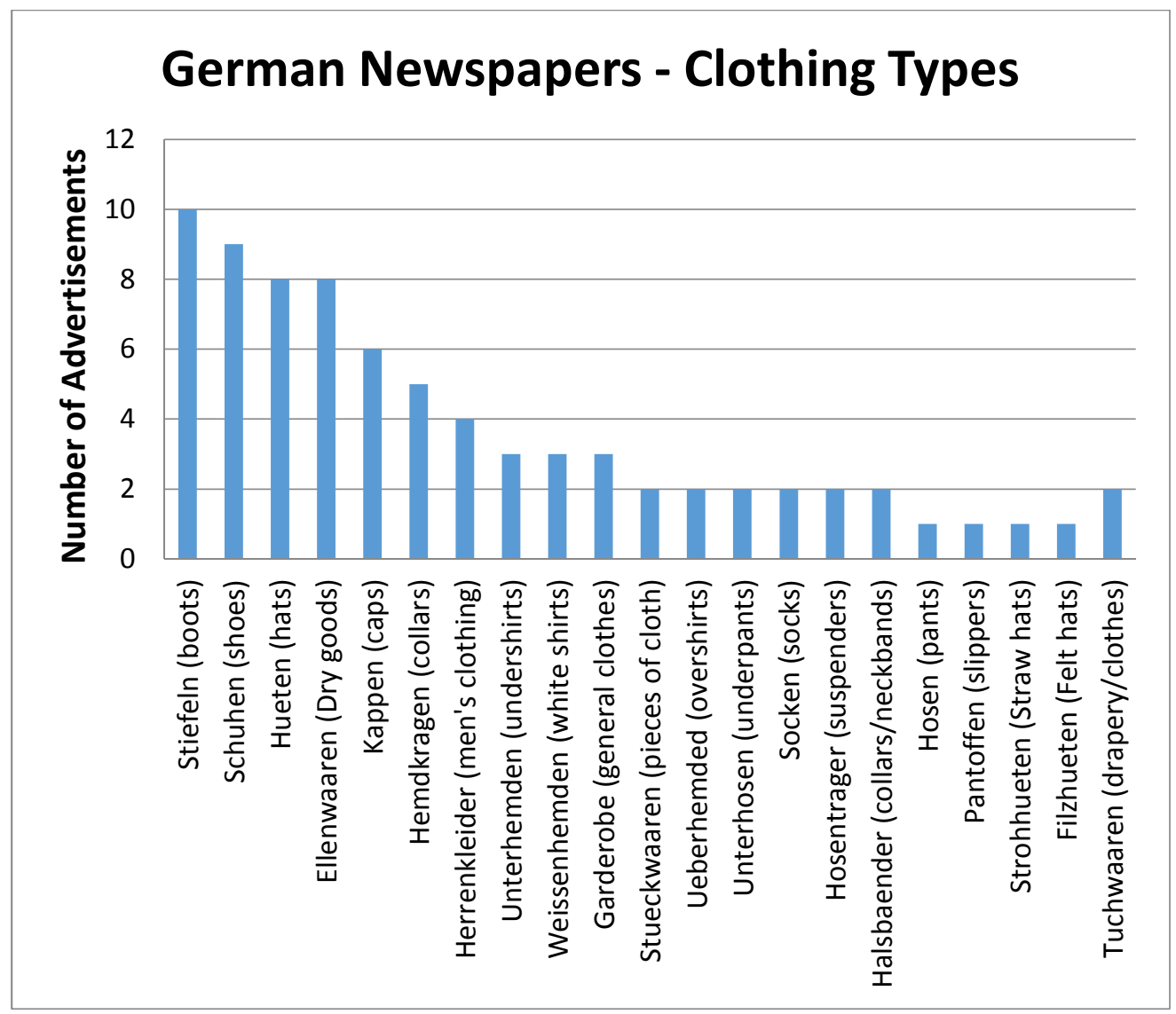

Table 4.13. German Newspapers Clothing Types

The English newspapers advertised for both men and women's clothing, such as lace, dresses and bonnets for the women, and neckties for the men (Figure 4.4), while the German newspapers only advertise for men's clothing (Herrenkleider) items, such as suspenders (Hosentrager), caps (Kappen), and pants (Hosen) (Figure 4.5). 
Mrs. F. Leavenworth,

\section{MILLINER :}

AND DEALER IN

Ladies Furnishing Goods,

Bonnets Hat Cossistiva oy

Plumps, Tibbons, Collarb.

$\rightarrow$ Embroidery, \&c.

Cuil and examine her syock. t. 2is.

Figure 4.4. Mrs. F. Leavenworth Milliner! from Fair Play 23, 1875

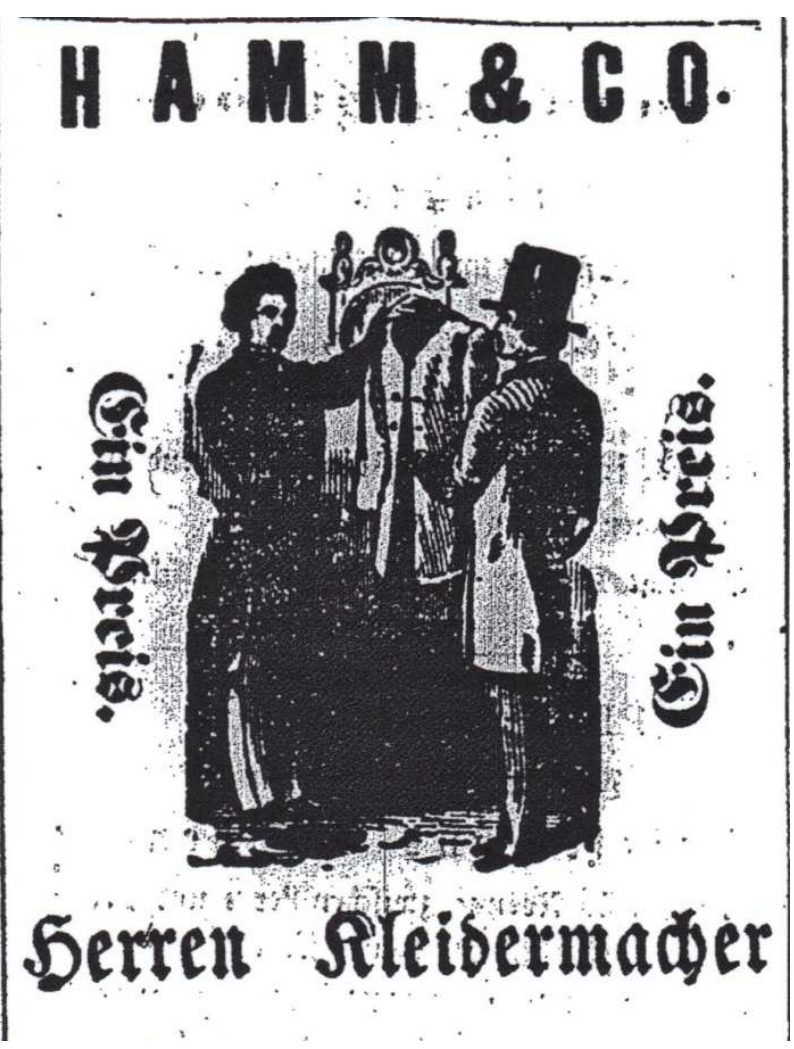

Figure 4.5. Hamm \& Co. advertisement from Freie Presse July 22, 1876

The German-English Herold newspaper did not specify gendered clothing.

Purely based on the newspaper analysis, it was suggested two things: First, perhaps the emphasis on men's clothing in the stores reflects the fact that there were a lot of single German men who did not have a mother or wife to make their 
clothing, or perhaps German women were making the majority of their family's clothing, or at least making their own and their children's clothing while the men bought ready-made clothing. This observation also comes from the eight newspaper advertisements listing Ellenwaaren. Ellenwaaren stood for dry goods, mainly cloth that could be measured from the tip of the finger to the elbow. This word was commonly used throughout the nineteenth and twentieth century by Germans and German-Americans but the term is no longer used today. The English newspapers did not have advertisements for anything similar to Ellenwaaren. They did, however, advertise for "ready-made clothing."

Therefore, it appears as though English-speaking men and women simply bought most of their clothing rather than producing their own like their German speaking neighbors appear to have been doing. This hypothesis is not only based on the types of clothing that were advertised in the newspapers but also the difference in frequency between the English and German newspapers. English newspapers had six percent more clothing advertisements than the German newspapers. This suggests that it was probably more economical for English newspapers to advertise ready-made clothing when compared to the German newspapers. Therefore, there was a much greater demand for clothing in the English-speaking community than the German-speaking community.

Another interesting difference was in the advertisements for hats. Though both the English and German newspapers advertised for hats and caps, the types of hats or caps were different. The German newspapers advertised for straw and felt hats. The English advertisements did not specify the type of hat or 
cap. One reason for this may be that German speaking people were said to have set themselves apart from their English neighbors by their appearance, including the use of certain hats. According to Carl Wittke (1952:61), German people not only wore large beards and mustaches which was different from the clean shaven Americans, but they also "wore caps or soft, slouch felt hats, in contrast with the high, stiff hats popular in the United States at the time." The advertisement for felt hats shows that this custom was also common in Ste. Genevieve in the nineteenth century.

In the Ziegler probate inventory, no specific clothing or ready-made clothing was listed. However, it listed 12 different types of cloth as well as a flax hackle. A flax hackle was used for aligning the long fibers of the flax before spinning. This was a common item that German families brought with them when they immigrated to America (van Ravenswaay 1977:83, 86). Textile production remained popular amongst Germans living in America even in the years following the Civil War because the cost of fabric was so high (van Ravenswaay 1977:445).

The archaeological data includes 20 clothing items: nine straight pin fragments, four button fragments, three hook-and-eye fragments, two tinkling cones or bangles, and two eyelet fragments. After reviewing the archaeological data and the probate inventory along with the newspaper analysis, it appears as though the Ziegler family was producing much of their own clothing. This supports the newspaper analysis and what could be expected from a German family living in Ste. Genevieve. 


\section{Personal Items}

The next category in the newspaper analysis was Personal Items. The English newspapers advertised for Personal Items in 30 out of 187 advertisements (16\%). The German newspapers advertised for Personal Items in 33 out of 173 advertisements (19\%). The German-English newspaper advertised for Personal Items in 18 out of 86 advertisements (21\%). The English newspapers had many various Personal items listed from books, stationery, and watches (Table 4.14).

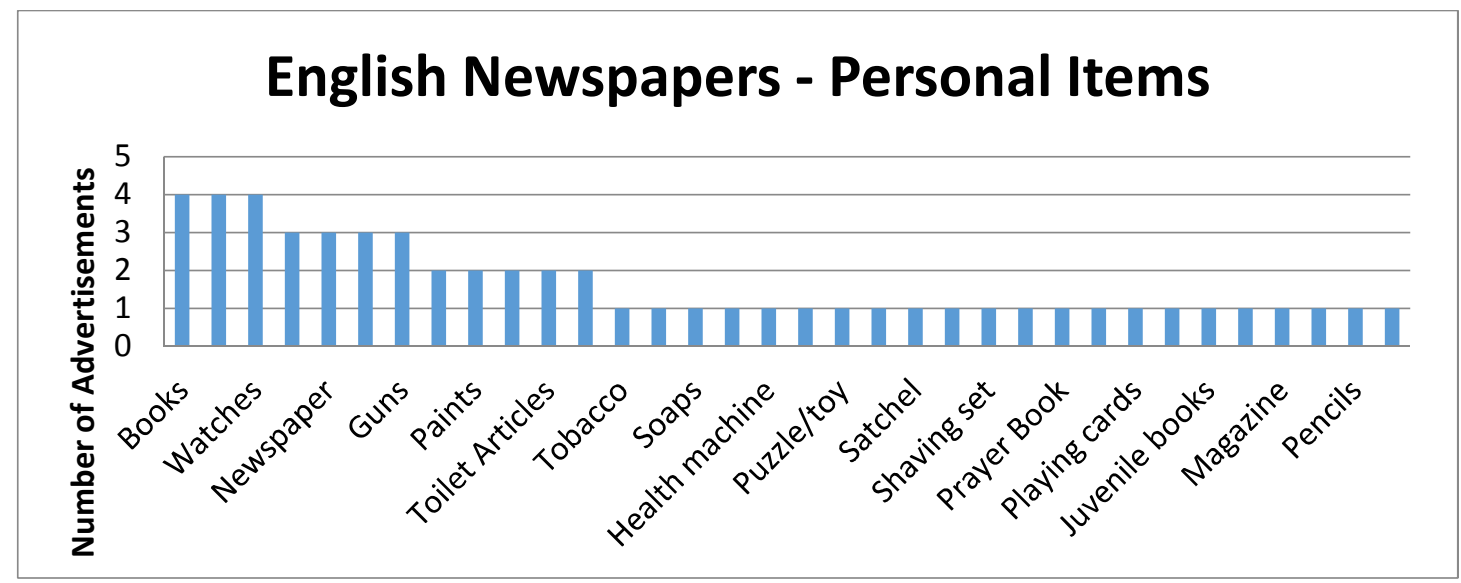

Table 4.14. English Newspapers Personal Items

The German newspapers had more advertisements for personal items, but considerably less variety. The main personal items advertised in the German newspapers were cigars, musical instruments, and pocket watches (Table 4.15). 


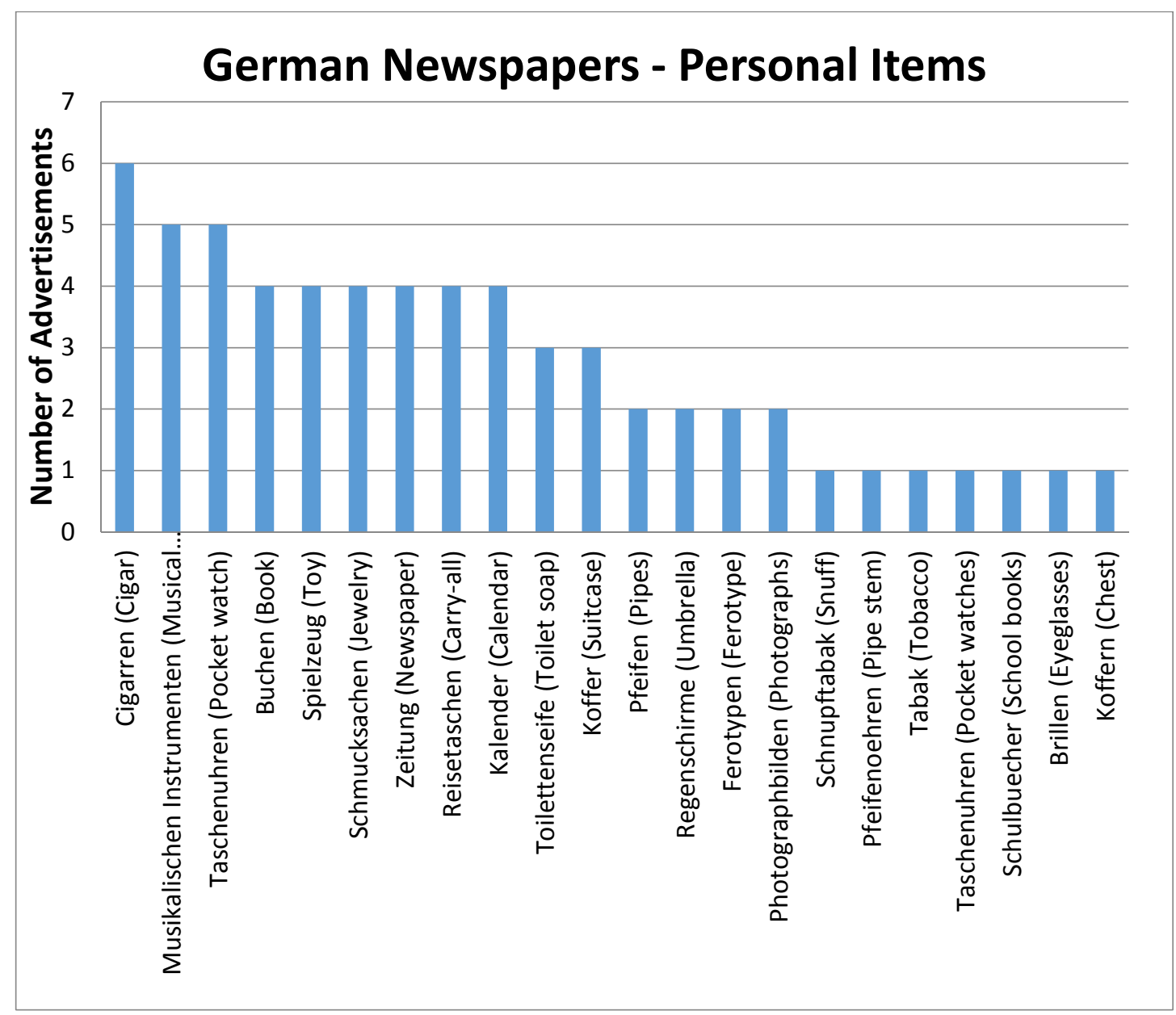

Table 4.15. German Newspapers Personal Items

The English newspapers advertised for guns, which the German and GermanEnglish newspapers did not. Figure 4.6 shows an advertisement for guns from one of the English newspapers.

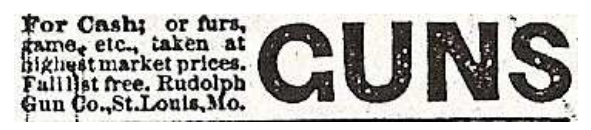

Figure 4.6. Rudolph Gun Co. from Fair Play December 23, 1875 
In addition, the German newspapers had two advertisements for pipes, and neither the English nor the German-English newspaper advertised for this item.

Figure 4.7 is an advertisement for pipes in the German newspaper.

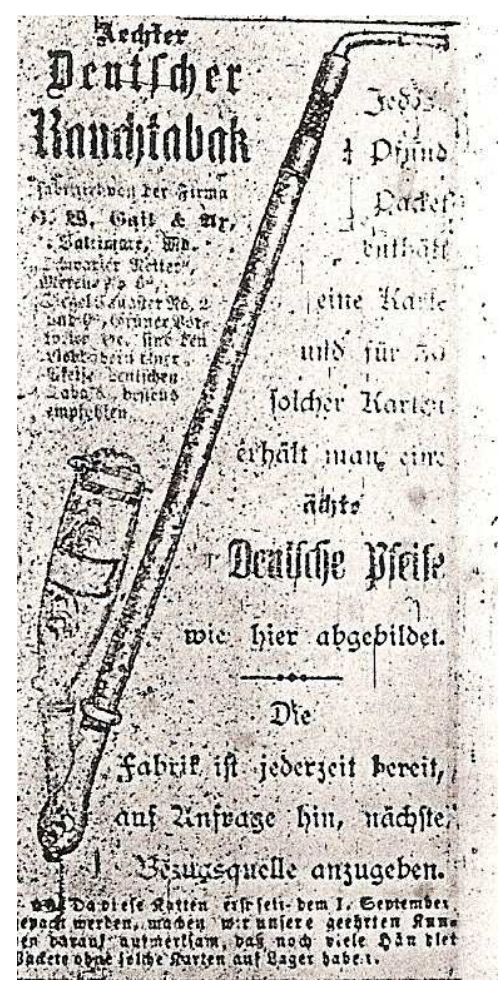

Figure 4.7. Dealsche Pfeife Advertisement from Herold Dezember 17, 1892

All three types of newspapers had advertisements for cigars and tobacco supplies. The Zieglers were known to have sold these products; however, they did not advertise their business in any of the newspapers that were reviewed considering the Ziegler family no longer ran their tobacco business after the 1850s.

The German newspapers advertised for calendars while other newspapers did not. German newspapers may have advertised for calendars 
because of the number of German farmers in the community. There were French and "American" farmers too, though the emphasis on calendars seems to have been a German practice. The German-speaking farmers may have used calendars and almanacs to track the planting and harvesting trends for their area. The German newspapers also advertised for musical instrument in five of the 33 personal item advertisements (15\%) versus two of the 30 personal item advertisements in the English newspapers (6.7\%). The advertising for musical instruments seems significant because many German choirs or musical groups were established upon arrival in America. Musical instruments were not only commonly transported with the Germans when they moved to America, but some craftsmen who produced these items moved to America and continued their work (van Ravenswaay 1977:395). Upon arrival in America, Germans worked as music teachers to wealthy families to make money. They introduced German terminology and a love for fine music to Americans (Wittke 1952:296).

The Ziegler probate inventory has only a few listings for Personal Items. The inventory includes: looking glasses, a silver watch, a cotton umbrella, volumes of Prussian wars, an atlas, a double barrel gun, "com. shotgun," and swords. However, the volumes of Prussian wars, show the Zieglers had an interest in European history, especially the history of other German speaking kingdoms. Books in general were a popular item that German immigrants transported with them to America (van Ravenswaay 1977:81). The inventory does not specify whether the books were in German or English or if they were books brought over from Germany; however, the books' inclusion in the inventory 
suggests their importance with the Ziegler family. Also, this shows that the Zieglers were able to read the German newspapers in the area, most probably the newspaper included in the research. Unfortunately, it cannot be determined whether any of these items were brought to America when the Ziegler family immigrated perhaps ten years earlier. The archaeology includes mainly jewelry items, such as beads and wire. Since the Ziegler family worked in the tobacco business, the discussion of the tobacco findings will be in the section of Household Production Activity-For Sale, since the Ziegler probate inventory included equipment for making cigars and snuff. One last note, the lack of pipes in the archaeological record at the Ziegler property suggests that perhaps the Zieglers might not have used their own tobacco or perhaps they did not use pipes, but cigars, snuff, or other tobacco products that would not turn up in the archaeological record.

\section{Household Production Activity}

The next category is Household Production Activity, that is, production activities for the use of the household. The English newspapers advertised for household production activity items in 12 out of 187 advertisements $(6.4 \%)$. The German newspapers have 23 advertisements for this category out of $173(13 \%)$. The German-English newspaper has three advertisements for household production activity items out of 86 (3.5\%). The English newspapers advertise for notions, saddlery, and sewing machines, as well as several other products (Table 
4.16). Figure 4.8 is an example of an English newspaper advertisement for saddlery.

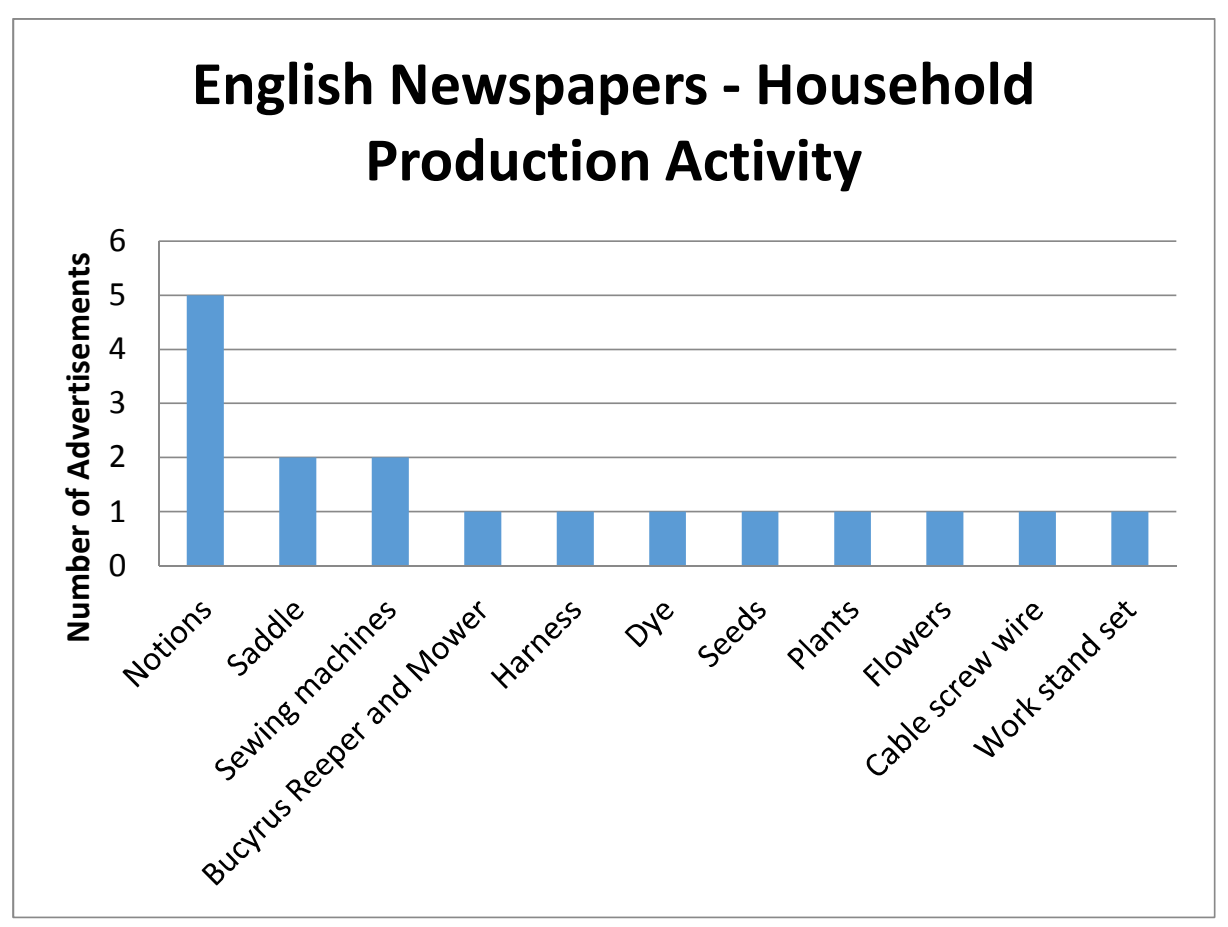

Table 4.16. English Newspapers Household Production Activity 


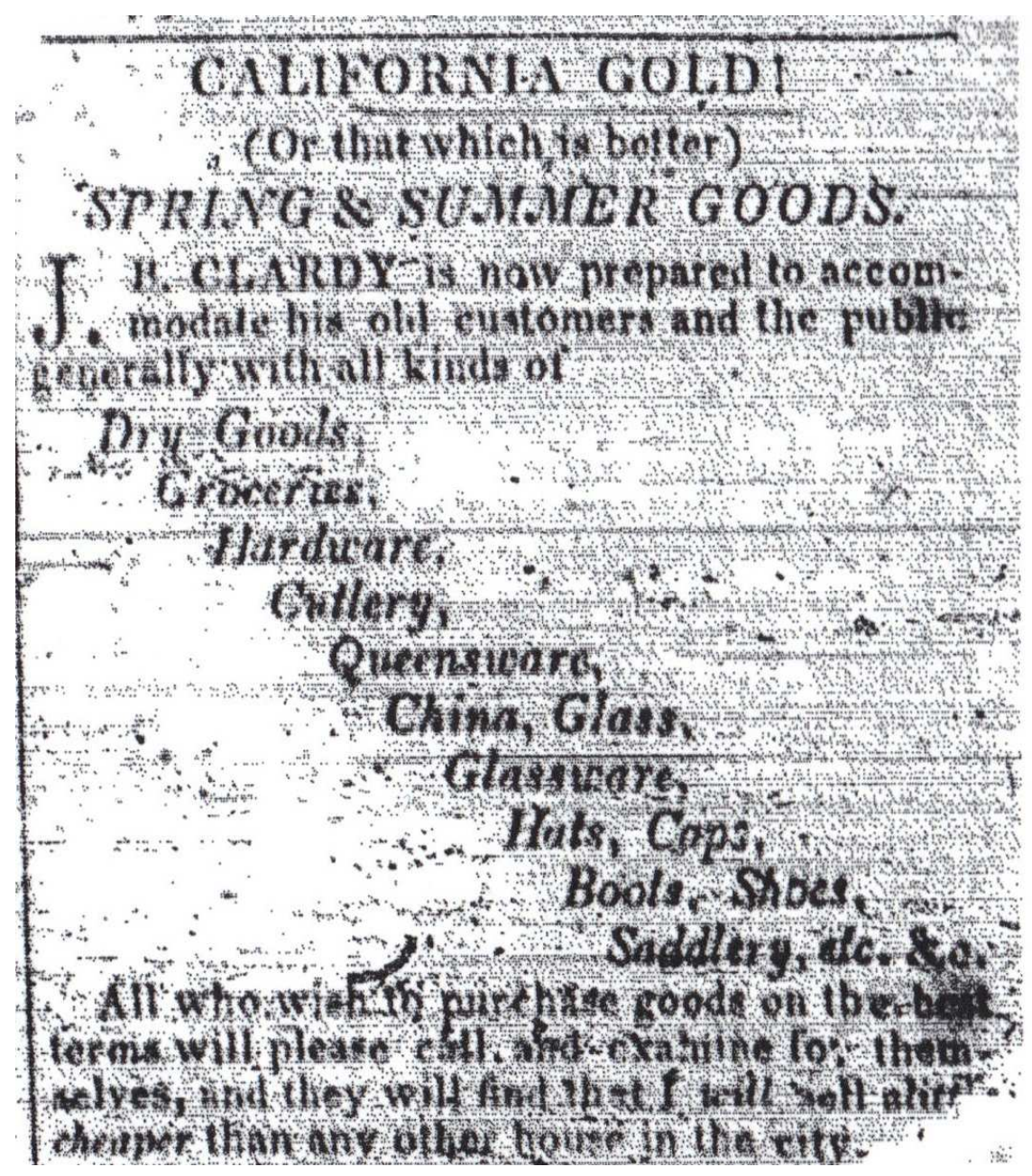

Figure 4.8. California Gold! Advertisement from Ste. Genevieve Democrat June 8,1850

The German newspapers advertised for considerably more farming equipment than the English newspapers (Table 4.17). 


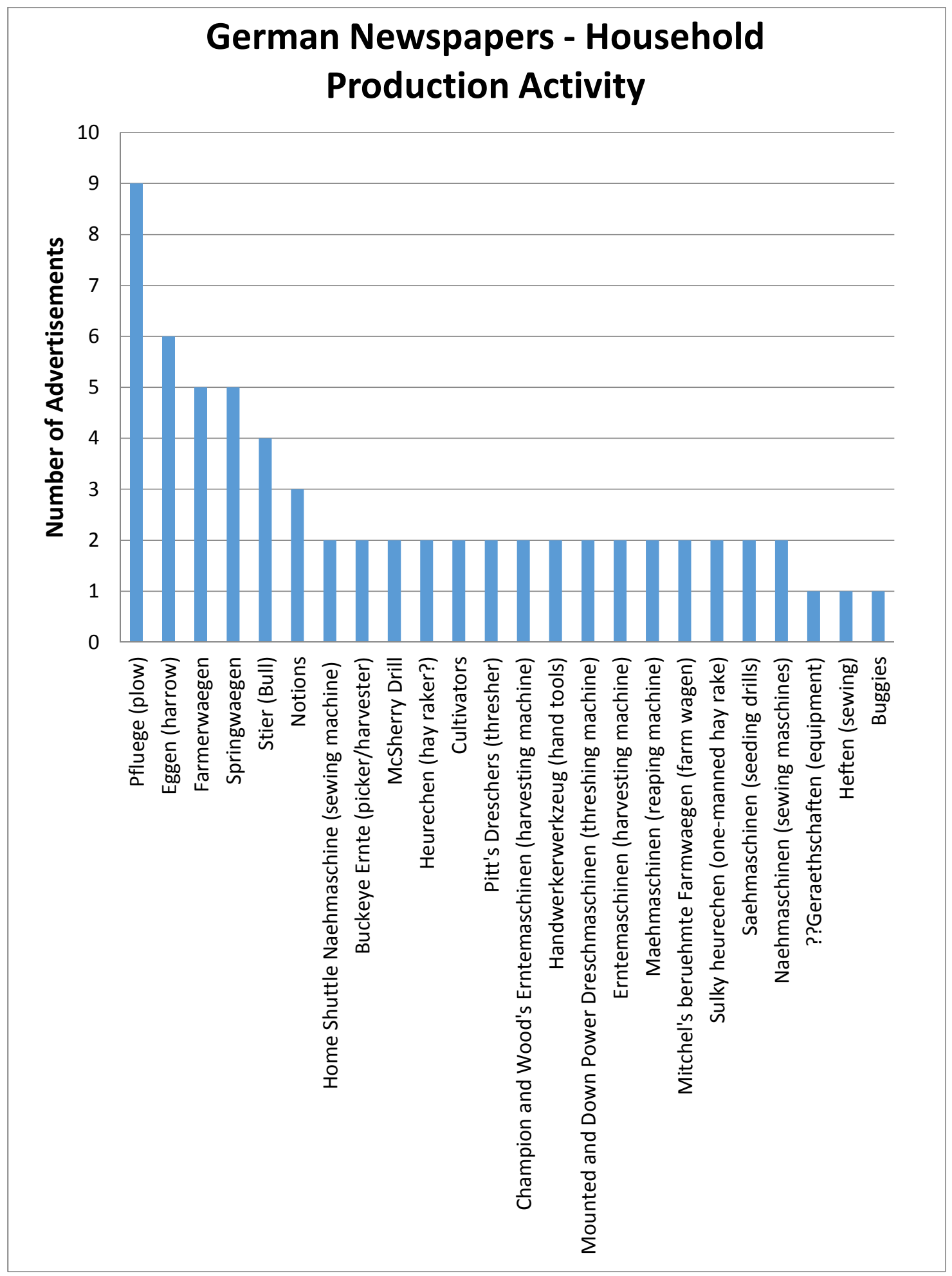

Table 4.17. German Newspapers Household Production Activity 
The English newspapers only advertised for one reaper and mower, while the German newspapers advertised for plows, harvesters, wagons, harrows, threshers, cultivators, and a hay-raker. Figure 4.9 is an example of the many kinds of farming equipment advertised in the German newspapers. 


\section{Agricultural Warehouse}

\section{, bon \\ Meter $\mathfrak{B i l d}$ er,}

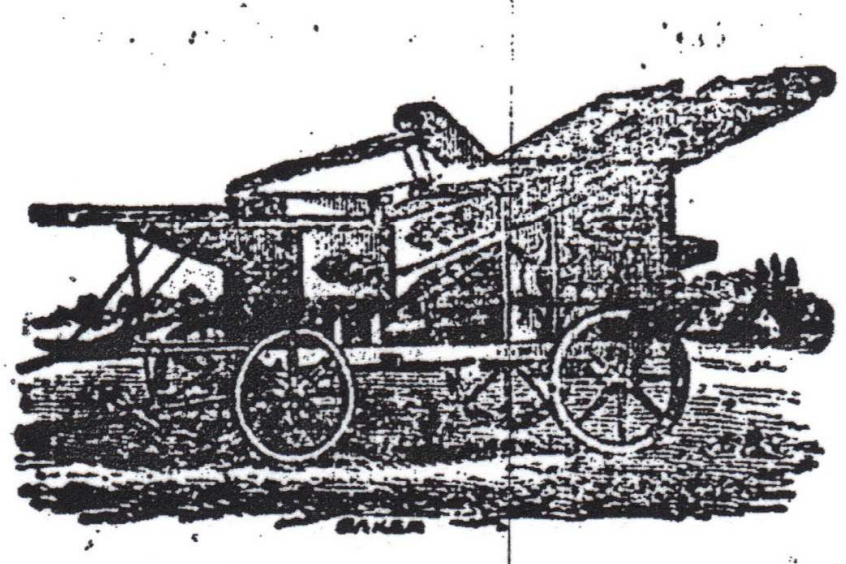

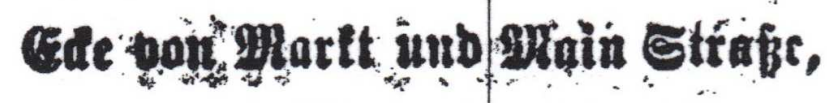
STE. GENE YIEVE, Mo.

hält ftet vorräthig :

Mounted and Down Power Drefd= majhinen, \$reis $\$ 450.00$ Biet veridiebene Sorten Ernte: und

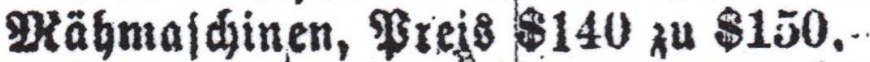

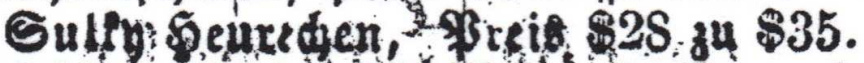
Säbmaldfinen (Drills), Gutey uno alle anbern Sorten Pflinge, Mritdel!'s

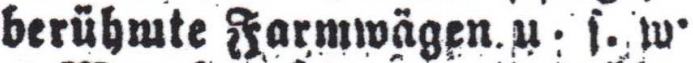
Dan fprede bôr uno prüfe.

Figure 4.9. Agricultural Warehouse Advertisement from Freie Presse July 22, 1876

The German-English newspaper advertised for only three items: barrows, sewing machines, and wagons. Based on the advertisements between the 
English and German newspapers, it appears that Germans were more focused on farming and buying more farming equipment than their English neighbors. This supports historical accounts that Germans were enticed to move into the area due to its rich farmland. For this reason, one would anticipate not only the advertisements for farming equipment but also the existence of farming equipment in the archaeological records of German family homes in Ste. Genevieve. Wagons were also a common item among German-American farmers (Wittke 1952:7). Fennell (2003:153) notes that in the mid-eighteenth century many German-American families owned some sort of wagon for the purpose of moving their surplus crops to larger markets in the city. This piece of equipment was vital for their economic sustainability and growth. For these reasons, farming equipment was perhaps more desired by the German families in Ste. Genevieve and was more frequently advertised in the German newspapers.

Notions and sewing machines were another area of focus in this category of Household Production Activity. The English newspapers have five advertisements for notions (29\%), and two advertisements for sewing machines (12\%). The German newspapers have four advertisements for notions $(7 \%)$ and four for sewing machines ( $7 \%)$. The reason for the lower percentage of these items in the German newspapers compared to the English newspapers was the abundance of farming equipment advertised in the German newspapers in this category. If the farming equipment is taken out of the picture, the household production advertisements in German newspapers would include 23 percent 
sewing machines and 22 percent for notions. Sewing machines appear to have been more popular with the German community than the English. When looking at this category, Germans appear to be performing more household production than the English. English may have used outside services, especially with food production. The German community appears to be more interested in farming and growing their own food, while the English may have been purchasing grown or produced food. As far as clothing production is concerned, the sewing machine correspond to the findings in the clothing category. The English were likely purchasing more pre-made clothing, using notions for small adjustments or the fixing of clothing, while German families may have been producing more of their own clothing in the home with sewing machines. Gottfried Duden (1980:69) reported that women made their own family's clothing throughout the 1820 s in Missouri, with concern for the popular fashions at the time. They would raise their own sheep, do their own spinning, as well as sewing their own clothes. This may have held true for much of the nineteenth century, according to the newspaper analysis. The archaeological data also shows evidence of clothing production or repair with hook and eye pieces, buttons, hooks, and eyelets; the flax hackle is listed in the inventory, which was explained more fully in the clothing category section.

The archaeological data also shows proof of food production at the Ziegler house. "Tumbled" materials make up 34 out of the 74 listings in the Household Production Activity category, amounting to 149 pieces found in the archaeological record. Gastroliths are stones ceramic sherds, or glass fragments 
which chickens, geese, and other types of poultry pick up and keep in the gizzard to break down food. Eventually these materials become smooth and have a polished appearance. The majority of the gastroliths were found in unit 150R0 (82 pieces), located on the southeast portion of the property. In unit 110R30, 56 gastroliths were found, located directly east of the house. Both of these units would be considered the "backyard" of the Ziegler house. Unit 40R60, located in front of the Ziegler house had only 11 gastroliths. This suggests that the Zieglers kept the geese in their "back yard," restricted perhaps by some sort of fence. With the appearance of these gastroliths in the archaeological data as well as the listing of geese in the inventory, it is clear that the Zieglers raised geese. Geese were a common sight in Pennsylvania German yards as well. They were used for their meat and goose grease, Gensfett. The fat was used for making shortening, medication, and spreads, while the feathers were used for making pillows (Long 1972:52). The inventory also lists beds and mattresses. These were probably made by the Zieglers using goose feathers. According to the Ziegler probate inventory of 1836, they owned 60 heads of geese, which would be a great source of feathers (SGA 1836). The inventory also lists wine bottles (apparently empty), which were often re-used, especially for food and medicine preservation; these will be discussed later in the chapter. Therefore, within the category of Household Production Activity, the Zieglers appear to have been participating in food and clothing production, supporting the newspaper analysis for a typical German speaking family living in Ste. Genevieve. 


\section{Household Production for Sale}

This category looks at production activities carried out for sale, outside of the household. Very few advertisements fit within the category of Household Production for Sale. In the English newspapers, only one advertisement out of 187 would be considered for Household Production for Sale (0.5\%). The German newspapers had two advertisements out of 173 (1.2\%). The GermanEnglish language newspaper did not have any advertisements for Household Production for Sale.

One advertisement that does stand out was in the German newspapers, where products for Wagenmacher und Schmiederbedarf (wagon maker and blacksmith needs) were advertised. This suggests that some German craftsmen immigrated to America and continued their trades or picked up trades upon arrival. Charles van Ravenswaay (1977:302-303) pointed out that several reports or letters from Germans living in Missouri, encouraged the emigration of German craftsmen because their skills would be of great use and economical in the New World. These crafts included wagon makers, blacksmiths, carpenters, tanners, and furniture makers.

Unlike the newspaper advertisements, the majority of the items in the 1836 probate inventory are related to household production for sale: the Zieglers' tobacco business. The inventory lists 42 tobacco business related items in various amounts. The Zieglers purchased tobacco from several locations, such as tobacco from Spain or Havanna, tobacco from St. Domingo, and Natchitoches snuff. The goods and products from Mathias Ziegler's tobacco business 
circulated throughout Ste. Genevieve from the Caribbean and lower Louisiana, aided by Ste. Genevieve's advantageous location on the Mississippi River. The archaeological data does not include any tobacco-related items, but the business ledgers and the inventory show clear evidence of the Ziegler tobacco business. The Zieglers could have brought their tobacco business to the New World with them, like the many other German craftsmen who were immigrating at this time to America. The archaeological data for the category of Household Production for Sale includes 232 pieces of lead shot found in the three units used in the research, so the Zieglers may have been making and selling lead shot along with their tobacco business. However, gun-related items only appear in the English newspapers ( 2 out of $187 ; 1.1 \%$ ) and not in the German newspapers, so this activity does not appear to be particularly "German" in association.

\section{Pharmaceuticals}

In one of the first archaeological studies of German immigrants, Elliott and Elliott (2002) interpret the archaeological appearance of pharmaceuticals as an ethnic marker for Germans, since they were ahead of their time in medicine development. For this reason, a pharmaceutical column was added to the data analysis. More advertisements were expected to be found in the German newspapers, versus the English papers, in addition to a plethora of pharmaceutical bottles and other items in the inventory and archaeological record. Surprisingly, quite the opposite has been found. In the German newspapers the only pharmaceutical-related advertisements were for medical 
pamphlets titled "Manhood" and "Womanhood" by Dr. Whittier, totaling only two advertisements for pharmaceuticals out of the 173 total advertisements (1.2\%) (Figure 4.10). 


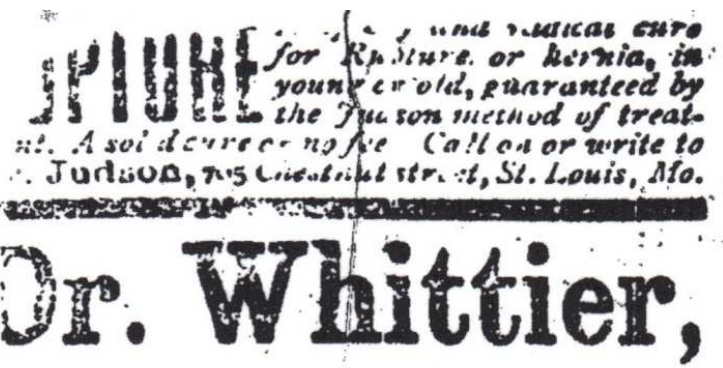

'7 St. Charles streel, St. Louis, Mon.

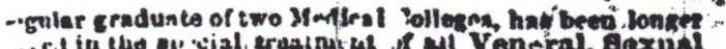

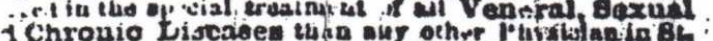

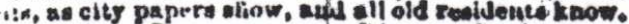

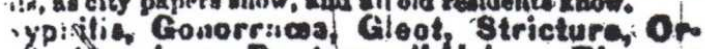
. fis. He pnis or Rupture all Urinary Diseseses 1 Syphil-tio or morcurial atioctions of the

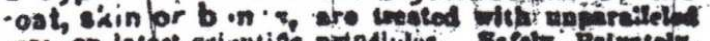

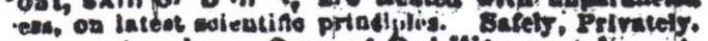
ipormaturrhos, Sexual Debitity and impoto

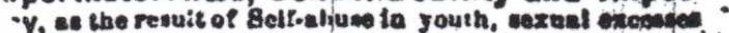
sturer y esch, or other onuwre, and which produos goeme

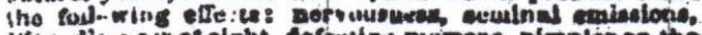

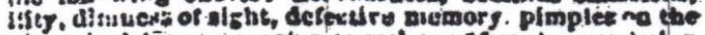

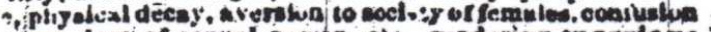

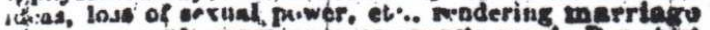

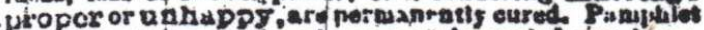
(2) pap

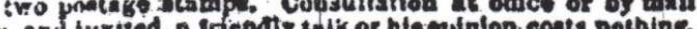
Juen

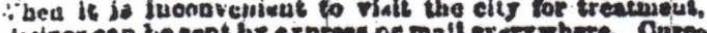
'beinew en hie ank bs express or mall erery where. Oure

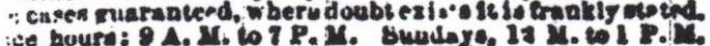
!'amphlet, to say address, for Two Stampe.

- ATTOOD All about 1t, nent by mell I ANHOOD Hor 10 conts TOM ANHOOD in i anhood and Wemanhood ia Berman, both jniher, Ithuirated, 15 Conts.

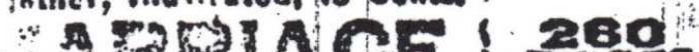

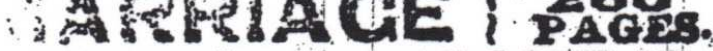

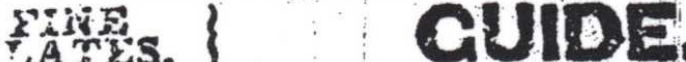

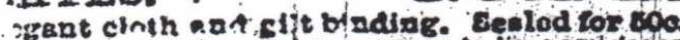

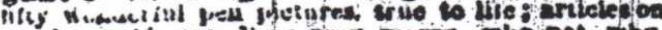

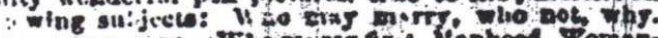

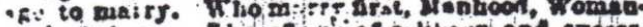

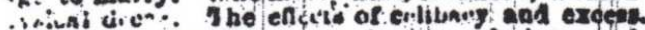

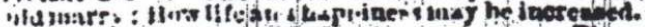

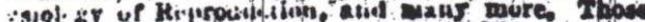

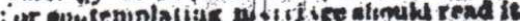

Cut a te to to

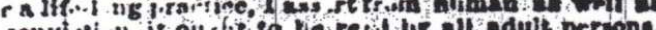

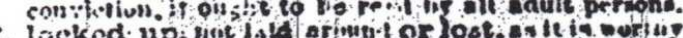
- locked up, vit lald arintit or lost, as fe if wortug

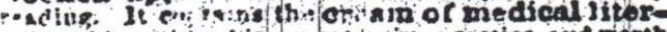

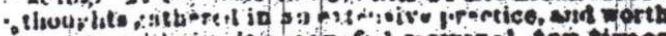
$\therefore \rightarrow$ uld wibl givo il a ciruful peruad, ten timen

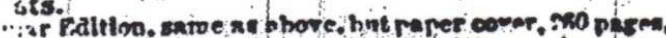

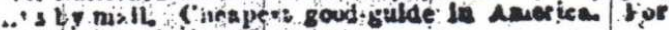

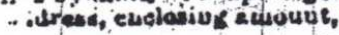

DR. MHITTIER,

$\because$ Charles strect, St. Louts, Mo.

Figure 4.10. Dr. Whittier Advertisement from Freie Presse July 22, 1876 
However, the English newspapers had 20 pharmaceutical advertisements (11\% of the total 187). Dr. Ayer \& Co. manufactured the majority of the pharmaceuticals advertised in the English newspapers. Figure 4.11 shows Ayer's Cherry Pectoral advertisement.

\section{CURED}

"About seren or eight months ago I was attacked by a cough, atit at once began to take a medicine much advertised as ${ }^{\top}$ an expectorant, and continued using it until $I$ had taken about six bottles. Instead of glving me relier, it only mado mo worse. I I tried several other remedies, but all in yain, and I don't think I had three whole nights' rest during my.llness. I began to think that

\section{Consumption}

had laid hold of me, and $\mathrm{my}$ hopes of rcoovery were all gono. I was a mere skeloton, but a friend of mine, who had been some timo away, called to seo me. - Ho recommeaded me to try A.yer's Cherry Pectoral, and kindly sending mo a bottle, I took it, but with littlo hopes of recovery. I am thankful, how. ever, to say that it cured me, and.I ain to-day enjoying tho best of bealth." J..Wilmot Payne, Monrovia, Liberia.

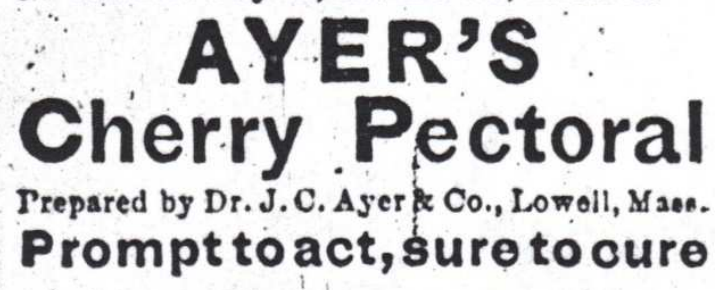

Figure 4.11. Ayer's Cherry Pectoral Advertisement from Herald December 17, 1892 
In the German-English newspaper, there were 16 advertisements for pharmaceuticals, and only one was in German (Table 4.18).

\begin{tabular}{|ll|}
\hline Medizinen & German \\
\hline Hood's Sarsaparilla & English \\
\hline Kenney's medical cure & English \\
\hline Dr. Taft's Asthmalene & English \\
\hline $\begin{array}{l}\text { Hale's Honey of [?dorehound] and Tar, Pike's } \\
\text { Toothache Drops cure }\end{array}$ & English \\
\hline Pike's Toothache Drops Cure & English \\
\hline "Brown's [Brauch?] [Troches?]" & English \\
\hline Chamberlain's Pain Balm & English \\
\hline The Sterling Remedy Co. Tobacco habit cure & English \\
\hline Cascarets candy cathartic cure & English \\
\hline opium, morphine habit cure & English \\
\hline opium and Whiskey habit cure & English \\
\hline Piso's Cure for Consumption & English \\
\hline Ayer's Sarsaparilla & English \\
\hline Ayer's Narsaparilla medicine & English \\
\hline Ayer's Pills & English \\
\hline
\end{tabular}

Table 4.18. German-English newspaper Pharmaceuticals

This means that pharmaceutical advertisements made up 17 percent of the advertisements in this newspaper, but only one listing was in German. This finding was unexpected because of the large number of German doctors in the United States in the nineteenth century. German doctors formed their own societies in major cities, including St. Louis, because many were excluded from Anglo-American doctors' societies and hospitals.

The Ziegler probate inventory shows a lack of pharmaceuticals as well. The record only lists one pharmaceutical-related item, a "Seringe" (SGA 1836). 
In the archaeological analysis, three pharmaceutical bottles were identifiable in the three units analyzed. It is interesting that Germans in Ste. Genevieve were not targeted for pharmaceuticals and the English-speaking population was.

Another explanation for the lack of pharmaceutical advertisements in German newspapers is that the Germans in Ste. Genevieve and elsewhere may have been depending on folk medicine rather than purchasing medicines. Don Yoder (1990) noted that folk medicine or "home remedies" were popular throughout the U.S., particularly in the Ozarks of Missouri. Folk healing otherwise called "powwowing" is best explained as a "magico-religious healing, on the folkcultural or traditional level, using words, charms, amulets, and physical manipulations in the attempt to heal the ills of man and beast" (Yoder 1990:96). Folk medicine was usually passed down from person to person or learned through recorded folk books. The first German folk medicine book was Romanus, first printed in 1788 (Yoder 1990:99). Many other books were produced in German and were later brought to America. The first of these books to appear in America was Der lang verborgene Freund, written by Johann Georg Hohman in 1820 (Yoder 1990:99).

Vance Randalph (1964) discussed how tobacco was used in the Ozarks to cure some common aches and pains:

Tobacco is used in other ways by the yarb doctors and granny-women. I have seen severe abdominal pain, later diagnosed as appendicitis and cured by surgery, apparently relieved at once with a poultice of tobacco leaves soaked in hot water. The Tobacco poultice is very generally used for cuts, stings, bites, bruises, and even bullet wounds. A poultice of tobacco leaves in cold water is often applied to "draw the pizen" out of a 
boil or a risen'. Some people think such a poultice is more effective if fresh mullein leaves are bound on outside the tobacco (1964:98-99).

This observation is more interesting when looking at the Ziegler family because they ran a tobacco business out of their home. Besides selling their tobacco products for smoking purposes, they could have sold it for medicinal purposes; they could also have been using their own product for home remedies, therefore explaining the lack of pharmaceuticals in the archaeological record. Other home remedies would also be less visible archaeologically, so the overall lack of pharmaceutical artifacts might be explained by this.

\section{Foodstuffs}

The next category in the newspaper analysis is Foodstuffs. The English newspapers had 23 advertisements out of 187 in this category (12\%). The German newspapers had 17 advertisements for Foodstuffs out of 173 (9.2\%). The German-English newspapers had 15 out of 86 advertisements (17\%). The English newspapers mainly advertised for general groceries, dry goods, and candies (Table 4.19). 


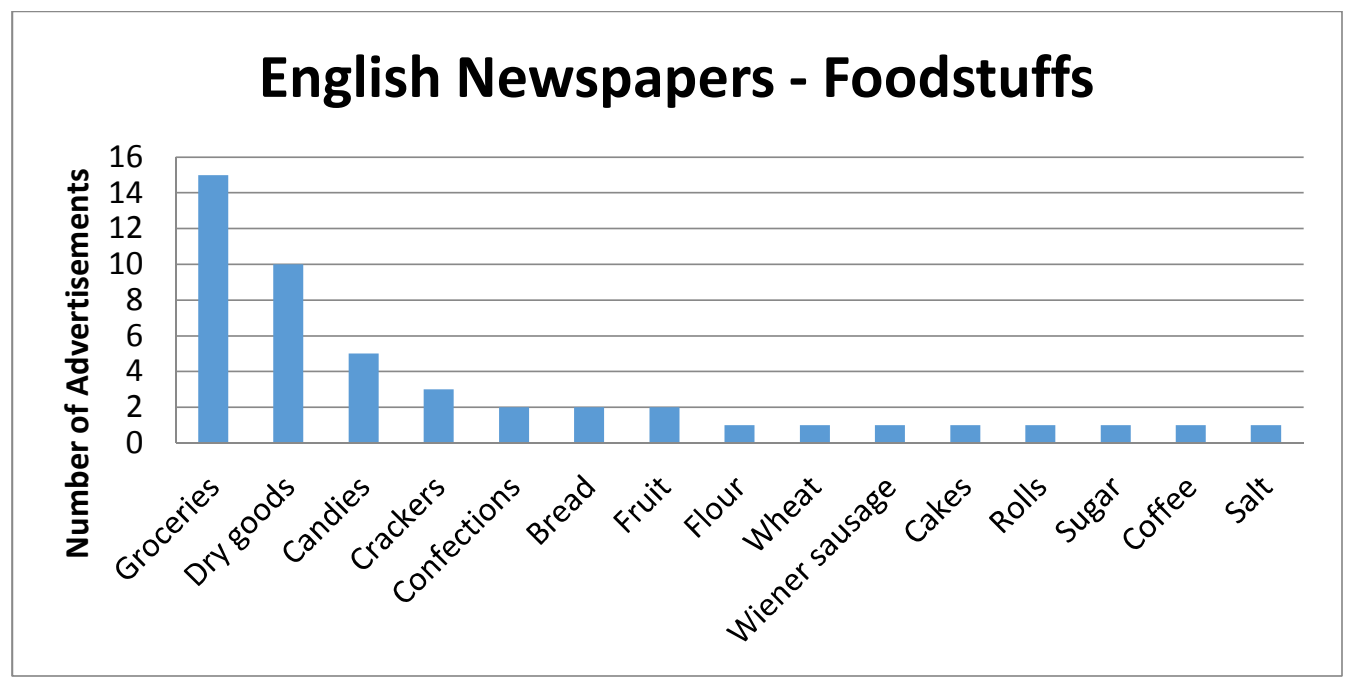

Table 4.19. English Newspapers Foodstuffs

They also included a large number of advertisements for baked goods such as cakes and breads, like the advertisement shown in Figure 4.12 for the Ste. Genevieve Bakery owned by M. Vieh. 


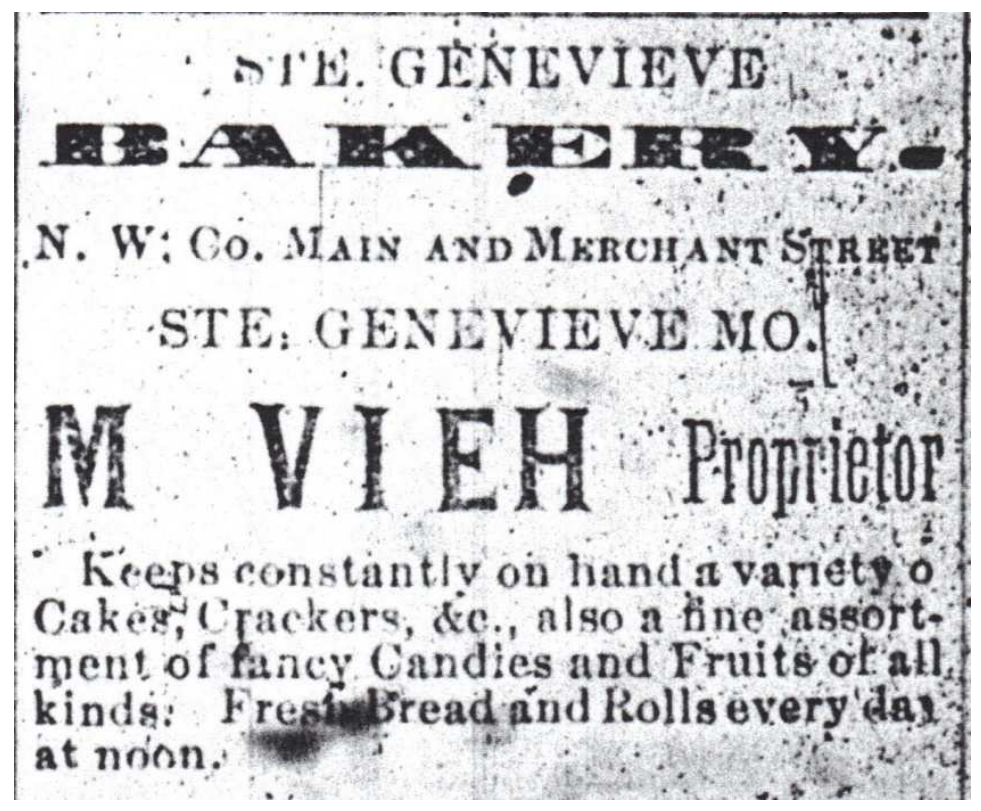

Figure 4.12. Ste. Genevieve Bakery advertisement from Herald December 17, 1892

The German newspapers advertised mainly for spices, general groceries, and coffee (Table 4.20), but they included many more specific products than the English newspapers. 


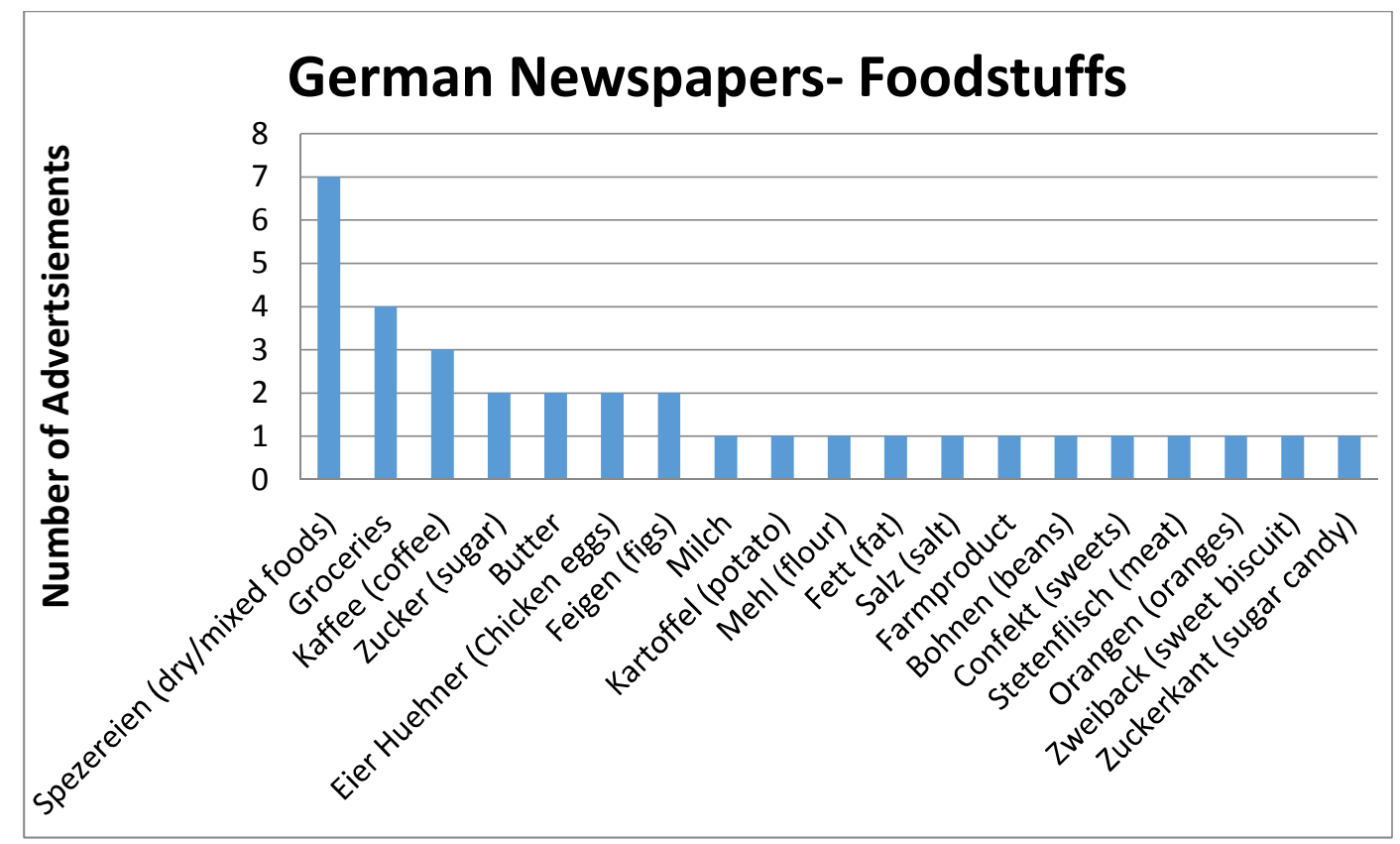

Table 4.20. German Newspapers Foodstuffs

One observation is that the German newspapers advertised for farm products such as eggs, potatoes and butter, while the English newspapers did not. This follows the pattern of the German newspapers advertising for farm equipment and the English newspapers not. The Germans appear to have done more farming and selling of their goods, using the newspapers to advertise their surplus goods. The English appear to have purchased already-made foods, such as breads. The German newspapers did not advertise for any baked goods, but 
rather the products that go into baked goods, such as flour, eggs, and butter.

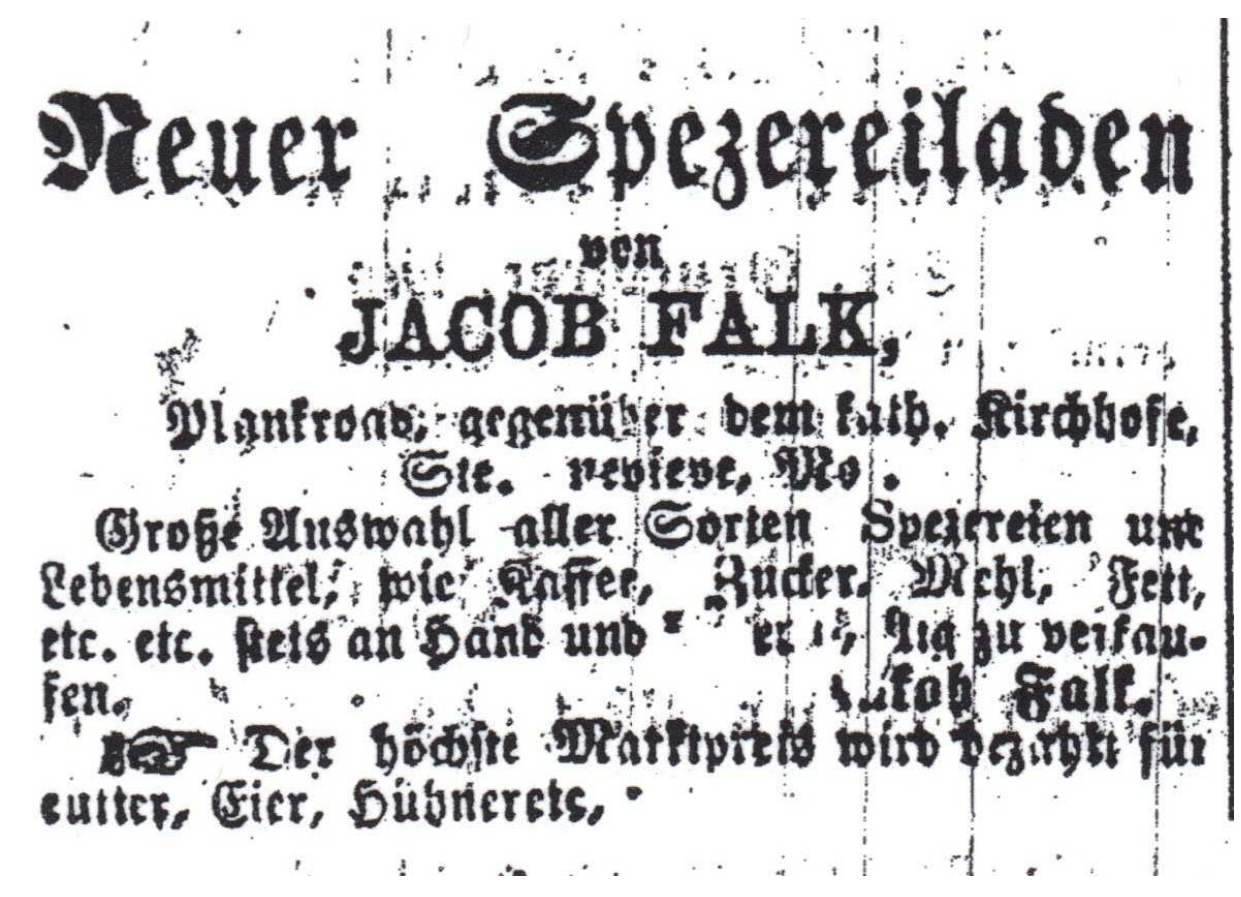

Figure 4.13. Neuer Spezereiladen advertisement from Freie Presse July 22, 1876

An example of this is shown in the advertisement for Neuer Spezereiladen by Jacob Falk (Figure 4.13). He advertised for items such as Zucker (sugar), Mehl (flour), and Fett (fat). This suggests that the German families may have produced more of their own baked goods rather than purchasing them like the English and French were doing. This agrees with other accounts of German families in the nineteenth century. Rachel Davis-DuBois and Emma Schweppe (1972:67) stated that "bakery and cooking are the pride of the Hausfrau [house wife]." Also, Gottfried Duden (1980:68) reported that fresh breads were produced daily in Missouri households in the 1820s. Faust (1927:2) also 
commented that, "The Germans have furnished the butchers and bakers in almost every large city of the United States, and that not alone within the German Belt. We need not single out large cities, for the same phenomenon can be observed in innumerable smaller towns" (Faust 1927:72). Therefore, many accounts verified that the German advertisements for Foodstuffs agreed with what was expected from the nineteenth century German family in not only Ste Genevieve, but other small towns and cities in the United States.

The inventory analysis for this category supports what one would expect from a German family in Ste. Genevieve. The Zieglers had four foodstuff items listed in their inventory: four Bels. dried apples, three flour barrels, and one lard stand. These items are farm related and are comparable to the observations from the German newspapers. Animal remains were recovered in the archaeological record, and while they are probably food remains, they have not been analyzed yet. Therefore, at least according to the Ziegler inventory, the family was most likely producing much of their own food, which one would expect from a German family in Ste. Genevieve at this time.

\section{Alcohol}

The last newspaper analysis category reviewed is Alcohol. In the English newspapers, nine advertisements out of 187 were for alcohol (4.8\%). In the German newspapers, 12 advertisements out of 173 were for alcohol (6.9\%). The German-English newspaper had five advertisements out of 86 for alcohol (5.8\%). 
The English newspapers advertised for general liquor, wine, and beer, as well as whiskies and brandies (Table 4.21).

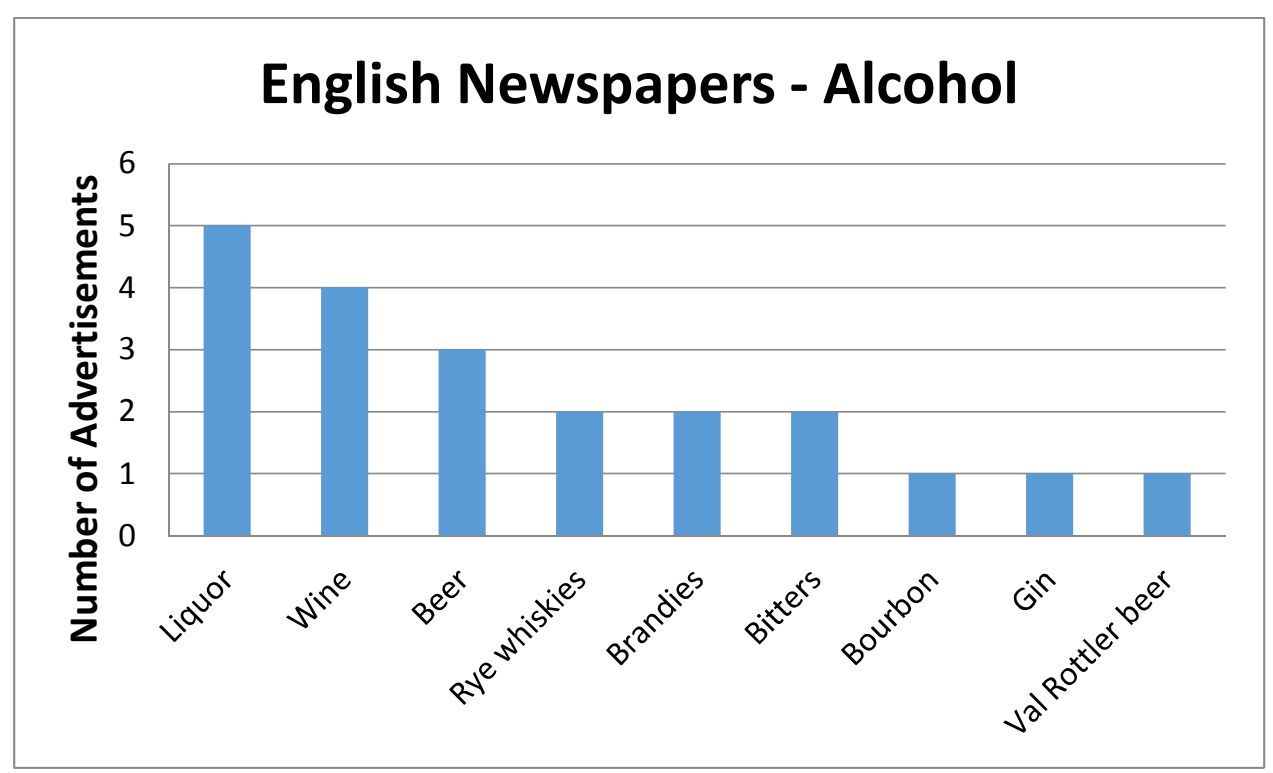

Table 4.21. English Newspapers Alcohol

The German advertisements were very general. They also advertised for mainly liquor, wine and beer; however, the English newspapers advertised a whole range of specific liquors, which the German newspapers did not (Table 4.22). 


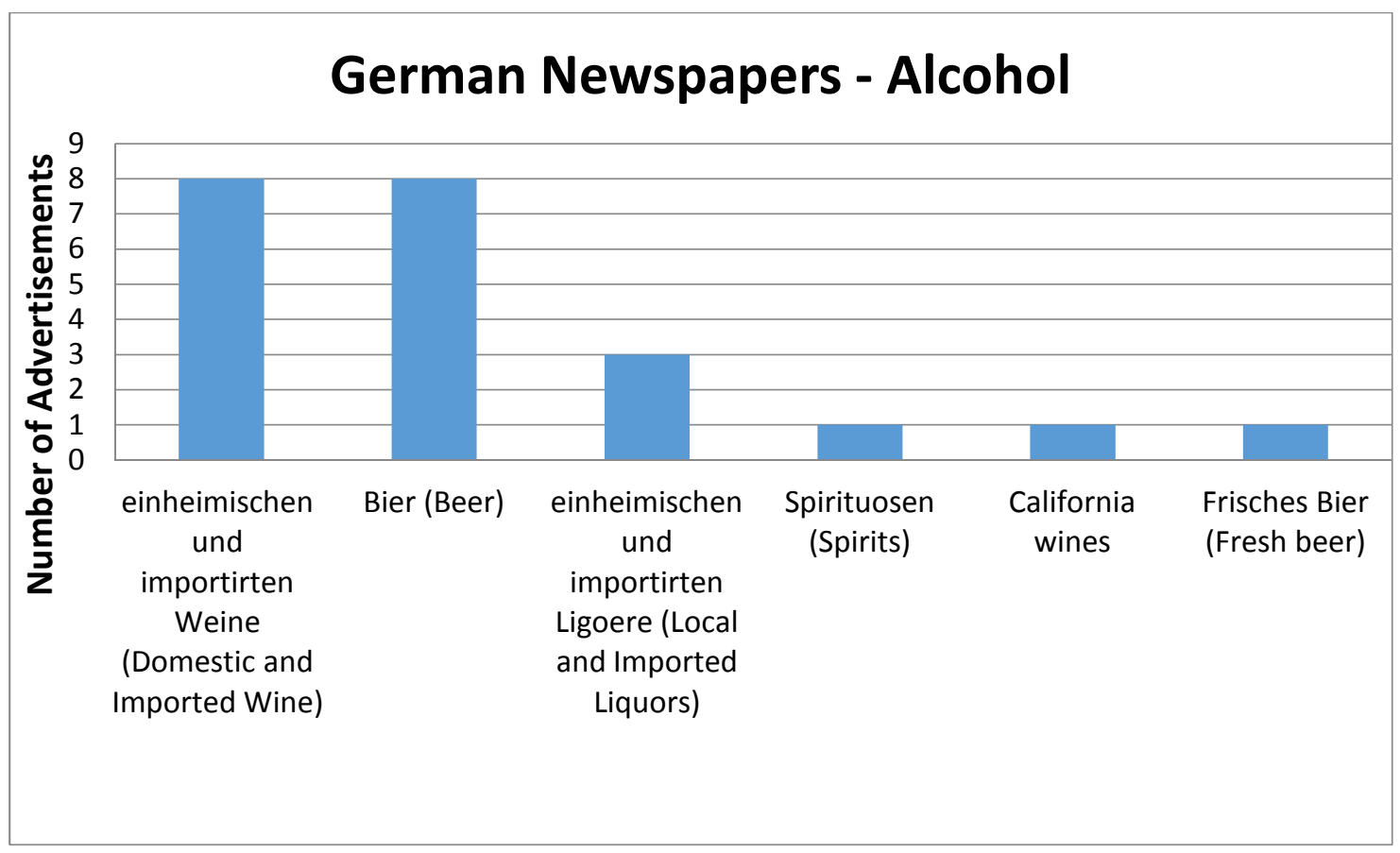

Table 4.22. German Newspaper Alcohol

In the German newspapers, $82 \%$ of the alcohol advertisements were for wine and beer. Only $38 \%$ of the English newspaper alcohol advertisements were for wine and beer. The rest were various types of liquors and bitters. Perhaps the Germans mainly drank beer and wine, while the English drank more liquor. The German newspapers also had advertisements for "fresh beer," which could be from a local brewer, or possibly homemade, as well as advertisements for imported wine and liquors. However, it is uncertain whether the Germanspeaking families were purchasing imported wine and liquor from their homeland. The English newspapers only specify one type of beer, Val Rottler's beer, who was a local brewer in Ste. Genevieve (Figure 4.14). Provided below is an 
advertisement for Val Rottler's beer from the Ste. Genevieve Herald on

December $17^{\text {th }}, 1892$.

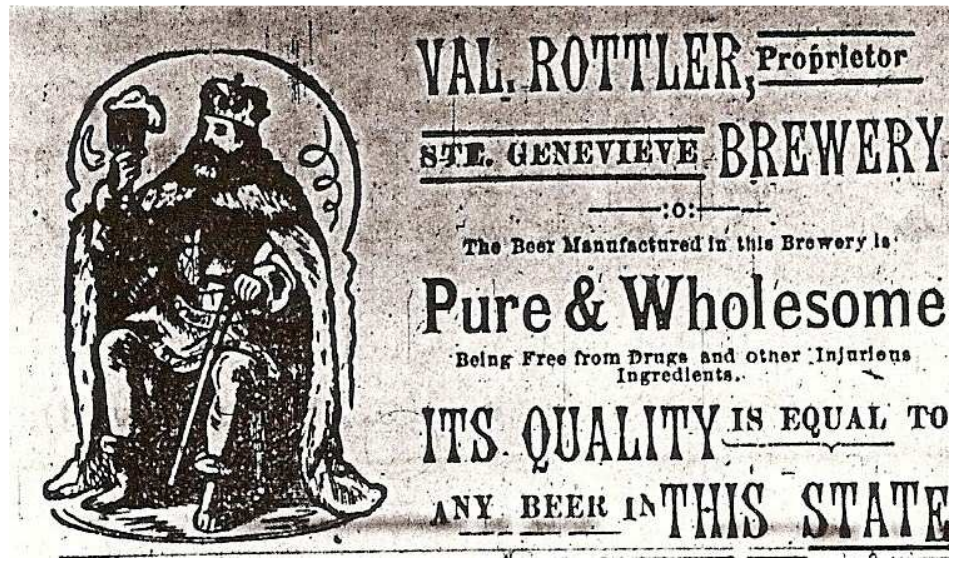

Figure 4.14. Val Rottler's Beer Advertisement from Ste. Genevieve Herald, December $17^{\text {th }}, 1892$.

The Ziegler probate inventory lists four demijohns, which were bottles encased in wickerwork. The fragments of bottle glass found in the archaeological record are too small to reveal whether they were filled with any alcohol or other substances. The probate inventory also lists 120 wine bottles. The storing of glass bottles for re-use was common in the nineteenth century, especially amongst housekeepers who used them for storing other foods in the fall (Busch 1987:71). Also, merchants kept bottles as permanent fixtures in their stores, to re-use and fill with products for sale (Busch 1987:69). Therefore, the bottles in the Ziegler inventory may have originally been used for wine, but without more information, it is not clear how the Zieglers were using the bottles. No other types of alcohol are identified in the inventory or archaeological record. Both English and German newspapers advertise for beer and wine. However, the 
Ziegler family did not list other types of alcoholic containers, like gin, brandy, or bourbon, which were advertised in the English newspapers. Perhaps the lack of alcoholic containers shows that the Zieglers were neither French or English.

\section{Inheritance Practices}

Besides the newspaper analysis, archaeological evidence, and probate inventory, I looked at the inheritance practices of the Ziegler family over several generations, hoping to find further evidence of German ethnic practices. Inheritance is about the passing on of property, or the materials possessed by the deceased owner. Therefore, the study of inheritance practices can tell archaeologists and other scholars much about past people and how they used their property. Inheritance practices were not only important for the individuals directly involved, but also the communities or other groups (Penner 1997:299). Stephen John Gross (1996:195) stated that "inheritance strategies, being broadly reflective of culturally prescribed values and attitudes, would be of greater utility in explaining how social structures were maintained and reproduced." Inheritance practices can affect several aspects of a person's and/or a culture's way of living, including "residency patterns, agricultural productivity, architecture, social standing, and familial relationships" (Penner 1997:301). Studying the different inheritance strategies a people use can also be another method for looking at the ethnic boundaries a group may set up while living amongst other groups. 
Inheritance practices can be studied through the research of not only wills, but also probate records and other personal writings. Wills and probate records can tell researchers so much more than just inheritance practices and have been a "valuable tool" in historical studies (Gross 1996:198). For this reason, the Ziegler family probate records and wills were analyzed to reveal whether or not the Ziegler family showed ethnic preferences through their inheritance practices, as well as how their inheritance practices may have changed over time.

There are two basic types of inheritance strategies that people use: partible and impartible inheritance. Partible inheritance can either be the equal distribution of inheritance to all heirs, regardless of gender, or, the male heirs inheriting land equally, with the other "movable" properties divided equally amongst all heirs, both male and female (Penner 1997:300). Impartible inheritance is when a single heir, usually the eldest son, receives all property (Penner 1997:300). Even with impartible inheritance, the primary heir was "still responsible for either maintaining or employing the other children" (Gross 1996:204). Though these inheritance strategies appear to affect only the individuals who are given the property, this is not necessarily true. Inheritance was important to the family's future well-being; in particular, impartible inheritance was important to "enhance family stability in the community" (Gross 1996:209).

Studying inheritance practices does not come without its biases and problems. Stephen John Gross (1996:195) points out that studying inheritance with wills alone may not fully identify how families distributed their assets. Wills 
leave out the transfer of land or money that might take place while the person is still alive or even post mortem. Therefore, including the research of probate records and other family documents is important to get the full picture of how each family distributed its assets, and which inheritance practices were used.

The inheritance practices were different from one region in Germany to another throughout the nineteenth century and before. Most of Germany used impartible (Anerbenrecht) inheritance but transferred assets inter vivos, as was practiced in the region of Bavaria. However, people from the region of Baden tended to use partible inheritance (Realteilungserbrecht) (Gross 1996:197). Among German peasants it was popular for them to give property to their children; in return they would be taken care of in old age (Gross 1996:197). The Swiss Appenzellers practiced partible inheritance. They believed that through this form of inheritance, each child would have equal opportunity for "economic and social stability and mobility" (Penner 1997:302). Despite distributing property equally, most Appenzellers would give the home-place to one heir, in order to keep it intact for future (Penner 1997:303). However, even their traditional inheritance practices from the Old World changed once they moved to the colony of South Carolina in the eighteenth century. In fact, most of the later generations of Appenzellers living in South Carolina began using impartible inheritance, much like most other German-speaking people.

The German people from St. Blasien in southern Baden also used partible inheritance. For this reason, many of them emigrated due to the small land allotments available after several generations of splitting up the family land 
(Fogleman 1996:27). The people of St. Peter changed from partible inheritance to a form of impartible inheritance called ultimogeniture, which was the passing down of land to the youngest son so that he would take care of the parents in their old age (Fogleman 1996:27). With this strategy, it was thought that the older siblings would have more time to find wealth and their own land. Eventually these German-speaking people ran into the same problem, overpopulation and division of landholdings, leading to emigration (Fogleman 1996:28). For this reason, most Germans appeared to be practicing impartible inheritance strategies while in Germany and as well as when they moved to America, so that their heirs would not have the same land problems that they themselves had to endure.

Several nineteenth-century wills and probate inventories related to the Ziegler family were analyzed: the petition by Mathias and Barbara Ziegler for inheritance from the father of Barbara Ziegler, Thomas Hefner (1827); Mathew Ziegler's will (1835) and probate inventory (1836); and the will of Francis Ziegler (1897). Though Thomas Hefner was not a Ziegler, his daughter became a Ziegler upon marriage to Matthias. According to a legal document from 1827, Barbara Ziegler, formerly Barbary Hefner the daughter of Thomas Hefner, and her husband Mathias Ziegler were heirs of Thomas Hefner. After Thomas Hefner died, both Barbara and her husband were to have received a share of his estate (Petition by Mathias and Barbara Ziegler 1827).

The fact that Barbara Ziegler received some share in her father's estate when he died shows that women were important for the future growth of the 
family. Stephen John Gross (1996:202) stated that "although wives were clearly junior partners in the family farm enterprise, their treatment in wills reflect their pronounced and important economic roles." Women had many roles to fill, taking care of the property, managing the household, and helping out in many other ways. For this reason, women were not always glanced over when it came to wills. Though the sons were favored in wills over daughters as far as real estate is concerned, daughters received other support such as dowries or financial help for their husbands (Gross 1996:203). Therefore, it is not surprising that Barbara Ziegler and her husband received a share of the estate when her father died.

Mathew Ziegler known as Mathias Ziegler, left his last will and testament in December of 1835. In his will he left all of his property to his wife Barbara, who was then to be the sole guardian of their children. If she were to marry, then half of the property would go to the children divided evenly. If she were to die, all the property was to be divided evenly among the surviving children. This being said, Mathias Ziegler appeared to have used a form of impartible inheritance. By doing this, Mathias followed in the same inheritance practices as other Bavarians. It was not uncommon for a man to leave his widow at least some of the estate. The Swiss-Appenzellers typically left at least one third of the estate to the widow. The English in the surrounding South Carolina colony did not follow this custom, and were not accustomed to willing widows any of the estate. Another reason Mathias Ziegler may have willed the inheritance to his wife was because of the young age of all his children. At the time of his death, his oldest child John was 13 years old and the youngest was only three years old. Overall, 
Mathias Ziegler followed what other German-speaking immigrants from Bavaria traditionally practiced.

A probate inventory was also produced after his death in 1836 . The document lists all the valuable property belonging to the Ziegler family, specifically Barbara, since she was the heir to the estate after Matthias's death. The probate inventory also lists the items that Barbara sold by 1840 , to settle debts against the estate. This document shows a more defined listing of the property and its value, compared to the will. Based on the probate inventory, the Ziegler property wealth was valued at $\$ 3,660.88$ in 1836 . Because the inventory listed the property sold by Barbara by 1840 , it shows that Barbara did remain heir of the property as well as the family tobacco business at least until 1840. She is listed as the head of household in the 1850 U.S. census (U.S. Bureau of the Census 1850). Barbara Ziegler, in fact, owned the house and property until she sold it to Francis Ziegler, her son, in 1851 for the amount of $\$ 1,760$, a debt he did not pay in full until 1860 (SGA 1860).

In 1897, Francis Ziegler died. Before his death he made his last will and testament on July 28,1897 . In his will he gave to his daughters Barbara Ziegler, Blanche Ziegler, Isabella Ziegler and Corinne Ziegler all his property of every sort. This property included: the house and land that was attached to survey no. 352 and lots on survey no. 331 , survey no. 212 , and survey no. 221 . He also gave Barbara, the oldest daughter, lot no. 15 and half of lot no. 14 on block 3 in town. To his son, Joseph Ziegler and to his four daughters he also gave part of surveys no. 20 and 21 , the west part of survey no. 22,23 , and 24 , and the 
undivided east part of surveys no. 23. Joseph was appointed the executor of his father's last will and testament (SGA 1897).

Francis gave most of his property to his four daughters and not his son, Joseph. He practiced a form of partible inheritance, giving equal distribution of property to his children. This does not follow in the customary inheritance practices of other Bavarians. This may show another way the Ziegler family was assimilating into their community. It also may just be a method of convenience. Joseph Ziegler, the only son of Francis, married while the remaining four daughters never married. Therefore, Francis may have distributed his property to his daughters because they were not married and he felt it necessary to take care of them even after his death. According to the census from 1900, Joseph was no longer living on the Ziegler property with his sisters (U.S. Bureau of the Census 1900). This supports the argument that Francis may have been looking out for his daughters after his death. Whatever the reason, the Ziegler family first used impartible inheritance then, by the next generation switched to partible inheritance, changing from the inheritance practices that their Bavarian ancestors used.

\section{Landscape Analysis}

Landscape can also reveal how an ethnic group used their space, and how this may be different from other ethnic groups. Unfortunately the JanisZiegler house/Green Tree Tavern was not built by the Ziegler family. If that were the case, the research could analyze the decisions or choices put into the 
building of the house and how it compares with other German houses. However, the Ziegler family did choose the house and made alterations throughout the years, which can reveal some of their thought processes. For example, once the Ziegler family moved into the Janis-Ziegler house/Green Tree Tavern, they more than likely changed the tavern into a tobacco business based on the 1836 probate inventory, listing a large variety of tobacco supplies in one location of the house (SGA 1836).

Most German families had homes built close to the street so their back yard property could be used for a garden (Coggeshall 1986:194), something also found with French families. The Ziegler house is much like this, with the house sitting right along the street with a large back yard, which they used for several purposes. German-speaking people used space differently than other groups, especially the back yard or back property of their house. Instead of using the front door to enter the house, German people customarily use the back or side door. John Coggeshall (1986:194) stated that this is true "regardless of direction of approach, type of weather, or degree of familiarity with the occupants." In the backyard, the garden was such an important part of a German person's property that "the kitchen back door usually led into the garden." (Long 1972:38). This is most likely true with the Ziegler family. A stone walkway is still in place leading from the back door to the archaeological remains of an outbuilding behind the house.

The garden was an area of great pride in a German household (Long 1972:42). The garden was typically cared for by the woman in the household, 
although the men helped with some of the gardening, mainly with laying out manure and plowing (Long 1972: 38). Germans used orchards mainly to produce fruit for juices and preserves (Long 1972:49). They usually used their garden to grow vegetables, herbs, and fruits and preserved most of these for use over the winter season (Long 1972:37). Germans were also given credit for founding vegetable horticulture in America (Faust 1927:62). John Coggeshall (1986:194) made note when an old gentleman told him that "the German people did love flowers, but they were also practical that they wanted to raise something to put into their cellars for over winter." Perhaps this is the reason there were no flower advertisements in the German newspapers, only in the English newspapers. German families were less likely to purchase flowers or their seeds than the English households.

Unfortunately, not a lot is known about the Ziegler garden, but through historical photographs and the probate inventory, one can suggest a few things concerning the Ziegler family and how they compare to their German counterparts. As mentioned previously, Germans were known for their cleanliness and orderly homes. This goes for their gardens as well. "[Their] buildings were kept in repair and weeds and brush were kept under control" (Long 1972:2). Usually the garden beds were rectangular shaped with pathways in between each garden bed (Long 1972:39). These pathways were important to the German family garden. "Many hours were spent weeding and hoeing such pathways by various members of the farms family, particularly the children (Long 1972:40)." In the Ziegler inventory, the Ziegler family owned a hoe and spade. 
Perhaps they used these implements to keep the pathways in their garden clear of weeds and other debris, like other German families. The fence surrounding the German garden was also important because it was used to keep animals away from the produce. This can also be seen in the Ziegler home picture from the 1880s (Figure 1.1). In the photograph, you can also see many trees and plants to the right of the property, most likely part of the garden or orchard. They also owned a barn, on the left side of the photograph, to keep any of their farm goods and equipment. Since it appears the Zieglers put the garden/orchard to the right of their property, they made sure to separate it from their outbuildings in the back of the house, keeping everything clean and separated much like their German ancestors.

On a regional landscape level, I would suggest that one of the reasons the Ziegler family moved to Ste. Genevieve, Missouri, was its close access to the major city of St. Louis, as well as the river accessibility of the Mississippi. The Ziegler family, similar to so many other German families, lived close to St. Louis so they were close to a good trading location. The Ziegler family most likely traded their tobacco, similar to other German families were known for trading and selling their farm products. The practice of producing goods outside of cities and then bringing them into the city to sell was a common one for German people. Christopher Fennell (2003:153) stated that many German-American families owned wagons so that they could transport their goods to trading centers in cities. The Ziegler family themselves did not have a wagon listed in their inventory. However, according to the newspaper analysis, the use of wagons 
was common for Germans in the area. Seven newspaper advertisements in the German Household Production Activity category listed wagons. However, no English advertisements listed wagons. Therefore, the transportation of goods on wagons and the use of wagons for other purposes was more common by German families than English.

\section{Summary}

By comparing the nineteenth-century English newspaper advertisements and the German newspaper advertisements from Ste. Genevieve, Missouri, several ethnic characteristics appeared. With the newspaper advertisement analysis, observations were made about the Ziegler archaeological assemblage, probate records, and wills. Several categories of analysis showed that the Ziegler family lived much like their English-speaking neighbors, while continuing to follow in some of their traditional German ways.

Some of the ways in which the Zieglers lived like their English-speaking neighbors had to do with ceramics and architecture. Their archaeological assemblage produced more queensware or white-colored refined earthenwares, which was popular in the English newspaper advertisements. Only a little bit of porcelain was found, which was advertised for much more in the German newspapers. The architecture of the Ziegler home and other buildings on their property were also more in line with those of other English-speakers. The German newspaper advertisements go along with historical accounts, that many German carpenters moved to North America or some Germans became 
carpenters once they moved here. The German advertisements were for heavy household repairs and building, unlike the English newspaper advertisements that were for general or small repairs and hardware. The Ziegler inventory and archaeological record only shows tools and items for small repairs. This is likely because the family moved into the already built house. Not much is known about the other buildings on the property, whether the Zieglers built them or not, since they are currently undergoing excavation and analysis.

Despite the ways in which the Zieglers lived like their English-speaking neighbors, the Zieglers still lived much like their German-speaking neighbors in Ste. Genevieve. The Zieglers owned many wooden furnishings according to the probate inventory, which was a popular German trait. The Zieglers probably made most of their own clothing, much like the other German-speakers. The German newspaper advertisements focused on advertising for men's clothing and the selling of cloth. The Ziegler probate inventory had listings for 12 types of cloth and a flax hackle, showing that the Zieglers made much of their own clothing. The Zieglers also owned books on the Prussian wars. They may have brought these from Germany, or bought them in the United States. Either way, they had an interest still in German history.

The Zieglers did what many of their other German-speaking neighbors did in food production. The German newspapers advertised for an abundance of farming equipment compared to the English newspapers. This was expected considering the historical accounts of Germans immigrating to America to be farmers. The Zieglers raised their own geese, using the fat, meat, and feathers 
for different household products and food. This was also common for other German families, according to historical accounts in Virginia. The German newspapers also advertised for farm and baking products, while the English newspapers advertised for already baked goods. The Zieglers had many baking and farm goods listed in their inventory, making them most like their Germanspeaking neighbors.

The Zieglers also ran a tobacco business out of their home, which could be considered a German ethnic trait since so many German craftsmen carried their skills over to America and continued in their practice. However, it is not certain whether the Ziegler family participated in the tobacco business over in Germany before they moved to America. Also, some of their tobacco could have been used for folk medicine, which was still common amongst German speaking people in Missouri in the nineteenth century.

The Zieglers interacted with their English-neighbors often, which can be seen through their business ledgers, which are written mostly in English; their relationships, according to the census records; and through architecture and ceramic use. Though the Zieglers had business and marriage ties with a prominent French family in town and conducted business in English, they continued to act much like their German-speaking neighbors throughout the nineteenth century.

German ethnicity can be seen in not only the materials German-speaking people used, but how they used these materials. German people tend to use similar inheritance strategies, especially people from the same regions of 
Germany. When looking at the Ziegler wills, census records, and probate records, one would expect that the Ziegler family would utilize an impartible inheritance strategy like other Bavarian immigrants. The Ziegler family began using impartible inheritance, but by the next generation had begun using partible inheritance. This shows that the Ziegler family began to follow some of the practices of their English-speaking neighbors rather than those of their Bavarian ancestors.

Landscape archaeology also has contributed to identifying ethnicity in the archaeological record. Though the Ziegler family did not build their own house, they modified and managed their home. They most likely began running their wholesale tobacco business out of their home, possibly using the tavern side of the house as the location for all the supplies and the business. Another area in particular was the use of the backyard. The Ziegler family used their backyard space much like other German-speaking people. They had a garden and used their backyard to access their home. Also, the location of the Ziegler house and their tobacco business was close to trading routes, much like other Germanspeaking farmers used. The Ziegler use of their landscape shows that their ethnic tendencies were similar to those of their German-speaking neighbors. 


\section{CHAPTER V}

\section{CONCLUSIONS}

The primary focus of this research has been to answer two questions. First, to what degree did the German Ziegler family express their ethnicity while living at the Janis-Ziegler house? Second, is it possible to identify the German ethnicity of the Ziegler family from the material culture of the Janis-Ziegler site? In order to answer these questions, I reviewed historical data, which included nineteenth-century German and English newspapers from Ste. Genevieve, probate records and wills from the Ziegler family, census records, and other historical accounts of Germans living in the United States. Along with the historical data, I also looked at the archaeological evidence left behind by the Ziegler family at the Janis-Ziegler/Green Tree Tavern site.

The first part of the research involved reviewing several German and English newspapers from nineteenth-century Ste. Genevieve, Fair Play (in English), the Ste. Genevieve Democrat (in English), Herald (in English and German), Freie Presse (in German), and Herold (in German). The advertisements from the newspaper issues that were dating closest to Christmas were analyzed and split into categories: Household Maintenance-Ceramics, Household MaintenanceGlass, Household Maintenance-Other, Architecture, Household Furnishings, Clothing, Personal Items, Household Production Activity, Household Production 
Activity For Sale, Pharmaceuticals, Foodstuffs, and Alcohol. After comparing the German, English, and German-English newspapers, several observations came apparent. The Ziegler family purchased and used some items in similar to ways that their English-speaking neighbors did, while continuing in some of their traditional German ways. The archaeological assemblage from the site, dating to the Ziegler occupation, looked closer to what was expected from an Englishspeaking resident of Ste. Genevieve. Along with ceramics, the home they chose to inhabit was in the French style, and therefore, shows a form of assimilation. The artifacts that pertain to the architecture category were also similar to what was expected of the English or French speakers in Ste. Genevieve.

However, the Ziegler family also continued to follow in their German ethnic traditions in the categories of Household Furnishings, Clothing, Personal Items and Foodstuffs. According to the probate inventory, the Ziegler family owned several pieces of wooden furniture which was a common trait amongst other German-speaking families in Ste. Genevieve. Whether they brought it from their home in Germany or bought it in the United States, is unknown. According to the newspaper analysis, the Ziegler family also made much of their clothing, which was similar to other German-speaking families in Ste. Genevieve. The Zieglers owned a flax hackle and many other items to produce or mend clothing. The Ziegler family also owned books about the Prussian wars, which show their interest in German history even while living in the United States. Finally, the Ziegler family produced much of their own food, instead of purchasing already made foodstuffs, which is similar to other German-speaking families. They 
tended to their orchard and garden, took care of geese, and owned many cooking products to produce their own food and baked goods.

They also showed some German ethnic preferences by their inheritance practices and their use of landscape. Mathias Ziegler used impartible inheritance, as had his Bavarian ancestors. However, later generations of the Ziegler family began using partible inheritance, much like their English-speaking neighbors. The Ziegler family's use of landscape also reveals their German ethnic tendencies. The probate inventory suggests that the Ziegler family transitioned the tavern into their tobacco business, continuing their craft of tobacco supplying which was popular amongst other German-speaking immigrants. Also, the separation of their orchard and garden from other uses of their property and outbuildings, are similar to how their German ancestors kept their space separated and clean. They also used their backyard space more than the front, as can be seen by the stone pathways in the back and the lack of steps leading to the front door.

After researching several Ste. Genevieve newspapers, the archaeological record, U.S. census records, and the Ziegler family wills and probate records, the Zieglers appear to be more similar to their German-speaking neighbors than previously thought. They may have appeared on the outside to follow with their English-speaking neighbors, moving into a French style house, working with another wealthy French family, and marrying into that family. However, they continued to use what they owned and to make purchases similar to their German heritage. After some time, the later Ziegler generations began to use 
their property more like their English-speaking neighbors, by changing their inheritance practices, using English in their business ledgers, and no longer running their tobacco business. Much of this can be equated to Francis Ziegler marrying Josephine Moreau, from a French family living in Ste. Genevieve. This does not necessarily mean the Ziegler family assimilated, but rather, best stated by Penner (1997:298), the Ziegler family “integrated" to gain "power, prestige, and wealth" (296).

\section{Potential for Further Research}

Much research can still be done on the Janis-Ziegler House/Green Tree Tavern site as well as other German-American sites. Further excavations of the Janis-Ziegler House/Green Tree Tavern could reveal more information about the Ziegler family's use of the backyard and potentially more about their tobacco business. More comparative work can also be conducted between the Ziegler occupation at the Janis-Ziegler house and other German-American sites in and around Ste. Genevieve, such as the Delassus-Kern house. This may reveal further ethnic tendencies of the Ziegler family as well as other German sites. Further newspaper analysis of German newspapers in the Unites States could reveal whether similar advertising campaigns were done in other cities, or whether there were distinct differences from one city to another. Lastly, more research and excavation should be conducted on German-American sites. Five and a half million German people immigrated to the United States from 1816 to 
1914 (Moltmann 1985:14), and German-American sites are still under researched in the field of archaeology today.

This study has not only revealed the ethnic preferences of the Ziegler family but also other German families in Ste. Genevieve. The research focused on newspaper, archaeological, other historical analysis, showing that ethnic markers are not the only means to identify the ethnicity in the historical and archaeological record. By looking at the historical and archaeological record together, a more comprehensive background of the Ziegler family and others in the future, can be revealed. 


\section{REFERENCES}

Babson, David W.

1990 The Archaeology of Racism and Ethnicity on Southern Plantations. Historical Archaeology 24(4):20-28.

Busch, Jane

1987 Second Time Around: A Look at Bottle Reuse. Historical

Archaeology 21(1):67-80.

Coggeshall, John M.

1986 "One of Those Intangibles": The Manifestation of Ethnic Identity in

Southwestern Illinois. The Journal of American Folklore 99(392):177-207.

Cook, Lauren, Rebecca Yamin, and John P. McCarthy

1996 Shopping as Meaningful Action: Toward a Redefinition of

Consumption in Historical Archaeology. Historical Archaeology 30(4):5065.

Cox, Matthew

2009 Living Beyond Their Means: an Archaeological Investigation of Consumption Patterns at the Janis-Ziegler Site (23SG272). Unpublished Master's Thesis. Illinois State University.

Crane, Brian D. 2000 Filth, Garbage, and Rubbish: Refuse Disposal, Sanitary Reform, and Nineteenth-Century Yard Deposits in Washington, D.C. Historical Archaeology 34(1):20-38

Cusick, James G.

2000 Creolization and the Borderlands. Historical Archaeology 34(3):4655.

Deposki, Richard 2008 Images of America: Ste. Genevieve. Arcadia Publishing, Charleston, South Carolina.

Dietler, Michael

2007 Culinary Encounters: Food, Identity, and Colonialism. In The 
Archaeology of Food and Identity, edited by Katheryn C. Twiss, pp. 218236. Center for Archaeological Investigations, Occasional Paper No.34. Southern llinois University, Carbondale.

DuBois, Rachel D. and Emma Schweppe 1936 The Germans in American Life. Books for Libraries Press, Freeport, New York.

Duden, Gottfried

1980 Report on a Journey to the Western States of North America and a Stay of Several Years Along the Missouri (During the Years 1824, '25, '26, and 1827). Translated by James W. Goodrich, the State Historical Society of Missouri, Columbia.

Ekberg, Carl J. 1985 Colonial Ste. Genevieve: An Adventure on the Mississippi Frontier. The Practice Press, Tucson.

1996 Colonial Ste. Genevieve: an Adventure on the Mississippi Frontier. Patrice Press, Tucson.

Elliott, Rita Folse and Daniel T. Elliott 2002 Guten Tag Bubba: German in the Colonial South. In Another's Country: Archaeological and Historical Perspectives on Cultural Interactions in the Southern Colonies, edited by J.W. Joseph and Martha Zierden, pp.79-2. The University of Alabama Press, Tuscaloosa and London.

Eriksen, Thomas Hylland 1992 Us and Them in Modern Societies: Ethnicity and Nationalism in Mauritius, Trinidad and Beyond. Scandinavian University Press, Oslo.

Faulkner, Charles $\mathrm{H}$.

1988 The Gibbs House: Excavation of a late $18^{\text {th }}$ century GermanAmerican farmstead in Knox County, Tennessee. Ohio Valley Historical Archaeology 6:1-8.

Faust, Albert Bernhardt 1927 The German Element in the United States: with Special Reference to its Political, Moral, Social, and Education Influence. 2 volumes. The Steuben Society of America, New York.

Fennell, Christopher C.

2000 Conjuring Boundaries: Inferring Past Identities from Religious Artifacts. International Journal of Historical Archaeology 4(4):281-313. 
2003 Group Identity, Individual Creativity, and Symbolic Generation in a BaKongo Diaspora. International Journal of Historical Archeology 7(1):131.

Ferguson, Leland

1992 Uncommon Ground: Archaeology and Early African America, 1650-

1800. Smithsonian Institution Press, Washington and London.

Fogleman, Aaron Spencer

1996 Hopeful Journeys: German Immigration, Settlement, and Political Culture in Colonial America, 1717-1775. University of Pennsylvania Press,Philadelphia.

Franzwa, Gregory M. 1998 The Story of Old Ste. Genevieve: An Account of an Old French Town in Upper Louisiana: its People and their Homes, sixth edition. The Practice Press, Tucson.

Fries, Adelaide L. 1922 Records of the Moravians in North Carolina. Edwards \& Broughton Print, Co., Raleigh.

Groover, Mark D. 1994 Evidence for Folkways and Cultural Exchange in the $18^{\text {th }}$-Century South Carolina Backcountry. Historical Archaeology 28(1):41-64.

2003 An Archaeological Study of Rural Capitalism and Material Life: The Gibbs Farmstead in Southern Appalachia, 1790-1920. Kluwer Academic/Plenum Publishers, New York.

2005 The Gibbs Farmstead: Household Archaeology in an Internal Periphery. International Journal of Historical Archaeology 9(4)229-289.

Gross, Stephen J. 1996 Handing Down the Farm: Values, Strategies, and Outcomes in Inheritance Practices Among Rural German Americans. Journal of Family History 21:192-217.

Gums, Bonnie L. 2009 Archaeological Investigations at Three Nineteenth-Century Sites Along the Kaskaska Trail in Monroe County, Illinois. Illinois Transportation Archaeological Research Program, No. 82.

Hawkins, Meredith M. 
2007 The Janis-Ziegler Site (23SG272): The Archaeological Investigations of Houselot in a French Colonial Village. Unpublished Master's Thesis.

Illinois State University.

Henry, Susan L.

1991 Consumers, Commodities, and Choices: A General Model of Consumer Behavior. Historical Archaeology 25(2):3-14.

Ste. Genevieve Herold

1892 December 17. Sainte Genevieve, Missouri.

1895 December 21. Sainte Genevieve, Missouri.

Idol, Bruce S. and Stephen T. Trage

1995 Excavations at the Dobb's Parish Cemetery and Bethabara

Stanger's Graveyard, Forsyth County, North Carolina. Wake Forest

University Archaeology Laboratories: Winston-Salem, North Carolina

Idol, Bruce S., Stephen T. Trage, and Roger W. Kirchen

1996 Report on Excavation at the Bethabara 1754 Sleeping Hall Site, Forsyth County, North Carolina. Wake Forest University Archaeology Laboratories: Winston-Salem, North Carolina.

Jones, Olive

1993 Commercial Foods, 1740-1820. Historical Archaeology 27(2):25-41.

Kamphoefner, Walter D.

1988 Chain Migration and Local Homogeneity of Immigration: Cape Girardeau County Germans in Comparative Perspective. In French and Germans in the Mississippi Valley: Landscape and Cultural Traditions, edited by Michael Roark, pp.179-189. Center for Regional History and Cultural Heritage: Cape Girardeau, Missouri.

Long Jr., Amos

1972 The Pennsylvania German Family Farm. The Pennsylvania German Society: Breinigsville, Pennsylvania.

Mann, Rob

2008 From Ethnogenesis to Ethnic Segmentation in the Wabash Valley: Constructing Identity and Houses in Great Lakes Fur Trade Society. International Journal of Historical Archaeology 12(3):319-337.

Martin, Ann Smart

1996 Material Culture in Early America. VA Institute of Early American History and Culture, Williamsburg. 
McCracken

1988 Culture and Consumption: new Approaches to the Symbolic Character of Consumer Goods and Activities. Indiana University Press:

Bloomington, Indiana.

McDavid, C.

2002 Archaeologies That Hurt; Descendants That Matter: A Pragmatic Approach to Collaboration in the Public Interpretation of African-American Archaeology. World Archaeology 34(2):303-314.

Meskell, Lynn

2001 Archaeologies of Identity. In Archaeological Theory Today, edited by I. Hodder, pp. 187-213. Polity Press, Cambridge.

Miller, George L.

1980 Classification and Economic Scaling of $19^{\text {th }}$ Century Ceramics.

Historical Archaeology 14(1):1-40.

Miller, George L.

1991 A Revised Set of CC Index Values and Economic Scaling of English Ceramics from 1787 to 1880. Historical Archaeology 25(1):1-25.

Moltmann, Günter

1985 The Pattern of German Emigration to the United States in the Nineteenth Century. In America and the Germans, an Assessment of a Three-Hundred-Year History, edited by Frank Trommler and Joseph McVeigh, pp. 14-24. University of Pennsylvania Press: Philadelphia.

Mullins, Paul R. 2008 Marketing in a Multicultural Neighborhood: An Archaeology of Corner Stores in the Urban Midwest. Historical Archaeology 42(1):88-96.

Murtagh, William J.

1967 Moravian Architecture and Town Planning; Bethlehem, Pennsylvania, and Other Eighteenth-Century American Settlements. University of North Carolina Press, Chapel Hill.

Nassaney, Michael S. 2008 Identity Formation at a French Colonial Outpost in the North American Interior. International Journal of Historical Archaeology 12:297318.

Orser, Charles 2004 Race and Practice in Archaeological Interpretations. University of Pennsylvania Press, Philadelphia. 
Penner, Bruce R.

1997 Old World Traditions, New World Landscapes: Ethnicity and Archaeology of Swiss-Appenzellers in the Colonial South Carolina Backcountry. International Journal of Historical Archaeology 1(4):257-321.

Pendery, Steven R.

1992 Consumer Behavior in Colonial Charlestown, Massachusetts, 16301760. Historical Archaeology 26 (3):57-72.

Pogue, Dennis J.

2001 The Transformation of America: Georgian Sensibility, Capitalist Conspiracy, or Consumer Revolution? Historical Archaeology 35(2): 4157.

Randalph

1964 Ozark Magic and Folklore. Dover Publishers.

Rowan and Primm

1983 Germans for a Free Missouri: Translations from the St. Louis Radical Press, 1857-1862. University of Missouri Press, Columbia.

Sainte Genevieve Archives

1827 Petition by Mathias and Barbara Ziegler for inheritance from the father of Barbara Ziegler, Thomas Hefner. Microfilm Roll 12, Folder 190, Ozark Regional Library. Ste. Genevieve, Missouri.

1835 Will of Mathew Ziegler (deceased 1835). Transcribed by Elizabeth Scott. Call No. MF567g, Microfilm Roll No. 76. Western Manuscript Collection, Ozark Regional Library. Ste. Genevieve, Missouri.

1836 Inventory and appraisement of the estate of Mathew Ziegler, deceased. Description of property inventoried and appraised. Transcribed by Elizabeth M.Scott.

1851 Sale of property by Barbara Ziegler on courthouse steps (September 1, 1851) and the deed transfer of property to Francis Ziegler (February 1860).Book N, pp.356-360.

1860. Transfer of property to Francis Ziegler by Barbara Ziegler (February 1860). Deed Book N, pp.356-360. Office of the Recorder of Deed, Ste. Genevieve, Missouri.

1897 Will of Francis J. Ziegler (July 28, 1897). Book 55, p. 377. 
Schrader, Frederick Franklin

1924 The Germans in the Making of America. The Stratford Co., Boston.

Schiffman, Leon and Leslie L. Kanuk

1983 Consumer Behavior. Prentice-Hall, Englewood Cliffs, New Jersey.

Scott, Elizabeth M.

2001a "An Indolent Slothfull Set of Vagabonds": Ethnicity and Race in a Colonial Fur-Trading Community. In Race and the Archaeology of Identity, edited by Charles E. Orser, Jr., pp. 14-33. University of Utah Press, Salt Lake City.

2001b Food and Social Relations at Nina Plantation. American Anthropologist 103(3):671-691.

Shackel, Paul A. 2003 Archaeology, Memory, and Landscapes of Conflict. Historical Archaeology 37(3):3-13.

Shackel, Paul A. and Barbara J. Little 1992 Post-Processual Approaches to Meanings and Uses of material Culture in Historical Archaeology. Historical Archaeology 26(3):5-11.

South, Stanley

1977 Methods and Theory in Historical Archaeology. Academic Press, New York.

1978 Research Strategies for Archaeological Pattern Recognition on Historic Sites. World Archaeology 10(1):36-50.

1999 Historical Archaeology In Wachovia: Excavating Eighteenth-century Bethabara and Moravian Pottery. New York: Kluwer Academic/Plenum Publishers.

State Historical Society of Missouri

1880 Francois Janis house (1790), image 4 (R338). Sainte Genevieve (Mo.) photographs, State Historical Society of Missouri.

Ste. Genevieve Democrat 1850 June 8. Sainte Genevieve, Missouri.

Ste. Genevieve Fair Play

1875 December 23. Sainte Genevieve, Missouri.

Ste. Genevieve Freie Presse 
1876 July 22. Sainte Genevieve, Missouri.

Steen, Carl

2002 John de la Howe and the Second Wave of French Refugees in the South Carolina Colony: Defining, Maintaining, and Losing Ethnicity on the Passing Frontier. In Another's Country: Archaeological and Historical Perspectives on Cultural Interactions in the Southern Colonies, edited by J.W. Joseph and Martha Zierden, pp.79-92. The University of Alabama Press, Tuscaloosa and London.

Thomas, Brian W.

1994 Inclusion and Exclusion in the Moravian Settlement in North

Carolina, 1770-1790. Historical Archaeology 28(3):15-29.

US Bureau of Census

1830 Federal Census for City of Ste. Genevieve in the County of Ste. Genevieve, State of Missouri.

1850 Federal Census for City of Ste. Genevieve in the County of Ste. Genevieve, State of Missouri. August 1850.

1860 Federal Census for City of Ste. Genevieve in the County of Ste. Genevieve, State of Missouri. June 1860.

1900 Federal Census for Ste. Genevieve County, Missouri. Twelfth Census of the United States, National Archives Microfilm Publications, Microcopy No. 623, Roll No. 886.

van Ravenswaay, Charles

1977 The Arts and Architecture of German Settlements in Missouri: A Survey of a Vanishing Culture. University of Missouri Press: Columbia, Missouri.

Werner, George S. 1977 Bavaria in the German Confederation, 1820-1848. Fairleigh Dickinson University Press: Rutherford, New Jersey.

Wesler, Kit W., Bonnie Stepenoff, N. Renae Farris, and Carol A. Morrow 1999 Archaeological Test Excavations at The Delassus-Kern House, Ste. Genevieve, Missouri. Ohio Valley Historical Archaeology 14:67-88.

Wilhelm, Hugh 1988 The Route West: German Immigration in Ohio before 1850 with Emphasis on the Riverine Area. In French and Germans in the Mississippi Valley: Landscape and Cultural Traditions, edited by Michael 
Roark, pp. 209-228. Center for Regional History and Cultural Heritage: Cape Girardeau, Missouri.

Willey, Gorden

1953 Prehistoric Settlement Patterns in the Viru Valley, Peru. U.S. Government Print, Washington.

Wittke, Carl

1952 Refugees of Revolution: the German Forty-Eighters in America. University of Pennsylvania Press: Philadelphia.

Wyman, Mark

1984 Immigrants in the Valley: Irish, Germans, and Americans in the Upper Mississippi Country, 1830-1860. Nelson-Hall, Inc.: Chicago.

Yentsch, Anne and Mary C. Beaudry 2001 American Material Culture in Mind, Thought, and Deed. In American Material Culture, edited by lan Hodder, pp. 214-240. Polity Press, Cambridge.

Yoder, Don

1990 Discovering American Folklife: Studies in Ethnic, Religious, and Regional Culture. U.M.I Research Press: Ann Arbor. 
APPENDIX A

NEWSPAPER DATABASES 


\section{English Newspapers}

\begin{tabular}{|c|c|c|c|c|c|c|}
\hline \multicolumn{7}{|c|}{ English Newspapers } \\
\hline $\begin{array}{c}\text { Newspape } \\
\text { r name }\end{array}$ & Date & $\left|\begin{array}{c}\text { advertisement } \\
\text { title }\end{array}\right|$ & \begin{tabular}{|} 
Household \\
Maintenanc \\
e-Ceramic
\end{tabular} & $\begin{array}{c}\text { Household } \\
\text { Maintenanc } \\
\text { e-glass }\end{array}$ & $\begin{array}{l}\text { Household } \\
\text { Maintenanc } \\
\text { e-other }\end{array}$ & $\begin{array}{c}\text { Architectur } \\
\text { al items }\end{array}$ \\
\hline Fair Play & \begin{tabular}{|r|}
$12 / 19 / 187$ \\
2
\end{tabular} & Paul L. Lempke & & & & \\
\hline Fair Play & \begin{tabular}{|r|}
$12 / 19 / 187$ \\
2
\end{tabular} & $\begin{array}{l}\text { Dr. C. S. } \\
\text { Hertich }\end{array}$ & & & & \\
\hline Fair Play & \begin{tabular}{|r|}
$12 / 19 / 187$ \\
2
\end{tabular} & $\begin{array}{l}\text { Chas. F. } \\
\text { Carssow, M.D. }\end{array}$ & & & & \\
\hline Fair Play & \begin{tabular}{|r|}
$12 / 19 / 187$ \\
2 \\
\end{tabular} & $\begin{array}{l}\text { R.F. Lanning, } \\
\text { M.D. }\end{array}$ & & & & \\
\hline Fair Play & \begin{tabular}{|r|}
$12 / 19 / 187$ \\
2
\end{tabular} & $\begin{array}{l}\text { Dr. J.W. } \\
\text { Braham }\end{array}$ & & & & \\
\hline Fair Play & \begin{tabular}{|r|}
$12 / 19 / 187$ \\
2
\end{tabular} & H. Knieriem & & & & \\
\hline Fair Play & \begin{tabular}{|r|}
$12 / 19 / 187$ \\
2
\end{tabular} & A.F. Beitrami & & & & \\
\hline Fair Play & \begin{tabular}{|r|}
$12 / 19 / 187$ \\
2 \\
\end{tabular} & Hamm \& Co. & & & & \\
\hline Fair Play & \begin{tabular}{|r|}
$12 / 19 / 187$ \\
2
\end{tabular} & $\begin{array}{l}\text { Edward } \\
\text { Seyssler }\end{array}$ & & & & \\
\hline Fair Play & \begin{tabular}{|r|}
$12 / 19 / 187$ \\
2
\end{tabular} & $\begin{array}{l}\text { Mrs. F. } \\
\text { Leavenworth }\end{array}$ & & & & \\
\hline Fair Play & \begin{tabular}{|r|}
$12 / 19 / 187$ \\
2
\end{tabular} & Janis \& Cox & 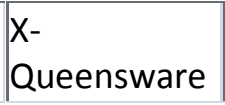 & & & X-Hardware \\
\hline Fair Play & \begin{tabular}{|r|}
$12 / 19 / 187$ \\
2
\end{tabular} & $\begin{array}{l}\text { United States } \\
\text { Mail Line }\end{array}$ & & & & \\
\hline Fair Play & \begin{tabular}{|r|}
$12 / 19 / 187$ \\
2 \\
\end{tabular} & Gambrinus Hall & & & & \\
\hline Fair Play & \begin{tabular}{|r|}
$12 / 19 / 187$ \\
2
\end{tabular} & Cone Mills & & & & \\
\hline Fair Play & \begin{tabular}{|r|}
$12 / 19 / 187$ \\
2
\end{tabular} & $\begin{array}{l}\text { Francis C. } \\
\text { Rozier \& Son }\end{array}$ & $\begin{array}{l}\text { X- } \\
\text { Queensware }\end{array}$ & & & X-Hardware \\
\hline Fair Play & $\begin{array}{r}12 / 19 / 187 \\
2\end{array}$ & $\begin{array}{l}\text { New Store, } \\
\text { New Goods, } \\
\text { C.F. Lawrence }\end{array}$ & $\begin{array}{l}\text { X-stoneware, } \\
\text { queensware }\end{array}$ & X-Glass & & X-Hardware \\
\hline Fair Play & $\begin{array}{r}12 / 19 / 187 \\
2\end{array}$ & $\begin{array}{l}\text { Notice } \\
\text { Farmers! Ste. } \\
\text { Mary Mills }\end{array}$ & & & & \\
\hline Fair Play & $\mid 12 / 19 / 187$ & Joseph Weiler & & & & \\
\hline
\end{tabular}




\begin{tabular}{|c|c|c|c|c|c|c|}
\hline \multicolumn{7}{|c|}{ English Newspapers } \\
\hline $\begin{array}{c}\text { Newspape } \\
\text { r name }\end{array}$ & Date & $\begin{array}{c}\text { advertisement } \\
\text { title }\end{array}$ & $\begin{array}{l}\text { Household } \\
\text { Maintenanc } \\
\text { e-Ceramic }\end{array}$ & \begin{tabular}{|c|} 
Household \\
Maintenanc \\
e-glass
\end{tabular} & $\begin{array}{c}\text { Household } \\
\text { Maintenanc } \\
\text { e-other }\end{array}$ & \begin{tabular}{|l} 
Architectur \\
al items
\end{tabular} \\
\hline & 2 & & & & & \\
\hline Fair Play & $\begin{array}{r}12 / 19 / 187 \\
2 \\
\end{array}$ & $\begin{array}{l}\text { Employment! } \\
\text { "up the heights } \\
\text { of fame and } \\
\text { Fortune" }\end{array}$ & & & & \\
\hline Fair Play & $\begin{array}{r}12 / 19 / 187 \\
2\end{array}$ & $\begin{array}{l}\text { Jubilee! New } \\
\text { York Observer }\end{array}$ & & & & \\
\hline Fair Play & \begin{tabular}{|r|}
$12 / 19 / 187$ \\
2 \\
\end{tabular} & $\begin{array}{l}\text { Tricks and } \\
\text { Traps of } \\
\text { America }\end{array}$ & & & & \\
\hline Fair Play & \begin{tabular}{|r|}
$12 / 19 / 187$ \\
2 \\
\end{tabular} & Accidents & & & & \\
\hline Fair Play & \begin{tabular}{|r|}
$12 / 19 / 187$ \\
2
\end{tabular} & \begin{tabular}{|l} 
Patronize \\
Home Industry
\end{tabular} & & & & \\
\hline Fair Play & \begin{tabular}{|r|}
$12 / 19 / 187$ \\
2 \\
\end{tabular} & E. Seckinger & & & & \\
\hline Fair Play & \begin{tabular}{|r|}
$12 / 19 / 187$ \\
2 \\
\end{tabular} & Michael Chene & $\begin{array}{l}\text { X-Japanned- } \\
\text { ware }\end{array}$ & & & \\
\hline Fair Play & $\begin{array}{r}12 / 19 / 187 \\
2\end{array}$ & $\begin{array}{l}\text { Andrew } \\
\text { Remlinger }\end{array}$ & & & & \\
\hline Fair Play & $\begin{array}{r}12 / 19 / 187 \\
2\end{array}$ & Le Boucher & & & & \\
\hline Fair Play & \begin{tabular}{|r|}
$12 / 19 / 187$ \\
2 \\
\end{tabular} & J.S. Whitlock & & & & \\
\hline Fair Play & \begin{tabular}{|r|}
$12 / 19 / 187$ \\
2
\end{tabular} & $\begin{array}{l}\text { Charles A. } \\
\text { Mueller }\end{array}$ & & & X-Tin-ware & \\
\hline Fair Play & \begin{tabular}{|r|}
$12 / 19 / 187$ \\
2
\end{tabular} & \begin{tabular}{|l} 
Jokerst \& \\
Boverie
\end{tabular} & & & & \\
\hline Fair Play & \begin{tabular}{|r|}
$12 / 19 / 187$ \\
2
\end{tabular} & A. Anderson & 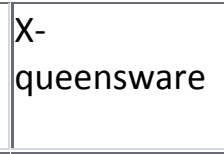 & & & $\begin{array}{l}\text { X-hardware, } \\
\text { tools, iron, } \\
\text { nails }\end{array}$ \\
\hline Fair Play & $\begin{array}{r}12 / 19 / 187 \\
2\end{array}$ & $\begin{array}{l}\text { The Fair Play } \\
\text { printing office }\end{array}$ & & & & \\
\hline Fair Play & \begin{tabular}{r|}
$12 / 19 / 187$ \\
2 \\
\end{tabular} & Dr. F. Quibourn & & & X-coal, oil, & \\
\hline Fair Play & \begin{tabular}{|r|}
$12 / 19 / 187$ \\
2
\end{tabular} & Job Printing & & & & \\
\hline Fair Play & $\mid 12 / 19 / 187$ & The Southeast & & & & \\
\hline
\end{tabular}




\begin{tabular}{|c|c|c|c|c|c|c|}
\hline \multicolumn{7}{|c|}{ English Newspapers } \\
\hline $\begin{array}{c}\text { Newspape } \\
\text { r name }\end{array}$ & Date & $\begin{array}{c}\text { advertisement } \\
\text { title }\end{array}$ & \begin{tabular}{|l} 
Household \\
Maintenanc \\
e-Ceramic
\end{tabular} & \begin{tabular}{|c|} 
Household \\
Maintenanc \\
e-glass \\
\end{tabular} & $\begin{array}{c}\text { Household } \\
\text { Maintenanc } \\
\text { e-other }\end{array}$ & \begin{tabular}{|c} 
Architectur \\
al items
\end{tabular} \\
\hline & 2 & Reporter & & & & \\
\hline Fair Play & \begin{tabular}{|r|}
$12 / 19 / 187$ \\
2
\end{tabular} & $\begin{array}{l}\text { Laclede County } \\
\text { Leader }\end{array}$ & & & & \\
\hline Fair Play & $\begin{array}{r}12 / 19 / 187 \\
2\end{array}$ & $\begin{array}{l}\text { Farmington } \\
\text { New Era }\end{array}$ & & & & \\
\hline Fair Play & \begin{tabular}{r|}
$\mid 12 / 19 / 187$ \\
2
\end{tabular} & $\begin{array}{l}\text { The Jefferson } \\
\text { County } \\
\text { Republican }\end{array}$ & & & & \\
\hline Fair Play & $\begin{array}{r}12 / 19 / 187 \\
2\end{array}$ & T. Morice & & & & \\
\hline Fair Play & \begin{tabular}{|r|}
$12 / 19 / 187$ \\
2
\end{tabular} & The Fair Play & & & & \\
\hline Fair Play & $\begin{array}{r}\mid 12 / 19 / 187 \\
2\end{array}$ & $\begin{array}{l}\text { The Gayoso } \\
\text { Democrat }\end{array}$ & & & & \\
\hline Fair Play & \begin{tabular}{|r|}
$12 / 19 / 187$ \\
2
\end{tabular} & The Bee & & & & \\
\hline Fair Play & \begin{tabular}{|r|}
$12 / 19 / 187$ \\
2
\end{tabular} & C. Bisch & $\begin{array}{l}\text { X- } \\
\text { Queensware }\end{array}$ & & & X-hardware \\
\hline Fair Play & \begin{tabular}{|r|}
$12 / 23 / 187$ \\
5
\end{tabular} & $\begin{array}{l}\text { More Cheap } \\
\text { Boots \& Shoes }\end{array}$ & & & & \\
\hline Fair Play & \begin{tabular}{|r|}
$12 / 23 / 187$ \\
5
\end{tabular} & $\begin{array}{l}\text { J.B. Robbins } \\
\text { Attorney at law }\end{array}$ & & & & \\
\hline Fair Play & \begin{tabular}{|r|}
$12 / 23 / 187$ \\
5
\end{tabular} & $\begin{array}{l}\text { H.S. Shaw } \\
\text { Attorney at } \\
\text { Law }\end{array}$ & & & & \\
\hline Fair Play & $\begin{array}{r}12 / 23 / 187 \\
5 \\
\end{array}$ & $\begin{array}{l}\text { Firmin A. } \\
\text { Rozier } \\
\text { Attorney at } \\
\text { Law }\end{array}$ & & & & \\
\hline Fair Play & \begin{tabular}{r|}
$12 / 23 / 187$ \\
5 \\
\end{tabular} & $\begin{array}{l}\text { Chas. C. Rozier } \\
\text { Attorney at } \\
\text { Law }\end{array}$ & & & & \\
\hline Fair Play & \begin{tabular}{|r|}
$12 / 23 / 187$ \\
5
\end{tabular} & $\begin{array}{l}\text { Dr. C. S. } \\
\text { Carssow, M.D. }\end{array}$ & & & & \\
\hline Fair Play & \begin{tabular}{|r|}
$12 / 23 / 187$ \\
5
\end{tabular} & $\begin{array}{l}\text { R.F. Lanning, } \\
\text { M.D. }\end{array}$ & & & & \\
\hline Fair Play & $\begin{array}{r}12 / 23 / 187 \\
5\end{array}$ & $\begin{array}{l}\text { M.F. Spalding, } \\
\text { M.D. }\end{array}$ & & & & \\
\hline
\end{tabular}




\begin{tabular}{|c|c|c|c|c|c|c|}
\hline \multicolumn{7}{|c|}{ English Newspapers } \\
\hline $\begin{array}{c}\text { Newspape } \\
r \text { name }\end{array}$ & Date & $\begin{array}{c}\text { advertisement } \\
\text { title }\end{array}$ & $\begin{array}{l}\text { Household } \\
\text { Maintenanc } \\
\text { e-Ceramic }\end{array}$ & \begin{tabular}{|c|} 
Household \\
Maintenanc \\
e-glass \\
\end{tabular} & $\begin{array}{c}\text { Household } \\
\text { Maintenanc } \\
\text { e-other }\end{array}$ & \begin{tabular}{|c} 
Architectur \\
al items
\end{tabular} \\
\hline Fair Play & $\begin{array}{r}12 / 23 / 187 \\
5\end{array}$ & $\begin{array}{l}\text { Dr. J.W. } \\
\text { Braham }\end{array}$ & & & & \\
\hline Fair Play & \begin{tabular}{|r|}
$12 / 23 / 187$ \\
5
\end{tabular} & Dr. F. Guibourd & & & & \\
\hline Fair Play & $\begin{array}{r}12 / 23 / 187 \\
5\end{array}$ & F.C. Albert & & & & \\
\hline Fair Play & $\begin{array}{r}12 / 23 / 187 \\
5\end{array}$ & $\begin{array}{l}\text { Read this: Rev. } \\
\text { Father Stein }\end{array}$ & & & & \\
\hline Fair Play & $\begin{array}{r}12 / 23 / 187 \\
5\end{array}$ & Gambrinus Hall & & & & \\
\hline Fair Play & \begin{tabular}{|r|}
$12 / 23 / 187$ \\
5
\end{tabular} & $\begin{array}{l}\text { Ste. Genevieve } \\
\text { House }\end{array}$ & & & & \\
\hline Fair Play & \begin{tabular}{|r|}
$12 / 23 / 187$ \\
5
\end{tabular} & $\begin{array}{l}\text { Bogy \& } \\
\text { Guignon }\end{array}$ & & & & \\
\hline Fair Play & \begin{tabular}{|r|}
$12 / 23 / 187$ \\
5
\end{tabular} & J.S. Whitlock & & & & \\
\hline Fair Play & \begin{tabular}{|r|}
$12 / 23 / 187$ \\
5
\end{tabular} & Lumber Yard & & & & X-lumber \\
\hline Fair Play & \begin{tabular}{|r|}
$12 / 23 / 187$ \\
5
\end{tabular} & P.U. Jaccard & & & & \\
\hline Fair Play & \begin{tabular}{|r|}
$12 / 23 / 187$ \\
5
\end{tabular} & $\begin{array}{l}\text { Mrs. F. } \\
\text { Leavenworth }\end{array}$ & & & & \\
\hline Fair Play & $\begin{array}{r}12 / 23 / 187 \\
5 \\
\end{array}$ & $\begin{array}{l}\text { Christian } \\
\text { Baum, Boots \& } \\
\text { Shoes }\end{array}$ & & & & \\
\hline Fair Play & \begin{tabular}{r|}
$12 / 23 / 187$ \\
5 \\
\end{tabular} & $\begin{array}{l}\text { Aug. } \\
\text { Becquette, } \\
\text { Blacksmith }\end{array}$ & & & & \\
\hline Fair Play & \begin{tabular}{|r|}
$12 / 23 / 187$ \\
5
\end{tabular} & $\begin{array}{l}\text { Mrs. Maria } \\
\text { Wilder }\end{array}$ & $\begin{array}{l}\text { X-china, } \\
\text { queensware }\end{array}$ & $x$ & & \\
\hline Fair Play & $\begin{array}{r}12 / 23 / 187 \\
5 \\
\end{array}$ & $\begin{array}{l}\text { At the Ste. } \\
\text { Genevieve One } \\
\text { price clothing }\end{array}$ & & & & \\
\hline Fair Play & \begin{tabular}{|r|}
$12 / 23 / 187$ \\
5
\end{tabular} & $\begin{array}{l}\text { Harris \& Janis } \\
\text { bankers }\end{array}$ & & & & \\
\hline Fair Play & \begin{tabular}{|r|}
$12 / 23 / 187$ \\
5.
\end{tabular} & $\begin{array}{l}\text { New Firm, } \\
\text { John F. } \\
\text { Schuchert }\end{array}$ & & & & \\
\hline
\end{tabular}




\begin{tabular}{|c|c|c|c|c|c|c|}
\hline \multicolumn{7}{|c|}{ English Newspapers } \\
\hline $\begin{array}{c}\text { Newspape } \\
\text { r name }\end{array}$ & Date & $\begin{array}{c}\text { advertisement } \\
\text { title }\end{array}$ & \begin{tabular}{|c|} 
Household \\
Maintenanc \\
e-Ceramic
\end{tabular} & $\begin{array}{c}\text { Household } \\
\text { Maintenanc } \\
\text { e-glass }\end{array}$ & $\begin{array}{c}\text { Household } \\
\text { Maintenanc } \\
\text { e-other }\end{array}$ & \begin{tabular}{|l} 
Architectur \\
al items
\end{tabular} \\
\hline Fair Play & $\begin{array}{r}12 / 23 / 187 \\
5\end{array}$ & $\begin{array}{l}\text { Rozier \& } \\
\text { Jokerst }\end{array}$ & $\begin{array}{l}\text { X- } \\
\text { queensware }\end{array}$ & & & X-hardware \\
\hline Fair Play & \begin{tabular}{|r|}
$12 / 23 / 187$ \\
5
\end{tabular} & Fitzkams & & & & \\
\hline Fair Play & \begin{tabular}{|r|}
$12 / 23 / 187$ \\
5
\end{tabular} & Mrs. A. Kempf & & & & \\
\hline Fair Play & $\begin{array}{r}12 / 23 / 187 \\
5\end{array}$ & P.U. Jaccard & & & & \\
\hline Fair Play & \begin{tabular}{|r|}
$12 / 23 / 187$ \\
5
\end{tabular} & $\begin{array}{l}\text { Terpsichorean } \\
\text { Hall }\end{array}$ & & & & \\
\hline Fair Play & \begin{tabular}{|r|}
$12 / 23 / 187$ \\
5
\end{tabular} & $\begin{array}{l}\text { Edward } \\
\text { Seyssler }\end{array}$ & & & & \\
\hline Fair Play & \begin{tabular}{|r|}
$12 / 23 / 187$ \\
5
\end{tabular} & $\begin{array}{l}\text { Regular } \\
\text { Connect'n }\end{array}$ & & & & \\
\hline Fair Play & \begin{tabular}{|r|}
$12 / 23 / 187$ \\
5
\end{tabular} & $\begin{array}{l}\text { Ste. Genevieve } \\
\text { Livery, Feed }\end{array}$ & & & & \\
\hline Fair Play & \begin{tabular}{|r|}
$12 / 23 / 187$ \\
5
\end{tabular} & Daily Hack Line & & & & \\
\hline Fair Play & \begin{tabular}{|r|}
$12 / 23 / 187$ \\
5 \\
\end{tabular} & $\begin{array}{l}\text { Ste. Genevieve } \\
\text { Accommodatio } \\
\text { n line }\end{array}$ & & & & \\
\hline Fair Play & $\begin{array}{r}12 / 23 / 187 \\
5\end{array}$ & $\begin{array}{l}\text { J No. B. Page \& } \\
\text { Son }\end{array}$ & & & & \\
\hline Fair Play & \begin{tabular}{|r|}
$12 / 23 / 187$ \\
5
\end{tabular} & $\$ 290$ a month & & & & \\
\hline Fair Play & \begin{tabular}{|r|}
$12 / 23 / 187$ \\
5
\end{tabular} & Asthma & & & & \\
\hline Fair Play & \begin{tabular}{|r|}
$12 / 23 / 187$ \\
5
\end{tabular} & $\begin{array}{l}\text { Zells' } \\
\text { encyclopedia }\end{array}$ & & & & \\
\hline Fair Play & \begin{tabular}{|r|}
$12 / 23 / 187$ \\
5
\end{tabular} & $\begin{array}{l}1000 \text { Outfits } \\
\text { Free Footprints }\end{array}$ & & & & \\
\hline Fair Play & \begin{tabular}{|r|}
$12 / 23 / 187$ \\
5
\end{tabular} & Revolvers!! & & & & \\
\hline Fair Play & \begin{tabular}{|r|}
$12 / 23 / 187$ \\
5
\end{tabular} & $\begin{array}{l}\text { The Only Live- } \\
\text { Stock paper }\end{array}$ & & & & \\
\hline Fair Play & \begin{tabular}{|r|}
$12 / 23 / 187$ \\
5
\end{tabular} & Health lift & & & & \\
\hline Fair Play & $12 / 23 / 187$ & Tubular Hand & & & & \\
\hline
\end{tabular}




\begin{tabular}{|c|c|c|c|c|c|c|}
\hline \multicolumn{7}{|c|}{ English Newspapers } \\
\hline $\begin{array}{c}\text { Newspape } \\
\text { r name }\end{array}$ & Date & $\begin{array}{c}\text { advertisement } \\
\text { title }\end{array}$ & \begin{tabular}{|c|} 
Household \\
Maintenanc \\
e-Ceramic \\
\end{tabular} & $\begin{array}{c}\text { Household } \\
\text { Maintenanc } \\
\text { e-glass }\end{array}$ & $\begin{array}{c}\text { Household } \\
\text { Maintenanc } \\
\text { e-other }\end{array}$ & $\begin{array}{c}\text { Architectur } \\
\text { al items }\end{array}$ \\
\hline & & lamp & & & & \\
\hline Fair Play & $\begin{array}{r}12 / 23 / 187 \\
5\end{array}$ & Cancer & & & & \\
\hline Fair Play & \begin{tabular}{|r|}
$12 / 23 / 187$ \\
5 \\
\end{tabular} & The Nursery & & & & \\
\hline Fair Play & $\begin{array}{r}12 / 23 / 187 \\
5\end{array}$ & Asthma & & & & \\
\hline Fair Play & \begin{tabular}{|r|}
$12 / 23 / 187$ \\
5
\end{tabular} & $\begin{array}{l}\text { Meriden } \\
\text { Cutlery Co. }\end{array}$ & & & $\begin{array}{l}\text { X-Cutlery- } \\
\text { ivory handles }\end{array}$ & \\
\hline Fair Play & \begin{tabular}{|r|}
$12 / 23 / 187$ \\
5
\end{tabular} & $\begin{array}{l}\text { Driscoll, } \\
\text { Church \& hall }\end{array}$ & & & & \\
\hline Fair Play & \begin{tabular}{|r|}
$12 / 23 / 187$ \\
5
\end{tabular} & Toledo Blade & & & & \\
\hline Fair Play & $\begin{array}{r}12 / 23 / 187 \\
5\end{array}$ & $\begin{array}{l}\text { Silver Tipped } \\
\text { Shoes }\end{array}$ & & & & \\
\hline Fair Play & $\begin{array}{r}12 / 23 / 187 \\
5\end{array}$ & $\begin{array}{l}\text { Gable Screw } \\
\text { Wire }\end{array}$ & & & & \\
\hline Fair Play & \begin{tabular}{|r|}
$12 / 23 / 187$ \\
5
\end{tabular} & $\$ 40$ a Day & & & & X-well auger \\
\hline Fair Play & $\begin{array}{r}12 / 23 / 187 \\
5\end{array}$ & Opium & & & & \\
\hline Fair Play & \begin{tabular}{r|}
$12 / 23 / 187$ \\
5 \\
\end{tabular} & $\begin{array}{l}\text { "Domestic" } \\
\text { sewing } \\
\text { machines }\end{array}$ & & & & \\
\hline Fair Play & $\begin{array}{r}12 / 23 / 187 \\
5\end{array}$ & $\begin{array}{l}\text { Washington, } \\
\text { D.C. }\end{array}$ & & & & \\
\hline Fair Play & \begin{tabular}{|r|}
$12 / 23 / 187$ \\
5
\end{tabular} & Opium & & & & \\
\hline Fair Play & $\begin{array}{r}12 / 23 / 187 \\
5\end{array}$ & $\begin{array}{l}\text { Smith Organ } \\
\text { Co. }\end{array}$ & & & & \\
\hline Fair Play & \begin{tabular}{|r|}
$12 / 23 / 187$ \\
5
\end{tabular} & Guns & & & & \\
\hline Fair Play & $\begin{array}{r}12 / 23 / 187 \\
5\end{array}$ & $\begin{array}{l}\text { Cincinnati } \\
\text { dollar weekly } \\
\text { star }\end{array}$ & & & & \\
\hline Fair Play & \begin{tabular}{|r|}
$12 / 23 / 187$ \\
5
\end{tabular} & $\$ 10$ to $\$ 500$ & & & & \\
\hline Fair Play & $|12 / 23 / 187|$ & Helio-telluric & & & & \\
\hline
\end{tabular}




\begin{tabular}{|c|c|c|c|c|c|c|}
\hline \multicolumn{7}{|c|}{ English Newspapers } \\
\hline $\begin{array}{c}\text { Newspape } \\
\mathrm{r} \text { name }\end{array}$ & Date & $\begin{array}{c}\text { advertisement } \\
\text { title }\end{array}$ & \begin{tabular}{|c|} 
Household \\
Maintenanc \\
e-Ceramic \\
\end{tabular} & \begin{tabular}{|c|} 
Household \\
Maintenanc \\
e-glass \\
\end{tabular} & $\begin{array}{c}\text { Household } \\
\text { Maintenanc } \\
\text { e-other }\end{array}$ & \begin{tabular}{|l} 
Architectur \\
al items
\end{tabular} \\
\hline & & treatment & & & & \\
\hline Fair Play & $\begin{array}{r}12 / 23 / 187 \\
5\end{array}$ & $\begin{array}{l}\text { Prof. hall's } \\
\text { Magic } \\
\text { Compound }\end{array}$ & & & & \\
\hline Fair Play & $\begin{array}{r}12 / 23 / 187 \\
5\end{array}$ & $\begin{array}{l}\text { Puff! Puff!! } \\
\text { Puff!!! }\end{array}$ & & & & \\
\hline Fair Play & $\begin{array}{r}12 / 23 / 187 \\
5 \\
\end{array}$ & $\begin{array}{l}\text { Dr. Sanford's } \\
\text { Liver } \\
\text { Invigorator }\end{array}$ & & & & \\
\hline Fair Play & $\begin{array}{r}12 / 23 / 187 \\
5\end{array}$ & Elastic truss & & & & \\
\hline Fair Play & $\begin{array}{r}12 / 23 / 187 \\
5 \\
\end{array}$ & Fair Play & & & & \\
\hline Fair Play & $\begin{array}{r}12 / 23 / 187 \\
5\end{array}$ & $\begin{array}{l}\text { Don't Neglect } \\
\text { your teeth: } \\
\text { Sozodont }\end{array}$ & & & & \\
\hline Fair Play & $\begin{array}{r}12 / 23 / 187 \\
5 \\
\end{array}$ & & & & & \\
\hline Fair Play & $\begin{array}{r}12 / 19 / 187 \\
2\end{array}$ & $\begin{array}{l}\text { Firmin A. } \\
\text { Rozier }\end{array}$ & & & & \\
\hline Fair Play & $\begin{array}{r}12 / 19 / 187 \\
2\end{array}$ & Chas. C. Rozier & & & & \\
\hline Fair Play & $\begin{array}{r}12 / 19 / 187 \\
2 \\
\end{array}$ & J.B. Robbins & & & & \\
\hline Fair Play & $\begin{array}{r}12 / 19 / 187 \\
2 \\
\end{array}$ & F.J. Moreau & & & & \\
\hline Fair Play & $\begin{array}{r}12 / 19 / 187 \\
2\end{array}$ & $\begin{array}{l}\text { Robinson \& } \\
\text { Clardy }\end{array}$ & & & & \\
\hline Fair Play & $\begin{array}{r}12 / 23 / 187 \\
5\end{array}$ & $\begin{array}{l}\text { Dr. C. S. } \\
\text { Hertich }\end{array}$ & & & & \\
\hline \begin{tabular}{|l|} 
Ste. \\
Genevieve \\
Democrat \\
\end{tabular} & $6 / 8 / 1850$ & $\begin{array}{l}\text { Ste. Genevieve } \\
\text { Marble Yard }\end{array}$ & & & & $\begin{array}{l}\text { X-Marble } \\
\text { and Stone }\end{array}$ \\
\hline \begin{tabular}{|l|} 
Ste. \\
Genevieve \\
Democrat
\end{tabular} & $6 / 8 / 1850$ & $\begin{array}{l}\text { Ste. Genevieve } \\
\text { Clothing Store }\end{array}$ & & & & \\
\hline \begin{tabular}{|l|} 
Ste. \\
Genevieve
\end{tabular} & $6 / 8 / 1850$ & $\begin{array}{l}\text { House and Sign } \\
\text { Painter }\end{array}$ & & & & \\
\hline
\end{tabular}




\begin{tabular}{|c|c|c|c|c|c|c|}
\hline \multicolumn{7}{|c|}{ English Newspapers } \\
\hline $\begin{array}{c}\text { Newspape } \\
\text { r name }\end{array}$ & Date & $\begin{array}{c}\text { advertisement } \\
\text { title }\end{array}$ & \begin{tabular}{|c|} 
Household \\
Maintenanc \\
e-Ceramic
\end{tabular} & \begin{tabular}{|c|} 
Household \\
Maintenanc \\
e-glass \\
\end{tabular} & $\begin{array}{c}\text { Household } \\
\text { Maintenanc } \\
\text { e-other }\end{array}$ & \begin{tabular}{|l} 
Architectur \\
al items
\end{tabular} \\
\hline \multicolumn{7}{|l|}{ Democrat } \\
\hline \begin{tabular}{|l} 
Ste. \\
Genevieve \\
Democrat
\end{tabular} & $6 / 8 / 1850$ & $\begin{array}{l}\text { W.K. Bradly } \\
\text { Attorney at } \\
\text { Law }\end{array}$ & & & & \\
\hline \begin{tabular}{|l} 
Ste. \\
Genevieve \\
Democrat
\end{tabular} & $6 / 8 / 1850$ & $\begin{array}{l}\text { R. Beauvais } \\
\text { Importer and } \\
\text { Manufacturer }\end{array}$ & & & $\begin{array}{l}\text { X-silver } \\
\text { spoons }\end{array}$ & \\
\hline \begin{tabular}{|l} 
Ste. \\
Genevieve \\
Democrat
\end{tabular} & $6 / 8 / 1850$ & $\begin{array}{l}\text { Cowan } \\
\text { Vegetable } \\
\text { Lithontriptic }\end{array}$ & & & & \\
\hline \begin{tabular}{|l} 
Ste. \\
Genevieve \\
Democrat \\
\end{tabular} & $6 / 8 / 1850$ & New Clothing & & & & \\
\hline $\begin{array}{l}\text { Ste. } \\
\text { Genevieve } \\
\text { Democrat }\end{array}$ & $6 / 8 / 1850$ & $\begin{array}{l}\text { Carpentering } \\
\text { and House } \\
\text { Joining }\end{array}$ & & & & \\
\hline $\begin{array}{l}\text { Ste. } \\
\text { Genevieve } \\
\text { Democrat }\end{array}$ & $6 / 8 / 1850$ & $\begin{array}{l}\text { Notice:Dofler's } \\
\text { Bee Palace }\end{array}$ & & & & \\
\hline \begin{tabular}{|l} 
Ste. \\
Genevieve \\
Democrat
\end{tabular} & $6 / 8 / 1850$ & Cheap Goods & $\begin{array}{l}\text { X- } \\
\text { Queensware }\end{array}$ & X-Glassware & & X-Hardware \\
\hline $\begin{array}{l}\text { Ste. } \\
\text { Genevieve } \\
\text { Democrat }\end{array}$ & $6 / 8 / 1850$ & Whiskey & & & & \\
\hline $\begin{array}{l}\text { Ste. } \\
\text { Genevieve } \\
\text { Democrat }\end{array}$ & $6 / 8 / 1850$ & $\begin{array}{l}\text { Kanawha \& } \\
\text { Alum Salt }\end{array}$ & & & & \\
\hline $\begin{array}{l}\text { Ste. } \\
\text { Genevieve } \\
\text { Democrat }\end{array}$ & $6 / 8 / 1850$ & Iron & & & & X-Iron \\
\hline $\begin{array}{l}\text { Ste. } \\
\text { Genevieve } \\
\text { Democrat }\end{array}$ & $6 / 8 / 1850$ & Nails & & & & X-Nails \\
\hline \begin{tabular}{|l} 
Ste. \\
Genevieve \\
Democrat
\end{tabular} & $6 / 8 / 1850$ & Brandy & & & & \\
\hline \begin{tabular}{|l} 
Ste. \\
Genevieve
\end{tabular} & $6 / 8 / 1850$ & $\begin{array}{l}\text { Law } \\
\text { Department }\end{array}$ & & & & \\
\hline
\end{tabular}




\begin{tabular}{|c|c|c|c|c|c|c|}
\hline \multicolumn{7}{|c|}{ English Newspapers } \\
\hline $\begin{array}{c}\text { Newspape } \\
\text { r name }\end{array}$ & Date & $\begin{array}{c}\text { advertisement } \\
\text { title }\end{array}$ & \begin{tabular}{|c|} 
Household \\
Maintenanc \\
e-Ceramic
\end{tabular} & $\begin{array}{c}\text { Household } \\
\text { Maintenanc } \\
\text { e-glass }\end{array}$ & $\begin{array}{c}\text { Household } \\
\text { Maintenanc } \\
\text { e-other }\end{array}$ & \begin{tabular}{|l} 
Architectur \\
al items
\end{tabular} \\
\hline \multicolumn{7}{|l|}{ Democrat } \\
\hline \begin{tabular}{|l} 
Ste. \\
Genevieve \\
Democrat
\end{tabular} & $6 / 8 / 1850$ & New Clothing & & & & \\
\hline $\begin{array}{l}\text { Ste. } \\
\text { Genevieve } \\
\text { Democrat }\end{array}$ & $6 / 8 / 1850$ & $\begin{array}{l}\text { For Sale Cheap: } \\
\text { Farm }\end{array}$ & & & & \\
\hline \begin{tabular}{|l} 
Ste. \\
Genevieve \\
Democrat
\end{tabular} & $6 / 8 / 1850$ & California Gold & $\begin{array}{l}\text { X- } \\
\text { Queensware, } \\
\text { China }\end{array}$ & $\begin{array}{l}\text { X-Glass, } \\
\text { Glassware }\end{array}$ & & X-Hardware \\
\hline \begin{tabular}{|l} 
Ste. \\
Genevieve \\
Democrat \\
\end{tabular} & $6 / 8 / 1850$ & Hats & & & & \\
\hline $\begin{array}{l}\text { Ste. } \\
\text { Genevieve } \\
\text { Democrat }\end{array}$ & $6 / 8 / 1850$ & $\begin{array}{l}\text { Ready made } \\
\text { Clothing }\end{array}$ & & & & \\
\hline $\begin{array}{l}\text { Ste. } \\
\text { Genevieve } \\
\text { Democrat }\end{array}$ & $6 / 8 / 1850$ & Sugar \& Coffee & & & & \\
\hline \begin{tabular}{|l} 
Ste. \\
Genevieve \\
Democrat
\end{tabular} & $6 / 8 / 1850$ & $\begin{array}{l}\text { Ladies Fine } \\
\text { Dress Goods }\end{array}$ & & & & \\
\hline \begin{tabular}{|l} 
Ste. \\
Genevieve \\
Democrat
\end{tabular} & $6 / 8 / 1850$ & $C(?)$ Boots & & & & \\
\hline $\begin{array}{l}\text { Ste. } \\
\text { Genevieve } \\
\text { Democrat }\end{array}$ & $6 / 8 / 1850$ & Salt & & & & \\
\hline $\begin{array}{l}\text { Ste. } \\
\text { Genevieve } \\
\text { Democrat }\end{array}$ & $6 / 8 / 1850$ & $\begin{array}{l}\text { Hats, Caps, \& } \\
\text { Straw Goods }\end{array}$ & & & & \\
\hline $\begin{array}{l}\text { Ste. } \\
\text { Genevieve } \\
\text { Democrat }\end{array}$ & $6 / 8 / 1850$ & $\begin{array}{l}\text { Spring and } \\
\text { Summer } \\
\text { Clothing at } \\
\text { Wholesale }\end{array}$ & & & & \\
\hline \begin{tabular}{|l} 
Ste. \\
Genevieve \\
Democrat \\
\end{tabular} & $6 / 8 / 1850$ & $\begin{array}{l}\text { Fancy Letter } \\
\text { and Note } \\
\text { Paper }\end{array}$ & & & & \\
\hline Ste. & $6 / 8 / 1850$ & Fancy and & & & & \\
\hline
\end{tabular}




\begin{tabular}{|c|c|c|c|c|c|c|}
\hline \multicolumn{7}{|c|}{ English Newspapers } \\
\hline $\begin{array}{c}\text { Newspape } \\
\text { r name }\end{array}$ & Date & $\begin{array}{c}\text { advertisement } \\
\text { title }\end{array}$ & $\begin{array}{l}\text { Household } \\
\text { Maintenanc } \\
\text { e-Ceramic }\end{array}$ & \begin{tabular}{|c|} 
Household \\
Maintenanc \\
e-glass
\end{tabular} & $\begin{array}{c}\text { Household } \\
\text { Maintenanc } \\
\text { e-other }\end{array}$ & \begin{tabular}{|c} 
Architectur \\
al items
\end{tabular} \\
\hline $\begin{array}{l}\text { Genevieve } \\
\text { Democrat }\end{array}$ & & $\begin{array}{l}\text { common } \\
\text { Envelopes }\end{array}$ & & & & \\
\hline $\begin{array}{l}\text { Ste. } \\
\text { Genevieve } \\
\text { Democrat }\end{array}$ & $6 / 8 / 1850$ & $\begin{array}{l}\text { New Goods! } \\
\text { New Goods! }\end{array}$ & & & & \\
\hline $\begin{array}{l}\text { Ste. } \\
\text { Genevieve } \\
\text { Democrat }\end{array}$ & $6 / 8 / 1850$ & Coats & & & & \\
\hline \begin{tabular}{|l} 
Ste. \\
Genevieve \\
Democrat
\end{tabular} & $6 / 8 / 1850$ & Shawls & & & & \\
\hline $\begin{array}{l}\text { Ste. } \\
\text { Genevieve } \\
\text { Harold }\end{array}$ & $\begin{array}{r}12 / 17 / 189 \\
2\end{array}$ & Christmas & $\begin{array}{l}\text { X-water } \\
\text { pitcher, } \\
\text { dinner set, } \\
\text { butter dish, } \\
\text { pickel dish }\end{array}$ & & $\begin{array}{l}\text { X-towels, } \\
\text { silver } \\
\text { spoons, } \\
\text { carving kife } \\
\text { and fork, } \\
\text { table knife } \\
\text { and forks }\end{array}$ & $\begin{array}{l}\text { X-home, } \\
\text { wall } \\
\text { brackets, } \\
\text { clock } \\
\text { shelves }\end{array}$ \\
\hline $\begin{array}{l}\text { Ste. } \\
\text { Genevieve } \\
\text { Harold }\end{array}$ & \begin{tabular}{r|}
$\mid 12 / 17 / 189$ \\
2
\end{tabular} & Meyer \& Vorst & & & & \\
\hline $\begin{array}{l}\text { Ste. } \\
\text { Genevieve } \\
\text { Harold } \\
\end{array}$ & \begin{tabular}{r|}
$12 / 17 / 189$ \\
2
\end{tabular} & $\begin{array}{l}\text { Guaranteed } \\
\text { cured }\end{array}$ & & & & \\
\hline $\begin{array}{l}\text { Ste. } \\
\text { Genevieve } \\
\text { Harold }\end{array}$ & \begin{tabular}{|r|}
$\mid 12 / 17 / 189$ \\
2
\end{tabular} & M. Vier & & & & \\
\hline $\begin{array}{l}\text { Ste. } \\
\text { Genevieve } \\
\text { Harold }\end{array}$ & \begin{tabular}{|r|}
$\mid 12 / 17 / 189$ \\
2
\end{tabular} & $\begin{array}{l}\text { Hugh \& } \\
\text { Hunold's }\end{array}$ & & & & \\
\hline $\begin{array}{l}\text { Ste. } \\
\text { Genevieve } \\
\text { Harold }\end{array}$ & $\begin{array}{r}12 / 17 / 189 \\
2\end{array}$ & John Koetting & & & & \\
\hline $\begin{array}{l}\text { Ste. } \\
\text { Genevieve } \\
\text { Harold }\end{array}$ & \begin{tabular}{|r|}
$\mid 12 / 17 / 189$ \\
2
\end{tabular} & $\begin{array}{l}\text { Henry } \\
\text { Okenfuss }\end{array}$ & & & & \\
\hline $\begin{array}{l}\text { Ste. } \\
\text { Genevieve } \\
\text { Harold }\end{array}$ & \begin{tabular}{|r|}
$\mid 12 / 17 / 189$ \\
2
\end{tabular} & Mary E. Kern's & & & & \\
\hline
\end{tabular}




\begin{tabular}{|c|c|c|c|c|c|c|}
\hline \multicolumn{7}{|c|}{ English Newspapers } \\
\hline $\begin{array}{c}\text { Newspape } \\
r \text { name }\end{array}$ & Date & $\begin{array}{c}\text { advertisement } \\
\text { title }\end{array}$ & \begin{tabular}{|l} 
Household \\
Maintenanc \\
e-Ceramic
\end{tabular} & \begin{tabular}{|c|} 
Household \\
Maintenanc \\
e-glass \\
\end{tabular} & $\begin{array}{c}\text { Household } \\
\text { Maintenanc } \\
\text { e-other }\end{array}$ & \begin{tabular}{|l} 
Architectur \\
al items
\end{tabular} \\
\hline $\begin{array}{l}\text { Ste. } \\
\text { Genevieve } \\
\text { Harold } \\
\end{array}$ & $\begin{array}{r}12 / 17 / 189 \\
2 \\
\end{array}$ & $\begin{array}{l}\text { Usefule } \\
\text { Christmas } \\
\text { Presents! }\end{array}$ & & & & \\
\hline \begin{tabular}{|l} 
Ste. \\
Genevieve \\
Harold \\
\end{tabular} & \begin{tabular}{|r|}
$12 / 17 / 189$ \\
2
\end{tabular} & $\begin{array}{l}\text { Strength and } \\
\text { Health }\end{array}$ & & & & \\
\hline \begin{tabular}{|l} 
Ste. \\
Genevieve \\
Harold \\
\end{tabular} & \begin{tabular}{|r|}
$12 / 17 / 189$ \\
2
\end{tabular} & $\begin{array}{l}\text { Deserving } \\
\text { Praise }\end{array}$ & & & & \\
\hline $\begin{array}{l}\text { Ste. } \\
\text { Genevieve } \\
\text { Harold }\end{array}$ & $\begin{array}{r}12 / 17 / 189 \\
2\end{array}$ & $\begin{array}{l}\text { The Weedly } \\
\text { Globe } \\
\text { Democrat }\end{array}$ & & & & \\
\hline \begin{tabular}{|l} 
Ste. \\
Genevieve \\
Harold
\end{tabular} & \begin{tabular}{|r|}
$12 / 17 / 189$ \\
2
\end{tabular} & Easy to Take & & & & \\
\hline $\begin{array}{l}\text { Ste. } \\
\text { Genevieve } \\
\text { Harold }\end{array}$ & \begin{tabular}{|r|}
$12 / 17 / 189$ \\
2
\end{tabular} & $\begin{array}{l}\text { Bucklen's } \\
\text { Arnica Salve }\end{array}$ & & & & \\
\hline $\begin{array}{l}\text { Ste. } \\
\text { Genevieve } \\
\text { Herald } \\
\end{array}$ & \begin{tabular}{|r|}
$12 / 17 / 189$ \\
2
\end{tabular} & Val. Rottler & & & & \\
\hline \begin{tabular}{|l} 
Ste. \\
Genevieve \\
Herald \\
\end{tabular} & \begin{tabular}{|r|}
$12 / 17 / 189$ \\
2
\end{tabular} & $\begin{array}{l}\text { Ayer's Cherry } \\
\text { Pectoral }\end{array}$ & & & & \\
\hline \begin{tabular}{|l} 
Ste. \\
Genevieve \\
Herald \\
\end{tabular} & \begin{tabular}{|r|}
$12 / 17 / 189$ \\
2
\end{tabular} & $\begin{array}{l}\text { My Stock: } \\
\text { Chas. H. Biel }\end{array}$ & $\begin{array}{l}\text { X-China- } \\
\text { ware }\end{array}$ & & & X-hardware \\
\hline $\begin{array}{l}\text { Ste. } \\
\text { Genevieve } \\
\text { Herald }\end{array}$ & $\begin{array}{r}12 / 17 / 189 \\
2\end{array}$ & $\begin{array}{l}\text { Joseph Weixel, } \\
\text { St. Louis House }\end{array}$ & & & & \\
\hline \begin{tabular}{|l} 
Ste. \\
Genevieve \\
Herald \\
\end{tabular} & \begin{tabular}{|r|}
$12 / 17 / 189$ \\
2
\end{tabular} & E.A. Rozier & & & & \\
\hline $\begin{array}{l}\text { Ste. } \\
\text { Genevieve } \\
\text { Herald }\end{array}$ & \begin{tabular}{|r|}
$12 / 17 / 189$ \\
2
\end{tabular} & Henry Hohman & & & & \\
\hline $\begin{array}{l}\text { Ste. } \\
\text { Genevieve } \\
\text { Herald }\end{array}$ & $\begin{array}{r}\mid 12 / 17 / 189 \\
2\end{array}$ & Albert A. Boyer & & & & \\
\hline
\end{tabular}




\begin{tabular}{|c|c|c|c|c|c|c|}
\hline \multicolumn{7}{|c|}{ English Newspapers } \\
\hline $\begin{array}{c}\text { Newspape } \\
r \text { name }\end{array}$ & Date & $\begin{array}{c}\text { advertisement } \\
\text { title }\end{array}$ & \begin{tabular}{|l} 
Household \\
Maintenanc \\
e-Ceramic
\end{tabular} & \begin{tabular}{|c|} 
Household \\
Maintenanc \\
e-glass \\
\end{tabular} & $\begin{array}{c}\text { Household } \\
\text { Maintenanc } \\
\text { e-other }\end{array}$ & \begin{tabular}{|l} 
Architectur \\
al items
\end{tabular} \\
\hline $\begin{array}{l}\text { Ste. } \\
\text { Genevieve } \\
\text { Herald } \\
\end{array}$ & \begin{tabular}{|r|}
$12 / 17 / 189$ \\
2
\end{tabular} & $\begin{array}{l}\text { Hoffman \& } \\
\text { Karst }\end{array}$ & & & & \\
\hline \begin{tabular}{|l} 
Ste. \\
Genevieve \\
Herald \\
\end{tabular} & \begin{tabular}{|r|}
$12 / 17 / 189$ \\
2
\end{tabular} & $\begin{array}{l}\text { Drs. Lanning \& } \\
\text { Ruledge }\end{array}$ & & & & \\
\hline \begin{tabular}{|l} 
Ste. \\
Genevieve \\
Herald
\end{tabular} & \begin{tabular}{|r|}
$12 / 17 / 189$ \\
2
\end{tabular} & Meyer's Hotel & & & & \\
\hline $\begin{array}{l}\text { Ste. } \\
\text { Genevieve } \\
\text { Herald } \\
\end{array}$ & \begin{tabular}{|r|}
$12 / 17 / 189$ \\
2
\end{tabular} & F. \& L. U. Store & $\begin{array}{l}\text { X- } \\
\text { Queensware }\end{array}$ & X-Glassware & & X-Hardware \\
\hline \begin{tabular}{|l} 
Ste. \\
Genevieve \\
Herald
\end{tabular} & \begin{tabular}{|r|}
$12 / 17 / 189$ \\
2
\end{tabular} & TENT's Albums & & & & \\
\hline $\begin{array}{l}\text { Ste. } \\
\text { Genevieve } \\
\text { Herald }\end{array}$ & \begin{tabular}{|r|}
$12 / 17 / 189$ \\
2
\end{tabular} & City Mills & & & & \\
\hline $\begin{array}{l}\text { Ste. } \\
\text { Genevieve } \\
\text { Herald } \\
\end{array}$ & \begin{tabular}{r|}
$12 / 17 / 189$ \\
2 \\
\end{tabular} & \begin{tabular}{|l|} 
Mich. \\
Beauchamp \\
Tonsorial Artist
\end{tabular} & & & & \\
\hline \begin{tabular}{|l} 
Ste. \\
Genevieve \\
Herald \\
\end{tabular} & \begin{tabular}{|r|}
$12 / 17 / 189$ \\
2
\end{tabular} & $\begin{array}{l}\text { American } \\
\text { Farmer }\end{array}$ & & & & \\
\hline \begin{tabular}{|l} 
Ste. \\
Genevieve \\
Herald \\
\end{tabular} & \begin{tabular}{|r|}
$12 / 17 / 189$ \\
2
\end{tabular} & Mrs. E. Kern & & & & \\
\hline $\begin{array}{l}\text { Ste. } \\
\text { Genevieve } \\
\text { Herald }\end{array}$ & \begin{tabular}{|r|}
$12 / 17 / 189$ \\
2
\end{tabular} & For Men only & & & & \\
\hline \begin{tabular}{|l} 
Ste. \\
Genevieve \\
Herald \\
\end{tabular} & \begin{tabular}{|r|}
$12 / 17 / 189$ \\
2
\end{tabular} & $\begin{array}{l}\text { Patents, C.A. } \\
\text { Snow \& Co. }\end{array}$ & & & & \\
\hline \begin{tabular}{|l} 
Ste. \\
Genevieve \\
Herald
\end{tabular} & \begin{tabular}{|r|}
$12 / 17 / 189$ \\
2
\end{tabular} & A.J. Tower & & & & \\
\hline $\begin{array}{l}\text { Ste. } \\
\text { Genevieve } \\
\text { Herald }\end{array}$ & $\begin{array}{r}\mid 12 / 17 / 189 \\
2\end{array}$ & $\begin{array}{l}\text { Tower's fish } \\
\text { brand }\end{array}$ & & & & \\
\hline
\end{tabular}




\begin{tabular}{|c|c|c|c|c|c|c|}
\hline \multicolumn{7}{|c|}{ English Newspapers } \\
\hline $\begin{array}{c}\text { Newspape } \\
\mathrm{r} \text { name }\end{array}$ & Date & $\begin{array}{c}\text { advertisement } \\
\text { title }\end{array}$ & $\begin{array}{l}\text { Household } \\
\text { Maintenanc } \\
\text { e-Ceramic }\end{array}$ & \begin{tabular}{|c} 
Household \\
Maintenanc \\
e-glass
\end{tabular} & $\begin{array}{c}\text { Household } \\
\text { Maintenanc } \\
\text { e-other }\end{array}$ & $\begin{array}{c}\text { Architectur } \\
\text { al items }\end{array}$ \\
\hline \begin{tabular}{|l|} 
Ste. \\
Genevieve \\
Herald
\end{tabular} & \begin{tabular}{|r|}
$12 / 17 / 189$ \\
2
\end{tabular} & $\begin{array}{l}\text { Prof. Harris' } \\
\text { Pastilles }\end{array}$ & & & & \\
\hline \begin{tabular}{|l|} 
Ste. \\
Genevieve \\
Herald
\end{tabular} & \begin{tabular}{r|}
$12 / 17 / 189$ \\
2
\end{tabular} & $\begin{array}{l}\text { Patents- } \\
\text { Scientific } \\
\text { American }\end{array}$ & & & & \\
\hline \begin{tabular}{|l|} 
Ste. \\
Gevevieve \\
Herald
\end{tabular} & \begin{tabular}{|r|}
$12 / 17 / 189$ \\
2
\end{tabular} & $\begin{array}{l}\text { Ste. Genevieve } \\
\text { Bakery }\end{array}$ & & & & \\
\hline
\end{tabular}

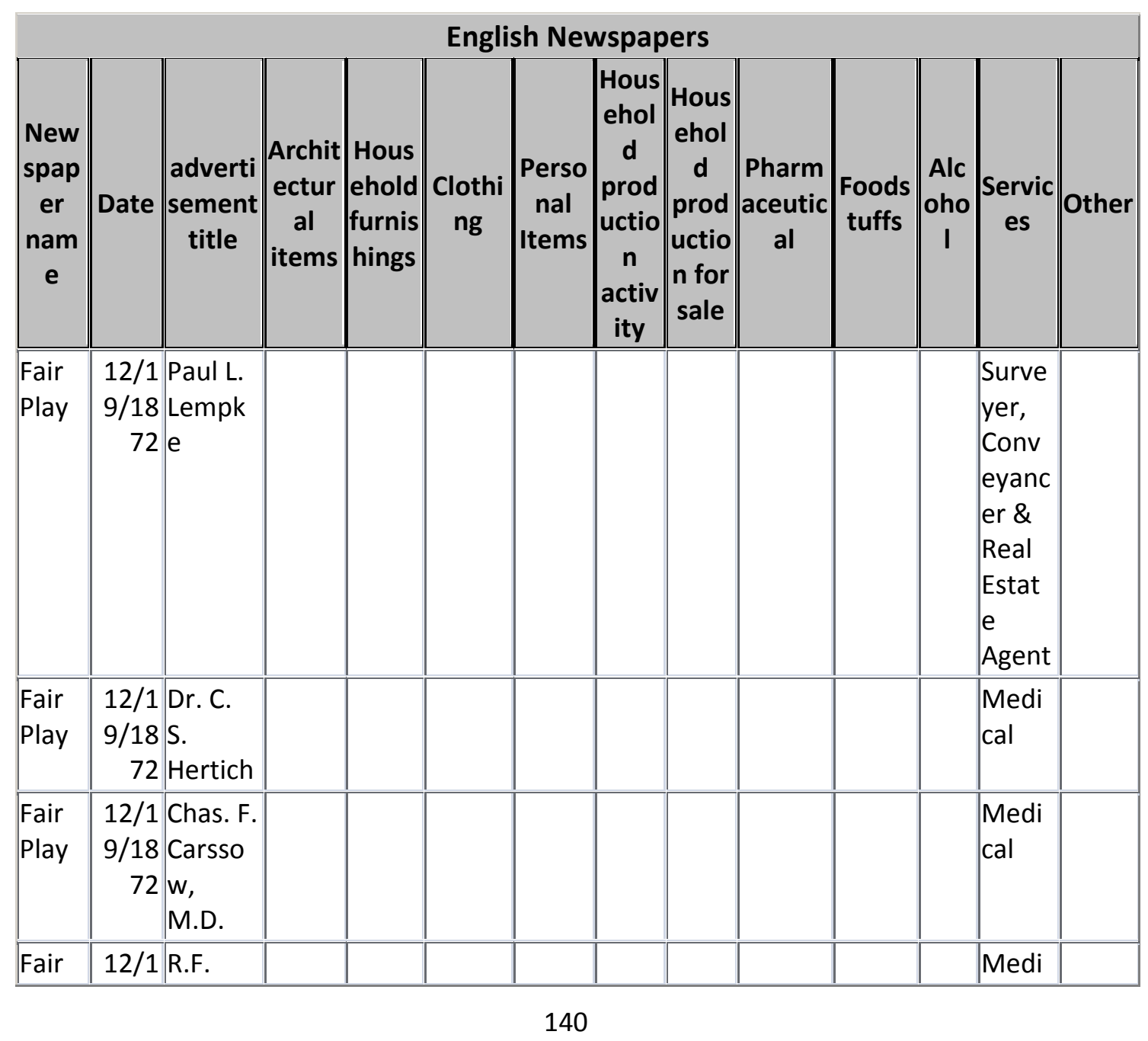




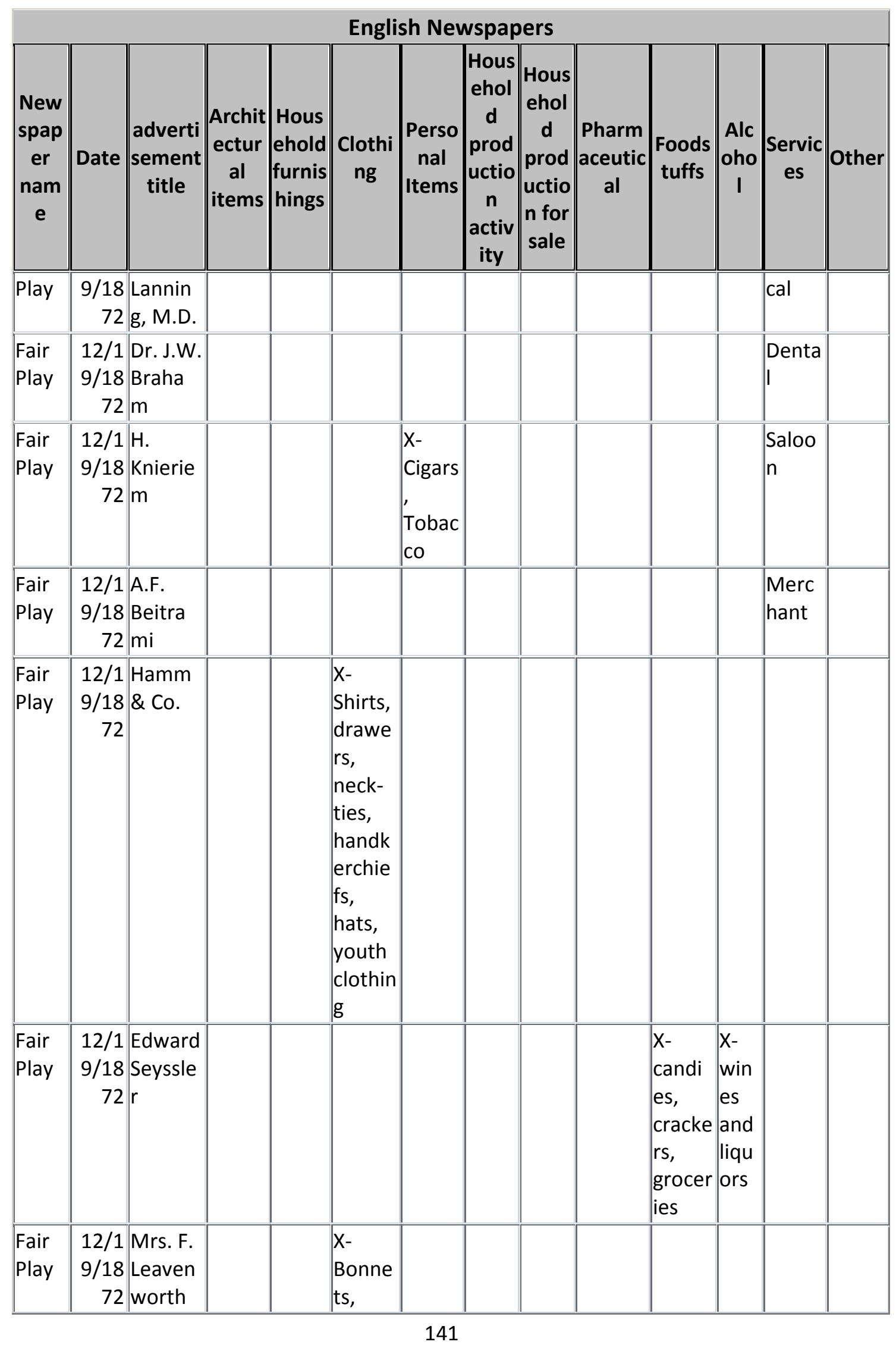




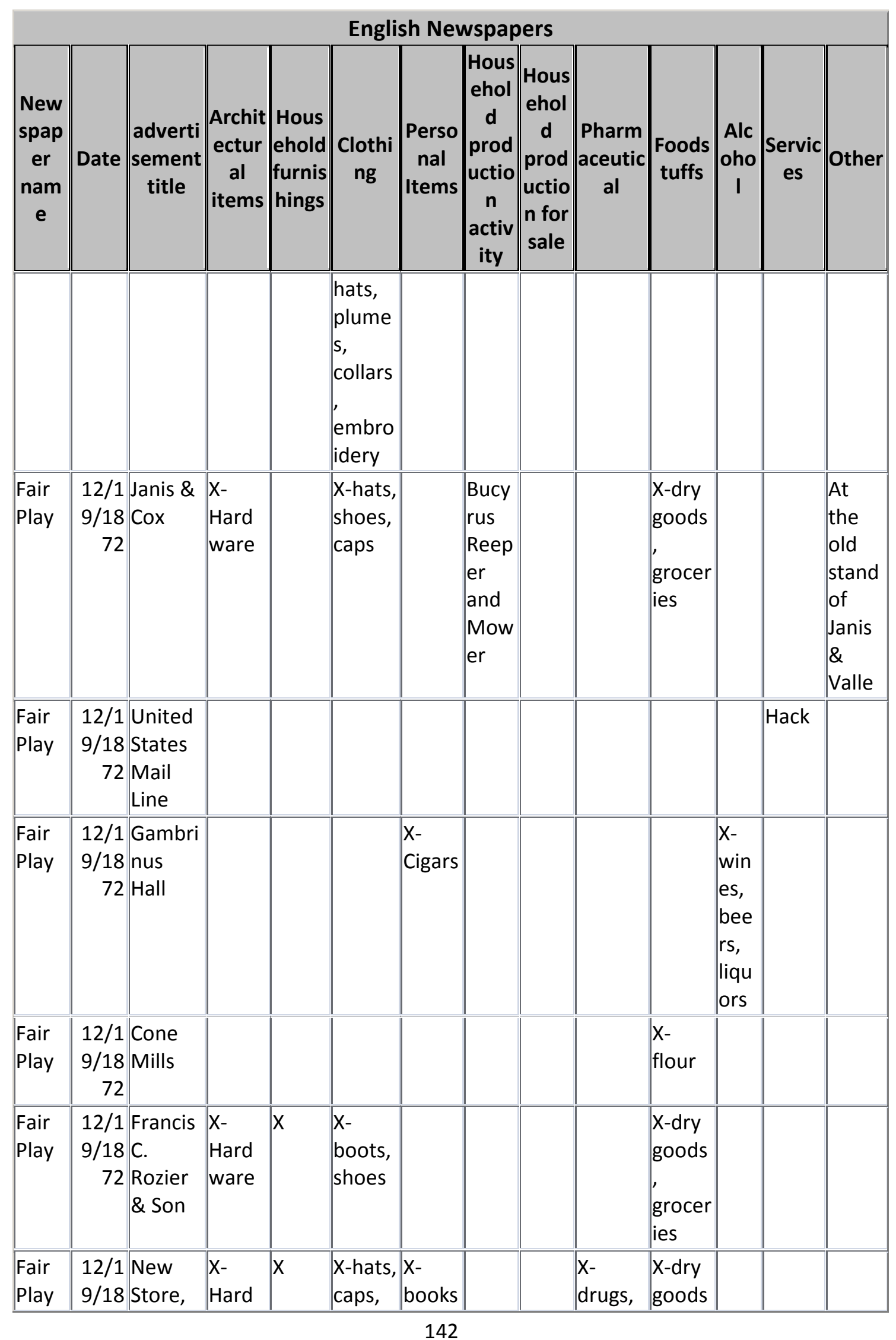




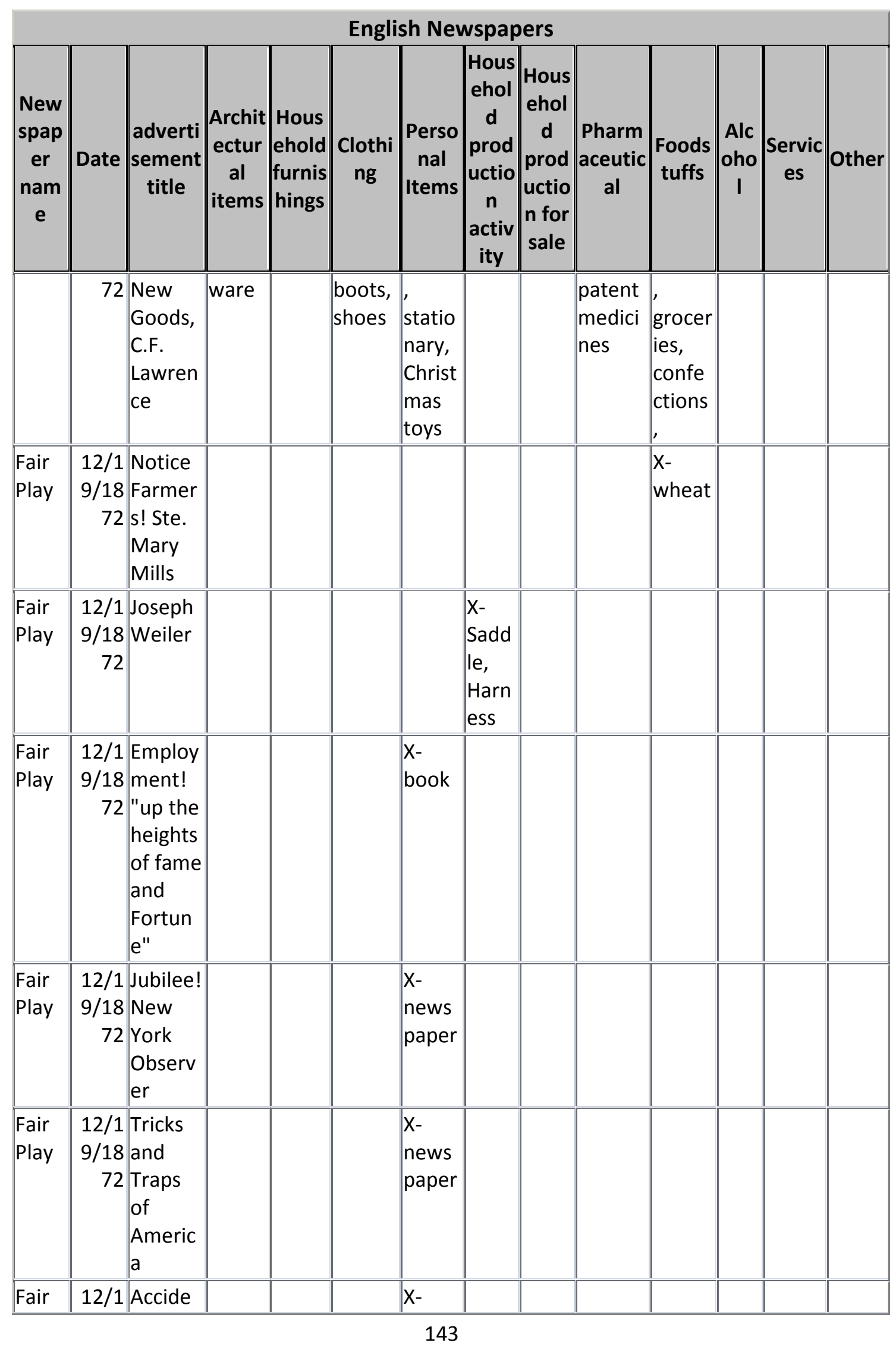




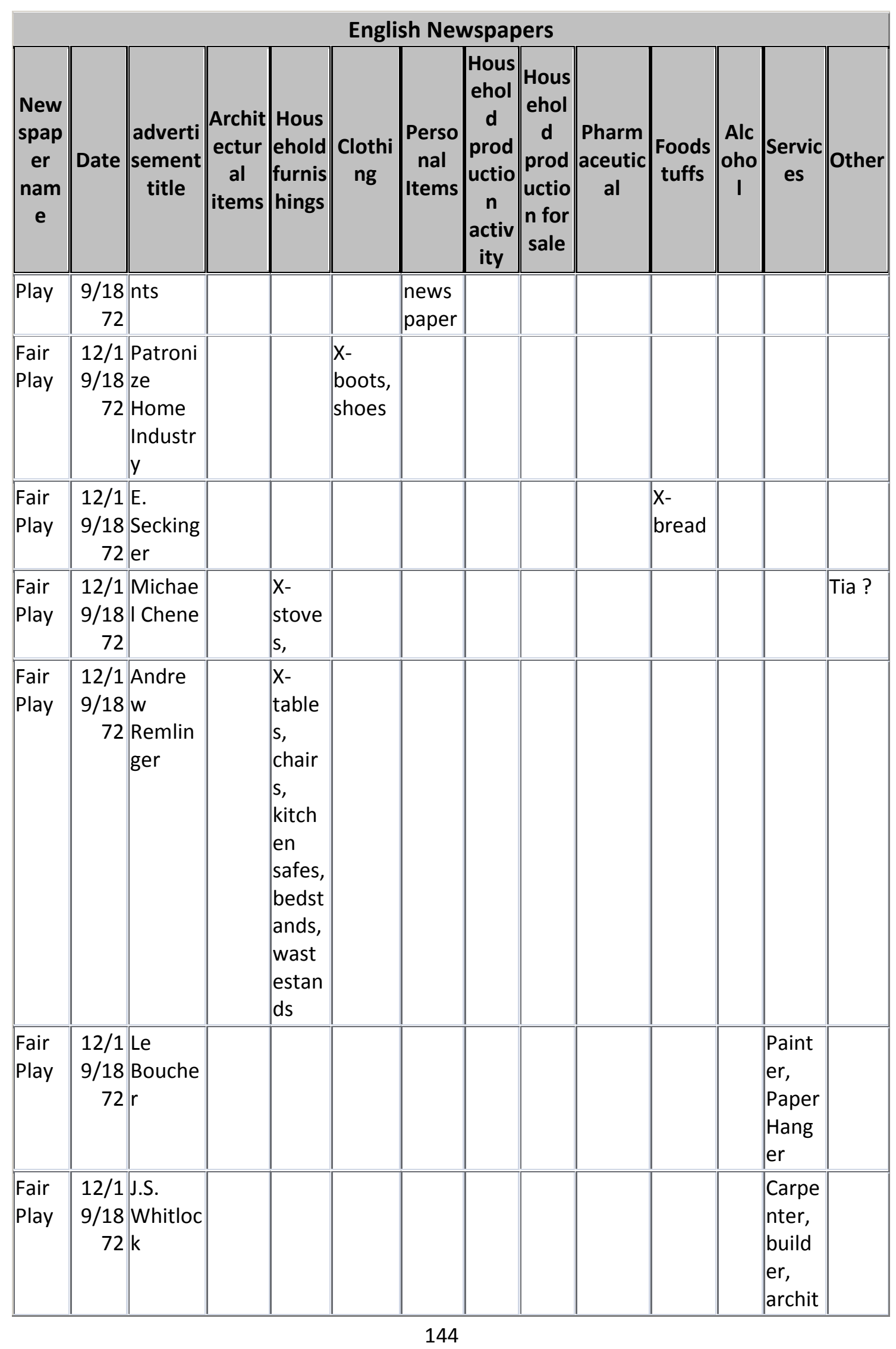




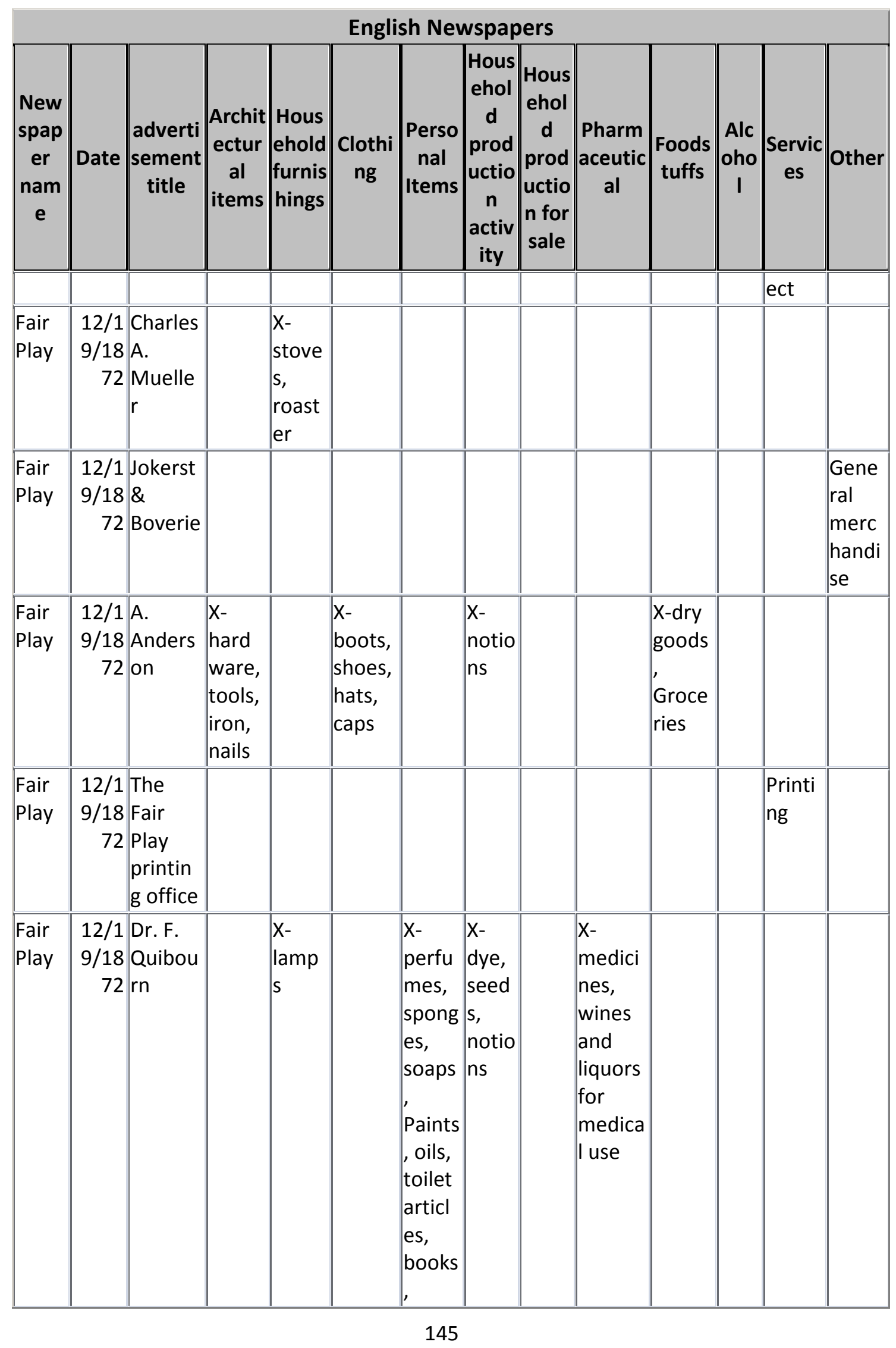




\begin{tabular}{|c|c|c|c|c|c|c|c|c|c|c|c|c|c|}
\hline \multicolumn{14}{|c|}{ English Newspapers } \\
\hline $\begin{array}{c}\text { New } \\
\text { spap } \\
\text { er } \\
\text { nam } \\
\text { e }\end{array}$ & Date & $\mid \begin{array}{c}\text { adverti } \\
\text { sement } \\
\text { title }\end{array}$ & $\mid \begin{array}{c}\text { Archit } \\
\text { ectur } \\
\text { al } \\
\text { items }\end{array}$ & $\begin{array}{l}\text { Hous } \\
\text { ehold } \\
\text { furnis } \\
\text { hings }\end{array}$ & $\begin{array}{c}\text { Clothi } \\
\text { ng }\end{array}$ & \begin{tabular}{|c} 
Perso \\
nal \\
Items
\end{tabular} & \begin{tabular}{|c|} 
Hous \\
ehol \\
d \\
prod \\
uctio \\
n \\
activ \\
ity
\end{tabular} & $\mid \begin{array}{c}\text { Hous } \\
\text { ehol } \\
d \\
\text { prod } \\
\text { uctio } \\
\text { n for } \\
\text { sale }\end{array}$ & \begin{tabular}{|c} 
Pharm \\
aceutic \\
al
\end{tabular} & $\mid \begin{array}{c}\text { Foods } \\
\text { tuffs }\end{array}$ & $\mid \begin{array}{c}\text { Alc } \\
\text { oho } \\
\text { I }\end{array}$ & $\begin{array}{c}\text { Servic } \\
\text { es }\end{array}$ & Other \\
\hline & & & & & & $\begin{array}{l}\text { statio } \\
\text { nary }\end{array}$ & & & & & & & \\
\hline $\begin{array}{l}\text { Fair } \\
\text { Play }\end{array}$ & \begin{tabular}{|r|}
$12 / 1$ \\
$9 / 18$ \\
72 \\
\end{tabular} & $\begin{array}{l}\text { Job } \\
\text { Printin } \\
\text { g }\end{array}$ & & & & & & & & & & $\begin{array}{l}\text { Printi } \\
\text { ng }\end{array}$ & \\
\hline \begin{tabular}{|l} 
Fair \\
Play
\end{tabular} & \begin{tabular}{|r|}
$12 / 1$ \\
$9 / 18$ \\
72 \\
\end{tabular} & \begin{tabular}{|l|} 
The \\
Southe \\
ast \\
Report \\
er
\end{tabular} & & & & & & & & & & $\begin{array}{l}\text { News } \\
\text { paper }\end{array}$ & \\
\hline $\begin{array}{l}\text { Fair } \\
\text { Play }\end{array}$ & \begin{tabular}{|r|}
$12 / 1$ \\
$9 / 18$ \\
72 \\
\end{tabular} & $\begin{array}{l}\text { Laclede } \\
\text { County } \\
\text { Leader }\end{array}$ & & & & & & & & & & $\begin{array}{l}\text { News } \\
\text { paper }\end{array}$ & \\
\hline $\begin{array}{l}\text { Fair } \\
\text { Play }\end{array}$ & \begin{tabular}{|r|}
$12 / 1$ \\
$9 / 18$ \\
72 \\
\end{tabular} & \begin{tabular}{|l|} 
Farmin \\
gton \\
New \\
Era
\end{tabular} & & & & & & & & & & $\begin{array}{l}\text { News } \\
\text { paper }\end{array}$ & \\
\hline $\begin{array}{l}\text { Fair } \\
\text { Play }\end{array}$ & \begin{tabular}{|r|}
$12 / 1$ \\
$9 / 18$ \\
72 \\
\end{tabular} & \begin{tabular}{|l|} 
The \\
Jeffers \\
on \\
County \\
Republi \\
can
\end{tabular} & & & & & & & & & & $\begin{array}{l}\text { News } \\
\text { paper }\end{array}$ & \\
\hline $\begin{array}{l}\text { Fair } \\
\text { Play }\end{array}$ & \begin{tabular}{|r|}
$12 / 1$ \\
$9 / 18$ \\
72 \\
\end{tabular} & T. & & & & & & & & $\begin{array}{l}\text { X-dry } \\
\text { goods } \\
\text { grocer } \\
\text { ies }\end{array}$ & & & \\
\hline $\begin{array}{l}\text { Fair } \\
\text { Play }\end{array}$ & \begin{tabular}{|r|}
$12 / 1$ \\
$9 / 18$ \\
72 \\
\end{tabular} & $\begin{array}{l}\text { The } \\
\text { Fair } \\
\text { Play }\end{array}$ & & & & & & & & & & $\begin{array}{l}\text { News } \\
\text { paper }\end{array}$ & \\
\hline $\begin{array}{l}\text { Fair } \\
\text { Play }\end{array}$ & \begin{tabular}{|r|}
$12 / 1$ \\
$9 / 18$ \\
72 \\
\end{tabular} & \begin{tabular}{|l|} 
The \\
Gayoso \\
Democ \\
rat
\end{tabular} & & & & & & & & & & $\begin{array}{l}\text { News } \\
\text { paper }\end{array}$ & \\
\hline
\end{tabular}




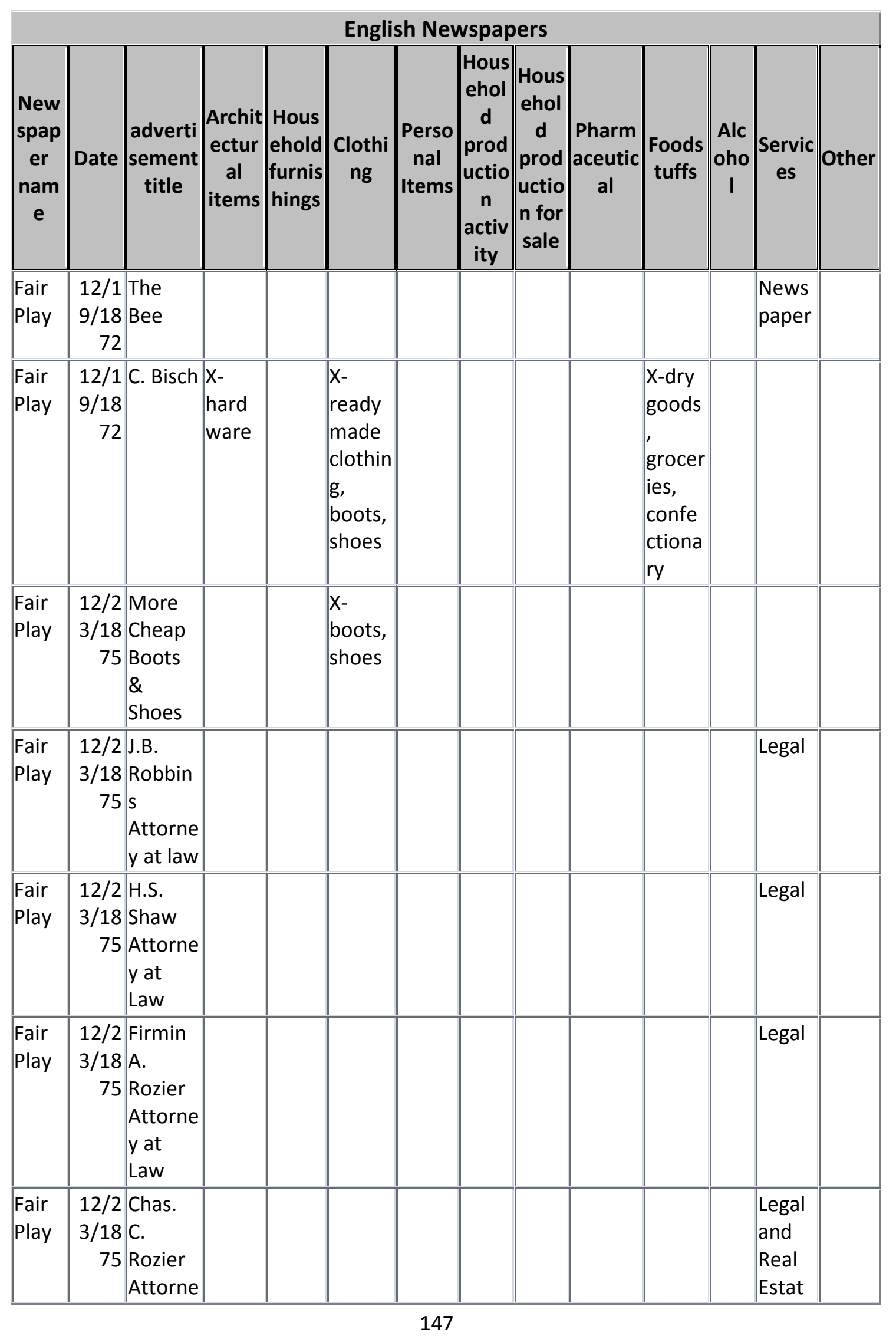




\begin{tabular}{|c|c|c|c|c|c|c|c|c|c|c|c|c|c|}
\hline \multicolumn{14}{|c|}{ English Newspapers } \\
\hline $\begin{array}{c}\text { New } \\
\text { spap } \\
\text { er } \\
\text { nam } \\
\text { e }\end{array}$ & Date & $\mid \begin{array}{c}\text { adverti } \\
\text { sement } \\
\text { title }\end{array}$ & $\mid \begin{array}{c}\text { Archit } \\
\text { ectur } \\
\text { al } \\
\text { items }\end{array}$ & $\begin{array}{l}\text { Hous } \\
\text { ehold } \\
\text { furnis } \\
\text { hings }\end{array}$ & $\begin{array}{c}\text { Clothi } \\
\text { ng }\end{array}$ & \begin{tabular}{|c} 
Perso \\
nal \\
Items
\end{tabular} & \begin{tabular}{|c|} 
Hous \\
ehol \\
d \\
prod \\
uctio \\
n \\
activ \\
ity
\end{tabular} & $\mid \begin{array}{c}\text { Hous } \\
\text { ehol } \\
d \\
\text { prod } \\
\text { uctio } \\
\text { n for } \\
\text { sale }\end{array}$ & $\mid \begin{array}{c}\text { Pharm } \\
\text { aceutic } \\
\text { al }\end{array}$ & $\begin{array}{c}\text { Foods } \\
\text { tuffs }\end{array}$ & $\mid \begin{array}{c}\text { Alc } \\
\text { oho } \\
\text { I }\end{array}$ & $\mid \begin{array}{c}\text { Servic } \\
\text { es }\end{array}$ & Other \\
\hline & & $\begin{array}{l}\text { y at } \\
\text { Law }\end{array}$ & & & & & & & & & & e & \\
\hline \begin{tabular}{|l} 
Fair \\
Play
\end{tabular} & $\begin{array}{r}12 / 2 \\
3 / 18 \\
75\end{array}$ & \begin{tabular}{|l} 
Dr. C. \\
S. \\
Carsso \\
w, \\
M.D.
\end{tabular} & & & & & & & & & & $\begin{array}{l}\text { Medi } \\
\text { cal }\end{array}$ & \\
\hline $\begin{array}{l}\text { Fair } \\
\text { Play }\end{array}$ & \begin{tabular}{r|}
$12 / 2$ \\
$3 / 18$ \\
75
\end{tabular} & $\begin{array}{l}\text { R.F. } \\
\text { Lannin } \\
\text { g, M.D. }\end{array}$ & & & & & & & & & & $\begin{array}{l}\text { Medi } \\
\text { cal }\end{array}$ & \\
\hline \begin{tabular}{|l} 
Fair \\
Play
\end{tabular} & \begin{tabular}{r|}
$12 / 2$ \\
$3 / 18$ \\
75 \\
\end{tabular} & $\begin{array}{l}\text { M.F. } \\
\text { Spaldin } \\
\text { g, M.D. }\end{array}$ & & & & & & & & & & $\begin{array}{l}\text { Medi } \\
\text { cal }\end{array}$ & \\
\hline \begin{tabular}{|l} 
Fair \\
Play
\end{tabular} & $\begin{array}{r}12 / 2 \\
3 / 18 \\
75 \\
\end{array}$ & \begin{tabular}{|l} 
Dr. J.W. \\
Braha \\
m
\end{tabular} & & & & & & & & & & Denta & \\
\hline \begin{tabular}{|l} 
Fair \\
Play
\end{tabular} & \begin{tabular}{r|}
$12 / 2$ \\
$3 / 18$ \\
75
\end{tabular} & $\begin{array}{l}\text { Dr. F. } \\
\text { Guibou } \\
\text { rd }\end{array}$ & & & & \begin{tabular}{|l} 
X- \\
Paints \\
oils
\end{tabular} & & & \begin{tabular}{|l|} 
X- \\
drugs, \\
medici \\
nes
\end{tabular} & & & & \\
\hline \begin{tabular}{|l} 
Fair \\
Play
\end{tabular} & $\begin{array}{r}12 / 2 \\
3 / 18 \\
75\end{array}$ & $\begin{array}{l}\text { F.C. } \\
\text { Albert }\end{array}$ & & & & & & & & & & $\begin{array}{l}\text { Unde } \\
\text { rtaker }\end{array}$ & \\
\hline \begin{tabular}{|l} 
Fair \\
Play
\end{tabular} & $\begin{array}{r}12 / 2 \\
3 / 18 \\
75 \\
\end{array}$ & $\begin{array}{l}\text { Read } \\
\text { this: } \\
\text { Rev. } \\
\text { Father } \\
\text { Stein } \\
\end{array}$ & & & & & \begin{tabular}{|l|} 
X- \\
plant \\
S, \\
flow \\
ers \\
\end{tabular} & & & & & & \\
\hline \begin{tabular}{|l} 
Fair \\
Play
\end{tabular} & \begin{tabular}{r|}
$12 / 2$ \\
$3 / 18$ \\
75
\end{tabular} & $\begin{array}{l}\text { Gambri } \\
\text { nus } \\
\text { Hall }\end{array}$ & & & & & & & & & $\begin{array}{l}X- \\
\text { bee } \\
r, \\
\text { liqu } \\
\text { or } \\
\end{array}$ & & \\
\hline \begin{tabular}{|l} 
Fair \\
Play
\end{tabular} & $\begin{array}{l}12 / 2 \\
3 / 18\end{array}$ & $\begin{array}{l}\text { Ste. } \\
\text { Genevi }\end{array}$ & & & & & & & & & & Hotel & \\
\hline
\end{tabular}




\begin{tabular}{|c|c|c|c|c|c|c|c|c|c|c|c|c|c|}
\hline \multicolumn{14}{|c|}{ English Newspapers } \\
\hline \multirow[t]{2}{*}{$\begin{array}{c}\text { New } \\
\text { spap } \\
\text { er } \\
\text { nam } \\
\text { e }\end{array}$} & Date & \begin{tabular}{|c} 
adverti \\
sement \\
title
\end{tabular} & $\begin{array}{c}\text { Archit } \\
\text { ectur } \\
\text { al } \\
\text { items }\end{array}$ & $\begin{array}{l}\text { Hous } \\
\text { ehold } \\
\text { furnis } \\
\text { hings }\end{array}$ & $\begin{array}{c}\text { Clothi } \\
\text { ng }\end{array}$ & $\begin{array}{c}\text { Perso } \\
\text { nal } \\
\text { Items }\end{array}$ & $\begin{array}{l}\text { Hous } \\
\text { ehol } \\
\text { d } \\
\text { prod } \\
\text { uctio } \\
\text { n } \\
\text { activ } \\
\text { ity }\end{array}$ & \begin{tabular}{|l} 
Hous \\
ehol \\
d \\
prod \\
uctio \\
n for \\
sale
\end{tabular} & $\begin{array}{c}\text { Pharm } \\
\text { aceutic } \\
\text { al }\end{array}$ & $\begin{array}{c}\text { Foods } \\
\text { tuffs }\end{array}$ & $\mid \begin{array}{c}\text { Alc } \\
\text { oho } \\
\text { I }\end{array}$ & $\begin{array}{c}\text { Servic } \\
\text { es }\end{array}$ & Other \\
\hline & 75 & $\begin{array}{l}\text { eve } \\
\text { House }\end{array}$ & & & & & & & & & & & \\
\hline $\begin{array}{l}\text { Fair } \\
\text { Play }\end{array}$ & $\begin{array}{r}12 / 2 \\
3 / 18 \\
75\end{array}$ & $\begin{array}{l}\text { Bogy \& } \\
\text { Guigno } \\
\text { n }\end{array}$ & & & & & & & & & & $\begin{array}{l}\text { Real } \\
\text { Estat } \\
\text { e }\end{array}$ & \\
\hline $\begin{array}{l}\text { Fair } \\
\text { Play }\end{array}$ & $\begin{array}{r}12 / 2 \\
3 / 18 \\
75\end{array}$ & $\begin{array}{l}\text { J.S. } \\
\text { Whitloc } \\
\mathrm{k}\end{array}$ & & & & & & & & & & $\begin{array}{l}\text { Carpe } \\
\text { nter, } \\
\text { archit } \\
\text { ect }\end{array}$ & \\
\hline $\begin{array}{l}\text { Fair } \\
\text { Play }\end{array}$ & $\begin{array}{r}12 / 2 \\
3 / 18 \\
75 \\
\end{array}$ & $\begin{array}{l}\text { Lumber } \\
\text { Yard }\end{array}$ & $\begin{array}{l}\text { X- } \\
\text { lumb } \\
\text { er }\end{array}$ & & & & & & & & & & \\
\hline $\begin{array}{l}\text { Fair } \\
\text { Play }\end{array}$ & $\begin{array}{r}12 / 2 \\
3 / 18 \\
75\end{array}$ & $\begin{array}{l}\text { P.U. } \\
\text { Jaccard }\end{array}$ & & $\begin{array}{l}\text { clock } \\
\text { s }\end{array}$ & & \begin{tabular}{|l} 
X- \\
watch \\
es, \\
jewel \\
ry,
\end{tabular} & & & & & & & \\
\hline $\begin{array}{l}\text { Fair } \\
\text { Play }\end{array}$ & $\begin{array}{r}12 / 2 \\
3 / 18 \\
75\end{array}$ & $\begin{array}{l}\text { Mrs. F. } \\
\text { Leaven } \\
\text { worth }\end{array}$ & & & \begin{tabular}{|l} 
X- \\
bonne \\
ts, \\
hats, \\
plume \\
s, \\
ribbins \\
, laces, \\
collars \\
embro \\
idery
\end{tabular} & & & & & & & & \\
\hline $\begin{array}{l}\text { Fair } \\
\text { Play }\end{array}$ & $\begin{array}{r}12 / 2 \\
3 / 18 \\
75 \\
\end{array}$ & \begin{tabular}{|l} 
Christia \\
n \\
Baum, \\
Boots \\
$\&$ \\
Shoes
\end{tabular} & & & \begin{tabular}{|l|} 
X- \\
boots, \\
shoes, \\
slipper \\
s
\end{tabular} & & & & & & & & \\
\hline
\end{tabular}




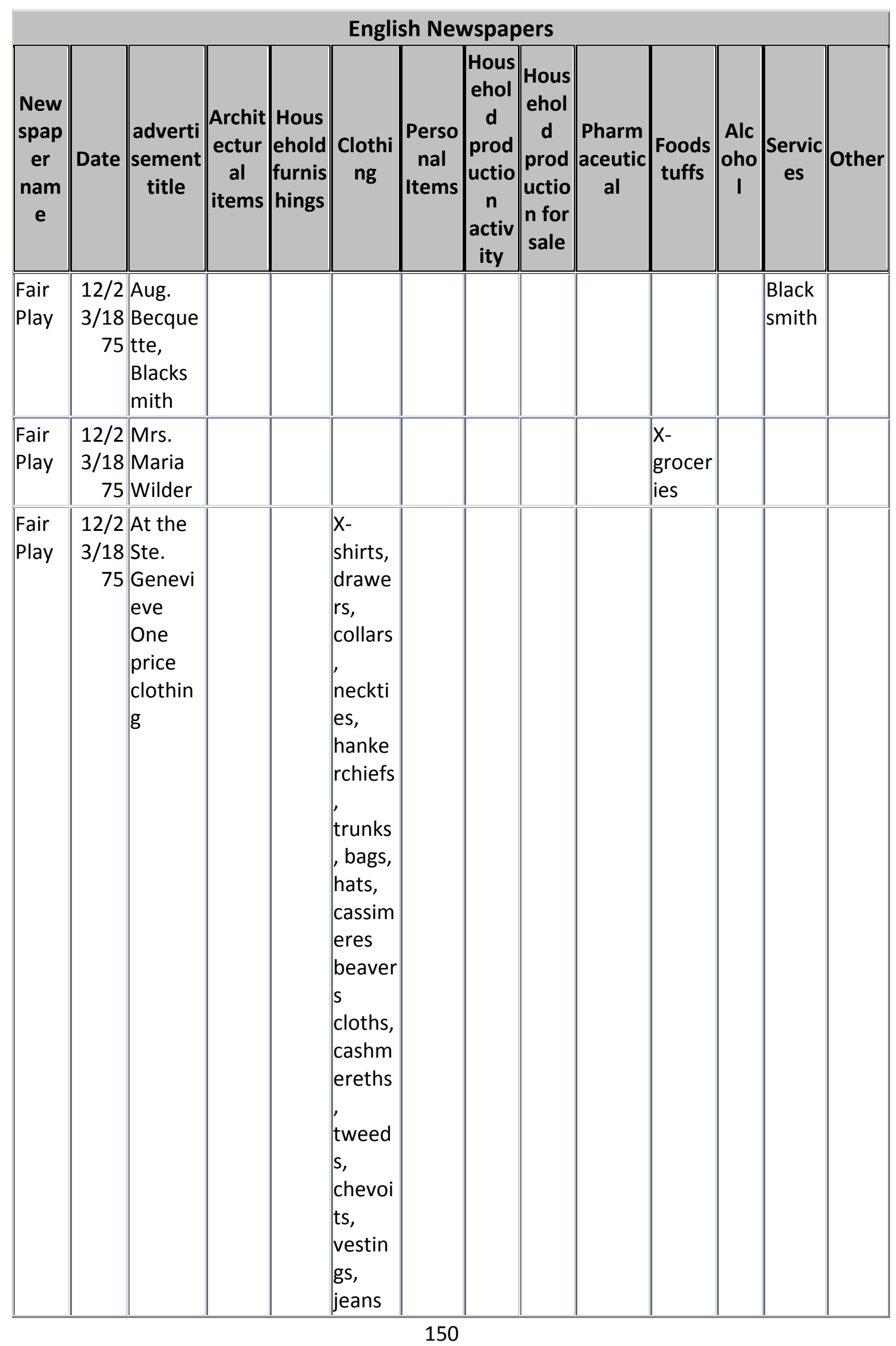




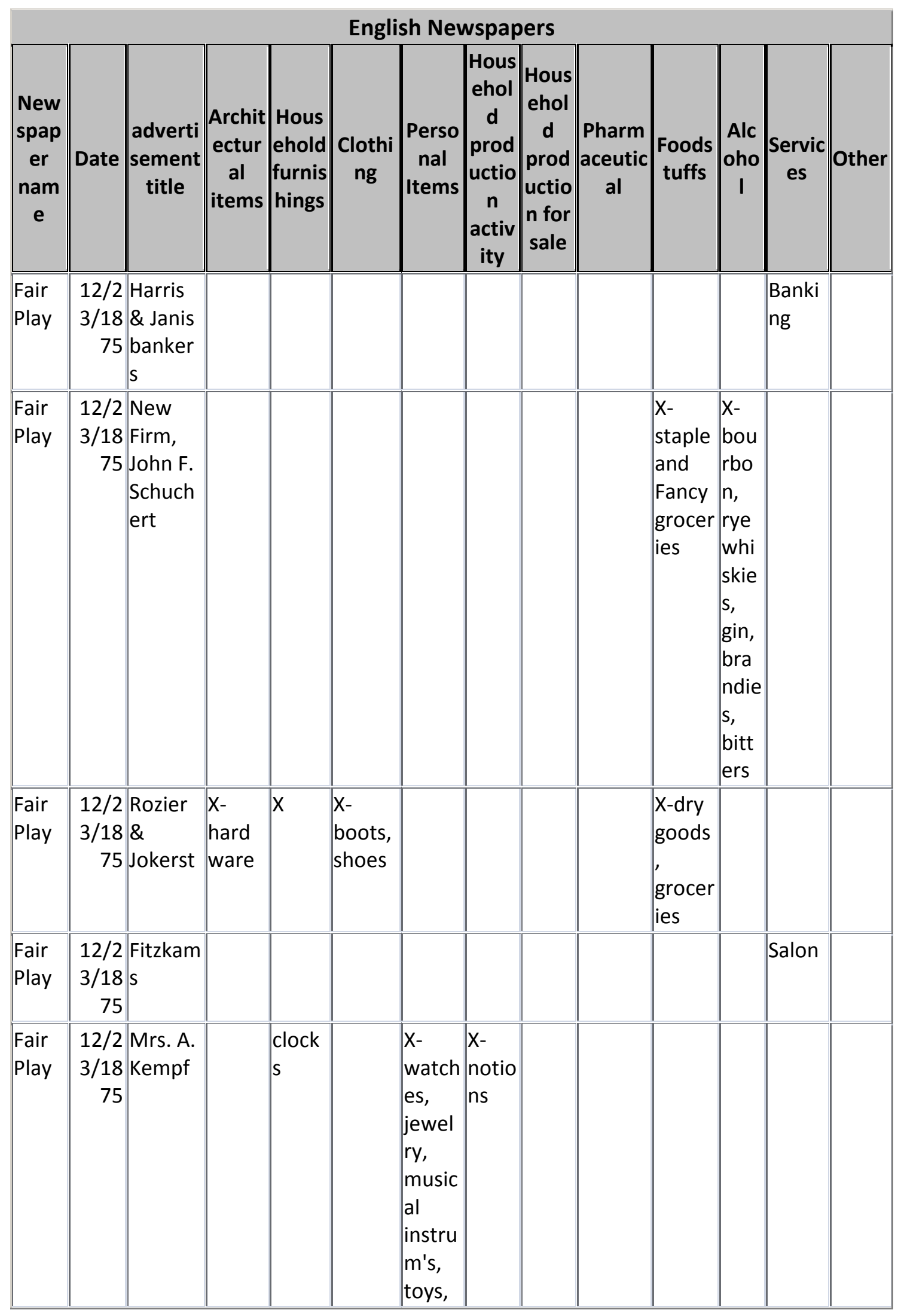




\begin{tabular}{|c|c|c|c|c|c|c|c|c|c|c|c|c|c|}
\hline \multicolumn{14}{|c|}{ English Newspapers } \\
\hline $\begin{array}{c}\text { New } \\
\text { spap } \\
\text { er } \\
\text { nam } \\
\text { e }\end{array}$ & Date & $\begin{array}{c}\text { adverti } \\
\text { sement } \\
\text { title }\end{array}$ & \begin{tabular}{|} 
Archit \\
ectur \\
al \\
items
\end{tabular} & $\mid \begin{array}{l}\text { Hous } \\
\text { ehold } \\
\text { furnis } \\
\text { hings }\end{array}$ & $\begin{array}{c}\text { Clothi } \\
\text { ng }\end{array}$ & \begin{tabular}{|c} 
Perso \\
nal \\
Items
\end{tabular} & $\begin{array}{l}\text { Hous } \\
\text { ehol } \\
\text { d } \\
\text { prod } \\
\text { uctio } \\
\text { n } \\
\text { activ } \\
\text { ity }\end{array}$ & $\mid \begin{array}{c}\text { Hous } \\
\text { ehol } \\
d \\
\text { prod } \\
\text { uctio } \\
n \text { for } \\
\text { sale }\end{array}$ & $\mid \begin{array}{c}\text { Pharm } \\
\text { aceutic } \\
\text { al }\end{array}$ & $\begin{array}{c}\text { Foods } \\
\text { tuffs }\end{array}$ & $\mid \begin{array}{c}\text { Alc } \\
\text { oho } \\
\text { I }\end{array}$ & $\begin{array}{c}\text { Servic } \\
\text { es }\end{array}$ & Other \\
\hline & & & & & & $\begin{array}{l}\text { toilet } \\
\text { soaps } \\
\text { perfu } \\
\text { mes }\end{array}$ & & & & & & & \\
\hline \begin{tabular}{|l} 
Fair \\
Play
\end{tabular} & $\begin{array}{r}12 / 2 \\
3 / 18 \\
75\end{array}$ & $\begin{array}{l}\text { P.U. } \\
\text { Jaccard }\end{array}$ & & & & $\begin{array}{l}\text { X- } \\
\text { watch }\end{array}$ & & & & & & & \\
\hline $\begin{array}{l}\text { Fair } \\
\text { Play }\end{array}$ & $\begin{array}{r}12 / 2 \\
3 / 18 \\
75\end{array}$ & $\begin{array}{l}\text { Terpsic } \\
\text { horean } \\
\text { Hall }\end{array}$ & & & & $\begin{array}{l}\text { X- } \\
\text { cigars }\end{array}$ & & & & & $\begin{array}{l}X- \\
\text { win } \\
e, \\
\text { bee } \\
r, \\
\text { liqu } \\
\text { ors }\end{array}$ & $\begin{array}{l}\text { Saloo } \\
n\end{array}$ & \\
\hline \begin{tabular}{|l} 
Fair \\
Play
\end{tabular} & \begin{tabular}{r|}
$12 / 2$ \\
$3 / 18$ \\
75
\end{tabular} & \begin{tabular}{|l|} 
Edward \\
Seyssle \\
$r$
\end{tabular} & & & & & & & & \begin{tabular}{|l|} 
X- \\
grocer \\
ies, \\
candi \\
es, \\
cracke \\
rs \\
\end{tabular} & $\begin{array}{l}\text { X- } \\
\text { bitt } \\
\text { ers, } \\
\text { win } \\
\text { es, } \\
\text { liqu } \\
\text { ors }\end{array}$ & & \\
\hline \begin{tabular}{|l} 
Fair \\
Play
\end{tabular} & \begin{tabular}{r|}
$12 / 2$ \\
$3 / 18$ \\
75 \\
\end{tabular} & $\begin{array}{l}\text { Regular } \\
\text { Connec } \\
\text { t'n }\end{array}$ & & & & & & & & & & Hack & \\
\hline \begin{tabular}{|l} 
Fair \\
Play
\end{tabular} & $\begin{array}{r}12 / 2 \\
3 / 18 \\
75 \\
\end{array}$ & \begin{tabular}{|l|} 
Ste. \\
Genevi \\
eve \\
Livery, \\
Feed
\end{tabular} & & & & & & $\begin{array}{l}\text { X- } \\
\text { Liver } \\
y, \\
\text { feed, } \\
\text { stabl } \\
\text { es } \\
\end{array}$ & & & & & \\
\hline \begin{tabular}{|l} 
Fair \\
Play
\end{tabular} & \begin{tabular}{r|}
$12 / 2$ \\
$3 / 18$ \\
75 \\
\end{tabular} & $\begin{array}{l}\text { Daily } \\
\text { Hack } \\
\text { Line }\end{array}$ & & & & & & & & & & $\begin{array}{l}\text { Hack, } \\
\text { Mail }\end{array}$ & \\
\hline Fair & $12 / 2$ & Ste. & & & & & & & & & & hack & \\
\hline
\end{tabular}




\begin{tabular}{|c|c|c|c|c|c|c|c|c|c|c|c|c|c|}
\hline \multicolumn{14}{|c|}{ English Newspapers } \\
\hline $\begin{array}{c}\text { New } \\
\text { spap } \\
\text { er } \\
\text { nam } \\
\text { e }\end{array}$ & Date & $\begin{array}{c}\text { adverti } \\
\text { sement } \\
\text { title }\end{array}$ & $\mid \begin{array}{c}\text { Archit } \\
\text { ectur } \\
\text { al } \\
\text { items }\end{array}$ & $\begin{array}{l}\text { Hous } \\
\text { ehold } \\
\text { furnis } \\
\text { hings }\end{array}$ & $\begin{array}{c}\text { Clothi } \\
\text { ng }\end{array}$ & $\mid \begin{array}{c}\text { Perso } \\
\text { nal } \\
\text { Items }\end{array}$ & \begin{tabular}{|c|} 
Hous \\
ehol \\
d \\
prod \\
uctio \\
n \\
activ \\
ity
\end{tabular} & $\mid \begin{array}{c}\text { Hous } \\
\text { ehol } \\
d \\
\text { prod } \\
\text { uctio } \\
\text { n for } \\
\text { sale }\end{array}$ & $\mid \begin{array}{c}\text { Pharm } \\
\text { aceutic } \\
\text { al }\end{array}$ & $\begin{array}{l}\text { Foods } \\
\text { tuffs }\end{array}$ & $\begin{array}{c}\text { Alc } \\
\text { oho } \\
\text { I }\end{array}$ & $\begin{array}{c}\text { Servic } \\
\text { es }\end{array}$ & Other \\
\hline Play & $\begin{array}{r}3 / 18 \\
75 \\
\end{array}$ & $\begin{array}{l}\text { Genevi } \\
\text { eve } \\
\text { Accom } \\
\text { modati } \\
\text { on line }\end{array}$ & & & & & & & & & & & \\
\hline $\begin{array}{l}\text { Fair } \\
\text { Play }\end{array}$ & $\begin{array}{r}12 / 2 \\
3 / 18 \\
75\end{array}$ & $\begin{array}{l}\text { J No. B. } \\
\text { Page \& } \\
\text { Son }\end{array}$ & & & & & & & & & & $\begin{array}{l}\text { Merc } \\
\text { hant }\end{array}$ & \\
\hline $\begin{array}{l}\text { Fair } \\
\text { Play }\end{array}$ & \begin{tabular}{r|}
$12 / 2$ \\
$3 / 18$ \\
75
\end{tabular} & $\begin{array}{l}\$ 290 \text { a } \\
\text { month }\end{array}$ & & & & & & & & & & $\begin{array}{l}\text { News } \\
\text { paper }\end{array}$ & \\
\hline $\begin{array}{l}\text { Fair } \\
\text { Play }\end{array}$ & \begin{tabular}{r|}
$12 / 2$ \\
$3 / 18$ \\
75
\end{tabular} & Asthma & & & & & & & $\begin{array}{l}\text { X- } \\
\text { asthma } \\
\text { cure }\end{array}$ & & & & \\
\hline $\begin{array}{l}\text { Fair } \\
\text { Play }\end{array}$ & $\begin{array}{r}12 / 2 \\
3 / 18 \\
75\end{array}$ & \begin{tabular}{|l|} 
Zells' \\
encyclo \\
pedia
\end{tabular} & & & & \begin{tabular}{|l|} 
X- \\
Encyc \\
loped \\
ia
\end{tabular} & & & & & & & \\
\hline $\begin{array}{l}\text { Fair } \\
\text { Play }\end{array}$ & \begin{tabular}{r|}
$12 / 2$ \\
$3 / 18$ \\
75
\end{tabular} & \begin{tabular}{|l|}
1000 \\
Outfits \\
Free \\
Footpri \\
nts
\end{tabular} & & & & $\begin{array}{l}\text { X- } \\
\text { book }\end{array}$ & & & & & & & \\
\hline $\begin{array}{l}\text { Fair } \\
\text { Play }\end{array}$ & \begin{tabular}{r|}
$12 / 2$ \\
$3 / 18$ \\
75
\end{tabular} & $\begin{array}{l}\text { Revolv } \\
\text { ers!! }\end{array}$ & & & & \begin{tabular}{|l|} 
X- \\
Revol \\
vers
\end{tabular} & & & & & & & \\
\hline $\begin{array}{l}\text { Fair } \\
\text { Play }\end{array}$ & $\begin{array}{r}12 / 2 \\
3 / 18 \\
75 \\
\end{array}$ & $\begin{array}{l}\text { The } \\
\text { Only } \\
\text { Live- } \\
\text { Stock } \\
\text { paper }\end{array}$ & & & & & & & & & & $\begin{array}{l}\text { Journ } \\
\text { al }\end{array}$ & \\
\hline $\begin{array}{l}\text { Fair } \\
\text { Play }\end{array}$ & \begin{tabular}{r|}
$12 / 2$ \\
$3 / 18$ \\
75
\end{tabular} & $\begin{array}{l}\text { Health } \\
\text { lift }\end{array}$ & & & & \begin{tabular}{|l|} 
X- \\
healt \\
h \\
machi
\end{tabular} & & & & & & & \\
\hline
\end{tabular}




\begin{tabular}{|c|c|c|c|c|c|c|c|c|c|c|c|c|c|}
\hline \multicolumn{14}{|c|}{ English Newspapers } \\
\hline $\begin{array}{c}\text { New } \\
\text { spap } \\
\text { er } \\
\text { nam } \\
\text { e }\end{array}$ & Date & $\mid \begin{array}{c}\text { adverti } \\
\text { sement } \\
\text { title }\end{array}$ & $\begin{array}{c}\text { Archit } \\
\text { ectur } \\
\text { al } \\
\text { items }\end{array}$ & $\begin{array}{l}\text { Hous } \\
\text { ehold } \\
\text { furnis } \\
\text { hings }\end{array}$ & $\begin{array}{c}\text { Clothi } \\
\text { ng }\end{array}$ & \begin{tabular}{|c} 
Perso \\
nal \\
Items
\end{tabular} & $\begin{array}{c}\text { Hous } \\
\text { ehol } \\
\text { d } \\
\text { prod } \\
\text { uctio } \\
\text { n } \\
\text { activ } \\
\text { ity }\end{array}$ & $\begin{array}{l}\text { Hous } \\
\text { ehol } \\
\text { d } \\
\text { prod } \\
\text { uctio } \\
\text { n for } \\
\text { sale }\end{array}$ & $\begin{array}{c}\text { Pharm } \\
\text { aceutic } \\
\text { al }\end{array}$ & $\begin{array}{c}\text { Foods } \\
\text { tuffs }\end{array}$ & $\begin{array}{c}\text { Alc } \\
\text { oho } \\
\text { I }\end{array}$ & $\begin{array}{c}\text { Servic } \\
\text { es }\end{array}$ & Other \\
\hline & & & & & & nes? & & & & & & & \\
\hline $\begin{array}{l}\text { Fair } \\
\text { Play }\end{array}$ & $\begin{array}{r}12 / 2 \\
3 / 18 \\
75\end{array}$ & $\begin{array}{l}\text { Tubular } \\
\text { Hand } \\
\text { lamp }\end{array}$ & & $\begin{array}{l}\text { X- } \\
\text { Tubul } \\
\text { ar } \\
\text { hand } \\
\text { lamp }\end{array}$ & & & & & & & & & \\
\hline $\begin{array}{l}\text { Fair } \\
\text { Play }\end{array}$ & $\begin{array}{r}12 / 2 \\
3 / 18 \\
75\end{array}$ & Cancer & & & & & & & & & & & $\begin{array}{l}\text { Tape } \\
\text { worm } \\
?\end{array}$ \\
\hline \begin{tabular}{|l} 
Fair \\
Play
\end{tabular} & $\begin{array}{r}12 / 2 \\
3 / 18 \\
75 \\
\end{array}$ & $\begin{array}{l}\text { The } \\
\text { Nurser } \\
\text { y }\end{array}$ & & & & & & & & & & $\begin{array}{l}\text { Maga } \\
\text { zine }\end{array}$ & \\
\hline $\begin{array}{l}\text { Fair } \\
\text { Play }\end{array}$ & $\begin{array}{r}12 / 2 \\
3 / 18 \\
75\end{array}$ & Asthma & & & & & & & $\begin{array}{l}\text { X- } \\
\text { asthma } \\
\text { cure }\end{array}$ & & & & \\
\hline $\begin{array}{l}\text { Fair } \\
\text { Play }\end{array}$ & $\begin{array}{r}12 / 2 \\
3 / 18 \\
75\end{array}$ & $\begin{array}{l}\text { Meride } \\
\text { n } \\
\text { Cutlery } \\
\text { Co. }\end{array}$ & & & & & & & & & & & \\
\hline \begin{tabular}{|l|} 
Fair \\
Play
\end{tabular} & $\begin{array}{r}12 / 2 \\
3 / 18 \\
75\end{array}$ & $\begin{array}{l}\text { Driscoll } \\
\text { Church } \\
\& \text { hall }\end{array}$ & & & & & & & & & & & $\begin{array}{l}\text { Sea } \\
\text { Foam } \\
?\end{array}$ \\
\hline \begin{tabular}{|l} 
Fair \\
Play
\end{tabular} & $\begin{array}{r}12 / 2 \\
3 / 18 \\
75\end{array}$ & $\begin{array}{l}\text { Toledo } \\
\text { Blade }\end{array}$ & & & & & & & & & & $\begin{array}{l}\text { News } \\
\text { paper }\end{array}$ & \\
\hline \begin{tabular}{|l} 
Fair \\
Play
\end{tabular} & $\begin{array}{r}12 / 2 \\
3 / 18 \\
75\end{array}$ & $\begin{array}{l}\text { Silver } \\
\text { Tipped } \\
\text { Shoes }\end{array}$ & & & \begin{tabular}{|l}
$\mathrm{X}-$ \\
silver \\
tipped \\
shoes
\end{tabular} & & & & & & & & \\
\hline $\begin{array}{l}\text { Fair } \\
\text { Play }\end{array}$ & $\begin{array}{r}12 / 2 \\
3 / 18 \\
75\end{array}$ & $\begin{array}{l}\text { Gable } \\
\text { Screw } \\
\text { Wire }\end{array}$ & & & & & $\begin{array}{l}\text { X- } \\
\text { cable } \\
\text { scre } \\
\text { w } \\
\text { wire }\end{array}$ & & & & & & \\
\hline
\end{tabular}




\begin{tabular}{|c|c|c|c|c|c|c|c|c|c|c|c|c|c|}
\hline \multicolumn{14}{|c|}{ English Newspapers } \\
\hline $\begin{array}{c}\text { New } \\
\text { spap } \\
\text { er } \\
\text { nam } \\
\text { e }\end{array}$ & Date & $\begin{array}{c}\text { adverti } \\
\text { sement } \\
\text { title }\end{array}$ & $\mid \begin{array}{c}\text { Archit } \\
\text { ectur } \\
\text { al } \\
\text { items }\end{array}$ & $\begin{array}{l}\text { Hous } \\
\text { ehold } \\
\text { furnis } \\
\text { hings }\end{array}$ & $\begin{array}{c}\text { Clothi } \\
\mathrm{ng}\end{array}$ & \begin{tabular}{|c} 
Perso \\
nal \\
Items
\end{tabular} & \begin{tabular}{|} 
Hous \\
ehol \\
d \\
prod \\
uctio \\
n \\
activ \\
ity
\end{tabular} & \begin{tabular}{|l|} 
Hous \\
ehol \\
$d$ \\
prod \\
uctio \\
n for \\
sale
\end{tabular} & $\begin{array}{c}\text { Pharm } \\
\text { aceutic } \\
\text { al }\end{array}$ & $\begin{array}{c}\text { Foods } \\
\text { tuffs }\end{array}$ & $\begin{array}{c}\text { Alc } \\
\text { oho } \\
\text { I }\end{array}$ & $\begin{array}{c}\text { Servic } \\
\text { es }\end{array}$ & Other \\
\hline $\begin{array}{l}\text { Fair } \\
\text { Play }\end{array}$ & $\begin{array}{r}12 / 2 \\
3 / 18 \\
75\end{array}$ & $\begin{array}{l}\$ 40 \text { a } \\
\text { Day }\end{array}$ & $\begin{array}{l}\text { X-well } \\
\text { auger }\end{array}$ & & & & & & & & & & \\
\hline $\begin{array}{l}\text { Fair } \\
\text { Play }\end{array}$ & $\begin{array}{r}12 / 2 \\
3 / 18 \\
75\end{array}$ & Opium & & & & & & & $\begin{array}{l}\text { X- } \\
\text { opium, } \\
\text { morphi } \\
\text { ne }\end{array}$ & & & & \\
\hline $\begin{array}{l}\text { Fair } \\
\text { Play }\end{array}$ & $\begin{array}{r}12 / 2 \\
3 / 18 \\
75 \\
\end{array}$ & $\begin{array}{l}\text { "Dome } \\
\text { stic" } \\
\text { sewing } \\
\text { machin } \\
\text { es }\end{array}$ & & & $\begin{array}{l}\text { X- } \\
\text { paper } \\
\text { fashio } \\
\text { ns }\end{array}$ & & $\begin{array}{l}\text { X- } \\
\text { sewi } \\
\text { ng } \\
\text { mac } \\
\text { hine } \\
\text { s }\end{array}$ & & & & & & \\
\hline $\begin{array}{l}\text { Fair } \\
\text { Play }\end{array}$ & $\begin{array}{r}12 / 2 \\
3 / 18 \\
75\end{array}$ & $\begin{array}{l}\text { Washin } \\
\text { gton, } \\
\text { D.C. }\end{array}$ & & & & & & & & & & legal & \\
\hline $\begin{array}{l}\text { Fair } \\
\text { Play }\end{array}$ & $\begin{array}{r}12 / 2 \\
3 / 18 \\
75\end{array}$ & Opium & & & & & & & $\begin{array}{l}\text { X- } \\
\text { opium }\end{array}$ & & & & \\
\hline $\begin{array}{l}\text { Fair } \\
\text { Play }\end{array}$ & $\begin{array}{r}12 / 2 \\
3 / 18 \\
75\end{array}$ & $\begin{array}{l}\text { Smith } \\
\text { Organ } \\
\text { Co. }\end{array}$ & & & & $\begin{array}{l}\text { X- } \\
\text { music } \\
\text { instru } \\
\text { ment } \\
\text { s }\end{array}$ & & & & & & & \\
\hline $\begin{array}{l}\text { Fair } \\
\text { Play }\end{array}$ & $\begin{array}{r}12 / 2 \\
3 / 18 \\
75\end{array}$ & Guns & & & & $\begin{array}{l}\text { X- } \\
\text { guns }\end{array}$ & & & & & & & \\
\hline $\begin{array}{l}\text { Fair } \\
\text { Play }\end{array}$ & $\begin{array}{r}12 / 2 \\
3 / 18 \\
75\end{array}$ & $\begin{array}{l}\text { Cincinn } \\
\text { ati } \\
\text { dollar } \\
\text { weekly } \\
\text { star }\end{array}$ & & & & & & & & & & $\begin{array}{l}\text { news } \\
\text { paper }\end{array}$ & \\
\hline $\begin{array}{l}\text { Fair } \\
\text { Play }\end{array}$ & $\begin{array}{r}12 / 2 \\
3 / 18 \\
75\end{array}$ & 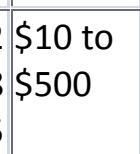 & & & & $\begin{array}{l}\text { X- } \\
\text { stock } \\
\text { s }\end{array}$ & & & & & & & \\
\hline
\end{tabular}




\begin{tabular}{|c|c|c|c|c|c|c|c|c|c|c|c|c|c|}
\hline \multicolumn{14}{|c|}{ English Newspapers } \\
\hline $\begin{array}{c}\text { New } \\
\text { spap } \\
\text { er } \\
\text { nam } \\
\text { e }\end{array}$ & |Date & $\mid \begin{array}{c}\text { adverti } \\
\text { sement } \\
\text { title }\end{array}$ & $\begin{array}{c}\text { Archit } \\
\text { ectur } \\
\text { al } \\
\text { items }\end{array}$ & $\begin{array}{l}\text { Hous } \\
\text { ehold } \\
\text { furnis } \\
\text { hings }\end{array}$ & $\begin{array}{c}\text { Clothi } \\
\text { ng }\end{array}$ & \begin{tabular}{|c} 
Perso \\
nal \\
Items
\end{tabular} & $\begin{array}{c}\text { Hous } \\
\text { ehol } \\
\text { d } \\
\text { prod } \\
\text { uctio } \\
\text { n } \\
\text { activ } \\
\text { ity }\end{array}$ & $\mid \begin{array}{c}\text { Hous } \\
\text { ehol } \\
d \\
\text { prod } \\
\text { uctio } \\
\text { n for } \\
\text { sale }\end{array}$ & $\begin{array}{c}\text { Pharm } \\
\text { aceutic } \\
\text { al }\end{array}$ & $\begin{array}{c}\text { Foods } \\
\text { tuffs }\end{array}$ & $\mid \begin{array}{c}\text { Alc } \\
\text { oho } \\
\text { I }\end{array}$ & $\begin{array}{c}\text { Servic } \\
\text { es }\end{array}$ & Other \\
\hline & & & & & & book & & & & & & & \\
\hline $\begin{array}{l}\text { Fair } \\
\text { Play }\end{array}$ & \begin{tabular}{r|}
$12 / 2$ \\
$3 / 18$ \\
75
\end{tabular} & \begin{tabular}{|l|} 
Helio- \\
telluric \\
treatm \\
ent
\end{tabular} & & & & & & & \begin{tabular}{|l|} 
X- \\
helio- \\
telluric \\
treatm \\
ent
\end{tabular} & & & & \\
\hline $\begin{array}{l}\text { Fair } \\
\text { Play }\end{array}$ & \begin{tabular}{r|}
$12 / 2$ \\
$3 / 18$ \\
75
\end{tabular} & \begin{tabular}{|l|} 
Prof. \\
hall's \\
Magic \\
Compo \\
und
\end{tabular} & & & & & & & $\begin{array}{l}\text { X-hair } \\
\text { growth }\end{array}$ & & & & \\
\hline $\begin{array}{l}\text { Fair } \\
\text { Play }\end{array}$ & \begin{tabular}{r|}
$12 / 2$ \\
$3 / 18$ \\
75 \\
\end{tabular} & \begin{tabular}{|l} 
Puff! \\
Puff!! \\
Puff!!!
\end{tabular} & & & & $\begin{array}{l}\text { X- } \\
\text { puzzl } \\
\text { e/toy }\end{array}$ & & & & & & & \\
\hline $\begin{array}{l}\text { Fair } \\
\text { Play }\end{array}$ & \begin{tabular}{r|}
$12 / 2$ \\
$3 / 18$ \\
75 \\
\end{tabular} & \begin{tabular}{|l|} 
Dr. \\
Sanfor \\
d's \\
Liver \\
Invigor \\
ator
\end{tabular} & & & & & & & $\begin{array}{l}\text { X-live } \\
\text { invigor } \\
\text { ator }\end{array}$ & & & & \\
\hline $\begin{array}{l}\text { Fair } \\
\text { Play }\end{array}$ & \begin{tabular}{r|}
$12 / 2$ \\
$3 / 18$ \\
75 \\
\end{tabular} & $\begin{array}{l}\text { Elastic } \\
\text { truss }\end{array}$ & & & \begin{tabular}{|l|}
$\mathrm{X}-$ \\
elastic \\
truss
\end{tabular} & & & & & & & & \\
\hline $\begin{array}{l}\text { Fair } \\
\text { Play }\end{array}$ & \begin{tabular}{r|}
$12 / 2$ \\
$3 / 18$ \\
75 \\
\end{tabular} & $\begin{array}{l}\text { Fair } \\
\text { Play }\end{array}$ & & & & & & & & & & $\begin{array}{l}\text { news } \\
\text { paper }\end{array}$ & \\
\hline $\begin{array}{l}\text { Fair } \\
\text { Play }\end{array}$ & \begin{tabular}{r|}
$12 / 2$ \\
$3 / 18$ \\
75 \\
\end{tabular} & \begin{tabular}{|l|} 
Don't \\
Neglect \\
your \\
teeth: \\
Sozodo \\
nt
\end{tabular} & & & & & & & \begin{tabular}{|l|} 
X- \\
sozodo \\
nt \\
(tooth \\
paste)
\end{tabular} & & & & \\
\hline $\begin{array}{l}\text { Fair } \\
\text { Play }\end{array}$ & \begin{tabular}{r|}
$12 / 2$ \\
$3 / 18$ \\
75
\end{tabular} & & & & & & & & & & & & \\
\hline
\end{tabular}




\begin{tabular}{|c|c|c|c|c|c|c|c|c|c|c|c|c|c|}
\hline \multicolumn{14}{|c|}{ English Newspapers } \\
\hline $\begin{array}{c}\text { New } \\
\text { spap } \\
\text { er } \\
\text { nam } \\
\text { e }\end{array}$ & Date & $\mid \begin{array}{c}\text { adverti } \\
\text { sement } \\
\text { title }\end{array}$ & $\begin{array}{c}\text { Archit } \\
\text { ectur } \\
\text { al } \\
\text { items }\end{array}$ & $\begin{array}{l}\text { Hous } \\
\text { ehold } \\
\text { furnis } \\
\text { hings }\end{array}$ & $\begin{array}{c}\text { Clothi } \\
\text { ng }\end{array}$ & \begin{tabular}{|c} 
Perso \\
nal \\
Items
\end{tabular} & \begin{tabular}{|} 
Hous \\
ehol \\
d \\
prod \\
uctio \\
n \\
activ \\
ity
\end{tabular} & $\mid \begin{array}{c}\text { Hous } \\
\text { ehol } \\
d \\
\text { prod } \\
\text { uctio } \\
\text { n for } \\
\text { sale }\end{array}$ & $\begin{array}{c}\text { Pharm } \\
\text { aceutic } \\
\text { al }\end{array}$ & $\begin{array}{c}\text { Foods } \\
\text { tuffs }\end{array}$ & $\begin{array}{c}\text { Alc } \\
\text { oho } \\
\text { I }\end{array}$ & $\begin{array}{c}\text { Servic } \\
\text { es }\end{array}$ & Other \\
\hline $\begin{array}{l}\text { Fair } \\
\text { Play }\end{array}$ & \begin{tabular}{|r|}
$12 / 1$ \\
$9 / 18$ \\
72
\end{tabular} & $\begin{array}{l}\text { Firmin } \\
\text { A. } \\
\text { Rozier }\end{array}$ & & & & & & & & & & Legal & \\
\hline $\begin{array}{l}\text { Fair } \\
\text { Play }\end{array}$ & $\begin{array}{r}12 / 1 \\
9 / 18 \\
72\end{array}$ & $\begin{array}{l}\text { Chas. } \\
\text { C. } \\
\text { Rozier }\end{array}$ & & & & & & & & & & Legal & \\
\hline $\begin{array}{l}\text { Fair } \\
\text { Play }\end{array}$ & \begin{tabular}{|r}
$12 / 1$ \\
$9 / 18$ \\
72
\end{tabular} & $\begin{array}{l}\text { J.B. } \\
\text { Robbin } \\
\text { S }\end{array}$ & & & & & & & & & & Legal & \\
\hline $\begin{array}{l}\text { Fair } \\
\text { Play }\end{array}$ & $\begin{array}{r}12 / 1 \\
9 / 18 \\
72\end{array}$ & $\begin{array}{l}\text { F.J. } \\
\text { Morea } \\
\text { u }\end{array}$ & & & & & & & & & & Legal & \\
\hline $\begin{array}{l}\text { Fair } \\
\text { Play }\end{array}$ & $\begin{array}{r}12 / 1 \\
9 / 18 \\
72\end{array}$ & $\begin{array}{l}\text { Robins } \\
\text { on \& } \\
\text { Clardy }\end{array}$ & & & & & & & & & & Legal & \\
\hline \begin{tabular}{|l} 
Fair \\
Play`
\end{tabular} & $\begin{array}{r}12 / 2 \\
3 / 18 \\
75\end{array}$ & $\begin{array}{l}\text { Dr. C. } \\
\text { S. } \\
\text { Hertich }\end{array}$ & & & & & & & & & & $\begin{array}{l}\text { Medi } \\
\text { cal }\end{array}$ & \\
\hline \begin{tabular}{|l} 
Ste. \\
Gene \\
vieve \\
Dem \\
ocrat
\end{tabular} & $\begin{array}{r}6 / 8 / \\
1850\end{array}$ & $\begin{array}{l}\text { Ste. } \\
\text { Genevi } \\
\text { eve } \\
\text { Marble } \\
\text { Yard }\end{array}$ & $\begin{array}{l}\text { X- } \\
\text { Marbl } \\
\text { e and } \\
\text { Stone }\end{array}$ & & & & & & & & & & \\
\hline \begin{tabular}{|l} 
Ste. \\
Gene \\
vieve \\
Dem \\
ocrat
\end{tabular} & $\begin{array}{r}6 / 8 / \\
1850\end{array}$ & $\begin{array}{l}\text { Ste. } \\
\text { Genevi } \\
\text { eve } \\
\text { Clothin } \\
\text { g Store }\end{array}$ & & & $x$ & & & & & & & & \\
\hline \begin{tabular}{|l} 
Ste. \\
Gene \\
vieve \\
Dem \\
ocrat
\end{tabular} & $\begin{array}{c}6 / 8 / \\
1850\end{array}$ & $\begin{array}{l}\text { House } \\
\text { and } \\
\text { Sign } \\
\text { Painter }\end{array}$ & & & & & & & & & & $\begin{array}{l}\text { Paint } \\
\text { er }\end{array}$ & \\
\hline $\begin{array}{l}\text { Ste. } \\
\text { Gene }\end{array}$ & $\begin{array}{c}6 / 8 / \\
1850\end{array}$ & $\begin{array}{l}\text { W.K. } \\
\text { Bradly }\end{array}$ & & & & & & & & & & Legal & \\
\hline
\end{tabular}




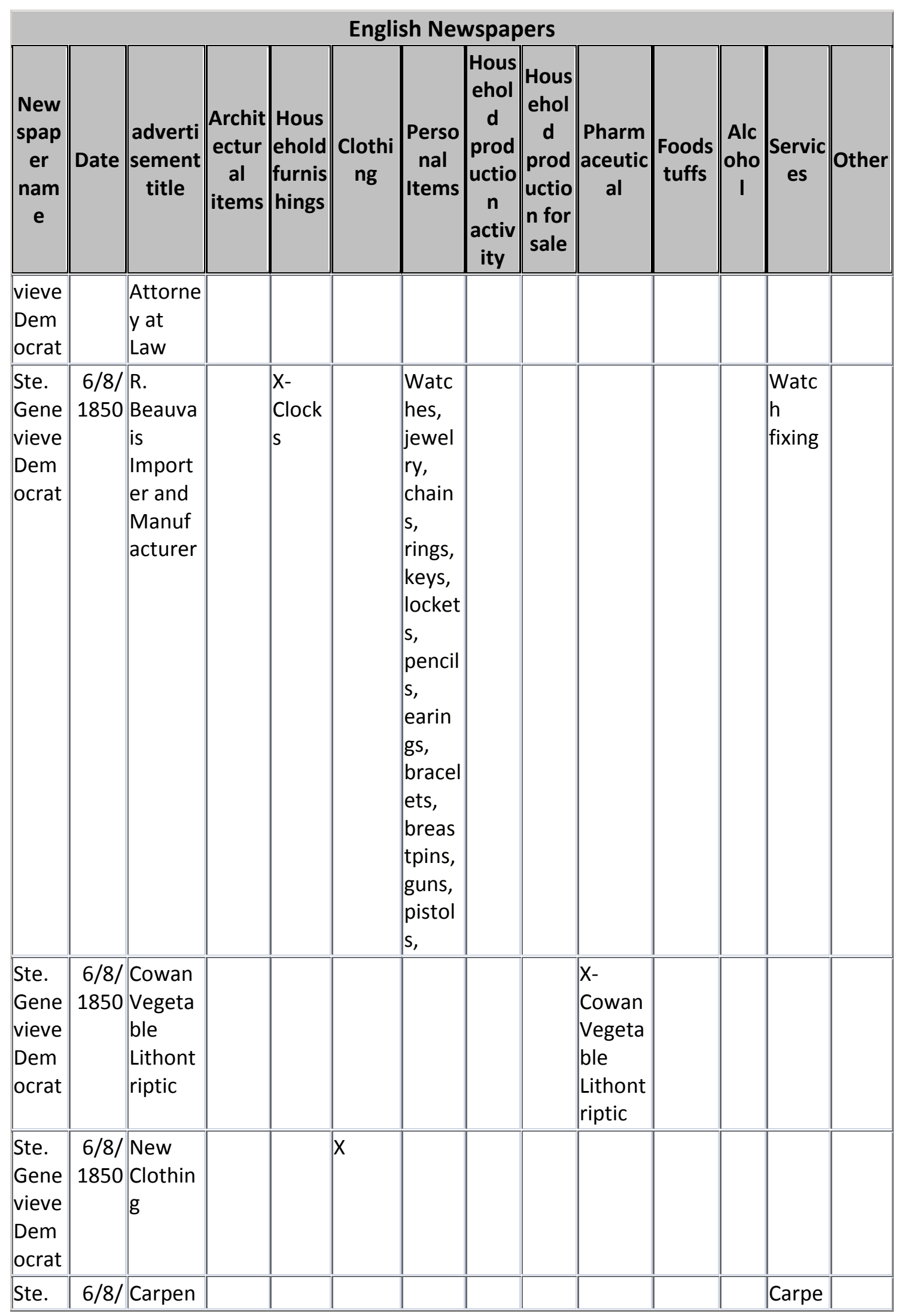




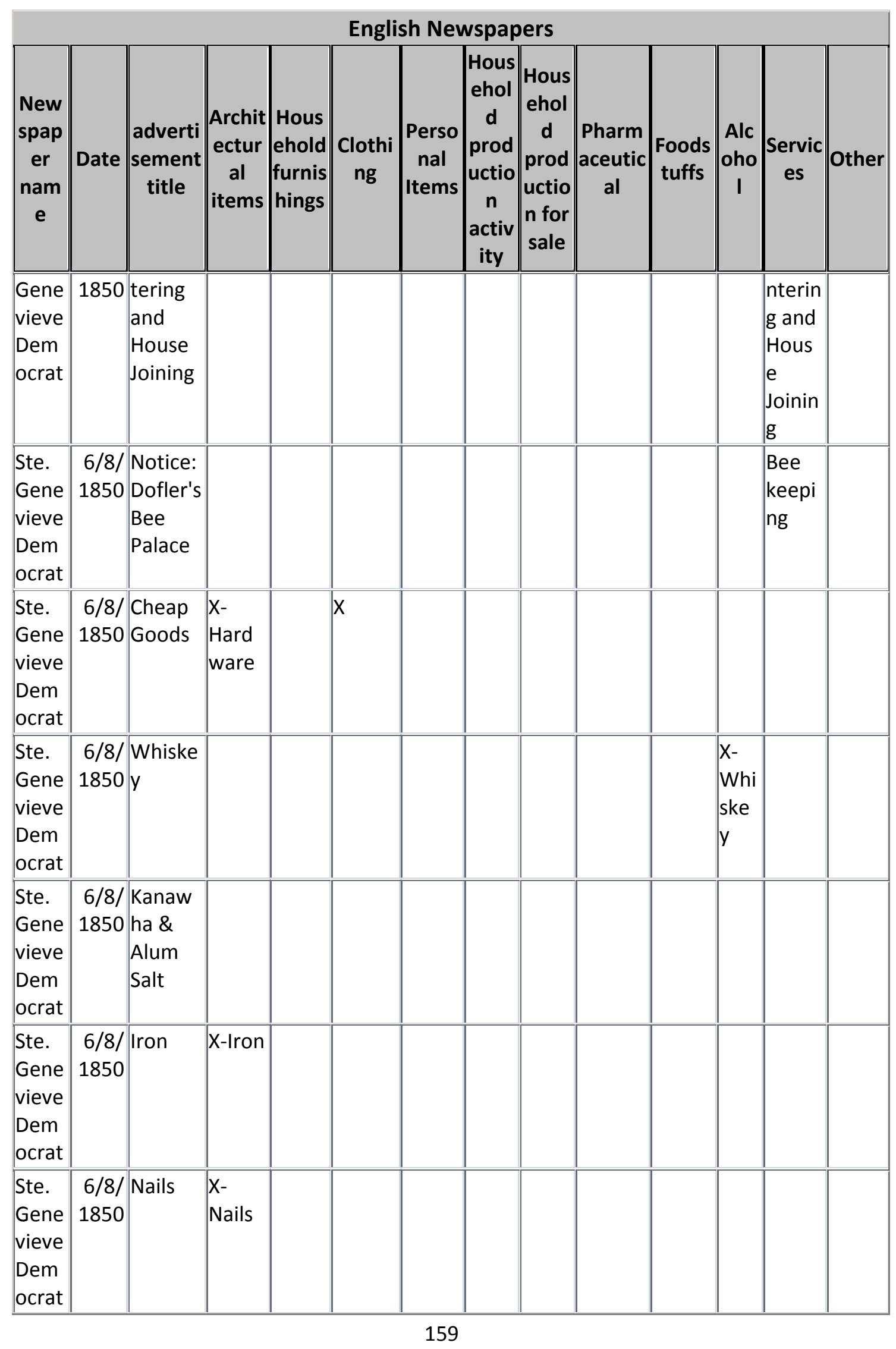




\begin{tabular}{|c|c|c|c|c|c|c|c|c|c|c|c|c|c|}
\hline \multicolumn{14}{|c|}{ English Newspapers } \\
\hline $\begin{array}{c}\text { New } \\
\text { spap } \\
\text { er } \\
\text { nam } \\
\text { e }\end{array}$ & Date & \begin{tabular}{|c} 
adverti \\
sement \\
title
\end{tabular} & $\begin{array}{c}\text { Archit } \\
\text { ectur } \\
\text { al } \\
\text { items }\end{array}$ & $\begin{array}{l}\text { Hous } \\
\text { ehold } \\
\text { furnis } \\
\text { hings }\end{array}$ & $\begin{array}{c}\text { Clothi } \\
\text { ng }\end{array}$ & $\begin{array}{c}\text { Perso } \\
\text { nal } \\
\text { Items }\end{array}$ & $\begin{array}{l}\text { Hous } \\
\text { ehol } \\
\text { d } \\
\text { prod } \\
\text { uctio } \\
\text { n } \\
\text { activ } \\
\text { ity }\end{array}$ & $\mid \begin{array}{c}\text { Hous } \\
\text { ehol } \\
d \\
\text { prod } \\
\text { uctio } \\
n \text { for } \\
\text { sale }\end{array}$ & \begin{tabular}{|c} 
Pharm \\
aceutic \\
al
\end{tabular} & $\begin{array}{l}\text { Foods } \\
\text { tuffs }\end{array}$ & $\mid \begin{array}{c}\text { Alc } \\
\text { oho } \\
\text { I }\end{array}$ & $\begin{array}{c}\text { Servic } \\
\text { es }\end{array}$ & Other \\
\hline $\begin{array}{l}\text { Ste. } \\
\text { Gene } \\
\text { vieve } \\
\text { Dem } \\
\text { ocrat }\end{array}$ & $\begin{array}{r}6 / 8 / \\
1850\end{array}$ & Brandy & & & & & & & & & $\begin{array}{l}\text { X- } \\
\text { Bra } \\
\text { ndy }\end{array}$ & & \\
\hline $\begin{array}{l}\text { Ste. } \\
\text { Gene } \\
\text { vieve } \\
\text { Dem } \\
\text { ocrat }\end{array}$ & $\begin{array}{r}6 / 8 / \\
1850 \\
\end{array}$ & $\begin{array}{l}\text { Law } \\
\text { Depart } \\
\text { ment }\end{array}$ & & & & & & & & & & Legal & \\
\hline $\begin{array}{l}\text { Ste. } \\
\text { Gene } \\
\text { vieve } \\
\text { Dem } \\
\text { ocrat }\end{array}$ & $\begin{array}{r}6 / 8 / \\
1850\end{array}$ & $\begin{array}{l}\text { New } \\
\text { Clothin } \\
g\end{array}$ & & & $x$ & & & & & & & & \\
\hline \begin{tabular}{|l} 
Ste. \\
Gene \\
vieve \\
Dem \\
ocrat
\end{tabular} & $\begin{array}{r}6 / 8 / \\
1850 \\
\end{array}$ & \begin{tabular}{|l} 
For \\
Sale \\
Cheap: \\
Farm
\end{tabular} & & & & & & & & & & & Farm \\
\hline $\begin{array}{l}\text { Ste. } \\
\text { Gene } \\
\text { vieve } \\
\text { Dem } \\
\text { ocrat } \\
\end{array}$ & $\begin{array}{r}6 / 8 / \\
1850\end{array}$ & $\begin{array}{l}\text { Califor } \\
\text { nia } \\
\text { Gold }\end{array}$ & $\begin{array}{l}\text { X- } \\
\text { Hard } \\
\text { ware }\end{array}$ & & $\begin{array}{l}\text { X- } \\
\text { Hats, } \\
\text { Caps, } \\
\text { Boots, } \\
\text { Shoes }\end{array}$ & & $\begin{array}{l}\text { X- } \\
\text { Sadd } \\
\text { lery }\end{array}$ & & & $\begin{array}{l}\text { X-Dry } \\
\text { goods } \\
\text { grocer } \\
\text { ies }\end{array}$ & & & \\
\hline \begin{tabular}{|l} 
Ste. \\
Gene \\
vieve \\
Dem \\
ocrat
\end{tabular} & $\begin{array}{r}6 / 8 / \\
1850\end{array}$ & Hats & & & X-Hats & & & & & & & & \\
\hline \begin{tabular}{|l} 
Ste. \\
Gene \\
vieve \\
Dem \\
ocrat \\
\end{tabular} & $\begin{array}{r}6 / 8 / \\
1850 \\
\end{array}$ & $\begin{array}{l}\text { Ready } \\
\text { made } \\
\text { Clothin } \\
\text { g }\end{array}$ & & & $x$ & & & & & & & & \\
\hline
\end{tabular}




\begin{tabular}{|c|c|c|c|c|c|c|c|c|c|c|c|c|c|}
\hline \multicolumn{14}{|c|}{ English Newspapers } \\
\hline $\begin{array}{c}\text { New } \\
\text { spap } \\
\text { er } \\
\text { nam } \\
\text { e }\end{array}$ & Date & $\begin{array}{c}\text { adverti } \\
\text { sement } \\
\text { title }\end{array}$ & $\mid \begin{array}{c}\text { Archit } \\
\text { ectur } \\
\text { al } \\
\text { items }\end{array}$ & $\begin{array}{l}\text { Hous } \\
\text { ehold } \\
\text { furnis } \\
\text { hings }\end{array}$ & $\begin{array}{c}\text { Clothi } \\
\text { ng }\end{array}$ & $\mid \begin{array}{c}\text { Perso } \\
\text { nal } \\
\text { Items }\end{array}$ & \begin{tabular}{|} 
Hous \\
ehol \\
d \\
prod \\
uctio \\
$n$ \\
activ \\
ity
\end{tabular} & $\mid \begin{array}{c}\text { Hous } \\
\text { ehol } \\
d \\
\text { prod } \\
\text { uctio } \\
n \text { for } \\
\text { sale }\end{array}$ & $\mid \begin{array}{c}\text { Pharm } \\
\text { aceutic } \\
\text { al }\end{array}$ & $\begin{array}{c}\text { Foods } \\
\text { tuffs }\end{array}$ & $\begin{array}{c}\text { Alc } \\
\text { oho } \\
\text { I }\end{array}$ & $\begin{array}{c}\text { Servic } \\
\text { es }\end{array}$ & Other \\
\hline \begin{tabular}{|l} 
Ste. \\
Gene \\
vieve \\
Dem \\
ocrat
\end{tabular} & $\begin{array}{r}6 / 8 / \\
1850 \\
\end{array}$ & $\begin{array}{l}\text { Sugar } \\
\& \\
\text { Coffee }\end{array}$ & & & & & & & & $\begin{array}{l}\text { X- } \\
\text { Sugar } \\
\text { and } \\
\text { Coffe } \\
\text { e }\end{array}$ & & & \\
\hline \begin{tabular}{|l} 
Ste. \\
Gene \\
vieve \\
Dem \\
ocrat
\end{tabular} & $\begin{array}{r}6 / 8 / \\
1850\end{array}$ & $\begin{array}{l}\text { Ladies } \\
\text { Fine } \\
\text { Dress } \\
\text { Goods }\end{array}$ & & & \begin{tabular}{|l|} 
X- \\
Ladies \\
dresse \\
$\mathrm{s}$
\end{tabular} & & & & & & & & \\
\hline \begin{tabular}{|l} 
Ste. \\
Gene \\
vieve \\
Dem \\
ocrat
\end{tabular} & $\begin{array}{r}6 / 8 / \\
1850\end{array}$ & $\begin{array}{l}C(?) \\
\text { Boots }\end{array}$ & & & $\begin{array}{l}X- \\
\text { boots }\end{array}$ & & & & & & & & \\
\hline \begin{tabular}{|l|} 
Ste. \\
Gene \\
vieve \\
Dem \\
ocrat \\
\end{tabular} & $\begin{array}{r}6 / 8 / \\
1850\end{array}$ & Salt & & & & & & & & X-Salt & & & \\
\hline \begin{tabular}{|l|} 
Ste. \\
Gene \\
vieve \\
Dem \\
ocrat \\
\end{tabular} & $\begin{array}{r}6 / 8 / \\
1850 \\
\end{array}$ & $\begin{array}{l}\text { Hats, } \\
\text { Caps, \& } \\
\text { Straw } \\
\text { Goods }\end{array}$ & & & $\begin{array}{l}\text { X-Hats } \\
\text { and } \\
\text { Caps }\end{array}$ & & & & & & & & \\
\hline \begin{tabular}{|l|} 
Ste. \\
Gene \\
vieve \\
Dem \\
ocrat \\
\end{tabular} & $\begin{array}{r}6 / 8 / \\
1850 \\
\\
\end{array}$ & \begin{tabular}{|l} 
Spring \\
and \\
Summe \\
$r$ \\
Clothin \\
g at \\
Wholes \\
ale
\end{tabular} & & & $x$ & & & & & & & & \\
\hline \begin{tabular}{|l|} 
Ste. \\
Gene \\
vieve
\end{tabular} & $\begin{array}{r}6 / 8 / \\
1850\end{array}$ & $\begin{array}{l}\text { Fancy } \\
\text { Letter } \\
\text { and }\end{array}$ & & & & \begin{tabular}{|l|} 
Fancy \\
Letter \\
and
\end{tabular} & & & & & & & \\
\hline
\end{tabular}




\begin{tabular}{|c|c|c|c|c|c|c|c|c|c|c|c|c|c|}
\hline \multicolumn{14}{|c|}{ English Newspapers } \\
\hline $\begin{array}{c}\text { New } \\
\text { spap } \\
\text { er } \\
\text { nam } \\
\text { e }\end{array}$ & Date & \begin{tabular}{|c} 
adverti \\
sement \\
title
\end{tabular} & $\mid \begin{array}{c}\text { Archit } \\
\text { ectur } \\
\text { al } \\
\text { items }\end{array}$ & $\begin{array}{l}\text { Hous } \\
\text { ehold } \\
\text { furnis } \\
\text { hings }\end{array}$ & $\begin{array}{c}\text { Clothi } \\
\text { ng }\end{array}$ & $\mid \begin{array}{c}\text { Perso } \\
\text { nal } \\
\text { Items }\end{array}$ & \begin{tabular}{|c|} 
Hous \\
ehol \\
d \\
prod \\
uctio \\
n \\
activ \\
ity \\
\end{tabular} & \begin{tabular}{|c|} 
Hous \\
ehol \\
$d$ \\
prod \\
uctio \\
$n$ for \\
sale
\end{tabular} & $\begin{array}{c}\text { Pharm } \\
\text { aceutic } \\
\text { al }\end{array}$ & $\begin{array}{c}\text { Foods } \\
\text { tuffs }\end{array}$ & $\begin{array}{c}\text { Alc } \\
\text { oho } \\
\text { I }\end{array}$ & $\begin{array}{c}\text { Servic } \\
\text { es }\end{array}$ & Other \\
\hline $\begin{array}{l}\text { Dem } \\
\text { ocrat }\end{array}$ & & $\begin{array}{l}\text { Note } \\
\text { Paper }\end{array}$ & & & & \begin{tabular}{|l||} 
Note \\
Paper
\end{tabular} & & & & & & & \\
\hline $\begin{array}{l}\text { Ste. } \\
\text { Gene } \\
\text { vieve } \\
\text { Dem } \\
\text { ocrat }\end{array}$ & $\begin{array}{r}6 / 8 / \\
1850 \\
\\
\end{array}$ & $\begin{array}{l}\text { Fancy } \\
\text { and } \\
\text { commo } \\
\text { n } \\
\text { Envelo } \\
\text { pes }\end{array}$ & & & & \begin{tabular}{|l|} 
Fancy \\
and \\
com \\
mon \\
envel \\
ope
\end{tabular} & & & & & & & \\
\hline $\begin{array}{l}\text { Ste. } \\
\text { Gene } \\
\text { vieve } \\
\text { Dem } \\
\text { ocrat }\end{array}$ & $\begin{array}{r}6 / 8 / \\
1850 \\
\end{array}$ & $\begin{array}{l}\text { New } \\
\text { Goods! } \\
\text { New } \\
\text { Goods! }\end{array}$ & & & & & & & & & & & \\
\hline $\begin{array}{l}\text { Ste. } \\
\text { Gene } \\
\text { vieve } \\
\text { Dem } \\
\text { ocrat }\end{array}$ & $\begin{array}{r}6 / 8 / \\
1850\end{array}$ & Coats & & & $\begin{array}{l}\text { X- } \\
\text { Coats }\end{array}$ & & & & & & & & \\
\hline \begin{tabular}{|l} 
Ste. \\
Gene \\
vieve \\
Dem \\
ocrat
\end{tabular} & \begin{tabular}{|r|}
$6 / 8 /$ \\
1850
\end{tabular} & Shawls & & & \begin{tabular}{|l} 
X- \\
Shawls
\end{tabular} & & & & & & & & \\
\hline $\begin{array}{l}\text { Ste. } \\
\text { Gene } \\
\text { vieve } \\
\text { Harol } \\
\text { d }\end{array}$ & $\begin{array}{r}12 / 1 \\
7 / 18 \\
92\end{array}$ & $\begin{array}{l}\text { Christm } \\
\text { as }\end{array}$ & $\begin{array}{l}\text { X- } \\
\text { home } \\
\text {, wall } \\
\text { brack } \\
\text { ets, } \\
\text { clock } \\
\text { shelv } \\
\text { es }\end{array}$ & \begin{tabular}{|l} 
X- \\
hangi \\
ng \\
lamp, \\
plush \\
boxes \\
odor \\
cases \\
table \\
cover \\
,
\end{tabular} & \begin{tabular}{|l} 
X- \\
shoes, \\
slipper \\
s, pair \\
gloves, \\
silk \\
mitten \\
s, silk \\
handk \\
erchie \\
f, \\
neck- \\
tie,
\end{tabular} & \begin{tabular}{|l|} 
X- \\
smok \\
er's \\
set, \\
cigars \\
satch \\
el, \\
lolog \\
ne, \\
shavi \\
ng \\
sets,b
\end{tabular} & \begin{tabular}{|l|} 
X- \\
work \\
stan \\
d \\
set, \\
Whe \\
eler \\
$\&$ \\
Wils \\
on \\
sewi \\
ng \\
mac
\end{tabular} & & & $\begin{array}{l}\text { X-box } \\
\text { candy }\end{array}$ & & $\begin{array}{l}\text { expre } \\
\text { ss } \\
\text { wago } \\
\text { n }\end{array}$ & \\
\hline
\end{tabular}




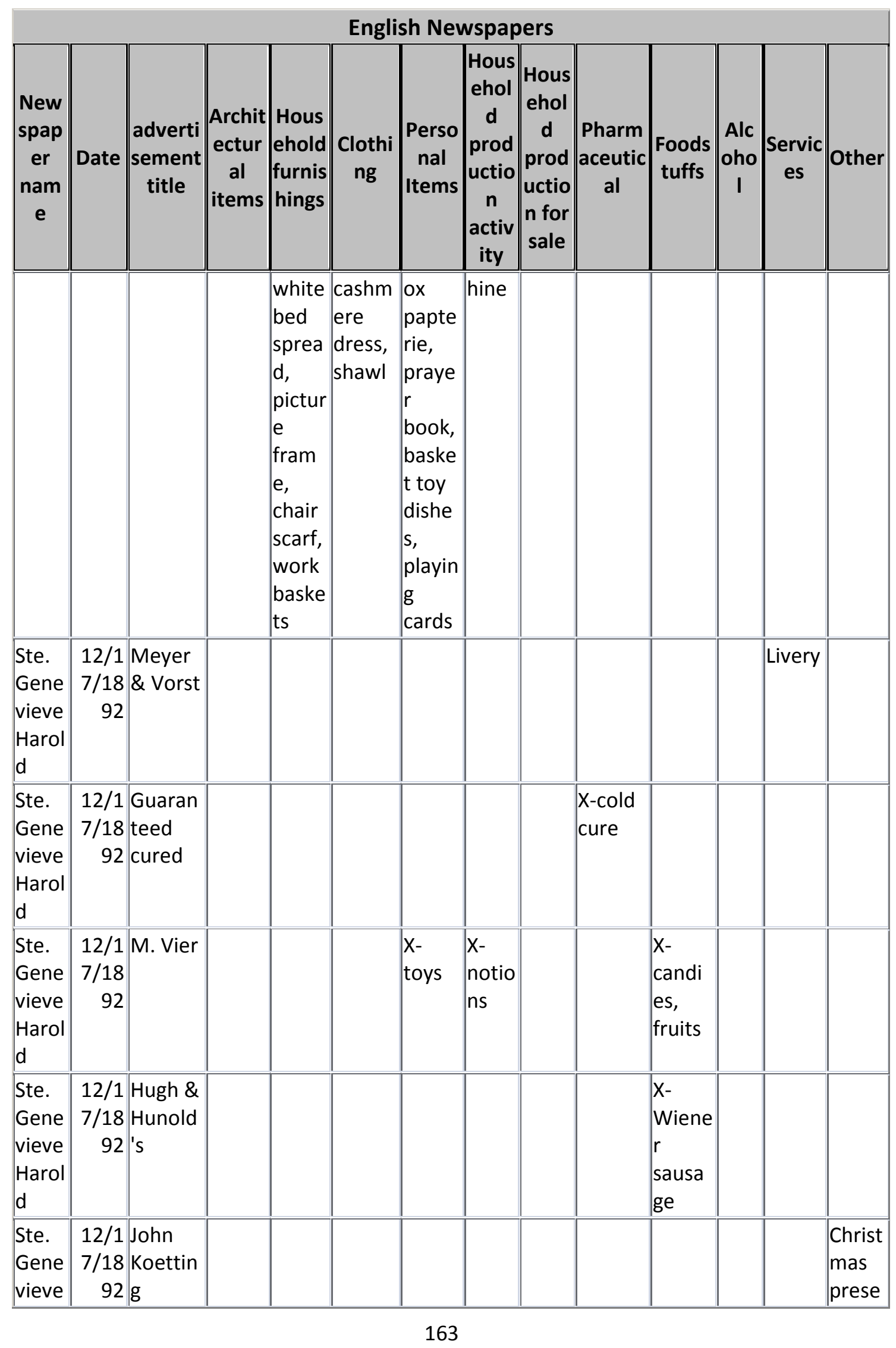




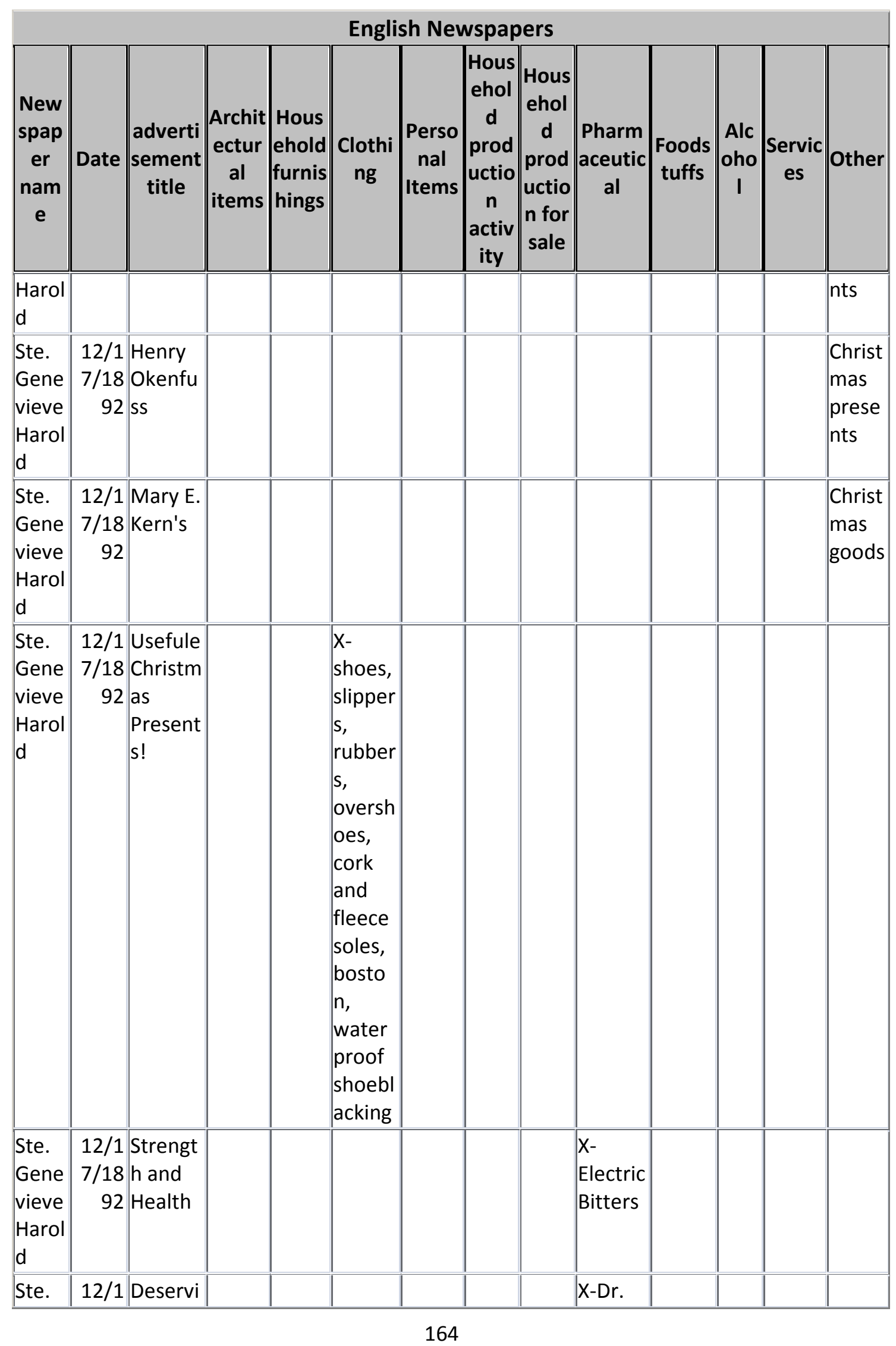




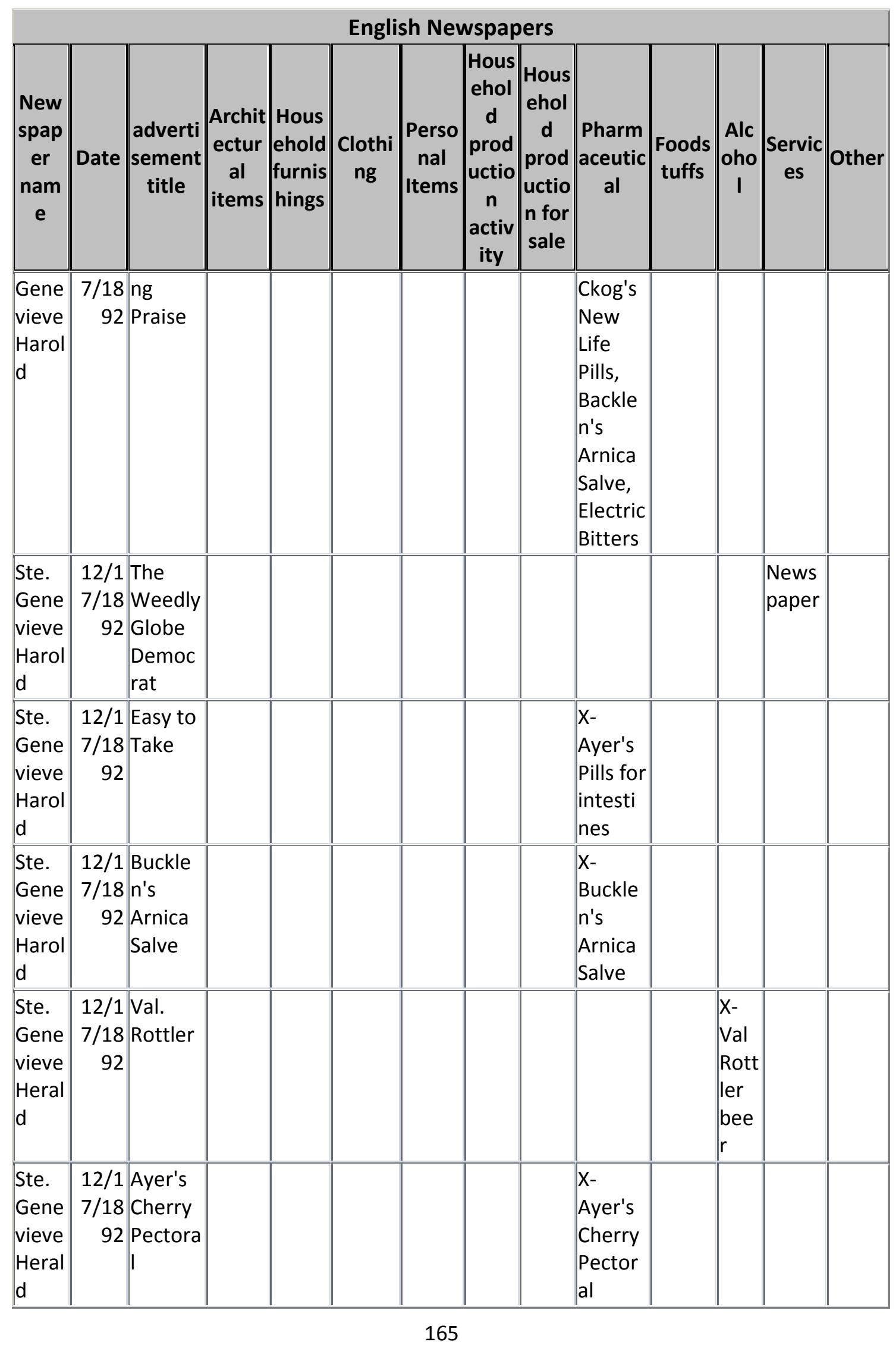




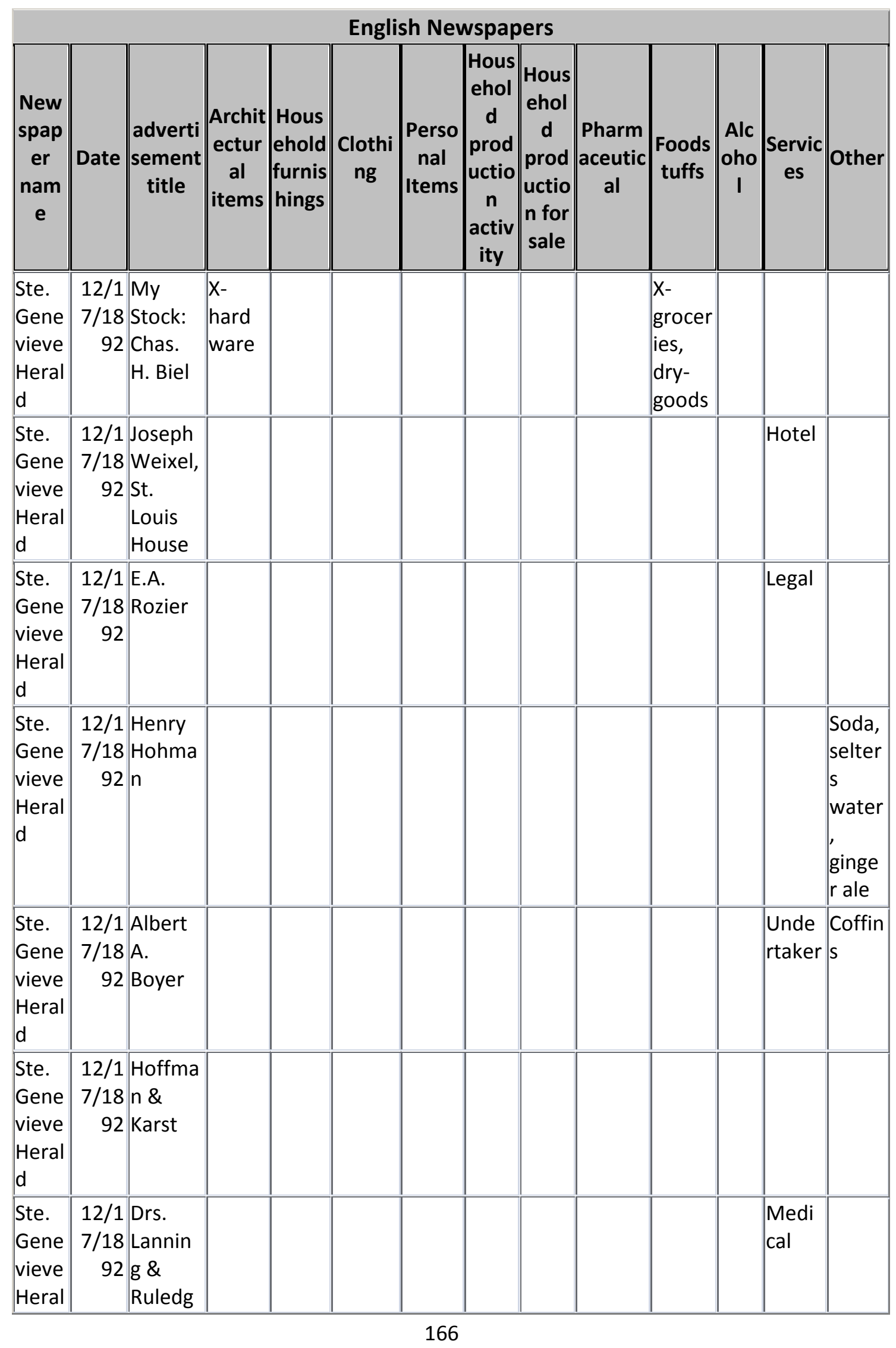




\begin{tabular}{|c|c|c|c|c|c|c|c|c|c|c|c|c|c|}
\hline \multicolumn{14}{|c|}{ English Newspapers } \\
\hline $\begin{array}{c}\text { New } \\
\text { spap } \\
\text { er } \\
\text { nam } \\
\text { e }\end{array}$ & Date & $\mid \begin{array}{c}\text { adverti } \\
\text { sement } \\
\text { title }\end{array}$ & $\begin{array}{c}\text { Archit } \\
\text { ectur } \\
\text { al } \\
\text { items }\end{array}$ & $\begin{array}{l}\text { Hous } \\
\text { ehold } \\
\text { furnis } \\
\text { hings }\end{array}$ & $\begin{array}{c}\text { Clothi } \\
\text { ng }\end{array}$ & $\begin{array}{c}\text { Perso } \\
\text { nal } \\
\text { Items }\end{array}$ & \begin{tabular}{|} 
Hous \\
ehol \\
d \\
prod \\
uctio \\
$n$ \\
activ \\
ity
\end{tabular} & \begin{tabular}{|} 
Hous \\
ehol \\
$d$ \\
prod \\
uctio \\
$n$ for \\
sale
\end{tabular} & \begin{tabular}{|c} 
Pharm \\
aceutic \\
al
\end{tabular} & $\mid \begin{array}{c}\text { Foods } \\
\text { tuffs }\end{array}$ & $\begin{array}{c}\text { Alc } \\
\text { oho } \\
\text { I }\end{array}$ & $\begin{array}{c}\text { Servic } \\
\text { es }\end{array}$ & Other \\
\hline d & & e & & & & & & & & & & & \\
\hline $\begin{array}{l}\text { Ste. } \\
\text { Gene } \\
\text { vieve } \\
\text { Heral } \\
\text { d }\end{array}$ & $\begin{array}{r}12 / 1 \\
7 / 18 \\
92\end{array}$ & $\begin{array}{l}\text { Meyer' } \\
\text { s Hotel }\end{array}$ & & & & & & & & & & $\begin{array}{l}\text { Hotel, } \\
\text { Omni } \\
\text { bus }\end{array}$ & \\
\hline $\begin{array}{l}\text { Ste. } \\
\text { Gene } \\
\text { vieve } \\
\text { Heral } \\
\text { d }\end{array}$ & \begin{tabular}{|r|}
$12 / 1$ \\
$7 / 18$ \\
92
\end{tabular} & $\begin{array}{l}\text { F. \& L. } \\
\text { U. } \\
\text { Store }\end{array}$ & $\begin{array}{l}\text { X- } \\
\text { Hard } \\
\text { ware }\end{array}$ & & $\begin{array}{l}\text { X-hats, } \\
\text { caps, } \\
\text { shoes, } \\
\text { boots }\end{array}$ & & & & & $\begin{array}{l}\text { X-dry } \\
\text { goods } \\
\text { grocer } \\
\text { ies }\end{array}$ & & & \\
\hline $\begin{array}{l}\text { Ste. } \\
\text { Gene } \\
\text { vieve } \\
\text { Heral } \\
\text { d }\end{array}$ & $\begin{array}{r}12 / 1 \\
7 / 18 \\
92\end{array}$ & $\begin{array}{l}\text { TENT's } \\
\text { Albums }\end{array}$ & & & & $\begin{array}{l}\text { X- } \\
\text { albu } \\
\text { ms, } \\
\text { juveni } \\
\text { le } \\
\text { books }\end{array}$ & & & & & & & \\
\hline \begin{tabular}{|l} 
Ste. \\
Gene \\
vieve \\
Heral \\
d \\
\end{tabular} & $\begin{array}{r}12 / 1 \\
7 / 18 \\
92\end{array}$ & $\begin{array}{l}\text { City } \\
\text { Mills }\end{array}$ & & & & & & & & & & Mill & \\
\hline \begin{tabular}{|l} 
Ste. \\
Gene \\
vieve \\
Heral \\
d \\
\end{tabular} & $\begin{array}{r}12 / 1 \\
7 / 18 \\
92 \\
\end{array}$ & $\begin{array}{l}\text { Mich. } \\
\text { Beauch } \\
\text { amp } \\
\text { Tonsori } \\
\text { al Artist }\end{array}$ & & & & $\begin{array}{l}\text { X- } \\
\text { cigars } \\
\text { cigare } \\
\text { ttes } \\
\end{array}$ & & & & & & $\begin{array}{l}\text { Shave } \\
r, \\
\text { Salon }\end{array}$ & \\
\hline \begin{tabular}{|l} 
Ste. \\
Gene \\
vieve \\
Heral \\
d \\
\end{tabular} & $\begin{array}{r}12 / 1 \\
7 / 18 \\
92\end{array}$ & $\begin{array}{l}\text { Americ } \\
\text { an } \\
\text { Farmer }\end{array}$ & & & & $\begin{array}{l}\text { X- } \\
\text { Maga } \\
\text { zine }\end{array}$ & & & & & & & \\
\hline $\begin{array}{l}\text { Ste. } \\
\text { Gene } \\
\text { vieve }\end{array}$ & $\begin{array}{r}12 / 1 \\
7 / 18 \\
92\end{array}$ & $\begin{array}{l}\text { Mrs. E. } \\
\text { Kern }\end{array}$ & & & & & $\begin{array}{l}\text { X- } \\
\text { notio } \\
\text { ns }\end{array}$ & & & & & & \\
\hline
\end{tabular}




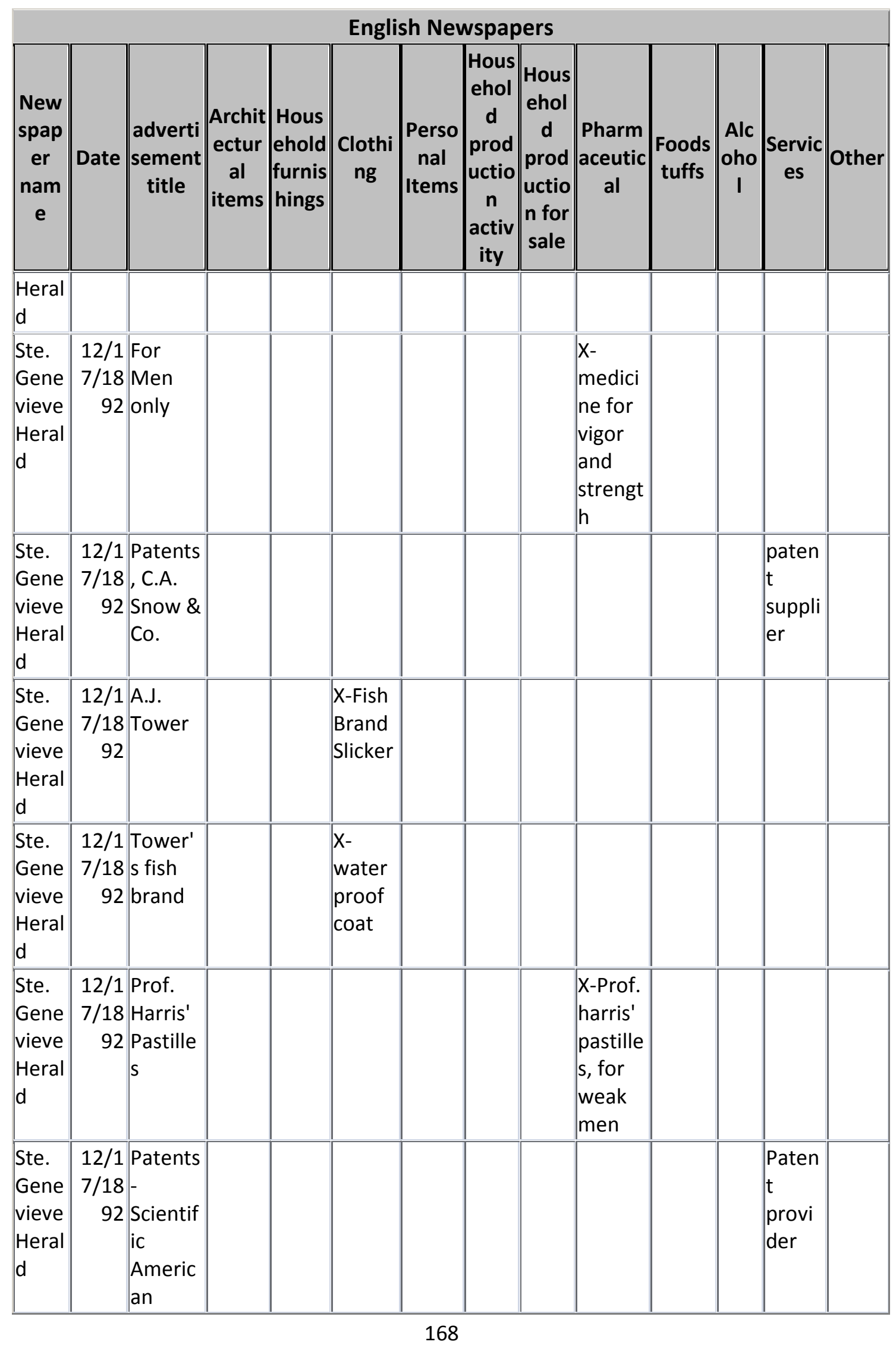




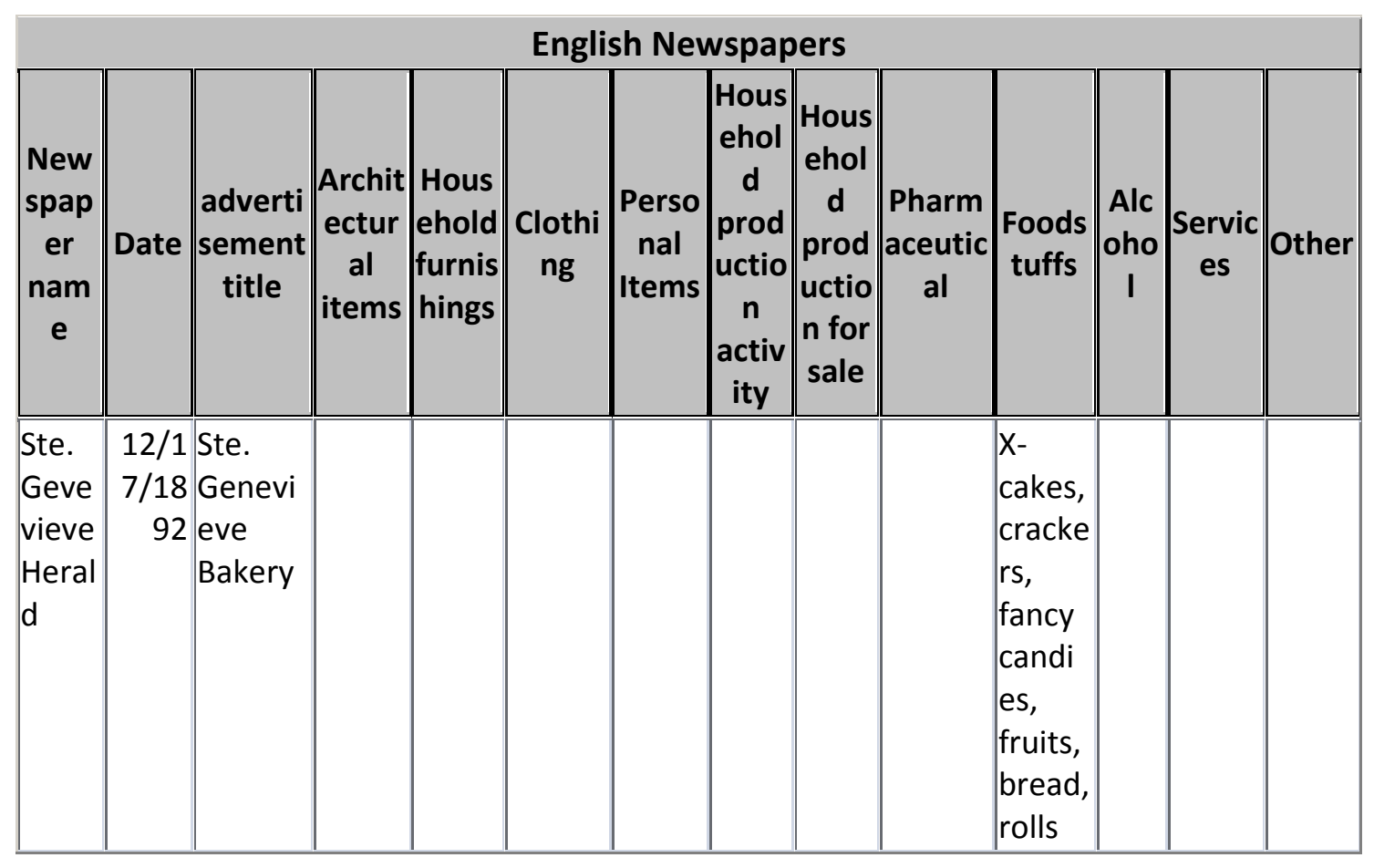

\section{German Newspapers}

\begin{tabular}{|c|c|c|c|c|c|c|c|c|}
\hline \multicolumn{9}{|c|}{ German Newspapers } \\
\hline$\left|\begin{array}{c}\text { News } \\
\text { pape } \\
r\end{array}\right|$ & Date & $\left|\begin{array}{c}\text { advertisem } \\
\text { ent title }\end{array}\right|$ & $\begin{array}{c}\text { Househ } \\
\text { old } \\
\text { Mainten } \\
\text { ance- } \\
\text { Ceramic }\end{array}$ & \begin{tabular}{|c} 
Househ \\
old \\
Mainte \\
nance- \\
glass
\end{tabular} & $\begin{array}{c}\text { House } \\
\text { hold } \\
\text { Maint } \\
\text { enanc } \\
\text { e- } \\
\text { other }\end{array}$ & $\begin{array}{l}\text { Architectur } \\
\text { al Items }\end{array}$ & $\begin{array}{l}\text { Household } \\
\text { Furnishings }\end{array}$ & Clothing \\
\hline \begin{tabular}{|l|} 
Freie \\
Press \\
e \\
\end{tabular} & $\begin{array}{r}7 / 22 / 18 \\
7 €\end{array}$ & $\begin{array}{l}\text { Chas C. } \\
\text { Rozier }\end{array}$ & & & & & & \\
\hline $\begin{array}{l}\text { Freie } \\
\text { Press } \\
\text { e }\end{array}$ & $\begin{array}{r}7 / 22 / 1 \varepsilon \\
7 €\end{array}$ & $\begin{array}{l}\text { 7ie Auflage } \\
\text { (7th } \\
\text { edition) }\end{array}$ & & & & & & \\
\hline \begin{tabular}{|l} 
Freie \\
Press \\
e \\
\end{tabular} & $\begin{array}{r}7 / 22 / 18 \\
7 \epsilon \\
\end{array}$ & $\begin{array}{l}\text { Fährboot } \\
\text { (Ferry } \\
\text { Boat) }\end{array}$ & & & & & & \\
\hline \begin{tabular}{|l} 
Freie \\
Press \\
e
\end{tabular} & $\begin{array}{r}7 / 22 / 18 \\
7 € \\
\end{array}$ & $\begin{array}{l}\text { Rheumatis } \\
\text { buch } \\
\text { (Rheumatis } \\
\text { m) }\end{array}$ & & & & & & \\
\hline \begin{tabular}{|l} 
Freie \\
Press
\end{tabular} & $\begin{array}{r}7 / 22 / 18 \\
76\end{array}$ & $\begin{array}{l}\text { Peter } \\
\text { Schumert }\end{array}$ & & & & & & $\begin{array}{l}\text { X-hosen (pants), } \\
\text { unterhemden }\end{array}$ \\
\hline
\end{tabular}




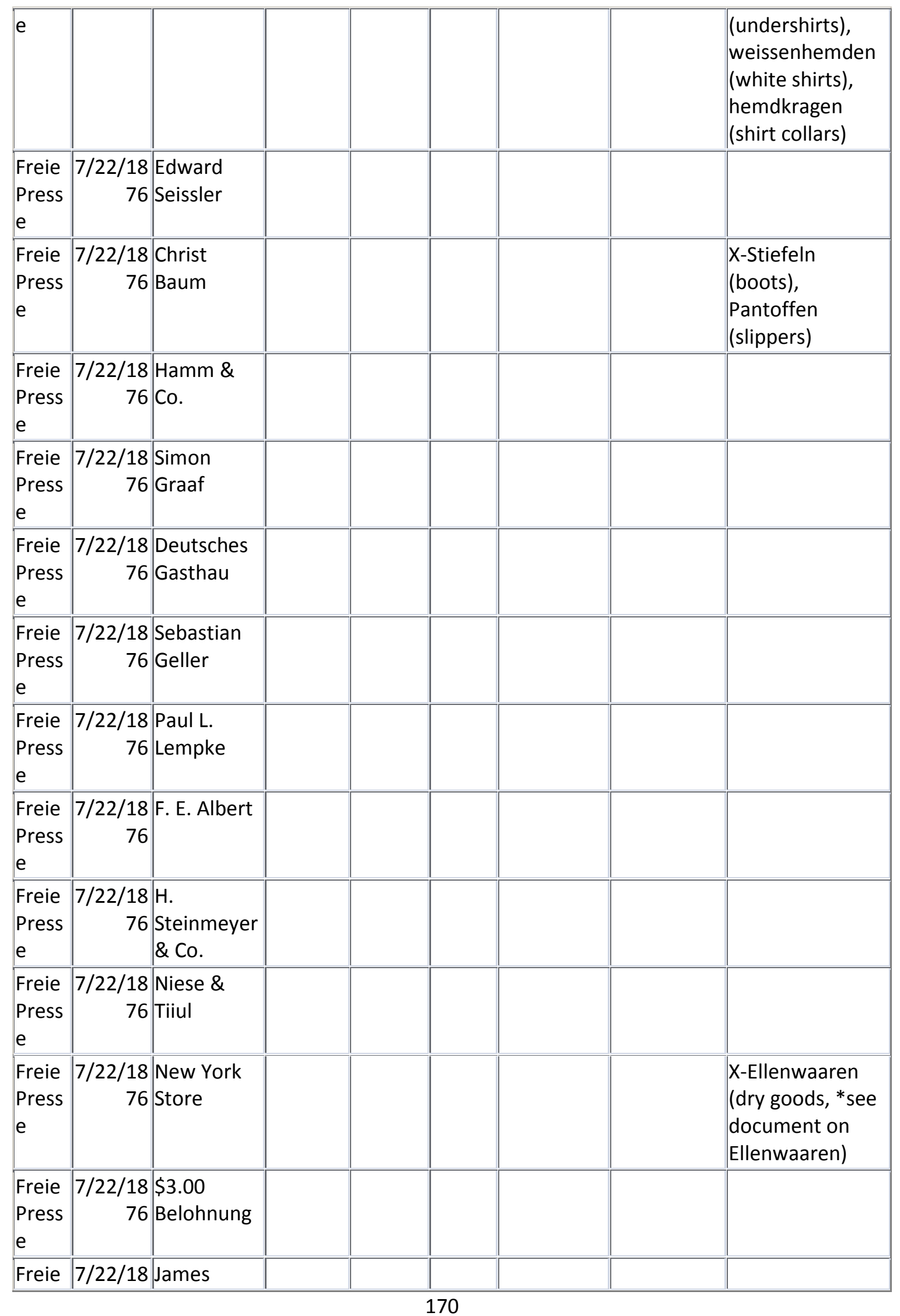




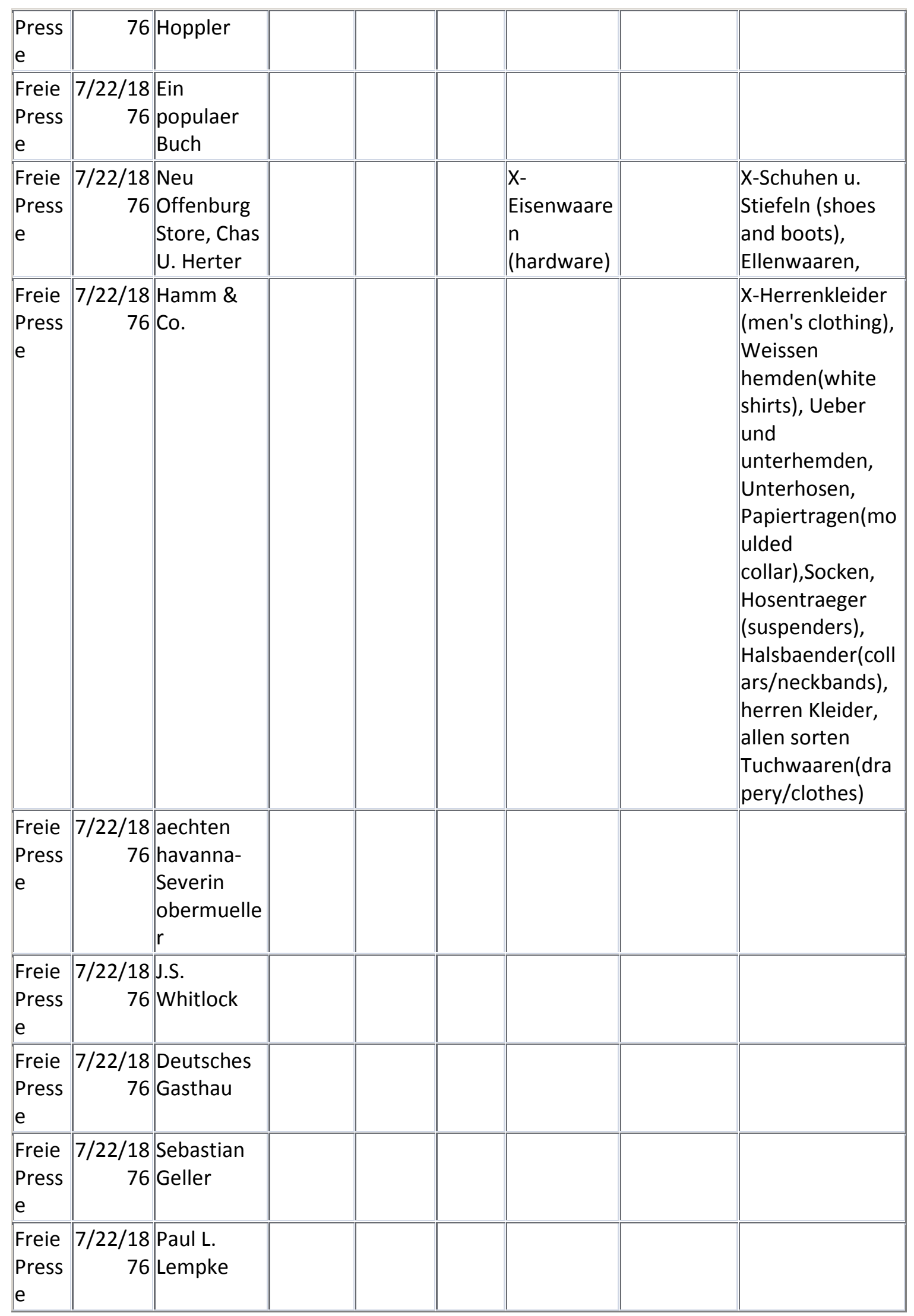




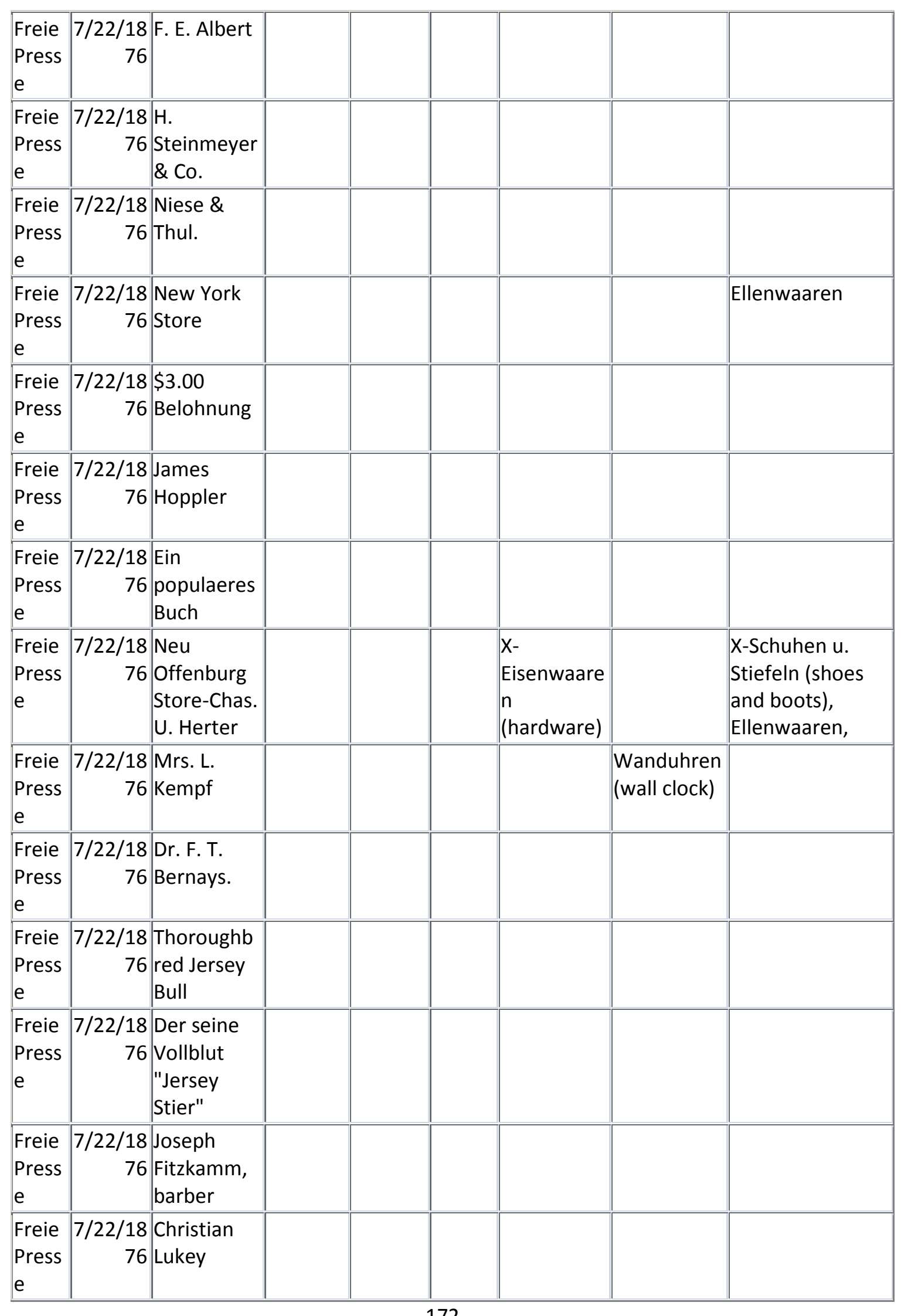




\begin{tabular}{|c|c|c|c|c|c|c|}
\hline \begin{tabular}{|l|} 
Freie \\
Press \\
e
\end{tabular} & $\begin{array}{r}7 / 22 / 18 \\
76\end{array}$ & $\begin{array}{l}\text { Southern } \\
\text { Hotel }\end{array}$ & & & & \\
\hline \begin{tabular}{|l|} 
Freie \\
Press \\
e
\end{tabular} & $\begin{array}{r}7 / 22 / 18 \\
76\end{array}$ & $\begin{array}{l}\text { Andrew } \\
\text { Andersen }\end{array}$ & $\begin{array}{l}\text { X- } \\
\text { Porzeila } \\
n\end{array}$ & $\begin{array}{l}\text { Glaswa } \\
\text { aren }\end{array}$ & \begin{tabular}{|l} 
X- \\
Eisenwaare \\
$n$ \\
(hardware)
\end{tabular} & $\begin{array}{l}\text { X-Hueten (hats), } \\
\text { Kappen (caps), } \\
\text { Schuhen, Stiefel } \\
\text { (boots), } \\
\text { Ellenwaaren }\end{array}$ \\
\hline \begin{tabular}{|l|} 
Freie \\
Press \\
e
\end{tabular} & $\begin{array}{r}7 / 22 / 18 \\
76\end{array}$ & Rupture, & & & & \\
\hline \begin{tabular}{|l|} 
Freie \\
Press \\
e
\end{tabular} & $\begin{array}{r}7 / 22 / 18 \\
76\end{array}$ & $\begin{array}{l}\text { Dr. } \\
\text { Whittier }\end{array}$ & & & & \\
\hline \begin{tabular}{|l|} 
Freie \\
Press \\
e
\end{tabular} & \begin{tabular}{|r|}
$7 / 22 / 18$ \\
76 \\
\end{tabular} & $\begin{array}{l}\text { Washingto } \\
\text { n Marble } \\
\text { Works- } \\
\text { James } \\
\text { Douglas } \\
\text { and George } \\
\text { Douglas }\end{array}$ & & & & \\
\hline \begin{tabular}{|l|} 
Freie \\
Press \\
$\mathrm{e}$
\end{tabular} & $\begin{array}{r}7 / 22 / 18 \\
76\end{array}$ & U.S. Mail & & & & \\
\hline \begin{tabular}{|l|} 
Freie \\
Press \\
e
\end{tabular} & $\begin{array}{r}7 / 22 / 18 \\
76\end{array}$ & $\begin{array}{l}\text { St. Clair } \\
\text { Hotel }\end{array}$ & & & & \\
\hline \begin{tabular}{|l|} 
Freie \\
Press \\
e
\end{tabular} & $\begin{array}{r}7 / 22 / 18 \\
76\end{array}$ & $\begin{array}{l}\text { Mette \& } \\
\text { Kanne, } \\
\text { Retifiers }\end{array}$ & & & & \\
\hline \begin{tabular}{|l|} 
Freie \\
Press \\
e
\end{tabular} & $\begin{array}{r}7 / 22 / 18 \\
76 \\
\end{array}$ & $\begin{array}{l}\text { California } \\
\text { Wines- } \\
\text { Napa \& } \\
\text { Sonoma } \\
\text { Wine Co. }\end{array}$ & & & & \\
\hline \begin{tabular}{|l|} 
Freie \\
Press \\
e
\end{tabular} & $\begin{array}{r}7 / 22 / 18 \\
76\end{array}$ & $\begin{array}{l}\text { Freie } \\
\text { Presse }\end{array}$ & & & & \\
\hline \begin{tabular}{|l|} 
Freie \\
Press \\
e
\end{tabular} & \begin{tabular}{|r|}
$7 / 22 / 18$ \\
76
\end{tabular} & Jos. Borst & & & & \\
\hline \begin{tabular}{|l|} 
Freie \\
Press \\
e
\end{tabular} & $\begin{array}{r}7 / 22 / 18 \\
76\end{array}$ & $\begin{array}{l}\mathrm{H} . \\
\text { Schwartz }\end{array}$ & & & & \\
\hline
\end{tabular}




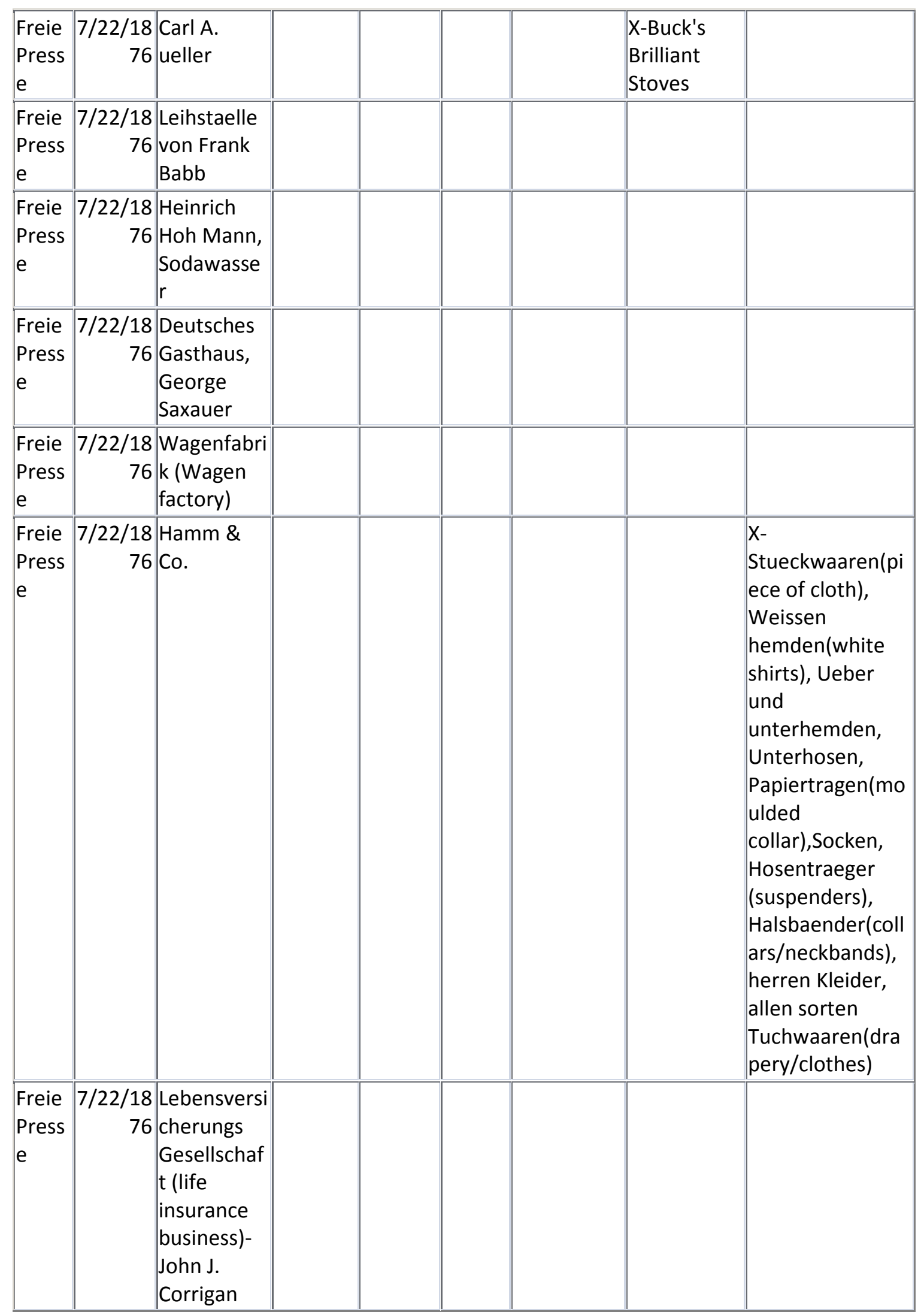




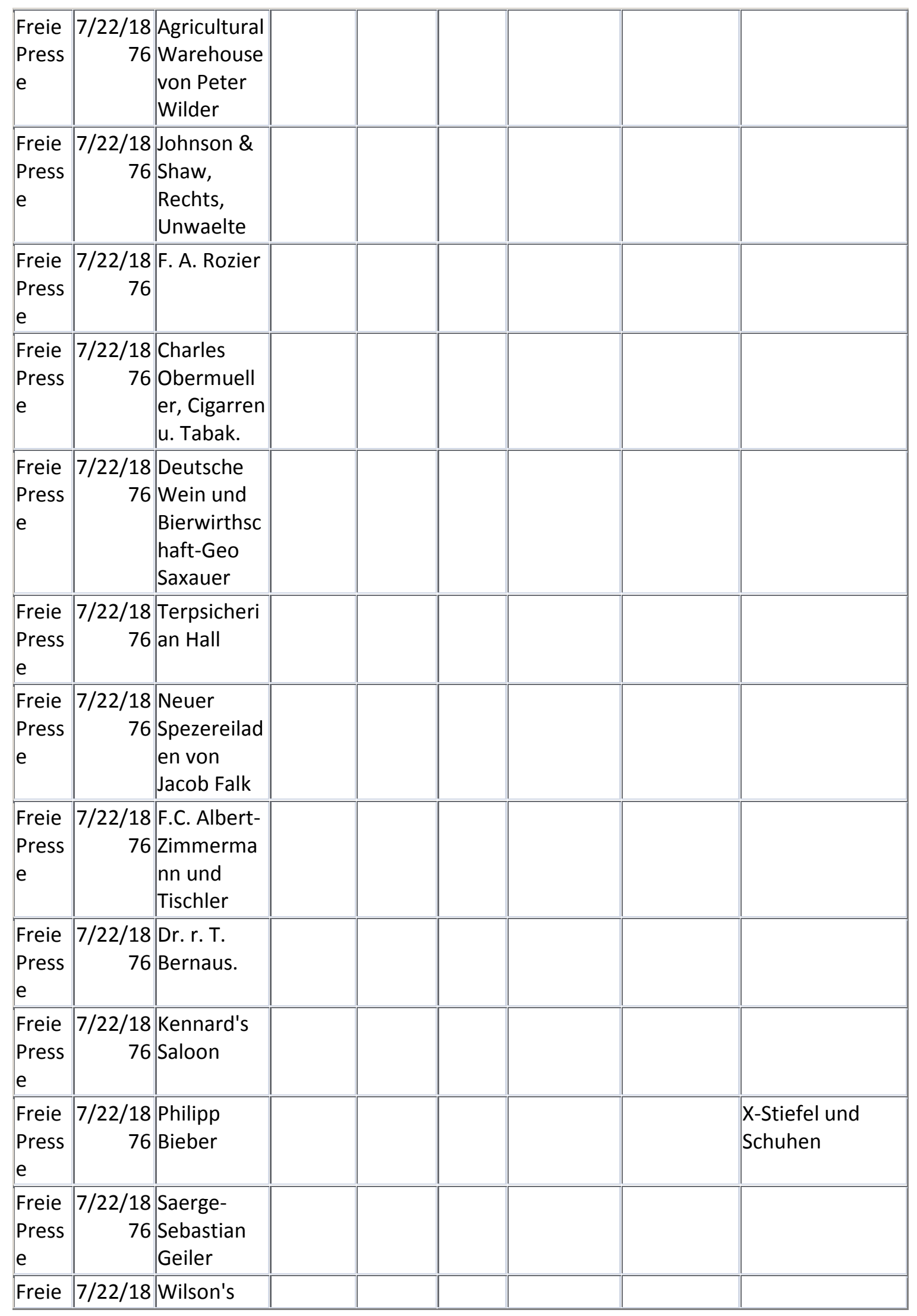




\begin{tabular}{|c|c|c|c|c|c|c|}
\hline \begin{tabular}{|l|} 
Press \\
e
\end{tabular} & & $\begin{array}{l}\text { Naehmasch } \\
\text { inen-Herr } \\
\text { August } \\
\text { Baechle }\end{array}$ & & & & \\
\hline \begin{tabular}{|l|} 
Freie \\
Press \\
e
\end{tabular} & $\begin{array}{r}7 / 22 / 18 \\
76 \\
\end{array}$ & $\begin{array}{l}\text { Joseph } \\
\text { Fitzkamm- } \\
\text { Deutscher } \\
\text { Barbier }\end{array}$ & & & & \\
\hline \begin{tabular}{|l|} 
Freie \\
Press \\
$\mathrm{e}$
\end{tabular} & $\begin{array}{r}7 / 22 / 18 \\
76\end{array}$ & $\begin{array}{l}\text { Dr. F. } \\
\text { Guibourd, } \\
\text { Apotheker }\end{array}$ & & & & \\
\hline \begin{tabular}{|l|} 
Freie \\
Press \\
e
\end{tabular} & $\begin{array}{r}7 / 22 / 18 \\
76\end{array}$ & $\begin{array}{l}\text { Harris und } \\
\text { Janis } \\
\text { Bankier }\end{array}$ & & & & \\
\hline \begin{tabular}{|l|} 
Freie \\
Press \\
e
\end{tabular} & $\begin{array}{r}7 / 22 / 18 \\
76\end{array}$ & $\begin{array}{l}\text { P.U. } \\
\text { Jaccard }\end{array}$ & & & \begin{tabular}{|l|} 
gold \\
und \\
silber \\
waare \\
n \\
(gold \\
and \\
silver \\
goods)
\end{tabular} & $\begin{array}{l}\text { Standuhren } \\
\text { (grandfathe } \\
\text { r clocks) }\end{array}$ \\
\hline \begin{tabular}{|l|} 
Freie \\
Press \\
e
\end{tabular} & $\begin{array}{r}7 / 22 / 18 \\
76 \\
\end{array}$ & $\begin{array}{l}\text { Taube und } \\
\text { Schwerhoe } \\
\text { rige-H. } \\
\text { Schwartz }\end{array}$ & & & & \\
\hline \begin{tabular}{|l|} 
Freie \\
Press \\
e
\end{tabular} & $\begin{array}{r}7 / 22 / 18 \\
76\end{array}$ & $\begin{array}{l}\text { Roesch \& } \\
\text { Efferin }\end{array}$ & & & & \\
\hline \begin{tabular}{|l|} 
Freie \\
Press \\
e
\end{tabular} & $\begin{array}{r}7 / 22 / 18 \\
76\end{array}$ & $\begin{array}{l}\text { Spezereilad } \\
\text { en-Mrs. M. } \\
\text { Wilder. }\end{array}$ & $\begin{array}{l}\text { X- } \\
\text { Porzelan }\end{array}$ & $\begin{array}{l}\text { Glaswar } \\
\text { en }\end{array}$ & & \\
\hline \begin{tabular}{|l|} 
Freie \\
Press \\
e
\end{tabular} & $\begin{array}{r}7 / 22 / 18 \\
76 \\
\end{array}$ & \begin{tabular}{|l|} 
Wm. H. \\
Bantz, \\
Receiving \& \\
Forwarding
\end{tabular} & & & & \\
\hline \begin{tabular}{|l|} 
Freie \\
Press \\
e
\end{tabular} & $\begin{array}{r}7 / 22 / 18 \\
76\end{array}$ & $\begin{array}{l}\text { Mrs. L. } \\
\text { Kempf }\end{array}$ & & & & X-clocks \\
\hline \begin{tabular}{|l|} 
Freie \\
Press \\
e
\end{tabular} & $\begin{array}{r}7 / 22 / 18 \\
76\end{array}$ & \begin{tabular}{|l} 
Ste. \\
Genevieve, \\
Bilder:Galle \\
rie-Franklin
\end{tabular} & & & & \\
\hline
\end{tabular}




\begin{tabular}{|c|c|c|c|c|c|c|c|}
\hline & & $\begin{array}{l}\text { Leavenwor } \\
\text { th }\end{array}$ & & & & & \\
\hline \begin{tabular}{|l} 
Freie \\
Press \\
e
\end{tabular} & $\begin{array}{r}7 / 22 / 18 \\
76\end{array}$ & $\begin{array}{l}\text { Paul L. } \\
\text { Lempke }\end{array}$ & & & & & \\
\hline $\begin{array}{l}\text { Freie } \\
\text { Press } \\
\text { e }\end{array}$ & $\begin{array}{r}7 / 22 / 18 \\
76\end{array}$ & $\begin{array}{l}\text { Paul L. } \\
\text { Lempke }\end{array}$ & & & & & \\
\hline $\begin{array}{l}\text { Freie } \\
\text { Press } \\
\text { e }\end{array}$ & $\begin{array}{r}7 / 22 / 18 \\
76\end{array}$ & $\begin{array}{l}\text { Matthew } \\
\text { Klein }\end{array}$ & & & & & X-boots, shoes \\
\hline \begin{tabular}{|l} 
Freie \\
Press \\
e
\end{tabular} & $\begin{array}{r}7 / 22 / 18 \\
76\end{array}$ & $\begin{array}{l}\text { Sebastan } \\
\text { Geiler }\end{array}$ & & & & $\begin{array}{l}\text { X-Moebel } \\
\text { (cabinet) }\end{array}$ & \\
\hline $\begin{array}{l}\text { Freie } \\
\text { Press } \\
\text { e }\end{array}$ & $\begin{array}{r}7 / 22 / 18 \\
76\end{array}$ & $\begin{array}{l}\text { Mrs. L. } \\
\text { Kempf }\end{array}$ & & & & $\begin{array}{l}\text { X- } \\
\text { Wanduhren } \\
\text { (wall clocks) }\end{array}$ & \\
\hline $\begin{array}{l}\text { Freie } \\
\text { Press } \\
\text { e }\end{array}$ & $\begin{array}{r}7 / 22 / 18 \\
76\end{array}$ & $\begin{array}{l}\text { Dr. F. T. } \\
\text { Bernans }\end{array}$ & & & & & \\
\hline $\begin{array}{l}\text { Freie } \\
\text { Press } \\
\text { e }\end{array}$ & $\begin{array}{r}7 / 22 / 18 \\
76\end{array}$ & $\begin{array}{l}\text { Thoroughb } \\
\text { red Jersey } \\
\text { Bull- } \\
\text { Strathmore } \\
\text { - Henry } \\
\text { Grobe }\end{array}$ & & & & & \\
\hline $\begin{array}{l}\text { Freie } \\
\text { Press } \\
\text { e }\end{array}$ & $\begin{array}{r}7 / 22 / 18 \\
76 \\
\end{array}$ & $\begin{array}{l}\text { Der seine } \\
\text { Bollbluth } \\
\text { "Jersey } \\
\text { Stier"- } \\
\text { Strathmore } \\
\text { - Heinrich } \\
\text { Grobe }\end{array}$ & & & & & \\
\hline \begin{tabular}{|l} 
Freie \\
Press \\
e
\end{tabular} & $\begin{array}{r}7 / 22 / 18 \\
76\end{array}$ & $\begin{array}{l}\text { Joseph } \\
\text { Fitzkamm, } \\
\text { Barber }\end{array}$ & & & & & \\
\hline $\begin{array}{l}\text { Freie } \\
\text { Press } \\
\text { e }\end{array}$ & $\begin{array}{r}7 / 22 / 18 \\
76\end{array}$ & $\begin{array}{l}\text { Christian } \\
\text { Lukey }\end{array}$ & & & & & \\
\hline $\begin{array}{l}\text { Freie } \\
\text { Press } \\
\text { e }\end{array}$ & $\begin{array}{r}7 / 22 / 18 \\
76\end{array}$ & $\begin{array}{l}\text { Andrew } \\
\text { Andersen }\end{array}$ & $\begin{array}{l}\text { X- } \\
\text { Porzeila } \\
n\end{array}$ & $\begin{array}{l}\text { X- } \\
\text { Glaswa } \\
\text { aren }\end{array}$ & $\begin{array}{l}\text { X- } \\
\text { Eisenwaare } \\
n \\
\text { (hardware) }\end{array}$ & & $\begin{array}{l}\text { X-Hueten (hats), } \\
\text { Kappen (caps), } \\
\text { Schuhen, Stiefel } \\
\text { (boots), } \\
\text { Ellenwaaren }\end{array}$ \\
\hline
\end{tabular}




\begin{tabular}{|c|c|c|c|c|c|c|c|}
\hline \begin{tabular}{|l|} 
Freie \\
Press \\
e
\end{tabular} & $\begin{array}{r}7 / 22 / 18 \\
76 \\
\end{array}$ & $\begin{array}{l}\text { Southern } \\
\text { hotel - } \\
\text { Joseph } \\
\text { Vorst }\end{array}$ & & & & & \\
\hline \begin{tabular}{|l|} 
Freie \\
Press \\
e
\end{tabular} & $\begin{array}{r}7 / 22 / 18 \\
76 \\
\end{array}$ & $\begin{array}{l}\text { Ste. } \\
\text { Genevieve } \\
\text { Photograp } \\
\text { h gallery- } \\
\text { Franklin } \\
\text { Leavenwor } \\
\text { th }\end{array}$ & & & & & \\
\hline $\begin{array}{l}\text { Freie } \\
\text { Press } \\
\text { e }\end{array}$ & $\begin{array}{r}7 / 22 / 18 \\
76 \\
\end{array}$ & $\begin{array}{l}\text { Edmund } \\
\text { Price's } \\
\text { Speisehaus } \\
\text { (eating } \\
\text { house) }\end{array}$ & & & & & \\
\hline $\begin{array}{l}\text { Freie } \\
\text { Press } \\
\text { e }\end{array}$ & $\begin{array}{r}7 / 22 / 18 \\
76\end{array}$ & $\begin{array}{l}\text { John L. } \\
\text { Boverie }\end{array}$ & $\begin{array}{l}\text { X- } \\
\text { Porzelan } \\
\text { Steingut } \\
\text { (stonew } \\
\text { are) }\end{array}$ & $\begin{array}{l}\text { X- } \\
\text { Glaswa } \\
\text { aren }\end{array}$ & $\begin{array}{l}\text { X- } \\
\text { Eisenwaare } \\
n\end{array}$ & $\begin{array}{l}\text { X- Farben } \\
\text { und Oelen } \\
\text { Moebeln } \\
\text { (colored } \\
\text { and oil } \\
\text { furniture?), } \\
\text { Korbwaare } \\
\text { n } \\
\text { (basketry), } \\
\text { Drechslerw } \\
\text { aaren } \\
\text { (wood } \\
\text { work) }\end{array}$ & $\begin{array}{l}\text { X-Hueten, } \\
\text { Kappen, Schuhe, } \\
\text { Stiefel,Ellenwaar } \\
\text { en }\end{array}$ \\
\hline $\begin{array}{l}\text { Freie } \\
\text { Press } \\
\text { e }\end{array}$ & $\begin{array}{r}7 / 22 / 18 \\
76 \\
\end{array}$ & $\begin{array}{l}\text { Wagenfabri } \\
\text { k (Wagen } \\
\text { factory) }\end{array}$ & & & & & \\
\hline \begin{tabular}{|l|} 
Freie \\
Press \\
e
\end{tabular} & $\begin{array}{r}7 / 22 / 18 \\
76\end{array}$ & $\begin{array}{l}\text { Hamm \& } \\
\text { Co. }\end{array}$ & & & & & $\begin{array}{l}\text { X-Stueckwaaren } \\
\text { (piece of cloth), } \\
\text { TuchWeissen } \\
\text { hemden (white } \\
\text { shirts), Ueber } \\
\text { und } \\
\text { unterhemden, } \\
\text { Unterhosen, } \\
\text { Papiertragen } \\
\text { (moulded collar), } \\
\text { Socken, } \\
\text { Hosentraeger } \\
\text { (suspenders), }\end{array}$ \\
\hline
\end{tabular}




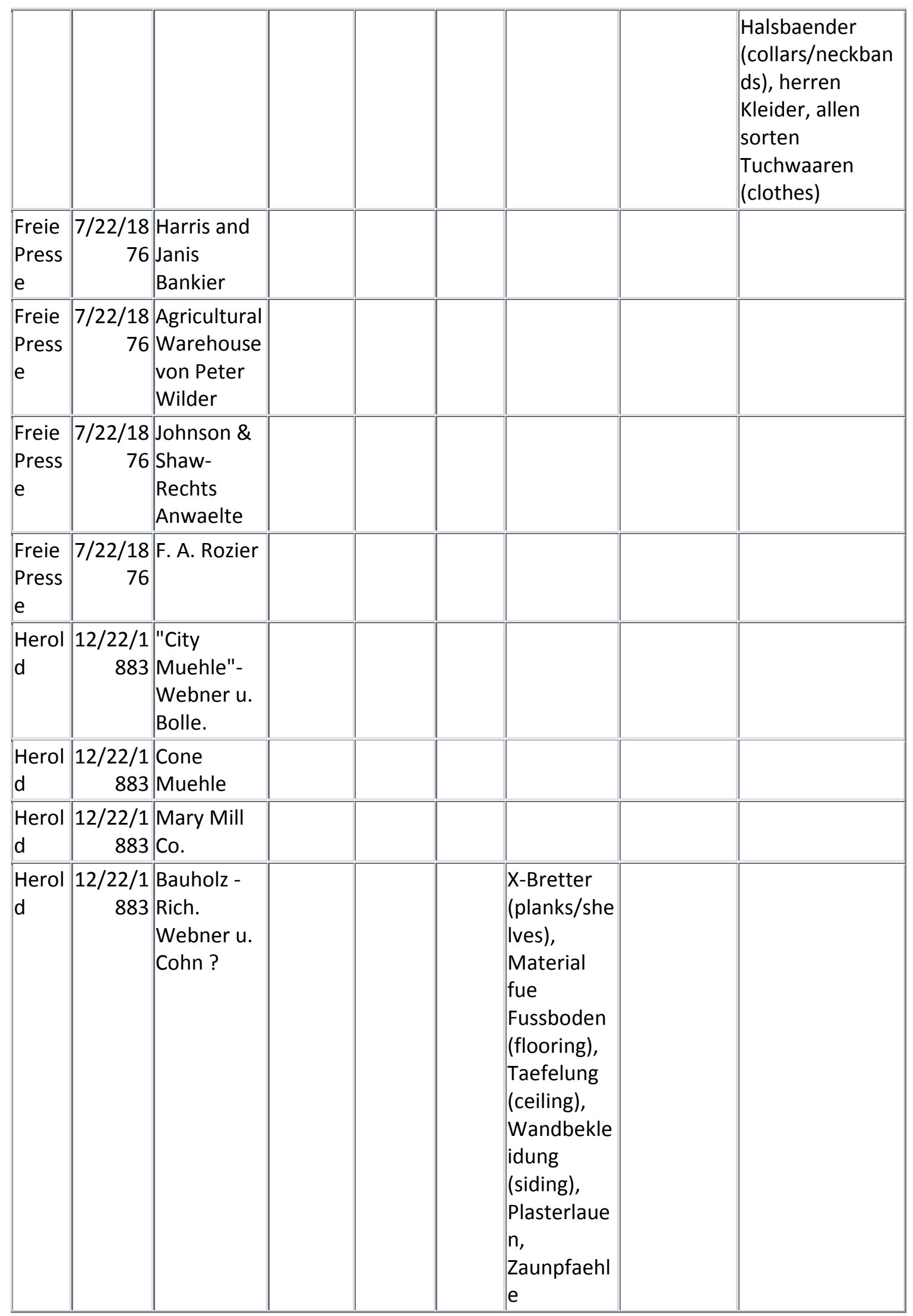




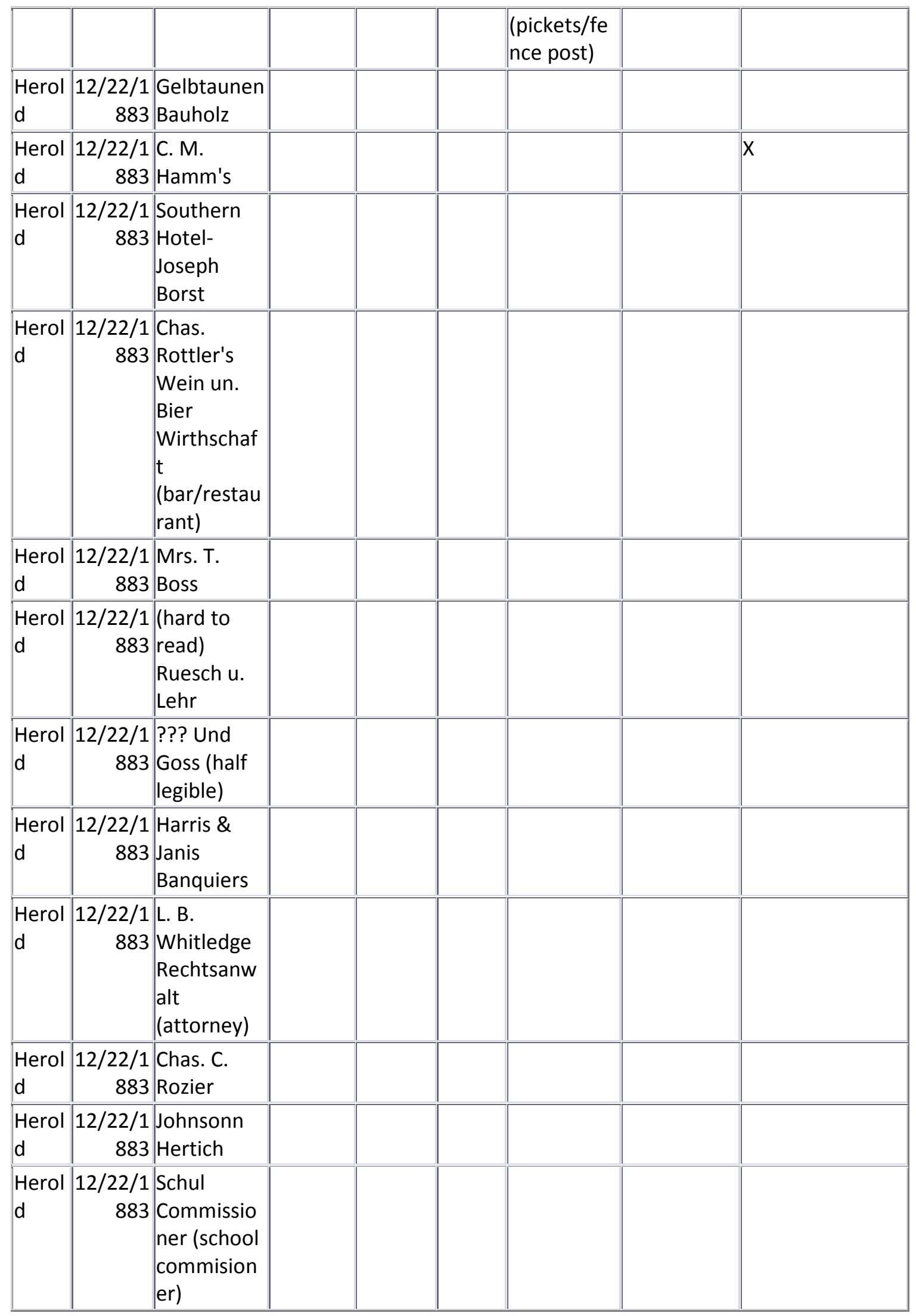




\begin{tabular}{|c|c|c|c|c|c|c|}
\hline \begin{tabular}{|l} 
Herol \\
d
\end{tabular} & $\begin{array}{r}12 / 22 / 1 \\
883 \\
\end{array}$ & $\begin{array}{l}\text { Wilhelm } \\
\text { Palmer- } \\
\text { Groceries } \\
\text { aller Art } \\
\text { (groceries } \\
\text { of all } \\
\text { forms) }\end{array}$ & & & & \\
\hline $\begin{array}{l}\text { Herol } \\
\mathrm{d}\end{array}$ & $\begin{array}{r}12 / 22 / 1 \\
883\end{array}$ & $\begin{array}{l}\text { Brauerei, B. } \\
\text { Rottler }\end{array}$ & & & & \\
\hline $\begin{array}{l}\text { Herol } \\
\text { d }\end{array}$ & $\begin{array}{r}12 / 22 / 1 \\
883\end{array}$ & $\begin{array}{l}\text { John G. } \\
\text { Whitlock, } \\
\text { Schreiner } \\
\text { (carpenter) }\end{array}$ & & & & \\
\hline $\begin{array}{l}\text { Herol } \\
d\end{array}$ & $\begin{array}{r}12 / 22 / 1 \\
883\end{array}$ & $\begin{array}{l}\text { Dr. F. } \\
\text { Guibourd }\end{array}$ & & & & \\
\hline \begin{tabular}{|l|} 
Herol \\
d
\end{tabular} & $\begin{array}{r}12 / 22 / 1 \\
883\end{array}$ & $\begin{array}{l}\text { Pilliusses } \\
\text { Inccard }\end{array}$ & $\begin{array}{l}\text { Gold } \\
\text { und } \\
\text { Silber } \\
\text { waarr } \\
\text { en }\end{array}$ & & Uhr & $\begin{array}{l}\text { Filzhueten (felt } \\
\text { hats), } \\
\text { Strohhueten } \\
\text { (straw hats) }\end{array}$ \\
\hline $\begin{array}{l}\text { Herol } \\
\text { d }\end{array}$ & $\begin{array}{r}12 / 22 / 1 \\
883\end{array}$ & $\begin{array}{l}\text { Christian } \\
\text { Baum }\end{array}$ & & & & $\begin{array}{l}\text { X-Stiefeln, } \\
\text { Schuhen, } \\
\text { Lederhaten }\end{array}$ \\
\hline \begin{tabular}{|l|} 
Herol \\
d
\end{tabular} & $\begin{array}{r}12 / 22 / 1 \\
883\end{array}$ & $\begin{array}{l}\text { Gottlieb } \\
\text { Kammerer }\end{array}$ & & $\begin{array}{l}\text { X- } \\
\text { Blechwaare } \\
\text { n (metal } \\
\text { goods), } \\
\text { Gusseisen } \\
\text { waaren } \\
\text { (cast iron } \\
\text { goods) }\end{array}$ & $\begin{array}{l}\text { X-Oefen } \\
\text { (stove/furn } \\
\text { ace), } \\
\text { Lampen } \\
\text { (lamps) }\end{array}$ & \\
\hline $\begin{array}{l}\text { Herol } \\
\text { d }\end{array}$ & $\begin{array}{r}12 / 22 / 1 \\
883\end{array}$ & $\begin{array}{l}\text { Joseph } \\
\text { Fitzkam, } \\
\text { Haar } \\
\text { Kuenstler }\end{array}$ & & & & \\
\hline $\begin{array}{l}\text { Herol } \\
\text { d }\end{array}$ & $\begin{array}{r}12 / 22 / 1 \\
883 \\
\end{array}$ & $\begin{array}{l}\text { Rozier u. } \\
\text { Tokerf (?), } \\
\text { Zahlen } \\
\text { luegen } \\
\text { nicht (the } \\
\text { numbers } \\
\text { do not lie) }\end{array}$ & & & & \\
\hline $\begin{array}{l}\text { Herol } \\
\text { d }\end{array}$ & $\begin{array}{r}12 / 22 / 1 \\
883\end{array}$ & $\begin{array}{l}\text { (hard to } \\
\text { read) Ihr }\end{array}$ & & & & \\
\hline
\end{tabular}




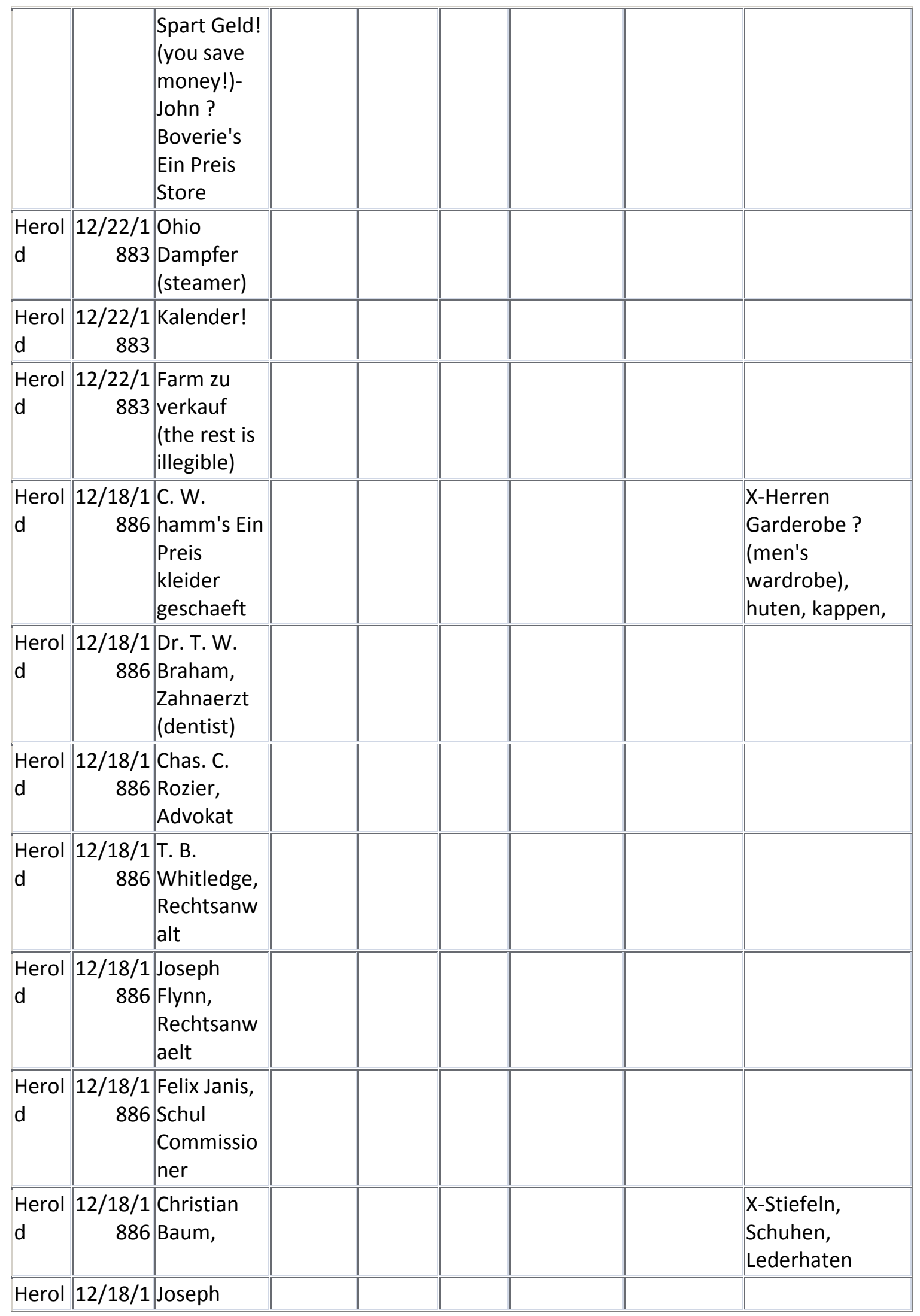




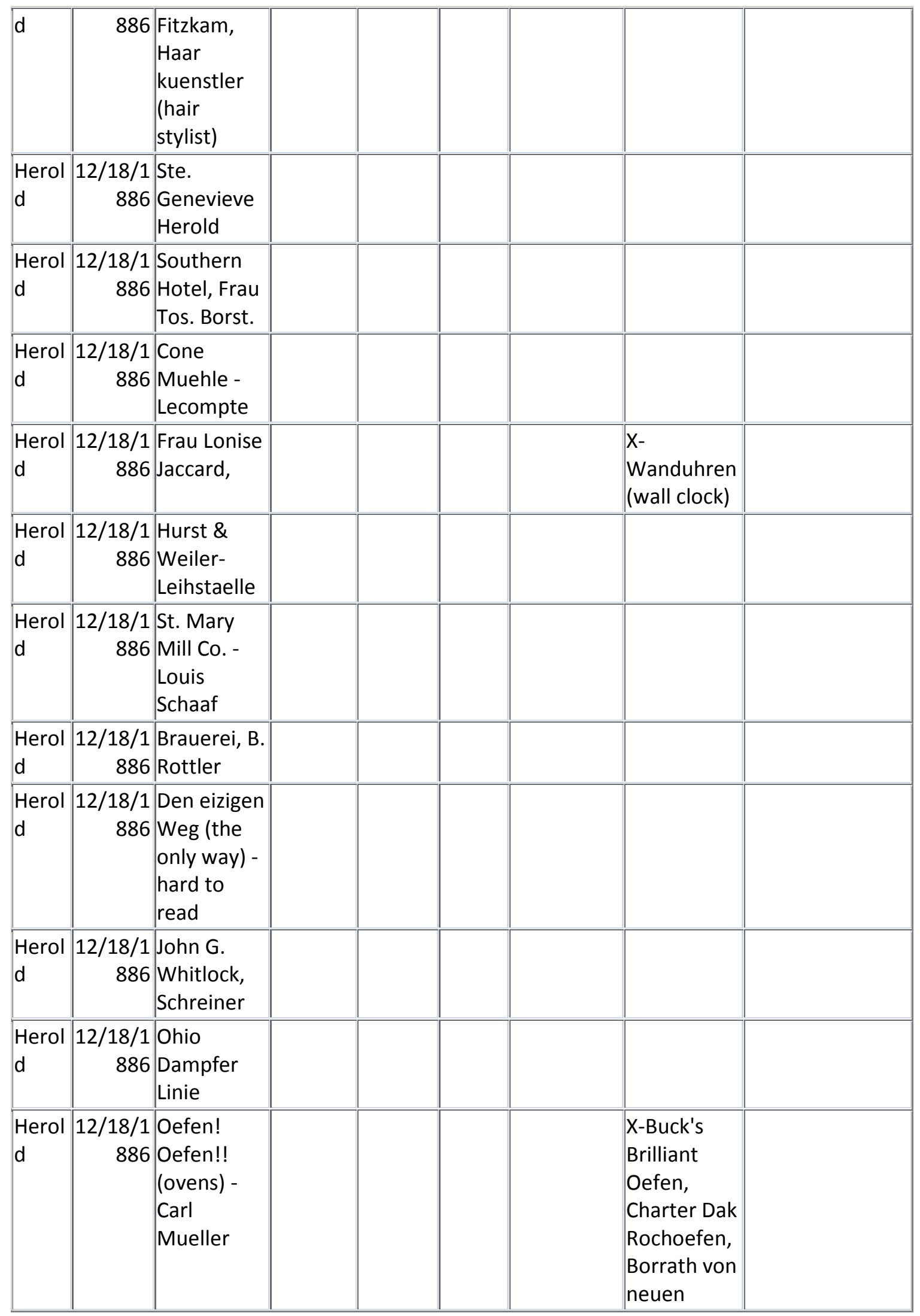




\begin{tabular}{|c|c|c|c|c|c|c|}
\hline & & & & & $\begin{array}{l}\text { Rochoefen, } \\
\text { Heiz Oefen }\end{array}$ & \\
\hline $\begin{array}{l}\text { Herol } \\
\text { d }\end{array}$ & $\begin{array}{r}12 / 18 / 1 \\
886\end{array}$ & ? Biel & & & & \\
\hline $\begin{array}{l}\text { Herol } \\
\text { d }\end{array}$ & \begin{tabular}{|r|}
$12 / 18 / 1$ \\
886
\end{tabular} & Herold & & & & \\
\hline $\begin{array}{l}\text { Herol } \\
\text { d }\end{array}$ & $\begin{array}{r}12 / 18 / 1 \\
886\end{array}$ & $\begin{array}{l}\text { Henry } \\
\text { Baum's } \\
\text { Barbierstan } \\
\text { de (barbor } \\
\text { stand) }\end{array}$ & & & & \\
\hline $\begin{array}{l}\text { Herol } \\
\text { d }\end{array}$ & $\begin{array}{r}12 / 18 / 1 \\
886\end{array}$ & $\begin{array}{l}\text { Die Lumber } \\
\text { Yard von R. } \\
\text { Wehner } \\
\text { und Sohn }\end{array}$ & & X-Lumber & & \\
\hline $\begin{array}{l}\text { Herol } \\
\text { d }\end{array}$ & $\begin{array}{r}\mid 12 / 18 / 1 \\
886\end{array}$ & $\begin{array}{l}\text { St. Louis } \\
\text { Tribuene }\end{array}$ & & & & \\
\hline $\begin{array}{l}\text { Herol } \\
\text { d }\end{array}$ & $\begin{array}{r}12 / 18 / 1 \\
886\end{array}$ & Carl Biel & $\begin{array}{l}\text { X- } \\
\text { Porzella } \\
n \\
\text { waaren }\end{array}$ & & & Ellenwaaren \\
\hline $\begin{array}{l}\text { Herol } \\
\text { d }\end{array}$ & $\begin{array}{r}12 / 18 / 1 \\
886\end{array}$ & ? Wilder & & & & \\
\hline $\begin{array}{l}\text { Herol } \\
\text { d }\end{array}$ & $\begin{array}{r}12 / 18 / 1 \\
886\end{array}$ & $\begin{array}{l}\text { Frau Falk u. } \\
\text { Sohn }\end{array}$ & & $\begin{array}{l}\text { X- } \\
\text { Bauholzes } \\
\text { (timber) }\end{array}$ & & \\
\hline $\begin{array}{l}\text { Herol } \\
\text { d }\end{array}$ & $\begin{array}{r}\mid 12 / 18 / 1 \\
886\end{array}$ & $\begin{array}{l}\text { Gottlob } \\
\text { Kammerer }\end{array}$ & & & \begin{tabular}{|l|} 
X-Lampen, \\
Oefen, \\
Birchwaare \\
n (wood \\
goods), \\
Gusseisenw \\
aaren (cast \\
iron goods) \\
\end{tabular} & \\
\hline $\begin{array}{l}\text { Herol } \\
\text { d }\end{array}$ & $\begin{array}{r}12 / 18 / 1 \\
886\end{array}$ & $\begin{array}{l}\text { Wehner u. } \\
\text { Bolle, City } \\
\text { Mill }\end{array}$ & & & & \\
\hline $\begin{array}{l}\text { Herol } \\
\text { d }\end{array}$ & $\begin{array}{r}12 / 18 / 1 \\
886\end{array}$ & $\begin{array}{l}\text { Der } \\
\text { Pelznickel } \\
\text { is da! } \\
\text { (Santa } \\
\text { Claus is } \\
\text { there!) }\end{array}$ & & & & \\
\hline Herol & $12 / 18 / 1$ & Frau Falk u. & & $x-$ & & \\
\hline
\end{tabular}




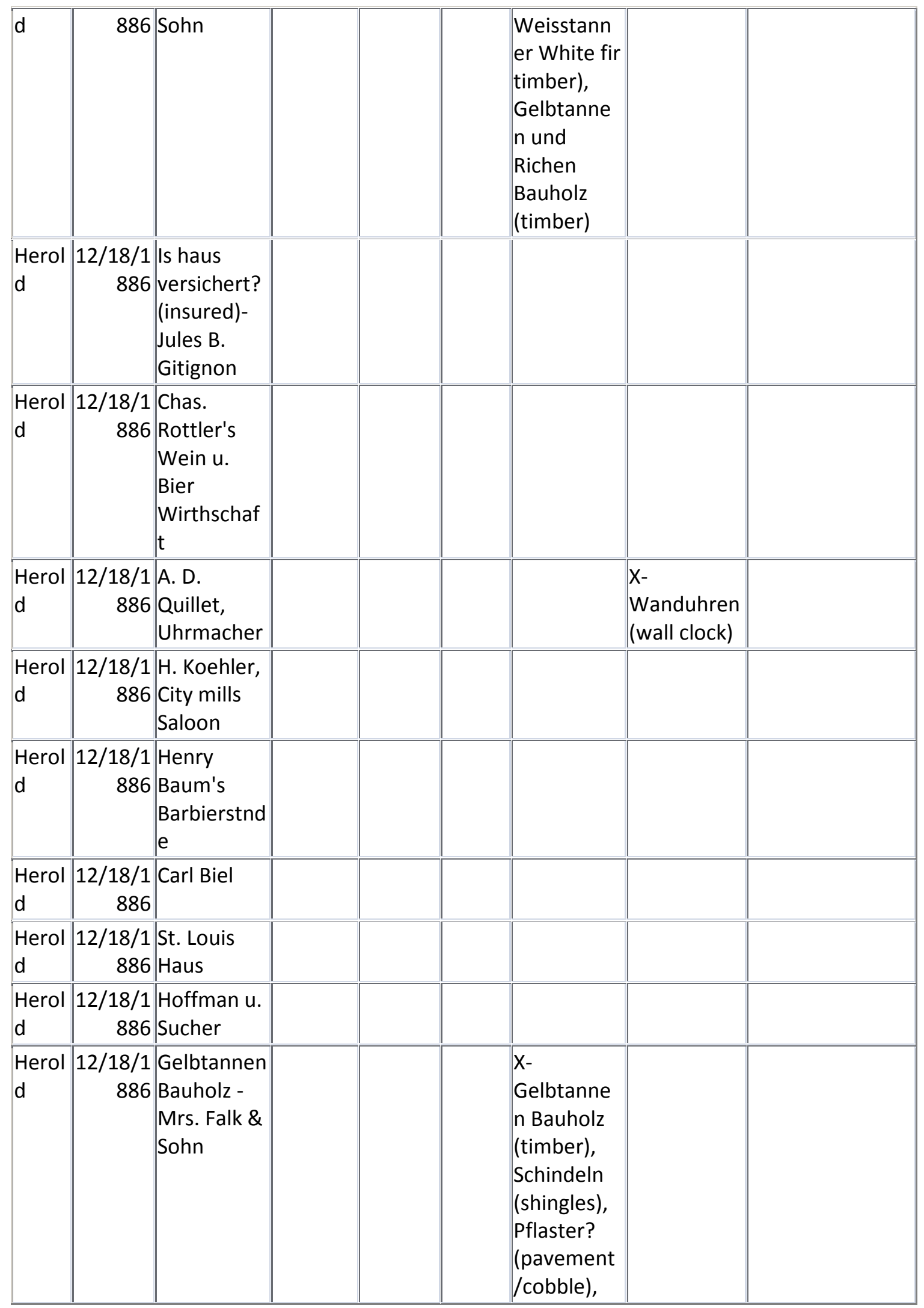




\begin{tabular}{|c|c|c|c|c|c|c|c|c|c|c|}
\hline & & & & & & \multicolumn{2}{|c|}{$\begin{array}{l}\text { Thueren } \\
\text { (doors), } \\
\text { Fenster } \\
\text { (windows), } \\
\text { Fensterlae } \\
\text { den } \\
\text { (shutters), } \\
\text { Eisen und } \\
\text { Stahlwaare } \\
\text { n (iron and } \\
\text { steel } \\
\text { goods), } \\
\text { Naegel } \\
\text { (nails) }\end{array}$} & & & \\
\hline $\begin{array}{l}\text { Herol } \\
\text { d }\end{array}$ & \multicolumn{3}{|c|}{\begin{tabular}{|l|l|}
$12 / 18 / 1$ & Kalender \\
886 & fuer 1887 - \\
& Tos. \\
& Fitzkam
\end{tabular}} & & & & & & & \\
\hline \multicolumn{11}{|c|}{ German Newspapers } \\
\hline $\begin{array}{c}\text { New } \\
\text { spap } \\
\text { er }\end{array}$ & Date & $\begin{array}{l}\text { advertis } \\
\text { ement } \\
\text { title }\end{array}$ & $\begin{array}{l}\text { Personal } \\
\text { Items }\end{array}$ & \begin{tabular}{|} 
Househo \\
Id \\
producti \\
on \\
activity
\end{tabular} & $\begin{array}{c}\text { House } \\
\text { hold } \\
\text { produc } \\
\text { tion } \\
\text { for } \\
\text { sale }\end{array}$ & \begin{tabular}{|} 
Pharm \\
aceutic \\
al
\end{tabular} & $\begin{array}{c}\text { Foodst } \\
\text { uffs }\end{array}$ & $\begin{array}{c}\text { Alcoho } \\
\text { I }\end{array}$ & $\begin{array}{c}\text { Service } \\
\text { s }\end{array}$ & Other \\
\hline \begin{tabular}{|l|} 
Frei \\
e \\
Pres \\
se
\end{tabular} & $\begin{array}{r}7 / 22 \\
/ 187 \\
6\end{array}$ & $\begin{array}{l}\text { Chas C. } \\
\text { Rozier }\end{array}$ & & & & & & & Legal & \\
\hline $\begin{array}{l}\text { Frei } \\
\text { e } \\
\text { Pres } \\
\text { se }\end{array}$ & $\begin{array}{r}7 / 22 \\
/ 187 \\
6\end{array}$ & $\begin{array}{l}\text { 7ie } \\
\text { Auflage } \\
\text { (7th } \\
\text { edition) }\end{array}$ & $\begin{array}{l}\text { Buch } \\
\text { (Book) }\end{array}$ & & & & & & & \\
\hline \begin{tabular}{|l|} 
Frei \\
e \\
Pres \\
se
\end{tabular} & $\begin{array}{r}7 / 22 \\
/ 187 \\
6\end{array}$ & $\begin{array}{l}\text { Fährboo } \\
\text { t (Ferry } \\
\text { Boat) }\end{array}$ & & & & & & & $\begin{array}{l}\text { Ferry } \\
\text { Boat }\end{array}$ & \\
\hline \begin{tabular}{|l|} 
Frei \\
e \\
Pres \\
se
\end{tabular} & $\begin{array}{r}7 / 22 \\
/ 187 \\
6\end{array}$ & $\begin{array}{l}\text { Rheuma } \\
\text { tisbuch } \\
\text { (Rheuma } \\
\text { tism) }\end{array}$ & Book & & & & & & & \\
\hline \begin{tabular}{|l|} 
Frei \\
e \\
Pres \\
se
\end{tabular} & $\begin{array}{r}7 / 22 \\
/ 187 \\
6\end{array}$ & $\begin{array}{l}\text { Peter } \\
\text { Schumer } \\
t\end{array}$ & & & & & & & $\begin{array}{l}\text { clothin } \\
\text { g } \\
\text { alterati } \\
\text { ons }\end{array}$ & \\
\hline
\end{tabular}




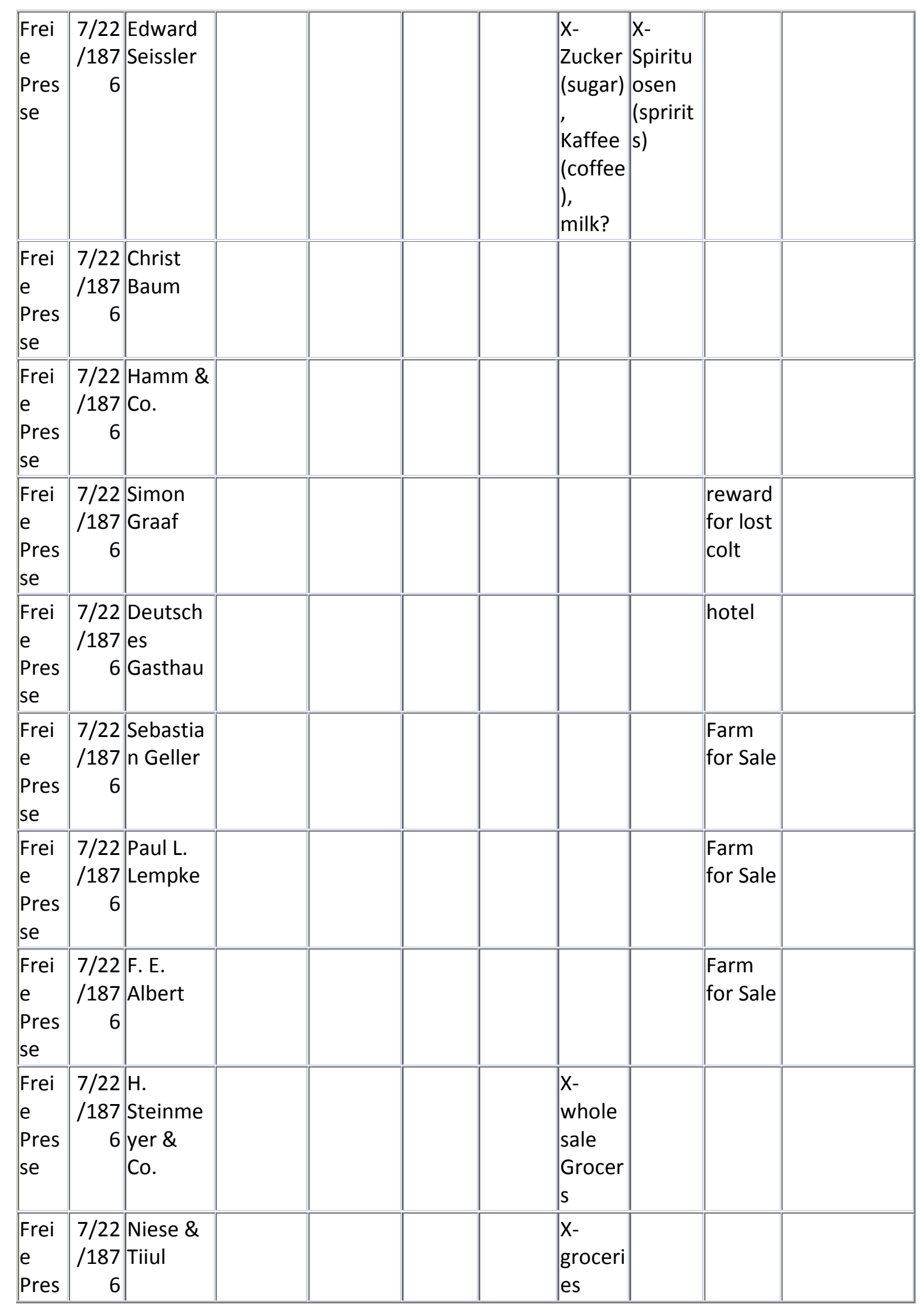




\begin{tabular}{|c|c|c|c|c|c|c|c|}
\hline se & & & & & & & \\
\hline $\begin{array}{l}\text { Frei } \\
\text { e } \\
\text { Pres } \\
\text { se }\end{array}$ & $\begin{array}{r}7 / 22 \\
/ 187 \\
6\end{array}$ & $\begin{array}{l}\text { New } \\
\text { York } \\
\text { Store }\end{array}$ & & & & & $\begin{array}{l}\text { kaufmannsw } \\
\text { aaren } \\
\text { (merchandise } \\
\text { ) }\end{array}$ \\
\hline \begin{tabular}{|l} 
Frei \\
e \\
Pres \\
se
\end{tabular} & \begin{tabular}{|r|}
$7 / 22$ \\
$/ 187$ \\
6 \\
\end{tabular} & $\begin{array}{l}\$ 3.00 \\
\text { Belohnu } \\
\text { ng }\end{array}$ & & & & $\begin{array}{l}\text { Reward } \\
\text { for lost } \\
\text { colt }\end{array}$ & \\
\hline $\begin{array}{l}\text { Frei } \\
\text { e } \\
\text { Pres } \\
\text { se }\end{array}$ & $\begin{array}{r}7 / 22 \\
/ 187 \\
6\end{array}$ & $\begin{array}{l}\text { James } \\
\text { Hoppler }\end{array}$ & & & & Agent & \\
\hline $\begin{array}{l}\text { Frei } \\
\text { e } \\
\text { Pres } \\
\text { se }\end{array}$ & $\begin{array}{r}7 / 22 \\
/ 187 \\
6\end{array}$ & $\begin{array}{l}\text { Ein } \\
\text { populaer } \\
\text { Buch }\end{array}$ & Book & & & & \\
\hline \begin{tabular}{|l|} 
Frei \\
e \\
Pres \\
se
\end{tabular} & \begin{tabular}{|r|}
$7 / 22$ \\
$/ 187$ \\
6 \\
\end{tabular} & $\begin{array}{l}\text { Neu } \\
\text { Offenbur } \\
\text { g Store, } \\
\text { Chas U. } \\
\text { Herter }\end{array}$ & & $\begin{array}{l}\text { X-Home } \\
\text { Shuttle } \\
\text { Naehmas } \\
\text { chine } \\
\text { (sewing } \\
\text { machine) } \\
\text { Buckeye } \\
\text { Ernte } \\
\text { (picker/h } \\
\text { arvester) } \\
\text { McSherr } \\
\text { y Drill }\end{array}$ & $\begin{array}{l}\text { X- } \\
\text { Spezer } \\
\text { eien } \\
\text { (dry/m } \\
\text { ixed } \\
\text { foods) }\end{array}$ & & \\
\hline \begin{tabular}{|l} 
Frei \\
e \\
Pres \\
se
\end{tabular} & \begin{tabular}{|r|}
$7 / 22$ \\
$/ 187$ \\
6
\end{tabular} & $\begin{array}{l}\text { Hamm \& } \\
\text { Co. }\end{array}$ & & & & & \\
\hline \begin{tabular}{|l} 
Frei \\
e \\
Pres \\
se
\end{tabular} & \begin{tabular}{|r|}
$7 / 22$ \\
$/ 187$ \\
6 \\
\end{tabular} & $\begin{array}{l}\text { aechten } \\
\text { havanna } \\
\text {-Severin } \\
\text { obermu } \\
\text { eller }\end{array}$ & \begin{tabular}{|l} 
X- \\
Cigarren, \\
Schnupft \\
abak \\
(snuff), \\
Pfeifen \\
(pipes), \\
Pfeifeno \\
ehren \\
(pipe
\end{tabular} & & & & \\
\hline
\end{tabular}




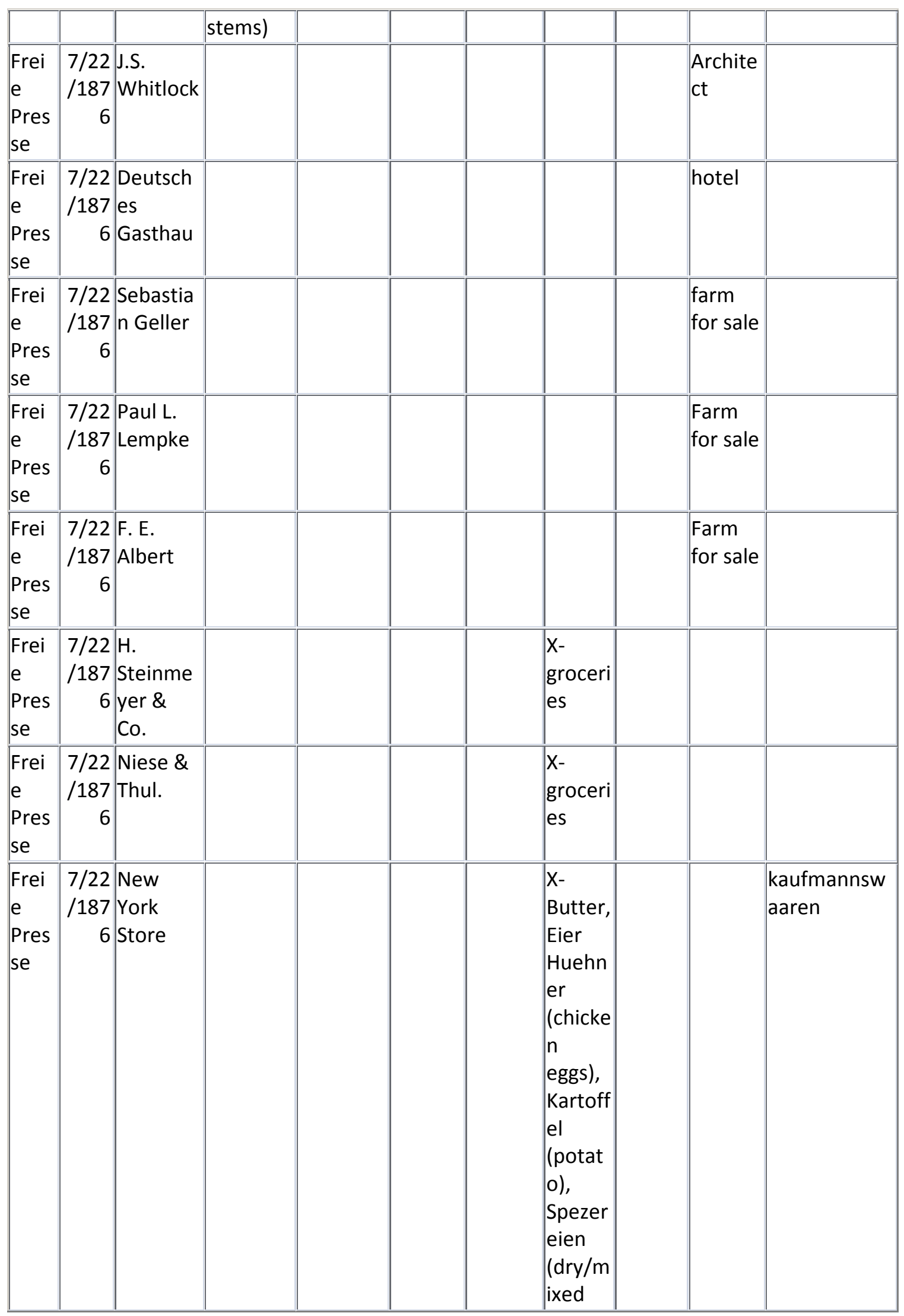




\begin{tabular}{|c|c|c|c|c|c|c|}
\hline & & & & & foods) & \\
\hline $\begin{array}{l}\text { Frei } \\
\text { e } \\
\text { Pres } \\
\text { se }\end{array}$ & $\begin{array}{r}7 / 22 \\
/ 187 \\
6\end{array}$ & $\begin{array}{l}\$ 3.00 \\
\text { Belohnu } \\
\text { ng }\end{array}$ & & & & $\begin{array}{l}\text { Reward } \\
\text { for lost } \\
\text { colt }\end{array}$ \\
\hline $\begin{array}{l}\text { Frei } \\
\text { e } \\
\text { Pres } \\
\text { se }\end{array}$ & $\begin{array}{r}7 / 22 \\
/ 187 \\
6\end{array}$ & $\begin{array}{l}\text { James } \\
\text { Hoppler }\end{array}$ & & & & Agent \\
\hline $\begin{array}{l}\text { Frei } \\
\text { e } \\
\text { Pres } \\
\text { se }\end{array}$ & $\begin{array}{r}7 / 22 \\
/ 187 \\
6\end{array}$ & $\begin{array}{l}\text { Ein } \\
\text { populaer } \\
\text { es Buch }\end{array}$ & book & & & \\
\hline $\begin{array}{l}\text { Frei } \\
\text { e } \\
\text { Pres } \\
\text { se }\end{array}$ & $\begin{array}{r}7 / 22 \\
/ 187 \\
6 \\
\end{array}$ & $\begin{array}{l}\text { Neu } \\
\text { Offenbur } \\
\text { g Store- } \\
\text { Chas. U. } \\
\text { Herter }\end{array}$ & & $\begin{array}{l}\text { X-Home } \\
\text { Shuttle } \\
\text { Naehmas } \\
\text { chine } \\
\text { (sewing } \\
\text { machine) } \\
\text {, } \\
\text { Buckeye } \\
\text { Ernte } \\
\text { (picker/h } \\
\text { arvester) } \\
\text { McSherr } \\
\text { y Drill }\end{array}$ & $\begin{array}{l}\text { X- } \\
\text { Spezer } \\
\text { eien } \\
\text { (dry/m } \\
\text { ixed } \\
\text { foods) }\end{array}$ & \\
\hline $\begin{array}{l}\text { Frei } \\
\text { e } \\
\text { Pres } \\
\text { se }\end{array}$ & $\begin{array}{r}7 / 22 \\
/ 187 \\
6\end{array}$ & $\begin{array}{l}\text { Mrs. L. } \\
\text { Kempf }\end{array}$ & $\begin{array}{l}\text { X- } \\
\text { musikalis } \\
\text { chen } \\
\text { Instrume } \\
\text { nten } \\
\text { (musical } \\
\text { instrume } \\
\text { nts), } \\
\text { Spielzeu } \\
\text { g (toy), } \\
\text { Toiletten } \\
\text { seife } \\
\text { (toilet } \\
\text { soap), } \\
\text { Taschen } \\
\text { uhren } \\
\text { (pocket } \\
\text { watch), }\end{array}$ & Notions & & \\
\hline
\end{tabular}




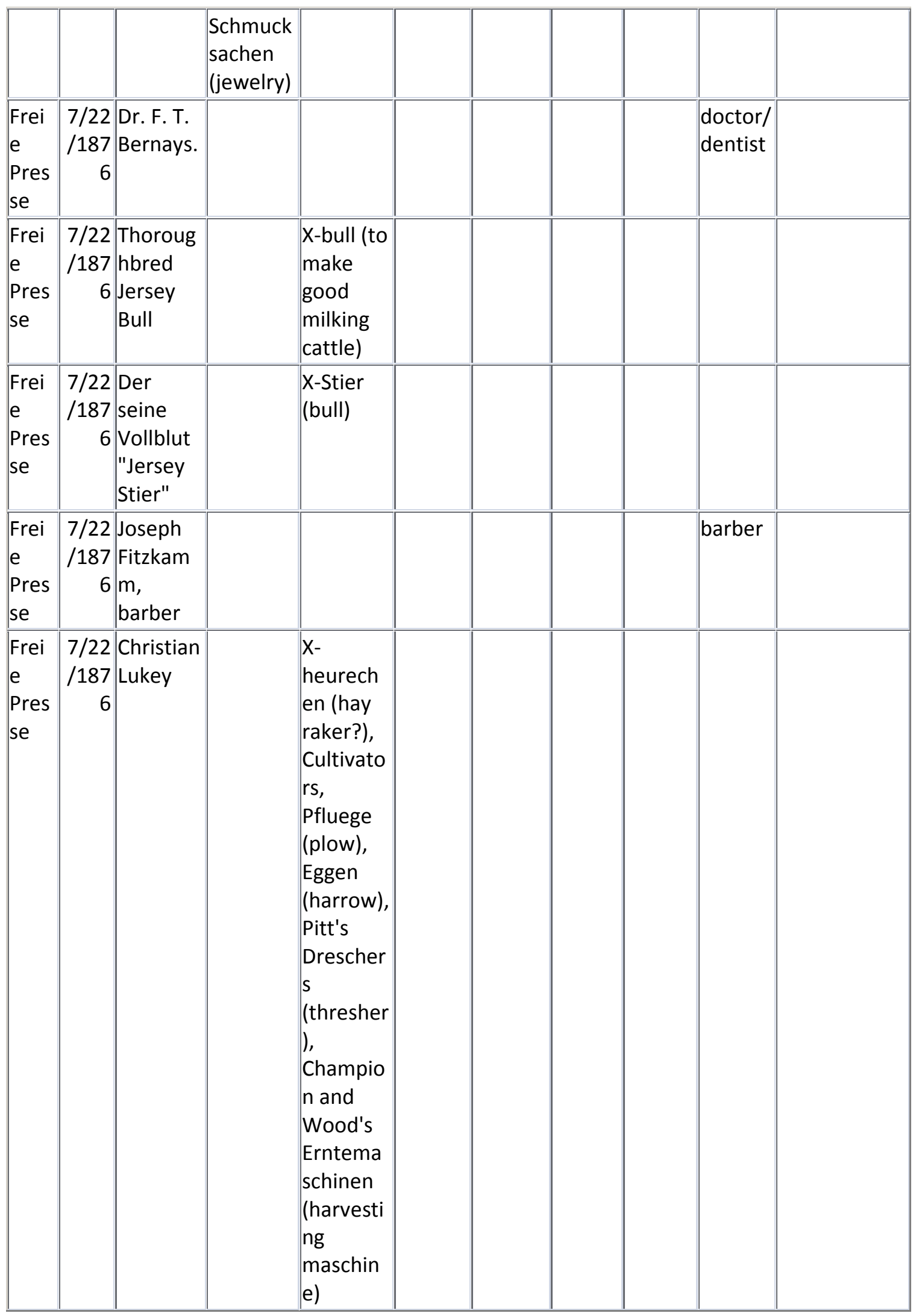




\begin{tabular}{|c|c|c|c|c|c|c|c|c|}
\hline \begin{tabular}{|l} 
Frei \\
e \\
Pres \\
se
\end{tabular} & \begin{tabular}{|r|}
$7 / 22$ \\
$/ 187$ \\
6 \\
\end{tabular} & $\begin{array}{l}\text { Souther } \\
\text { n Hotel }\end{array}$ & & & & & hotel & \\
\hline $\begin{array}{l}\text { Frei } \\
\text { e } \\
\text { Pres } \\
\text { se }\end{array}$ & $\begin{array}{r}7 / 22 \\
/ 187 \\
6\end{array}$ & $\begin{array}{l}\text { Andrew } \\
\text { Anderse } \\
n\end{array}$ & $\begin{array}{l}\text { X- } \\
\text { handwer } \\
\text { kerwerkz } \\
\text { eug } \\
\text { (hand } \\
\text { tools) }\end{array}$ & $\begin{array}{l}\text { X- } \\
\text { Wagen } \\
\text { macher } \\
\text { und } \\
\text { Schmie } \\
\text { derbed } \\
\text { arf (car } \\
\text { maker } \\
\text { and } \\
\text { blacks } \\
\text { mith } \\
\text { needs) }\end{array}$ & & $\begin{array}{l}\text { X- } \\
\text { Spezer } \\
\text { eiwaar } \\
\text { en }\end{array}$ & & $\begin{array}{l}\text { Galanteriewa } \\
\text { aren (fancies) }\end{array}$ \\
\hline \begin{tabular}{|l|} 
Frei \\
e \\
Pres \\
se
\end{tabular} & \begin{tabular}{|r|}
$7 / 22$ \\
$/ 187$ \\
6
\end{tabular} & Rupture, & & & & & $\begin{array}{l}\text { Medica } \\
\text { I- } \\
\text { hernia }\end{array}$ & \\
\hline $\begin{array}{l}\text { Frei } \\
\text { e } \\
\text { Pres } \\
\text { se }\end{array}$ & $\begin{array}{r}7 / 22 \\
/ 187 \\
6\end{array}$ & $\begin{array}{l}\text { Dr. } \\
\text { Whittier }\end{array}$ & & & $\begin{array}{l}\text { X- } \\
\text { medical } \\
\text { pamphl } \\
\text { ets } \\
\text { "Manh } \\
\text { ood" } \\
\text { and } \\
\text { "Woma } \\
\text { nhood" }\end{array}$ & & $\begin{array}{l}\text { Medica } \\
1\end{array}$ & \\
\hline \begin{tabular}{|l} 
Frei \\
e \\
Pres \\
se
\end{tabular} & \begin{tabular}{|r|}
$7 / 22$ \\
$/ 187$ \\
6 \\
\\
\\
\\
\end{tabular} & $\begin{array}{l}\text { Washing } \\
\text { ton } \\
\text { Marble } \\
\text { Works- } \\
\text { James } \\
\text { Douglas } \\
\text { and } \\
\text { George } \\
\text { Douglas }\end{array}$ & & & & & & $\begin{array}{l}\text { Italian and } \\
\text { American } \\
\text { Marble } \\
\text { Monuments, } \\
\text { Gravestones, } \\
\text { scotch } \\
\text { granite } \\
\text { monuments, } \\
\text { lime, cement, } \\
\text { fire brick, } \\
\text { plaster paris, } \\
\text { plastering } \\
\text { hair }\end{array}$ \\
\hline \begin{tabular}{|l|} 
Frei \\
e \\
Pres
\end{tabular} & \begin{tabular}{|r|}
$7 / 22$ \\
$/ 187$ \\
6
\end{tabular} & U.S. Mail & & & & & $\begin{array}{l}\text { mail } \\
\text { service }\end{array}$ & \\
\hline
\end{tabular}




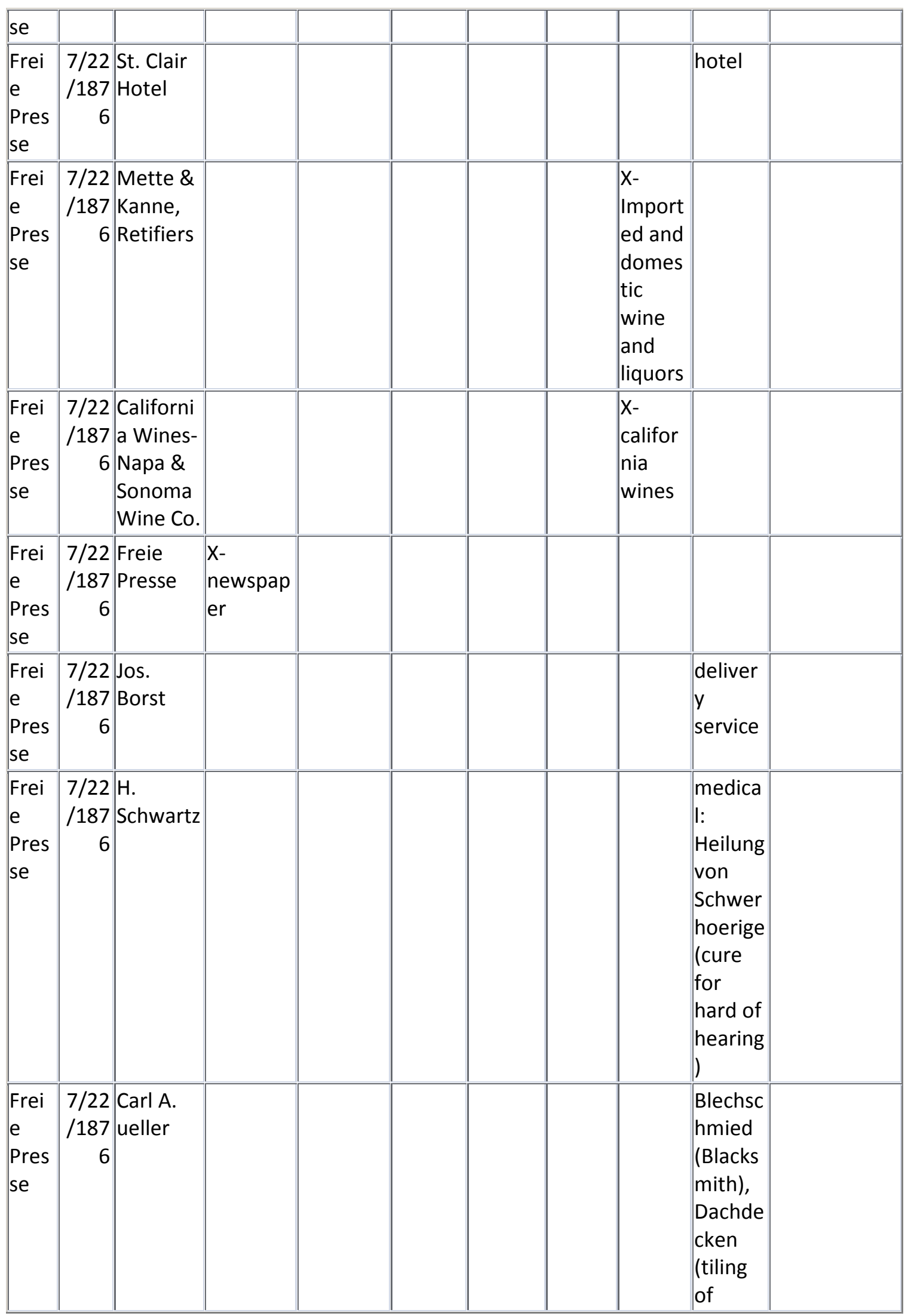




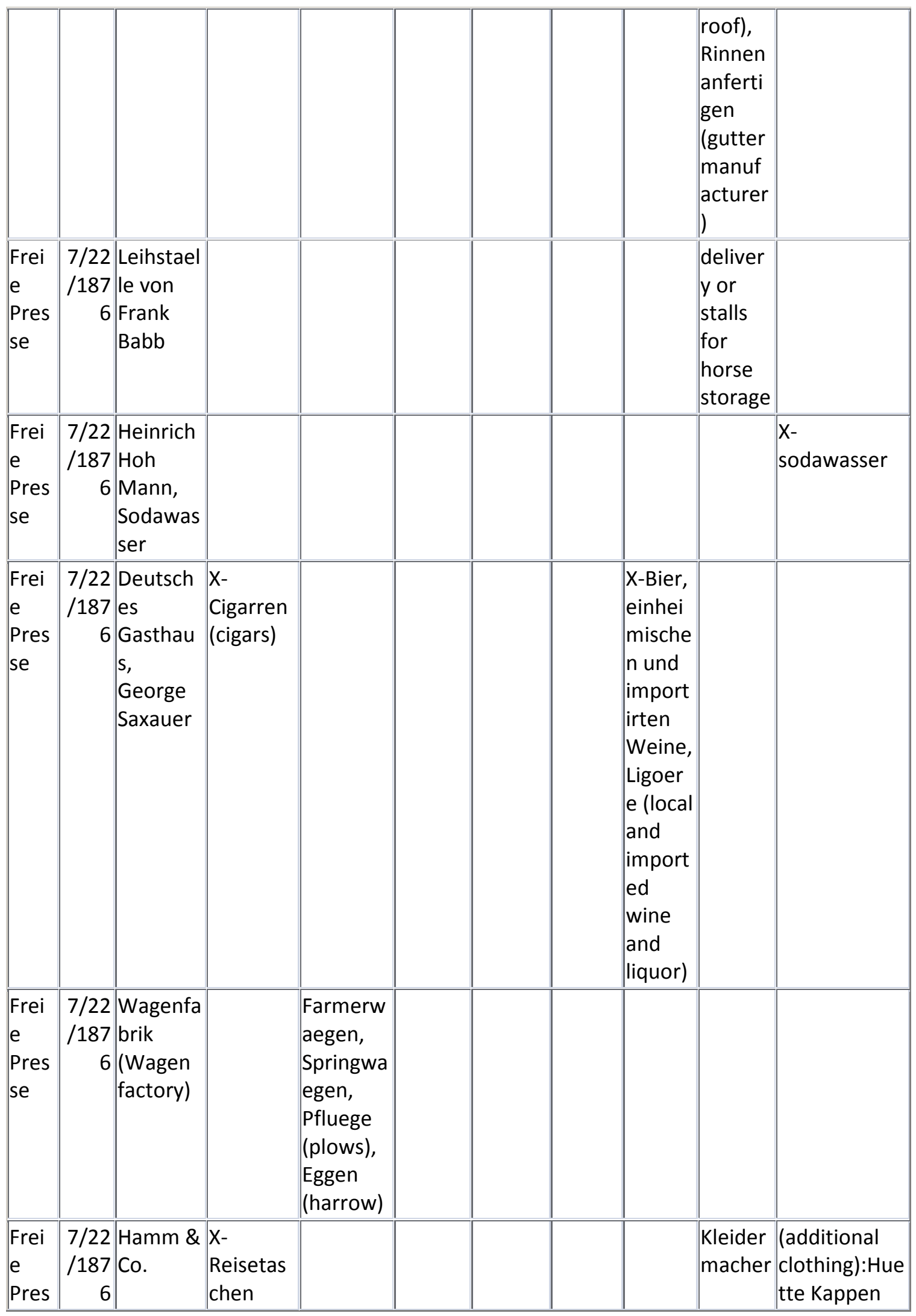




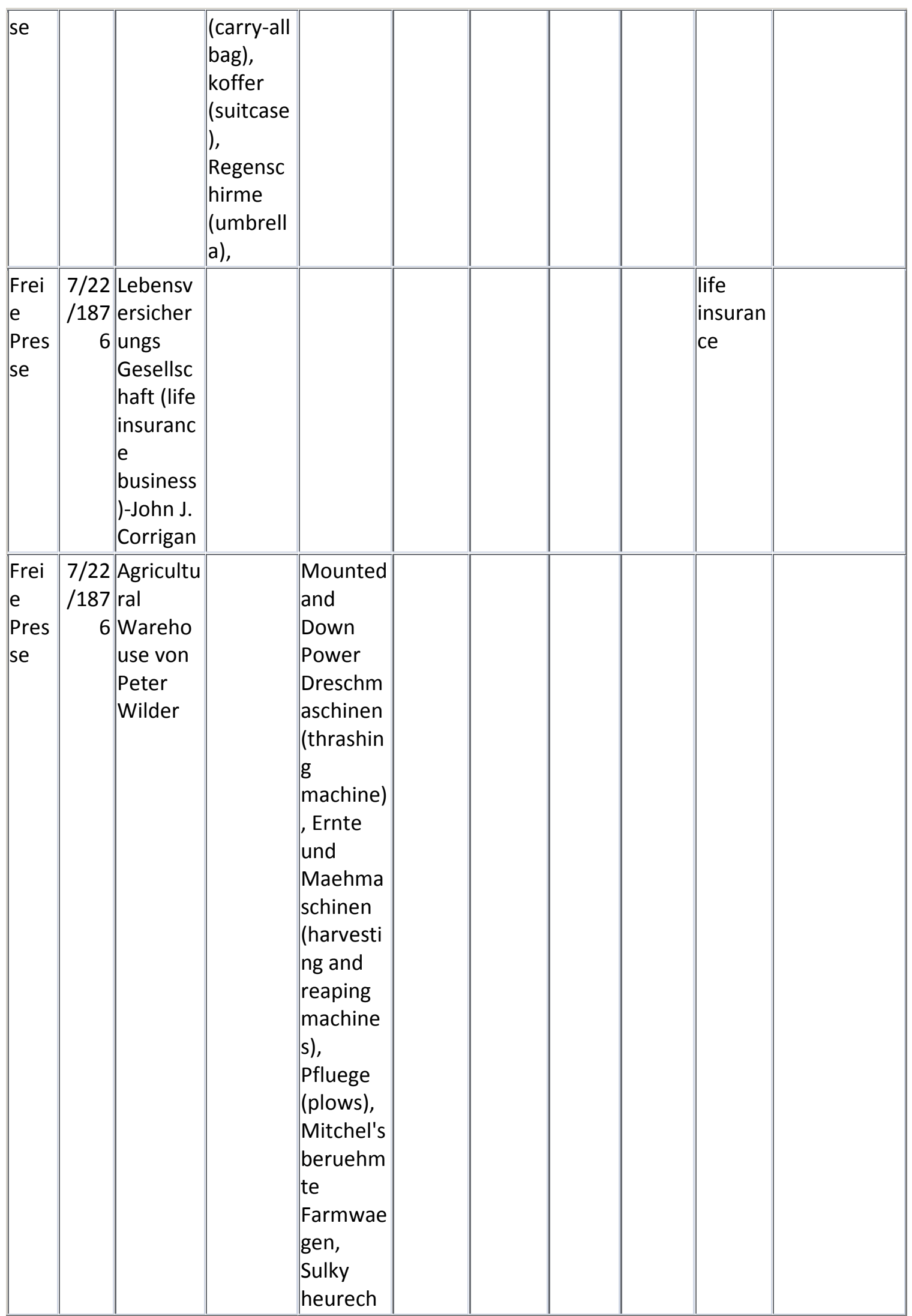




\begin{tabular}{|c|c|c|c|c|c|c|c|c|}
\hline & & & & $\begin{array}{l}\text { en (one- } \\
\text { manned } \\
\text { hay } \\
\text { rake), } \\
\text { Saehmas } \\
\text { chinen } \\
\text { (Drills) } \\
\text { (seeding } \\
\text { drill) }\end{array}$ & & & & \\
\hline $\begin{array}{l}\text { Frei } \\
\text { e } \\
\text { Pres } \\
\text { se }\end{array}$ & $\begin{array}{r}7 / 22 \\
/ 187 \\
6 \\
\end{array}$ & $\begin{array}{l}\text { Johnson } \\
\text { \& Shaw, } \\
\text { Rechts, } \\
\text { Unwaelt } \\
\text { e }\end{array}$ & & & & & Legal & \\
\hline \begin{tabular}{|l} 
Frei \\
e \\
Pres \\
se
\end{tabular} & \begin{tabular}{|r|}
$7 / 22$ \\
$/ 187$ \\
6 \\
\end{tabular} & $\begin{array}{l}\text { F. A. } \\
\text { Rozier }\end{array}$ & & & & & Legal & \\
\hline \begin{tabular}{|l} 
Frei \\
e \\
Pres \\
se
\end{tabular} & \begin{tabular}{|r|}
$7 / 22$ \\
$/ 187$ \\
6 \\
\end{tabular} & $\begin{array}{l}\text { Charles } \\
\text { Obermu } \\
\text { eller, } \\
\text { Cigarren } \\
\text { u. Tabak. }\end{array}$ & $\begin{array}{l}\text { Cigarren } \\
\text { u. Tabak } \\
\text { (Cigars } \\
\text { and } \\
\text { Tobacco) } \\
\text {, Pfeifen } \\
\text { (pipes) }\end{array}$ & & & & & \\
\hline \begin{tabular}{|l} 
Frei \\
e \\
Pres \\
se
\end{tabular} & \begin{tabular}{|r|}
$7 / 22$ \\
$/ 187$ \\
6 \\
\\
\end{tabular} & \begin{tabular}{|l|} 
Deutsch \\
e Wein \\
und \\
Bierwirt \\
hschaft- \\
Geo \\
Saxauer
\end{tabular} & & & & $\begin{array}{l}\text { X-Wein } \\
\text { und } \\
\text { Bier }\end{array}$ & \begin{tabular}{|l} 
Wein \\
und \\
Bierwir \\
tschaft \\
und \\
Billiard \\
halle \\
(pub \\
and \\
billiard \\
hall)
\end{tabular} & \\
\hline \begin{tabular}{|l} 
Frei \\
e \\
Pres \\
se
\end{tabular} & \begin{tabular}{|r|}
$7 / 22$ \\
$/ 187$ \\
6
\end{tabular} & $\begin{array}{l}\text { Terpsich } \\
\text { erian } \\
\text { Hall }\end{array}$ & & & & $\begin{array}{l}\text { Wein } \\
\text { und } \\
\text { Bier }\end{array}$ & $\begin{array}{l}\text { Wein } \\
\text { und } \\
\text { Bierwir } \\
\text { tschaft, } \\
\text { tanzhal } \\
\text { le }\end{array}$ & \\
\hline \begin{tabular}{|l|} 
Frei \\
e \\
Pres
\end{tabular} & \begin{tabular}{|r|}
$7 / 22$ \\
$/ 187$ \\
6
\end{tabular} & $\begin{array}{l}\text { Neuer } \\
\text { Spezereil } \\
\text { aden }\end{array}$ & & & $\begin{array}{l}\text { X- } \\
\text { Spezer } \\
\text { ei }\end{array}$ & & & \\
\hline
\end{tabular}




\begin{tabular}{|c|c|c|c|c|c|c|c|}
\hline se & & $\begin{array}{l}\text { von } \\
\text { Jacob } \\
\text { Falk }\end{array}$ & & $\begin{array}{l}\text { (spices } \\
\text { ), } \\
\text { Kaffee, } \\
\text { Zucker } \\
\text {, Mehl } \\
\text { (flour), } \\
\text { Fett, } \\
\text { Butter, } \\
\text { Eier, }\end{array}$ & & & \\
\hline $\begin{array}{l}\text { Frei } \\
\text { e } \\
\text { Pres } \\
\text { se }\end{array}$ & $\begin{array}{r}7 / 22 \\
/ 187 \\
6 \\
\end{array}$ & $\begin{array}{l}\text { F.C. } \\
\text { Albert- } \\
\text { Zimmer } \\
\text { mann } \\
\text { und } \\
\text { Tischler }\end{array}$ & & & & $\begin{array}{l}\text { Tischle } \\
r \\
\text { (carpen } \\
\text { ter) }\end{array}$ & \\
\hline $\begin{array}{l}\text { Frei } \\
\text { e } \\
\text { Pres } \\
\text { se }\end{array}$ & $\begin{array}{r}7 / 22 \\
/ 187 \\
6\end{array}$ & $\begin{array}{l}\text { Dr. r. T. } \\
\text { Bernaus. }\end{array}$ & & & & $\begin{array}{l}\text { Arzt } \\
\text { (doctor } \\
\text { )- } \\
\text { Augen } \\
\text { und } \\
\text { Ohren } \\
\text { (eyes } \\
\text { and } \\
\text { ears) }\end{array}$ & \\
\hline \begin{tabular}{|l} 
Frei \\
e \\
Pres \\
se
\end{tabular} & \begin{tabular}{|r|}
$7 / 22$ \\
$/ 187$ \\
6 \\
\end{tabular} & $\begin{array}{l}\text { Kennard' } \\
\text { s Saloon }\end{array}$ & & & $\begin{array}{l}\text { X-Bier, } \\
\text { Wein }\end{array}$ & Saloon & Soda \\
\hline \begin{tabular}{|l} 
Frei \\
e \\
Pres \\
se
\end{tabular} & \begin{tabular}{|r|}
$7 / 22$ \\
$/ 187$ \\
6 \\
\end{tabular} & $\begin{array}{l}\text { Philipp } \\
\text { Bieber }\end{array}$ & & & & & \\
\hline \begin{tabular}{|l} 
Frei \\
e \\
Pres \\
se
\end{tabular} & \begin{tabular}{|r|}
$7 / 22$ \\
$/ 187$ \\
6 \\
\end{tabular} & $\begin{array}{l}\text { Saerge- } \\
\text { Sebastia } \\
\text { n Geiler }\end{array}$ & & & & & $\begin{array}{l}\text { Saerge } \\
\text { (coffins) }\end{array}$ \\
\hline \begin{tabular}{|l|} 
Frei \\
e \\
Pres \\
se
\end{tabular} & \begin{tabular}{|r|}
$7 / 22$ \\
$/ 187$ \\
6 \\
\\
\end{tabular} & $\begin{array}{l}\text { Wilson's } \\
\text { Naehma } \\
\text { schinen- } \\
\text { Herr } \\
\text { August } \\
\text { Baechle }\end{array}$ & $\begin{array}{l}\text { X- } \\
\text { Naehmas } \\
\text { chinen } \\
\text { (sewing } \\
\text { maschin } \\
\text { es) }\end{array}$ & & & & \\
\hline \begin{tabular}{|l|} 
Frei \\
e \\
Pres \\
\end{tabular} & \begin{tabular}{|r|}
$7 / 22$ \\
$/ 187$ \\
6 \\
\end{tabular} & $\begin{array}{l}\text { Joseph } \\
\text { Fitzkam } \\
\text { m- }\end{array}$ & & & & Barbier & \\
\hline
\end{tabular}




\begin{tabular}{|c|c|c|c|c|c|c|}
\hline se & & $\begin{array}{l}\text { Deutsch } \\
\text { er } \\
\text { Barbier }\end{array}$ & & & & \\
\hline $\begin{array}{l}\text { Frei } \\
\text { e } \\
\text { Pres } \\
\text { se }\end{array}$ & $\begin{array}{r}7 / 22 \\
/ 187 \\
6 \\
\end{array}$ & $\begin{array}{l}\text { Dr. F. } \\
\text { Guibour } \\
\text { d, } \\
\text { Apothek } \\
\text { er }\end{array}$ & & & $x$ & $\begin{array}{l}\text { Apothe } \\
\text { ker }\end{array}$ \\
\hline $\begin{array}{l}\text { Frei } \\
\text { e } \\
\text { Pres } \\
\text { se }\end{array}$ & $\begin{array}{r}7 / 22 \\
/ 187 \\
6\end{array}$ & $\begin{array}{l}\text { Harris } \\
\text { und } \\
\text { Janis } \\
\text { Bankier }\end{array}$ & & & & \begin{tabular}{|l|} 
Feuer \\
und \\
Lebens \\
Versich \\
erungs \\
(fire \\
and life \\
insuran \\
ce)
\end{tabular} \\
\hline \begin{tabular}{|l} 
Frei \\
e \\
Pres \\
se
\end{tabular} & \begin{tabular}{|r|}
$7 / 22$ \\
$/ 187$ \\
6
\end{tabular} & $\begin{array}{l}\text { P.U. } \\
\text { Jaccard }\end{array}$ & $\begin{array}{l}\text { X- } \\
\text { Taschen } \\
\text { uhr } \\
\text { (pocket } \\
\text { watch) }\end{array}$ & & & \begin{tabular}{|l|} 
Uhren \\
macher \\
, Gold \\
u. \\
Silbersc \\
hmied \\
(clock \\
macker \\
, gold \\
and \\
silver \\
smith)
\end{tabular} \\
\hline $\begin{array}{l}\text { Frei } \\
\text { e } \\
\text { Pres } \\
\text { se }\end{array}$ & $\begin{array}{r}7 / 22 \\
/ 187 \\
6 \\
\end{array}$ & $\begin{array}{l}\text { Taube } \\
\text { und } \\
\text { Schwerh } \\
\text { oerige- } \\
\text { H. } \\
\text { Schwartz }\end{array}$ & & & & medica \\
\hline \begin{tabular}{|l} 
Frei \\
e \\
Pres \\
se
\end{tabular} & \begin{tabular}{|r|}
$7 / 22$ \\
$/ 187$ \\
6 \\
\end{tabular} & $\begin{array}{l}\text { Roesch } \\
\text { \& Efferin }\end{array}$ & & $\begin{array}{l}\text { X- } \\
\text { Springwa } \\
\text { egen, } \\
\text { Farmerw } \\
\text { aegen, } \\
\text { Pfluege } \\
\text { (plows), } \\
\text { Eggen }\end{array}$ & & \\
\hline $\begin{array}{l}\text { Frei } \\
\mathrm{e}\end{array}$ & \begin{tabular}{|l|}
$7 / 22$ \\
$/ 187$
\end{tabular} & $\begin{array}{l}\text { Spezereil } \\
\text { aden- }\end{array}$ & & $\begin{array}{l}\text { X-home } \\
\text { Nuehma }\end{array}$ & & \\
\hline
\end{tabular}




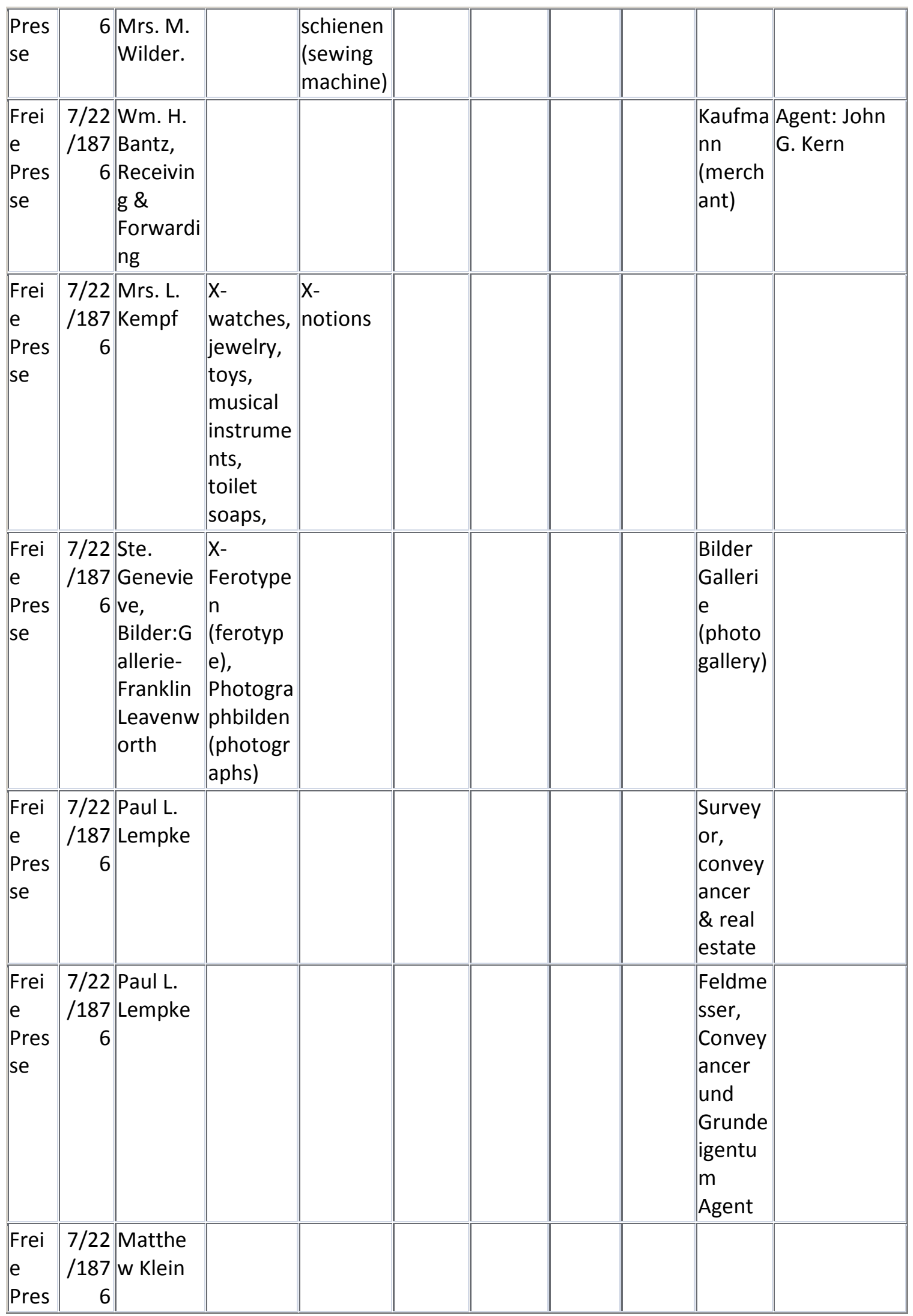




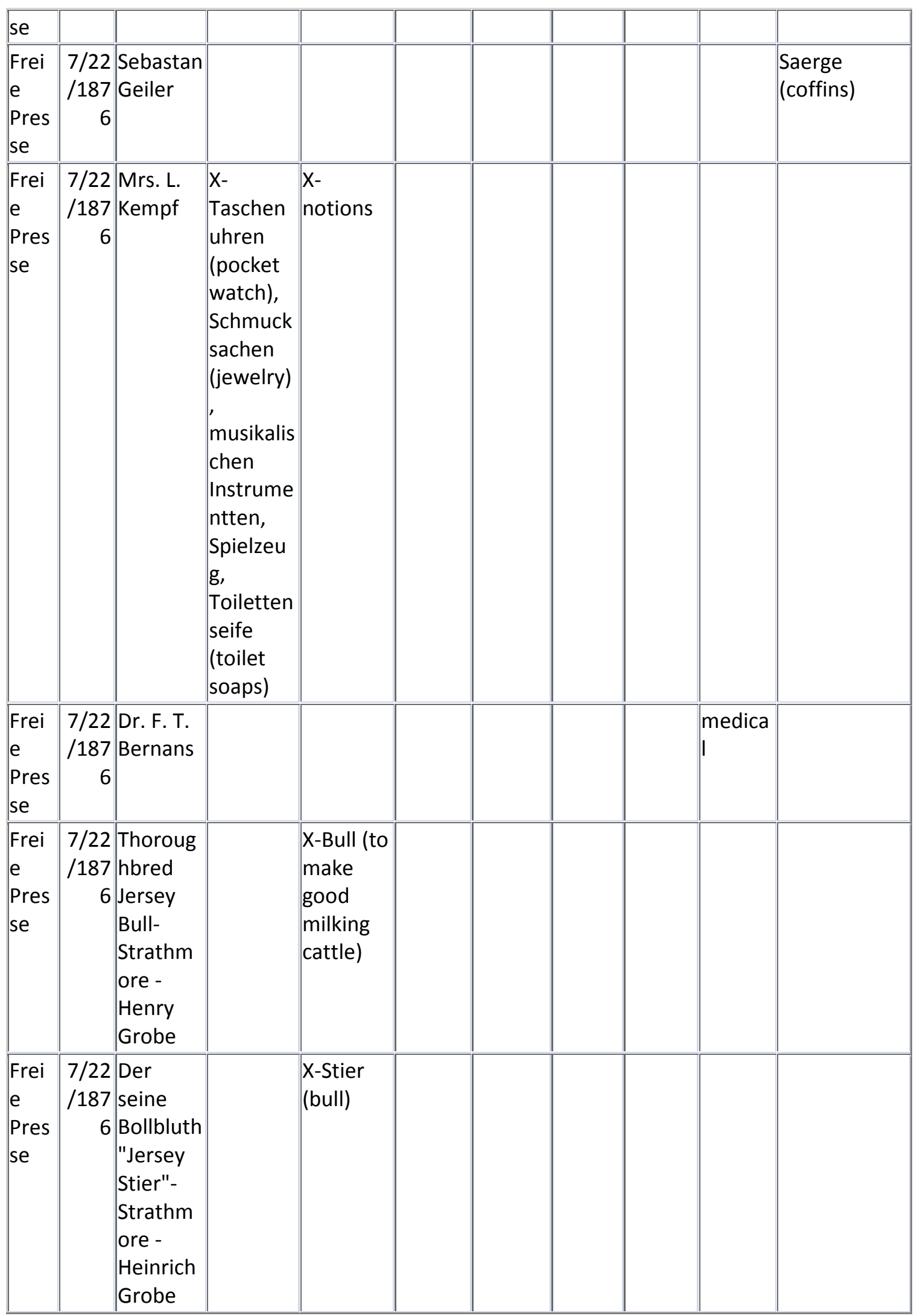




\begin{tabular}{|c|c|c|c|c|c|c|c|c|}
\hline \begin{tabular}{|l} 
Frei \\
e \\
Pres \\
se
\end{tabular} & \begin{tabular}{|r|}
$7 / 22$ \\
$/ 187$ \\
6 \\
\end{tabular} & $\begin{array}{l}\text { Joseph } \\
\text { Fitzkam } \\
\text { m, } \\
\text { Barber }\end{array}$ & & & & & barber & \\
\hline \begin{tabular}{|l} 
Frei \\
e \\
Pres \\
se
\end{tabular} & \begin{tabular}{|r|}
$7 / 22$ \\
$/ 187$ \\
6 \\
\end{tabular} & $\begin{array}{l}\text { Christian } \\
\text { Lukey }\end{array}$ & & $\begin{array}{l}\text { X- } \\
\text { heurech } \\
\text { en (hay } \\
\text { raker?), } \\
\text { Cultivato } \\
\text { rs, } \\
\text { Pfluege } \\
\text { (plow), } \\
\text { Eggen } \\
\text { (harrow), } \\
\text { Pitt's } \\
\text { Drescher } \\
\text { s } \\
\text { (thresher } \\
\text { ), } \\
\text { Champio } \\
\text { n and } \\
\text { Wood's } \\
\text { Erntema } \\
\text { schinen } \\
\text { (harvesti } \\
\text { ng } \\
\text { maschin } \\
\text { e) }\end{array}$ & & & & \\
\hline \begin{tabular}{|l} 
Frei \\
e \\
Pres \\
se
\end{tabular} & \begin{tabular}{|r|}
$7 / 22$ \\
$/ 187$ \\
6
\end{tabular} & $\begin{array}{l}\text { Andrew } \\
\text { Anderse } \\
n\end{array}$ & & $\begin{array}{l}\text { X- } \\
\text { handwer } \\
\text { kerwerkz } \\
\text { eug } \\
\text { (hand } \\
\text { tools) }\end{array}$ & \begin{tabular}{|l|} 
X- \\
Wagen \\
macher \\
und \\
Schmie \\
derbed \\
arf (car \\
maker \\
and \\
blacks \\
mith \\
needs)
\end{tabular} & $\begin{array}{l}\text { X- } \\
\text { Spezer } \\
\text { eiwaar } \\
\text { en }\end{array}$ & & $\begin{array}{l}\text { Galanteriewa } \\
\text { aren (fancies) }\end{array}$ \\
\hline \begin{tabular}{|l} 
Frei \\
e \\
Pres \\
se
\end{tabular} & \begin{tabular}{|r|}
$7 / 22$ \\
$/ 187$ \\
6 \\
\end{tabular} & $\begin{array}{l}\text { Souther } \\
\text { n hotel - } \\
\text { Joseph } \\
\text { Vorst }\end{array}$ & & & & & hotel & \\
\hline $\begin{array}{l}\text { Frei } \\
\text { e }\end{array}$ & \begin{tabular}{|l|}
$7 / 22$ \\
$/ 187$
\end{tabular} & $\begin{array}{l}\text { Ste. } \\
\text { Genevie }\end{array}$ & $\begin{array}{l}\text { X- } \\
\text { Ferotype }\end{array}$ & & & & \begin{tabular}{|l|} 
Photog \\
raph
\end{tabular} & \\
\hline
\end{tabular}




\begin{tabular}{|c|c|c|c|c|c|c|c|}
\hline \begin{tabular}{|l} 
Pres \\
se
\end{tabular} & & \begin{tabular}{|l|} 
ve \\
Photogr \\
aph \\
gallery- \\
Franklin \\
Leavenw \\
orth
\end{tabular} & $\begin{array}{l}\text { s \& } \\
\text { Photogra } \\
\text { phs }\end{array}$ & & & Gallery & \\
\hline \begin{tabular}{|l} 
Frei \\
e \\
Pres \\
se
\end{tabular} & \begin{tabular}{|r|}
$7 / 22$ \\
$/ 187$ \\
6 \\
\\
\end{tabular} & $\begin{array}{l}\text { Edmund } \\
\text { Price's } \\
\text { Speiseha } \\
\text { us } \\
\text { (eating } \\
\text { house) }\end{array}$ & & & & $\begin{array}{l}\text { Seiseha } \\
\text { us } \\
\text { (eating } \\
\text { house) }\end{array}$ & \\
\hline \begin{tabular}{|l} 
Frei \\
e \\
Pres \\
se
\end{tabular} & \begin{tabular}{|r|}
$7 / 22$ \\
$/ 187$ \\
6
\end{tabular} & $\begin{array}{l}\text { John L. } \\
\text { Boverie }\end{array}$ & $\begin{array}{l}\text { X- } \\
\text { Schulbue } \\
\text { cher } \\
\text { (school } \\
\text { books) }\end{array}$ & & $\begin{array}{l}\text { X-Salz } \\
\text { (salt), } \\
\text { Zpezer } \\
\text { eien, }\end{array}$ & & \\
\hline \begin{tabular}{|l} 
Frei \\
e \\
Pres \\
se
\end{tabular} & \begin{tabular}{|r|}
$7 / 22$ \\
$/ 187$ \\
6 \\
\end{tabular} & $\begin{array}{l}\text { Wagenfa } \\
\text { brik } \\
\text { (Wagen } \\
\text { factory) }\end{array}$ & & \begin{tabular}{|l|} 
X- \\
Farmerw \\
aegen, \\
Springwa \\
egen, \\
Pfluege \\
(plows), \\
Eggen \\
(harrow)
\end{tabular} & & & \\
\hline \begin{tabular}{|l} 
Frei \\
e \\
Pres \\
se
\end{tabular} & \begin{tabular}{|r|}
$7 / 22$ \\
$/ 187$ \\
6
\end{tabular} & $\begin{array}{l}\text { Hamm \& } \\
\text { Co. }\end{array}$ & $\begin{array}{l}\text { X- } \\
\text { Reisetas } \\
\text { chen } \\
\text { (carry-all } \\
\text { bag), } \\
\text { koffer } \\
\text { (suitcase } \\
\text { ), } \\
\text { Regensc } \\
\text { hirme } \\
\text { (umbrell } \\
\text { a) }\end{array}$ & & & $\begin{array}{l}\text { Kleider } \\
\text { macher }\end{array}$ & $\begin{array}{l}\text { (additional } \\
\text { clothing): } \\
\text { Huette } \\
\text { Kappen }\end{array}$ \\
\hline \begin{tabular}{|l} 
Frei \\
e \\
Pres \\
se
\end{tabular} & \begin{tabular}{|r|}
$7 / 22$ \\
$/ 187$ \\
6 \\
\end{tabular} & $\begin{array}{l}\text { Harris } \\
\text { and } \\
\text { Janis } \\
\text { Bankier }\end{array}$ & & & & Bankier & \\
\hline $\begin{array}{l}\text { Frei } \\
\mathrm{e}\end{array}$ & \begin{tabular}{|l|}
$7 / 22$ \\
$/ 187$
\end{tabular} & $\begin{array}{l}\text { Agricultu } \\
\text { ral }\end{array}$ & & $\begin{array}{l}\text { X- } \\
\text { Mounted }\end{array}$ & & & \\
\hline
\end{tabular}




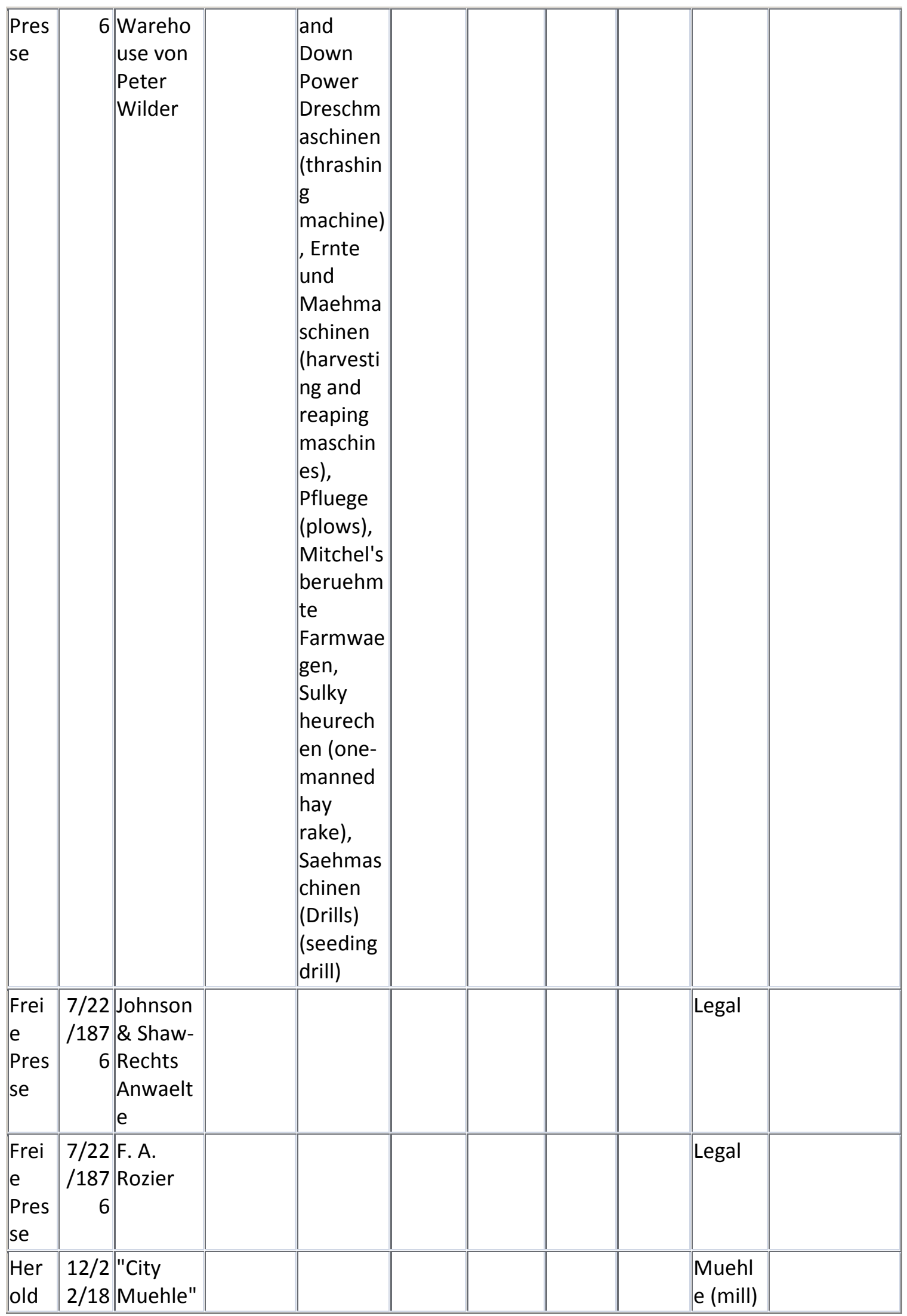




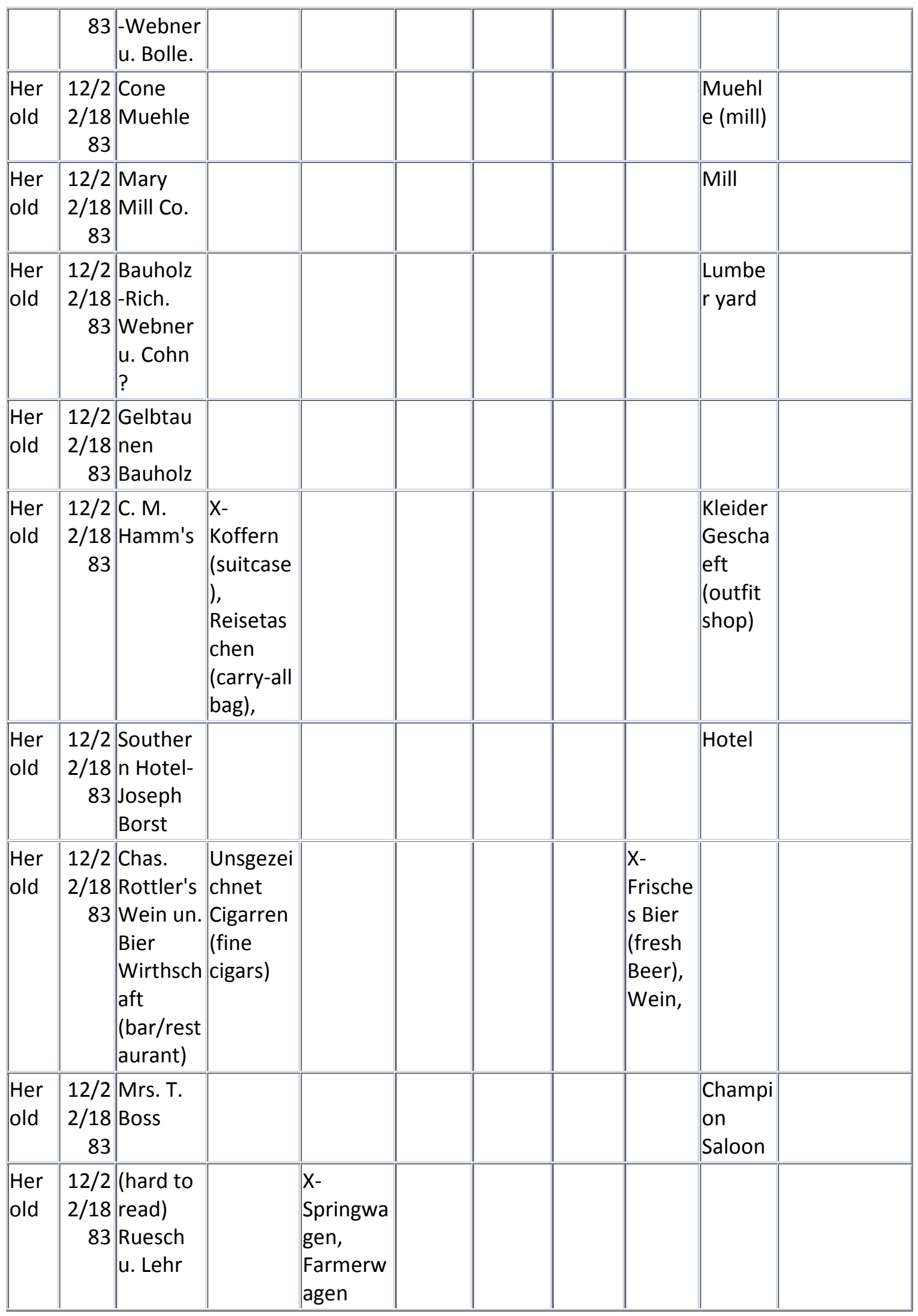




\begin{tabular}{|c|c|c|c|c|c|c|}
\hline $\begin{array}{l}\text { Her } \\
\text { old }\end{array}$ & \begin{tabular}{|r|}
$12 / 2$ \\
$2 / 18$ \\
83 \\
\end{tabular} & \begin{tabular}{|l} 
??? Und \\
Goss \\
(half \\
legible)
\end{tabular} & $\begin{array}{l}\text { X- } \\
\text { Pfluege } \\
\text { (plow), } \\
\text { ?? } \\
\text { Geraeths } \\
\text { chaften } \\
\text { (equipm } \\
\text { ent) }\end{array}$ & & & \\
\hline $\begin{array}{l}\text { Her } \\
\text { old }\end{array}$ & $\begin{array}{r}12 / 2 \\
2 / 18 \\
83 \\
\end{array}$ & $\begin{array}{l}\text { Harris \& } \\
\text { Janis } \\
\text { Banquier } \\
\text { S }\end{array}$ & & & & $\begin{array}{l}\text { Banqui } \\
\text { ers } \\
\text { (Banke } \\
\text { rs) } \\
\end{array}$ \\
\hline $\begin{array}{l}\text { Her } \\
\text { old }\end{array}$ & $\begin{array}{r}12 / 2 \\
2 / 18 \\
83 \\
\end{array}$ & $\begin{array}{l}\text { L. B. } \\
\text { Whitled } \\
\text { ge } \\
\text { Rechtsa } \\
\text { nwalt } \\
\text { (attorne } \\
\text { y) }\end{array}$ & & & & Legal \\
\hline $\begin{array}{l}\text { Her } \\
\text { old }\end{array}$ & \begin{tabular}{|r|}
$12 / 2$ \\
$2 / 18$ \\
83 \\
\end{tabular} & $\begin{array}{l}\text { Chas. C. } \\
\text { Rozier }\end{array}$ & & & & $\begin{array}{l}\text { Advoka } \\
\mathrm{t} \\
\text { (lawyer } \\
\text { ) }\end{array}$ \\
\hline $\begin{array}{l}\text { Her } \\
\text { old }\end{array}$ & \begin{tabular}{|r|}
$12 / 2$ \\
$2 / 18$ \\
83 \\
\end{tabular} & $\begin{array}{l}\text { Johnson } \\
\text { n } \\
\text { Hertich }\end{array}$ & & & & \begin{tabular}{|l} 
Rechts \\
anwael \\
$\mathrm{t}$ \\
(attorn \\
ey)
\end{tabular} \\
\hline $\begin{array}{l}\text { Her } \\
\text { old }\end{array}$ & \begin{tabular}{|r|}
$12 / 2$ \\
$2 / 18$ \\
83 \\
\end{tabular} & $\begin{array}{l}\text { Schul } \\
\text { Commiss } \\
\text { ioner } \\
\text { (school } \\
\text { commisi } \\
\text { oner) }\end{array}$ & & & & \\
\hline $\begin{array}{l}\text { Her } \\
\text { old }\end{array}$ & \begin{tabular}{|r|}
$12 / 2$ \\
$2 / 18$ \\
83 \\
\\
\\
\\
\end{tabular} & $\begin{array}{l}\text { Wilhelm } \\
\text { Palmer- } \\
\text { Grocerie } \\
\text { s aller } \\
\text { Art } \\
\text { (grocerie } \\
\text { s of all } \\
\text { forms) }\end{array}$ & & $\begin{array}{l}\text { X- } \\
\text { Farmp } \\
\text { roduct }\end{array}$ & & \\
\hline $\begin{array}{l}\text { Her } \\
\text { old }\end{array}$ & \begin{tabular}{|r|}
$12 / 2$ \\
$2 / 18$ \\
83 \\
\end{tabular} & $\begin{array}{l}\text { Brauerei } \\
\text { B. } \\
\text { Rottler }\end{array}$ & & & X-Beer & $\begin{array}{l}\text { Brauer } \\
\text { ei } \\
\text { (brewe }\end{array}$ \\
\hline
\end{tabular}




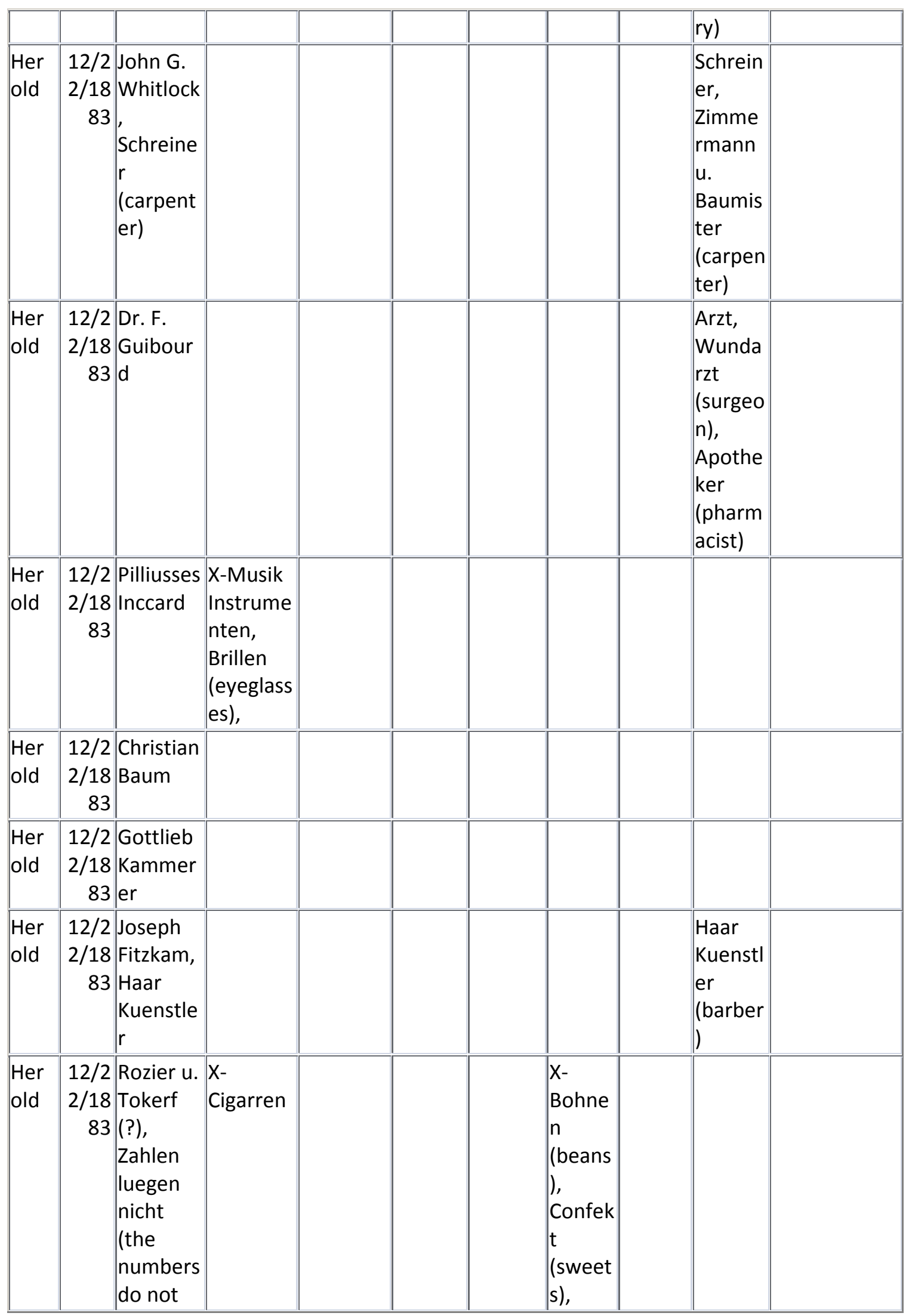




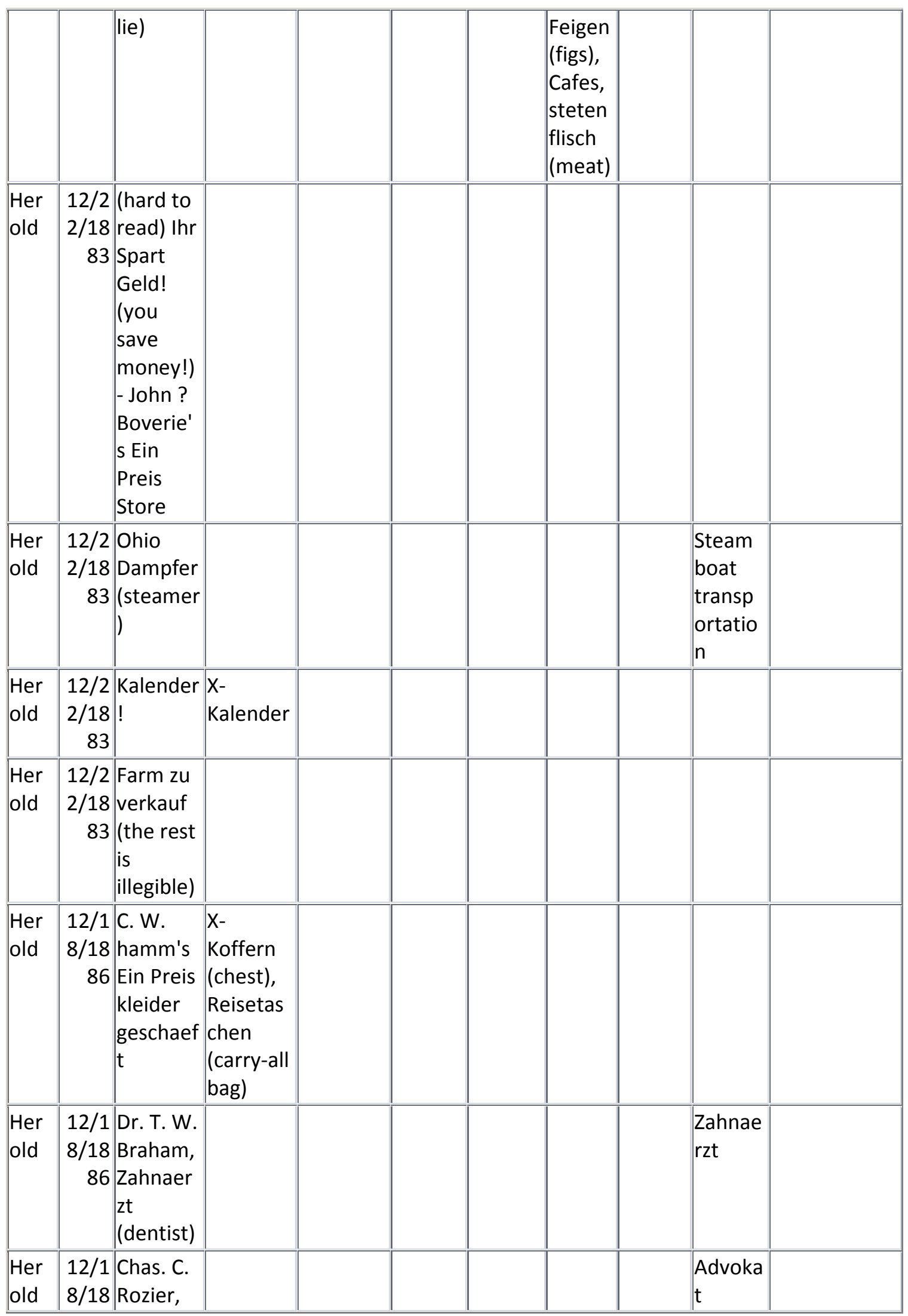




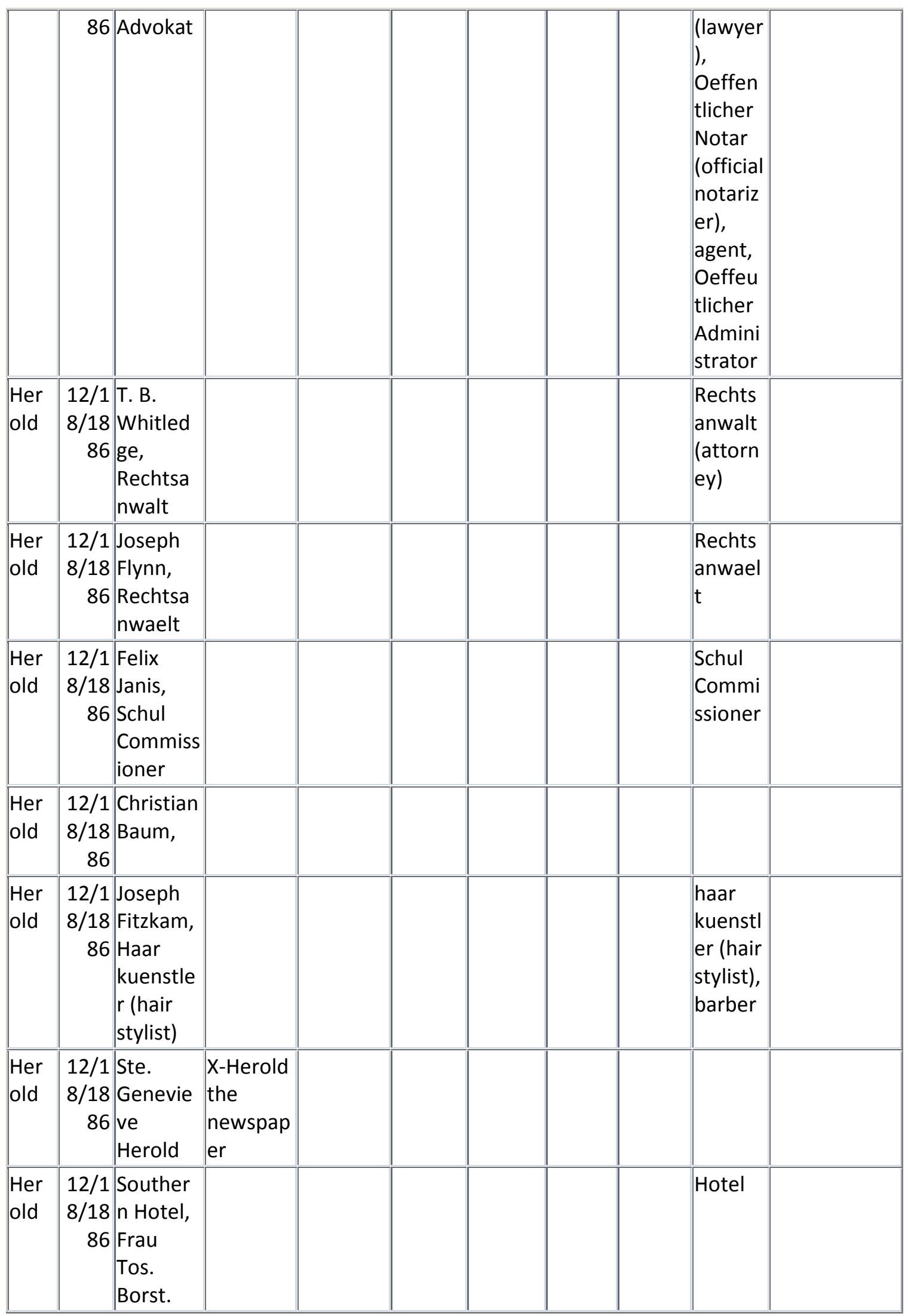




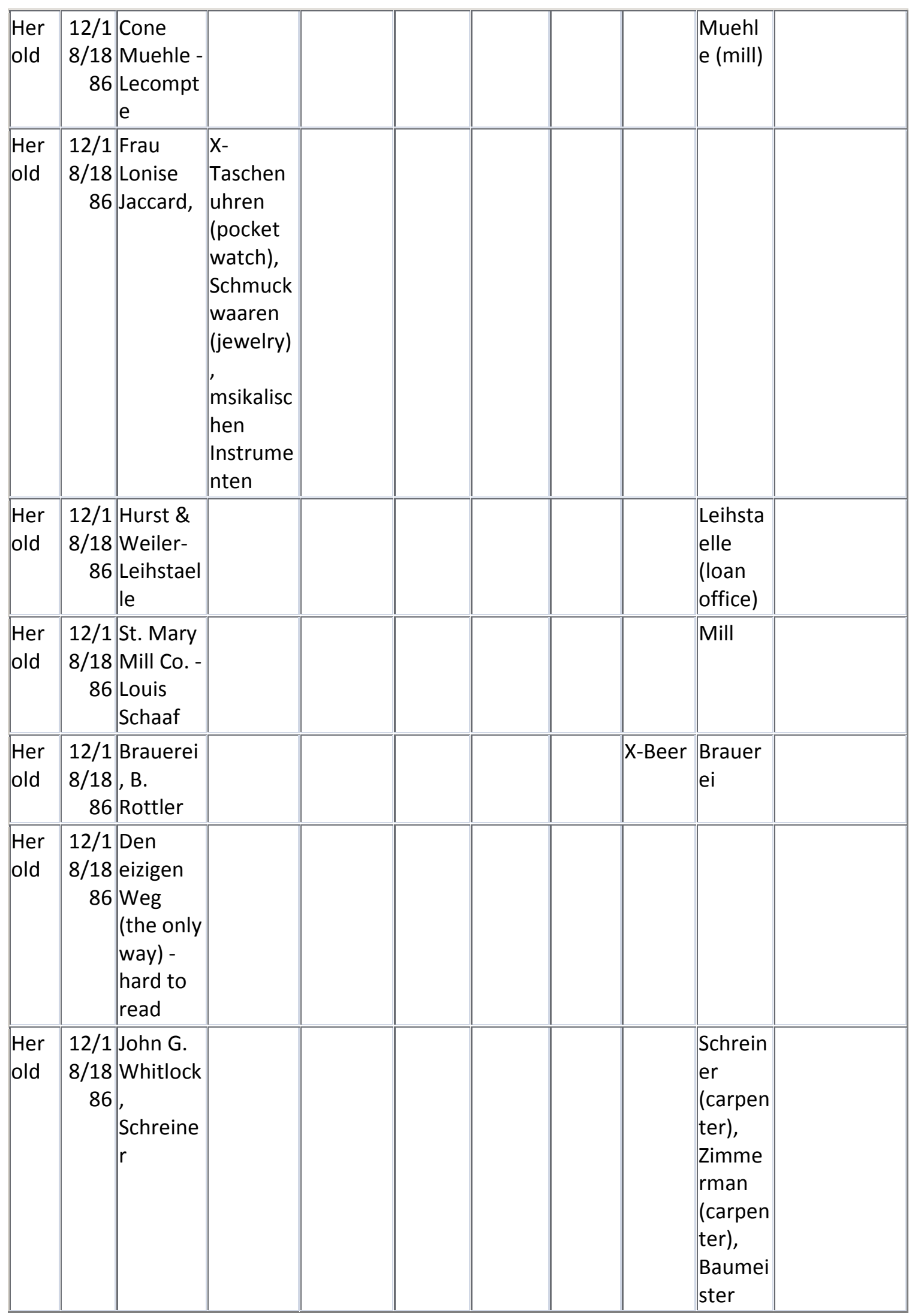




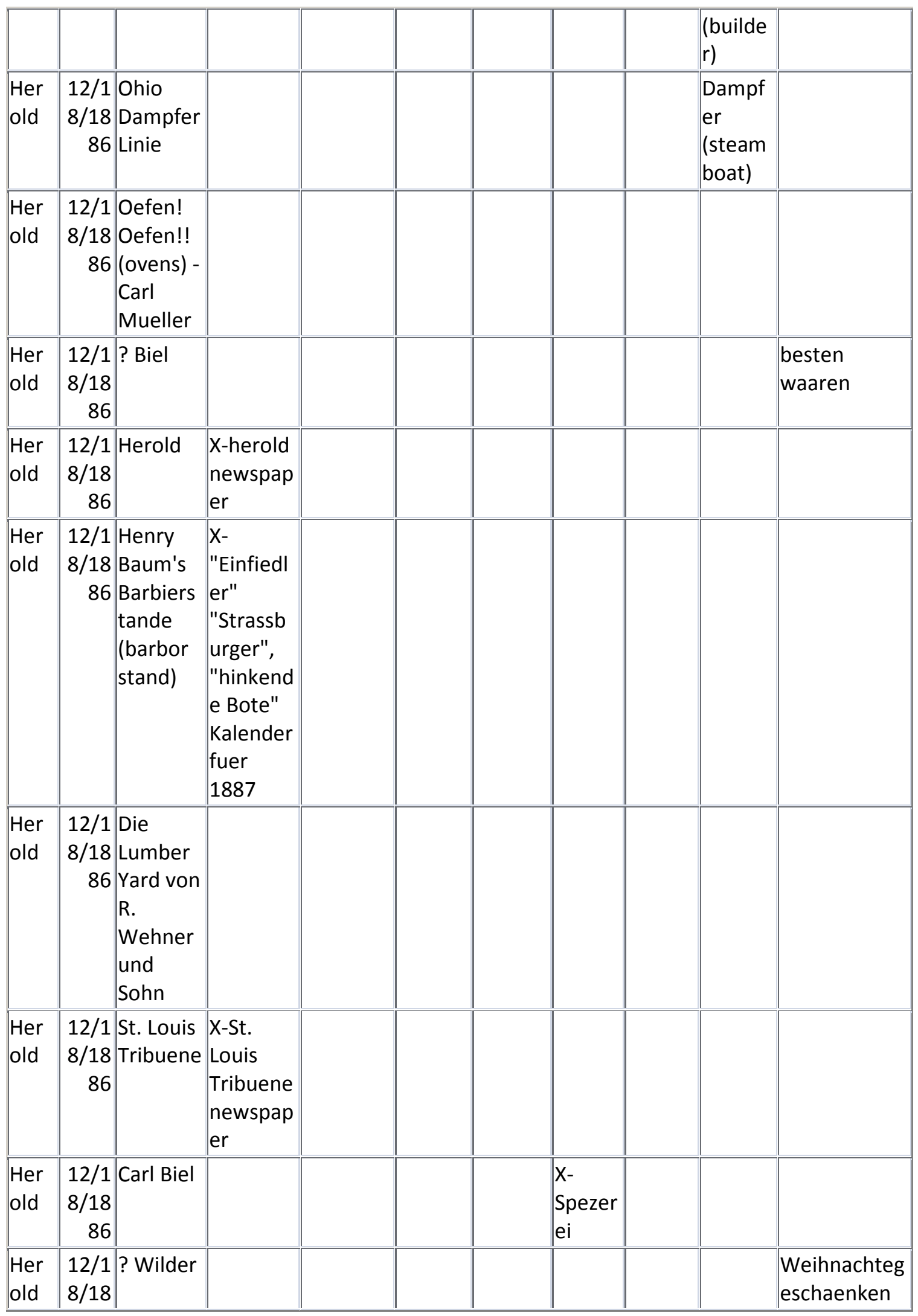




\begin{tabular}{|c|c|c|c|c|c|c|c|c|}
\hline & 86 & & & & & & & $\begin{array}{l}\text { (christmas } \\
\text { gifts) }\end{array}$ \\
\hline $\begin{array}{l}\text { Her } \\
\text { old }\end{array}$ & \begin{tabular}{|r|}
$12 / 1$ \\
$8 / 18$ \\
86
\end{tabular} & $\begin{array}{l}\text { Frau Falk } \\
\text { u. Sohn }\end{array}$ & & & & & & \\
\hline $\begin{array}{l}\text { Her } \\
\text { old }\end{array}$ & \begin{tabular}{|r|}
$12 / 1$ \\
$8 / 18$ \\
86 \\
\end{tabular} & $\begin{array}{l}\text { Gottlob } \\
\text { Kammer } \\
\text { er }\end{array}$ & & $\begin{array}{l}\text { X-Heften } \\
\text { (sewing) }\end{array}$ & & & & $\begin{array}{l}\text { Puempen? } \\
\text { (pump) }\end{array}$ \\
\hline $\begin{array}{l}\text { Her } \\
\text { old }\end{array}$ & \begin{tabular}{|r|}
$12 / 1$ \\
$8 / 18$ \\
86 \\
\end{tabular} & $\begin{array}{l}\text { Wehner } \\
\text { u. Bolle, } \\
\text { City Mill }\end{array}$ & & & & & Mille & \\
\hline $\begin{array}{l}\text { Her } \\
\text { old }\end{array}$ & $\begin{array}{r}12 / 1 \\
8 / 18 \\
86 \\
\end{array}$ & $\begin{array}{l}\text { Der } \\
\text { Pelznick } \\
\text { el is da! } \\
\text { (Santa } \\
\text { Claus is } \\
\text { there!) }\end{array}$ & $\begin{array}{l}\text { X- } \\
\text { Spielwaa } \\
\text { ren } \\
\text { (toys) }\end{array}$ & & \begin{tabular}{|l} 
X- \\
Zucker \\
kand \\
(suger \\
candy) \\
Feigen \\
(fig), \\
Orang \\
en, \\
Zwieb \\
ack \\
(sweet \\
biscuit \\
)
\end{tabular} & & & $\begin{array}{l}\text { Weihnachtsg } \\
\text { eshenke }\end{array}$ \\
\hline $\begin{array}{l}\text { Her } \\
\text { old }\end{array}$ & \begin{tabular}{|r|}
$12 / 1$ \\
$8 / 18$ \\
86 \\
\end{tabular} & $\begin{array}{l}\text { Frau Falk } \\
\text { u. Sohn }\end{array}$ & & & & & & \\
\hline $\begin{array}{l}\text { Her } \\
\text { old }\end{array}$ & \begin{tabular}{|r|}
$12 / 1$ \\
$8 / 18$ \\
86 \\
\\
\end{tabular} & $\begin{array}{l}\text { Is haus } \\
\text { versiche } \\
\text { rt? } \\
\text { (insured) } \\
\text {-Jules B. } \\
\text { Gitignon }\end{array}$ & & & & & $\begin{array}{l}\text { Insuran } \\
\text { ce }\end{array}$ & \\
\hline $\begin{array}{l}\text { Her } \\
\text { old }\end{array}$ & \begin{tabular}{|r|}
$12 / 1$ \\
$8 / 18$ \\
86 \\
\end{tabular} & $\begin{array}{l}\text { Chas. } \\
\text { Rottler's } \\
\text { Wein u. } \\
\text { Bier } \\
\text { Wirthsch } \\
\text { aft }\end{array}$ & $\begin{array}{l}\text { X- } \\
\text { Cigarren }\end{array}$ & & & $\begin{array}{l}\text { X- } \\
\text { Wein, } \\
\text { Bier }\end{array}$ & $\begin{array}{l}\text { Wirtsc } \\
\text { haft } \\
\text { (pub) }\end{array}$ & \\
\hline $\begin{array}{l}\text { Her } \\
\text { old }\end{array}$ & \begin{tabular}{|r|}
$12 / 1$ \\
$8 / 18$ \\
86
\end{tabular} & $\begin{array}{l}\text { A. D. } \\
\text { Quillet, } \\
\text { Uhrmac } \\
\text { her }\end{array}$ & $\begin{array}{l}\text { X- } \\
\text { taschenu } \\
\text { hren(poc } \\
\text { ket }\end{array}$ & & & & & \\
\hline
\end{tabular}




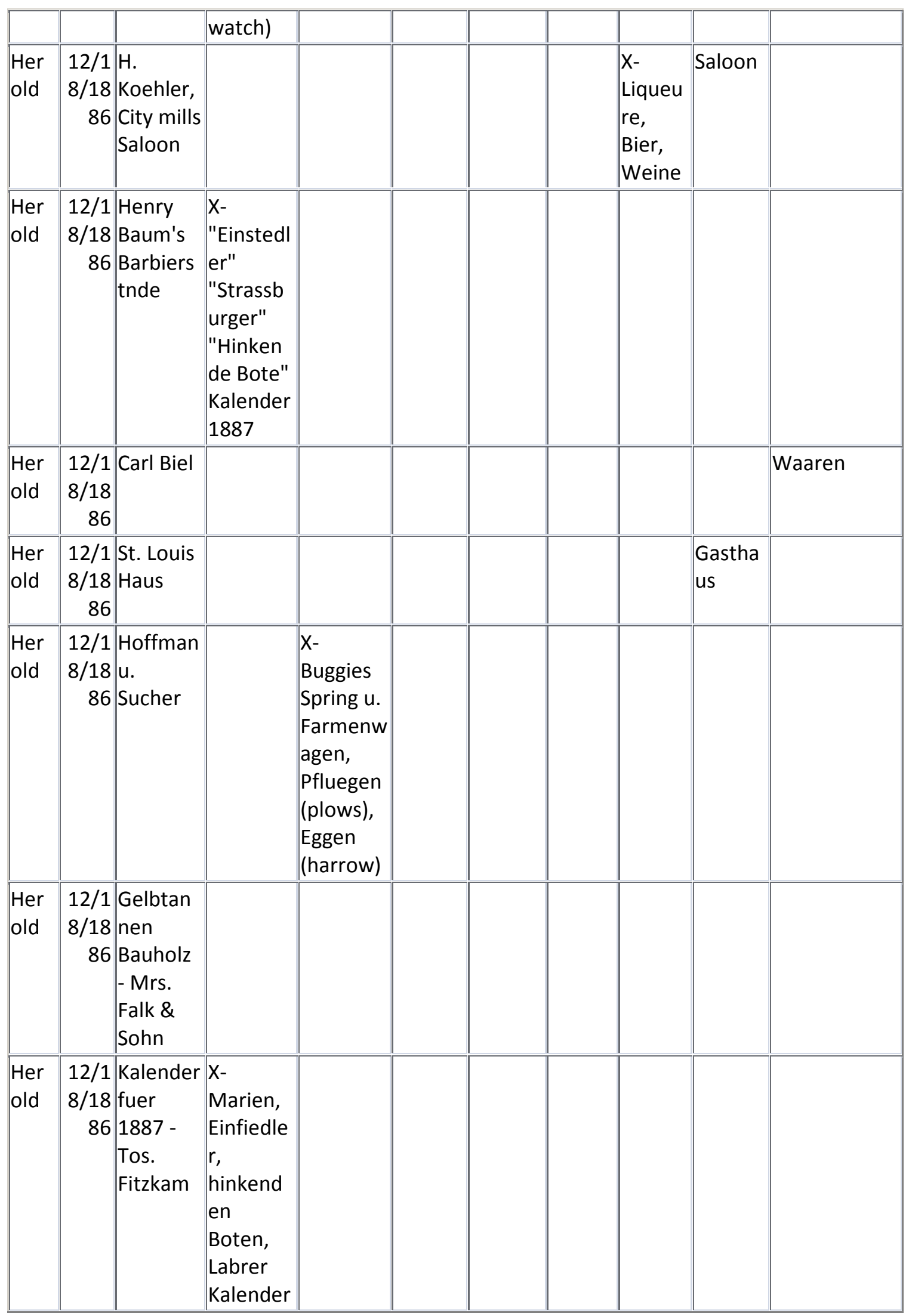




\begin{tabular}{|c|c|c|c|c|c|c|c|}
\hline \multicolumn{8}{|c|}{ German-English Newpaper } \\
\hline \multicolumn{8}{|c|}{ German-English Newspaper } \\
\hline $\begin{array}{c}\text { Newspa } \\
\text { per }\end{array}$ & Date & $\begin{array}{c}\text { advertiseme } \\
\text { nt title }\end{array}$ & $\begin{array}{c}\text { German/Eng } \\
\text { lish }\end{array}$ & \begin{tabular}{|l|} 
Household \\
Maintenan \\
ce-Ceramic
\end{tabular} & \begin{tabular}{|c|}
$\begin{array}{c}\text { Household } \\
\text { Maintenan } \\
\text { ce-glass }\end{array}$ \\
\end{tabular} & \begin{tabular}{|c|}
$\begin{array}{c}\text { Household } \\
\text { Maintenan } \\
\text { ce-other }\end{array}$ \\
\end{tabular} & $\begin{array}{l}\text { Architect } \\
\text { ural Items }\end{array}$ \\
\hline Herald & $\begin{array}{r}12 / 21 / 18 \\
95 \\
\end{array}$ & $\begin{array}{l}\text { Ste. } \\
\text { Geenevieve } \\
\text { Herald \& } \\
\text { Demorest's } \\
\text { magazine }\end{array}$ & $\mathrm{E}$ & & & & \\
\hline Herald & $\begin{array}{r}12 / 21 / 18 \\
95\end{array}$ & $\begin{array}{l}\text { Meyer's } \\
\text { Hotel - Wm. } \\
\text { Baumstark }\end{array}$ & $G$ & & & & \\
\hline Herald & $\begin{array}{r}12 / 21 / 18 \\
95\end{array}$ & $\begin{array}{l}\text { Seraner's, } \\
\text { Wein, Bier u. } \\
\text { Billiard } \\
\text { Saloon }\end{array}$ & $G$ & & & & \\
\hline Herald & $\begin{array}{r}12 / 21 / 18 \\
95 \\
\end{array}$ & $\begin{array}{l}\text { Gasthaus - } \\
\text { Carl Rottler }\end{array}$ & G & & & & \\
\hline Herald & $\begin{array}{r}12 / 21 / 18 \\
95 \\
\end{array}$ & $\begin{array}{l}\text { Dr. Price's } \\
\text { Cream } \\
\text { Baking } \\
\text { Powder }\end{array}$ & G & & & & \\
\hline Herald & $\begin{array}{r}12 / 21 / 18 \\
95\end{array}$ & $\begin{array}{l}\text { Zahn Arzt - } \\
\text { Dr. C. J. } \\
\text { Hertich }\end{array}$ & G & & & & \\
\hline Herald & $\begin{array}{r}12 / 21 / 18 \\
95 \\
\end{array}$ & $\begin{array}{l}\text { Peter H. } \\
\text { Huck, } \\
\text { Dentischer } \\
\text { Rechtsenwal } \\
\text { t }\end{array}$ & G & & & & \\
\hline Herald & $\begin{array}{r}12 / 21 / 18 \\
95\end{array}$ & $\begin{array}{l}\text { Meyer's } \\
\text { Hotel }\end{array}$ & $\mathrm{E}$ & & & & \\
\hline Herald & $\begin{array}{r}12 / 21 / 18 \\
95\end{array}$ & $\begin{array}{l}\text { Mich. } \\
\text { Beauchamp, }\end{array}$ & $\mathrm{E}$ & & & & \\
\hline
\end{tabular}




\begin{tabular}{|c|c|c|c|c|c|c|c|}
\hline \multicolumn{8}{|c|}{ German-English Newspaper } \\
\hline $\begin{array}{c}\text { Newspa } \\
\text { per }\end{array}$ & Date & $\begin{array}{c}\text { advertiseme } \\
\text { nt title }\end{array}$ & \begin{tabular}{|} 
German/Eng \\
lish
\end{tabular} & \begin{tabular}{|l|} 
Household \\
Maintenan \\
ce-Ceramic
\end{tabular} & \begin{tabular}{|c|} 
Household \\
Maintenan \\
ce-glass
\end{tabular} & \begin{tabular}{|c|}
$\begin{array}{c}\text { Household } \\
\text { Maintenan } \\
\text { ce-other }\end{array}$ \\
\end{tabular} & $\begin{array}{l}\text { Architect } \\
\text { ural Items }\end{array}$ \\
\hline & & $\begin{array}{l}\text { Tonsorial } \\
\text { Artist }\end{array}$ & & & & & \\
\hline Herald & $\begin{array}{r}12 / 21 / 18 \\
95 \\
\end{array}$ & $\begin{array}{l}\text { T. B. } \\
\text { Whitledge } \\
\text { Rechtsanwal } \\
t\end{array}$ & G & & & & \\
\hline Herald & $\begin{array}{r}12 / 21 / 18 \\
95 \\
\end{array}$ & $\begin{array}{l}\text { R. W. } \\
\text { Lanning, } \\
\text { Apotheker } \\
\text { (difficult to } \\
\text { read) }\end{array}$ & G & & & & \\
\hline Herald & \begin{tabular}{|r|}
$12 / 21 / 18$ \\
95
\end{tabular} & $\begin{array}{l}\text { Hood's } \\
\text { Sarsaparilla }\end{array}$ & $E$ & & & & \\
\hline Herald & $\begin{array}{r}\mid 12 / 21 / 18 \\
95\end{array}$ & $\begin{array}{l}\text { Kennedy's } \\
\text { Medical } \\
\text { Discovery }\end{array}$ & $E$ & & & & \\
\hline Herald & $\begin{array}{r}12 / 21 / 18 \\
95 \\
\end{array}$ & $\begin{array}{l}\text { Imperial } \\
\text { Granum - } \\
\text { John Carle \& } \\
\text { Sons, New } \\
\text { York }\end{array}$ & $E$ & & & & \\
\hline Herald & \begin{tabular}{|r|}
$12 / 21 / 18$ \\
95 \\
\end{tabular} & $\begin{array}{l}\text { Asthma, Dr. } \\
\text { Taft's } \\
\text { Asthmalene }\end{array}$ & $\mathrm{E}$ & & & & \\
\hline Herald & $\begin{array}{r}12 / 21 / 18 \\
95 \\
\end{array}$ & $\begin{array}{l}\text { See that } \\
\text { hump? - } \\
\text { Richardson \& } \\
\text { DeLong Bros, } \\
\text { Philada. }\end{array}$ & $\mathrm{E}$ & & & & \\
\hline Herald & $\begin{array}{r}12 / 21 / 18 \\
95\end{array}$ & \begin{tabular}{|l} 
The Pilgrim, \\
Geo. \\
Heavford, \\
Chicago
\end{tabular} & $\mathrm{E}$ & & & & \\
\hline Herald & \begin{tabular}{|r|}
$12 / 21 / 18$ \\
95
\end{tabular} & Hale's Honey & $\mathrm{E}$ & & & & \\
\hline Herald & $\begin{array}{r}12 / 21 / 18 \\
95\end{array}$ & $\begin{array}{l}\text { Brown's } \\
\text { Brauch } \\
\text { Troches" ? }\end{array}$ & $\mathrm{E}$ & & & & \\
\hline Herald & \begin{tabular}{|r|}
$12 / 21 / 18$ \\
95
\end{tabular} & $\begin{array}{l}\text { Aeromotore } \\
\text { Co. }\end{array}$ & $E$ & & & & $\begin{array}{l}\text { X- } \\
\text { pumping, }\end{array}$ \\
\hline
\end{tabular}




\begin{tabular}{|c|c|c|c|c|c|c|c|}
\hline \multicolumn{8}{|c|}{ German-English Newspaper } \\
\hline $\begin{array}{c}\text { Newspa } \\
\text { per }\end{array}$ & Date & $\begin{array}{c}\text { advertiseme } \\
\text { nt title }\end{array}$ & \begin{tabular}{|} 
German/Eng \\
lish
\end{tabular} & \begin{tabular}{|l|} 
Household \\
Maintenan \\
ce-Ceramic
\end{tabular} & \begin{tabular}{|c} 
Household \\
Maintenan \\
ce-glass \\
\end{tabular} & \begin{tabular}{|c|}
$\begin{array}{c}\text { Household } \\
\text { Maintenan } \\
\text { ce-other }\end{array}$ \\
\end{tabular} & $\begin{array}{l}\text { Architect } \\
\text { ural Items }\end{array}$ \\
\hline & & & & & & & $\begin{array}{l}\text { Geared, } \\
\text { steel, } \\
\text { Galvanize } \\
\text { d } \\
\text { Windmills } \\
\text {, Fixed } \\
\text { steel } \\
\text { Towers, } \\
\text { steel Buzz } \\
\text { saw, } \\
\text { Frames, } \\
\text { Steel Feed } \\
\text { Cutters, } \\
\text { Feed } \\
\text { Grinders }\end{array}$ \\
\hline Herald & $\begin{array}{r}12 / 21 / 18 \\
95\end{array}$ & $\begin{array}{l}\text { The Best, } \\
\text { The rest, the } \\
\text { test }\end{array}$ & $E$ & & & & \\
\hline Herald & $\begin{array}{r}12 / 21 / 18 \\
95\end{array}$ & $\begin{array}{l}\text { Burns or } \\
\text { Scalds }\end{array}$ & $E$ & & & & \\
\hline Herald & $\begin{array}{r}12 / 21 / 18 \\
95\end{array}$ & No-To-Bac & E & & & & \\
\hline Herald & $\begin{array}{r}12 / 21 / 18 \\
95\end{array}$ & Cascarets & $E$ & & & & \\
\hline Herald & $\begin{array}{r}12 / 21 / 18 \\
95\end{array}$ & $\begin{array}{l}\text { Rising Sun } \\
\text { Stove Polish }\end{array}$ & $E$ & & & $\begin{array}{l}\text { Rising Sun } \\
\text { Stove } \\
\text { polish }\end{array}$ & \\
\hline Herald & $\begin{array}{r}12 / 21 / 18 \\
95 \\
\end{array}$ & $\begin{array}{l}\text { Opium - J. } \\
\text { Stephens, } \\
\text { Lebanon, } \\
\text { Ohio }\end{array}$ & $\mathrm{E}$ & & & & \\
\hline Herald & $\begin{array}{r}12 / 21 / 18 \\
95\end{array}$ & $\begin{array}{l}\text { Opium - } \\
\text { Woolley, } \\
\text { Atlanta }\end{array}$ & $\mathrm{E}$ & & & & \\
\hline Herald & $\begin{array}{r}12 / 21 / 18 \\
95 \\
\end{array}$ & $\begin{array}{l}\text { Piso's Cure } \\
\text { for } \\
\text { Consumptio } \\
\text { n }\end{array}$ & $\mathrm{E}$ & & & & \\
\hline Herald & $\begin{array}{r}12 / 21 / 18 \\
95\end{array}$ & $\begin{array}{l}\text { Warnung - } \\
\text { Gebrueder }\end{array}$ & G & & & & \\
\hline
\end{tabular}




\begin{tabular}{|c|c|c|c|c|c|c|c|}
\hline \multicolumn{8}{|c|}{ German-English Newspaper } \\
\hline $\begin{array}{c}\text { Newspa } \\
\text { per }\end{array}$ & Date & $\begin{array}{c}\text { advertiseme } \\
\text { nt title }\end{array}$ & \begin{tabular}{|} 
German/Eng \\
lish
\end{tabular} & $\begin{array}{l}\text { Household } \\
\text { Maintenan } \\
\text { ce-Ceramic }\end{array}$ & $\begin{array}{c}\text { Household } \\
\text { Maintenan } \\
\text { ce-glass }\end{array}$ & $\begin{array}{c}\text { Household } \\
\text { Maintenan } \\
\text { ce-other }\end{array}$ & \begin{tabular}{|c|} 
Architect \\
ural Items
\end{tabular} \\
\hline & & Wilder & & & & & \\
\hline Herald & $\begin{array}{r}12 / 21 / 18 \\
95\end{array}$ & $\begin{array}{l}\text { Lanning's } \\
\text { Apotheke }\end{array}$ & G & & & & \\
\hline Herald & \begin{tabular}{|r|}
$12 / 21 / 18$ \\
95
\end{tabular} & $\begin{array}{l}\text { Siebert \& } \\
\text { Sohn }\end{array}$ & G & & & & \\
\hline Herald & \begin{tabular}{|r|}
$12 / 21 / 18$ \\
95
\end{tabular} & $\begin{array}{l}\text { Frau } \\
\text { Rennard? }\end{array}$ & G & & & & \\
\hline Herald & $\begin{array}{r}12 / 21 / 18 \\
95 \\
\end{array}$ & $\begin{array}{l}\text { Schoettler } \\
\text { und } \\
\text { Scheuring, } \\
\text { Leichenbesta } \\
\text { tter und } \\
\text { Einbalsamire } \\
\text { r (mortician } \\
\text { and } \\
\text { embalmer) }\end{array}$ & G & & & & \\
\hline Herald & $\begin{array}{r}12 / 21 / 18 \\
95 \\
\end{array}$ & $\begin{array}{l}\text { Trifft euch } \\
\text { die } \\
\text { Wahrheit? - } \\
\text { Tokerst u. } \\
\text { Bruder }\end{array}$ & G & & & & \\
\hline Herald & $\begin{array}{r}12 / 21 / 18 \\
95\end{array}$ & $\begin{array}{l}\text { Geht zu } \\
\text { Henry } \\
\text { Okenfuss }\end{array}$ & G & & & $\begin{array}{l}\text { Schlitten } \\
\text { (Coasters) }\end{array}$ & \\
\hline Herald & $\begin{array}{r}12 / 17 / 18 \\
98\end{array}$ & $\begin{array}{l}\text { Genuine } \\
\text { Bargains in } \\
\text { Capes,Capes } \\
\text { - Jokerst } \\
\text { Bros. \& } \\
\text { Yealy. }\end{array}$ & $\mathrm{E}$ & & & & \\
\hline Herald & $\begin{array}{r}12 / 17 / 18 \\
98 \\
\end{array}$ & $\begin{array}{l}\text { For } \\
\text { Christmas. } \\
10 \text { \& } 15 \\
\text { percent off } \\
\text { on all shoes, } \\
\text { hy. Herzog } \\
\text { Shoe Co. }\end{array}$ & $E$ & & & & \\
\hline Herald & $\begin{array}{r}12 / 17 / 18 \\
98\end{array}$ & $\begin{array}{l}\text { John } \\
\text { Koetting }\end{array}$ & $E$ & & & & \\
\hline Herald & $12 / 17 / 18$ & The semi- & $E$ & & & & \\
\hline
\end{tabular}




\begin{tabular}{|c|c|c|c|c|c|c|c|}
\hline \multicolumn{8}{|c|}{ German-English Newspaper } \\
\hline $\begin{array}{c}\text { Newspa } \\
\text { per }\end{array}$ & Date & $\begin{array}{c}\text { advertiseme } \\
\text { nt title }\end{array}$ & $\begin{array}{c}\text { German/Eng } \\
\text { lish }\end{array}$ & \begin{tabular}{|l|} 
Household \\
Maintenan \\
ce-Ceramic
\end{tabular} & \begin{tabular}{|c|} 
Household \\
Maintenan \\
ce-glass
\end{tabular} & \begin{tabular}{|c|}
$\begin{array}{c}\text { Household } \\
\text { Maintenan } \\
\text { ce-other }\end{array}$ \\
\end{tabular} & $\begin{array}{l}\text { Architect } \\
\text { ural Items }\end{array}$ \\
\hline & 98 & $\begin{array}{l}\text { weekly } \\
\text { republic }\end{array}$ & & & & & \\
\hline Herald & $\begin{array}{r}\mid 12 / 17 / 18 \\
98 \\
\end{array}$ & $\begin{array}{l}\text { St. Louis } \\
\text { Globe- } \\
\text { Democrat }\end{array}$ & $E$ & & & & \\
\hline Herald & \begin{tabular}{|r|}
$12 / 17 / 18$ \\
98
\end{tabular} & $\begin{array}{l}\text { Rozier's Cash } \\
\text { Store }\end{array}$ & $E$ & & & & \\
\hline Herald & $\begin{array}{r}\mid 12 / 17 / 18 \\
98\end{array}$ & $\begin{array}{l}\text { Gus L. } \\
\text { Schoettler }\end{array}$ & $\mathrm{E}$ & & & & \\
\hline Herald & \begin{tabular}{|r|}
$12 / 17 / 18$ \\
98
\end{tabular} & $\begin{array}{l}\text { Hy. Herz? } \\
\text { Shoe Co. }\end{array}$ & $E$ & & & & \\
\hline Herald & $\begin{array}{r}\mid 12 / 17 / 18 \\
98 \\
\end{array}$ & \begin{tabular}{|l} 
Christmas \\
Trees -Felix \\
Thomure \\
\end{tabular} & $E$ & & & & \\
\hline Herald & \begin{tabular}{|r|}
$12 / 17 / 18$ \\
98
\end{tabular} & Koetting's & $E$ & & & & \\
\hline Herald & $\begin{array}{r}\mid 12 / 17 / 18 \\
98\end{array}$ & Herald & $E$ & & & & \\
\hline Herald & $\begin{array}{r}12 / 17 / 18 \\
98 \\
\end{array}$ & $\begin{array}{l}\text { Rutledge \& } \\
\text { Co. and F. E. } \\
\text { Delassus }\end{array}$ & $\mathrm{E}$ & & & & \\
\hline Herald & \begin{tabular}{|r|}
$12 / 17 / 18$ \\
98
\end{tabular} & $\begin{array}{l}\text { Rutledge \& } \\
\text { Co. }\end{array}$ & $E$ & & & & \\
\hline Herald & \begin{tabular}{|r|}
$12 / 17 / 18$ \\
98
\end{tabular} & $\begin{array}{l}\text { Rutledge \& } \\
\text { Co. }\end{array}$ & $E$ & & & & \\
\hline Herald & $\begin{array}{r}\mid 12 / 17 / 18 \\
98 \\
\end{array}$ & $\begin{array}{l}\text { J. Tlapek } \\
\text { Imp. \& } \\
\text { Lumber Co. }\end{array}$ & $E$ & & & & $\begin{array}{l}\text { X-Kendall } \\
\text { Wire } \\
\text { Fence }\end{array}$ \\
\hline Herald & \begin{tabular}{|r|}
$12 / 17 / 18$ \\
98
\end{tabular} & M.Vieh & E & & & & \\
\hline Herald & \begin{tabular}{|r|}
$12 / 17 / 18$ \\
98
\end{tabular} & $\begin{array}{l}\text { Rutledge \& } \\
\text { Co's }\end{array}$ & $\mathrm{E}$ & & & & \\
\hline Herald & $\begin{array}{r}12 / 17 / 18 \\
98 \\
\end{array}$ & $\begin{array}{l}\text { Mrs. } \\
\text { Kennard's } \\
\text { restaurant }\end{array}$ & $E$ & & & & \\
\hline Herald & \begin{tabular}{r|}
$12 / 21 / 18$ \\
95 \\
\end{tabular} & $\begin{array}{l}\text { Warning! } \\
\text { Warning! - } \\
\text { Wilder Bros }\end{array}$ & $\mathrm{E}$ & & & & \\
\hline
\end{tabular}




\begin{tabular}{|c|c|c|c|c|c|c|c|}
\hline \multicolumn{8}{|c|}{ German-English Newspaper } \\
\hline $\begin{array}{c}\text { Newspa } \\
\text { per }\end{array}$ & Date & $\begin{array}{c}\text { advertiseme } \\
\text { nt title }\end{array}$ & $\begin{array}{c}\text { German/Eng } \\
\text { lish }\end{array}$ & $\begin{array}{l}\text { Household } \\
\text { Maintenan } \\
\text { ce-Ceramic }\end{array}$ & \begin{tabular}{|c|} 
Household \\
Maintenan \\
ce-glass
\end{tabular} & \begin{tabular}{|c} 
Household \\
Maintenan \\
ce-other
\end{tabular} & $\begin{array}{l}\text { Architect } \\
\text { ural Items }\end{array}$ \\
\hline Herald & $\begin{array}{r}12 / 21 / 18 \\
95\end{array}$ & $\begin{array}{l}\text { Schoettler \& } \\
\text { Scheuring }\end{array}$ & $\mathrm{E}$ & & & & \\
\hline Herald & $\begin{array}{r}12 / 21 / 18 \\
95\end{array}$ & $\begin{array}{l}\text { Does it Strike } \\
\text { You? -Jokerst } \\
\& \text { Bro. }\end{array}$ & E & & & & \\
\hline Herald & $\begin{array}{r}12 / 21 / 18 \\
95\end{array}$ & $\begin{array}{l}\text { Go to Hy. } \\
\text { Okenfuss }\end{array}$ & $E$ & $\begin{array}{l}\text { X-Fancy } \\
\text { Cups and } \\
\text { Saucers, } \\
\text { Dinner } \\
\text { Sets,Tea } \\
\text { Sets, } \\
\text { Chamber } \\
\text { Sets }\end{array}$ & & $\begin{array}{l}\text { Coasters, } \\
\text { Fine Vases }\end{array}$ & $\begin{array}{l}\text { X-Saws } \\
\text { and Bucks }\end{array}$ \\
\hline Herald & $\begin{array}{r}12 / 21 / 18 \\
95\end{array}$ & Koetting's & $E$ & & & & \\
\hline Herald & $\begin{array}{r}12 / 21 / 18 \\
95\end{array}$ & $\begin{array}{l}\text { Siebert \& } \\
\text { Son }\end{array}$ & $E$ & & & & \\
\hline Herald & $\begin{array}{r}12 / 21 / 18 \\
95\end{array}$ & $\begin{array}{l}\text { Baum Stark's } \\
\text { Saloon }\end{array}$ & $E$ & & & & \\
\hline Herald & $\begin{array}{r}12 / 21 / 18 \\
95\end{array}$ & $\begin{array}{l}\text { Koetting's } \\
\text { Jewelry } \\
\text { Store }\end{array}$ & $E$ & & & & \\
\hline Herald & $\begin{array}{r}12 / 21 / 18 \\
95\end{array}$ & $\begin{array}{l}\text { Mrs. } \\
\text { Kennard }\end{array}$ & $E$ & & & & \\
\hline Herald & $\begin{array}{r}12 / 21 / 18 \\
95\end{array}$ & $\begin{array}{l}\text { Siebert \& } \\
\text { Son }\end{array}$ & $E$ & & & & \\
\hline Herald & $\begin{array}{r}12 / 21 / 18 \\
95\end{array}$ & \begin{tabular}{|l} 
Lanning's \\
Drug Store
\end{tabular} & $E$ & & & & \\
\hline Herald & $\begin{array}{r}12 / 21 / 18 \\
95\end{array}$ & $\begin{array}{l}\text { Siebert \& } \\
\text { Son }\end{array}$ & $E$ & & & & \\
\hline Herald & $\begin{array}{r}12 / 21 / 18 \\
95\end{array}$ & $\begin{array}{l}\text { Gus L. } \\
\text { Schoettler }\end{array}$ & $E$ & & & & \\
\hline Herald & $\begin{array}{r}12 / 21 / 18 \\
95\end{array}$ & $\begin{array}{l}\text { Ayer's } \\
\text { Narsaparilla } \\
\text { medicine }\end{array}$ & $E$ & & & & \\
\hline Herald & $\begin{array}{r}12 / 21 / 18 \\
95\end{array}$ & $\begin{array}{l}\text { Kohm \& } \\
\text { Petrequin }\end{array}$ & $E$ & & & & $\begin{array}{l}\text { X-Feed } \\
\text { Cutters, } \\
\text { Wood } \\
\text { saws }\end{array}$ \\
\hline
\end{tabular}




\begin{tabular}{|c|c|c|c|c|c|c|c|}
\hline \multicolumn{8}{|c|}{ German-English Newspaper } \\
\hline $\begin{array}{c}\text { Newspa } \\
\text { per }\end{array}$ & Date & $\begin{array}{c}\text { advertiseme } \\
\text { nt title }\end{array}$ & $\begin{array}{c}\text { German/Eng } \\
\text { lish }\end{array}$ & \begin{tabular}{|l|} 
Household \\
Maintenan \\
ce-Ceramic
\end{tabular} & \begin{tabular}{|c|} 
Household \\
Maintenan \\
ce-glass
\end{tabular} & \begin{tabular}{|c|} 
Household \\
Maintenan \\
ce-other
\end{tabular} & $\begin{array}{l}\text { Architect } \\
\text { ural Items }\end{array}$ \\
\hline Herald & $\begin{array}{r}12 / 21 / 18 \\
95\end{array}$ & $\begin{array}{l}\text { Lanning's } \\
\text { Drub Store }\end{array}$ & $\mathrm{E}$ & & & & \\
\hline Herald & $\begin{array}{r}12 / 21 / 18 \\
95\end{array}$ & $\begin{array}{l}\text { Siebert \& } \\
\text { Son }\end{array}$ & $E$ & & & & \\
\hline Herald & $\begin{array}{r}12 / 21 / 18 \\
95\end{array}$ & $\begin{array}{l}\text { Koetting \& } \\
\text { Hunold }\end{array}$ & $E$ & & & & \\
\hline Herald & $\begin{array}{r}12 / 21 / 18 \\
95\end{array}$ & $\begin{array}{l}\text { Siebert \& } \\
\text { Son }\end{array}$ & $E$ & & & & \\
\hline Herald & $\begin{array}{r}12 / 21 / 18 \\
95 \\
\end{array}$ & $\begin{array}{l}\text { A.B.C. } \\
\text { Bohemian ?- } \\
\text { sold at Ed. } \\
\text { Sielert's } \\
\text { saloon }\end{array}$ & $E$ & & & & \\
\hline Herald & $\begin{array}{r}12 / 21 / 18 \\
95\end{array}$ & $\begin{array}{l}\text { J.H. Rottman } \\
\text { Distilling Co. }\end{array}$ & $E$ & & & & \\
\hline Herald & $\begin{array}{r}12 / 21 / 18 \\
95\end{array}$ & J.W.Shaw & $E$ & & & & \\
\hline Herald & $\begin{array}{r}12 / 21 / 18 \\
95\end{array}$ & Ayer's Pills & $E$ & & & & \\
\hline Herald & $\begin{array}{r}12 / 21 / 18 \\
95\end{array}$ & $\begin{array}{l}\text { Frank U. } \\
\text { Geiler }\end{array}$ & G & & & & \\
\hline Herald & $\begin{array}{r}12 / 21 / 18 \\
95\end{array}$ & $\begin{array}{l}\text { Lumber Yard } \\
\text { - R. Wehner } \\
\text { und Sohn }\end{array}$ & G & & & & X-Lumber \\
\hline Herald & $\begin{array}{r}12 / 21 / 18 \\
95\end{array}$ & $\begin{array}{l}\text { Great } \\
\text { Chicago } \\
\text { Tailors (hard } \\
\text { to read) }\end{array}$ & $E$ & & & & \\
\hline Herald & $\begin{array}{r}12 / 21 / 18 \\
95\end{array}$ & $\begin{array}{l}\text { Brewing \& } \\
\text { Lighting } \\
\text { Ass'n. }\end{array}$ & $E$ & & & & \\
\hline Herald & $\begin{array}{r}12 / 21 / 18 \\
95\end{array}$ & $\begin{array}{l}\text { Brau u. } \\
\text { Leucht } \\
\text { Association }\end{array}$ & G & & & & \\
\hline Herald & $\begin{array}{r}12 / 21 / 18 \\
95\end{array}$ & $\begin{array}{l}\text { Joseph } \\
\text { Fitzkam Haar } \\
\text { Kuenstler }\end{array}$ & $G$ & & & & \\
\hline Herald & $\begin{array}{r}12 / 21 / 18 \\
95\end{array}$ & $\begin{array}{l}\text { Union Halle } \\
\text { Saloon- }\end{array}$ & $G$ & & & & \\
\hline
\end{tabular}




\begin{tabular}{|c|c|c|c|c|c|c|c|}
\hline \multicolumn{8}{|c|}{ German-English Newspaper } \\
\hline $\begin{array}{c}\text { Newspa } \\
\text { per }\end{array}$ & Date & $\begin{array}{c}\text { advertiseme } \\
\text { nt title }\end{array}$ & $\begin{array}{c}\text { German/Eng } \\
\text { lish }\end{array}$ & $\begin{array}{l}\text { Household } \\
\text { Maintenan } \\
\text { ce-Ceramic }\end{array}$ & $\begin{array}{l}\text { Household } \\
\text { Maintenan } \\
\text { ce-glass }\end{array}$ & $\begin{array}{c}\text { Household } \\
\text { Maintenan } \\
\text { ce-other }\end{array}$ & $\begin{array}{l}\text { Architect } \\
\text { ural Items }\end{array}$ \\
\hline & & $\begin{array}{l}\text { Eduard } \\
\text { Siebert }\end{array}$ & & & & & \\
\hline Herald & $\begin{array}{r}12 / 21 / 18 \\
95\end{array}$ & $\begin{array}{l}\text { Chas Rozier } \\
\text { Advokat }\end{array}$ & G & & & & \\
\hline Herald & $\begin{array}{r}12 / 21 / 18 \\
95\end{array}$ & $\begin{array}{l}\text { Henry L. } \\
\text { Rozier, } \\
\text { Bankier }\end{array}$ & G & & & & \\
\hline Herald & $\begin{array}{r}12 / 21 / 18 \\
95\end{array}$ & $\begin{array}{l}\text { Demorest's } \\
\text { Magazine }\end{array}$ & $\mathrm{E}$ & & & & \\
\hline
\end{tabular}

\begin{tabular}{|c|c|c|c|c|c|c|c|c|c|c|c|c|}
\hline \multicolumn{13}{|c|}{ German-English newspaper } \\
\hline Date & $\begin{array}{c}\text { advertis } \\
\text { ement } \\
\text { title }\end{array}$ & \begin{tabular}{|c} 
Germa \\
n/Engli \\
sh
\end{tabular} & $\begin{array}{c}\text { Hous } \\
\text { ehol } \\
d \\
\text { furni } \\
\text { shing } \\
\text { s }\end{array}$ & $\begin{array}{l}\text { Clot } \\
\text { hing }\end{array}$ & $\begin{array}{c}\text { Person } \\
\text { al } \\
\text { Items }\end{array}$ & $\begin{array}{c}\text { Househ } \\
\text { old } \\
\text { produc } \\
\text { tion } \\
\text { activity }\end{array}$ & $\begin{array}{l}\text { Hous } \\
\text { ehol } \\
\text { d } \\
\text { prod } \\
\text { uctio } \\
\text { n for } \\
\text { sale }\end{array}$ & $\begin{array}{c}\text { Pharm } \\
\text { aceutic } \\
\text { al }\end{array}$ & $\begin{array}{l}\text { Food } \\
\text { stuffs }\end{array}$ & $\begin{array}{c}\text { Alcoh } \\
\text { ol }\end{array}$ & $\mid \begin{array}{c}\text { Servic } \\
\text { es }\end{array}$ & Other \\
\hline $\begin{array}{r}12 / 2 \\
1 / 18 \\
95 \\
\\
\end{array}$ & $\begin{array}{l}\text { Ste. } \\
\text { Geenevi } \\
\text { eve } \\
\text { Herald } \\
\& \\
\text { Demore } \\
\text { st's } \\
\text { magazi } \\
\text { ne }\end{array}$ & $E$ & & & $\begin{array}{l}\text { X- } \\
\text { Herald } \\
\text { Demor } \\
\text { est's } \\
\text { magazi } \\
\text { ne }\end{array}$ & & & & & & & \\
\hline $\begin{array}{r}12 / 2 \\
1 / 18 \\
95\end{array}$ & $\begin{array}{l}\text { Meyer's } \\
\text { Hotel - } \\
\text { Wm. } \\
\text { Baumst } \\
\text { ark }\end{array}$ & G & & & & & & & & & Hotel & \\
\hline $\begin{array}{r}12 / 2 \\
1 / 18 \\
95 \\
\end{array}$ & $\begin{array}{l}\text { Seraner } \\
\text { 's, } \\
\text { Wein, } \\
\text { Bier u. } \\
\text { Billiard } \\
\text { Saloon }\end{array}$ & G & & & & & & & & $\begin{array}{l}\text { X- } \\
\text { Wein, } \\
\text { Bier }\end{array}$ & Saloon & \\
\hline $\begin{array}{l}12 / 2 \\
1 / 18\end{array}$ & $\begin{array}{l}\text { Gastha } \\
\text { us -Carl }\end{array}$ & G & & & $\begin{array}{l}\text { X- } \\
\text { Cigarr }\end{array}$ & & & & & & & \\
\hline
\end{tabular}




\begin{tabular}{|c|c|c|c|c|c|c|c|c|c|c|c|c|}
\hline \multicolumn{13}{|c|}{ German-English newspaper } \\
\hline Date & \begin{tabular}{|c||} 
advertis \\
ement \\
title
\end{tabular} & $\begin{array}{c}\text { Germa } \\
\text { n/Engli } \\
\text { sh }\end{array}$ & $\begin{array}{c}\text { Hous } \\
\text { ehol } \\
d \\
\text { furni } \\
\text { shing } \\
\text { s }\end{array}$ & $\begin{array}{l}\text { Clot } \\
\text { hing }\end{array}$ & $\begin{array}{c}\text { Person } \\
\text { al } \\
\text { Items }\end{array}$ & $\begin{array}{c}\text { Househ } \\
\text { old } \\
\text { produc } \\
\text { tion } \\
\text { activity }\end{array}$ & \begin{tabular}{|l} 
Hous \\
ehol \\
d \\
prod \\
uctio \\
n for \\
sale
\end{tabular} & $\begin{array}{c}\text { Pharm } \\
\text { aceutic } \\
\text { al }\end{array}$ & $\begin{array}{l}\text { Food } \\
\text { stuffs }\end{array}$ & $\begin{array}{c}\text { Alcoh } \\
\text { ol }\end{array}$ & \begin{tabular}{|c} 
Servic \\
es
\end{tabular} & Other \\
\hline 95 & Rottler & & & & en & & & & & & & \\
\hline $\begin{array}{r}12 / 2 \\
1 / 18 \\
95 \\
\end{array}$ & \begin{tabular}{|l} 
Dr. \\
Price's \\
Cream \\
Baking \\
Powder
\end{tabular} & G & & & & & & & $\begin{array}{l}\text { X-Dr. } \\
\text { Price' } \\
\text { s } \\
\text { Crea } \\
\text { m } \\
\text { Bakin } \\
\text { g } \\
\text { Powd } \\
\text { er }\end{array}$ & & & \\
\hline $\begin{array}{r}12 / 2 \\
1 / 18 \\
95 \\
\end{array}$ & \begin{tabular}{|l} 
Zahn \\
Arzt - \\
Dr. C. J. \\
Hertich
\end{tabular} & G & & & & & & & & & $\begin{array}{l}\text { Medic } \\
\text { al }\end{array}$ & \\
\hline $\begin{array}{r}12 / 2 \\
1 / 18 \\
95\end{array}$ & \begin{tabular}{|l} 
Peter H. \\
Huck, \\
Dentisc \\
her \\
Rechtse \\
nwalt
\end{tabular} & G & & & & & & & & & legal & \\
\hline $\begin{array}{r}12 / 2 \\
1 / 18 \\
95\end{array}$ & $\begin{array}{l}\text { Meyer's } \\
\text { Hotel }\end{array}$ & $\mathrm{E}$ & & & & & & & & & hotel & \\
\hline $\begin{array}{r}12 / 2 \\
1 / 18 \\
95\end{array}$ & \begin{tabular}{|l} 
Mich. \\
Beauch \\
amp, \\
Tonsori \\
al Artist
\end{tabular} & $\mathrm{E}$ & & & & & & & & & $\begin{array}{l}\text { Cuttin } \\
\text { g/styli } \\
\text { ng hair }\end{array}$ & \\
\hline $\begin{array}{r}12 / 2 \\
1 / 18 \\
95\end{array}$ & \begin{tabular}{|l} 
T. B. \\
Whitled \\
ge \\
Rechtsa \\
nwalt
\end{tabular} & G & & & & & & & & & legal & \\
\hline $\begin{array}{r}12 / 2 \\
1 / 18 \\
95\end{array}$ & $\begin{array}{l}\text { R. W. } \\
\text { Lanning }\end{array}$ & G & & & $\begin{array}{l}\text { X- } \\
\text { Toilett } \\
\text { en }\end{array}$ & & & $\begin{array}{l}\text { X- } \\
\text { Medizi } \\
\text { nen }\end{array}$ & & & & $\begin{array}{l}\text { Chem } \\
\text { ikal'n } \\
\text { (chem }\end{array}$ \\
\hline
\end{tabular}




\begin{tabular}{|c|c|c|c|c|c|c|c|c|c|c|c|c|}
\hline \multicolumn{13}{|c|}{ German-English newspaper } \\
\hline Date & \begin{tabular}{|c||} 
advertis \\
ement \\
title
\end{tabular} & $\begin{array}{c}\text { Germa } \\
\text { n/Engli } \\
\text { sh }\end{array}$ & $\begin{array}{c}\text { Hous } \\
\text { ehol } \\
d \\
\text { furni } \\
\text { shing } \\
\text { s }\end{array}$ & $\begin{array}{l}\text { Clot } \\
\text { hing }\end{array}$ & $\begin{array}{c}\text { Person } \\
\text { al } \\
\text { Items }\end{array}$ & $\begin{array}{c}\text { Househ } \\
\text { old } \\
\text { produc } \\
\text { tion } \\
\text { activity }\end{array}$ & $\begin{array}{l}\text { Hous } \\
\text { ehol } \\
\text { d } \\
\text { prod } \\
\text { uctio } \\
\text { n for } \\
\text { sale }\end{array}$ & $\begin{array}{c}\text { Pharm } \\
\text { aceutic } \\
\text { al }\end{array}$ & $\begin{array}{l}\text { Food } \\
\text { stuffs }\end{array}$ & $\begin{array}{c}\text { Alcoh } \\
\text { ol }\end{array}$ & $\begin{array}{c}\text { Servic } \\
\text { es }\end{array}$ & Other \\
\hline & $\begin{array}{l}\text { Apothe } \\
\text { ker } \\
\text { (difficul } \\
\text { t to } \\
\text { read) }\end{array}$ & & & & $\begin{array}{l}\text { Artikel } \\
\text { Bucher }\end{array}$ & & & & & & & icals) \\
\hline $\begin{array}{r}12 / 2 \\
1 / 18 \\
95\end{array}$ & $\begin{array}{l}\text { Hood's } \\
\text { Sarsapa } \\
\text { rilla }\end{array}$ & $E$ & & & & & & $\begin{array}{l}\text { X- } \\
\text { Hood's } \\
\text { Sarsap } \\
\text { arilla }\end{array}$ & & & & \\
\hline $\begin{array}{r}12 / 2 \\
1 / 18 \\
95 \\
\end{array}$ & \begin{tabular}{|l} 
Kenned \\
y's \\
Medical \\
Discove \\
ry
\end{tabular} & $E$ & & & & & & \begin{tabular}{|l} 
X- \\
Kenney \\
's \\
medica \\
I cure
\end{tabular} & & & & \\
\hline $\begin{array}{r}12 / 2 \\
1 / 18 \\
95 \\
\end{array}$ & \begin{tabular}{|l|} 
Imperial \\
Granum \\
-John \\
Carle \& \\
Sons, \\
New \\
York
\end{tabular} & E & & & & & & & $\begin{array}{l}\text { X- } \\
\text { Impe } \\
\text { rial } \\
\text { Gran } \\
\text { um }\end{array}$ & & & \\
\hline $\begin{array}{r}12 / 2 \\
1 / 18 \\
95\end{array}$ & \begin{tabular}{|l} 
Asthma, \\
Dr. \\
Taft's \\
Asthmal \\
ene
\end{tabular} & E & & & & & & $\begin{array}{l}\text { X-Dr. } \\
\text { Taft's } \\
\text { Asthma } \\
\text { lene }\end{array}$ & & & & \\
\hline $\begin{array}{r}12 / 2 \\
1 / 18 \\
95 \\
\end{array}$ & \begin{tabular}{|l} 
See that \\
hump? \\
Richard \\
son \& \\
DeLong \\
Bros, \\
Philada.
\end{tabular} & $\mathrm{E}$ & & $\begin{array}{l}\text { X- } \\
\text { DeLo } \\
\text { ng } \\
\text { Pat } \\
\text { hook } \\
\text { and } \\
\text { Eye }\end{array}$ & & & & & & & & \\
\hline $\begin{array}{r}12 / 2 \\
1 / 18 \\
95\end{array}$ & $\begin{array}{l}\text { The } \\
\text { Pilgrim, } \\
\text { Geo. }\end{array}$ & $E$ & & & X-book & & & & & & & \\
\hline
\end{tabular}




\begin{tabular}{|c|c|c|c|c|c|c|c|c|c|c|c|c|}
\hline \multicolumn{13}{|c|}{ German-English newspaper } \\
\hline Date & $\begin{array}{c}\text { advertis } \\
\text { ement } \\
\text { title }\end{array}$ & $\begin{array}{c}\text { Germa } \\
\text { n/Engli } \\
\text { sh }\end{array}$ & $\begin{array}{c}\text { Hous } \\
\text { ehol } \\
d \\
\text { furni } \\
\text { shing } \\
\text { s }\end{array}$ & $\begin{array}{l}\text { Clot } \\
\text { hing }\end{array}$ & $\begin{array}{c}\text { Person } \\
\text { al } \\
\text { Items }\end{array}$ & $\begin{array}{c}\text { Househ } \\
\text { old } \\
\text { produc } \\
\text { tion } \\
\text { activity }\end{array}$ & \begin{tabular}{|l} 
Hous \\
ehol \\
$d$ \\
prod \\
uctio \\
n for \\
sale
\end{tabular} & \begin{tabular}{||c} 
Pharm \\
aceutic \\
al
\end{tabular} & $\begin{array}{l}\text { Food } \\
\text { stuffs }\end{array}$ & $\begin{array}{c}\text { Alcoh } \\
\text { ol }\end{array}$ & $\begin{array}{c}\text { Servic } \\
\text { es }\end{array}$ & Other \\
\hline & $\begin{array}{l}\text { Heavfor } \\
\text { d, } \\
\text { Chicago }\end{array}$ & & & & & & & & & & & \\
\hline $\begin{array}{r}12 / 2 \\
1 / 18 \\
95\end{array}$ & $\begin{array}{l}\text { Hale's } \\
\text { Honey }\end{array}$ & $E$ & & & & & & \begin{tabular}{|l|} 
X- \\
Hale's \\
Honey \\
of \\
?doreh \\
ound \\
and \\
Tar, \\
Pike's \\
Tootha \\
che \\
Drops \\
cure
\end{tabular} & & & & \\
\hline $\begin{array}{r}12 / 2 \\
1 / 18 \\
95\end{array}$ & $\begin{array}{l}\text { Brown's } \\
\text { Brauch } \\
\text { Troches } \\
\text { " ? }\end{array}$ & $E$ & & & & & & \begin{tabular}{|l|} 
X- \\
?"Brow \\
n's \\
Brauch \\
? \\
Troche \\
s"
\end{tabular} & & & & \\
\hline $\begin{array}{r}12 / 2 \\
1 / 18 \\
95\end{array}$ & $\begin{array}{l}\text { Aeromo } \\
\text { tore Co. }\end{array}$ & E & & & & & & & & & & \\
\hline $\begin{array}{r}12 / 2 \\
1 / 18 \\
95\end{array}$ & $\begin{array}{l}\text { The } \\
\text { Best, } \\
\text { The } \\
\text { rest, } \\
\text { the test }\end{array}$ & $\mathrm{E}$ & & & & & & $\begin{array}{l}\text { X- } \\
\text { Ayer's } \\
\text { Sarsap } \\
\text { arilla }\end{array}$ & & & & \\
\hline $\begin{array}{r}12 / 2 \\
1 / 18 \\
95 \\
\end{array}$ & $\begin{array}{l}\text { Burns } \\
\text { or } \\
\text { Scalds }\end{array}$ & $E$ & & & & & & & & & & $\begin{array}{l}\text { X-St. } \\
\text { Jacob } \\
\text { s Oil }\end{array}$ \\
\hline $\begin{array}{r}12 / 2 \\
1 / 18 \\
95\end{array}$ & $\begin{array}{l}\text { No-To- } \\
\text { Bac }\end{array}$ & $E$ & & & & & & \begin{tabular}{|l|} 
X-The \\
Sterling \\
Remed
\end{tabular} & & & & \\
\hline
\end{tabular}




\begin{tabular}{|c|c|c|c|c|c|c|c|c|c|c|c|c|}
\hline \multicolumn{13}{|c|}{ German-English newspaper } \\
\hline Date & $\begin{array}{c}\text { advertis } \\
\text { ement } \\
\text { title }\end{array}$ & $\begin{array}{c}\text { Germa } \\
\text { n/Engli } \\
\text { sh }\end{array}$ & $\begin{array}{c}\text { Hous } \\
\text { ehol } \\
d \\
\text { furni } \\
\text { shing } \\
\text { s }\end{array}$ & $\begin{array}{l}\text { Clot } \\
\text { hing }\end{array}$ & $\begin{array}{c}\text { Person } \\
\text { al } \\
\text { Items }\end{array}$ & $\begin{array}{l}\text { Househ } \\
\text { old } \\
\text { produc } \\
\text { tion } \\
\text { activity }\end{array}$ & \begin{tabular}{|l} 
Hous \\
ehol \\
d \\
prod \\
uctio \\
n for \\
sale
\end{tabular} & \begin{tabular}{|c||} 
Pharm \\
aceutic \\
al
\end{tabular} & $\begin{array}{l}\text { Food } \\
\text { stuffs }\end{array}$ & $\begin{array}{c}\text { Alcoh } \\
\text { ol }\end{array}$ & $\begin{array}{c}\text { Servic } \\
\text { es }\end{array}$ & Other \\
\hline & & & & & & & & \begin{tabular}{|l|} 
y Co. \\
Tabacc \\
o habit \\
cure
\end{tabular} & & & & \\
\hline $\begin{array}{r}12 / 2 \\
1 / 18 \\
95\end{array}$ & $\begin{array}{l}\text { Cascare } \\
\text { ts }\end{array}$ & E & & & & & & $\begin{array}{l}\text { X- } \\
\text { Cascare } \\
\text { ts } \\
\text { candy } \\
\text { catharti } \\
\text { c cure }\end{array}$ & & & & \\
\hline $\begin{array}{r}12 / 2 \\
1 / 18 \\
95\end{array}$ & $\begin{array}{l}\text { Rising } \\
\text { Sun } \\
\text { Stove } \\
\text { Polish }\end{array}$ & $E$ & & & & & & & & & & \\
\hline $\begin{array}{r}12 / 2 \\
1 / 18 \\
95 \\
\end{array}$ & $\begin{array}{l}\text { Opium - } \\
\text { J. } \\
\text { Stephe } \\
\text { ns, } \\
\text { Lebano } \\
\text { n, Ohio }\end{array}$ & $\mathrm{E}$ & & & & & & \begin{tabular}{|l} 
X- \\
opium, \\
morphi \\
ne \\
habit \\
cure
\end{tabular} & & & & \\
\hline \begin{tabular}{r|}
$12 / 2$ \\
$1 / 18$ \\
95 \\
\end{tabular} & $\begin{array}{l}\text { Opium - } \\
\text { Woolley } \\
\text { Atlanta }\end{array}$ & $\mathrm{E}$ & & & & & & \begin{tabular}{|l} 
X- \\
opium \\
and \\
Whiske \\
y habit \\
cure
\end{tabular} & & & & \\
\hline $\begin{array}{r}12 / 2 \\
1 / 18 \\
95\end{array}$ & $\begin{array}{l}\text { Piso's } \\
\text { Cure for } \\
\text { Consum } \\
\text { ption }\end{array}$ & $\mathrm{E}$ & & & & & & $\begin{array}{l}\text { X-Piso's } \\
\text { Cure } \\
\text { for } \\
\text { Consu } \\
\text { mption }\end{array}$ & & & & \\
\hline $\begin{array}{r}12 / 2 \\
1 / 18 \\
95\end{array}$ & $\begin{array}{l}\text { Warnun } \\
\text { g - } \\
\text { Gebrue } \\
\text { der } \\
\text { Wilder }\end{array}$ & G & & & & & & & & & & $\begin{array}{l}\text { Waar } \\
\text { en }\end{array}$ \\
\hline
\end{tabular}




\begin{tabular}{|c|c|c|c|c|c|c|c|c|c|c|c|c|}
\hline \multicolumn{13}{|c|}{ German-English newspaper } \\
\hline Date & \begin{tabular}{|c||} 
advertis \\
ement \\
title
\end{tabular} & $\begin{array}{c}\text { Germa } \\
\text { n/Engli } \\
\text { sh }\end{array}$ & $\begin{array}{c}\text { Hous } \\
\text { ehol } \\
\text { d } \\
\text { furni } \\
\text { shing } \\
\text { s }\end{array}$ & $\begin{array}{l}\text { Clot } \\
\text { hing }\end{array}$ & $\begin{array}{c}\text { Person } \\
\text { al } \\
\text { Items }\end{array}$ & $\begin{array}{c}\text { Househ } \\
\text { old } \\
\text { produc } \\
\text { tion } \\
\text { activity }\end{array}$ & \begin{tabular}{|} 
Hous \\
ehol \\
$d$ \\
prod \\
uctio \\
n for \\
sale
\end{tabular} & $\begin{array}{c}\text { Pharm } \\
\text { aceutic } \\
\text { al }\end{array}$ & $\begin{array}{l}\text { Food } \\
\text { stuffs }\end{array}$ & $\begin{array}{l}\text { Alcoh } \\
\text { ol }\end{array}$ & $\begin{array}{c}\text { Servic } \\
\text { es }\end{array}$ & Other \\
\hline $\begin{array}{r}12 / 2 \\
1 / 18 \\
95\end{array}$ & \begin{tabular}{|l} 
Lanning \\
's \\
Apothe \\
ke
\end{tabular} & G & & & & & & & $\begin{array}{l}\text { X- } \\
\text { Oake } \\
\text { s's } \\
\text { Zuck } \\
\text { erko } \\
\text { nt? } \\
\text { (suga } \\
\text { r } \\
\text { cand } \\
\text { y) }\end{array}$ & & & \\
\hline $\begin{array}{r}12 / 2 \\
1 / 18 \\
95 \\
\end{array}$ & $\begin{array}{l}\text { Siebert } \\
\text { \& Sohn }\end{array}$ & G & & & & & & & $\begin{array}{l}\text { X- } \\
\text { Groc } \\
\text { eries }\end{array}$ & & & \\
\hline $\begin{array}{r}12 / 2 \\
1 / 18 \\
95\end{array}$ & $\begin{array}{l}\text { Frau } \\
\text { Rennar } \\
\text { d ? }\end{array}$ & G & & & $\begin{array}{l}\text { X- } \\
\text { Spielsa } \\
\text { chen } \\
\text { (toys) }\end{array}$ & & & & \begin{tabular}{|l} 
X- \\
Wein \\
achts \\
Zuck \\
erkan \\
t, \\
Fruec \\
hten \\
(fruit \\
), \\
Nues \\
sen \\
(nuts \\
)
\end{tabular} & & & \\
\hline $\begin{array}{r}12 / 2 \\
1 / 18 \\
95 \\
\end{array}$ & $\begin{array}{l}\text { Schoettl } \\
\text { er und } \\
\text { Scheuri } \\
\text { ng, } \\
\text { Leichen } \\
\text { bestatt } \\
\text { er und } \\
\text { Einbalsa } \\
\text { mirer } \\
\text { (mortici }\end{array}$ & G & & & & & & & & & & $\begin{array}{l}\text { Saerg } \\
\text { en } \\
\text { (coffi } \\
\text { n) }\end{array}$ \\
\hline
\end{tabular}




\begin{tabular}{|c|c|c|c|c|c|c|c|c|c|c|c|c|}
\hline \multicolumn{13}{|c|}{ German-English newspaper } \\
\hline Date & $\begin{array}{c}\text { advertis } \\
\text { ement } \\
\text { title }\end{array}$ & $\begin{array}{c}\text { Germa } \\
\text { n/Engli } \\
\text { sh }\end{array}$ & $\begin{array}{l}\text { Hous } \\
\text { ehol } \\
\text { d } \\
\text { furni } \\
\text { shing } \\
\text { s }\end{array}$ & $\begin{array}{l}\text { Clot } \\
\text { hing }\end{array}$ & $\mid \begin{array}{c}\text { Person } \\
\text { al } \\
\text { Items }\end{array}$ & $\begin{array}{l}\text { Househ } \\
\text { old } \\
\text { produc } \\
\text { tion } \\
\text { activity }\end{array}$ & \begin{tabular}{|c|} 
Hous \\
ehol \\
d \\
prod \\
uctio \\
n for \\
sale
\end{tabular} & $\begin{array}{c}\text { Pharm } \\
\text { aceutic } \\
\text { al }\end{array}$ & $\begin{array}{l}\text { Food } \\
\text { stuffs }\end{array}$ & $\begin{array}{c}\text { Alcoh } \\
\text { ol }\end{array}$ & $\begin{array}{c}\text { Servic } \\
\text { es }\end{array}$ & Other \\
\hline & $\begin{array}{l}\text { an and } \\
\text { embalm } \\
\text { er) }\end{array}$ & & & & & & & & & & & \\
\hline $\begin{array}{r}12 / 2 \\
1 / 18 \\
95 \\
\\
\end{array}$ & \begin{tabular}{|l} 
Trifft \\
euch \\
die \\
Wahrhe \\
it? - \\
Tokerst \\
u. \\
Bruder
\end{tabular} & G & & $\begin{array}{l}\text { X- } \\
\text { Kleid } \\
\text { er, } \\
\text { Gard } \\
\text { erob } \\
\text { e } \\
\text { waar } \\
\text { en } \\
\text { (clot } \\
\text { hes) }\end{array}$ & & & & & & & & \\
\hline $\begin{array}{r}12 / 2 \\
1 / 18 \\
95 \\
\end{array}$ & $\begin{array}{l}\text { Geht zu } \\
\text { Henry } \\
\text { Okenfus } \\
\mathrm{s}\end{array}$ & G & & & \begin{tabular}{|l} 
X- \\
Stecke \\
npferd \\
e \\
(hobby \\
- \\
horses \\
), \\
Mecha \\
nische \\
s \\
Spielze \\
ug \\
(Mech \\
anical \\
Toys),
\end{tabular} & $\begin{array}{l}\text { Schiebk } \\
\text { arren } \\
\text { (Barro } \\
\text { ws), } \\
\text { Raehm } \\
\text { aschine } \\
\text { n } \\
\text { (sewing } \\
\text { Machin } \\
\text { es), } \\
\text { Waege } \\
\text { n } \\
\text { (wagen } \\
\text { s) }\end{array}$ & & & & & & \\
\hline $\begin{array}{r}12 / 1 \\
7 / 18 \\
98 \\
\\
\end{array}$ & $\begin{array}{l}\text { Genuin } \\
\text { e } \\
\text { Bargain } \\
\text { s in } \\
\text { Capes, C } \\
\text { apes - } \\
\text { Jokerst } \\
\text { Bros. \& }\end{array}$ & $\mathrm{E}$ & & $\begin{array}{l}\text { X- } \\
\text { cape } \\
\text { s }\end{array}$ & & & & & & & & \\
\hline
\end{tabular}




\begin{tabular}{|c|c|c|c|c|c|c|c|c|c|c|c|c|}
\hline \multicolumn{13}{|c|}{ German-English newspaper } \\
\hline Date & $\begin{array}{c}\text { advertis } \\
\text { ement } \\
\text { title }\end{array}$ & $\begin{array}{c}\text { Germa } \\
\text { n/Engli } \\
\text { sh }\end{array}$ & $\begin{array}{c}\text { Hous } \\
\text { ehol } \\
\text { d } \\
\text { furni } \\
\text { shing } \\
\text { s }\end{array}$ & $\begin{array}{l}\text { Clot } \\
\text { hing }\end{array}$ & $\begin{array}{c}\text { Person } \\
\text { al } \\
\text { Items }\end{array}$ & $\begin{array}{c}\text { Househ } \\
\text { old } \\
\text { produc } \\
\text { tion } \\
\text { activity }\end{array}$ & \begin{tabular}{|c|} 
Hous \\
ehol \\
d \\
prod \\
uctio \\
n for \\
sale
\end{tabular} & $\begin{array}{l}\text { Pharm } \\
\text { aceutic } \\
\text { al }\end{array}$ & $\begin{array}{l}\text { Food } \\
\text { stuffs }\end{array}$ & $\begin{array}{c}\text { Alcoh } \\
\text { ol }\end{array}$ & $\begin{array}{c}\text { Servic } \\
\text { es }\end{array}$ & Other \\
\hline & Yealy. & & & & & & & & & & & \\
\hline $\begin{array}{r}12 / 1 \\
7 / 18 \\
98 \\
\end{array}$ & $\begin{array}{l}\text { For } \\
\text { Christm } \\
\text { as. } 10 \text { \& } \\
15 \\
\text { percent } \\
\text { off on } \\
\text { all } \\
\text { shoes, } \\
\text { hy. } \\
\text { Herzog } \\
\text { Shoe } \\
\text { Co. }\end{array}$ & $\mathrm{E}$ & & $\begin{array}{l}X- \\
\text { shoe } \\
\text { s }\end{array}$ & & & & & & & & \\
\hline \begin{tabular}{|r|}
$12 / 1$ \\
$7 / 18$ \\
98
\end{tabular} & $\begin{array}{l}\text { John } \\
\text { Koettin } \\
\text { g }\end{array}$ & $E$ & & & & & & & & & & $\begin{array}{l}\text { Holid } \\
\text { ay } \\
\text { goods }\end{array}$ \\
\hline \begin{tabular}{|r|}
$12 / 1$ \\
$7 / 18$ \\
98
\end{tabular} & $\begin{array}{l}\text { The } \\
\text { semi- } \\
\text { weekly } \\
\text { republic }\end{array}$ & $\mathrm{E}$ & & & $\begin{array}{l}\text { X- } \\
\text { Republ } \\
\text { ic } \\
\text { magazi } \\
\text { ne }\end{array}$ & & & & & & & \\
\hline $\begin{array}{r}12 / 1 \\
7 / 18 \\
98\end{array}$ & $\begin{array}{l}\text { St. } \\
\text { Louis } \\
\text { Globe- } \\
\text { Democr } \\
\text { at }\end{array}$ & $\mathrm{E}$ & & & $\begin{array}{l}\text { X-St. } \\
\text { Louis } \\
\text { Globe } \\
\text { democ } \\
\text { rat }\end{array}$ & & & & & & & \\
\hline $\begin{array}{r}12 / 1 \\
7 / 18 \\
98\end{array}$ & $\begin{array}{l}\text { Rozier's } \\
\text { Cash } \\
\text { Store }\end{array}$ & $\mathrm{E}$ & & & & & & & & & & $\begin{array}{l}\text { gener } \\
\text { al } \\
\text { merc } \\
\text { handi } \\
\text { se }\end{array}$ \\
\hline $\begin{array}{r}12 / 1 \\
7 / 18 \\
98\end{array}$ & $\begin{array}{l}\text { Gus L. } \\
\text { Schoettl } \\
\text { er }\end{array}$ & E & $\begin{array}{l}\text { X- } \\
\text { furni } \\
\text { ture }\end{array}$ & & & & & & & & & \\
\hline $\begin{array}{l}12 / 1 \\
7 / 18\end{array}$ & $\begin{array}{l}\text { Hy. } \\
\text { Herz ? }\end{array}$ & $E$ & & $\begin{array}{l}X- \\
\text { shoe }\end{array}$ & & & & & & & & \\
\hline
\end{tabular}




\begin{tabular}{|c|c|c|c|c|c|c|c|c|c|c|c|c|}
\hline \multicolumn{13}{|c|}{ German-English newspaper } \\
\hline Date & $\begin{array}{c}\text { advertis } \\
\text { ement } \\
\text { title }\end{array}$ & $\begin{array}{c}\text { Germa } \\
\text { n/Engli } \\
\text { sh }\end{array}$ & $\begin{array}{c}\text { Hous } \\
\text { ehol } \\
d \\
\text { furni } \\
\text { shing } \\
\text { s }\end{array}$ & $\begin{array}{l}\text { Clot } \\
\text { hing }\end{array}$ & $\mid \begin{array}{c}\text { Person } \\
\text { al } \\
\text { Items }\end{array}$ & $\begin{array}{c}\text { Househ } \\
\text { old } \\
\text { produc } \\
\text { tion } \\
\text { activity }\end{array}$ & \begin{tabular}{|} 
Hous \\
ehol \\
$d$ \\
prod \\
uctio \\
n for \\
sale
\end{tabular} & $\begin{array}{c}\text { Pharm } \\
\text { aceutic } \\
\text { al }\end{array}$ & \begin{tabular}{|l} 
Food \\
stuffs
\end{tabular} & $\begin{array}{c}\text { Alcoh } \\
\text { ol }\end{array}$ & $\begin{array}{c}\text { Servic } \\
\text { es }\end{array}$ & Other \\
\hline 98 & $\begin{array}{l}\text { Shoe } \\
\text { Co. }\end{array}$ & & & $s$ & & & & & & & & \\
\hline $\begin{array}{r}12 / 1 \\
7 / 18 \\
98\end{array}$ & $\begin{array}{l}\text { Christm } \\
\text { as Trees } \\
\text {-Felix } \\
\text { Thomur } \\
\text { e }\end{array}$ & E & & & & & & & & & & $\begin{array}{l}\text { Christ } \\
\text { mas } \\
\text { Trees }\end{array}$ \\
\hline $\begin{array}{r}12 / 1 \\
7 / 18 \\
98\end{array}$ & $\begin{array}{l}\text { Koettin } \\
\text { g's }\end{array}$ & $\mathrm{E}$ & & & $\begin{array}{l}\text { X- } \\
\text { men's } \\
\text { and } \\
\text { wome } \\
\text { n's } \\
\text { watch } \\
\text { es }\end{array}$ & & & & & & & \\
\hline $\begin{array}{r}12 / 1 \\
7 / 18 \\
98\end{array}$ & Herald & $E$ & & & $\begin{array}{l}\text { X- } \\
\text { Herald } \\
\text { newsp } \\
\text { aper }\end{array}$ & & & & & & & \\
\hline $\begin{array}{r}12 / 1 \\
7 / 18 \\
98\end{array}$ & $\begin{array}{l}\text { Rutledg } \\
\text { e \& Co. } \\
\text { and F. } \\
\text { E. } \\
\text { Delassu } \\
\text { s }\end{array}$ & E & & & & & & $\begin{array}{l}\text { X- } \\
\text { Chamb } \\
\text { erlain's } \\
\text { Pain } \\
\text { Balm }\end{array}$ & & & & \\
\hline $\begin{array}{r}12 / 1 \\
7 / 18 \\
98\end{array}$ & $\begin{array}{l}\text { Rutledg } \\
\text { e \& Co. }\end{array}$ & $E$ & & & $\begin{array}{l}\text { X- } \\
\text { holida } \\
\text { y } \\
\text { perfu } \\
\text { mes, } \\
\text { toilet } \\
\text { article } \\
\text { s }\end{array}$ & & & & & & & \\
\hline $\begin{array}{r}12 / 1 \\
7 / 18 \\
98\end{array}$ & $\begin{array}{l}\text { Rutledg } \\
\text { e \& Co. }\end{array}$ & $\mathrm{E}$ & & & & & & & & & & $\begin{array}{l}\text { Christ } \\
\text { mas } \\
\text { prese } \\
\text { nts }\end{array}$ \\
\hline
\end{tabular}




\begin{tabular}{|c|c|c|c|c|c|c|c|c|c|c|c|c|}
\hline \multicolumn{13}{|c|}{ German-English newspaper } \\
\hline Date & $\begin{array}{c}\text { advertis } \\
\text { ement } \\
\text { title }\end{array}$ & \begin{tabular}{|c} 
Germa \\
n/Engli \\
sh
\end{tabular} & \begin{tabular}{|} 
Hous \\
ehol \\
d \\
furni \\
shing \\
s
\end{tabular} & $\begin{array}{l}\text { Clot } \\
\text { hing }\end{array}$ & $\mid \begin{array}{c}\text { Person } \\
\text { al } \\
\text { Items }\end{array}$ & $\begin{array}{c}\text { Househ } \\
\text { old } \\
\text { produc } \\
\text { tion } \\
\text { activity }\end{array}$ & \begin{tabular}{|c} 
Hous \\
ehol \\
$d$ \\
prod \\
uctio \\
$n$ for \\
sale
\end{tabular} & $\begin{array}{c}\text { Pharm } \\
\text { aceutic } \\
\text { al }\end{array}$ & $\begin{array}{l}\text { Food } \\
\text { stuffs }\end{array}$ & $\begin{array}{c}\text { Alcoh } \\
\text { ol }\end{array}$ & $\begin{array}{c}\text { Servic } \\
\text { es }\end{array}$ & Other \\
\hline $\begin{array}{r}12 / 1 \\
7 / 18 \\
98 \\
\end{array}$ & $\begin{array}{l}\text { J. } \\
\text { Tlapek } \\
\text { Imp. \& } \\
\text { Lumber } \\
\text { Co. }\end{array}$ & $E$ & & & & & & & & & & \\
\hline $\begin{array}{r}12 / 1 \\
7 / 18 \\
98\end{array}$ & M.Vieh & $E$ & & & $\begin{array}{l}\text { X- } \\
\text { Christ } \\
\text { mas } \\
\text { Toys }\end{array}$ & & & & & & & \\
\hline $\begin{array}{r}12 / 1 \\
7 / 18 \\
98\end{array}$ & $\begin{array}{l}\text { Rutledg } \\
\text { e \& Co's }\end{array}$ & $E$ & & & $\begin{array}{l}\text { X- } \\
\text { Toilet } \\
\text { Sets, } \\
\text { Manic } \\
\text { ure } \\
\text { Sets, } \\
\text { work } \\
\text { boxes, } \\
\text { glove } \\
\text { boxes, } \\
\text { accord } \\
\text { eons, } \\
\text { novelti } \\
\text { es, } \\
\text { Christ } \\
\text { mas } \\
\text { cards }\end{array}$ & & & & & & & \\
\hline $\begin{array}{r}12 / 1 \\
7 / 18 \\
98 \\
\end{array}$ & $\begin{array}{l}\text { Mrs. } \\
\text { Kennar } \\
\text { d's } \\
\text { restaur } \\
\text { ant }\end{array}$ & $\mathrm{E}$ & & & & & & & $\begin{array}{l}\text { X- } \\
\text { Xmas } \\
\text { candi } \\
\text { es, } \\
\text { cakes } \\
\text { oran } \\
\text { oran, } \\
\text { ges, } \\
\text { bana } \\
\text { nas, } \\
\text { Figs, }\end{array}$ & & & \\
\hline
\end{tabular}




\begin{tabular}{|c|c|c|c|c|c|c|c|c|c|c|c|c|}
\hline \multicolumn{13}{|c|}{ German-English newspaper } \\
\hline Date & $\begin{array}{c}\text { advertis } \\
\text { ement } \\
\text { title }\end{array}$ & $\begin{array}{c}\text { Germa } \\
\text { n/Engli } \\
\text { sh }\end{array}$ & \begin{tabular}{|c} 
Hous \\
ehol \\
$d$ \\
furni \\
shing \\
s
\end{tabular} & $\begin{array}{l}\text { Clot } \\
\text { hing }\end{array}$ & $\mid \begin{array}{c}\text { Person } \\
\text { al } \\
\text { Items }\end{array}$ & $\begin{array}{l}\text { Househ } \\
\text { old } \\
\text { produc } \\
\text { tion } \\
\text { activity }\end{array}$ & \begin{tabular}{|c} 
Hous \\
ehol \\
$d$ \\
prod \\
uctio \\
n for \\
sale
\end{tabular} & $\begin{array}{l}\text { Pharm } \\
\text { aceutic } \\
\text { al }\end{array}$ & $\begin{array}{l}\text { Food } \\
\text { stuffs }\end{array}$ & $\begin{array}{c}\text { Alcoh } \\
\text { ol }\end{array}$ & \begin{tabular}{|c} 
Servic \\
es
\end{tabular} & Other \\
\hline & & & & & & & & & nuts & & & \\
\hline $\begin{array}{r}12 / 2 \\
1 / 18 \\
95 \\
\end{array}$ & $\begin{array}{l}\text { Warnin } \\
\text { g! } \\
\text { Warnin } \\
\text { g! - } \\
\text { Wilder } \\
\text { Bros }\end{array}$ & $\mathrm{E}$ & & & & & & & & & & $\begin{array}{l}\text { Holid } \\
\text { ay } \\
\text { gifts }\end{array}$ \\
\hline $\begin{array}{r}12 / 2 \\
1 / 18 \\
95\end{array}$ & $\begin{array}{l}\text { Schoettl } \\
\text { er \& } \\
\text { Scheuri } \\
\text { ng }\end{array}$ & E & & & & & & & & & $\begin{array}{l}\text { Undert } \\
\text { akers } \\
\& \\
\text { Embal } \\
\text { mers }\end{array}$ & $\begin{array}{l}\text { Caske } \\
\text { ts, } \\
\text { Coffin } \\
\text { s, } \\
\text { Burial } \\
\text { Robes }\end{array}$ \\
\hline $\begin{array}{r}12 / 2 \\
1 / 18 \\
95 \\
\end{array}$ & $\begin{array}{l}\text { Does it } \\
\text { Strike } \\
\text { You? - } \\
\text { Jokerst } \\
\text { \& Bro. }\end{array}$ & $\mathrm{E}$ & $x$ & $x$ & & & & & & & & \\
\hline $\begin{array}{r}12 / 2 \\
1 / 18 \\
95\end{array}$ & $\begin{array}{l}\text { Go to } \\
\text { Hy. } \\
\text { Okenfus } \\
\text { s }\end{array}$ & E & $\begin{array}{l}\text { X- } \\
\text { Banq } \\
\text { uet } \\
\text { Lamp } \\
\text { s, } \\
\text { Hang } \\
\text { ing } \\
\text { Lamp } \\
\text { s, } \\
\text { Stan } \\
\text { d } \\
\text { Lamp } \\
\text { s, }\end{array}$ & & $\begin{array}{l}\text { X- } \\
\text { Veloci } \\
\text { pedes, } \\
\text { Hobby } \\
\text { Horses } \\
\text {, } \\
\text { Mecha } \\
\text { nical } \\
\text { Toys }\end{array}$ & $\begin{array}{l}\text { X- } \\
\text { Wagon } \\
\text { s, } \\
\text { Barrow } \\
\text { s, } \\
\text { Sewing } \\
\text { Machin } \\
\text { es }\end{array}$ & & & & & & \\
\hline $\begin{array}{r}12 / 2 \\
1 / 18 \\
95\end{array}$ & $\begin{array}{l}\text { Koettin } \\
\text { g's }\end{array}$ & E & & & & & & & & & & $\begin{array}{l}\text { Xmas } \\
\text { Good } \\
\mathrm{s}\end{array}$ \\
\hline $\begin{array}{r}12 / 2 \\
1 / 18 \\
95\end{array}$ & $\begin{array}{l}\text { Siebert } \\
\& \text { Son }\end{array}$ & $\mathrm{E}$ & & & & & & & $\begin{array}{l}\text { X- } \\
\text { Coffe } \\
\text { e }\end{array}$ & & & \\
\hline
\end{tabular}




\begin{tabular}{|c|c|c|c|c|c|c|c|c|c|c|c|c|}
\hline \multicolumn{13}{|c|}{ German-English newspaper } \\
\hline Date & $\begin{array}{c}\text { advertis } \\
\text { ement } \\
\text { title }\end{array}$ & $\begin{array}{c}\text { Germa } \\
\text { n/Engli } \\
\text { sh }\end{array}$ & \begin{tabular}{|c} 
Hous \\
ehol \\
$d$ \\
furni \\
shing \\
s
\end{tabular} & $\begin{array}{l}\text { Clot } \\
\text { hing }\end{array}$ & $\begin{array}{c}\text { Person } \\
\text { al } \\
\text { Items }\end{array}$ & $\begin{array}{l}\text { Househ } \\
\text { old } \\
\text { produc } \\
\text { tion } \\
\text { activity }\end{array}$ & \begin{tabular}{|c|} 
Hous \\
ehol \\
$d$ \\
prod \\
uctio \\
$n$ for \\
sale
\end{tabular} & $\begin{array}{c}\text { Pharm } \\
\text { aceutic } \\
\text { al }\end{array}$ & $\mid \begin{array}{l}\text { Food } \\
\text { stuffs }\end{array}$ & $\begin{array}{c}\text { Alcoh } \\
\text { ol }\end{array}$ & $\begin{array}{c}\text { Servic } \\
\text { es }\end{array}$ & Other \\
\hline \begin{tabular}{r|}
$12 / 2$ \\
$1 / 18$ \\
95
\end{tabular} & $\begin{array}{l}\text { Baum } \\
\text { Stark's } \\
\text { Saloon }\end{array}$ & $\mathrm{E}$ & & & & & & & \begin{tabular}{|l} 
X- \\
Wien \\
er \\
Wurs \\
t
\end{tabular} & & & \\
\hline \begin{tabular}{|r|}
$12 / 2$ \\
$1 / 18$ \\
95 \\
\end{tabular} & $\begin{array}{l}\text { Koettin } \\
\text { g's } \\
\text { Jewelry } \\
\text { Store }\end{array}$ & $E$ & & & & & & & & & & $\begin{array}{l}\text { Xmas } \\
\text { Good } \\
\text { s }\end{array}$ \\
\hline \begin{tabular}{|r|}
$12 / 2$ \\
$1 / 18$ \\
95 \\
\end{tabular} & $\begin{array}{l}\text { Mrs. } \\
\text { Kennar } \\
\text { d }\end{array}$ & $E$ & & & $\begin{array}{l}X- \\
\text { Xmas } \\
\text { toys }\end{array}$ & & & & \begin{tabular}{|l|} 
X- \\
Xmas \\
candi \\
es, \\
fruits \\
, nuts
\end{tabular} & & & \\
\hline $\begin{array}{r}12 / 2 \\
1 / 18 \\
95\end{array}$ & $\begin{array}{l}\text { Siebert } \\
\& \text { Son }\end{array}$ & $\mathrm{E}$ & & & & & & & \begin{tabular}{|l} 
X- \\
oran \\
ges, \\
lemo \\
ns, \\
dates \\
, figs
\end{tabular} & & & \\
\hline \begin{tabular}{|r|}
$12 / 2$ \\
$1 / 18$ \\
95
\end{tabular} & $\begin{array}{l}\text { Lanning } \\
\text { 's Drug } \\
\text { Store }\end{array}$ & $E$ & & & & & & & \begin{tabular}{|l|} 
X- \\
Oake \\
s' $^{\prime}$ \\
Candi \\
es
\end{tabular} & & & \\
\hline $\begin{array}{r}12 / 2 \\
1 / 18 \\
95\end{array}$ & $\begin{array}{l}\text { Siebert } \\
\text { \& Son }\end{array}$ & $\mathrm{E}$ & & & & & & & \begin{tabular}{|l|} 
X- \\
groce \\
ries
\end{tabular} & & & \\
\hline $\begin{array}{r}12 / 2 \\
1 / 18 \\
95\end{array}$ & $\begin{array}{l}\text { Gus L. } \\
\text { Schoettl } \\
\text { er }\end{array}$ & $\mathrm{E}$ & & & & & & & & & $\begin{array}{l}\text { Uphols } \\
\text { tering } \\
\text { and } \\
\text { repair }\end{array}$ & \\
\hline $\begin{array}{l}12 / 2 \\
1 / 18\end{array}$ & $\begin{array}{l}\text { Ayer's } \\
\text { Narsapa }\end{array}$ & E & & & & & & $\begin{array}{l}\text { X- } \\
\text { Ayer's }\end{array}$ & & & & \\
\hline
\end{tabular}




\begin{tabular}{|c|c|c|c|c|c|c|c|c|c|c|c|c|}
\hline \multicolumn{13}{|c|}{ German-English newspaper } \\
\hline Date & $\begin{array}{c}\text { advertis } \\
\text { ement } \\
\text { title }\end{array}$ & $\begin{array}{c}\text { Germa } \\
\text { n/Engli } \\
\text { sh }\end{array}$ & $\begin{array}{c}\text { Hous } \\
\text { ehol } \\
d \\
\text { furni } \\
\text { shing } \\
s\end{array}$ & $\begin{array}{l}\text { Clot } \\
\text { hing }\end{array}$ & $\mid \begin{array}{c}\text { Person } \\
\text { al } \\
\text { Items }\end{array}$ & $\begin{array}{c}\text { Househ } \\
\text { old } \\
\text { produc } \\
\text { tion } \\
\text { activity }\end{array}$ & \begin{tabular}{|c|} 
Hous \\
ehol \\
$d$ \\
prod \\
uctio \\
n for \\
sale
\end{tabular} & $\begin{array}{c}\text { Pharm } \\
\text { aceutic } \\
\text { al }\end{array}$ & $\begin{array}{l}\text { Food } \\
\text { stuffs }\end{array}$ & $\begin{array}{c}\text { Alcoh } \\
\text { ol }\end{array}$ & \begin{tabular}{|c} 
Servic \\
es
\end{tabular} & Other \\
\hline 95 & $\begin{array}{l}\text { rilla } \\
\text { medicin } \\
\text { e }\end{array}$ & & & & & & & $\begin{array}{l}\text { Narsap } \\
\text { arilla } \\
\text { medici } \\
\text { ne }\end{array}$ & & & & \\
\hline $\begin{array}{r}12 / 2 \\
1 / 18 \\
95\end{array}$ & $\begin{array}{l}\text { Kohm \& } \\
\text { Petrequ } \\
\text { in }\end{array}$ & $\mathrm{E}$ & & & & & & & & & & \\
\hline $\begin{array}{r}12 / 2 \\
1 / 18 \\
95\end{array}$ & $\begin{array}{l}\text { Lanning } \\
\text { 's Drub } \\
\text { Store }\end{array}$ & E & & & & & & & $\begin{array}{l}\text { X- } \\
\text { Chris } \\
\text { tmas } \\
\text { Candi } \\
\text { es }\end{array}$ & & & $\begin{array}{l}\text { Christ } \\
\text { mas } \\
\text { gifts }\end{array}$ \\
\hline $\begin{array}{r}12 / 2 \\
1 / 18 \\
95\end{array}$ & $\begin{array}{l}\text { Siebert } \\
\& \text { Son }\end{array}$ & $\mathrm{E}$ & & & & & & & $\begin{array}{l}\text { X- } \\
\text { xmas } \\
\text { candi } \\
\text { es }\end{array}$ & & & \\
\hline $\begin{array}{r}12 / 2 \\
1 / 18 \\
95\end{array}$ & $\begin{array}{l}\text { Koettin } \\
\text { g \& } \\
\text { Hunold }\end{array}$ & $E$ & & & & & & & & & $\begin{array}{l}\text { Undert } \\
\text { akers }\end{array}$ & $\begin{array}{l}\text { Coffin } \\
\text { s, } \\
\text { caske } \\
\text { ts, } \\
\text { robes, } \\
\text { trimm } \\
\text { ings }\end{array}$ \\
\hline $\begin{array}{r}12 / 2 \\
1 / 18 \\
95\end{array}$ & $\begin{array}{l}\text { Siebert } \\
\& \text { Son }\end{array}$ & $\mathrm{E}$ & & & & & & & $\begin{array}{l}\text { X- } \\
\text { groce } \\
\text { ries }\end{array}$ & & & \\
\hline $\begin{array}{r}12 / 2 \\
1 / 18 \\
95 \\
\end{array}$ & $\begin{array}{l}\text { A.B.C. } \\
\text { Bohemi } \\
\text { an ?- } \\
\text { sold at } \\
\text { Ed. } \\
\text { Sielert's } \\
\text { saloon }\end{array}$ & $E$ & & & & & & & & $\begin{array}{l}\text { X- } \\
\text { A.B.C. } \\
\text { Bohe } \\
\text { mian } \\
\text { Beer }\end{array}$ & & \\
\hline $\begin{array}{r}12 / 2 \\
1 / 18 \\
95\end{array}$ & $\begin{array}{l}\text { J.H. } \\
\text { Rottma } \\
n\end{array}$ & E & & & & & & & & $\begin{array}{l}\text { X- } \\
\text { Wiske } \\
\text { ys, }\end{array}$ & & \\
\hline
\end{tabular}




\begin{tabular}{|c|c|c|c|c|c|c|c|c|c|c|c|c|}
\hline \multicolumn{13}{|c|}{ German-English newspaper } \\
\hline Date & \begin{tabular}{|c||} 
advertis \\
ement \\
title
\end{tabular} & $\begin{array}{c}\text { Germa } \\
\text { n/Engli } \\
\text { sh }\end{array}$ & $\begin{array}{c}\text { Hous } \\
\text { ehol } \\
d \\
\text { furni } \\
\text { shing } \\
\text { s }\end{array}$ & $\begin{array}{l}\text { Clot } \\
\text { hing }\end{array}$ & $\begin{array}{c}\text { Person } \\
\text { al } \\
\text { Items }\end{array}$ & $\begin{array}{c}\text { Househ } \\
\text { old } \\
\text { produc } \\
\text { tion } \\
\text { activity }\end{array}$ & $\begin{array}{l}\text { Hous } \\
\text { ehol } \\
\text { d } \\
\text { prod } \\
\text { uctio } \\
\text { n for } \\
\text { sale }\end{array}$ & $\begin{array}{c}\text { Pharm } \\
\text { aceutic } \\
\text { al }\end{array}$ & $\begin{array}{l}\text { Food } \\
\text { stuffs }\end{array}$ & $\begin{array}{c}\text { Alcoh } \\
\text { ol }\end{array}$ & $\begin{array}{c}\text { Servic } \\
\text { es }\end{array}$ & Other \\
\hline & $\begin{array}{l}\text { Distillin } \\
\text { g Co. }\end{array}$ & & & & & & & & & \begin{tabular}{|l} 
Rottm \\
ann's \\
celebr \\
ated \\
"stein \\
haege \\
r" \\
(germ \\
an \\
gin)
\end{tabular} & & \\
\hline $\begin{array}{r}12 / 2 \\
1 / 18 \\
95\end{array}$ & $\begin{array}{l}\text { J.W.Sha } \\
\text { w }\end{array}$ & $\mathrm{E}$ & & & & $\begin{array}{l}\text { X- } \\
\text { Sewing } \\
\text { machin } \\
\text { es }\end{array}$ & & & & & & \\
\hline $\begin{array}{r}12 / 2 \\
1 / 18 \\
95\end{array}$ & $\begin{array}{l}\text { Ayer's } \\
\text { Pills }\end{array}$ & $E$ & & & & & & $\begin{array}{l}\text { X- } \\
\text { Ayer's } \\
\text { Pills }\end{array}$ & & & & \\
\hline $\begin{array}{r}12 / 2 \\
1 / 18 \\
95\end{array}$ & $\begin{array}{l}\text { Frank } \\
\text { U. } \\
\text { Geiler }\end{array}$ & G & & & & & & & & & $\begin{array}{l}\text { Contra } \\
\text { ctor } \\
\text { und } \\
\text { Baume } \\
\text { ister }\end{array}$ & \\
\hline $\begin{array}{r}12 / 2 \\
1 / 18 \\
95 \\
\end{array}$ & \begin{tabular}{|l} 
Lumber \\
Yard - \\
R. \\
Wehner \\
und \\
Sohn
\end{tabular} & G & & & & & & & & & & \\
\hline $\begin{array}{r}12 / 2 \\
1 / 18 \\
95\end{array}$ & $\begin{array}{l}\text { Great } \\
\text { Chicago } \\
\text { Tailors } \\
\text { (hard to } \\
\text { read) }\end{array}$ & E & & & & & & & & & & \\
\hline $\begin{array}{r}12 / 2 \\
1 / 18 \\
95\end{array}$ & \begin{tabular}{|l} 
Brewing \\
$\&$ \\
Lighting \\
Ass'n.
\end{tabular} & $\mathrm{E}$ & & & & & & & & X-Beer & & $\begin{array}{l}\text { pure } \\
\text { ice }\end{array}$ \\
\hline
\end{tabular}




\begin{tabular}{|c|c|c|c|c|c|c|c|c|c|c|c|c|}
\hline \multicolumn{13}{|c|}{ German-English newspaper } \\
\hline Date & $\begin{array}{c}\text { advertis } \\
\text { ement } \\
\text { title }\end{array}$ & $\begin{array}{c}\text { Germa } \\
\text { n/Engli } \\
\text { sh }\end{array}$ & $\begin{array}{c}\text { Hous } \\
\text { ehol } \\
\text { d } \\
\text { furni } \\
\text { shing } \\
\text { s }\end{array}$ & $\begin{array}{l}\text { Clot } \\
\text { hing }\end{array}$ & $\begin{array}{c}\text { Person } \\
\text { al } \\
\text { Items }\end{array}$ & $\begin{array}{l}\text { Househ } \\
\text { old } \\
\text { produc } \\
\text { tion } \\
\text { activity }\end{array}$ & \begin{tabular}{|l} 
Hous \\
ehol \\
d \\
prod \\
uctio \\
n for \\
sale
\end{tabular} & $\begin{array}{c}\text { Pharm } \\
\text { aceutic } \\
\text { al }\end{array}$ & $\begin{array}{l}\text { Food } \\
\text { stuffs }\end{array}$ & $\begin{array}{c}\text { Alcoh } \\
\text { ol }\end{array}$ & $\mid \begin{array}{c}\text { Servic } \\
\text { es }\end{array}$ & Other \\
\hline $\begin{array}{r}12 / 2 \\
1 / 18 \\
95\end{array}$ & $\begin{array}{l}\text { Brau u. } \\
\text { Leucht } \\
\text { Associat } \\
\text { ion }\end{array}$ & G & & & & & & & & X-Beer & & $\begin{array}{l}\text { Reine } \\
\mathrm{m} \text { Eis }\end{array}$ \\
\hline $\begin{array}{r}12 / 2 \\
1 / 18 \\
95 \\
\end{array}$ & $\begin{array}{l}\text { Joseph } \\
\text { Fitzkam } \\
\text { Haar } \\
\text { Kuenstl } \\
\text { er }\end{array}$ & G & & & $\begin{array}{l}\text { X- } \\
\text { Cigarr } \\
\text { en }\end{array}$ & & & & & & Barber & \\
\hline $\begin{array}{r}12 / 2 \\
1 / 18 \\
95 \\
\end{array}$ & $\begin{array}{l}\text { Union } \\
\text { Halle } \\
\text { Saloon- } \\
\text { Eduard } \\
\text { Siebert }\end{array}$ & G & & & $\begin{array}{l}\text { X- } \\
\text { Cigarr } \\
\text { en }\end{array}$ & & & & & & & \\
\hline $\begin{array}{r}12 / 2 \\
1 / 18 \\
95\end{array}$ & $\begin{array}{l}\text { Chas } \\
\text { Rozier } \\
\text { Advokat }\end{array}$ & G & & & & & & & & & Legal & \\
\hline $\begin{array}{r}12 / 2 \\
1 / 18 \\
95\end{array}$ & $\begin{array}{l}\text { Henry L. } \\
\text { Rozier, } \\
\text { Bankier }\end{array}$ & G & & & & & & & & & Banker & \\
\hline $\begin{array}{r}12 / 2 \\
1 / 18 \\
95\end{array}$ & $\begin{array}{l}\text { Demore } \\
\text { st's } \\
\text { Magazi } \\
\text { ne }\end{array}$ & $\mathrm{E}$ & & & $\begin{array}{l}\text { X- } \\
\text { Demor } \\
\text { est's } \\
\text { magazi } \\
\text { ne }\end{array}$ & & & & & & & \\
\hline
\end{tabular}


APPENDIX B

ARCHAEOLOGICAL DATABASES 


\begin{tabular}{|c|c|c|c|c|c|c|c|c|c|}
\hline \multicolumn{10}{|c|}{ Archaeological Analysis } \\
\hline $\begin{array}{c}\text { Catalog } \\
\text { ue \# }\end{array}$ & Unit & 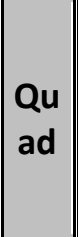 & $\mid \begin{array}{c}\text { Lev } \\
\text { el }\end{array}$ & $\begin{array}{c}\text { Time } \\
\text { peri } \\
\text { od }\end{array}$ & Amou & $\begin{array}{l}\text { Household } \\
\text { Maintenan } \\
\text { ce-Other }\end{array}$ & \begin{tabular}{|c|} 
Househol \\
d \\
Maintena \\
nce- \\
Ceramic
\end{tabular} & $\begin{array}{c}\text { Household } \\
\text { Maintenance- } \\
\text { Glass }\end{array}$ & $\begin{array}{c}\text { Architectural } \\
\text { Items }\end{array}$ \\
\hline $\begin{array}{l}23 S G 27 \\
2-14\end{array}$ & $\begin{array}{l}110 R \\
30\end{array}$ & 2 & & ZLO & 1 & & & & \\
\hline $\begin{array}{l}23 S G 27 \\
2-14\end{array}$ & $\begin{array}{l}110 R \\
30\end{array}$ & 2 & 2 & ZLO & 1 & & & & \\
\hline $\begin{array}{l}23 S G 27 \\
2-14\end{array}$ & $\begin{array}{l}110 R \\
30\end{array}$ & 2 & 2 & ZLO & 6 & & & & \\
\hline $\begin{array}{l}23 S G 27 \\
2-14\end{array}$ & $\begin{array}{l}110 R \\
30\end{array}$ & 2 & 2 & ZLO & 1 & & & & \\
\hline \begin{tabular}{|l|}
$23 S G 27$ \\
$2-14$
\end{tabular} & $\begin{array}{l}110 R \\
30\end{array}$ & 2 & 2 & ZLO & 1 & & & & \\
\hline $\begin{array}{l}23 S G 27 \\
2-14\end{array}$ & $\begin{array}{l}110 R \\
30\end{array}$ & 2 & 2 & ZLO & 1 & & & & \\
\hline \begin{tabular}{|l|} 
23SG27 \\
2-14 \\
Comlar \\
a Park \\
Mount \\
ain \\
Bike \\
Trail \\
\end{tabular} & $\begin{array}{l}110 R \\
30\end{array}$ & 2 & 2 & ZLO & & $\begin{array}{l}\text { frags. Brass } \\
\text { scrap }\end{array}$ & & & \\
\hline \begin{tabular}{|l|}
$23 S G 27$ \\
$2-14$
\end{tabular} & $\begin{array}{l}110 R \\
30\end{array}$ & 2 & 2 & ZLO & & $\begin{array}{l}\text { frag. Lead; } \\
\text { sheet-like, } \\
\text { not sprue }\end{array}$ & & & \\
\hline $\begin{array}{l}23 S G 27 \\
2-14\end{array}$ & $\begin{array}{l}110 R \\
30\end{array}$ & 2 & 2 & ZLO & 11 & & & & \\
\hline \begin{tabular}{|l|}
$23 S G 27$ \\
$2-14$
\end{tabular} & $\begin{array}{l}110 R \\
30\end{array}$ & 2 & 2 & ZLO & 2 & & & & \\
\hline \begin{tabular}{|l|}
$23 S G 27$ \\
$2-14$
\end{tabular} & $\begin{array}{l}110 R \\
30\end{array}$ & 2 & 2 & ZLO & 6 & & & & \\
\hline \begin{tabular}{|l|}
$23 S G 27$ \\
$2-14$
\end{tabular} & $\begin{array}{l}110 R \\
30\end{array}$ & 2 & 2 & ZLO & 1 & & & & \\
\hline $\begin{array}{l}23 S G 27 \\
2-14\end{array}$ & $\begin{array}{l}110 R \\
30\end{array}$ & 2 & 2 & ZLO & 2 & & & & \\
\hline \begin{tabular}{|l|}
$23 S G 27$ \\
$2-14$
\end{tabular} & $\begin{array}{l}110 R \\
30\end{array}$ & 2 & 2 & ZLO & 58 & & & & $\begin{array}{l}\text { frags, lime } \\
\text { mortar/plaste } \\
\text { r (20 are gray) }\end{array}$ \\
\hline $23 S G 27$ & $110 R$ & 2 & 2 & ZLO & 7 & & & & \\
\hline
\end{tabular}




\begin{tabular}{|c|c|c|c|c|c|c|c|c|c|}
\hline \multicolumn{10}{|c|}{ Archaeological Analysis } \\
\hline $\begin{array}{c}\text { Catalog } \\
\text { ue \# }\end{array}$ & Unit & 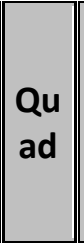 & $\left(\begin{array}{c}\text { Lev } \\
\text { el }\end{array}\right.$ & $\mid \begin{array}{c}\text { Time } \\
\text { peri } \\
\text { od }\end{array}$ & \begin{tabular}{c||} 
Amou \\
$\mathrm{nt}$
\end{tabular} & \begin{tabular}{|c||} 
Household \\
Maintenan \\
ce-Other
\end{tabular} & \begin{tabular}{|c|} 
Househol \\
d \\
Maintena \\
nce- \\
Ceramic \\
\end{tabular} & $\begin{array}{l}\text { Household } \\
\text { Maintenance- } \\
\text { Glass }\end{array}$ & $\begin{array}{l}\text { Architectural } \\
\text { Items }\end{array}$ \\
\hline $2-14$ & 30 & & & & & & & & \\
\hline \begin{tabular}{|l|}
$23 S G 27$ \\
$2-14$
\end{tabular} & $\begin{array}{l}110 \mathrm{R} \\
30\end{array}$ & 2 & & 2 ZLO & 7 & & & & \\
\hline \begin{tabular}{|l|}
$23 S G 27$ \\
$2-15$
\end{tabular} & $\begin{array}{l}110 R \\
30\end{array}$ & 2 & & 3 ZLO & 1 & & & $\begin{array}{l}\text { body frag. } \\
\text { Medium- } \\
\text { green/yellow- } \\
\text { green vessel } \\
\text { glass (probably } \\
\text { bottle) }\end{array}$ & \\
\hline $\begin{array}{l}23 S G 27 \\
2-15\end{array}$ & $\begin{array}{l}110 \mathrm{R} \\
30\end{array}$ & 2 & & 3 ZLO & 4 & & & $\begin{array}{l}\text { frags, clear } \\
\text { vessel glass }\end{array}$ & \\
\hline \begin{tabular}{|l|}
$23 S G 27$ \\
$2-15$
\end{tabular} & $\begin{array}{l}110 \mathrm{R} \\
30\end{array}$ & 2 & & 3 ZLO & 6 & & & & $\begin{array}{l}\text { frags, light to } \\
\text { medium blue } \\
\text { window glass }\end{array}$ \\
\hline \begin{tabular}{|l|}
$23 S G 27$ \\
$2-15$
\end{tabular} & $\begin{array}{l}110 R \\
30\end{array}$ & 2 & & $3 \mathrm{ZLO}$ & 1 & & & frag, clear glass & \\
\hline \begin{tabular}{|l|}
$23 S G 27$ \\
$2-15$
\end{tabular} & $\begin{array}{l}110 R \\
30\end{array}$ & 2 & & ZZLO & 1 & & & & \\
\hline \begin{tabular}{|l|}
$23 S G 27$ \\
$2-15$
\end{tabular} & $\begin{array}{l}110 \mathrm{R} \\
30\end{array}$ & 2 & & 3 ZLO & 1 & & & & \\
\hline \begin{tabular}{|l|}
$23 S G 27$ \\
$2-15$
\end{tabular} & $\begin{array}{l}110 R \\
30\end{array}$ & 2 & & 3 ZLO & 3 & & & & \\
\hline \begin{tabular}{|l|}
$23 S G 27$ \\
$2-15$
\end{tabular} & $\begin{array}{l}110 \mathrm{R} \\
30\end{array}$ & 2 & & 3 ZLO & & $\begin{array}{l}\text { frags, sheet } \\
\text { brass scrap }\end{array}$ & & & \\
\hline \begin{tabular}{|l|}
$23 S G 27$ \\
$2-15$
\end{tabular} & $\begin{array}{l}110 \mathrm{R} \\
30\end{array}$ & 2 & & 3 ZLO & 1 & & & & \\
\hline \begin{tabular}{|l|}
$23 S G 27$ \\
$2-15$
\end{tabular} & $\begin{array}{l}110 \mathrm{R} \\
30\end{array}$ & 2 & & 3 ZLO & 1 & & & & \\
\hline $\begin{array}{l}23 S G 27 \\
2-15\end{array}$ & $\begin{array}{l}110 R \\
30\end{array}$ & 2 & & ZLO & 1 & & & & \\
\hline \begin{tabular}{|l|}
$23 S G 27$ \\
$2-15$
\end{tabular} & $\begin{array}{l}110 \mathrm{R} \\
30\end{array}$ & 2 & & ZZLO & 1 & & & & \\
\hline \begin{tabular}{|l|}
$23 S G 27$ \\
$2-15$
\end{tabular} & $\begin{array}{l}110 \mathrm{R} \\
30\end{array}$ & 2 & & 3 ZLO & 2 & & & & \\
\hline $\begin{array}{l}23 S G 27 \\
2-15\end{array}$ & $\begin{array}{l}110 R \\
30\end{array}$ & 2 & & $3 \mathrm{ZLO}$ & 21 & & & & $\begin{array}{l}\text { frags, lime } \\
\text { plaster/morta } \\
r\end{array}$ \\
\hline
\end{tabular}




\begin{tabular}{|c|c|c|c|c|c|c|c|c|c|}
\hline \multicolumn{10}{|c|}{ Archaeological Analysis } \\
\hline $\begin{array}{c}\text { Catalog } \\
\text { ue \# }\end{array}$ & Unit & 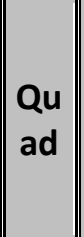 & \begin{tabular}{||c} 
Lev \\
el
\end{tabular} & $\begin{array}{c}\text { Time } \\
\text { peri } \\
\text { od }\end{array}$ & Amou & $\mid \begin{array}{c}\text { Household } \\
\text { Maintenan } \\
\text { ce-Other }\end{array}$ & \begin{tabular}{|l} 
Househol \\
d \\
Maintena \\
nce- \\
Ceramic
\end{tabular} & $\begin{array}{l}\text { Household } \\
\text { Maintenance- } \\
\text { Glass }\end{array}$ & $\begin{array}{l}\text { Architectural } \\
\text { Items }\end{array}$ \\
\hline \begin{tabular}{|l}
$23 S G 27$ \\
$2-15$
\end{tabular} & $\begin{array}{l}110 R \\
30\end{array}$ & 2 & & 3 ZLO & 12 & & & & \\
\hline $\begin{array}{l}\text { 23SG27 } \\
2-14\end{array}$ & $\begin{array}{l}110 R \\
30\end{array}$ & 2 & & 2 ZLO & 1 & & & $\begin{array}{l}\text { frag. Light blue } \\
\text { vessel glass; } \\
\text { burned }\end{array}$ & \\
\hline $\begin{array}{l}23 S G 27 \\
2-14\end{array}$ & $\begin{array}{l}110 R \\
30\end{array}$ & 2 & & 2 ZLO & 2 & & & $\begin{array}{l}\text { clear glass } \\
\text { frags., burned }\end{array}$ & \\
\hline $\begin{array}{l}23 S G 27 \\
2-14\end{array}$ & $\begin{array}{l}110 R \\
30\end{array}$ & 2 & & 2 ZLO & 1 & & & $\begin{array}{l}\text { light blue glass } \\
\text { frag. }\end{array}$ & \\
\hline $\begin{array}{l}23 S G 27 \\
2-14\end{array}$ & $\begin{array}{l}110 R \\
30\end{array}$ & 2 & & 2 ZLO & 1 & & & $\begin{array}{l}\text { frag. Thick blue- } \\
\text { green glass }\end{array}$ & \\
\hline $\begin{array}{l}23 S G 27 \\
2-14\end{array}$ & $\begin{array}{l}110 R \\
30\end{array}$ & 2 & & 2 ZLO & 1 & & & $\begin{array}{l}\text { frag. Medium- } \\
\text { green/yellow- } \\
\text { green bottle } \\
\text { glass }\end{array}$ & \\
\hline \begin{tabular}{|l}
$23 S G 27$ \\
$2-14$
\end{tabular} & $\begin{array}{l}110 \mathrm{R} \\
30\end{array}$ & 2 & & 2 ZLO & 1 & & & $\begin{array}{l}\text { frag. Clear glass; } \\
\text { probably from a } \\
\text { flat-sided bottle }\end{array}$ & \\
\hline \begin{tabular}{|l}
$23 S G 27$ \\
$2-14$
\end{tabular} & $\begin{array}{l}110 \mathrm{R} \\
30\end{array}$ & 2 & & 2 ZLO & 2 & & & $\begin{array}{l}\text { frags, clear } \\
\text { vessel glass }\end{array}$ & \\
\hline $\begin{array}{l}23 S G 27 \\
2-14\end{array}$ & $\begin{array}{l}110 R \\
30\end{array}$ & 2 & & 2 ZLO & 9 & & & & $\begin{array}{l}\text { frag. Blue- } \\
\text { green } \\
\text { window glass }\end{array}$ \\
\hline $\begin{array}{l}23 S G 27 \\
2-14\end{array}$ & $\begin{array}{l}110 R \\
30\end{array}$ & 2 & & 2 ZLO & 1 & & & & \\
\hline \begin{tabular}{|l|}
$23 S G 27$ \\
$2-15$
\end{tabular} & $\begin{array}{l}110 \mathrm{R} \\
30\end{array}$ & 2 & & $3 Z \mathrm{ZLO}$ & 9 & & & & \\
\hline \begin{tabular}{|l}
$23 S G 27$ \\
$2-16$
\end{tabular} & $\begin{array}{l}110 \mathrm{R} \\
30\end{array}$ & 2 & & $4 Z \mathrm{ZLO}$ & 2 & & & $\begin{array}{l}\text { frags. Of glass, } \\
\text { probably green; } \\
\text { heavy patinated }\end{array}$ & \\
\hline $\begin{array}{l}23 S G 27 \\
2-16\end{array}$ & $\begin{array}{l}110 R \\
30\end{array}$ & 2 & & 4 ZLO & 2 & & & $\begin{array}{l}\text { frags, olive- } \\
\text { green glass }\end{array}$ & \\
\hline \begin{tabular}{|l}
$23 S G 27$ \\
$2-16$
\end{tabular} & $\begin{array}{l}110 R \\
30\end{array}$ & 2 & & $4 Z \mathrm{ZLO}$ & 3 & & & $\begin{array}{l}\text { frags, clear } \\
\text { vessel glass }\end{array}$ & \\
\hline \begin{tabular}{|l}
$23 S G 27$ \\
$2-16$
\end{tabular} & $\begin{array}{l}110 R \\
30\end{array}$ & 2 & & 4 ZLO & 9 & & & & $\begin{array}{l}\text { frags, window } \\
\text { pane glass } \\
\text { (clear to blue- }\end{array}$ \\
\hline
\end{tabular}




\begin{tabular}{|c|c|c|c|c|c|c|c|c|c|}
\hline \multicolumn{10}{|c|}{ Archaeological Analysis } \\
\hline \begin{tabular}{c|} 
Catalog \\
ue \#
\end{tabular} & Unit & $\begin{array}{l}\text { Qu } \\
\text { ad }\end{array}$ & \begin{tabular}{||c} 
Lev \\
el
\end{tabular} & $\begin{array}{c}\text { Time } \\
\text { peri } \\
\text { od }\end{array}$ & Amou & $\begin{array}{c}\text { Household } \\
\text { Maintenan } \\
\text { ce-Other }\end{array}$ & $\begin{array}{l}\text { Househol } \\
\text { d } \\
\text { Maintena } \\
\text { nce- } \\
\text { Ceramic }\end{array}$ & $\begin{array}{l}\text { Household } \\
\text { Maintenance- } \\
\text { Glass }\end{array}$ & $\begin{array}{c}\text { Architectural } \\
\text { Items }\end{array}$ \\
\hline & & & & & & & & & green to blue) \\
\hline \begin{tabular}{|l|}
$23 S G 27$ \\
$2-16$
\end{tabular} & $\begin{array}{l}110 R \\
30\end{array}$ & 2 & & ZLO & 1 & & & frags, clear glass & \\
\hline $\begin{array}{l}23 S G 27 \\
2-16\end{array}$ & $\begin{array}{l}110 R \\
30\end{array}$ & 2 & & ZLO & 1 & & & $\begin{array}{l}\text { frags light blue- } \\
\text { green glass }\end{array}$ & \\
\hline \begin{tabular}{|l|}
$23 S G 27$ \\
$2-16$
\end{tabular} & $\begin{array}{l}110 R \\
30\end{array}$ & 2 & & ZLO & 1 & & & & \\
\hline $\begin{array}{l}23 S G 27 \\
2-16\end{array} \mid$ & $\begin{array}{l}110 R \\
30\end{array}$ & 2 & 4 & ZLO & 1 & & & & \\
\hline \begin{tabular}{|l|}
$23 S G 27$ \\
$2-16$
\end{tabular} & $\begin{array}{l}110 R \\
30\end{array}$ & 2 & 4 & ZLO & 3 & & & & \\
\hline \begin{tabular}{|l|}
$23 S G 27$ \\
$2-16$
\end{tabular} & $\begin{array}{l}110 R \\
30\end{array}$ & 2 & 4 & ZLO & 1 & & & & \\
\hline \begin{tabular}{|l|}
$23 S G 27$ \\
$2-16$
\end{tabular} & $\begin{array}{l}110 R \\
30\end{array}$ & 2 & 4 & ZLO & 1 & & & & \\
\hline \begin{tabular}{|l|}
$23 S G 27$ \\
$2-16$
\end{tabular} & $\begin{array}{l}110 R \\
30\end{array}$ & 2 & 4 & ZLO & 1 & & & & \\
\hline \begin{tabular}{|l|}
$23 S G 27$ \\
$2-16$
\end{tabular} & $\begin{array}{l}110 R \\
30\end{array}$ & 2 & & ZLO & 36 & & & & $\begin{array}{l}\text { frags, lime } \\
\text { mortar/plaste } \\
r\end{array}$ \\
\hline $\begin{array}{l}23 \mathrm{SG} 27 \\
2-16\end{array}$ & $\begin{array}{l}110 R \\
30\end{array}$ & 2 & 4 & ZLO & 13 & & & & \\
\hline \begin{tabular}{|l|}
$23 S G 27$ \\
$2-16$
\end{tabular} & $\begin{array}{l}110 R \\
30\end{array}$ & 2 & 4 & ZLO & 2 & & & & \\
\hline \begin{tabular}{|l|}
$23 S G 27$ \\
$2-16$
\end{tabular} & $\begin{array}{l}110 R \\
30\end{array}$ & 2 & 4 & ZLO & 1 & & & & \\
\hline \begin{tabular}{|l|}
$23 S G 27$ \\
$2-17$
\end{tabular} & $\begin{array}{l}110 R \\
30\end{array}$ & 2 & & 20 & 1 & & & $\begin{array}{l}\text { frag. Olive-green } \\
\text { vessel glass, } \\
\text { highly patinated }\end{array}$ & \\
\hline $\begin{array}{l}23 S G 27 \\
2-17\end{array}$ & $\begin{array}{l}110 R \\
30\end{array}$ & 2 & & $\mathrm{ZO}$ & 1 & & & $\begin{array}{l}\text { frag, medium- } \\
\text { green glass }\end{array}$ & \\
\hline \begin{tabular}{|l|}
$23 S G 27$ \\
$2-17$
\end{tabular} & $\begin{array}{l}110 R \\
30\end{array}$ & 2 & & 20 & 1 & & & $\begin{array}{l}\text { frag, light blue } \\
\text { glass }\end{array}$ & \\
\hline \begin{tabular}{|l|}
$23 S G 27$ \\
$2-17$
\end{tabular} & $\begin{array}{l}110 R \\
30\end{array}$ & 2 & & 20 & 2 & & & $\begin{array}{l}\text { frag, clear vessel } \\
\text { glass }\end{array}$ & \\
\hline $23 S G 27$ & $110 R$ & 2 & & 80 & 1 & & & & \\
\hline
\end{tabular}




\begin{tabular}{|c|c|c|c|c|c|c|c|c|c|}
\hline \multicolumn{10}{|c|}{ Archaeological Analysis } \\
\hline $\begin{array}{c}\text { Catalog } \\
\text { ue \# }\end{array}$ & Unit & $\begin{array}{l}\text { Qu } \\
\text { ad }\end{array}$ & \begin{tabular}{||c} 
Lev \\
el
\end{tabular} & $\begin{array}{c}\text { Time } \\
\text { peri } \\
\text { od }\end{array}$ & Amou & $\mid \begin{array}{c}\text { Household } \\
\text { Maintenan } \\
\text { ce-Other }\end{array}$ & \begin{tabular}{|c|} 
Househol \\
d \\
Maintena \\
nce- \\
Ceramic \\
\end{tabular} & $\begin{array}{c}\text { Household } \\
\text { Maintenance- } \\
\text { Glass }\end{array}$ & $\begin{array}{c}\text { Architectural } \\
\text { Items }\end{array}$ \\
\hline $2-17$ & 30 & & & & & & & & \\
\hline \begin{tabular}{|l|}
$23 S G 27$ \\
$2-17$
\end{tabular} & $\begin{array}{l}110 R \\
30\end{array}$ & 2 & & 20 & 1 & & & & $\begin{array}{l}\text { frag, light } \\
\text { blue-green } \\
\text { window glass }\end{array}$ \\
\hline \begin{tabular}{|l|}
$23 S G 27$ \\
$2-17$
\end{tabular} & $\begin{array}{l}110 \mathrm{R} \\
30\end{array}$ & 2 & & 20 & 1 & & & & \\
\hline \begin{tabular}{|l|}
$23 S G 27$ \\
$2-17$
\end{tabular} & $\begin{array}{l}110 R \\
30\end{array}$ & 2 & & 20 & 1 & & & & \\
\hline \begin{tabular}{|l|}
$23 S G 27$ \\
$2-17$
\end{tabular} & $\begin{array}{l}110 \mathrm{R} \\
30\end{array}$ & 2 & & $\mathrm{ZO}$ & 1 & & & & \\
\hline \begin{tabular}{|l|}
$23 S G 27$ \\
$2-17$
\end{tabular} & $\begin{array}{l}110 R \\
30\end{array}$ & 2 & & 20 & 1 & & & & \\
\hline \begin{tabular}{|l|}
$23 S G 27$ \\
$2-17$
\end{tabular} & $\begin{array}{l}110 R \\
30\end{array}$ & 2 & & 20 & 1 & & & & \\
\hline \begin{tabular}{|l|}
$23 S G 27$ \\
$2-17$
\end{tabular} & $\begin{array}{l}110 \mathrm{R} \\
30\end{array}$ & 2 & & 80 & 1 & & & & \\
\hline \begin{tabular}{|l|}
$23 S G 27$ \\
$2-17$
\end{tabular} & $\begin{array}{l}110 \mathrm{R} \\
30\end{array}$ & 2 & & 20 & 1 & & & & \\
\hline \begin{tabular}{|l|}
$23 S G 27$ \\
$2-17$
\end{tabular} & $\begin{array}{l}110 R \\
30\end{array}$ & 2 & & 20 & 11 & & & & $\begin{array}{l}\text { frags, lime } \\
\text { mortar/plaste } \\
r\end{array}$ \\
\hline \begin{tabular}{|l|}
$23 S G 27$ \\
$2-17$
\end{tabular} & $\begin{array}{l}110 \mathrm{R} \\
30\end{array}$ & 2 & & 20 & 3 & & & & \\
\hline \begin{tabular}{|l|}
$23 S G 27$ \\
$2-17$
\end{tabular} & $\begin{array}{l}110 R \\
30\end{array}$ & 2 & & 20 & 1 & & & & \\
\hline \begin{tabular}{|l|}
$23 S G 27$ \\
$2-18$
\end{tabular} & $\begin{array}{l}110 R \\
30\end{array}$ & 2 & & $5 \mathrm{ZO}$ & 1 & & & $\begin{array}{l}\text { frag, clear to } \\
\text { light blue-green } \\
\text { glass }\end{array}$ & \\
\hline \begin{tabular}{|l|}
$23 S G 27$ \\
$2-18$
\end{tabular} & $\begin{array}{l}110 R \\
30\end{array}$ & 2 & & $5 \mathrm{ZO}$ & 1 & & & & \\
\hline \begin{tabular}{|l|}
$23 S G 27$ \\
$2-18$
\end{tabular} & $\begin{array}{l}110 \mathrm{R} \\
30\end{array}$ & 2 & & 20 & 1 & & & & \\
\hline \begin{tabular}{|l|}
$23 S G 27$ \\
$2-18$
\end{tabular} & $\begin{array}{l}110 \mathrm{R} \\
30\end{array}$ & 2 & & 20 & 1 & \begin{tabular}{|l|} 
handle- \\
bowl \\
fragment of \\
a pewter \\
spoon
\end{tabular} & & & \\
\hline
\end{tabular}




\begin{tabular}{|c|c|c|c|c|c|c|c|c|c|}
\hline \multicolumn{10}{|c|}{ Archaeological Analysis } \\
\hline $\begin{array}{c}\text { Catalog } \\
\text { ue \# }\end{array}$ & Unit & Qu & \begin{tabular}{||c} 
Lev \\
el
\end{tabular} & $\mid \begin{array}{c}\text { Time } \\
\text { peri } \\
\text { od }\end{array}$ & Amou & \begin{tabular}{|c||} 
Household \\
Maintenan \\
ce-Other
\end{tabular} & \begin{tabular}{|c|} 
Househol \\
d \\
Maintena \\
nce- \\
Ceramic
\end{tabular} & $\begin{array}{l}\text { Household } \\
\text { Maintenance- } \\
\text { Glass }\end{array}$ & $\begin{array}{c}\text { Architectural } \\
\text { Items }\end{array}$ \\
\hline $\begin{array}{l}23 S G 27 \\
2-18\end{array}$ & $\begin{array}{l}110 R \\
30\end{array}$ & 2 & & $6 Z O$ & 2 & & & & $\begin{array}{l}\text { frags, lime } \\
\text { plaster }\end{array}$ \\
\hline $\begin{array}{l}23 S G 27 \\
2-18\end{array}$ & $\begin{array}{l}110 R \\
30\end{array}$ & 2 & & $6 Z 0$ & 1 & & & & \\
\hline $\begin{array}{l}23 \mathrm{SG} 27 \\
2-18\end{array}$ & $\begin{array}{l}110 R \\
30\end{array}$ & 2 & & $6 Z O$ & 1 & & & & \\
\hline $\begin{array}{l}23 S G 27 \\
2-2\end{array}$ & $\begin{array}{l}150 \mathrm{R} \\
0\end{array}$ & 1 & & 1 ZLO & 1 & & & $\begin{array}{l}\text { frag, olive-green } \\
\text { bottle glass }\end{array}$ & \\
\hline $\begin{array}{l}23 S G 27 \\
2-2\end{array}$ & $\begin{array}{l}150 \mathrm{R} \\
0\end{array}$ & 1 & & 1 ZLO & & $\begin{array}{l}\text { frag, of } \\
\text { brass- } \\
\text { plated iron } \\
\text { molded } \\
\text { object }\end{array}$ & & & \\
\hline $\begin{array}{l}23 S G 27 \\
2-2\end{array}$ & $\begin{array}{l}150 \mathrm{R} \\
0\end{array}$ & 1 & & 1 ZLO & 19 & & & & $\begin{array}{l}\text { white frags, } \\
\text { lime } \\
\text { plaster/morta } \\
r\end{array}$ \\
\hline $\begin{array}{l}23 S G 27 \\
2-2\end{array}$ & $\begin{array}{l}150 \mathrm{R} \\
0\end{array}$ & 1 & & 1 ZLO & 108 & & & & $\begin{array}{l}\text { gray frags, } \\
\text { lime } \\
\text { plaster/morta } \\
\text { r }\end{array}$ \\
\hline $\begin{array}{l}23 S G 27 \\
2-2\end{array}$ & $\begin{array}{l}150 \mathrm{R} \\
0\end{array}$ & 1 & & 1 ZLO & 2 & & & & $\begin{array}{l}\text { gray and } \\
\text { white frags, } \\
\text { lime } \\
\text { plaster/morta } \\
\text { r }\end{array}$ \\
\hline $\begin{array}{l}23 S G 27 \\
2-2\end{array}$ & $\begin{array}{l}150 \mathrm{R} \\
0\end{array}$ & 1 & & 1 ZLO & 1 & & & & \\
\hline $\begin{array}{l}23 S G 27 \\
2-3\end{array}$ & $\begin{array}{l}150 \mathrm{R} \\
0\end{array}$ & 1 & & 2 ZLO & 1 & & & $\begin{array}{l}\text { frag, thin, clear } \\
\text { vessel glass }\end{array}$ & \\
\hline $\begin{array}{l}23 S G 27 \\
2-3\end{array}$ & $\begin{array}{l}150 R \\
0\end{array}$ & 1 & & 2 ZLO & 1 & & & $\begin{array}{l}\text { frag, clear vessel } \\
\text { glass }\end{array}$ & \\
\hline $\begin{array}{l}23 S G 27 \\
2-3\end{array}$ & $\begin{array}{l}150 \mathrm{R} \\
0\end{array}$ & 1 & & 2 ZLO & 1 & & & $\begin{array}{l}\text { frag, blue-green } \\
\text { vessel glass }\end{array}$ & \\
\hline $\begin{array}{l}23 S G 27 \\
2-3\end{array}$ & $\begin{array}{l}150 \mathrm{R} \\
0\end{array}$ & 1 & & 2 ZLO & 1 & & & $\begin{array}{l}\text { frag, thick, clear } \\
\text { vessel glass }\end{array}$ & \\
\hline $23 S G 27$ & $150 \mathrm{R}$ & 1 & & 2 ZLO & 2 & & & tiny frags, clear & \\
\hline
\end{tabular}




\begin{tabular}{|c|c|c|c|c|c|c|c|c|c|}
\hline \multicolumn{10}{|c|}{ Archaeological Analysis } \\
\hline $\begin{array}{c}\text { Catalog } \\
\text { ue \# }\end{array}$ & Unit & $\begin{array}{l}\text { Qu } \\
\text { ad }\end{array}$ & \begin{tabular}{|c} 
Lev \\
el
\end{tabular} & $\mid \begin{array}{c}\text { Time } \\
\text { peri } \\
\text { od }\end{array}$ & Amou & $\begin{array}{c}\text { Household } \\
\text { Maintenan } \\
\text { ce-Other }\end{array}$ & \begin{tabular}{|c|} 
Househol \\
d \\
Maintena \\
nce- \\
Ceramic
\end{tabular} & $\begin{array}{c}\text { Household } \\
\text { Maintenance- } \\
\text { Glass }\end{array}$ & $\begin{array}{l}\text { Architectural } \\
\text { Items }\end{array}$ \\
\hline $2-3$ & 0 & & & & & & & glass & \\
\hline $\begin{array}{l}23 S G 27 \\
2-3\end{array}$ & $\begin{array}{l}150 \mathrm{R} \\
0\end{array}$ & 1 & & 2 ZLO & 1 & & & & $\begin{array}{l}\text { large frag, } \\
\text { thick, light } \\
\text { blue-green } \\
\text { flat glass, } \\
\text { window glass }\end{array}$ \\
\hline $\begin{array}{l}23 S G 27 \\
2-3\end{array}$ & $\begin{array}{l}150 R \\
0\end{array}$ & 1 & & $2 \mathrm{ZLO}$ & 1 & & & & $\begin{array}{l}\text { frag, light } \\
\text { blue-green } \\
\text { window glass }\end{array}$ \\
\hline $\begin{array}{l}23 S G 27 \\
2-3\end{array}$ & $\begin{array}{l}150 R \\
0\end{array}$ & 1 & & 2 ZLO & 1 & & & & $\begin{array}{l}\text { frag, light } \\
\text { blue-green } \\
\text { window glass }\end{array}$ \\
\hline $\begin{array}{l}23 S G 27 \\
2-3\end{array}$ & $\begin{array}{l}150 \mathrm{R} \\
0\end{array}$ & 1 & 2 & 2 ZLO & 1 & & & & \\
\hline $\begin{array}{l}23 S G 27 \\
2-3\end{array}$ & $\begin{array}{l}150 R \\
0\end{array}$ & 1 & & 2 ZLO & & $\begin{array}{l}\text { brass- } \\
\text { plated and } \\
\text { silver- } \\
\text { plated iron } \\
\text { hook }\end{array}$ & & & \\
\hline $\begin{array}{l}23 S G 27 \\
2-3\end{array}$ & $\begin{array}{l}150 \mathrm{R} \\
0\end{array}$ & 1 & 2 & 2 ZLO & 1 & & & & \\
\hline $\begin{array}{l}23 S G 27 \\
2-3\end{array}$ & $\begin{array}{l}150 \mathrm{R} \\
0\end{array}$ & 1 & & 2 ZLO & & $\begin{array}{l}\text { frags, very } \\
\text { thin brass- } \\
\text { plated iron } \\
\text { wire }\end{array}$ & & & \\
\hline $\begin{array}{l}23 S G 27 \\
2-3\end{array}$ & $\begin{array}{l}150 \mathrm{R} \\
0\end{array}$ & 1 & & 2 ZLO & 142 & & & & $\begin{array}{l}\text { white frags, } \\
\text { lime } \\
\text { mortar/plaste } \\
r\end{array}$ \\
\hline $\begin{array}{l}23 S G 27 \\
2-3\end{array}$ & $\begin{array}{l}150 R \\
0\end{array}$ & 1 & & 2 ZLO & 26 & & & & $\begin{array}{l}\text { gray frags, } \\
\text { lime } \\
\text { mortar/plaste } \\
r\end{array}$ \\
\hline $\begin{array}{l}23 S G 27 \\
2-3\end{array}$ & $\begin{array}{l}150 \mathrm{R} \\
0\end{array}$ & 1 & & 2 ZLO & 3 & & & & \\
\hline $\begin{array}{l}\text { 23SG27 } \\
2-3\end{array}$ & $\begin{array}{l}150 R \\
0\end{array}$ & 1 & & 2 ZLO & 2 & & & & \\
\hline
\end{tabular}




\begin{tabular}{|c|c|c|c|c|c|c|c|c|c|}
\hline \multicolumn{10}{|c|}{ Archaeological Analysis } \\
\hline $\begin{array}{c}\text { Catalog } \\
\text { ue \# }\end{array}$ & Unit & $\begin{array}{l}\text { Qu } \\
\text { ad }\end{array}$ & Lev & $\begin{array}{c}\text { Time } \\
\text { peri } \\
\text { od }\end{array}$ & Amou & $\begin{array}{c}\text { Household } \\
\text { Maintenan } \\
\text { ce-Other }\end{array}$ & \begin{tabular}{|l} 
Househol \\
d \\
Maintena \\
nce- \\
Ceramic
\end{tabular} & $\begin{array}{c}\text { Household } \\
\text { Maintenance- } \\
\text { Glass }\end{array}$ & $\begin{array}{c}\text { Architectural } \\
\text { Items }\end{array}$ \\
\hline $\begin{array}{l}23 S G 27 \\
2-4\end{array}$ & $\begin{array}{l}150 R \\
0\end{array}$ & 1 & 3 & ZLO & 1 & & & $\begin{array}{l}\text { frag, clear vessel } \\
\text { glass }\end{array}$ & \\
\hline $\begin{array}{l}23 S G 27 \\
2-4\end{array}$ & $\begin{array}{l}150 \mathrm{R} \\
0\end{array}$ & 1 & 3 & ZLO & 1 & & & $\begin{array}{l}\text { frag, bottle } \\
\text { glass, heavily } \\
\text { patinated, olive- } \\
\text { green or yellow- } \\
\text { green }\end{array}$ & \\
\hline $\begin{array}{l}23 S G 27 \\
2-4\end{array}$ & $\begin{array}{l}150 R \\
0\end{array}$ & 1 & 3 & ZLO & 2 & & & & $\begin{array}{l}\text { large frags, } \\
\text { light blue- } \\
\text { green flat } \\
\text { glass, window } \\
\text { glass, patina }\end{array}$ \\
\hline \begin{tabular}{|l|}
$23 S G 27$ \\
$2-4$
\end{tabular} & $\begin{array}{l}150 \mathrm{R} \\
0\end{array}$ & 1 & 3 & ZLO & 5 & & & $\begin{array}{l}\text { frags, blue- } \\
\text { green flat glass }\end{array}$ & \\
\hline \begin{tabular}{|l|}
$23 S G 27$ \\
$2-4$
\end{tabular} & $\begin{array}{l}150 \mathrm{R} \\
0\end{array}$ & 1 & 3 & ZLO & 1 & & & & \\
\hline \begin{tabular}{|l|}
$23 S G 27$ \\
$2-4$
\end{tabular} & $\begin{array}{l}150 \mathrm{R} \\
0\end{array}$ & 1 & 3 & ZLO & 1 & & & & \\
\hline \begin{tabular}{|l|}
$23 S G 27$ \\
$2-4$
\end{tabular} & $\begin{array}{l}150 \mathrm{R} \\
0\end{array}$ & 1 & 3 & ZLO & 300 & & & & $\begin{array}{l}\text { white frags, } \\
\text { lime } \\
\text { mortar/plaste } \\
r\end{array}$ \\
\hline $\begin{array}{l}23 S G 27 \\
2-4\end{array}$ & $\begin{array}{l}150 R \\
0\end{array}$ & 1 & 3 & ZLO & 9 & & & & $\begin{array}{l}\text { gray frags, } \\
\text { lime } \\
\text { mortar/plaste } \\
r\end{array}$ \\
\hline \begin{tabular}{|l|}
$23 S G 27$ \\
$2-4$
\end{tabular} & $\begin{array}{l}150 \mathrm{R} \\
0\end{array}$ & 1 & 3 & ZLO & 20 & & & & $\begin{array}{l}\text { grey and } \\
\text { white frags, } \\
\text { lime } \\
\text { mortar/plaste } \\
\text { r }\end{array}$ \\
\hline $\begin{array}{l}23 S G 27 \\
2-4\end{array} \mid$ & $\begin{array}{l}150 R \\
0\end{array}$ & 1 & 3 & ZLO & 5 & & & & \\
\hline \begin{tabular}{|l|}
$23 S G 27$ \\
$2-5$
\end{tabular} & $\begin{array}{l}150 R \\
0\end{array}$ & 1 & 4 & ZLO & 1 & & & $\begin{array}{l}\text { frag, mirror, } \\
\text { light blue-green } \\
\text { flat glass with } \\
\text { silver backing }\end{array}$ & \\
\hline $23 S G 27$ & $150 R$ & 1 & 4 & ZLO & 1 & & & frag, clear vessel & \\
\hline
\end{tabular}




\begin{tabular}{|c|c|c|c|c|c|c|c|c|c|}
\hline \multicolumn{10}{|c|}{ Archaeological Analysis } \\
\hline $\begin{array}{c}\text { Catalog } \\
\text { ue \# }\end{array}$ & Unit & $\begin{array}{l}\text { Qu } \\
\text { ad }\end{array}$ & \begin{tabular}{|c} 
Lev \\
el
\end{tabular} & $\mid \begin{array}{c}\text { Time } \\
\text { peri } \\
\text { od }\end{array}$ & Amou & $\begin{array}{c}\text { Household } \\
\text { Maintenan } \\
\text { ce-Other }\end{array}$ & $\begin{array}{c}\text { Househol } \\
d \\
\text { Maintena } \\
\text { nce- } \\
\text { Ceramic }\end{array}$ & $\begin{array}{c}\text { Household } \\
\text { Maintenance- } \\
\text { Glass }\end{array}$ & $\begin{array}{l}\text { Architectural } \\
\text { Items }\end{array}$ \\
\hline $2-5$ & 0 & & & & & & & $\begin{array}{l}\text { glass, } \\
\text { shoulder/neck } \\
\text { of bottle }\end{array}$ & \\
\hline $\begin{array}{l}23 S G 27 \\
2-5\end{array}$ & $\begin{array}{l}150 \mathrm{R} \\
0\end{array}$ & 1 & & $4 \mathrm{ZLO}$ & 1 & & & & \\
\hline $\begin{array}{l}23 S G 27 \\
2-5\end{array}$ & $\begin{array}{l}150 \mathrm{R} \\
0\end{array}$ & 1 & & 4 ZLO & 3 & & & $\begin{array}{l}\text { small frags, clear } \\
\text { glass; probably } \\
\text { vessel glass }\end{array}$ & \\
\hline $\begin{array}{l}23 S G 27 \\
2-5\end{array}$ & $\begin{array}{l}150 \mathrm{R} \\
0\end{array}$ & 1 & & 4 ZLO & 8 & & & & $\begin{array}{l}\text { frags, light } \\
\text { blue-green } \\
\text { window glass }\end{array}$ \\
\hline $\begin{array}{l}23 S G 27 \\
2-5\end{array}$ & $\begin{array}{l}150 R \\
0\end{array}$ & 1 & & 4 ZLO & 5 & & & $\begin{array}{l}\text { frags, burned } \\
\text { glass, clear }\end{array}$ & \\
\hline $\begin{array}{l}23 S G 27 \\
2-5\end{array}$ & $\begin{array}{l}150 R \\
0\end{array}$ & 1 & & 4 ZLO & 3 & & & $\begin{array}{l}\text { frags, burned } \\
\text { glass, light blue }\end{array}$ & \\
\hline $\begin{array}{l}23 S G 27 \\
2-5\end{array}$ & $\begin{array}{l}150 \mathrm{R} \\
0\end{array}$ & 1 & & 4 ZLO & 1 & & & $\begin{array}{l}\text { small, thin piece } \\
\text { of glass, heavily } \\
\text { patinated }\end{array}$ & \\
\hline $\begin{array}{l}23 S G 27 \\
2-5\end{array}$ & $\begin{array}{l}150 \mathrm{R} \\
0\end{array}$ & 1 & & 4 ZLO & 1 & & & & \\
\hline $\begin{array}{l}23 S G 27 \\
2-5\end{array}$ & $\begin{array}{l}150 R \\
0\end{array}$ & 1 & & 4 ZLO & 1 & & & & \\
\hline $\begin{array}{l}23 S G 27 \\
2-5\end{array}$ & $\begin{array}{l}150 R \\
0\end{array}$ & 1 & & 4 ZLO & 1 & & & & \\
\hline $\begin{array}{l}23 S G 27 \\
2-5\end{array}$ & $\begin{array}{l}150 R \\
0\end{array}$ & 1 & & 4 ZLO & 1 & & & & \\
\hline $\begin{array}{l}23 S G 27 \\
2-5\end{array}$ & $\begin{array}{l}150 \mathrm{R} \\
0\end{array}$ & 1 & & $4 \mathrm{ZLO}$ & & $\begin{array}{l}\text { brass- } \\
\text { plated iron } \\
\text { eye-screw } \\
\text { hook } \\
\text { (perhaps } \\
\text { for } \\
\text { furniture or } \\
\text { box) }\end{array}$ & & & \\
\hline $\begin{array}{l}23 S G 27 \\
2-5\end{array}$ & $\begin{array}{l}150 R \\
0\end{array}$ & 1 & & 4 ZLO & 1 & & & & \\
\hline $23 S G 27$ & $150 R$ & 1 & & 4 ZLO & 1 & & & & \\
\hline
\end{tabular}




\begin{tabular}{|c|c|c|c|c|c|c|c|c|c|}
\hline \multicolumn{10}{|c|}{ Archaeological Analysis } \\
\hline $\begin{array}{c}\text { Catalog } \\
\text { ue \# }\end{array}$ & Unit & Qu & $\begin{array}{c}\text { Lev } \\
\text { el }\end{array}$ & $\begin{array}{c}\text { Time } \\
\text { peri } \\
\text { od }\end{array}$ & Amou & $\mid \begin{array}{c}\text { Household } \\
\text { Maintenan } \\
\text { ce-Other }\end{array}$ & \begin{tabular}{|l} 
Househol \\
d \\
Maintena \\
nce- \\
Ceramic
\end{tabular} & $\begin{array}{c}\text { Household } \\
\text { Maintenance- } \\
\text { Glass }\end{array}$ & $\begin{array}{l}\text { Architectural } \\
\text { Items }\end{array}$ \\
\hline $2-5$ & 0 & & & & & & & & \\
\hline $\begin{array}{l}23 S G 27 \\
2-5\end{array}$ & $\begin{array}{l}150 \mathrm{R} \\
0\end{array}$ & 1 & 4 & ZLO & 1 & & & & \\
\hline $\begin{array}{l}23 S G 27 \\
2-5\end{array}$ & $\begin{array}{l}150 \mathrm{R} \\
0\end{array}$ & 1 & & ZLO & 160 & & & & $\begin{array}{l}\text { white frags, } \\
\text { lime } \\
\text { plaster/morta } \\
\text { r }\end{array}$ \\
\hline $\begin{array}{l}23 S G 27 \\
2-5\end{array}$ & $\begin{array}{l}150 \mathrm{R} \\
0\end{array}$ & 1 & & ZLO & 25 & & & & $\begin{array}{l}\text { gray frags, } \\
\text { lime } \\
\text { plaster/morta } \\
\text { r }\end{array}$ \\
\hline $\begin{array}{l}23 S G 27 \\
2-5\end{array}$ & $\begin{array}{l}150 \mathrm{R} \\
0\end{array}$ & 1 & & ZLO & 5 & & & & $\begin{array}{l}\text { white and } \\
\text { gray frags, } \\
\text { lime } \\
\text { plaster/morta } \\
\text { r }\end{array}$ \\
\hline $\begin{array}{l}23 S G 27 \\
2-5\end{array}$ & $\begin{array}{l}150 \mathrm{R} \\
0\end{array}$ & 1 & 4 & ZLO & 11 & & & & \\
\hline $\begin{array}{l}23 S G 27 \\
2-5\end{array}$ & $\begin{array}{l}150 R \\
0\end{array}$ & 1 & & ZLO & 13 & & & & \\
\hline $\begin{array}{l}23 S G 27 \\
2-6\end{array}$ & $\begin{array}{l}150 \mathrm{R} \\
0\end{array}$ & 1 & & 20 & 1 & & & & $\begin{array}{l}\text { frag, blue- } \\
\text { green } \\
\text { window glass }\end{array}$ \\
\hline $\begin{array}{l}23 S G 27 \\
2-6\end{array}$ & $\begin{array}{l}150 \mathrm{R} \\
0\end{array}$ & 1 & & 20 & 1 & & & & $\begin{array}{l}\text { frag, light } \\
\text { blue-green } \\
\text { window glass }\end{array}$ \\
\hline $\begin{array}{l}23 S G 27 \\
2-6\end{array}$ & $\begin{array}{l}150 \mathrm{R} \\
0\end{array}$ & 1 & & 20 & 1 & & & $\begin{array}{l}\text { tiny frag, light } \\
\text { green- } \\
\text { blue/green glass }\end{array}$ & \\
\hline $\begin{array}{l}23 S G 27 \\
2-6\end{array}$ & $\begin{array}{l}150 \mathrm{R} \\
0\end{array}$ & 1 & & 20 & 1 & & & $\begin{array}{l}\text { tiny frag, clear } \\
\text { glass }\end{array}$ & \\
\hline $\begin{array}{l}23 S G 27 \\
2-6\end{array}$ & $\begin{array}{l}150 \mathrm{R} \\
0\end{array}$ & 1 & & 20 & 1 & & & $\begin{array}{l}\text { small frag, clear } \\
\text { to light blue- } \\
\text { green glass }\end{array}$ & \\
\hline $\begin{array}{l}23 S G 27 \\
2-6\end{array}$ & $\begin{array}{l}150 R \\
0\end{array}$ & 1 & & 20 & 1 & & & & \\
\hline $23 S G 27$ & $150 \mathrm{R}$ & 1 & & 20 & & broken & & & \\
\hline
\end{tabular}




\begin{tabular}{|c|c|c|c|c|c|c|c|c|c|}
\hline \multicolumn{10}{|c|}{ Archaeological Analysis } \\
\hline $\begin{array}{c}\text { Catalog } \\
\text { ue \# }\end{array}$ & Unit & $\begin{array}{l}\text { Qu } \\
\text { ad }\end{array}$ & \begin{tabular}{||c||} 
Lev \\
el
\end{tabular} & $\begin{array}{c}\text { Time } \\
\text { peri } \\
\text { od }\end{array}$ & Amou & $\begin{array}{l}\text { Household } \\
\text { Maintenan } \\
\text { ce-Other }\end{array}$ & \begin{tabular}{|} 
Househol \\
d \\
Maintena \\
nce- \\
Ceramic
\end{tabular} & $\begin{array}{c}\text { Household } \\
\text { Maintenance- } \\
\text { Glass }\end{array}$ & $\begin{array}{c}\text { Architectural } \\
\text { Items }\end{array}$ \\
\hline $2-6$ & 0 & & & & & $\begin{array}{l}\text { ?hook; cast } \\
\text { brass; 19th } \\
\text { century }\end{array}$ & & & \\
\hline \begin{tabular}{|l|}
$23 S G 27$ \\
$2-6$
\end{tabular} & $\begin{array}{l}150 \mathrm{R} \\
0\end{array}$ & 1 & & ZO & & $\begin{array}{l}\text { frag, brass } \\
\text { scrap or } \\
\text { brass-plate } \\
\text { scrap }\end{array}$ & & & \\
\hline \begin{tabular}{|l|}
$23 S G 27$ \\
$2-6$
\end{tabular} & $\begin{array}{l}150 \mathrm{R} \\
0\end{array}$ & 1 & & 20 & 10 & & & & $\begin{array}{l}\text { frags, lime } \\
\text { mortar/plaste } \\
r\end{array}$ \\
\hline $\begin{array}{l}23 S G 27 \\
2-6\end{array}$ & $\begin{array}{l}150 \mathrm{R} \\
0\end{array}$ & 1 & & 20 & 11 & & & & \\
\hline \begin{tabular}{|l|}
$23 S G 27$ \\
$2-6$
\end{tabular} & $\begin{array}{l}150 \mathrm{R} \\
0\end{array}$ & 1 & & 20 & 2 & & & & \\
\hline \begin{tabular}{|l|}
$23 S G 27$ \\
$2-7$
\end{tabular} & $\begin{array}{l}150 \mathrm{R} \\
0\end{array}$ & 1 & & 20 & 1 & & & $\begin{array}{l}\text { frag, yellow- } \\
\text { green vessel } \\
\text { glass }\end{array}$ & \\
\hline \begin{tabular}{|l|}
$23 S G 27$ \\
$2-7$
\end{tabular} & $\begin{array}{l}150 \mathrm{R} \\
0\end{array}$ & 1 & & 20 & 1 & & & $\begin{array}{l}\text { frag, clear vessel } \\
\text { glass }\end{array}$ & \\
\hline \begin{tabular}{|l|}
$23 S G 27$ \\
$2-7$
\end{tabular} & $\begin{array}{l}150 \mathrm{R} \\
0\end{array}$ & 1 & & 20 & 1 & & & $\begin{array}{l}\text { tiny frag, clear } \\
\text { glass }\end{array}$ & \\
\hline \begin{tabular}{|l|}
$23 S G 27$ \\
$2-7$
\end{tabular} & $\begin{array}{l}150 \mathrm{R} \\
0\end{array}$ & 1 & & $\mathrm{ZO}$ & 7 & & & & $\begin{array}{l}\text { frags, lime } \\
\text { mortar/plaste } \\
r\end{array}$ \\
\hline \begin{tabular}{|l|}
$23 S G 27$ \\
$2-7$
\end{tabular} & $\begin{array}{l}150 \mathrm{R} \\
0\end{array}$ & 1 & & 20 & 8 & & & & \\
\hline \begin{tabular}{|l|}
$23 S G 27$ \\
$2-7$
\end{tabular} & $\begin{array}{l}150 \mathrm{R} \\
0\end{array}$ & 1 & & $\mathrm{ZO}$ & 9 & & & & \\
\hline \begin{tabular}{|l|}
$23 S G 27$ \\
$2-8$
\end{tabular} & $\begin{array}{l}150 R \\
0\end{array}$ & 1 & & 20 & 1 & & & $\begin{array}{l}\text { small frag, of } \\
\text { blue glass }\end{array}$ & \\
\hline \begin{tabular}{|l|}
$23 S G 27$ \\
$2-8$
\end{tabular} & $\begin{array}{l}150 \mathrm{R} \\
0\end{array}$ & 1 & & 20 & 1 & & & $\begin{array}{l}\text { small frag, flat } \\
\text { glass }\end{array}$ & \\
\hline \begin{tabular}{|l|}
$23 S G 27$ \\
$2-8$
\end{tabular} & $\begin{array}{l}150 R \\
0\end{array}$ & 1 & & 20 & 4 & & & & $\begin{array}{l}\text { frags, lime } \\
\text { plaster/morta } \\
\text { r }\end{array}$ \\
\hline \begin{tabular}{|l|}
$23 S G 27$ \\
$2-8$
\end{tabular} & $\begin{array}{l}150 \mathrm{R} \\
0\end{array}$ & 1 & & 20 & 5 & & & & \\
\hline
\end{tabular}




\begin{tabular}{|c|c|c|c|c|c|c|c|c|c|}
\hline \multicolumn{10}{|c|}{ Archaeological Analysis } \\
\hline $\begin{array}{c}\text { Catalog } \\
\text { ue \# }\end{array}$ & Unit & $\begin{array}{l}\text { Qu } \\
\text { ad }\end{array}$ & $\begin{array}{c}\text { Lev } \\
\text { el }\end{array}$ & $\begin{array}{c}\text { Time } \\
\text { peri } \\
\text { od }\end{array}$ & Amou & $\mid \begin{array}{c}\text { Household } \\
\text { Maintenan } \\
\text { ce-Other }\end{array}$ & \begin{tabular}{|c|} 
Househol \\
d \\
Maintena \\
nce- \\
Ceramic
\end{tabular} & $\begin{array}{l}\text { Household } \\
\text { Maintenance- } \\
\text { Glass }\end{array}$ & $\begin{array}{c}\text { Architectural } \\
\text { Items }\end{array}$ \\
\hline $\begin{array}{l}23 S G 27 \\
2-8\end{array}$ & $\begin{array}{l}150 R \\
0\end{array}$ & 1 & & 20 & 4 & & & & \\
\hline $\begin{array}{l}23 S G 27 \\
2-8\end{array}$ & $\begin{array}{l}150 R \\
0\end{array}$ & 1 & & $\mathrm{ZO}$ & 1 & & & & \\
\hline $\begin{array}{l}23 S G 27 \\
2-9\end{array}$ & $\begin{array}{l}150 R \\
0\end{array}$ & 1 & & 20 & 1 & & & & \\
\hline $\begin{array}{l}23 S G 27 \\
2-9\end{array}$ & $\begin{array}{l}150 \mathrm{R} \\
0\end{array}$ & 1 & & 20 & 1 & & & $\begin{array}{l}\text { tiny frag, light } \\
\text { blue-green glass }\end{array}$ & \\
\hline $\begin{array}{l}23 S G 27 \\
2-9\end{array}$ & $\begin{array}{l}150 R \\
0\end{array}$ & 1 & & 20 & 1 & & & & \\
\hline $\begin{array}{l}23 S G 27 \\
2-9\end{array}$ & $\begin{array}{l}150 \mathrm{R} \\
0\end{array}$ & 1 & & $Z O$ & 11 & & & & $\begin{array}{l}\text { small frags, } \\
\text { lime } \\
\text { mortar/plaste } \\
r\end{array}$ \\
\hline $\begin{array}{l}23 S G 27 \\
2-9\end{array}$ & $\begin{array}{l}150 \mathrm{R} \\
0\end{array}$ & 1 & & 20 & 2 & & & & \\
\hline $\begin{array}{l}23 S G 27 \\
2-9\end{array}$ & $\begin{array}{l}150 R \\
0\end{array}$ & 1 & & 20 & 3 & & & & \\
\hline $\begin{array}{l}23 S G 27 \\
2-9\end{array}$ & $\begin{array}{l}150 \mathrm{R} \\
0\end{array}$ & 1 & & 20 & 1 & & & & \\
\hline $\begin{array}{l}23 S G 27 \\
2-10\end{array}$ & $\begin{array}{l}150 \mathrm{R} \\
0\end{array}$ & 1 & & 20 & 1 & & & & $\begin{array}{l}\text { frag, blue- } \\
\text { green } \\
\text { window glass }\end{array}$ \\
\hline $\begin{array}{l}23 S G 27 \\
2-10\end{array}$ & $\begin{array}{l}150 \mathrm{R} \\
0\end{array}$ & 1 & & 20 & 5 & & & & $\begin{array}{l}\text { frags, lime } \\
\text { mortar/plaste } \\
r\end{array}$ \\
\hline $\begin{array}{l}23 S G 27 \\
2-10\end{array}$ & $\begin{array}{l}150 \mathrm{R} \\
0\end{array}$ & 1 & & 20 & 2 & & & & \\
\hline $\begin{array}{l}23 S G 27 \\
2-162 \\
\end{array}$ & $\begin{array}{l}40 \mathrm{R} 6 \\
0\end{array}$ & 3 & 7 & ZLO & 1 & & & & \\
\hline $\begin{array}{l}23 S G 27 \\
2-162\end{array}$ & $\begin{array}{l}40 R 6 \\
0\end{array}$ & 3 & & ZLO & 1 & & & & $\begin{array}{l}\text { frag. Gray } \\
\text { lime mortar }\end{array}$ \\
\hline $\begin{array}{l}23 S G 27 \\
2-162\end{array}$ & $\begin{array}{l}40 R 6 \\
0\end{array}$ & 3 & 7 & ZLO & 9 & & & & $\begin{array}{l}\text { frags. Flake of } \\
\text { whitewash/w } \\
\text { hite paint }\end{array}$ \\
\hline $\begin{array}{l}23 S G 27 \\
2-163\end{array}$ & $\begin{array}{l}40 R 6 \\
0\end{array}$ & 3 & 8 & ZLO & 4 & & & & $\begin{array}{l}\text { window glass } \\
\text { frags, light }\end{array}$ \\
\hline
\end{tabular}




\begin{tabular}{|c|c|c|c|c|c|c|c|c|c|}
\hline \multicolumn{10}{|c|}{ Archaeological Analysis } \\
\hline $\begin{array}{c}\text { Catalog } \\
\text { ue \# }\end{array}$ & Unit & 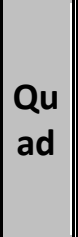 & $\mid \begin{array}{c}\text { Lev } \\
\text { el }\end{array}$ & $\mid \begin{array}{c}\text { Time } \\
\text { peri } \\
\text { od }\end{array}$ & Amou & $\begin{array}{l}\text { Household } \\
\text { Maintenan } \\
\text { ce-Other }\end{array}$ & \begin{tabular}{|l} 
Househol \\
d \\
Maintena \\
nce- \\
Ceramic
\end{tabular} & $\begin{array}{c}\text { Household } \\
\text { Maintenance- } \\
\text { Glass }\end{array}$ & $\begin{array}{l}\text { Architectural } \\
\text { Items }\end{array}$ \\
\hline & & & & & & & & & $\begin{array}{l}\text { blue-green to } \\
\text { medium blue- } \\
\text { green }\end{array}$ \\
\hline $\begin{array}{l}23 S G 27 \\
2-163\end{array}$ & $\begin{array}{l}40 R 6 \\
0\end{array}$ & 3 & & 8 ZLO & 3 & & & $\begin{array}{l}\text { frags, light } \\
\text { yellow-green } \\
\text { vessel glass, } \\
\text { possible } \\
\text { straight-sided } \\
\text { bottle }\end{array}$ & \\
\hline \begin{tabular}{|l|}
$23 S G 27$ \\
$2-163$
\end{tabular} & $\begin{array}{l}40 \mathrm{R} 6 \\
0\end{array}$ & 3 & & $8 \mathrm{ZLO}$ & 3 & & & $\begin{array}{l}\text { small frags, clear } \\
\text { glass }\end{array}$ & \\
\hline $\begin{array}{l}23 S G 27 \\
2-164\end{array}$ & $\begin{array}{l}40 \mathrm{R} 6 \\
0\end{array}$ & 3 & & 8 ZLO & 2 & & & $\begin{array}{l}\text { frags of pressed, } \\
\text { molded clear } \\
\text { glass }\end{array}$ & \\
\hline \begin{tabular}{|l|}
$23 S G 27$ \\
$2-164$
\end{tabular} & $\begin{array}{l}40 \mathrm{R} 6 \\
0\end{array}$ & 3 & & 8 ZLO & 2 & & & $\begin{array}{l}\text { frags, fairly } \\
\text { thick, clear glass }\end{array}$ & \\
\hline $\begin{array}{l}23 S G 27 \\
2-164\end{array}$ & $\begin{array}{l}40 \mathrm{R} 6 \\
0\end{array}$ & 3 & & $8 \mathrm{ZLO}$ & 1 & & & $\begin{array}{l}\text { tiny frag, dark } \\
\text { aqua } \\
\text { translucent glass }\end{array}$ & \\
\hline \begin{tabular}{|l|}
$23 S G 27$ \\
$2-164$
\end{tabular} & $\begin{array}{l}40 \mathrm{R} 6 \\
0\end{array}$ & 3 & & 8 ZLO & 11 & & & & $\begin{array}{l}\text { frags, window } \\
\text { glass, light } \\
\text { blue-green to } \\
\text { light blue }\end{array}$ \\
\hline $\begin{array}{l}23 S G 27 \\
2-164\end{array}$ & $\begin{array}{l}40 R 6 \\
0\end{array}$ & 3 & & 8 ZLO & 2 & & & $\begin{array}{l}\text { tiny clear glass } \\
\text { frags }\end{array}$ & \\
\hline $\begin{array}{l}23 S G 27 \\
2-164\end{array}$ & $\begin{array}{l}40 R 6 \\
0\end{array}$ & 3 & & 8 ZLO & 2 & & & & \\
\hline $\begin{array}{l}23 S G 27 \\
2-164\end{array}$ & $\begin{array}{l}40 R 6 \\
0\end{array}$ & 3 & & 8 ZLO & 1 & & & & \\
\hline \begin{tabular}{|l|}
$23 S G 27$ \\
$2-164$
\end{tabular} & $\begin{array}{l}40 \mathrm{R} 6 \\
0\end{array}$ & 3 & & 8 ZLO & 2 & & & & \\
\hline $\begin{array}{l}23 S G 27 \\
2-164\end{array}$ & $\begin{array}{l}40 \mathrm{R} 6 \\
0\end{array}$ & 3 & & $8 \mathrm{ZLO}$ & 1 & & & & \\
\hline $\begin{array}{l}23 S G 27 \\
2-164\end{array}$ & $\begin{array}{l}40 R 6 \\
0\end{array}$ & 3 & & $8 \mathrm{ZLO}$ & 1 & & & & \\
\hline \begin{tabular}{|l|}
$23 S G 27$ \\
$2-164$
\end{tabular} & $\begin{array}{l}40 R 6 \\
0\end{array}$ & 3 & & 8 ZLO & 1 & & & & \\
\hline
\end{tabular}




\begin{tabular}{|c|c|c|c|c|c|c|c|c|c|}
\hline \multicolumn{10}{|c|}{ Archaeological Analysis } \\
\hline $\begin{array}{c}\text { Catalog } \\
\text { ue \# }\end{array}$ & Unit & 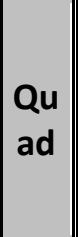 & $\begin{array}{c}\text { Lev } \\
\text { el }\end{array}$ & $\begin{array}{c}\text { Time } \\
\text { peri } \\
\text { od }\end{array}$ & Amou & \begin{tabular}{|c||} 
Household \\
Maintenan \\
ce-Other
\end{tabular} & \begin{tabular}{|c|} 
Househol \\
d \\
Maintena \\
nce- \\
Ceramic
\end{tabular} & $\begin{array}{c}\text { Household } \\
\text { Maintenance- } \\
\text { Glass }\end{array}$ & $\begin{array}{c}\text { Architectural } \\
\text { Items }\end{array}$ \\
\hline $\begin{array}{l}23 S G 27 \\
2-164\end{array}$ & $\begin{array}{l}40 R 6 \\
0\end{array}$ & 3 & & ZLO & 1 & & & & \\
\hline $\begin{array}{l}23 S G 27 \\
2-164\end{array}$ & $\begin{array}{l}40 R 6 \\
0\end{array}$ & 3 & & ZLO & 1 & & & & \\
\hline $\begin{array}{l}23 S G 27 \\
2-164\end{array}$ & $\begin{array}{l}40 \mathrm{R} 6 \\
0\end{array}$ & 3 & & ZLO & 11 & & & & \\
\hline $\begin{array}{l}23 S G 27 \\
2-164\end{array}$ & $\begin{array}{l}40 R 6 \\
0\end{array}$ & 3 & & ZLO & 1 & & & & \\
\hline $\begin{array}{l}23 S G 27 \\
2-164\end{array}$ & $\begin{array}{l}40 R 6 \\
0\end{array}$ & 3 & & ZLO & & $\begin{array}{l}\text { small frag, } \\
\text { brass scrap }\end{array}$ & & & \\
\hline $\begin{array}{l}23 S G 27 \\
2-164\end{array}$ & $\begin{array}{l}40 R 6 \\
0\end{array}$ & 3 & 8 & ZLO & 1 & & & & \\
\hline $\begin{array}{l}23 S G 27 \\
2-164\end{array}$ & $\begin{array}{l}40 \mathrm{R} 6 \\
0\end{array}$ & 3 & & ZLO & 1 & & & & \\
\hline $\begin{array}{l}23 S G 27 \\
2-164\end{array}$ & $\begin{array}{l}40 \mathrm{R} 6 \\
0\end{array}$ & 3 & & ZLO & 5 & & & & $\begin{array}{l}\text { frags, dark } \\
\text { gray lime } \\
\text { mortar }\end{array}$ \\
\hline $\begin{array}{l}23 S G 27 \\
2-164\end{array}$ & $\begin{array}{l}40 R 6 \\
0\end{array}$ & 3 & & ZLO & 1 & & & & $\begin{array}{l}\text { frag, white } \\
\text { lime mortar, } \\
\text { with large } \\
\text { stones } \\
\text { adhering }\end{array}$ \\
\hline $\begin{array}{l}23 S G 27 \\
2-164\end{array}$ & $\begin{array}{l}40 R 6 \\
0\end{array}$ & 3 & 8 & ZLO & 33 & & & & $\begin{array}{l}\text { frags, } \\
\text { whitewash/w } \\
\text { hite paint }\end{array}$ \\
\hline $\begin{array}{l}23 S G 27 \\
2-164\end{array}$ & $\begin{array}{l}40 R 6 \\
0\end{array}$ & 3 & 8 & ZLO & 1 & & & & $\begin{array}{l}\text { large frag, } \\
\text { white lime } \\
\text { plaster with } \\
\text { traces of gray } \\
\text { paint on } \\
\text { surface }\end{array}$ \\
\hline $\begin{array}{l}23 S G 27 \\
2-164\end{array}$ & $\begin{array}{l}40 R 6 \\
0\end{array}$ & 3 & & ZLO & 13 & & & & $\begin{array}{l}\text { frags, gray } \\
\text { and green } \\
\text { paint over } \\
\text { lime plaster }\end{array}$ \\
\hline $\begin{array}{l}23 S G 27 \\
2-164\end{array}$ & $\begin{array}{l}40 R 6 \\
0\end{array}$ & 3 & 8 & ZLO & 2 & & & & \\
\hline
\end{tabular}




\begin{tabular}{|c|c|c|c|c|c|c|c|c|c|}
\hline \multicolumn{10}{|c|}{ Archaeological Analysis } \\
\hline $\begin{array}{c}\text { Catalog } \\
\text { ue \# }\end{array}$ & Unit & $\begin{array}{l}\text { Qu } \\
\text { ad }\end{array}$ & $\begin{array}{c}\text { Lev } \\
\text { el }\end{array}$ & $\begin{array}{c}\text { Time } \\
\text { peri } \\
\text { od }\end{array}$ & Amou & $\begin{array}{c}\text { Household } \\
\text { Maintenan } \\
\text { ce-Other }\end{array}$ & \begin{tabular}{|c|} 
Househol \\
d \\
Maintena \\
nce- \\
Ceramic
\end{tabular} & $\begin{array}{l}\text { Household } \\
\text { Maintenance- } \\
\text { Glass }\end{array}$ & $\begin{array}{l}\text { Architectural } \\
\text { Items }\end{array}$ \\
\hline $\begin{array}{l}23 S G 27 \\
2-165\end{array}$ & $\begin{array}{l}40 \mathrm{R} 6 \\
0\end{array}$ & 3 & & ZLO & 1 & & & $\begin{array}{l}\text { frag, lightly } \\
\text { curved clear } \\
\text { glass, "frosted" } \\
\text { on surface }\end{array}$ & \\
\hline $\begin{array}{l}23 S G 27 \\
2-165\end{array}$ & $\begin{array}{l}40 R 6 \\
0\end{array}$ & 3 & & ZLO & 1 & & & & $\begin{array}{l}\text { frag, window } \\
\text { glass, light } \\
\text { blue-green }\end{array}$ \\
\hline $\begin{array}{l}23 S G 27 \\
2-165\end{array}$ & $\begin{array}{l}40 R 6 \\
0\end{array}$ & 3 & & ZLO & 2 & & & & \\
\hline $\begin{array}{l}23 S G 27 \\
2-165\end{array}$ & $\begin{array}{l}40 R 6 \\
0\end{array}$ & 3 & & ZLO & 2 & & & & \\
\hline $\begin{array}{l}23 S G 27 \\
2-165\end{array}$ & $\begin{array}{l}40 R 6 \\
0\end{array}$ & 3 & & ZLO & & $\begin{array}{l}\text { frag, brass } \\
\text { scrap or } \\
\text { "nugget" }\end{array}$ & & & \\
\hline $\begin{array}{l}23 S G 27 \\
2-165\end{array}$ & $\begin{array}{l}40 \mathrm{R} 6 \\
0\end{array}$ & 3 & & ZLO & & $\begin{array}{l}\text { tiny flakes } \\
\text { of brass } \\
\text { scrap/oxida } \\
\text { tion }\end{array}$ & & & \\
\hline $\begin{array}{l}23 S G 27 \\
2-165\end{array}$ & $\begin{array}{l}40 R 6 \\
0\end{array}$ & 3 & 8 & ZLO & 3 & & & & $\begin{array}{l}\text { frags, dark } \\
\text { gray lime } \\
\text { mortar }\end{array}$ \\
\hline $\begin{array}{l}23 S G 27 \\
2-165\end{array}$ & $\begin{array}{l}40 R 6 \\
0\end{array}$ & 3 & 8 & ZLO & 82 & & & & $\begin{array}{l}\text { frags, } \\
\text { whitewash/w } \\
\text { hite paint } \\
\text { chips }\end{array}$ \\
\hline $\begin{array}{l}23 S G 27 \\
2-165\end{array}$ & $\begin{array}{l}40 \mathrm{R} 6 \\
0\end{array}$ & 3 & 8 & ZLO & 1 & & & & \\
\hline $\begin{array}{l}23 S G 27 \\
2-166\end{array}$ & $\begin{array}{l}40 \mathrm{R} 6 \\
0\end{array}$ & 3 & & ZLO & 1 & & & $\begin{array}{l}\text { frag, clear glass } \\
\text { vessel }\end{array}$ & \\
\hline $\begin{array}{l}23 S G 27 \\
2-166\end{array}$ & $\begin{array}{l}40 \mathrm{R} 6 \\
0\end{array}$ & 3 & 8 & ZLO & 1 & & & $\begin{array}{l}\text { frag, olive green } \\
\text { vessel glass, } \\
\text { probably bottle } \\
\text { glass }\end{array}$ & \\
\hline $\begin{array}{l}23 S G 27 \\
2-166\end{array}$ & $\begin{array}{l}40 \mathrm{R} 6 \\
0\end{array}$ & 3 & 8 & ZLO & 1 & & & $\begin{array}{l}\text { small frag, olive } \\
\text { green vessel } \\
\text { glass }\end{array}$ & \\
\hline $23 S G 27$ & $40 R 6$ & 3 & & ZLO & 1 & & & frag, thin, light & \\
\hline
\end{tabular}




\begin{tabular}{|c|c|c|c|c|c|c|c|c|c|}
\hline \multicolumn{10}{|c|}{ Archaeological Analysis } \\
\hline $\begin{array}{c}\text { Catalog } \\
\text { ue \# }\end{array}$ & Unit & $\begin{array}{l}\text { Qu } \\
\text { ad }\end{array}$ & \begin{tabular}{||c||} 
Lev \\
el
\end{tabular} & $\begin{array}{c}\text { Time } \\
\text { peri } \\
\text { od }\end{array}$ & Amou & $\begin{array}{c}\text { Household } \\
\text { Maintenan } \\
\text { ce-Other }\end{array}$ & \begin{tabular}{|l} 
Househol \\
d \\
Maintena \\
nce- \\
Ceramic
\end{tabular} & $\begin{array}{c}\text { Household } \\
\text { Maintenance- } \\
\text { Glass }\end{array}$ & $\begin{array}{c}\text { Architectural } \\
\text { Items }\end{array}$ \\
\hline $2-166$ & 0 & & & & & & & $\begin{array}{l}\text { blue-green } \\
\text { glass, possibly } \\
\text { from } \\
\text { pharmaceutical } \\
\text { bottle }\end{array}$ & \\
\hline \begin{tabular}{|l|}
$23 S G 27$ \\
$2-166$
\end{tabular} & $\begin{array}{l}40 \mathrm{R} 6 \\
0\end{array}$ & 3 & 8 & ZLO & 2 & & & $\begin{array}{l}\text { frags, light blue- } \\
\text { green glass, } \\
\text { window or } \\
\text { vessel glass }\end{array}$ & \\
\hline \begin{tabular}{|l|}
$23 S G 27$ \\
$2-166$
\end{tabular} & $\begin{array}{l}40 \mathrm{R} 6 \\
0\end{array}$ & 3 & 8 & ZLO & 1 & & & & $\begin{array}{l}\text { frag, window } \\
\text { glass, light } \\
\text { blue-green }\end{array}$ \\
\hline \begin{tabular}{|l|}
$23 S G 27$ \\
$2-166$
\end{tabular} & $\begin{array}{l}40 \mathrm{R} 6 \\
0\end{array}$ & 3 & 8 & ZLO & 1 & & & & \\
\hline \begin{tabular}{|l|}
$23 S G 27$ \\
$2-167$
\end{tabular} & $\begin{array}{l}40 \mathrm{R} 6 \\
0\end{array}$ & 3 & 8 & ZLO & 7 & & & & $\begin{array}{l}\text { flakes, } \\
\text { whitewash/w } \\
\text { hite paint }\end{array}$ \\
\hline \begin{tabular}{|l|}
$23 S G 27$ \\
$2-168$
\end{tabular} & $\begin{array}{l}40 \mathrm{R} 6 \\
0\end{array}$ & 3 & 9 & ZLO & 90 & & & & $\begin{array}{l}\text { window glass } \\
\text { frags, light } \\
\text { blue green to } \\
\text { blue }\end{array}$ \\
\hline \begin{tabular}{|l|}
$23 S G 27$ \\
$2-168$
\end{tabular} & $\begin{array}{l}40 \mathrm{R} 6 \\
0\end{array}$ & 3 & 9 & ZLO & 1 & & & $\begin{array}{l}\text { medium blue- } \\
\text { green vessel } \\
\text { glass, molded } \\
\text { decoration on } \\
\text { exterior }\end{array}$ & \\
\hline \begin{tabular}{|l|}
$23 S G 27$ \\
$2-168$
\end{tabular} & $\begin{array}{l}40 \mathrm{R} 6 \\
0\end{array}$ & 3 & 9 & ZLO & 1 & & & $\begin{array}{l}\text { light blue-green } \\
\text { curved glass, } \\
\text { probably a } \\
\text { bottle neck frag. }\end{array}$ & \\
\hline $\begin{array}{l}23 S G 27 \\
2-168\end{array}$ & $\begin{array}{l}40 \mathrm{R} 6 \\
0\end{array}$ & 3 & 9 & ZLO & 2 & & & $\begin{array}{l}\text { frags, light blue- } \\
\text { green vessel } \\
\text { glass, molded } \\
\text { design }\end{array}$ & \\
\hline \begin{tabular}{|l|}
$23 S G 27$ \\
$2-168$
\end{tabular} & $\begin{array}{l}40 \mathrm{R} 6 \\
0\end{array}$ & 3 & 9 & ZLO & 8 & & & $\begin{array}{l}\text { frags, light blue- } \\
\text { green vessel } \\
\text { glass }\end{array}$ & \\
\hline $23 S G 27$ & $40 R 6$ & 3 & 9 & ZLO & 2 & & & frags, light blue & \\
\hline
\end{tabular}




\begin{tabular}{|c|c|c|c|c|c|c|c|c|c|}
\hline \multicolumn{10}{|c|}{ Archaeological Analysis } \\
\hline $\begin{array}{c}\text { Catalog } \\
\text { ue \# }\end{array}$ & Unit & Qu & $\begin{array}{c}\text { Lev } \\
\text { el }\end{array}$ & $\left|\begin{array}{c}\text { Time } \\
\text { peri } \\
\text { od }\end{array}\right|$ & Amou & $\begin{array}{c}\text { Household } \\
\text { Maintenan } \\
\text { ce-Other }\end{array}$ & \begin{tabular}{|l} 
Househol \\
d \\
Maintena \\
nce- \\
Ceramic
\end{tabular} & $\begin{array}{c}\text { Household } \\
\text { Maintenance- } \\
\text { Glass }\end{array}$ & $\begin{array}{l}\text { Architectural } \\
\text { Items }\end{array}$ \\
\hline $2-168$ & 0 & & & & & & & $\begin{array}{l}\text { vessel glass, } \\
\text { possibly } \\
\text { pharmaceutical } \\
\text { bottle }\end{array}$ & \\
\hline $\begin{array}{l}23 S G 27 \\
2-168\end{array}$ & $\begin{array}{l}40 R 6 \\
0\end{array}$ & 3 & & ZLO & 2 & & & $\begin{array}{l}\text { frags, clear } \\
\text { vessel glass, } \\
\text { possibily } \\
\text { pharmaceutical } \\
\text { bottle }\end{array}$ & \\
\hline $\begin{array}{l}23 S G 27 \\
2-168\end{array}$ & $\begin{array}{l}40 R 6 \\
0\end{array}$ & 3 & 9 & ZLO & 1 & & & $\begin{array}{l}\text { frag, clear vessel } \\
\text { glass, possibly } \\
\text { wineglass bowl } \\
\text { or other vessel }\end{array}$ & \\
\hline $\begin{array}{l}23 S G 27 \\
2-168\end{array}$ & $\begin{array}{l}40 R 6 \\
0\end{array}$ & 3 & & ZLO & 1 & & & $\begin{array}{l}\text { bottle frag, clear } \\
\text { glass, mold } \\
\text { seam present }\end{array}$ & \\
\hline $\begin{array}{l}23 S G 27 \\
2-168\end{array}$ & $\begin{array}{l}40 R 6 \\
0\end{array}$ & 3 & 9 & ZLO & 9 & & & $\begin{array}{l}\text { frags, clear } \\
\text { vessel glass }\end{array}$ & \\
\hline $\begin{array}{l}23 S G 27 \\
2-168\end{array}$ & $\begin{array}{l}40 \mathrm{R} 6 \\
0\end{array}$ & 3 & & ZLO & 2 & & & $\begin{array}{l}\text { frags, dark olive } \\
\text { green case } \\
\text { bottle glass }\end{array}$ & \\
\hline $\begin{array}{l}23 S G 27 \\
2-168\end{array}$ & $\begin{array}{l}40 R 6 \\
0\end{array}$ & 3 & & ZLO & 6 & & & $\begin{array}{l}\text { frags, olive } \\
\text { green vessel } \\
\text { glass, probably } \\
\text { bottle glass }\end{array}$ & \\
\hline $\begin{array}{l}23 S G 27 \\
2-168\end{array}$ & $\begin{array}{l}40 R 6 \\
0\end{array}$ & 3 & 9 & ZLO & 5 & & & $\begin{array}{l}\text { frags, olive- } \\
\text { green and dark } \\
\text { olive-green } \\
\text { vessel glass }\end{array}$ & \\
\hline $\begin{array}{l}23 S G 27 \\
2-168\end{array}$ & $\begin{array}{l}40 R 6 \\
0\end{array}$ & 3 & 9 & ZLO & 5 & & & $\begin{array}{l}\text { frags, light } \\
\text { green/yellow } \\
\text { green vessel } \\
\text { glass }\end{array}$ & \\
\hline $\begin{array}{l}23 S G 27 \\
2-168\end{array}$ & $\begin{array}{l}40 R 6 \\
0\end{array}$ & 3 & 9 & ZLO & 12 & & & $\begin{array}{l}\text { frags. Clear to } \\
\text { light blue-green } \\
\text { curved glass, } \\
\text { with crackling } \\
\text { decoration }\end{array}$ & \\
\hline
\end{tabular}




\begin{tabular}{|c|c|c|c|c|c|c|c|c|c|}
\hline \multicolumn{10}{|c|}{ Archaeological Analysis } \\
\hline $\begin{array}{c}\text { Catalog } \\
\text { ue \# }\end{array}$ & Unit & 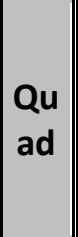 & $\begin{array}{c}\text { Lev } \\
\text { el }\end{array}$ & $\begin{array}{c}\text { Time } \\
\text { peri } \\
\text { od }\end{array}$ & Amou & $\begin{array}{c}\text { Household } \\
\text { Maintenan } \\
\text { ce-Other }\end{array}$ & \begin{tabular}{|l} 
Househol \\
d \\
Maintena \\
nce- \\
Ceramic
\end{tabular} & $\begin{array}{c}\text { Household } \\
\text { Maintenance- } \\
\text { Glass }\end{array}$ & $\begin{array}{l}\text { Architectural } \\
\text { Items }\end{array}$ \\
\hline \begin{tabular}{|l}
$23 S G 27$ \\
$2-168$
\end{tabular} & $\begin{array}{l}40 \mathrm{R} 6 \\
0\end{array}$ & 3 & & ZLO & 1 & & & \begin{tabular}{|l} 
frag of flat, light \\
blue-green \\
glass, with 2 \\
incised lines
\end{tabular} & \\
\hline $\begin{array}{l}23 S G 27 \\
2-168\end{array}$ & $\begin{array}{l}40 \mathrm{R} 6 \\
0\end{array}$ & 3 & & ZLO & 1 & & & $\begin{array}{l}\text { frags burned } \\
\text { glass, light blue } \\
\text { to blue-green }\end{array}$ & \\
\hline $\begin{array}{l}23 S G 27 \\
2-168\end{array}$ & $\begin{array}{l}40 R 6 \\
0\end{array}$ & 3 & & ZLO & 1 & & & $\begin{array}{l}\text { frags burned } \\
\text { glass, } \\
\text { clear/cloudy }\end{array}$ & \\
\hline $\begin{array}{l}23 S G 27 \\
2-168\end{array}$ & $\begin{array}{l}40 R 6 \\
0\end{array}$ & 3 & & ZLO & 1 & & & $\begin{array}{l}\text { frag, light amber } \\
\text { glass }\end{array}$ & \\
\hline $\begin{array}{l}23 S G 27 \\
2-168\end{array}$ & $\begin{array}{l}40 R 6 \\
0\end{array}$ & 3 & 9 & ZLO & 1 & & & frag, milk glass & \\
\hline $\begin{array}{l}23 S G 27 \\
2-168\end{array}$ & $\begin{array}{l}40 \mathrm{R} 6 \\
0\end{array}$ & 3 & 9 & ZLO & 5 & & & $\begin{array}{l}\text { frags, clear to } \\
\text { light blue-green } \\
\text { glass }\end{array}$ & \\
\hline $\begin{array}{l}23 S G 27 \\
2-168\end{array}$ & $\begin{array}{l}40 \mathrm{R} 6 \\
0\end{array}$ & 3 & 9 & ZLO & 4 & & & & \\
\hline $\begin{array}{l}23 S G 27 \\
2-168\end{array}$ & $\begin{array}{l}40 R 6 \\
0\end{array}$ & 3 & & ZLO & 1 & & & & \\
\hline $\begin{array}{l}23 S G 27 \\
2-168\end{array}$ & $\begin{array}{l}40 R 6 \\
0\end{array}$ & 3 & 9 & ZLO & 1 & & & & \\
\hline $\begin{array}{l}23 S G 27 \\
2-168\end{array}$ & $\begin{array}{l}40 R 6 \\
0\end{array}$ & 3 & 9 & ZLO & 1 & & & & \\
\hline \begin{tabular}{|l|}
$23 S G 27$ \\
$2-168$
\end{tabular} & $\begin{array}{l}40 \mathrm{R} 6 \\
0\end{array}$ & 3 & 9 & ZLO & 1 & & & & \\
\hline \begin{tabular}{|l|}
$23 S G 27$ \\
$2-168$
\end{tabular} & $\begin{array}{l}40 \mathrm{R} 6 \\
0\end{array}$ & 3 & 9 & ZLO & 8 & & & & \\
\hline \begin{tabular}{|l|}
$23 S G 27$ \\
$2-168$
\end{tabular} & $\begin{array}{l}40 \mathrm{R} 6 \\
0\end{array}$ & 3 & 9 & ZLO & 1 & & & & \\
\hline \begin{tabular}{|l|}
$23 S G 27$ \\
$2-168$
\end{tabular} & $\begin{array}{l}40 \mathrm{R} 6 \\
0\end{array}$ & 3 & 9 & ZLO & 1 & & & & \\
\hline \begin{tabular}{|l|}
$23 S G 27$ \\
$2-168$
\end{tabular} & $\begin{array}{l}40 \mathrm{R} 6 \\
0\end{array}$ & 3 & 9 & ZLO & 4 & & & & \\
\hline \begin{tabular}{|l|}
$23 S G 27$ \\
$2-168$
\end{tabular} & $\begin{array}{l}40 R 6 \\
0\end{array}$ & 3 & 9 & ZLO & 3 & & & & \\
\hline
\end{tabular}




\begin{tabular}{|c|c|c|c|c|c|c|c|c|c|}
\hline \multicolumn{10}{|c|}{ Archaeological Analysis } \\
\hline $\begin{array}{c}\text { Catalog } \\
\text { ue \# }\end{array}$ & Unit & $\begin{array}{l}\text { Qu } \\
\text { ad }\end{array}$ & \begin{tabular}{||c} 
Lev \\
el
\end{tabular} & $\begin{array}{c}\text { Time } \\
\text { peri } \\
\text { od }\end{array}$ & Amou & $\begin{array}{c}\text { Household } \\
\text { Maintenan } \\
\text { ce-Other }\end{array}$ & \begin{tabular}{|c|} 
Househol \\
d \\
Maintena \\
nce- \\
Ceramic \\
\end{tabular} & $\begin{array}{c}\text { Household } \\
\text { Maintenance- } \\
\text { Glass }\end{array}$ & $\begin{array}{l}\text { Architectural } \\
\text { Items }\end{array}$ \\
\hline \begin{tabular}{|l}
$23 S G 27$ \\
$2-168$
\end{tabular} & $\begin{array}{l}40 R 6 \\
0\end{array}$ & 3 & 9 & ZLO & 5 & & & & \\
\hline $\begin{array}{l}23 \mathrm{SG} 27 \\
2-168\end{array}$ & $\begin{array}{l}40 R 6 \\
0\end{array}$ & 3 & 9 & ZLO & 6 & & & & \\
\hline \begin{tabular}{|l|}
$23 S G 27$ \\
$2-168$
\end{tabular} & $\begin{array}{l}40 \mathrm{R} 6 \\
0\end{array}$ & 3 & 9 & ZLO & 3 & & & & \\
\hline \begin{tabular}{|l|}
$23 S G 27$ \\
$2-168$
\end{tabular} & $\begin{array}{l}40 R 6 \\
0\end{array}$ & 3 & 9 & ZLO & 2 & & & & \\
\hline \begin{tabular}{|l|}
$23 S G 27$ \\
$2-168$
\end{tabular} & $\begin{array}{l}40 R 6 \\
0\end{array}$ & 3 & 9 & ZLO & 1 & & & & \\
\hline \begin{tabular}{|l|}
$23 S G 27$ \\
$2-168$
\end{tabular} & $\begin{array}{l}40 R 6 \\
0\end{array}$ & 3 & 9 & ZLO & 3 & & & & \\
\hline $\begin{array}{l}23 S G 27 \\
2-168\end{array}$ & $\begin{array}{l}40 R 6 \\
0\end{array}$ & 3 & & ZLO & 1 & & & & \\
\hline \begin{tabular}{|l|}
$23 S G 27$ \\
$2-168$
\end{tabular} & $\begin{array}{l}40 \mathrm{R} 6 \\
0\end{array}$ & 3 & 9 & ZLO & 1 & & & & \\
\hline \begin{tabular}{|l|}
$23 S G 27$ \\
$2-168$
\end{tabular} & $\begin{array}{l}40 R 6 \\
0\end{array}$ & 3 & 9 & ZLO & 1 & & & & \\
\hline \begin{tabular}{|l|}
$23 S G 27$ \\
$2-168$
\end{tabular} & $\begin{array}{l}40 R 6 \\
0\end{array}$ & 3 & 9 & ZLO & 2 & & & & \\
\hline \begin{tabular}{|l|}
$23 S G 27$ \\
$2-168$
\end{tabular} & $\begin{array}{l}40 \mathrm{R} 6 \\
0\end{array}$ & 3 & 9 & ZLO & 1 & & & & \\
\hline \begin{tabular}{|l|}
$23 S G 27$ \\
$2-168$
\end{tabular} & $\begin{array}{l}40 \mathrm{R} 6 \\
0\end{array}$ & 3 & 9 & ZLO & 2 & & & & \\
\hline \begin{tabular}{|l|}
$23 S G 27$ \\
$2-168$
\end{tabular} & $\begin{array}{l}40 R 6 \\
0\end{array}$ & 3 & 9 & ZLO & 1 & & & & \\
\hline \begin{tabular}{|l|}
$23 S G 27$ \\
$2-168$
\end{tabular} & $\begin{array}{l}40 R 6 \\
0\end{array}$ & 3 & 9 & ZLO & 3 & & & & \\
\hline \begin{tabular}{|l|}
$23 S G 27$ \\
$2-168$
\end{tabular} & $\begin{array}{l}40 R 6 \\
0\end{array}$ & 3 & 9 & ZLO & 2 & & & & \\
\hline \begin{tabular}{|l|}
$23 S G 27$ \\
$2-168$
\end{tabular} & $\begin{array}{l}40 R 6 \\
0\end{array}$ & 3 & 9 & ZLO & 1 & & & & \\
\hline \begin{tabular}{|l|}
$23 S G 27$ \\
$2-168$
\end{tabular} & $\begin{array}{l}40 R 6 \\
0\end{array}$ & 3 & 9 & ZLO & 6 & & & & \\
\hline \begin{tabular}{|l|}
$23 S G 27$ \\
$2-168$
\end{tabular} & $\begin{array}{l}40 R 6 \\
0\end{array}$ & 3 & 9 & ZLO & 1 & & & & \\
\hline
\end{tabular}




\begin{tabular}{|c|c|c|c|c|c|c|c|c|c|}
\hline \multicolumn{10}{|c|}{ Archaeological Analysis } \\
\hline $\begin{array}{c}\text { Catalog } \\
\text { ue \# }\end{array}$ & Unit & 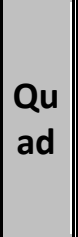 & $\mid \begin{array}{c}\text { Lev } \\
\text { el }\end{array}$ & $\begin{array}{c}\text { Time } \\
\text { peri } \\
\text { od }\end{array}$ & Amou & \begin{tabular}{|c} 
Household \\
Maintenan \\
ce-Other
\end{tabular} & \begin{tabular}{|c|} 
Househol \\
d \\
Maintena \\
nce- \\
Ceramic
\end{tabular} & $\begin{array}{c}\text { Household } \\
\text { Maintenance- } \\
\text { Glass }\end{array}$ & $\begin{array}{c}\text { Architectural } \\
\text { Items }\end{array}$ \\
\hline $\begin{array}{l}23 S G 27 \\
2-168\end{array}$ & $\begin{array}{l}40 R 6 \\
0\end{array}$ & 3 & & ZLO & 1 & & & & \\
\hline $\begin{array}{l}23 S G 27 \\
2-168\end{array}$ & $\begin{array}{l}40 R 6 \\
0\end{array}$ & 3 & & ZLO & 1 & & & & \\
\hline $\begin{array}{l}23 S G 27 \\
2-168\end{array}$ & $\begin{array}{l}40 \mathrm{R} 6 \\
0\end{array}$ & 3 & & ZLO & & $\begin{array}{l}\text { brass tack, } \\
\text { possibly } \\
\text { furniture } \\
\text { tack or gun } \\
\text { decoration }\end{array}$ & & & \\
\hline $\begin{array}{l}23 S G 27 \\
2-168\end{array}$ & $\begin{array}{l}40 R 6 \\
0\end{array}$ & 3 & & ZLO & 1 & & & & \\
\hline $\begin{array}{l}23 S G 27 \\
2-168\end{array}$ & $\begin{array}{l}40 R 6 \\
0\end{array}$ & 3 & & ZLO & 1 & & & & \\
\hline $\begin{array}{l}23 S G 27 \\
2-168\end{array}$ & $\begin{array}{l}40 R 6 \\
0\end{array}$ & 3 & & ZLO & & $\begin{array}{l}\text { frag, brass- } \\
\text { plated iron } \\
\text { wire }\end{array}$ & & & \\
\hline $\begin{array}{l}23 S G 27 \\
2-168\end{array}$ & $\begin{array}{l}40 R 6 \\
0\end{array}$ & 3 & & ZLO & 1 & & & & \\
\hline $\begin{array}{l}23 S G 27 \\
2-168\end{array}$ & $\begin{array}{l}40 R 6 \\
0\end{array}$ & 3 & 9 & ZLO & 4 & & & & \\
\hline $\begin{array}{l}23 S G 27 \\
2-168\end{array}$ & $\begin{array}{l}40 R 6 \\
0\end{array}$ & 3 & & ZLO & 20 & & & & \\
\hline $\begin{array}{l}23 S G 27 \\
2-168\end{array}$ & $\begin{array}{l}40 R 6 \\
0\end{array}$ & 3 & & ZLO & 1 & & & & $\begin{array}{l}\text { frag, } \\
\text { bousillage }\end{array}$ \\
\hline $\begin{array}{l}23 S G 27 \\
2-168\end{array}$ & $\begin{array}{l}40 R 6 \\
0\end{array}$ & 3 & & ZLO & 1 & & & & $\begin{array}{l}\text { frag, dark } \\
\text { gray lime } \\
\text { mortar; } \\
\text { coarse- } \\
\text { grained }\end{array}$ \\
\hline $\begin{array}{l}23 S G 27 \\
2-168\end{array}$ & $\begin{array}{l}40 R 6 \\
0\end{array}$ & 3 & 9 & ZLO & 5 & & & & $\begin{array}{l}\text { small frags, } \\
\text { dark gray lime } \\
\text { mortar; fine- } \\
\text { grained }\end{array}$ \\
\hline $\begin{array}{l}23 S G 27 \\
2-168\end{array}$ & $\begin{array}{l}40 R 6 \\
0\end{array}$ & 3 & & ZLO & 5 & & & & $\begin{array}{l}\text { frags, white } \\
\text { lime mortar }\end{array}$ \\
\hline $\begin{array}{l}23 S G 27 \\
2-168\end{array}$ & $\begin{array}{l}40 R 6 \\
0\end{array}$ & 3 & 9 & ZLO & 103 & & & & $\begin{array}{l}\text { flakes, } \\
\text { whitewash/w }\end{array}$ \\
\hline
\end{tabular}




\begin{tabular}{|c|c|c|c|c|c|c|c|c|c|}
\hline \multicolumn{10}{|c|}{ Archaeological Analysis } \\
\hline $\begin{array}{c}\text { Catalog } \\
\text { ue \# }\end{array}$ & Unit & Qu & $\begin{array}{c}\text { Lev } \\
\text { el }\end{array}$ & $\begin{array}{c}\text { Time } \\
\text { peri } \\
\text { od }\end{array}$ & Amou & $\mid \begin{array}{c}\text { Household } \\
\text { Maintenan } \\
\text { ce-Other }\end{array}$ & \begin{tabular}{|l} 
Househol \\
d \\
Maintena \\
nce- \\
Ceramic
\end{tabular} & $\begin{array}{l}\text { Household } \\
\text { Maintenance- } \\
\text { Glass }\end{array}$ & $\begin{array}{l}\text { Architectural } \\
\text { Items }\end{array}$ \\
\hline & & & & & & & & & hite paint \\
\hline $\begin{array}{l}23 S G 27 \\
2-168\end{array}$ & $\begin{array}{l}40 R 6 \\
0\end{array}$ & 3 & 9 & ZLO & 10 & & & & $\begin{array}{l}\text { flakes, green } \\
\text { pain over } \\
\text { white }\end{array}$ \\
\hline $\begin{array}{l}23 S G 27 \\
2-168\end{array}$ & $\begin{array}{l}40 R 6 \\
0\end{array}$ & 3 & & ZLO & 1 & & & & $\begin{array}{l}\text { flake, green } \\
\text { over white } \\
\text { over red paint }\end{array}$ \\
\hline $\begin{array}{l}23 S G 27 \\
2-168\end{array}$ & $\begin{array}{l}40 R 6 \\
0\end{array}$ & 3 & & ZLO & 1 & & & & \\
\hline $\begin{array}{l}23 S G 27 \\
2-168\end{array}$ & $\begin{array}{l}40 R 6 \\
0\end{array}$ & 3 & & ZLO & 1 & & & & \\
\hline $\begin{array}{l}23 S G 27 \\
2-168\end{array}$ & $\begin{array}{l}40 R 6 \\
0\end{array}$ & 3 & & ZLO & 2 & & & & \\
\hline $\begin{array}{l}23 S G 27 \\
2-169\end{array}$ & $\begin{array}{l}40 R 6 \\
0\end{array}$ & 3 & 9 & ZLO & 1 & & & & $\begin{array}{l}\text { frag, window } \\
\text { glass; light } \\
\text { blue-green }\end{array}$ \\
\hline $\begin{array}{l}23 S G 27 \\
2-169\end{array}$ & $\begin{array}{l}40 R 6 \\
0\end{array}$ & 3 & & ZLO & 1 & & & & \\
\hline $\begin{array}{l}23 S G 27 \\
2-170\end{array}$ & $\begin{array}{l}40 \mathrm{R} 6 \\
0\end{array}$ & 3 & 9 & ZLO & 2 & & & $\begin{array}{l}\text { frags, thick, } \\
\text { clear glass }\end{array}$ & \\
\hline $\begin{array}{l}23 S G 27 \\
2-170\end{array}$ & $\begin{array}{l}40 R 6 \\
0\end{array}$ & 3 & & ZLO & 1 & & & $\begin{array}{l}\text { rim and neck } \\
\text { frag, open- } \\
\text { mouth jar or } \\
\text { bottle; light } \\
\text { blue, thin glass; } \\
\text { pharmaceutical } \\
\text { bottle? }\end{array}$ & \\
\hline $\begin{array}{l}23 S G 27 \\
2-170\end{array}$ & $\begin{array}{l}40 R 6 \\
0\end{array}$ & 3 & 9 & ZLO & 2 & & & $\begin{array}{l}\text { body frags, light } \\
\text { blue, thin bottle }\end{array}$ & \\
\hline $\begin{array}{l}23 S G 27 \\
2-170\end{array}$ & $\begin{array}{l}40 R 6 \\
0\end{array}$ & 3 & 9 & ZLO & 3 & & & $\begin{array}{l}\text { body frags light } \\
\text { blue, thicker } \\
\text { bottle glass }\end{array}$ & \\
\hline $\begin{array}{l}23 S G 27 \\
2-170\end{array}$ & $\begin{array}{l}40 R 6 \\
0\end{array}$ & 3 & 9 & ZLO & 2 & & & $\begin{array}{l}\text { body frags, olive } \\
\text { green bottle } \\
\text { glass }\end{array}$ & \\
\hline $\begin{array}{l}23 S G 27 \\
2-170\end{array}$ & $\begin{array}{l}40 R 6 \\
0\end{array}$ & 3 & 9 & ZLO & 1 & & & $\begin{array}{l}\text { rim frag, } \\
\text { medium green }\end{array}$ & \\
\hline
\end{tabular}




\begin{tabular}{|c|c|c|c|c|c|c|c|c|c|}
\hline \multicolumn{10}{|c|}{ Archaeological Analysis } \\
\hline $\begin{array}{c}\text { Catalog } \\
\text { ue \# }\end{array}$ & Unit & 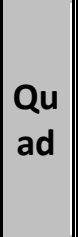 & $\begin{array}{c}\text { Lev } \\
\text { el }\end{array}$ & $\begin{array}{c}\text { Time } \\
\text { peri } \\
\text { od }\end{array}$ & Amou & $\begin{array}{c}\text { Household } \\
\text { Maintenan } \\
\text { ce-Other }\end{array}$ & \begin{tabular}{|l} 
Househol \\
d \\
Maintena \\
nce- \\
Ceramic
\end{tabular} & $\begin{array}{c}\text { Household } \\
\text { Maintenance- } \\
\text { Glass }\end{array}$ & $\begin{array}{c}\text { Architectural } \\
\text { Items }\end{array}$ \\
\hline & & & & & & & & $\begin{array}{l}\text { to yellow-green } \\
\text { vessel glass }\end{array}$ & \\
\hline $\begin{array}{l}23 S G 27 \\
2-170\end{array}$ & $\begin{array}{l}40 \mathrm{R} 6 \\
0\end{array}$ & 3 & 9 & ZLO & 4 & & & \begin{tabular}{|l} 
body frags, \\
medium green \\
to yellow-green \\
vessel glass
\end{tabular} & \\
\hline $\begin{array}{l}23 S G 27 \\
2-170\end{array}$ & $\begin{array}{l}40 \mathrm{R} 6 \\
0\end{array}$ & 3 & 9 & ZLO & 6 & & & $\begin{array}{l}\text { frags, light blue } \\
\text { glass }\end{array}$ & \\
\hline $\begin{array}{l}23 S G 27 \\
2-170\end{array}$ & $\begin{array}{l}40 \mathrm{R} 6 \\
0\end{array}$ & 3 & & ZLO & 1 & & & $\begin{array}{l}\text { frag, thick clear } \\
\text { glass, vessel } \\
\text { glass }\end{array}$ & \\
\hline $\begin{array}{l}23 S G 27 \\
2-170\end{array}$ & $\begin{array}{l}40 R 6 \\
0\end{array}$ & 3 & 9 & ZLO & 1 & & & $\begin{array}{l}\text { frag, thin clear } \\
\text { glass, vessel } \\
\text { glass }\end{array}$ & \\
\hline $\begin{array}{l}23 S G 27 \\
2-170\end{array}$ & $\begin{array}{l}40 \mathrm{R} 6 \\
0\end{array}$ & 3 & 9 & ZLO & 3 & & & frags, clear glass & \\
\hline $\begin{array}{l}23 S G 27 \\
2-170\end{array}$ & $\begin{array}{l}40 R 6 \\
0\end{array}$ & 3 & & ZLO & 2 & & & $\begin{array}{l}\text { frags, clear glass } \\
\text { that are cloudy }\end{array}$ & \\
\hline $\begin{array}{l}23 S G 27 \\
2-170\end{array}$ & $\begin{array}{l}40 \mathrm{R} 6 \\
0\end{array}$ & 3 & 9 & ZLO & 28 & & & & $\begin{array}{l}\text { frags, window } \\
\text { glass, light } \\
\text { blue-green }\end{array}$ \\
\hline $\begin{array}{l}23 S G 27 \\
2-170\end{array}$ & $\begin{array}{l}40 R 6 \\
0\end{array}$ & 3 & 9 & ZLO & 2 & & & & $\begin{array}{l}\text { frags, window } \\
\text { glass, clear }\end{array}$ \\
\hline $\begin{array}{l}23 S G 27 \\
2-170\end{array}$ & $\begin{array}{l}40 R 6 \\
0\end{array}$ & 3 & 9 & ZLO & 1 & & & & \\
\hline $\begin{array}{l}23 S G 27 \\
2-170\end{array}$ & $\begin{array}{l}40 \mathrm{R} 6 \\
0\end{array}$ & 3 & 9 & ZLO & 1 & & & & \\
\hline $\begin{array}{l}23 S G 27 \\
2-170\end{array}$ & $\begin{array}{l}40 \mathrm{R} 6 \\
0\end{array}$ & 3 & 9 & ZLO & 1 & & & & \\
\hline $\begin{array}{l}23 S G 27 \\
2-170\end{array}$ & $\begin{array}{l}40 \mathrm{R} 6 \\
0\end{array}$ & 3 & 9 & ZLO & 1 & & & & \\
\hline $\begin{array}{l}23 S G 27 \\
2-170\end{array}$ & $\begin{array}{l}40 \mathrm{R} 6 \\
0\end{array}$ & 3 & 9 & ZLO & 1 & & & & \\
\hline $\begin{array}{l}23 S G 27 \\
2-170\end{array}$ & $\begin{array}{l}40 \mathrm{R} 6 \\
0\end{array}$ & 3 & 9 & ZLO & 1 & & & & \\
\hline $\begin{array}{l}23 S G 27 \\
2-170\end{array}$ & $\begin{array}{l}40 R 6 \\
0\end{array}$ & 3 & 9 & ZLO & 1 & & & & \\
\hline
\end{tabular}




\begin{tabular}{|c|c|c|c|c|c|c|c|c|c|}
\hline \multicolumn{10}{|c|}{ Archaeological Analysis } \\
\hline $\begin{array}{c}\text { Catalog } \\
\text { ue \# }\end{array}$ & Unit & $\begin{array}{l}\text { Qu } \\
\text { ad }\end{array}$ & $\begin{array}{l}\text { Lev } \\
\text { el }\end{array}$ & \begin{tabular}{||c} 
Time \\
peri \\
od
\end{tabular} & Amou & $\begin{array}{c}\text { Household } \\
\text { Maintenan } \\
\text { ce-Other }\end{array}$ & \begin{tabular}{|} 
Househol \\
d \\
Maintena \\
nce- \\
Ceramic
\end{tabular} & $\begin{array}{c}\text { Household } \\
\text { Maintenance- } \\
\text { Glass }\end{array}$ & $\begin{array}{c}\text { Architectural } \\
\text { Items }\end{array}$ \\
\hline $\begin{array}{l}23 S G 27 \\
2-170\end{array}$ & $\begin{array}{l}40 R 6 \\
0\end{array}$ & 3 & & 9 ZLO & 3 & & & & \\
\hline $\begin{array}{l}23 S G 27 \\
2-170\end{array}$ & $\begin{array}{l}40 R 6 \\
0\end{array}$ & 3 & & 9 ZLO & 3 & & & & \\
\hline $\begin{array}{l}23 S G 27 \\
2-170\end{array}$ & $\begin{array}{l}40 R 6 \\
0\end{array}$ & 3 & & 9 ZLO & 1 & & & & \\
\hline $\begin{array}{l}23 S G 27 \\
2-170\end{array}$ & $\begin{array}{l}40 R 6 \\
0\end{array}$ & 3 & & 9 ZLO & 4 & & & & \\
\hline $\begin{array}{l}23 S G 27 \\
2-170\end{array}$ & $\begin{array}{l}40 R 6 \\
0\end{array}$ & 3 & & 9 ZLO & 1 & & & & \\
\hline $\begin{array}{l}23 S G 27 \\
2-170\end{array}$ & $\begin{array}{l}40 R 6 \\
0\end{array}$ & 3 & & 9 ZLO & 1 & & & & \\
\hline \begin{tabular}{|l|}
$23 S G 27$ \\
$2-170$
\end{tabular} & $\begin{array}{l}40 R 6 \\
0\end{array}$ & 3 & & 9 ZLO & 1 & & & & \\
\hline $\begin{array}{l}23 S G 27 \\
2-170\end{array}$ & $\begin{array}{l}40 \mathrm{R} 6 \\
0\end{array}$ & 3 & & 9 ZLO & 1 & & & & \\
\hline $\begin{array}{l}23 S G 27 \\
2-170\end{array}$ & $\begin{array}{l}40 R 6 \\
0\end{array}$ & 3 & & 9 ZLO & 1 & & & & \\
\hline $\begin{array}{l}23 S G 27 \\
2-170\end{array}$ & $\begin{array}{l}40 R 6 \\
0\end{array}$ & 3 & & 9 ZLO & 3 & & & & \\
\hline $\begin{array}{l}23 S G 27 \\
2-170\end{array}$ & $\begin{array}{l}40 \mathrm{R} 6 \\
0\end{array}$ & 3 & & 9 ZLO & 1 & & & & \\
\hline $\begin{array}{l}23 S G 27 \\
2-170\end{array}$ & $\begin{array}{l}40 \mathrm{R} 6 \\
0\end{array}$ & 3 & & 9 ZLO & 1 & & & & \\
\hline $\begin{array}{l}23 S G 27 \\
2-170\end{array}$ & $\begin{array}{l}40 R 6 \\
0\end{array}$ & 3 & & 9 ZLO & & $\begin{array}{l}\text { thin frag, } \\
\text { white } \\
\text { metal }\end{array}$ & & & \\
\hline $\begin{array}{l}23 S G 27 \\
2-170\end{array}$ & $\begin{array}{l}40 \mathrm{R} 6 \\
0\end{array}$ & 3 & & 9 ZLO & 1 & & & & \\
\hline $\begin{array}{l}23 S G 27 \\
2-170\end{array}$ & $\begin{array}{l}40 R 6 \\
0\end{array}$ & 3 & & 9 ZLO & 1 & & & & \\
\hline $\begin{array}{l}23 S G 27 \\
2-170\end{array}$ & $\begin{array}{l}40 \mathrm{R} 6 \\
0\end{array}$ & 3 & & 9 ZLO & 4 & & & & $\begin{array}{l}\text { frags, white } \\
\text { lime mortar }\end{array}$ \\
\hline $\begin{array}{l}23 S G 27 \\
2-170\end{array}$ & $\begin{array}{l}40 R 6 \\
0\end{array}$ & 3 & & 9 ZLO & 10 & & & & $\begin{array}{l}\text { frags, } \\
\text { whitewash/w } \\
\text { hite paint }\end{array}$ \\
\hline
\end{tabular}




\begin{tabular}{|c|c|c|c|c|c|c|c|c|c|}
\hline \multicolumn{10}{|c|}{ Archaeological Analysis } \\
\hline $\begin{array}{c}\text { Catalog } \\
\text { ue \# }\end{array}$ & Unit & $\begin{array}{l}\text { Qu } \\
\text { ad }\end{array}$ & $\begin{array}{c}\text { Lev } \\
\text { el }\end{array}$ & $\mid \begin{array}{c}\text { Time } \\
\text { peri } \\
\text { od }\end{array}$ & Amou & $\mid \begin{array}{c}\text { Household } \\
\text { Maintenan } \\
\text { ce-Other }\end{array}$ & \begin{tabular}{|c|} 
Househol \\
d \\
Maintena \\
nce- \\
Ceramic
\end{tabular} & $\begin{array}{l}\text { Household } \\
\text { Maintenance- } \\
\text { Glass }\end{array}$ & $\begin{array}{l}\text { Architectural } \\
\text { Items }\end{array}$ \\
\hline $\begin{array}{l}23 S G 27 \\
2-170\end{array}$ & $\begin{array}{l}40 R 6 \\
0\end{array}$ & 3 & & 9 ZLO & 1 & & & & $\begin{array}{l}\text { small frag, } \\
\text { green pain } \\
\text { over } \\
\text { whitewash or } \\
\text { lime plaster }\end{array}$ \\
\hline $\begin{array}{l}23 S G 27 \\
2-171\end{array}$ & $\begin{array}{l}40 \mathrm{R} 6 \\
0\end{array}$ & 3 & & 9 ZLO & 4 & & & & $\begin{array}{l}\text { frags, window } \\
\text { glass, light } \\
\text { blue-green }\end{array}$ \\
\hline $\begin{array}{l}23 S G 27 \\
2-171\end{array}$ & $\begin{array}{l}40 \mathrm{R} 6 \\
0\end{array}$ & 3 & & 9 ZLO & 1 & & & $\begin{array}{l}\text { small frag, } \\
\text { yellow-green } \\
\text { glass }\end{array}$ & \\
\hline $\begin{array}{l}23 S G 27 \\
2-171\end{array}$ & $\begin{array}{l}40 R 6 \\
0\end{array}$ & 3 & & 9 ZLO & 2 & & & frags, clear glass & \\
\hline $\begin{array}{l}23 S G 27 \\
2-171\end{array}$ & $\begin{array}{l}40 R 6 \\
0\end{array}$ & 3 & & 9 ZLO & 5 & & & $\begin{array}{l}\text { tiny slivers of } \\
\text { clear glass }\end{array}$ & \\
\hline $\begin{array}{l}23 S G 27 \\
2-171\end{array}$ & $\begin{array}{l}40 R 6 \\
0\end{array}$ & 3 & & 9 ZLO & 1 & & & & \\
\hline $\begin{array}{l}23 S G 27 \\
2-171\end{array}$ & $\begin{array}{l}40 R 6 \\
0\end{array}$ & 3 & & 9 ZLO & 1 & & & & \\
\hline $\begin{array}{l}23 S G 27 \\
2-171\end{array}$ & $\begin{array}{l}40 R 6 \\
0\end{array}$ & 3 & & 9 ZLO & 1 & & & & \\
\hline $\begin{array}{l}23 S G 27 \\
2-171\end{array}$ & $\begin{array}{l}40 R 6 \\
0\end{array}$ & 3 & & 9 ZLO & 1 & $\begin{array}{l}\text { small } \\
\text { tubular } \\
\text { frag., brass- } \\
\text { plated iron }\end{array}$ & & & \\
\hline $\begin{array}{l}23 S G 27 \\
2-171 \\
\end{array}$ & $\begin{array}{l}40 \mathrm{R} 6 \\
0\end{array}$ & 3 & & 9 ZLO & 1 & & & & $\begin{array}{l}\text { frag., lime } \\
\text { plaster }\end{array}$ \\
\hline $\begin{array}{l}23 S G 27 \\
2-171\end{array}$ & $\begin{array}{l}40 R 6 \\
0\end{array}$ & 3 & & 9 ZLO & 9 & & & & $\begin{array}{l}\text { frags., } \\
\text { whitewash/w } \\
\text { hite paint }\end{array}$ \\
\hline $\begin{array}{l}23 S G 27 \\
2-172\end{array}$ & $\begin{array}{l}40 R 6 \\
0\end{array}$ & 3 & 10 & $\mathrm{ZO}$ & 4 & & & & $\begin{array}{l}\text { frags, window } \\
\text { glass; light } \\
\text { blue-green }\end{array}$ \\
\hline $\begin{array}{l}23 S G 27 \\
2-172\end{array}$ & $\begin{array}{l}40 R 6 \\
0\end{array}$ & 3 & 10 & $\mathrm{ZO}$ & 1 & & & & $\begin{array}{l}\text { frag, window } \\
\text { glass; light } \\
\text { blue }\end{array}$ \\
\hline $23 S G 27$ & $40 R 6$ & 3 & 10 & 0 & 1 & & & frag. Light blue- & \\
\hline
\end{tabular}




\begin{tabular}{|c|c|c|c|c|c|c|c|c|c|}
\hline \multicolumn{10}{|c|}{ Archaeological Analysis } \\
\hline $\begin{array}{c}\text { Catalog } \\
\text { ue \# }\end{array}$ & Unit & 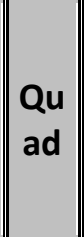 & $\begin{array}{c}\text { Lev } \\
\text { el }\end{array}$ & $\begin{array}{c}\text { Time } \\
\text { peri } \\
\text { od }\end{array}$ & Amou & $\begin{array}{c}\text { Household } \\
\text { Maintenan } \\
\text { ce-Other }\end{array}$ & \begin{tabular}{|l} 
Househol \\
d \\
Maintena \\
nce- \\
Ceramic
\end{tabular} & $\begin{array}{c}\text { Household } \\
\text { Maintenance- } \\
\text { Glass }\end{array}$ & $\begin{array}{c}\text { Architectural } \\
\text { Items }\end{array}$ \\
\hline $2-172$ & 0 & & & & & & & green glass & \\
\hline \begin{tabular}{|l|}
$23 S G 27$ \\
$2-172$
\end{tabular} & $\begin{array}{l}40 R 6 \\
0\end{array}$ & 3 & 10 & $\mathrm{ZO}$ & 2 & & & & \\
\hline \begin{tabular}{|l|}
$23 S G 27$ \\
$2-172$
\end{tabular} & $\begin{array}{l}40 R 6 \\
0\end{array}$ & 3 & 10 & $\mathrm{ZO}$ & 1 & & & & \\
\hline \begin{tabular}{|l|}
$23 S G 27$ \\
$2-172$
\end{tabular} & $\begin{array}{l}40 R 6 \\
0\end{array}$ & 3 & 10 & 20 & 1 & & & & \\
\hline \begin{tabular}{|l|}
$23 S G 27$ \\
$2-172$
\end{tabular} & $\begin{array}{l}40 R 6 \\
0\end{array}$ & 3 & 10 & 20 & 1 & & & & \\
\hline $\begin{array}{l}23 S G 27 \\
2-172\end{array}$ & $\begin{array}{l}40 R 6 \\
0\end{array}$ & 3 & 10 & 20 & 2 & & & & \\
\hline \begin{tabular}{|l|}
$23 S G 27$ \\
$2-172$
\end{tabular} & \begin{tabular}{|l}
$40 \mathrm{R} 6$ \\
0 \\
\end{tabular} & 3 & 10 & $\mathrm{ZO}$ & 2 & & & & \\
\hline$\left|\begin{array}{l}23 S G 27 \\
2-172\end{array}\right|$ & $\begin{array}{l}40 R 6 \\
0\end{array}$ & 3 & 10 & 20 & 2 & & & & \\
\hline \begin{tabular}{|l|}
$23 S G 27$ \\
$2-172$
\end{tabular} & $\begin{array}{l}40 R 6 \\
0\end{array}$ & 3 & 10 & 20 & 3 & & & & \\
\hline \begin{tabular}{|l|}
$23 S G 27$ \\
$2-172$
\end{tabular} & $\begin{array}{l}40 \mathrm{R} 6 \\
0\end{array}$ & 3 & 10 & $\mathrm{ZO}$ & 1 & & & & \\
\hline \begin{tabular}{|l|}
$23 S G 27$ \\
$2-172$
\end{tabular} & $\begin{array}{l}40 \mathrm{R} 6 \\
0\end{array}$ & 3 & 10 & 20 & 1 & & & & \\
\hline $\begin{array}{l}23 S G 27 \\
2-172\end{array}$ & $\begin{array}{l}40 R 6 \\
0\end{array}$ & 3 & 10 & ZO & 1 & & & & \\
\hline \begin{tabular}{|l|}
$23 S G 27$ \\
$2-172$
\end{tabular} & $\begin{array}{l}40 R 6 \\
0\end{array}$ & 3 & 10 & 20 & 1 & & & & \\
\hline \begin{tabular}{|l|}
$23 S G 27$ \\
$2-172$
\end{tabular} & $\begin{array}{l}40 R 6 \\
0\end{array}$ & 3 & 10 & 20 & 1 & & & & \\
\hline \begin{tabular}{|l|}
$23 S G 27$ \\
$2-172$
\end{tabular} & $\begin{array}{l}40 \mathrm{R} 6 \\
0\end{array}$ & 3 & 10 & $\mathrm{ZO}$ & 1 & & & & \\
\hline \begin{tabular}{|l|}
$23 S G 27$ \\
$2-172$
\end{tabular} & $\begin{array}{l}40 \mathrm{R} 6 \\
0\end{array}$ & 3 & 10 & $\mathrm{ZO}$ & 4 & & & & $\begin{array}{l}\text { frags lime } \\
\text { plaster } \\
\text { (whitewash) }\end{array}$ \\
\hline $\begin{array}{l}23 S G 27 \\
2-173\end{array}$ & $\begin{array}{l}40 R 6 \\
0\end{array}$ & 3 & 11 & $\mathrm{ZO}$ & 3 & & & $\begin{array}{l}\text { frags, dark olive- } \\
\text { green bottle } \\
\text { glass }\end{array}$ & \\
\hline $23 S G 27$ & 40R6 & 3 & 11 & 20 & 2 & & & frags, yellow- & \\
\hline
\end{tabular}




\begin{tabular}{|c|c|c|c|c|c|c|c|c|c|}
\hline \multicolumn{10}{|c|}{ Archaeological Analysis } \\
\hline $\begin{array}{c}\text { Catalog } \\
\text { ue \# }\end{array}$ & Unit & Qu & $\begin{array}{c}\text { Lev } \\
\text { el }\end{array}$ & $\begin{array}{c}\text { Time } \\
\text { peri } \\
\text { od }\end{array}$ & Amou & $\begin{array}{c}\text { Household } \\
\text { Maintenan } \\
\text { ce-Other }\end{array}$ & \begin{tabular}{|c|} 
Househol \\
d \\
Maintena \\
nce- \\
Ceramic
\end{tabular} & $\begin{array}{c}\text { Household } \\
\text { Maintenance- } \\
\text { Glass }\end{array}$ & $\begin{array}{c}\text { Architectural } \\
\text { Items }\end{array}$ \\
\hline $2-173$ & 0 & & & & & & & $\begin{array}{l}\text { green bottle } \\
\text { glass }\end{array}$ & \\
\hline $\begin{array}{l}23 S G 27 \\
2-173\end{array}$ & $\begin{array}{l}40 R 6 \\
0\end{array}$ & 3 & 11 & ZO & 1 & & & & \\
\hline $\begin{array}{l}23 S G 27 \\
2-173\end{array}$ & $\begin{array}{l}40 \mathrm{R} 6 \\
0\end{array}$ & 3 & 11 & 20 & 2 & & & $\begin{array}{l}\text { frags, thin light } \\
\text { blue/clear vessel } \\
\text { glass }\end{array}$ & \\
\hline $\begin{array}{l}23 S G 27 \\
2-173\end{array}$ & $\begin{array}{l}40 \mathrm{R} 6 \\
0\end{array}$ & 3 & 112 & ZO & 7 & & & & $\begin{array}{l}\text { frags, window } \\
\text { glass; light } \\
\text { blue-green }\end{array}$ \\
\hline \begin{tabular}{|l|}
$23 S G 27$ \\
$2-173$
\end{tabular} & $\begin{array}{l}40 R 6 \\
0\end{array}$ & 3 & 11 & ZO & 2 & & & $\begin{array}{l}\text { frags, } \\
\text { miscellaneous } \\
\text { glass; light blue- } \\
\text { green }\end{array}$ & \\
\hline $\begin{array}{l}23 S G 27 \\
2-173\end{array}$ & $\begin{array}{l}40 \mathrm{R} 6 \\
0\end{array}$ & 3 & 112 & ZO & 1 & & & $\begin{array}{l}\text { frag, burned } \\
\text { glass; appears } \\
\text { light blue-green }\end{array}$ & \\
\hline \begin{tabular}{|l|}
$23 S G 27$ \\
$2-173$
\end{tabular} & $\begin{array}{l}40 R 6 \\
0\end{array}$ & 3 & 11 & ZO & 1 & & & & \\
\hline $\begin{array}{l}23 S G 27 \\
2-173\end{array}$ & $\begin{array}{l}40 \mathrm{R} 6 \\
0\end{array}$ & 3 & 11 & ZO & 1 & & & & \\
\hline $\begin{array}{l}23 S G 27 \\
2-173\end{array}$ & $\begin{array}{l}40 \mathrm{R} 6 \\
0\end{array}$ & 3 & 112 & ZO & 1 & & & & \\
\hline $\begin{array}{l}23 S G 27 \\
2-173\end{array}$ & $\begin{array}{l}40 R 6 \\
0\end{array}$ & 3 & 11 & ZO & 1 & & & & \\
\hline \begin{tabular}{|l|}
$23 S G 27$ \\
$2-173$
\end{tabular} & $\begin{array}{l}40 \mathrm{R} 6 \\
0\end{array}$ & 3 & 11 & 20 & 3 & & & & \\
\hline $\begin{array}{l}23 S G 27 \\
2-173\end{array}$ & $\begin{array}{l}40 \mathrm{R} 6 \\
0\end{array}$ & 3 & 112 & ZO & 2 & & & & \\
\hline $\begin{array}{l}23 S G 27 \\
2-173\end{array}$ & $\begin{array}{l}40 R 6 \\
0\end{array}$ & 3 & 11 & ZO & 2 & & & & \\
\hline \begin{tabular}{|l|}
$23 S G 27$ \\
$2-173$
\end{tabular} & $\begin{array}{l}40 R 6 \\
0\end{array}$ & 3 & 112 & ZO & 2 & & & & \\
\hline $\begin{array}{l}23 S G 27 \\
2-173\end{array}$ & $\begin{array}{l}40 \mathrm{R} 6 \\
0\end{array}$ & 3 & 11 & ZO & 1 & & & & \\
\hline \begin{tabular}{|l|}
$23 S G 27$ \\
$2-173$
\end{tabular} & $\begin{array}{l}40 R 6 \\
0\end{array}$ & 3 & 11 & ZO & 1 & & & & \\
\hline
\end{tabular}




\begin{tabular}{|c|c|c|c|c|c|c|c|c|c|}
\hline \multicolumn{10}{|c|}{ Archaeological Analysis } \\
\hline $\begin{array}{c}\text { Catalog } \\
\text { ue \# }\end{array}$ & Unit & $\begin{array}{l}\text { Qu } \\
\text { ad }\end{array}$ & Lev & $\mid \begin{array}{c}\text { Time } \\
\text { peri } \\
\text { od }\end{array}$ & Amou & $\mid \begin{array}{c}\text { Household } \\
\text { Maintenan } \\
\text { ce-Other }\end{array}$ & \begin{tabular}{|c|} 
Househol \\
d \\
Maintena \\
nce- \\
Ceramic
\end{tabular} & $\begin{array}{c}\text { Household } \\
\text { Maintenance- } \\
\text { Glass }\end{array}$ & $\begin{array}{c}\text { Architectural } \\
\text { Items }\end{array}$ \\
\hline \begin{tabular}{|l|}
$23 S G 27$ \\
$2-173$
\end{tabular} & $\begin{array}{l}40 R 6 \\
0\end{array}$ & 3 & 11 & 20 & 1 & & & & \\
\hline $\begin{array}{l}23 S G 27 \\
2-173\end{array}$ & $\begin{array}{l}40 R 6 \\
0\end{array}$ & 3 & 11 & 20 & 1 & & & & \\
\hline $\begin{array}{l}23 S G 27 \\
2-173\end{array}$ & $\begin{array}{l}40 R 6 \\
0\end{array}$ & 3 & 11 & 20 & 1 & & & & \\
\hline \begin{tabular}{|l|}
$23 S G 27$ \\
$2-173$
\end{tabular} & $\begin{array}{l}40 R 6 \\
0\end{array}$ & 3 & 11 & 20 & 1 & & & & \\
\hline $\begin{array}{l}23 S G 27 \\
2-173\end{array}$ & $\begin{array}{l}40 R 6 \\
0\end{array}$ & 3 & 11 & 20 & 2 & & & & \\
\hline \begin{tabular}{|l|}
$23 S G 27$ \\
$2-173$
\end{tabular} & $\begin{array}{l}40 \mathrm{R} 6 \\
0\end{array}$ & 3 & 11 & 20 & & \begin{tabular}{|l|} 
frags scrap \\
copper/raw \\
copper
\end{tabular} & & & \\
\hline \begin{tabular}{|l|}
$23 S G 27$ \\
$2-173$
\end{tabular} & $\begin{array}{l}40 \mathrm{R} 6 \\
0 \\
\end{array}$ & 3 & 11 & 20 & 2 & & & & \\
\hline \begin{tabular}{|l|}
$23 S G 27$ \\
$2-173$
\end{tabular} & $\begin{array}{l}40 \mathrm{R} 6 \\
0 \\
\end{array}$ & 3 & 11 & 20 & 1 & & & & \\
\hline \begin{tabular}{|l|}
$23 S G 27$ \\
$2-173$
\end{tabular} & $\begin{array}{l}40 \mathrm{R} 6 \\
0 \\
\end{array}$ & 3 & 11 & 20 & 29 & & & & \\
\hline $\begin{array}{l}23 S G 27 \\
2-173\end{array}$ & $\begin{array}{l}40 R 6 \\
0\end{array}$ & 3 & 11 & 20 & 3 & & & & $\begin{array}{l}\text { thin frags } \\
\text { lime } \\
\text { plaster/white } \\
\text { wash }\end{array}$ \\
\hline \begin{tabular}{|l|}
$23 S G 27$ \\
$2-173$
\end{tabular} & $\begin{array}{l}40 \mathrm{R} 6 \\
0\end{array}$ & 3 & 11 & 20 & 1 & & & & \\
\hline $\begin{array}{l}23 S G 27 \\
2-173\end{array}$ & $\begin{array}{l}40 R 6 \\
0 \\
\end{array}$ & 3 & 11 & 20 & 2 & & & & \\
\hline \begin{tabular}{|l|}
$23 S G 27$ \\
$2-174$
\end{tabular} & $\begin{array}{l}40 \mathrm{R} 6 \\
0\end{array}$ & 3 & 12 & 20 & 1 & & & \begin{tabular}{|l} 
frag medium \\
green vessel \\
glass, possible \\
bottle glass
\end{tabular} & \\
\hline \begin{tabular}{|l|}
$23 S G 27$ \\
$2-174$
\end{tabular} & $\begin{array}{l}40 R 6 \\
0\end{array}$ & 3 & 12 & 20 & 7 & & & & $\begin{array}{l}\text { frags window } \\
\text { glass, light } \\
\text { blue-green }\end{array}$ \\
\hline \begin{tabular}{|l|}
$23 S G 27$ \\
$2-174$
\end{tabular} & $\begin{array}{l}40 R 6 \\
0\end{array}$ & 3 & 12 & 20 & 1 & & & & \\
\hline $23 S G 27$ & $40 R 6$ & 3 & 12 & & 1 & & & & \\
\hline
\end{tabular}




\begin{tabular}{|c|c|c|c|c|c|c|c|c|c|}
\hline \multicolumn{10}{|c|}{ Archaeological Analysis } \\
\hline $\begin{array}{c}\text { Catalog } \\
\text { ue \# }\end{array}$ & Unit & $\begin{array}{l}\text { Qu } \\
\text { ad }\end{array}$ & $\mid \begin{array}{c}\text { Lev } \\
\text { el }\end{array}$ & $\left|\begin{array}{c}\text { Time } \\
\text { peri } \\
\text { od }\end{array}\right|$ & Amou & \begin{tabular}{||c||} 
Household \\
Maintenan \\
ce-Other
\end{tabular} & \begin{tabular}{|c|} 
Househol \\
d \\
Maintena \\
nce- \\
Ceramic
\end{tabular} & $\begin{array}{c}\text { Household } \\
\text { Maintenance- } \\
\text { Glass }\end{array}$ & $\begin{array}{c}\text { Architectural } \\
\text { Items }\end{array}$ \\
\hline $2-174$ & 0 & & & & & & & & \\
\hline $\begin{array}{l}23 S G 27 \\
2-174\end{array}$ & $\begin{array}{l}40 \mathrm{R} 6 \\
0\end{array}$ & 3 & 12 & 20 & 1 & & & & \\
\hline $\begin{array}{l}23 S G 27 \\
2-174\end{array}$ & $\begin{array}{l}40 R 6 \\
0\end{array}$ & 3 & 12 & 20 & 1 & & & & \\
\hline $\begin{array}{l}23 S G 27 \\
2-174\end{array}$ & $\begin{array}{l}40 R 6 \\
0\end{array}$ & 3 & 12 & 20 & 4 & & & & \\
\hline $\begin{array}{l}23 S G 27 \\
2-174\end{array}$ & $\begin{array}{l}40 R 6 \\
0\end{array}$ & 3 & 12 & 20 & 1 & & & & \\
\hline $\begin{array}{l}23 S G 27 \\
2-174\end{array}$ & $\begin{array}{l}40 R 6 \\
0\end{array}$ & 3 & 12 & 20 & 3 & & & & \\
\hline $\begin{array}{l}23 S G 27 \\
2-174\end{array}$ & $\begin{array}{l}40 R 6 \\
0\end{array}$ & 3 & 12 & 20 & 1 & & & & \\
\hline $\begin{array}{l}23 S G 27 \\
2-174\end{array}$ & $\begin{array}{l}40 R 6 \\
0\end{array}$ & 3 & 12 & 20 & 2 & & & & \\
\hline $\begin{array}{l}23 S G 27 \\
2-174\end{array}$ & $\begin{array}{l}40 R 6 \\
0\end{array}$ & 3 & 12 & 20 & 1 & & & & \\
\hline $\begin{array}{l}23 S G 27 \\
2-174\end{array}$ & $\begin{array}{l}40 R 6 \\
0\end{array}$ & 3 & 12 & 20 & 1 & & & & \\
\hline $\begin{array}{l}23 S G 27 \\
2-174\end{array}$ & $\begin{array}{l}40 R 6 \\
0\end{array}$ & 3 & 12 & 20 & 2 & & & & \\
\hline $\begin{array}{l}23 S G 27 \\
2-174\end{array}$ & $\begin{array}{l}40 R 6 \\
0\end{array}$ & 3 & 12 & 20 & & $\begin{array}{l}\text { frag sheet } \\
\text { brass scrap }\end{array}$ & & & \\
\hline $\begin{array}{l}23 S G 27 \\
2-174\end{array}$ & $\begin{array}{l}40 R 6 \\
0\end{array}$ & 3 & 12 & 20 & 1 & & & & \\
\hline $\begin{array}{l}23 S G 27 \\
2-174\end{array}$ & $\begin{array}{l}40 R 6 \\
0\end{array}$ & 3 & 12 & 20 & 1 & & & & \\
\hline $\begin{array}{l}23 S G 27 \\
2-175\end{array}$ & $\begin{array}{l}40 R 6 \\
0\end{array}$ & 3 & 13 & 20 & 1 & & & $\begin{array}{l}\text { frag, yellow- } \\
\text { green bottle; } \\
\text { pharmaceutical/ } \\
\text { sauce bottle size }\end{array}$ & \\
\hline $\begin{array}{l}23 S G 27 \\
2-175\end{array}$ & $\begin{array}{l}40 R 6 \\
0\end{array}$ & 3 & 13 & 20 & 1 & & & $\begin{array}{l}\text { frag, yellow- } \\
\text { green bottle } \\
\text { (wine bottle } \\
\text { thickness) }\end{array}$ & \\
\hline $23 S G 27$ & $40 R 6$ & 3 & 13 & $\mathrm{ZO}$ & 5 & & & frags window & \\
\hline
\end{tabular}




\begin{tabular}{|c|c|c|c|c|c|c|c|c|c|}
\hline \multicolumn{10}{|c|}{ Archaeological Analysis } \\
\hline $\begin{array}{c}\text { Catalog } \\
\text { ue \# }\end{array}$ & Unit & $\begin{array}{l}\text { Qu } \\
\text { ad }\end{array}$ & $\mid \begin{array}{c}\text { Lev } \\
\text { el }\end{array}$ & $\begin{array}{c}\text { Time } \\
\text { peri } \\
\text { od }\end{array}$ & Amou & $\begin{array}{c}\text { Household } \\
\text { Maintenan } \\
\text { ce-Other }\end{array}$ & \begin{tabular}{|} 
Househol \\
d \\
Maintena \\
nce- \\
Ceramic
\end{tabular} & $\begin{array}{c}\text { Household } \\
\text { Maintenance- } \\
\text { Glass }\end{array}$ & $\begin{array}{c}\text { Architectural } \\
\text { Items }\end{array}$ \\
\hline $2-175$ & 0 & & & & & & & $\begin{array}{l}\text { glass; light blue- } \\
\text { green }\end{array}$ & \\
\hline $\begin{array}{l}23 S G 27 \\
2-175\end{array}$ & $\begin{array}{l}40 R 6 \\
0\end{array}$ & 3 & 13 & & 1 & & & & \\
\hline \begin{tabular}{|l|}
$23 S G 27$ \\
$2-175$
\end{tabular} & $\begin{array}{l}40 R 6 \\
0\end{array}$ & 3 & 13 & $\mathrm{ZO}$ & 3 & & & & \\
\hline $\begin{array}{l}23 S G 27 \\
2-175\end{array}$ & $\begin{array}{l}40 R 6 \\
0\end{array}$ & 3 & 13 & ZO & 1 & & & & \\
\hline $\begin{array}{l}23 S G 27 \\
2-175\end{array}$ & $\begin{array}{l}40 R 6 \\
0\end{array}$ & 3 & 13 & $\mathrm{ZO}$ & 1 & & & & \\
\hline $\begin{array}{l}23 S G 27 \\
2-175\end{array}$ & $\begin{array}{l}40 R 6 \\
0\end{array}$ & 3 & 13 & $\mathrm{ZO}$ & 1 & & & & \\
\hline $\begin{array}{l}23 S G 27 \\
2-175\end{array}$ & $\begin{array}{l}40 R 6 \\
0\end{array}$ & 3 & 13 & ZO & 2 & $\begin{array}{l}\text { strips of } \\
\text { sheet brass } \\
\text { scrap, } \\
\text { possible } \\
\text { brass- } \\
\text { plated } \\
\text { sheet tin }\end{array}$ & & & \\
\hline $\begin{array}{l}23 S G 27 \\
2-175\end{array}$ & $\begin{array}{l}40 R 6 \\
0\end{array}$ & 3 & 13 & $\mathrm{ZO}$ & 3 & & & & \\
\hline $\begin{array}{l}23 S G 27 \\
2-175\end{array}$ & $\begin{array}{l}40 R 6 \\
0\end{array}$ & 3 & 13 & $\mathrm{ZO}$ & 1 & & & & \\
\hline $\begin{array}{l}23 S G 27 \\
2-176\end{array}$ & $\begin{array}{l}40 R 6 \\
0\end{array}$ & 3 & 14 & $\mathrm{ZO}$ & 1 & & & $\begin{array}{l}\text { frag, olive-green } \\
\text { bottle glass }\end{array}$ & \\
\hline $\begin{array}{l}23 S G 27 \\
2-176\end{array}$ & $\begin{array}{l}40 R 6 \\
0\end{array}$ & 3 & 14 & ZO & 1 & & & $\begin{array}{l}\text { frag, yellow- } \\
\text { green bottle } \\
\text { glass }\end{array}$ & \\
\hline $\begin{array}{l}23 S G 27 \\
2-176\end{array}$ & $\begin{array}{l}40 R 6 \\
0\end{array}$ & 3 & 14 & $\mathrm{ZO}$ & 1 & & & $\begin{array}{l}\text { frag, light blue } \\
\text { bottle glass }\end{array}$ & \\
\hline $\begin{array}{l}23 S G 27 \\
2-176\end{array}$ & $\begin{array}{l}40 R 6 \\
0\end{array}$ & 3 & 14 & $\mathrm{ZO}$ & 3 & & & $\begin{array}{l}\text { frags, clear } \\
\text { glass, burned or } \\
\text { frosted }\end{array}$ & \\
\hline $\begin{array}{l}23 S G 27 \\
2-176\end{array}$ & $\begin{array}{l}40 R 6 \\
0\end{array}$ & 3 & 14 & $\mathrm{ZO}$ & 1 & & & $\begin{array}{l}\text { frag, light blue- } \\
\text { green to clear } \\
\text { glass }\end{array}$ & \\
\hline $23 S G 27$ & $40 R 6$ & 3 & 14 & $\mathrm{ZO}$ & 12 & & & & frags, window \\
\hline
\end{tabular}




\begin{tabular}{|c|c|c|c|c|c|c|c|c|c|}
\hline \multicolumn{10}{|c|}{ Archaeological Analysis } \\
\hline $\begin{array}{c}\text { Catalog } \\
\text { ue \# }\end{array}$ & Unit & $\begin{array}{l}\text { Qu } \\
\text { ad }\end{array}$ & Lev & $\begin{array}{c}\text { Time } \\
\text { peri } \\
\text { od }\end{array}$ & Amou & $\begin{array}{c}\text { Household } \\
\text { Maintenan } \\
\text { ce-Other }\end{array}$ & \begin{tabular}{|c|} 
Househol \\
d \\
Maintena \\
nce- \\
Ceramic
\end{tabular} & $\begin{array}{c}\text { Household } \\
\text { Maintenance- } \\
\text { Glass }\end{array}$ & $\begin{array}{l}\text { Architectural } \\
\text { Items }\end{array}$ \\
\hline $2-176$ & 0 & & & & & & & & $\begin{array}{l}\text { glass, light } \\
\text { blue-green }\end{array}$ \\
\hline \begin{tabular}{|l|}
$23 S G 27$ \\
$2-176$
\end{tabular} & $\begin{array}{l}40 \mathrm{R} 6 \\
0\end{array}$ & 3 & 14 & 20 & 2 & & & & $\begin{array}{l}\text { frags, window } \\
\text { glass; } \\
\text { medium blue }\end{array}$ \\
\hline \begin{tabular}{|l|}
$23 S G 27$ \\
$2-176$
\end{tabular} & $\begin{array}{l}40 \mathrm{R} 6 \\
0\end{array}$ & 3 & 14 & 20 & 1 & & & & \\
\hline \begin{tabular}{|l|}
$23 S G 27$ \\
$2-176$
\end{tabular} & $\begin{array}{l}40 \mathrm{R} 6 \\
0\end{array}$ & 3 & 14 & 20 & 1 & & & & \\
\hline \begin{tabular}{|l|}
$23 S G 27$ \\
$2-176$
\end{tabular} & $\begin{array}{l}40 \mathrm{R} 6 \\
0\end{array}$ & 3 & 14 & 20 & 1 & & & & \\
\hline \begin{tabular}{|l|}
$23 S G 27$ \\
$2-176$
\end{tabular} & $\begin{array}{l}40 R 6 \\
0\end{array}$ & 3 & 14 & 20 & 1 & & & & \\
\hline \begin{tabular}{|l|}
$23 S G 27$ \\
$2-176$
\end{tabular} & $\begin{array}{l}40 R 6 \\
0\end{array}$ & 3 & 14 & 20 & 1 & & & & \\
\hline \begin{tabular}{|l|}
$23 S G 27$ \\
$2-176$
\end{tabular} & $\begin{array}{l}40 \mathrm{R} 6 \\
0 \\
\end{array}$ & 3 & 14 & 20 & 1 & & & & \\
\hline \begin{tabular}{|l|}
$23 S G 27$ \\
$2-176$
\end{tabular} & \begin{tabular}{|l}
$40 R 6$ \\
0 \\
\end{tabular} & 3 & 14 & 20 & 1 & & & & \\
\hline \begin{tabular}{|l|}
$23 S G 27$ \\
$2-176$
\end{tabular} & $\begin{array}{l}40 \mathrm{R} 6 \\
0\end{array}$ & 3 & 14 & 20 & 1 & & & & \\
\hline \begin{tabular}{|l|}
$23 S G 27$ \\
$2-176$
\end{tabular} & $\begin{array}{l}40 \mathrm{R} 6 \\
0\end{array}$ & 3 & 14 & 20 & 1 & & & & \\
\hline \begin{tabular}{|l|}
$23 S G 27$ \\
$2-176$
\end{tabular} & $\begin{array}{l}40 R 6 \\
0\end{array}$ & 3 & 14 & 20 & 1 & & & & \\
\hline \begin{tabular}{|l|}
$23 S G 27$ \\
$2-176$
\end{tabular} & $\begin{array}{l}40 \mathrm{R} 6 \\
0\end{array}$ & 3 & 14 & 20 & & $\begin{array}{l}\text { frag, sheet } \\
\text { brass scrap }\end{array}$ & & & \\
\hline \begin{tabular}{|l|}
$23 S G 27$ \\
$2-176$
\end{tabular} & $\begin{array}{l}40 \mathrm{R} 6 \\
0\end{array}$ & 3 & 14 & 20 & 1 & & & & \\
\hline \begin{tabular}{|l|}
$23 S G 27$ \\
$2-176$
\end{tabular} & $\begin{array}{l}40 \mathrm{R} 6 \\
0\end{array}$ & 3 & 14 & 20 & 3 & & & & \\
\hline \begin{tabular}{|l|}
$23 S G 27$ \\
$2-176$
\end{tabular} & $\begin{array}{l}40 \mathrm{R} 6 \\
0\end{array}$ & 3 & 14 & 20 & 2 & & & & \\
\hline \begin{tabular}{|l|}
$23 S G 27$ \\
$2-178$
\end{tabular} & $\begin{array}{l}40 \mathrm{R} 6 \\
0\end{array}$ & 3 & 15 & ZO & 2 & & & & $\begin{array}{l}\text { frags, } \\
\text { whitewash/li } \\
\text { me plaster }\end{array}$ \\
\hline
\end{tabular}




\begin{tabular}{|c|c|c|c|c|c|c|c|c|c|}
\hline \multicolumn{10}{|c|}{ Archaeological Analysis } \\
\hline $\begin{array}{c}\text { Catalog } \\
\text { ue \# }\end{array}$ & Unit & 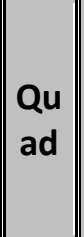 & $\mid \begin{array}{c}\text { Lev } \\
\text { el }\end{array}$ & $\begin{array}{c}\text { Time } \\
\text { peri } \\
\text { od }\end{array}$ & Amou & $\mid \begin{array}{c}\text { Household } \\
\text { Maintenan } \\
\text { ce-Other }\end{array}$ & \begin{tabular}{|l} 
Househol \\
d \\
Maintena \\
nce- \\
Ceramic
\end{tabular} & $\begin{array}{c}\text { Household } \\
\text { Maintenance- } \\
\text { Glass }\end{array}$ & $\begin{array}{c}\text { Architectural } \\
\text { Items }\end{array}$ \\
\hline $\begin{array}{l}23 S G 27 \\
2-179\end{array}$ & $\begin{array}{l}40 R 6 \\
0\end{array}$ & 3 & 15 & 20 & 1 & & & $\begin{array}{l}\text { frag, clear bottle } \\
\text { glass }\end{array}$ & \\
\hline $\begin{array}{l}23 S G 27 \\
2-179\end{array}$ & $\begin{array}{l}40 R 6 \\
0\end{array}$ & 3 & 15 & 20 & 2 & & & $\begin{array}{l}\text { frags, yellow- } \\
\text { green glass, } \\
\text { probably small } \\
\text { bottle }\end{array}$ & \\
\hline \begin{tabular}{|l|}
$23 S G 27$ \\
$2-179$
\end{tabular} & $\begin{array}{l}40 R 6 \\
0\end{array}$ & 3 & 15 & 20 & 1 & & & $\begin{array}{l}\text { frag, olive-green } \\
\text { bottle glass }\end{array}$ & \\
\hline $\begin{array}{l}23 S G 27 \\
2-179\end{array}$ & $\begin{array}{l}40 R 6 \\
0\end{array}$ & 3 & 15 & 20 & 6 & & & $\begin{array}{l}\text { frags, window } \\
\text { glass; light blue- } \\
\text { green }\end{array}$ & \\
\hline \begin{tabular}{|l}
$23 S G 27$ \\
$2-179$
\end{tabular} & $\begin{array}{l}40 R 6 \\
0\end{array}$ & 3 & 15 & 20 & 1 & & & & \\
\hline \begin{tabular}{|l}
$23 S G 27$ \\
$2-179$
\end{tabular} & $\begin{array}{l}40 R 6 \\
0\end{array}$ & 3 & 15 & 20 & 1 & & & & \\
\hline \begin{tabular}{|l}
$23 S G 27$ \\
$2-179$
\end{tabular} & $\begin{array}{l}40 R 6 \\
0\end{array}$ & 3 & 15 & 20 & 1 & & & & \\
\hline $\begin{array}{l}23 S G 27 \\
2-179\end{array}$ & $\begin{array}{l}40 \mathrm{R} 6 \\
0\end{array}$ & 3 & 15 & 20 & 1 & & & & \\
\hline $\begin{array}{l}23 S G 27 \\
2-179\end{array}$ & $\begin{array}{l}40 R 6 \\
0\end{array}$ & 3 & 15 & 20 & 1 & & & & \\
\hline \begin{tabular}{|l|}
$23 S G 27$ \\
$2-179$
\end{tabular} & $\begin{array}{l}40 R 6 \\
0\end{array}$ & 3 & 15 & 20 & 1 & & & & \\
\hline $\begin{array}{l}23 S G 27 \\
2-179\end{array}$ & $\begin{array}{l}40 R 6 \\
0\end{array}$ & 3 & 15 & 20 & 1 & & & & \\
\hline \begin{tabular}{|l|}
$23 S G 27$ \\
$2-179$
\end{tabular} & $\begin{array}{l}40 R 6 \\
0\end{array}$ & 3 & 15 & 20 & 1 & & & & \\
\hline \begin{tabular}{|l|}
$23 S G 27$ \\
$2-179$
\end{tabular} & $\begin{array}{l}40 R 6 \\
0\end{array}$ & 3 & 15 & 20 & 1 & & & & \\
\hline \begin{tabular}{|l|}
$23 S G 27$ \\
$2-179$
\end{tabular} & $\begin{array}{l}40 R 6 \\
0\end{array}$ & 3 & 15 & 20 & 1 & & & & \\
\hline \begin{tabular}{|l|}
$23 S G 27$ \\
$2-180$
\end{tabular} & $\begin{array}{l}40 \mathrm{R} 6 \\
0\end{array}$ & 3 & 15 & 20 & 1 & & & & $\begin{array}{l}\text { frag, window } \\
\text { glass; light } \\
\text { blue-green }\end{array}$ \\
\hline \begin{tabular}{|l|}
$23 S G 27$ \\
$2-13$
\end{tabular} & $\begin{array}{l}110 \mathrm{R} \\
30\end{array}$ & 2 & & ZLO & 1 & & & $\begin{array}{l}\text { glass bottle } \\
\text { either wine glass } \\
\text { bowl or }\end{array}$ & \\
\hline
\end{tabular}




\begin{tabular}{|c|c|c|c|c|c|c|c|c|c|}
\hline \multicolumn{10}{|c|}{ Archaeological Analysis } \\
\hline $\begin{array}{c}\text { Catalog } \\
\text { ue \# }\end{array}$ & Unit & $\begin{array}{l}\text { Qu } \\
\text { ad }\end{array}$ & $\mid \begin{array}{c}\text { Lev } \\
\text { el }\end{array}$ & $\mid \begin{array}{c}\text { Time } \\
\text { peri } \\
\text { od }\end{array}$ & Amou & $\mid \begin{array}{c}\text { Household } \\
\text { Maintenan } \\
\text { ce-Other }\end{array}$ & \begin{tabular}{|c|} 
Househol \\
d \\
Maintena \\
nce- \\
Ceramic \\
\end{tabular} & $\begin{array}{l}\text { Household } \\
\text { Maintenance- } \\
\text { Glass }\end{array}$ & $\begin{array}{c}\text { Architectural } \\
\text { Items }\end{array}$ \\
\hline & & & & & & & & $\begin{array}{l}\text { pharmaceutical } \\
\text { bottle frag. }\end{array}$ & \\
\hline $\begin{array}{l}23 S G 27 \\
2-13\end{array}$ & $\begin{array}{l}110 R \\
30\end{array}$ & 2 & & ZLO & 1 & & & white milk glass & \\
\hline \begin{tabular}{|l|}
$23 S G 27$ \\
$2-13$
\end{tabular} & $\begin{array}{l}110 \mathrm{R} \\
30\end{array}$ & 2 & 1 & ZLO & 1 & & & & \\
\hline \begin{tabular}{|l|}
$23 S G 27$ \\
$2-13$
\end{tabular} & $\begin{array}{l}110 R \\
30\end{array}$ & 2 & & ZLO & 1 & & & & \\
\hline \begin{tabular}{|l|}
$23 S G 27$ \\
$2-13$
\end{tabular} & $\begin{array}{l}110 \mathrm{R} \\
30\end{array}$ & 2 & & ZLO & 1 & & & & \\
\hline \begin{tabular}{|l|}
$23 S G 27$ \\
$2-13$
\end{tabular} & $\begin{array}{l}110 \mathrm{R} \\
30\end{array}$ & 2 & & ZLO & 17 & & & & $\begin{array}{l}\text { frags, lime } \\
\text { plaster/morta } \\
\text { r }\end{array}$ \\
\hline & & & & & 2320 & & & & \\
\hline
\end{tabular}

\begin{tabular}{|c|c|c|c|c|c|c|c|c|c|c|c|c|c|c|}
\hline \multicolumn{15}{|c|}{ Archaeological Analysis } \\
\hline $\begin{array}{c}\text { Catal } \\
\text { ogue } \\
\#\end{array}$ & $\begin{array}{c}\text { Uni } \\
t\end{array}$ & 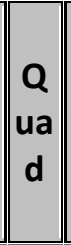 & $\begin{array}{c}\text { Le } \\
\text { ve } \\
\text { I }\end{array}$ & $\begin{array}{l}\text { Ti } \\
\text { me } \\
\text { per } \\
\text { iod }\end{array}$ & $\mid \begin{array}{c}\text { Am } \\
\text { oun } \\
t\end{array}$ & $\begin{array}{l}\text { Hous } \\
\text { ehold } \\
\text { Furnis } \\
\text { hings }\end{array}$ & Clothing & $\mid \begin{array}{c}\text { Perso } \\
\text { nal } \\
\text { Items }\end{array}$ & \begin{tabular}{|c|} 
Househ \\
old \\
product \\
ion \\
activity
\end{tabular} & \begin{tabular}{||c|}
$\begin{array}{c}\text { Househ } \\
\text { old } \\
\text { produc } \\
\text { tion-for } \\
\text { sale }\end{array}$ \\
\end{tabular} & $\mid \begin{array}{l}\text { Pharma } \\
\text { ceutical }\end{array}$ & $\mid \begin{array}{l}\text { Food } \\
\text { stuffs }\end{array}$ & $\mid \begin{array}{c}\text { Alc } \\
\text { oho } \\
\text { I }\end{array}$ & $\begin{array}{l}\text { Ot } \\
\text { her }\end{array}$ \\
\hline $\begin{array}{l}23 S G \\
272- \\
14\end{array}$ & $\begin{array}{l}110 \\
\text { R30 }\end{array}$ & 2 & & $\begin{array}{l}\mathrm{ZL} \\
\mathrm{O}\end{array}$ & 1 & & & $\begin{array}{l}\text { opaqu } \\
\text { e } \\
\text { black } \\
\text { seed } \\
\text { bead }\end{array}$ & & & & & & \\
\hline \begin{tabular}{|l|}
$23 S G$ \\
$272-$ \\
14
\end{tabular} & $\begin{array}{l}110 \\
\text { R30 }\end{array}$ & 2 & & $\begin{array}{l}Z L \\
O\end{array}$ & 1 & & & $\begin{array}{l}\text { neckl } \\
\text { ace } \\
\text { bead, } \\
\text { yello } \\
\text { w }\end{array}$ & & & & & & \\
\hline \begin{tabular}{|l|}
$23 S G$ \\
$272-$ \\
14
\end{tabular} & $\begin{array}{l}110 \\
\text { R30 }\end{array}$ & 2 & & $\begin{array}{l}Z L \\
O\end{array}$ & 6 & & & & \begin{tabular}{|l|} 
brass \\
straight \\
pin \\
frags
\end{tabular} & & & & & \\
\hline $\begin{array}{l}23 S G \\
272-\end{array}$ & $\begin{array}{l}110 \\
\text { R30 }\end{array}$ & 2 & & $\begin{array}{l}Z \mathrm{ZL} \\
\mathrm{O}\end{array}$ & 1 & & & & $\begin{array}{l}\text { brass } \\
\text { button }\end{array}$ & & & & & \\
\hline
\end{tabular}




\begin{tabular}{|c|c|c|c|c|c|c|c|c|c|c|c|c|c|c|}
\hline \multicolumn{15}{|c|}{ Archaeological Analysis } \\
\hline $\mid \begin{array}{c}\text { Catal } \\
\text { ogue } \\
\#\end{array}$ & $\begin{array}{c}\text { Uni } \\
t\end{array}$ & $\begin{array}{c}\text { Q } \\
\text { ua } \\
d\end{array}$ & $\begin{array}{c}\text { Le } \\
\text { ve } \\
\text { I }\end{array}$ & $\begin{array}{l}\text { Ti } \\
\text { me } \\
\text { per } \\
\text { iod }\end{array}$ & $\mid \begin{array}{c}\text { Am } \\
\text { oun } \\
t\end{array}$ & $\mid \begin{array}{l}\text { Hous } \\
\text { ehold } \\
\text { Furnis } \\
\text { hings }\end{array}$ & Clothing & $\begin{array}{c}\text { Perso } \\
\text { nal } \\
\text { Items }\end{array}$ & $\begin{array}{l}\text { Househ } \\
\text { old } \\
\text { product } \\
\text { ion } \\
\text { activity }\end{array}$ & \begin{tabular}{|c} 
Househ \\
old \\
produc \\
tion-for \\
sale
\end{tabular} & \begin{tabular}{|l} 
Pharma \\
ceutical
\end{tabular} & \begin{tabular}{||l} 
Food \\
stuffs
\end{tabular} & $\begin{array}{c}\text { Alc } \\
\text { oho } \\
\text { I }\end{array}$ & $\begin{array}{l}\text { Ot } \\
\text { her }\end{array}$ \\
\hline 14 & & & & & & & & & $\begin{array}{l}\text { with } \\
\text { gold gilt } \\
\text { and a } \\
\text { floral/st } \\
\text { ars/sun } \\
\text { pattern }\end{array}$ & & & & & \\
\hline $\begin{array}{l}23 S G \\
272- \\
14\end{array}$ & $\begin{array}{l}110 \\
\text { R30 }\end{array}$ & 2 & & $\begin{array}{l}Z L \\
O\end{array}$ & 1 & & & & $\begin{array}{l}\text { brass } \\
\text { eye } \\
\text { from } \\
\text { hook- } \\
\text { and-eye }\end{array}$ & & & & & \\
\hline $\begin{array}{l}23 S G \\
272- \\
14\end{array}$ & $\begin{array}{l}110 \\
\text { R30 }\end{array}$ & 2 & & $\begin{array}{l}\text { ZL } \\
0\end{array}$ & 1 & & & $\begin{array}{l}\text { brass } \\
\text { chain } \\
\text { sectio } \\
\text { n; } \\
\text { jewelr } \\
y- \\
\text { sized } \\
\text { chain }\end{array}$ & & & & & & \\
\hline $\begin{array}{l}23 S G \\
272- \\
14 \\
\text { Coml } \\
\text { ara } \\
\text { Park } \\
\text { Mou } \\
\text { ntain } \\
\text { Bike } \\
\text { Trail }\end{array}$ & $\begin{array}{l}110 \\
\text { R30 }\end{array}$ & 2 & & $\begin{array}{l}Z L \\
O\end{array}$ & 7 & & & & & & & & & \\
\hline \begin{tabular}{|l|}
$23 S G$ \\
$272-$ \\
14 \\
\end{tabular} & $\begin{array}{l}110 \\
\text { R30 }\end{array}$ & 2 & & $\begin{array}{l}Z L \\
O\end{array}$ & 1 & & & & & & & & & \\
\hline \begin{tabular}{|l}
$23 S G$ \\
$272-$ \\
14
\end{tabular} & $\begin{array}{l}110 \\
\text { R30 }\end{array}$ & 2 & & $\begin{array}{l}\text { ZL } \\
0\end{array}$ & 11 & & & & & $\begin{array}{l}\text { frags., } \\
\text { lead } \\
\text { splatter } \\
\text { /sprue, } \\
\text { waste } \\
\text { from } \\
\text { making }\end{array}$ & & & & \\
\hline
\end{tabular}




\begin{tabular}{|c|c|c|c|c|c|c|c|c|c|c|c|c|c|c|}
\hline \multicolumn{15}{|c|}{ Archaeological Analysis } \\
\hline $\mid \begin{array}{c}\text { Catal } \\
\text { ogue } \\
\#\end{array}$ & Uni & $\begin{array}{c}Q \\
\text { ua } \\
d\end{array}$ & \begin{tabular}{c|c} 
Le & \\
ve & $r$ \\
I & r \\
&
\end{tabular} & $\begin{array}{c}\mathrm{Ti} \\
\mathrm{me} \\
\mathrm{per} \\
\mathrm{iod}\end{array}$ & $\mid \begin{array}{c}\text { Am } \\
\text { oun } \\
t\end{array}$ & $\mid \begin{array}{l}\text { Hous } \\
\text { ehold } \\
\text { Furnis } \\
\text { hings }\end{array}$ & Clothing & \begin{tabular}{|c} 
Perso \\
nal \\
Items
\end{tabular} & $\begin{array}{c}\text { Househ } \\
\text { old } \\
\text { product } \\
\text { ion } \\
\text { activity }\end{array}$ & \begin{tabular}{|c} 
Househ \\
old \\
produc \\
tion-for \\
sale
\end{tabular} & $\begin{array}{l}\text { Pharma } \\
\text { ceutical }\end{array}$ & \begin{tabular}{|l|} 
Food \\
stuffs
\end{tabular} & $\begin{array}{c}\text { Alc } \\
\text { oho } \\
\text { I }\end{array}$ & $\begin{array}{l}\text { Ot } \\
\text { her }\end{array}$ \\
\hline & & & & & & & & & & shot & & & & \\
\hline \begin{tabular}{|l|}
$23 S G$ \\
$272-$ \\
14
\end{tabular} & $\begin{array}{l}110 \\
R 30\end{array}$ & 2 & & $\begin{array}{l}Z L \\
O\end{array}$ & 2 & & & & & $\begin{array}{l}\text { lead } \\
\text { shot, } \\
0.13 " \\
\text { caliber }\end{array}$ & & & & \\
\hline \begin{tabular}{|l|}
$23 S G$ \\
$272-$ \\
14
\end{tabular} & $\begin{array}{l}110 \\
R 30\end{array}$ & 2 & & $\begin{array}{l}Z \mathrm{~L} \\
\mathrm{O}\end{array}$ & 6 & & & & & $\begin{array}{l}\text { lead } \\
\text { shot, } \\
0.18 " \\
\text { caliber }\end{array}$ & & & & \\
\hline \begin{tabular}{|l|}
$23 S G$ \\
$272-$ \\
14 \\
\end{tabular} & $\begin{array}{l}110 \\
\text { R30 }\end{array}$ & 2 & & $\begin{array}{l}Z L \\
O\end{array}$ & 1 & & & & & $\begin{array}{l}\text { deform } \\
\text { ed lead } \\
\text { shot }\end{array}$ & & & & \\
\hline \begin{tabular}{|l|}
$23 S G$ \\
$272-$ \\
14
\end{tabular} & $\begin{array}{l}110 \\
\text { R30 }\end{array}$ & 2 & & $\begin{array}{l}Z L \\
O\end{array}$ & 2 & & & $\begin{array}{l}\text { "teeth } \\
\text { " } \\
\text { from } \\
\text { bone/ } \\
\text { wood } \\
\text { comb }\end{array}$ & & & & & & \\
\hline \begin{tabular}{|l|}
$23 S G$ \\
$272-$ \\
14 \\
\end{tabular} & $\begin{array}{l}110 \\
R 30\end{array}$ & 2 & & $\begin{array}{l}\mathrm{ZL} \\
\mathrm{O}\end{array}$ & 58 & & & & & & & & & \\
\hline \begin{tabular}{|l|}
$23 S G$ \\
$272-$ \\
14
\end{tabular} & $\begin{array}{l}110 \\
R 30\end{array}$ & 2 & & $\begin{array}{l}Z \mathrm{~L} \\
\mathrm{O}\end{array}$ & 7 & & & & $\begin{array}{l}\text { "tumble } \\
\text { d" } \\
\text { pieces } \\
\text { of glass }\end{array}$ & & & & & \\
\hline \begin{tabular}{|l|}
$23 S G$ \\
$272-$ \\
14
\end{tabular} & $\begin{array}{l}110 \\
R 30\end{array}$ & 2 & & $\begin{array}{l}\mathrm{ZL} \\
\mathrm{O}\end{array}$ & 7 & & & & $\begin{array}{l}\text { "tumble } \\
\text { d" } \\
\text { stones } \\
\text { or } \\
\text { pebbles }\end{array}$ & & & & & \\
\hline \begin{tabular}{|l|}
$23 S G$ \\
$272-$ \\
15 \\
\end{tabular} & $\begin{array}{l}110 \\
R 30\end{array}$ & 2 & & $\begin{array}{l}\mathrm{ZL} \\
\mathrm{O}\end{array}$ & 1 & & & & & & & & & \\
\hline \begin{tabular}{|l|}
$23 S G$ \\
$272-$ \\
15 \\
\end{tabular} & $\begin{array}{l}110 \\
R 30\end{array}$ & 2 & & $\begin{array}{l}\mathrm{ZL} \\
\mathrm{O}\end{array}$ & 4 & & & & & & & & & \\
\hline $\begin{array}{l}23 S G \\
272-\end{array}$ & $\begin{array}{l}110 \\
\text { R30 }\end{array}$ & 2 & & $\begin{array}{l}\mathrm{ZL} \\
\mathrm{O}\end{array}$ & 6 & & & & & & & & & \\
\hline
\end{tabular}




\begin{tabular}{|c|c|c|c|c|c|c|c|c|c|c|c|c|c|c|}
\hline \multicolumn{15}{|c|}{ Archaeological Analysis } \\
\hline$\left|\begin{array}{c}\text { Catal } \\
\text { ogue } \\
\#\end{array}\right|$ & Uni & $\mid \begin{array}{c}Q \\
\text { ua } \\
d\end{array}$ & \begin{tabular}{c||} 
Le \\
ve \\
I
\end{tabular} & $\begin{array}{c}\mathrm{Ti} \\
\mathrm{me} \\
\mathrm{per} \\
\mathrm{iod}\end{array}$ & $\mid \begin{array}{c}\text { Am } \\
\text { oun } \\
t\end{array}$ & $\mid \begin{array}{l}\text { Hous } \\
\text { ehold } \\
\text { Furnis } \\
\text { hings }\end{array}$ & Clothing & \begin{tabular}{|c} 
Perso \\
nal \\
Items
\end{tabular} & $\begin{array}{l}\text { Househ } \\
\text { old } \\
\text { product } \\
\text { ion } \\
\text { activity }\end{array}$ & \begin{tabular}{|c} 
Househ \\
old \\
produc \\
tion-for \\
sale
\end{tabular} & \begin{tabular}{|l} 
Pharma \\
ceutical
\end{tabular} & $\begin{array}{l}\text { Food } \\
\text { stuffs }\end{array}$ & $\begin{array}{c}\text { Alc } \\
\text { oho } \\
\text { I }\end{array}$ & $\begin{array}{l}\text { Ot } \\
\text { her }\end{array}$ \\
\hline \multicolumn{15}{|l|}{15} \\
\hline \begin{tabular}{|l|}
$23 S G$ \\
$272-$ \\
15 \\
\end{tabular} & $\begin{array}{l}110 \\
R 30\end{array}$ & 2 & & $\begin{array}{l}\text { ZL } \\
O\end{array}$ & 1 & & & & & & & & & \\
\hline \begin{tabular}{|l|}
$23 S G$ \\
$272-$ \\
15
\end{tabular} & $\begin{array}{l}110 \\
R 30\end{array}$ & 2 & & $\begin{array}{l}\mathrm{ZL} \\
\mathrm{O}\end{array}$ & 1 & & & & $\begin{array}{l}\text { brass } \\
\text { eye } \\
\text { from } \\
\text { hood- } \\
\text { and-eye }\end{array}$ & & & & & \\
\hline $\begin{array}{l}23 S G \\
272- \\
15\end{array}$ & $\begin{array}{l}110 \\
R 30\end{array}$ & 2 & & $\begin{array}{l}\text { ZL } \\
0\end{array}$ & 1 & & & $\begin{array}{l}\text { brass } \\
\text { loop; } \\
\text { possib } \\
\text { ly a } \\
\text { jewelr } \\
\text { y or } \\
\text { watch } \\
\text { chain } \\
\text { fasten } \\
\text { er }\end{array}$ & & & & & & \\
\hline \begin{tabular}{|l|}
$23 S G$ \\
$272-$ \\
15 \\
\end{tabular} & $\begin{array}{l}110 \\
\text { R30 }\end{array}$ & 2 & & $\begin{array}{l}\text { ZL } \\
\text { O }\end{array}$ & 3 & & & $\begin{array}{l}\text { frags, } \\
\text { brass } \\
\text { wire }\end{array}$ & & & & & & \\
\hline \begin{tabular}{|l|}
$23 S G$ \\
$272-$ \\
15
\end{tabular} & $\begin{array}{l}110 \\
\text { R30 }\end{array}$ & 2 & & $\begin{array}{l}Z \mathrm{~L} \\
\mathrm{O}\end{array}$ & 3 & & & & & & & & & \\
\hline \begin{tabular}{|l}
$23 S G$ \\
$272-$ \\
15
\end{tabular} & $\begin{array}{l}110 \\
R 30\end{array}$ & 2 & & $\begin{array}{l}\text { ZL } \\
\text { O }\end{array}$ & 1 & & & & & $\begin{array}{l}\text { frag, } \\
\text { sheet } \\
\text { lead } \\
\text { scrap }\end{array}$ & & & & \\
\hline \begin{tabular}{|l|}
$23 S G$ \\
$272-$ \\
15
\end{tabular} & $\begin{array}{l}110 \\
R 30\end{array}$ & 2 & & $\begin{array}{l}Z \mathrm{ZL} \\
\mathrm{O}\end{array}$ & 1 & & & & & $\begin{array}{l}\text { frag, of } \\
\text { lead, } \\
\text { (possibl } \\
\text { y the } \\
\text { missing } \\
\text { center } \\
\text { portion } \\
\text { of a } \\
\text { lead }\end{array}$ & & & & \\
\hline
\end{tabular}




\begin{tabular}{|c|c|c|c|c|c|c|c|c|c|c|c|c|c|c|}
\hline \multicolumn{15}{|c|}{ Archaeological Analysis } \\
\hline $\mid \begin{array}{c}\text { Catal } \\
\text { ogue } \\
\#\end{array}$ & Uni & $\mid \begin{array}{c}Q \\
\text { ua } \\
\text { d }\end{array}$ & Le & $\begin{array}{c}\mathrm{Ti} \\
\mathrm{me} \\
\mathrm{per} \\
\mathrm{iod}\end{array}$ & $\mid \begin{array}{c}\text { Am } \\
\text { oun } \\
t\end{array}$ & $\mid \begin{array}{l}\text { Hous } \\
\text { ehold } \\
\text { Furnis } \\
\text { hings }\end{array}$ & Clothing & $\begin{array}{c}\text { Perso } \\
\text { nal } \\
\text { Items }\end{array}$ & $\begin{array}{l}\text { Househ } \\
\text { old } \\
\text { product } \\
\text { ion } \\
\text { activity }\end{array}$ & \begin{tabular}{|} 
Househ \\
old \\
produc \\
tion-for \\
sale
\end{tabular} & \begin{tabular}{|l} 
Pharma \\
ceutical
\end{tabular} & \begin{tabular}{||l} 
Food \\
stuffs
\end{tabular} & $\begin{array}{c}\text { Alc } \\
\text { oho } \\
\text { I }\end{array}$ & $\begin{array}{l}\text { Ot } \\
\text { her }\end{array}$ \\
\hline & & & & & & & & & & $\begin{array}{l}\text { seal or } \\
\text { button) }\end{array}$ & & & & \\
\hline $\begin{array}{l}23 S G \\
272- \\
15\end{array}$ & $\begin{array}{l}110 \\
R 30\end{array}$ & 2 & & $\begin{array}{l}\text { ZL } \\
\text { O }\end{array}$ & 1 & & & & & $\begin{array}{l}\text { lead } \\
\text { shot, } \\
0.19 " \\
\text { caliber; } \\
\text { has the } \\
\text { "dimple } \\
\text { " of } \\
\text { Rupert } \\
\text { shot }\end{array}$ & & & & \\
\hline $\begin{array}{l}23 S G \\
272- \\
15\end{array}$ & $\begin{array}{l}110 \\
R 30\end{array}$ & 2 & & $\begin{array}{l}\mathrm{ZL} \\
\mathrm{O}\end{array}$ & 1 & & & & & $\begin{array}{l}\text { lead } \\
\text { shot, } \\
0.18 " \\
\text { caliber; } \\
\text { has the } \\
\text { "dimple } \\
\text { " of } \\
\text { Rupert } \\
\text { shot }\end{array}$ & & & & \\
\hline $\begin{array}{l}23 S G \\
272- \\
15\end{array}$ & $\begin{array}{l}110 \\
\text { R30 }\end{array}$ & 2 & & $\begin{array}{l}Z L \\
O\end{array}$ & 2 & & & & & $\begin{array}{l}\text { lead } \\
\text { shot, } \\
0.10 " \\
\text { caliber }\end{array}$ & & & & \\
\hline \begin{tabular}{|l|}
$23 S G$ \\
$272-$ \\
15 \\
\end{tabular} & $\begin{array}{l}110 \\
\text { R30 }\end{array}$ & 2 & & $\begin{array}{l}\mathrm{ZL} \\
\mathrm{O}\end{array}$ & 21 & & & & & & & & & \\
\hline \begin{tabular}{|l|}
$23 S G$ \\
$272-$ \\
15
\end{tabular} & $\begin{array}{l}110 \\
R 30\end{array}$ & 2 & & $\begin{array}{l}\text { ZL } \\
\text { O }\end{array}$ & 12 & & & & $\begin{array}{l}\text { "tumble } \\
\text { d" } \\
\text { pieces } \\
\text { of glass }\end{array}$ & & & & & \\
\hline \begin{tabular}{|l|}
$23 S G$ \\
$272-$ \\
14 \\
\end{tabular} & $\begin{array}{l}110 \\
R 30\end{array}$ & 2 & 2 & $\begin{array}{l}\mathrm{ZL} \\
\mathrm{O}\end{array}$ & 1 & & & & & & & & & \\
\hline \begin{tabular}{|l|}
$23 S G$ \\
$272-$ \\
14 \\
\end{tabular} & $\begin{array}{l}110 \\
R 30\end{array}$ & 2 & & $\begin{array}{l}\mathrm{ZL} \\
\mathrm{O}\end{array}$ & 2 & & & & & & & & & \\
\hline $23 S G$ & 110 & 2 & & ZL & 1 & & & & & & & & & \\
\hline
\end{tabular}




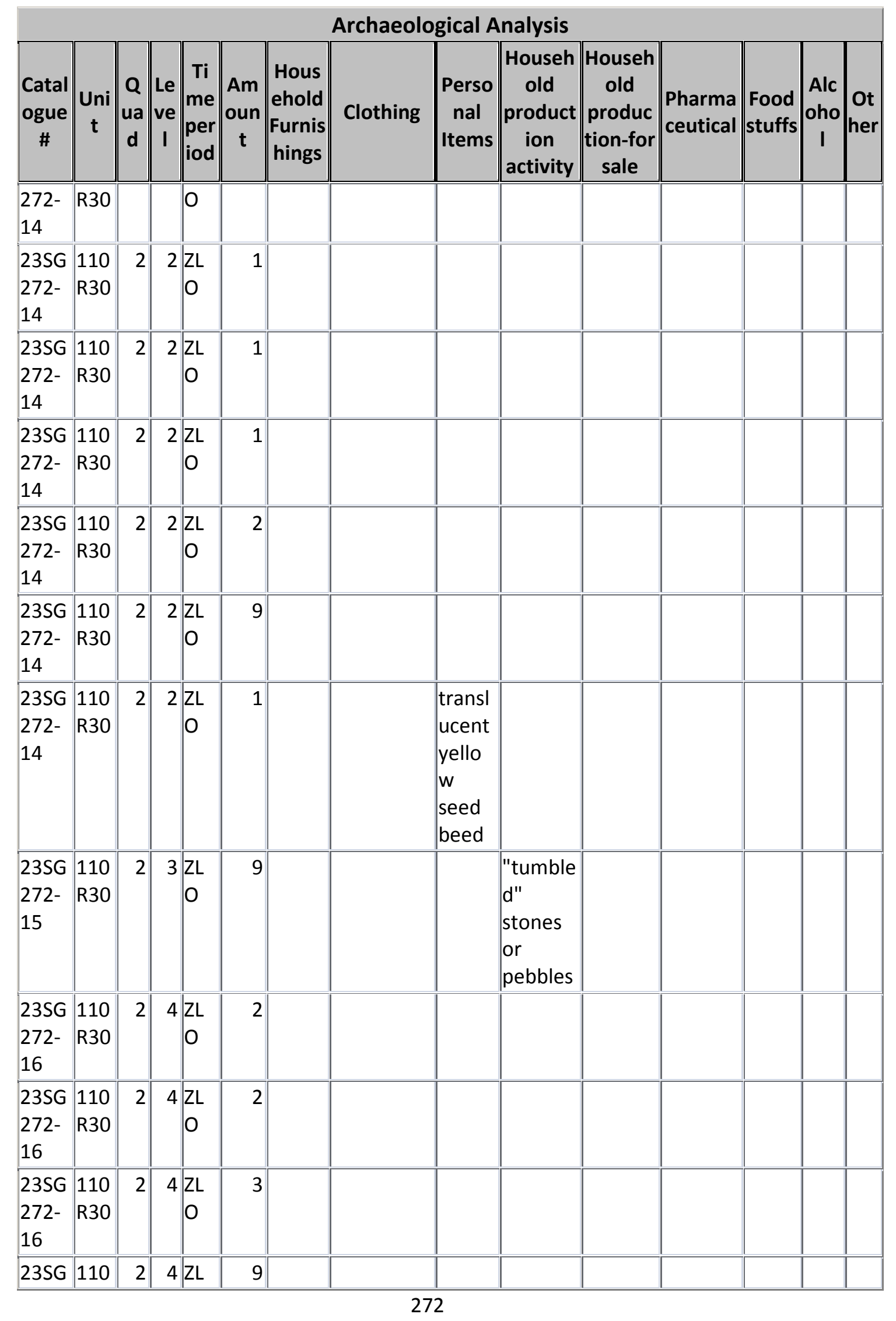




\begin{tabular}{|c|c|c|c|c|c|c|c|c|c|c|c|c|c|c|}
\hline \multicolumn{15}{|c|}{ Archaeological Analysis } \\
\hline $\begin{array}{c}\text { Catal } \\
\text { ogue } \\
\#\end{array}$ & Uni & $\begin{array}{c}\text { Q } \\
\text { ua } \\
\text { d }\end{array}$ & \begin{tabular}{c|c} 
Le & $r$ \\
ve & $r$ \\
I & i
\end{tabular} & $\begin{array}{c}\mathrm{Ti} \\
\mathrm{me} \\
\mathrm{per} \\
\mathrm{iod}\end{array}$ & $\mid \begin{array}{c}\text { Am } \\
\text { oun } \\
t\end{array}$ & $\mid \begin{array}{l}\text { Hous } \\
\text { ehold } \\
\text { Furnis } \\
\text { hings }\end{array}$ & Clothing & \begin{tabular}{|c} 
Perso \\
nal \\
Items
\end{tabular} & $\begin{array}{l}\text { Househ } \\
\text { old } \\
\text { product } \\
\text { ion } \\
\text { activity }\end{array}$ & \begin{tabular}{|c} 
Househ \\
old \\
produc \\
tion-for \\
sale
\end{tabular} & \begin{tabular}{|l} 
Pharma \\
ceutical
\end{tabular} & \begin{tabular}{||l} 
Food \\
stuffs
\end{tabular} & $\begin{array}{c}\text { Alc } \\
\text { oho } \\
\text { I }\end{array}$ & $\begin{array}{l}\text { Ot } \\
\text { her }\end{array}$ \\
\hline \begin{tabular}{|l|}
$272-$ \\
16
\end{tabular} & R30 & & & 0 & & & & & & & & & & \\
\hline \begin{tabular}{|l|}
$23 S G$ \\
$272-$ \\
16 \\
\end{tabular} & $\begin{array}{l}110 \\
R 30\end{array}$ & 2 & & $\begin{array}{l}\mathrm{ZL} \\
\mathrm{O}\end{array}$ & 1 & & & & & & & & & \\
\hline \begin{tabular}{|l|}
$23 S G$ \\
$272-$ \\
16 \\
\end{tabular} & $\begin{array}{l}110 \\
\text { R30 }\end{array}$ & 2 & & $\begin{array}{l}\mathrm{ZL} \\
\mathrm{O}\end{array}$ & 1 & & & & & & & & & \\
\hline \begin{tabular}{|l|}
$23 S G$ \\
$272-$ \\
16
\end{tabular} & $\begin{array}{l}110 \\
R 30\end{array}$ & 2 & & $\begin{array}{l}\mathrm{ZL} \\
\mathrm{O}\end{array}$ & 1 & & & & $\begin{array}{l}\text { frag. Of } \\
\text { brass } \\
\text { eye } \\
\text { from a } \\
\text { hood- } \\
\text { and-eye }\end{array}$ & & & & & \\
\hline \begin{tabular}{|l|}
$23 S G$ \\
$272-$ \\
16
\end{tabular} & $\begin{array}{l}110 \\
R 30\end{array}$ & 2 & & $\begin{array}{l}\mathrm{ZL} \\
\mathrm{O}\end{array}$ & 1 & & & & $\begin{array}{l}\text { brass } \\
\text { hook, } \\
\text { probabl } \\
\text { y from a } \\
\text { hood- } \\
\text { and- } \\
\text { eye, but } \\
\text { possibly } \\
\text { from } \\
\text { jewelry }\end{array}$ & & & & & \\
\hline \begin{tabular}{|l|}
$23 S G$ \\
$272-$ \\
16
\end{tabular} & $\begin{array}{l}110 \\
\text { R30 }\end{array}$ & 2 & & $\begin{array}{l}Z \mathrm{ZL} \\
\mathrm{O}\end{array}$ & 3 & & & & & $\begin{array}{l}\text { frags, } \\
\text { sheet } \\
\text { lead } \\
\text { scrap }\end{array}$ & & & & \\
\hline \begin{tabular}{|l|}
$23 S G$ \\
$272-$ \\
16
\end{tabular} & $\begin{array}{l}110 \\
\text { R30 }\end{array}$ & 2 & & $\begin{array}{l}Z \mathrm{~L} \\
\mathrm{O}\end{array}$ & 1 & & & & \begin{tabular}{|l|} 
gunflint \\
frag, \\
brown \\
gunflint
\end{tabular} & & & & & \\
\hline \begin{tabular}{|l|}
$23 S G$ \\
$272-$ \\
16
\end{tabular} & $\begin{array}{l}110 \\
\text { R30 }\end{array}$ & 2 & & $\begin{array}{l}\mathrm{ZL} \\
\mathrm{O}\end{array}$ & 1 & & & & & $\begin{array}{l}\text { lead } \\
\text { shot, } \\
0.12 " \\
\text { caliber }\end{array}$ & & & & \\
\hline $\begin{array}{l}23 S G \\
272-\end{array}$ & $\begin{array}{l}110 \\
\text { R30 }\end{array}$ & 2 & & $\begin{array}{l}\text { ZL } \\
O\end{array}$ & 1 & & & & & $\begin{array}{l}\text { lead } \\
\text { shot, }\end{array}$ & & & & \\
\hline
\end{tabular}




\begin{tabular}{|c|c|c|c|c|c|c|c|c|c|c|c|c|c|c|}
\hline \multicolumn{15}{|c|}{ Archaeological Analysis } \\
\hline $\mid \begin{array}{c}\text { Catal } \\
\text { ogue } \\
\#\end{array}$ & $\begin{array}{c}\text { Uni } \\
t\end{array}$ & $\begin{array}{c}\text { Q } \\
\text { ua } \\
\text { d }\end{array}$ & \begin{tabular}{|c||l} 
Le & $r$ \\
ve & $r$ \\
I & i
\end{tabular} & $\begin{array}{c}\mathrm{Ti} \\
\mathrm{me} \\
\mathrm{per} \\
\mathrm{iod}\end{array}$ & $\mid \begin{array}{c}\text { Am } \\
\text { oun } \\
t\end{array}$ & $\mid \begin{array}{l}\text { Hous } \\
\text { ehold } \\
\text { Furnis } \\
\text { hings }\end{array}$ & Clothing & $\begin{array}{c}\text { Perso } \\
\text { nal } \\
\text { Items }\end{array}$ & $\begin{array}{l}\text { Househ } \\
\text { old } \\
\text { product } \\
\text { ion } \\
\text { activity }\end{array}$ & \begin{tabular}{|} 
Househ \\
old \\
produc \\
tion-for \\
sale
\end{tabular} & $\begin{array}{l}\text { Pharma } \\
\text { ceutical }\end{array}$ & \begin{tabular}{||l} 
Food \\
stuffs
\end{tabular} & $\begin{array}{c}\text { Alc } \\
\text { oho } \\
\text { I }\end{array}$ & $\begin{array}{l}\text { Ot } \\
\text { her }\end{array}$ \\
\hline 16 & & & & & & & & & & $\begin{array}{l}0.18 " \\
\text { caliber }\end{array}$ & & & & \\
\hline \begin{tabular}{|l|}
$23 S G$ \\
$272-$ \\
16 \\
\end{tabular} & $\begin{array}{l}110 \\
\text { R30 }\end{array}$ & 2 & & $\begin{array}{l}\text { ZL } \\
O\end{array}$ & 36 & & & & & & & & & \\
\hline \begin{tabular}{|l|}
$23 S G$ \\
$272-$ \\
16
\end{tabular} & $\begin{array}{l}110 \\
\text { R30 }\end{array}$ & 2 & & $\begin{array}{l}\mathrm{ZL} \\
\mathrm{O}\end{array}$ & 13 & & & & $\begin{array}{l}\text { "tumble } \\
\text { d" } \\
\text { pieces } \\
\text { of glass }\end{array}$ & & & & & \\
\hline \begin{tabular}{|l|}
$23 S G$ \\
$272-$ \\
16 \\
\end{tabular} & $\begin{array}{l}110 \\
\text { R30 }\end{array}$ & 2 & & $\begin{array}{l}\mathrm{ZL} \\
\mathrm{O}\end{array}$ & 2 & & & & $\begin{array}{l}\text { "tumble } \\
\text { d" } \\
\text { stones }\end{array}$ & & & & & \\
\hline \begin{tabular}{|l|}
$23 S G$ \\
$272-$ \\
16
\end{tabular} & $\begin{array}{l}110 \\
\text { R30 }\end{array}$ & 2 & & $\begin{array}{l}\mathrm{ZL} \\
\mathrm{O}\end{array}$ & 1 & & & \begin{tabular}{|l} 
opaqu \\
e \\
black \\
shiny \\
spher \\
e; \\
bead \\
like \\
but \\
no \\
hole, \\
glass \\
or \\
porcel \\
ain \\
(deco \\
rative \\
object \\
from \\
most \\
likely \\
clothi \\
ng or \\
purse)
\end{tabular} & & & & & & \\
\hline \begin{tabular}{|l|}
$23 S G$ \\
$272-$ \\
17 \\
\end{tabular} & $\begin{array}{l}110 \\
\text { R30 }\end{array}$ & 2 & & 20 & 1 & & & & & & & & & \\
\hline
\end{tabular}




\begin{tabular}{|c|c|c|c|c|c|c|c|c|c|c|c|c|c|c|}
\hline \multicolumn{15}{|c|}{ Archaeological Analysis } \\
\hline $\begin{array}{c}\text { Cata } \\
\text { ogue } \\
\#\end{array}$ & Uni & $\begin{array}{c}\text { Q } \\
\text { ua } \\
\text { d }\end{array}$ & \begin{tabular}{c||} 
Le \\
ve \\
I
\end{tabular} & $\begin{array}{c}\mathrm{Ti} \\
\mathrm{me} \\
\mathrm{per} \\
\mathrm{iod}\end{array}$ & $\mid \begin{array}{c}\text { Am } \\
\text { oun } \\
t\end{array}$ & $\mid \begin{array}{l}\text { Hous } \\
\text { ehold } \\
\text { Furnis } \\
\text { hings }\end{array}$ & Clothing & $\mid \begin{array}{c}\text { Perso } \\
\text { nal } \\
\text { Items }\end{array}$ & $\begin{array}{l}\text { Househ } \\
\text { old } \\
\text { product } \\
\text { ion } \\
\text { activity }\end{array}$ & \begin{tabular}{|c} 
Househ \\
old \\
produc \\
tion-for \\
sale
\end{tabular} & \begin{tabular}{|l} 
Pharma \\
ceutical
\end{tabular} & \begin{tabular}{||l} 
Food \\
stuffs
\end{tabular} & $\begin{array}{c}\text { Alc } \\
\text { oho } \\
\text { I }\end{array}$ & $\begin{array}{l}\text { Ot } \\
\text { her }\end{array}$ \\
\hline \begin{tabular}{|l}
$23 S G$ \\
$272-$ \\
17
\end{tabular} & $\begin{array}{l}110 \\
\text { R30 }\end{array}$ & 2 & & 20 & 1 & & & & & & & & & \\
\hline \begin{tabular}{|l|}
$23 S G$ \\
$272-$ \\
17 \\
\end{tabular} & $\begin{array}{l}110 \\
\text { R30 }\end{array}$ & 2 & & ZO & 1 & & & & & & & & & \\
\hline \begin{tabular}{|l|}
$23 S G$ \\
$272-$ \\
17
\end{tabular} & $\begin{array}{l}110 \\
R 30\end{array}$ & 2 & 5 & 20 & 2 & & & & & & & & & \\
\hline \begin{tabular}{|l}
$23 S G$ \\
$272-$ \\
17
\end{tabular} & $\begin{array}{l}110 \\
\text { R30 }\end{array}$ & 2 & 5 & 20 & 1 & & & & & & $\begin{array}{l}\text { pharma } \\
\text { ceutical } \\
\text { bottle } \\
\text { or } \\
\text { winegla } \\
\text { ss }\end{array}$ & & & \\
\hline \begin{tabular}{|l}
$23 S G$ \\
$272-$ \\
17 \\
\end{tabular} & $\begin{array}{l}110 \\
\text { R30 }\end{array}$ & 2 & 5 & 20 & 1 & & & & & & & & & \\
\hline \begin{tabular}{|l}
$23 S G$ \\
$272-$ \\
17
\end{tabular} & $\begin{array}{l}110 \\
\text { R30 }\end{array}$ & 2 & & ZO & 1 & & & $\begin{array}{l}\text { seed } \\
\text { bead, } \\
\text { opaqu } \\
\text { e } \\
\text { white } \\
\text { glass }\end{array}$ & & & & & & \\
\hline \begin{tabular}{|l}
$23 S G$ \\
$272-$ \\
17
\end{tabular} & $\begin{array}{l}110 \\
\text { R30 }\end{array}$ & 2 & & 20 & 1 & & & & & $\begin{array}{l}\text { lead } \\
\text { shot, } \\
\text { flattene } \\
\text { d or } \\
\text { oval } \\
\text { shape }\end{array}$ & & & & \\
\hline \begin{tabular}{|l|}
$23 S G$ \\
$272-$ \\
17
\end{tabular} & $\begin{array}{l}110 \\
\text { R30 }\end{array}$ & 2 & & ZO & 1 & & & & $\begin{array}{l}\text { gunflint } \\
\text { frag, } \\
\text { thin } \\
\text { flake } \\
\text { clear- } \\
\text { honey } \\
\text { colored } \\
\end{array}$ & & & & & \\
\hline $23 S G$ & 110 & 2 & & ZO & 1 & & & & gunflint & & & & & \\
\hline
\end{tabular}




\begin{tabular}{|c|c|c|c|c|c|c|c|c|c|c|c|c|c|c|}
\hline \multicolumn{15}{|c|}{ Archaeological Analysis } \\
\hline $\begin{array}{c}\text { Catal } \\
\text { ogue } \\
\#\end{array}$ & Uni & $\begin{array}{c}Q \\
\text { ua } \\
\text { d }\end{array}$ & $\mid \begin{array}{c}\text { Le } \\
v \epsilon \\
\text { I }\end{array}$ & \begin{tabular}{l||l} 
e & e \\
me \\
per \\
iod
\end{tabular} & $\mid \begin{array}{c}\text { Am } \\
\text { oun } \\
t\end{array}$ & $\mid \begin{array}{l}\text { Hous } \\
\text { ehold } \\
\text { Furnis } \\
\text { hings }\end{array}$ & Clothing & \begin{tabular}{|c} 
Perso \\
nal \\
Items
\end{tabular} & $\begin{array}{l}\text { Househ } \\
\text { old } \\
\text { product } \\
\text { ion } \\
\text { activity }\end{array}$ & \begin{tabular}{|c} 
Househ \\
old \\
produc \\
tion-for \\
sale
\end{tabular} & \begin{tabular}{|l} 
Pharma \\
ceutical
\end{tabular} & \begin{tabular}{||l} 
Food \\
stuffs
\end{tabular} & $\begin{array}{c}\text { Alc } \\
\text { oho } \\
\text { I }\end{array}$ & $\begin{array}{l}\text { Ot } \\
\text { her }\end{array}$ \\
\hline \begin{tabular}{|l|}
$272-$ \\
17
\end{tabular} & R30 & & & & & & & & $\begin{array}{l}\text { frag, } \\
\text { honey- } \\
\text { colored }\end{array}$ & & & & & \\
\hline \begin{tabular}{|l|}
$23 S G$ \\
$272-$ \\
17
\end{tabular} & $\begin{array}{l}110 \\
\text { R30 }\end{array}$ & 2 & & $5 \mathrm{ZO}$ & 1 & & & & \begin{tabular}{|l} 
gunflint \\
frag, \\
gray- \\
tan- \\
colored
\end{tabular} & & & & & \\
\hline \begin{tabular}{|l|}
$23 S G$ \\
$272-$ \\
17
\end{tabular} & $\begin{array}{l}110 \\
R 30\end{array}$ & 2 & & $5 \mathrm{ZO}$ & 1 & & & & & $\begin{array}{l}\text { lead } \\
\text { frag, } \\
\text { either } \\
\text { sheet } \\
\text { lead or } \\
\text { sprue }\end{array}$ & & & & \\
\hline \begin{tabular}{|l|}
$23 S G$ \\
$272-$ \\
17 \\
\end{tabular} & $\begin{array}{l}110 \\
R 30\end{array}$ & 2 & & $5 \mathrm{ZO}$ & 1 & & & & & $\begin{array}{l}\text { sheet } \\
\text { lead } \\
\text { frag. }\end{array}$ & & & & \\
\hline \begin{tabular}{|l|}
$23 S G$ \\
$272-$ \\
17 \\
\end{tabular} & $\begin{array}{l}110 \\
R 30\end{array}$ & 2 & & $5 Z$ & 11 & & & & & & & & & \\
\hline \begin{tabular}{|l|}
$23 S G$ \\
$272-$ \\
17 \\
\end{tabular} & $\begin{array}{l}110 \\
\text { R30 }\end{array}$ & 2 & & $5 \mathrm{ZO}$ & 3 & & & & $\begin{array}{l}\text { "tumble } \\
\text { d" glass }\end{array}$ & & & & & \\
\hline \begin{tabular}{|l|}
$23 S G$ \\
$272-$ \\
17
\end{tabular} & $\begin{array}{l}110 \\
\text { R30 }\end{array}$ & 2 & & $5 \mathrm{ZO}$ & 1 & & & & \begin{tabular}{|l} 
"tumble \\
d" blue \\
glass; \\
possibil \\
y a \\
bead \\
fragme \\
nt
\end{tabular} & & & & & \\
\hline \begin{tabular}{|l|}
$23 S G$ \\
$272-$ \\
18 \\
\end{tabular} & $\begin{array}{l}110 \\
R 30\end{array}$ & 2 & & $6 \mathrm{ZO}$ & 1 & & & & & & & & & \\
\hline \begin{tabular}{|l|}
$23 S G$ \\
$272-$ \\
18
\end{tabular} & $\begin{array}{l}110 \\
R 30\end{array}$ & 2 & & 670 & 1 & & & $\begin{array}{l}\text { white } \\
\text { seed } \\
\text { bead; } \\
\text { opaqu }\end{array}$ & & & & & & \\
\hline
\end{tabular}




\begin{tabular}{|c|c|c|c|c|c|c|c|c|c|c|c|c|c|c|}
\hline \multicolumn{15}{|c|}{ Archaeological Analysis } \\
\hline $\begin{array}{c}\text { Catal } \\
\text { ogue } \\
\#\end{array}$ & Uni & $\mid \begin{array}{c}Q \\
\text { ua } \\
d\end{array}$ & $\begin{array}{c}\text { Le } \\
\text { ve } \\
\text { I }\end{array}$ & $\begin{array}{l}\mathrm{Ti} \\
\mathrm{me} \\
\mathrm{per} \\
\mathrm{iod}\end{array}$ & $\mid \begin{array}{c}\text { Am } \\
\text { oun } \\
t\end{array}$ & $\begin{array}{l}\text { Hous } \\
\text { ehold } \\
\text { Furnis } \\
\text { hings }\end{array}$ & Clothing & $\mid \begin{array}{c}\text { Perso } \\
\text { nal } \\
\text { Items }\end{array}$ & \begin{tabular}{|} 
Househ \\
old \\
product \\
ion \\
activity
\end{tabular} & \begin{tabular}{|c} 
Househ \\
old \\
produc \\
tion-for \\
sale
\end{tabular} & $\begin{array}{l}\text { Pharma } \\
\text { ceutical }\end{array}$ & Food & $\mid \begin{array}{c}\text { Alc } \\
\text { oho } \\
\text { I }\end{array}$ & Ot \\
\hline & & & & & & & & e & & & & & & \\
\hline $\begin{array}{l}23 S G \\
272- \\
18\end{array}$ & $\begin{array}{l}110 \\
\text { R30 }\end{array}$ & 2 & & ZO & 1 & & & & $\begin{array}{l}\text { gunflint } \\
\text { flake, } \\
\text { honey- } \\
\text { colored }\end{array}$ & & & & & \\
\hline \begin{tabular}{|l}
$23 S G$ \\
$272-$ \\
18
\end{tabular} & $\begin{array}{l}110 \\
\text { R30 }\end{array}$ & 2 & & ZO & 1 & & & & & & & & & \\
\hline \begin{tabular}{|l|}
$23 S G$ \\
$272-$ \\
18 \\
\end{tabular} & $\begin{array}{l}110 \\
\text { R30 }\end{array}$ & 2 & & ZO & 2 & & & & & & & & & \\
\hline \begin{tabular}{|l|}
$23 S G$ \\
$272-$ \\
18 \\
\end{tabular} & $\begin{array}{l}110 \\
\text { R30 }\end{array}$ & 2 & & ZO & 1 & & & & $\begin{array}{l}\text { "tumble } \\
\text { d" piece } \\
\text { of glass }\end{array}$ & & & & & \\
\hline \begin{tabular}{|l|}
$23 S G$ \\
$272-$ \\
18 \\
\end{tabular} & $\begin{array}{l}110 \\
\text { R30 }\end{array}$ & 2 & & ZO & 1 & & & & $\begin{array}{l}\text { "tumble } \\
\text { d" small } \\
\text { pebble }\end{array}$ & & & & & \\
\hline \begin{tabular}{|l|}
$23 S G$ \\
$272-$ \\
2 \\
\end{tabular} & $\begin{array}{l}150 \\
\text { RO }\end{array}$ & 1 & & $\begin{array}{l}\mathrm{ZL} \\
\mathrm{O}\end{array}$ & 1 & & & & & & & & & \\
\hline \begin{tabular}{|l|}
$23 S G$ \\
$272-$ \\
2
\end{tabular} & $\begin{array}{l}150 \\
\text { RO }\end{array}$ & 1 & & $\begin{array}{l}Z L \\
O\end{array}$ & 1 & & & & & & & & & \\
\hline \begin{tabular}{|l|}
$23 S G$ \\
$272-$ \\
2 \\
\end{tabular} & $\begin{array}{l}150 \\
\text { RO }\end{array}$ & 1 & & $\begin{array}{l}Z L \\
O\end{array}$ & 19 & & & & & & & & & \\
\hline \begin{tabular}{|l|}
$23 S G$ \\
$272-$ \\
2
\end{tabular} & $\begin{array}{l}150 \\
\text { RO }\end{array}$ & 1 & & $\begin{array}{l}\mathrm{ZL} \\
\mathrm{O}\end{array}$ & 108 & & & & & & & & & \\
\hline \begin{tabular}{|l|}
$23 S G$ \\
$272-$ \\
2
\end{tabular} & $\begin{array}{l}150 \\
\text { R0 }\end{array}$ & 1 & & $\begin{array}{l}\mathrm{ZL} \\
\mathrm{O}\end{array}$ & 2 & & & & & & & & & \\
\hline \begin{tabular}{|l|}
$23 S G$ \\
$272-$ \\
2
\end{tabular} & $\begin{array}{l}150 \\
\text { RO }\end{array}$ & 1 & & $\begin{array}{l}Z L \\
O\end{array}$ & 1 & & & & \begin{tabular}{|l|} 
"tumble \\
d" \\
stone/p \\
ebble
\end{tabular} & & & & & \\
\hline 23SG & 150 & 1 & & ZL & 1 & & & & & & & & & \\
\hline
\end{tabular}




\begin{tabular}{|c|c|c|c|c|c|c|c|c|c|c|c|c|c|c|}
\hline \multicolumn{15}{|c|}{ Archaeological Analysis } \\
\hline $\begin{array}{c}\text { Catal } \\
\text { ogue } \\
\#\end{array}$ & $\begin{array}{c}\text { Uni } \\
t\end{array}$ & $\begin{array}{c}\text { Q } \\
\text { ua } \\
d\end{array}$ & 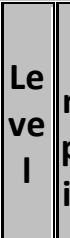 & $\begin{array}{c}\text { Ti } \\
\text { me } \\
\text { per } \\
\text { iod }\end{array}$ & $\mid \begin{array}{c}\text { Am } \\
\text { oun } \\
t\end{array}$ & \begin{tabular}{|l} 
Hous \\
ehold \\
Furnis \\
hings
\end{tabular} & Clothing & \begin{tabular}{|c} 
Perso \\
nal \\
Items
\end{tabular} & \begin{tabular}{|c|} 
Househ \\
old \\
product \\
ion \\
activity
\end{tabular} & \begin{tabular}{|l} 
Househ \\
old \\
produc \\
tion-for \\
sale
\end{tabular} & $\begin{array}{l}\text { Pharma } \\
\text { ceutical }\end{array}$ & $\mid \begin{array}{l}\text { Food } \\
\text { stuffs }\end{array}$ & $\mid \begin{array}{c}\text { Alc } \\
\text { oho } \\
\text { I }\end{array}$ & $\begin{array}{l}\text { Ot } \\
\text { her }\end{array}$ \\
\hline $\begin{array}{l}272- \\
3\end{array}$ & RO & & & $\mathrm{O}$ & & & & & & & & & & \\
\hline \begin{tabular}{|l|}
$23 S G$ \\
$272-$ \\
3
\end{tabular} & $\begin{array}{l}150 \\
\text { RO }\end{array}$ & 1 & 28 & $\begin{array}{l}\mathrm{ZL} \\
\mathrm{O}\end{array}$ & 1 & & & & & & & & & \\
\hline \begin{tabular}{|l|}
$23 S G$ \\
$272-$ \\
3
\end{tabular} & $\begin{array}{l}150 \\
\text { RO }\end{array}$ & 1 & $2 z$ & $\begin{array}{l}\text { ZL } \\
\mathrm{O}\end{array}$ & 1 & & & & & & & & & \\
\hline \begin{tabular}{|l|}
$23 S G$ \\
$272-$ \\
3
\end{tabular} & $\begin{array}{l}150 \\
\text { RO }\end{array}$ & 1 & $2 z$ & $\begin{array}{l}\mathrm{ZL} \\
\mathrm{O}\end{array}$ & 1 & & & & & & & & & \\
\hline \begin{tabular}{|l|}
$23 S G$ \\
$272-$ \\
3
\end{tabular} & $\begin{array}{l}150 \\
\text { RO }\end{array}$ & 1 & 2 & $\begin{array}{l}\mathrm{ZL} \\
\mathrm{O}\end{array}$ & 2 & & & & & & & & & \\
\hline \begin{tabular}{|l|}
$23 S G$ \\
$272-$ \\
3
\end{tabular} & $\begin{array}{l}150 \\
\text { RO }\end{array}$ & 1 & 2 & $\begin{array}{l}\mathrm{ZL} \\
\mathrm{O}\end{array}$ & 1 & & & & & & & & & \\
\hline \begin{tabular}{|l|}
$23 S G$ \\
$272-$ \\
3
\end{tabular} & $\begin{array}{l}150 \\
\text { RO }\end{array}$ & 1 & 2 & $\begin{array}{l}\mathrm{ZL} \\
\mathrm{O}\end{array}$ & 1 & & & & & & & & & \\
\hline \begin{tabular}{|l|}
$23 S G$ \\
$272-$ \\
3 \\
\end{tabular} & $\begin{array}{l}150 \\
\text { RO }\end{array}$ & 1 & 28 & $\begin{array}{l}\text { ZL } \\
\mathrm{O}\end{array}$ & 1 & & & & & & & & & \\
\hline \begin{tabular}{|l|}
$23 S G$ \\
$272-$ \\
3
\end{tabular} & $\begin{array}{l}150 \\
\text { RO }\end{array}$ & 1 & & $\begin{array}{l}\mathrm{ZL} \\
\mathrm{O}\end{array}$ & 1 & & & $\begin{array}{l}\text { red } \\
\text { facete } \\
\text { d } \\
\text { neckl } \\
\text { ace } \\
\text { bead }\end{array}$ & & & & & & \\
\hline \begin{tabular}{|l|}
$23 S G$ \\
$272-$ \\
3
\end{tabular} & $\begin{array}{l}150 \\
\text { RO }\end{array}$ & 1 & 2 & $\begin{array}{l}\mathrm{ZL} \\
\mathrm{O}\end{array}$ & 1 & & & & & & & & & \\
\hline \begin{tabular}{|l}
$23 S G$ \\
$272-$ \\
3
\end{tabular} & $\begin{array}{l}150 \\
\text { RO }\end{array}$ & 1 & 2 & $\begin{array}{l}\mathrm{ZL} \\
\mathrm{O}\end{array}$ & 1 & & & & \begin{tabular}{|l} 
silver- \\
plated \\
brass \\
straight \\
pin
\end{tabular} & & & & & \\
\hline $23 S G$ & 150 & 1 & $2 z$ & ZL & 7 & & & & & & & & & \\
\hline
\end{tabular}




\begin{tabular}{|c|c|c|c|c|c|c|c|c|c|c|c|c|c|c|}
\hline \multicolumn{15}{|c|}{ Archaeological Analysis } \\
\hline $\begin{array}{c}\text { Catal } \\
\text { ogue } \\
\#\end{array}$ & $\begin{array}{c}\text { Uni } \\
t\end{array}$ & $\begin{array}{c}\text { Q } \\
\text { ua } \\
\text { d }\end{array}$ & \begin{tabular}{c||} 
Le \\
ve \\
I
\end{tabular} & $\mid \begin{array}{c}\mathrm{Ti} \\
\mathrm{me} \\
\mathrm{per} \\
\mathrm{iod}\end{array}$ & \begin{tabular}{c||} 
Am \\
oun \\
$t$
\end{tabular} & $\mid \begin{array}{l}\text { Hous } \\
\text { ehold } \\
\text { Furnis } \\
\text { hings }\end{array}$ & Clothing & \begin{tabular}{|c|} 
Perso \\
nal \\
Items
\end{tabular} & \begin{tabular}{|c|}
$\begin{array}{c}\text { Househ } \\
\text { old } \\
\text { product } \\
\text { ion } \\
\text { activity }\end{array}$ \\
\end{tabular} & \begin{tabular}{||c|}
$\begin{array}{c}\text { Househ } \\
\text { old } \\
\text { produc } \\
\text { tion-for } \\
\text { sale }\end{array}$ \\
\end{tabular} & $\begin{array}{l}\text { Pharma } \\
\text { ceutical }\end{array}$ & $\begin{array}{l}\text { Food } \\
\text { stuffs }\end{array}$ & $\mid \begin{array}{c}\text { Alc } \\
\text { oho } \\
\text { I }\end{array}$ & $\begin{array}{c}\text { Ot } \\
\text { her }\end{array}$ \\
\hline $\begin{array}{l}272- \\
3\end{array}$ & RO & & & O & & & & & & & & & & \\
\hline \begin{tabular}{|l|}
$23 S G$ \\
$272-$ \\
3
\end{tabular} & $\begin{array}{l}150 \\
\text { RO }\end{array}$ & 1 & & $\begin{array}{l}Z L \\
O\end{array}$ & 142 & & & & & & & & & \\
\hline \begin{tabular}{|l|}
$23 S G$ \\
$272-$ \\
3
\end{tabular} & $\begin{array}{l}150 \\
\text { RO }\end{array}$ & 1 & & $\begin{array}{l}\mathrm{ZL} \\
\mathrm{O}\end{array}$ & 26 & & & & & & & & & \\
\hline \begin{tabular}{|l|}
$23 S G$ \\
$272-$ \\
3
\end{tabular} & $\begin{array}{l}150 \\
\text { RO }\end{array}$ & 1 & & $\begin{array}{l}Z \mathrm{ZL} \\
\mathrm{O}\end{array}$ & 3 & & & & $\begin{array}{l}\text { "tumble } \\
\text { d" glass }\end{array}$ & & & & & \\
\hline \begin{tabular}{|l|}
$23 S G$ \\
$272-$ \\
3
\end{tabular} & $\begin{array}{l}150 \\
\text { RO }\end{array}$ & 1 & & $\begin{array}{l}\mathrm{ZL} \\
\mathrm{O}\end{array}$ & 2 & & & & \begin{tabular}{|l} 
"tumble \\
d" \\
stones/ \\
pebbles
\end{tabular} & & & & & \\
\hline \begin{tabular}{|l|}
$23 S G$ \\
$272-$ \\
4
\end{tabular} & $\begin{array}{l}150 \\
\text { RO }\end{array}$ & 1 & & $\begin{array}{l}\mathrm{ZL} \\
\mathrm{O}\end{array}$ & 1 & & & & & & & & & \\
\hline \begin{tabular}{|l|}
$23 S G$ \\
$272-$ \\
4
\end{tabular} & $\begin{array}{l}150 \\
\text { RO }\end{array}$ & 1 & & $\begin{array}{l}\mathrm{ZL} \\
\mathrm{O}\end{array}$ & 1 & & & & & & & & & \\
\hline \begin{tabular}{|l|}
$23 S G$ \\
$272-$ \\
4
\end{tabular} & $\begin{array}{l}150 \\
\text { RO }\end{array}$ & 1 & & $\begin{array}{l}Z \mathrm{ZL} \\
\mathrm{O}\end{array}$ & 2 & & & & & & & & & \\
\hline \begin{tabular}{|l|}
$23 S G$ \\
$272-$ \\
4
\end{tabular} & $\begin{array}{l}150 \\
\text { RO }\end{array}$ & 1 & & $\begin{array}{l}Z \mathrm{ZL} \\
\mathrm{O}\end{array}$ & 5 & & & & & & & & & \\
\hline \begin{tabular}{|l|}
$23 S G$ \\
$272-$ \\
4
\end{tabular} & $\begin{array}{l}150 \\
\text { RO }\end{array}$ & 1 & & $\begin{array}{l}\mathrm{ZL} \\
\mathrm{O}\end{array}$ & 1 & & & & $\begin{array}{l}\text { brass- } \\
\text { plated } \\
\text { iron } \\
\text { button }\end{array}$ & & & & & \\
\hline \begin{tabular}{|l|}
$23 S G$ \\
$272-$ \\
4
\end{tabular} & $\begin{array}{l}150 \\
\text { RO }\end{array}$ & 1 & & $\begin{array}{l}\mathrm{ZL} \\
\mathrm{O}\end{array}$ & 1 & & & & $\begin{array}{l}\text { brass } \\
\text { hook }\end{array}$ & & & & & \\
\hline \begin{tabular}{|l|}
$23 S G$ \\
$272-$ \\
4
\end{tabular} & $\begin{array}{l}150 \\
\text { RO }\end{array}$ & 1 & & $\begin{array}{l}\mathrm{ZL} \\
\mathrm{O}\end{array}$ & 300 & & & & & & & & & \\
\hline
\end{tabular}




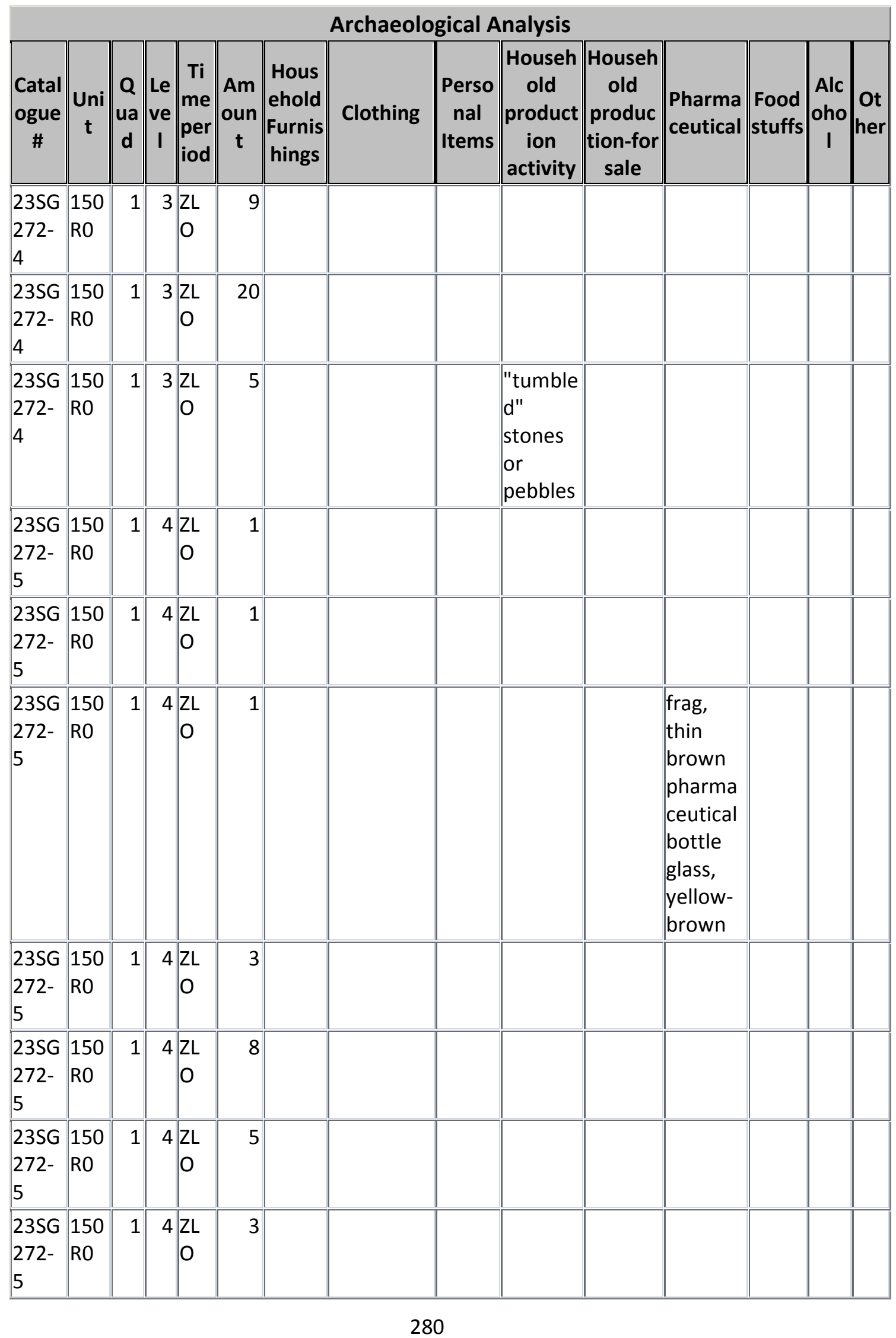




\begin{tabular}{|c|c|c|c|c|c|c|c|c|c|c|c|c|c|c|}
\hline \multicolumn{15}{|c|}{ Archaeological Analysis } \\
\hline $\begin{array}{c}\text { Catal } \\
\text { ogue } \\
\#\end{array}$ & Uni & $\mid \begin{array}{c}Q \\
\text { ua } \\
\text { d }\end{array}$ & \begin{tabular}{c|} 
Le \\
ve \\
I
\end{tabular} & $\begin{array}{c}\mathrm{Ti} \\
\mathrm{me} \\
\mathrm{per} \\
\mathrm{iod}\end{array}$ & $\mid \begin{array}{c}\text { Am } \\
\text { oun } \\
t\end{array}$ & $\mid \begin{array}{l}\text { Hous } \\
\text { ehold } \\
\text { Furnis } \\
\text { hings }\end{array}$ & Clothing & \begin{tabular}{|c} 
Perso \\
nal \\
Items
\end{tabular} & \begin{tabular}{|c|} 
Househ \\
old \\
product \\
ion \\
activity
\end{tabular} & \begin{tabular}{|c} 
Househ \\
old \\
produc \\
tion-for \\
sale
\end{tabular} & $\begin{array}{l}\text { Pharma } \\
\text { ceutical }\end{array}$ & $\begin{array}{l}\text { Food } \\
\text { stuffs }\end{array}$ & $\begin{array}{c}\text { Alc } \\
\text { oho } \\
\text { I }\end{array}$ & $\begin{array}{l}\text { Ot } \\
\text { her }\end{array}$ \\
\hline \begin{tabular}{|l|}
$23 S G$ \\
$272-$ \\
5
\end{tabular} & $\begin{array}{l}150 \\
\text { R0 }\end{array}$ & 1 & & $\begin{array}{l}\mathrm{ZL} \\
\mathrm{O}\end{array}$ & 1 & & & & & & & & & \\
\hline \begin{tabular}{|l|}
$23 S G$ \\
$272-$ \\
5
\end{tabular} & \begin{tabular}{|l|}
150 \\
R0
\end{tabular} & 1 & & $\begin{array}{l}\mathrm{ZL} \\
\mathrm{O}\end{array}$ & 1 & & & & & $\begin{array}{l}\text { lead } \\
\text { shot, } \\
0.18: \\
\text { caliber }\end{array}$ & & & & \\
\hline \begin{tabular}{|l|}
$23 S G$ \\
$272-$ \\
5
\end{tabular} & $\begin{array}{l}150 \\
\text { RO }\end{array}$ & 1 & & $\begin{array}{l}\mathrm{ZL} \\
\mathrm{O}\end{array}$ & 1 & & & & & $\begin{array}{l}\text { lead } \\
\text { shot, } \\
0.14: \\
\text { caliber }\end{array}$ & & & & \\
\hline \begin{tabular}{|l|}
$23 S G$ \\
$272-$ \\
5
\end{tabular} & $\begin{array}{l}150 \\
\text { RO }\end{array}$ & 1 & & $\begin{array}{l}\mathrm{ZL} \\
\mathrm{O}\end{array}$ & 1 & & & & \begin{tabular}{|l|} 
brass- \\
plated \\
iron \\
straight \\
pin
\end{tabular} & & & & & \\
\hline \begin{tabular}{|l|}
$23 S G$ \\
$272-$ \\
5
\end{tabular} & $\begin{array}{l}150 \\
\text { R0 }\end{array}$ & 1 & & $\begin{array}{l}Z L \\
O\end{array}$ & 1 & & & & $\begin{array}{l}\text { brass- } \\
\text { plated } \\
\text { iron } \\
\text { shoe } \\
\text { buckle } \\
\text { or belt } \\
\text { buckle } \\
\text { frag }\end{array}$ & & & & & \\
\hline \begin{tabular}{|l|}
$23 S G$ \\
$272-$ \\
5 \\
\end{tabular} & $\begin{array}{l}150 \\
\text { R0 }\end{array}$ & 1 & & $\begin{array}{l}\mathrm{ZL} \\
\mathrm{O}\end{array}$ & 1 & & & & & & & & & \\
\hline \begin{tabular}{|l|}
$23 S G$ \\
$272-$ \\
5
\end{tabular} & $\begin{array}{l}150 \\
\text { RO }\end{array}$ & 1 & & $\begin{array}{l}\mathrm{ZL} \\
\mathrm{O}\end{array}$ & 1 & & $\begin{array}{l}\text { brass } \\
\text { eyelet, } \\
\text { probably } \\
\text { from a } \\
\text { shoe }\end{array}$ & & & & & & & \\
\hline \begin{tabular}{|l|}
$23 S G$ \\
$272-$ \\
5
\end{tabular} & $\begin{array}{l}150 \\
\text { R0 }\end{array}$ & 1 & & $\begin{array}{l}\mathrm{ZL} \\
\mathrm{O}\end{array}$ & 1 & & & $\begin{array}{l}\text { mass } \\
\text { of } \\
\text { corro } \\
\text { ded } \\
\text { brass; } \\
\text { possib }\end{array}$ & & & & & & \\
\hline
\end{tabular}




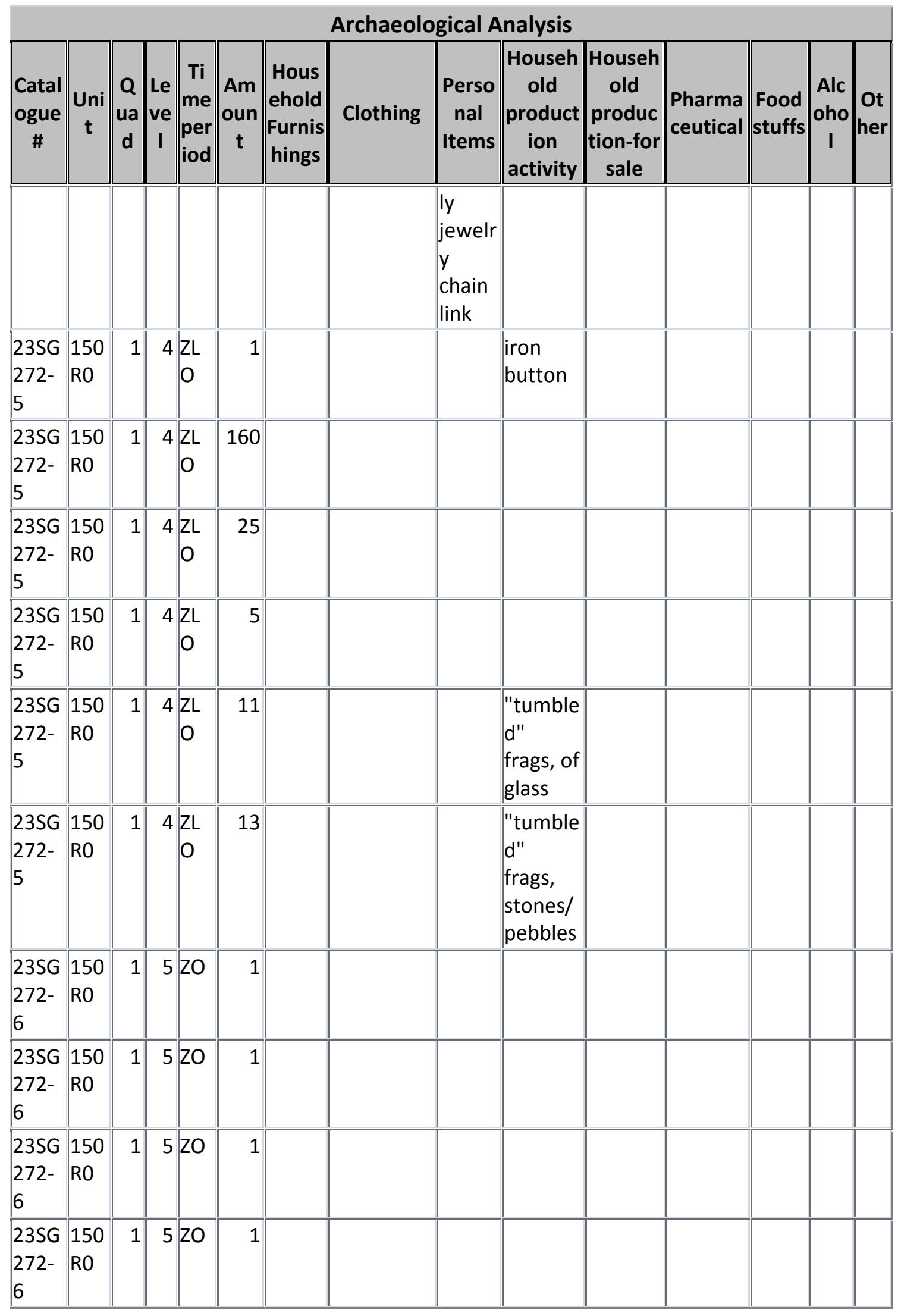




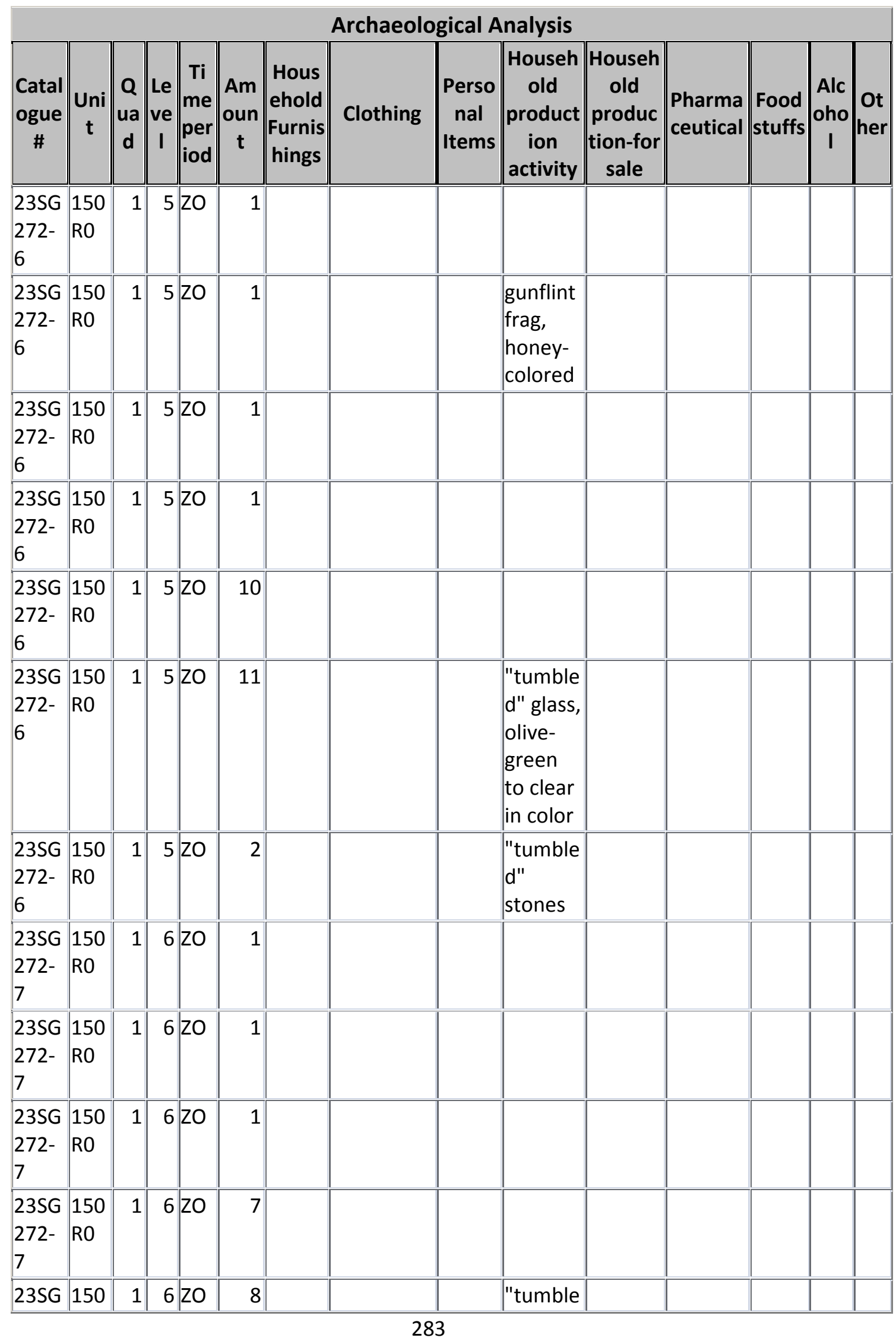




\begin{tabular}{|c|c|c|c|c|c|c|c|c|c|c|c|c|c|c|}
\hline \multicolumn{15}{|c|}{ Archaeological Analysis } \\
\hline $\begin{array}{c}\text { Catal } \\
\text { ogue } \\
\#\end{array}$ & Uni & $\begin{array}{c}\text { Q } \\
\text { ua } \\
\text { d }\end{array}$ & $\mid \begin{array}{c}\text { Le } \\
v \epsilon \\
\text { I }\end{array}$ & \begin{tabular}{l||l} 
e & e \\
me \\
per \\
iod
\end{tabular} & $\mid \begin{array}{c}\text { Am } \\
\text { oun } \\
t\end{array}$ & $\mid \begin{array}{l}\text { Hous } \\
\text { ehold } \\
\text { Furnis } \\
\text { hings }\end{array}$ & Clothing & \begin{tabular}{|c} 
Perso \\
nal \\
Items
\end{tabular} & $\begin{array}{l}\text { Househ } \\
\text { old } \\
\text { product } \\
\text { ion } \\
\text { activity }\end{array}$ & \begin{tabular}{|} 
Househ \\
old \\
produc \\
tion-for \\
sale
\end{tabular} & \begin{tabular}{|l} 
Pharma \\
ceutical
\end{tabular} & \begin{tabular}{||l} 
Food \\
stuffs
\end{tabular} & $\begin{array}{c}\text { Alc } \\
\text { oho } \\
\text { I }\end{array}$ & $\begin{array}{l}\text { Ot } \\
\text { her }\end{array}$ \\
\hline $\begin{array}{l}272- \\
7\end{array}$ & RO & & & & & & & & d" glass & & & & & \\
\hline \begin{tabular}{|l|}
$23 S G$ \\
$272-$ \\
7
\end{tabular} & $\begin{array}{l}150 \\
\text { R0 }\end{array}$ & 1 & & 670 & 9 & & & & $\begin{array}{l}\text { "tumble } \\
\text { d" } \\
\text { rocks/p } \\
\text { ebbles }\end{array}$ & & & & & \\
\hline \begin{tabular}{|l|}
$23 S G$ \\
$272-$ \\
8
\end{tabular} & $\begin{array}{l}150 \\
\text { RO }\end{array}$ & 1 & & $7 \mathrm{ZO}$ & 1 & & & & & & & & & \\
\hline \begin{tabular}{|l|}
$23 S G$ \\
$272-$ \\
8 \\
\end{tabular} & $\begin{array}{l}150 \\
\text { R0 }\end{array}$ & 1 & & $7 \mathrm{ZO}$ & 1 & & & & & & & & & \\
\hline \begin{tabular}{|l|}
$23 S G$ \\
$272-$ \\
8 \\
\end{tabular} & $\begin{array}{l}150 \\
\text { RO }\end{array}$ & 1 & & $7 \mathrm{ZO}$ & 4 & & & & & & & & & \\
\hline \begin{tabular}{|l|}
$23 S G$ \\
$272-$ \\
8 \\
\end{tabular} & $\begin{array}{l}150 \\
\text { RO }\end{array}$ & 1 & & $7 \mathrm{ZO}$ & 5 & & & & $\begin{array}{l}\text { "tumble } \\
\text { d" frags } \\
\text { of glass }\end{array}$ & & & & & \\
\hline \begin{tabular}{|l|}
$23 S G$ \\
$272-$ \\
8
\end{tabular} & $\begin{array}{l}150 \\
\text { RO }\end{array}$ & 1 & & $7 \mathrm{ZO}$ & 4 & & & & $\begin{array}{l}\text { "tumble } \\
\text { d" } \\
\text { rocks/p } \\
\text { ebbles }\end{array}$ & & & & & \\
\hline \begin{tabular}{|l|}
$23 S G$ \\
$272-$ \\
8
\end{tabular} & $\begin{array}{l}150 \\
\text { R0 }\end{array}$ & 1 & & $7 \mathrm{ZO}$ & 1 & & & & \begin{tabular}{|l} 
"tumble \\
d" frag \\
of sawn \\
bone
\end{tabular} & & & & & \\
\hline \begin{tabular}{|l|}
$23 S G$ \\
$272-$ \\
9
\end{tabular} & $\begin{array}{l}150 \\
\text { R0 }\end{array}$ & 1 & & $8 Z$ & 1 & & & $\begin{array}{l}\text { Jewel } \\
\text { ry } \\
\text { frag, } \\
\text { facete } \\
\text { d } \\
\text { clear } \\
\text { glass } \\
\text { "ston } \\
\text { e" } \\
\text { (could } \\
\text { be } \\
\text { ring, }\end{array}$ & & & & & & \\
\hline
\end{tabular}




\begin{tabular}{|c|c|c|c|c|c|c|c|c|c|c|c|c|c|c|}
\hline \multicolumn{15}{|c|}{ Archaeological Analysis } \\
\hline $\begin{array}{c}\text { Catal } \\
\text { ogue } \\
\#\end{array}$ & Uni & $\mid \begin{array}{c}\text { Q } \\
\text { ua } \\
\text { d }\end{array}$ & $\begin{array}{c}\text { Le } \\
\text { ve } \\
\text { I }\end{array}$ & $\begin{array}{c}\mathrm{Ti} \\
\mathrm{me} \\
\mathrm{per} \\
\mathrm{iod}\end{array}$ & $\mid \begin{array}{c}\text { Am } \\
\text { oun } \\
t\end{array}$ & $\mid \begin{array}{l}\text { Hous } \\
\text { ehold } \\
\text { Furnis } \\
\text { hings }\end{array}$ & Clothing & \begin{tabular}{|c} 
Perso \\
nal \\
Items
\end{tabular} & $\begin{array}{c}\text { Househ } \\
\text { old } \\
\text { product } \\
\text { ion } \\
\text { activity }\end{array}$ & \begin{tabular}{|c|} 
Househ \\
old \\
produc \\
tion-for \\
sale
\end{tabular} & $\begin{array}{l}\text { Pharma } \\
\text { ceutical }\end{array}$ & $\begin{array}{l}\text { Food } \\
\text { stuffs }\end{array}$ & $\begin{array}{c}\text { Alc } \\
\text { oho } \\
\text { I }\end{array}$ & $\begin{array}{l}\text { Ot } \\
\text { her }\end{array}$ \\
\hline & & & & & & & & $\begin{array}{l}\text { earrin } \\
\text { g, pin, } \\
\text { or } \\
\text { penda } \\
\text { nt }\end{array}$ & & & & & & \\
\hline $\begin{array}{l}23 S G \\
272- \\
9\end{array}$ & $\begin{array}{l}150 \\
\text { RO }\end{array}$ & 1 & 8 & ZO & 1 & & & & & & & & & \\
\hline \begin{tabular}{|l|}
$23 S G$ \\
$272-$ \\
9
\end{tabular} & $\begin{array}{l}150 \\
\text { R0 }\end{array}$ & 1 & 8 & ZO & 1 & & & & $\begin{array}{l}\text { tiny } \\
\text { flake of } \\
\text { gunflint } \\
\text {, tan- } \\
\text { colored }\end{array}$ & & & & & \\
\hline \begin{tabular}{|l|}
$23 S G$ \\
$272-$ \\
9 \\
\end{tabular} & $\begin{array}{l}150 \\
\text { RO }\end{array}$ & 1 & 8 & ZO & 11 & & & & & & & & & \\
\hline \begin{tabular}{|l|}
$23 S G$ \\
$272-$ \\
9
\end{tabular} & $\begin{array}{l}150 \\
\text { RO }\end{array}$ & 1 & 8 & ZO & 2 & & & & $\begin{array}{l}\text { "tumble } \\
\text { d" } \\
\text { frags, of } \\
\text { glass }\end{array}$ & & & & & \\
\hline $\begin{array}{l}23 S G \\
272- \\
9\end{array}$ & $\begin{array}{l}150 \\
\text { RO }\end{array}$ & 1 & & ZO & 3 & & & & $\begin{array}{l}\text { "tumble } \\
\text { d" small } \\
\text { stones }\end{array}$ & & & & & \\
\hline \begin{tabular}{|l|}
$23 S G$ \\
$272-$ \\
9
\end{tabular} & $\begin{array}{l}150 \\
\text { R0 }\end{array}$ & 1 & & 20 & 1 & & & & & & & & & $\begin{array}{l}\text { sm } \\
\text { all } \\
\text { fra }\end{array}$ \\
\hline $\begin{array}{l}23 S G \\
272-\end{array}$ & $\begin{array}{l}150 \\
\text { RO }\end{array}$ & 1 & 8 & ZO & 1 & & & & & & & & & \\
\hline
\end{tabular}




\begin{tabular}{|c|c|c|c|c|c|c|c|c|c|c|c|c|c|c|}
\hline \multicolumn{15}{|c|}{ Archaeological Analysis } \\
\hline $\begin{array}{c}\text { Catal } \\
\text { ogue } \\
\#\end{array}$ & $\begin{array}{c}\text { Uni } \\
t\end{array}$ & $\mid \begin{array}{c}Q \\
\text { ua } \\
d\end{array}$ & $\begin{array}{c}\text { Le } \\
\text { ve } \\
\text { I }\end{array}$ & $\begin{array}{l}\text { Ti } \\
\text { me } \\
\text { per } \\
\text { iod }\end{array}$ & $\begin{array}{c}\text { Am } \\
\text { oun } \\
t\end{array}$ & \begin{tabular}{|l} 
Hous \\
ehold \\
Furnis \\
hings
\end{tabular} & Clothing & $\mid \begin{array}{c}\text { Perso } \\
\text { nal } \\
\text { Items }\end{array}$ & \begin{tabular}{|c|}
$\begin{array}{c}\text { Househ } \\
\text { old } \\
\text { product } \\
\text { ion } \\
\text { activity }\end{array}$ \\
\end{tabular} & \begin{tabular}{|c} 
Househ \\
old \\
produc \\
tion-for \\
sale
\end{tabular} & $\begin{array}{l}\text { Pharma } \\
\text { ceutical }\end{array}$ & $\begin{array}{l}\text { Food } \\
\text { stuffs }\end{array}$ & $\mid \begin{array}{c}\text { Alc } \\
\text { oho } \\
\text { I }\end{array}$ & Ot \\
\hline 10 & & & & & & & & & & & & & & \\
\hline \begin{tabular}{|l|}
$23 S G$ \\
$272-$ \\
10
\end{tabular} & $\begin{array}{l}150 \\
\text { RO }\end{array}$ & 1 & & $Z O$ & 5 & & & & & & & & & \\
\hline \begin{tabular}{|l|}
$23 S G$ \\
$272-$ \\
10 \\
\end{tabular} & $\begin{array}{l}150 \\
\text { RO }\end{array}$ & 1 & & 30 & 2 & & & & $\begin{array}{l}\text { "tumble } \\
\text { d" tiny } \\
\text { stones }\end{array}$ & & & & & \\
\hline \begin{tabular}{|l|}
$23 S G$ \\
$272-$ \\
162 \\
\end{tabular} & $\begin{array}{l}40 R \\
60\end{array}$ & 3 & & $\begin{array}{l}Z \mathrm{Z} \\
\mathrm{O}\end{array}$ & 1 & & & & $\begin{array}{l}\text { gunflint } \\
\text { frag }\end{array}$ & & & & & \\
\hline $\begin{array}{l}23 S G \\
272- \\
162\end{array}$ & $\begin{array}{l}40 R \\
60\end{array}$ & 3 & & $\begin{array}{l}\text { ZL } \\
0\end{array}$ & 1 & & & & & & & & & \\
\hline $\begin{array}{l}23 S G \\
272- \\
162 \\
\end{array}$ & $\begin{array}{l}40 R \\
60\end{array}$ & 3 & & $\begin{array}{l}\text { ZL } \\
0\end{array}$ & 9 & & & & & & & & & \\
\hline \begin{tabular}{|l|}
$23 S G$ \\
$272-$ \\
163 \\
\end{tabular} & $\begin{array}{l}40 R \\
60\end{array}$ & 3 & & $\begin{array}{l}Z \mathrm{ZL} \\
\mathrm{O}\end{array}$ & 4 & & & & & & & & & \\
\hline \begin{tabular}{|l|}
$23 S G$ \\
$272-$ \\
163 \\
\end{tabular} & $\begin{array}{l}40 \mathrm{R} \\
60\end{array}$ & 3 & & $\begin{array}{l}Z \mathrm{ZL} \\
\mathrm{O}\end{array}$ & 3 & & & & & & & & & \\
\hline \begin{tabular}{|l|}
$23 S G$ \\
$272-$ \\
163
\end{tabular} & $\begin{array}{l}40 R \\
60\end{array}$ & 3 & & $\begin{array}{l}Z \mathrm{ZL} \\
\mathrm{O}\end{array}$ & 3 & & & & & & & & & \\
\hline \begin{tabular}{|l|}
$23 S G$ \\
$272-$ \\
164 \\
\end{tabular} & $\begin{array}{l}40 R \\
60\end{array}$ & 3 & & $\begin{array}{l}Z \mathrm{ZL} \\
\mathrm{O}\end{array}$ & 2 & & & & & & & & & \\
\hline \begin{tabular}{|l|}
$23 S G$ \\
$272-$ \\
164 \\
\end{tabular} & $\begin{array}{l}40 R \\
60\end{array}$ & 3 & & $\begin{array}{l}Z \mathrm{ZL} \\
\mathrm{O}\end{array}$ & 2 & & & & & & & & & \\
\hline \begin{tabular}{|l|}
$23 S G$ \\
$272-$ \\
164 \\
\end{tabular} & $\begin{array}{l}40 R \\
60\end{array}$ & 3 & & $\begin{array}{l}Z \mathrm{ZL} \\
\mathrm{O}\end{array}$ & 1 & & & & & & & & & \\
\hline $\begin{array}{l}23 S G \\
272- \\
164\end{array}$ & $\begin{array}{l}40 R \\
60\end{array}$ & 3 & & $\begin{array}{l}Z \mathrm{Z} \\
\mathrm{O}\end{array}$ & 11 & & & & & & & & & \\
\hline
\end{tabular}




\begin{tabular}{|c|c|c|c|c|c|c|c|c|c|c|c|c|c|c|}
\hline \multicolumn{15}{|c|}{ Archaeological Analysis } \\
\hline$\left|\begin{array}{c}\text { Catal } \\
\text { ogue } \\
\#\end{array}\right|$ & Uni & $\mid \begin{array}{c}Q \\
\text { ua } \\
d\end{array}$ & $\begin{array}{c}\text { Le } \\
\text { ve } \\
\text { I }\end{array}$ & $\begin{array}{c}\mathrm{Ti} \\
\mathrm{me} \\
\mathrm{per} \\
\mathrm{iod}\end{array}$ & $\mid \begin{array}{c}\text { Am } \\
\text { oun } \\
t\end{array}$ & $\mid \begin{array}{l}\text { Hous } \\
\text { ehold } \\
\text { Furnis } \\
\text { hings }\end{array}$ & Clothing & \begin{tabular}{|c} 
Perso \\
nal \\
Items
\end{tabular} & \begin{tabular}{|} 
Househ \\
old \\
product \\
ion \\
activity
\end{tabular} & \begin{tabular}{|c} 
Househ \\
old \\
produc \\
tion-for \\
sale
\end{tabular} & $\begin{array}{l}\text { Pharma } \\
\text { ceutical }\end{array}$ & \begin{tabular}{||l} 
Food \\
stuffs
\end{tabular} & $\begin{array}{c}\text { Alc } \\
\text { oho } \\
\text { I }\end{array}$ & $\begin{array}{l}\text { Ot } \\
\text { her }\end{array}$ \\
\hline $\begin{array}{l}23 S G \\
272- \\
164\end{array}$ & $\begin{array}{l}40 R \\
60\end{array}$ & 3 & & $\begin{array}{l}\mathrm{ZL} \\
\mathrm{O}\end{array}$ & 2 & & & & & & & & & \\
\hline \begin{tabular}{|l|}
$23 S G$ \\
$272-$ \\
164
\end{tabular} & \begin{tabular}{|l|}
$40 R$ \\
60
\end{tabular} & 3 & & $\begin{array}{l}\mathrm{ZL} \\
\mathrm{O}\end{array}$ & 2 & & & \begin{tabular}{|l} 
possib \\
le \\
jewelr \\
y- \\
small \\
frags, \\
either \\
clear \\
glass \\
or \\
quart \\
z \\
crysta \\
I
\end{tabular} & & & & & & \\
\hline $\begin{array}{l}23 S G \\
272- \\
164\end{array}$ & $\begin{array}{l}40 R \\
60\end{array}$ & 3 & & $\begin{array}{l}\text { ZL } \\
\text { O }\end{array}$ & 1 & & & $\begin{array}{l}\text { red, } \\
\text { opaqu } \\
\text { e } \\
\text { facete } \\
\text { d } \\
\text { neckl } \\
\text { ace } \\
\text { bead }\end{array}$ & & & & & & \\
\hline \begin{tabular}{|l|}
$23 S G$ \\
$272-$ \\
164
\end{tabular} & $\begin{array}{l}40 R \\
60\end{array}$ & 3 & & $\begin{array}{l}\mathrm{ZL} \\
\mathrm{O}\end{array}$ & 2 & & & \begin{tabular}{|l} 
black \\
opaqu \\
e \\
facete \\
d \\
neckl \\
ace \\
beads
\end{tabular} & & & & & & \\
\hline \begin{tabular}{|l|}
$23 S G$ \\
$272-$ \\
164
\end{tabular} & \begin{tabular}{|l|}
$40 R$ \\
60
\end{tabular} & 3 & & $\begin{array}{l}\text { ZL } \\
\text { O }\end{array}$ & 1 & & & $\begin{array}{l}\text { dark } \\
\text { purpl } \\
\text { e, } \\
\text { transl } \\
\text { ucent } \\
\text { seed }\end{array}$ & & & & & & \\
\hline
\end{tabular}




\begin{tabular}{|c|c|c|c|c|c|c|c|c|c|c|c|c|c|c|}
\hline \multicolumn{15}{|c|}{ Archaeological Analysis } \\
\hline $\begin{array}{c}\text { Catal } \\
\text { ogue } \\
\#\end{array}$ & $\begin{array}{c}\text { Uni } \\
t\end{array}$ & \begin{tabular}{c|c} 
Q & \\
ua & \\
d &
\end{tabular} & $\mid$\begin{tabular}{c|} 
Le \\
ve \\
I
\end{tabular} & $\begin{array}{l}\mathrm{Ti} \\
\mathrm{me} \\
\mathrm{per} \\
\mathrm{iod}\end{array}$ & $\mid \begin{array}{c}\text { Am } \\
\text { oun } \\
t\end{array}$ & \begin{tabular}{|l} 
Hous \\
ehold \\
Furnis \\
hings
\end{tabular} & Clothing & $\mid \begin{array}{c}\text { Perso } \\
\text { nal } \\
\text { Items }\end{array}$ & \begin{tabular}{|c|} 
Househ \\
old \\
product \\
ion \\
activity
\end{tabular} & \begin{tabular}{||c||} 
Househ \\
old \\
produc \\
tion-for \\
sale
\end{tabular} & | Pharma & Food & $\mid \begin{array}{c}\text { Alc } \\
\text { oho } \\
\text { I }\end{array}$ & $\begin{array}{c}\text { Ot } \\
\text { her }\end{array}$ \\
\hline & & & & & & & & bead & & & & & & \\
\hline \begin{tabular}{|l|}
$23 S G$ \\
$272-$ \\
164
\end{tabular} & $\begin{array}{l}40 R \\
60\end{array}$ & 3 & & $\begin{array}{l}Z L \\
O\end{array}$ & 1 & & $\begin{array}{l}\text { half a } \\
\text { bone } \\
\text { button }\end{array}$ & & & & & & & \\
\hline $\begin{array}{l}23 S G \\
272- \\
164\end{array}$ & $\begin{array}{l}40 R \\
60\end{array}$ & 3 & & $\begin{array}{l}Z \mathrm{~L} \\
0\end{array}$ & 1 & & & & & $\begin{array}{l}0.18 " \\
\text { caliber } \\
\text { lead } \\
\text { shot }\end{array}$ & & & & \\
\hline \begin{tabular}{|l|}
$23 S G$ \\
$272-$ \\
164
\end{tabular} & $\begin{array}{l}40 R \\
60\end{array}$ & 3 & & $\begin{array}{l}\text { ZL } \\
\mathrm{O}\end{array}$ & 1 & & & & & \begin{tabular}{|l} 
lead \\
shot, \\
approxi \\
mately \\
$0.18 "$ \\
caliber
\end{tabular} & & & & \\
\hline \begin{tabular}{|l|}
$23 S G$ \\
$272-$ \\
164
\end{tabular} & $\begin{array}{l}40 R \\
60\end{array}$ & 3 & & $\begin{array}{l}Z L \\
O\end{array}$ & 1 & & & & & $\begin{array}{l}0.13 " \\
\text { caliber } \\
\text { lead } \\
\text { shot }\end{array}$ & & & & \\
\hline $\begin{array}{l}23 S G \\
272- \\
164\end{array}$ & $\begin{array}{l}40 R \\
60\end{array}$ & 3 & & $\begin{array}{l}\text { ZL } \\
\mathrm{O}\end{array}$ & 11 & & & & $\begin{array}{l}\text { gunflint } \\
\text { frags }\end{array}$ & & & & & \\
\hline \begin{tabular}{|l|}
$23 S G$ \\
$272-$ \\
164
\end{tabular} & $\begin{array}{l}40 R \\
60\end{array}$ & 3 & & $\begin{array}{l}\text { ZL } \\
\text { O }\end{array}$ & 1 & & & & $\begin{array}{l}\text { brass- } \\
\text { plated } \\
\text { iron } \\
\text { safety } \\
\text { pin }\end{array}$ & & & & & \\
\hline \begin{tabular}{|l|}
$23 S G$ \\
$272-$ \\
164
\end{tabular} & $\begin{array}{l}40 R \\
60\end{array}$ & 3 & & $\begin{array}{l}Z L \\
O\end{array}$ & 1 & & & & & & & & & \\
\hline \begin{tabular}{|l|}
$23 S G$ \\
$272-$ \\
164
\end{tabular} & $\begin{array}{l}40 R \\
60\end{array}$ & 3 & & $\begin{array}{l}Z L \\
O\end{array}$ & 1 & & & & & \begin{tabular}{|l|} 
frag, \\
molten \\
lead \\
scrap
\end{tabular} & & & & \\
\hline \begin{tabular}{|l|}
$23 S G$ \\
$272-$ \\
164
\end{tabular} & $\begin{array}{l}40 R \\
60\end{array}$ & 3 & & $\begin{array}{l}\text { ZL } \\
\text { O }\end{array}$ & 1 & & & & & $\begin{array}{l}\text { frag, } \\
\text { sheet } \\
\text { lead } \\
\text { scrap }\end{array}$ & & & & \\
\hline $23 S G$ & $40 R$ & 3 & & ZL & 5 & & & & & & & & & \\
\hline
\end{tabular}




\begin{tabular}{|c|c|c|c|c|c|c|c|c|c|c|c|c|c|c|}
\hline \multicolumn{15}{|c|}{ Archaeological Analysis } \\
\hline $\begin{array}{c}\text { Catal } \\
\text { ogue } \\
\#\end{array}$ & $\begin{array}{c}\text { Uni } \\
t\end{array}$ & $\begin{array}{c}\text { Q } \\
\text { ua } \\
d\end{array}$ & \begin{tabular}{c||l} 
Le & \\
ve & i \\
I & i
\end{tabular} & $\begin{array}{c}\text { Ti } \\
\text { me } \\
\text { per } \\
\text { iod }\end{array}$ & $\begin{array}{c}\text { Am } \\
\text { oun } \\
t\end{array}$ & $\begin{array}{l}\text { Hous } \\
\text { ehold } \\
\text { Furnis } \\
\text { hings }\end{array}$ & Clothing & $\mid \begin{array}{c}\text { Perso } \\
\text { nal } \\
\text { Items }\end{array}$ & \begin{tabular}{|c|} 
Househ \\
old \\
product \\
ion \\
activity
\end{tabular} & \begin{tabular}{|l} 
Househ \\
old \\
produc \\
tion-for \\
sale
\end{tabular} & $\begin{array}{l}\text { Pharma } \\
\text { ceutical }\end{array}$ & $\mid \begin{array}{l}\text { Food } \\
\text { stuffs }\end{array}$ & $\mid \begin{array}{c}\text { Alc } \\
\text { oho } \\
\text { I }\end{array}$ & $\begin{array}{l}\text { Ot } \\
\text { her }\end{array}$ \\
\hline $\begin{array}{l}272- \\
164\end{array}$ & 60 & & & 0 & & & & & & & & & & \\
\hline \begin{tabular}{|l|}
$23 S G$ \\
$272-$ \\
164
\end{tabular} & $\begin{array}{l}40 R \\
60\end{array}$ & 3 & 8 & $\begin{array}{l}\mathrm{ZL} \\
\mathrm{O}\end{array}$ & 1 & & & & & & & & & \\
\hline \begin{tabular}{|l|}
$23 S G$ \\
$272-$ \\
164 \\
\end{tabular} & $\begin{array}{l}40 R \\
60\end{array}$ & 3 & 82 & $\begin{array}{l}\text { ZL } \\
\text { O }\end{array}$ & 33 & & & & & & & & & \\
\hline \begin{tabular}{|l|}
$23 S G$ \\
$272-$ \\
164 \\
\end{tabular} & $\begin{array}{l}40 R \\
60\end{array}$ & 3 & 82 & $\begin{array}{l}\mathrm{ZL} \\
\mathrm{O}\end{array}$ & 1 & & & & & & & & & \\
\hline $\begin{array}{l}23 S G \\
272- \\
164\end{array}$ & $\begin{array}{l}40 R \\
60\end{array}$ & 3 & 8 & $\begin{array}{l}\mathrm{ZL} \\
\mathrm{O}\end{array}$ & 13 & & & & & & & & & \\
\hline \begin{tabular}{|l|}
$23 S G$ \\
$272-$ \\
164
\end{tabular} & $\begin{array}{l}40 R \\
60\end{array}$ & 3 & 8 & $\begin{array}{l}\mathrm{ZL} \\
\mathrm{O}\end{array}$ & 2 & & & & $\begin{array}{l}\text { stones } \\
\text { that } \\
\text { have } \\
\text { been } \\
\text { "tumble } \\
\text { d" }\end{array}$ & & & & & \\
\hline $\begin{array}{l}23 S G \\
272- \\
165\end{array}$ & $\begin{array}{l}40 R \\
60\end{array}$ & 3 & 8 & $\begin{array}{l}\mathrm{ZL} \\
\mathrm{O}\end{array}$ & 1 & & & & & & & & & \\
\hline \begin{tabular}{|l|}
$23 S G$ \\
$272-$ \\
165 \\
\end{tabular} & $\begin{array}{l}40 R \\
60\end{array}$ & 3 & 82 & $\begin{array}{l}\mathrm{ZL} \\
\mathrm{O}\end{array}$ & 1 & & & & & & & & & \\
\hline \begin{tabular}{|l|}
$23 S G$ \\
$272-$ \\
165
\end{tabular} & $\begin{array}{l}40 R \\
60\end{array}$ & 3 & 8 & $\begin{array}{l}\text { ZL } \\
\text { O }\end{array}$ & 2 & & & & \begin{tabular}{|l} 
gunflint \\
frag, \\
honey \\
colored
\end{tabular} & & & & & \\
\hline $\begin{array}{l}23 S G \\
272- \\
165\end{array}$ & $\begin{array}{l}40 R \\
60\end{array}$ & 3 & 8 & $\begin{array}{l}\mathrm{ZL} \\
\mathrm{O}\end{array}$ & 2 & & & & \begin{tabular}{|l} 
gunflint \\
frag, \\
honey \\
colored
\end{tabular} & & & & & \\
\hline \begin{tabular}{|l|}
$23 S G$ \\
$272-$ \\
165 \\
\end{tabular} & $\begin{array}{l}40 R \\
60\end{array}$ & 3 & 8 & $\begin{array}{l}\mathrm{ZL} \\
\mathrm{O}\end{array}$ & 1 & & & & & & & & & \\
\hline $23 S G$ & $40 R$ & 3 & $8 z$ & & 3 & & & & & & & & & \\
\hline
\end{tabular}




\begin{tabular}{|c|c|c|c|c|c|c|c|c|c|c|c|c|c|c|}
\hline \multicolumn{15}{|c|}{ Archaeological Analysis } \\
\hline $\mid \begin{array}{c}\text { Catal } \\
\text { ogue } \\
\#\end{array}$ & Uni & $\begin{array}{c}\text { Q } \\
\text { ua } \\
d\end{array}$ & \begin{tabular}{c|} 
Le \\
ve \\
I
\end{tabular} & $\begin{array}{c}\mathrm{Ti} \\
\mathrm{me} \\
\mathrm{per} \\
\mathrm{iod}\end{array}$ & $\mid \begin{array}{c}\text { Am } \\
\text { oun } \\
t\end{array}$ & $\begin{array}{l}\text { Hous } \\
\text { ehold } \\
\text { Furnis } \\
\text { hings }\end{array}$ & Clothing & $\begin{array}{c}\text { Perso } \\
\text { nal } \\
\text { Items }\end{array}$ & \begin{tabular}{|c|} 
Househ \\
old \\
product \\
ion \\
activity
\end{tabular} & \begin{tabular}{|c|} 
Househ \\
old \\
produc \\
tion-for \\
sale
\end{tabular} & $\begin{array}{l}\text { Pharma } \\
\text { ceutical }\end{array}$ & \begin{tabular}{|l||} 
Food \\
stuffs
\end{tabular} & $\mid \begin{array}{c}\text { Alc } \\
\text { oho } \\
\text { I }\end{array}$ & $\begin{array}{l}\text { Ot } \\
\text { her }\end{array}$ \\
\hline $\begin{array}{l}272- \\
165\end{array}$ & 60 & & & 0 & & & & & & & & & & \\
\hline $\begin{array}{l}23 S G \\
272- \\
165\end{array}$ & $\begin{array}{l}40 R \\
60\end{array}$ & 3 & & $\begin{array}{l}\text { ZL } \\
0\end{array}$ & 3 & & & & & & & & & \\
\hline \begin{tabular}{|l|}
$23 S G$ \\
$272-$ \\
165
\end{tabular} & $\begin{array}{l}40 R \\
60\end{array}$ & 3 & & $\begin{array}{l}\mathrm{ZL} \\
\mathrm{O}\end{array}$ & 82 & & & & & & & & & \\
\hline \begin{tabular}{|l|}
$23 S G$ \\
$272-$ \\
165
\end{tabular} & $\begin{array}{l}40 R \\
60\end{array}$ & 3 & & $\begin{array}{l}\mathrm{ZL} \\
\mathrm{O}\end{array}$ & 1 & & & & \begin{tabular}{|l} 
stone, \\
possibly \\
"tumble \\
d"
\end{tabular} & & & & & \\
\hline \begin{tabular}{|l|}
$23 S G$ \\
$272-$ \\
166
\end{tabular} & $\begin{array}{l}40 R \\
60\end{array}$ & 3 & & $\begin{array}{l}\mathrm{ZL} \\
\mathrm{O}\end{array}$ & 1 & & & & & & & & & \\
\hline \begin{tabular}{|l|}
$23 S G$ \\
$272-$ \\
166 \\
\end{tabular} & \begin{tabular}{|l|}
$40 R$ \\
60
\end{tabular} & 3 & & $\begin{array}{l}\mathrm{ZL} \\
\mathrm{O}\end{array}$ & 1 & & & & & & & & & \\
\hline \begin{tabular}{|l|}
$23 S G$ \\
$272-$ \\
166 \\
\end{tabular} & $\begin{array}{l}40 R \\
60\end{array}$ & 3 & & $\begin{array}{l}\mathrm{ZL} \\
\mathrm{O}\end{array}$ & 1 & & & & & & & & & \\
\hline \begin{tabular}{|l|}
$23 S G$ \\
$272-$ \\
166 \\
\end{tabular} & $\begin{array}{l}40 R \\
60\end{array}$ & 3 & & $\begin{array}{l}\mathrm{ZL} \\
\mathrm{O}\end{array}$ & 1 & & & & & & & & & \\
\hline \begin{tabular}{|l|}
$23 S G$ \\
$272-$ \\
166 \\
\end{tabular} & \begin{tabular}{|l|}
$40 R$ \\
60
\end{tabular} & 3 & & $\begin{array}{l}\mathrm{ZL} \\
\mathrm{O}\end{array}$ & 2 & & & & & & & & & \\
\hline \begin{tabular}{|l|}
$23 S G$ \\
$272-$ \\
166 \\
\end{tabular} & $\begin{array}{l}40 R \\
60\end{array}$ & 3 & & $\begin{array}{l}\mathrm{ZL} \\
\mathrm{O}\end{array}$ & 1 & & & & & & & & & \\
\hline \begin{tabular}{|l|}
$23 S G$ \\
$272-$ \\
166 \\
\end{tabular} & $\begin{array}{l}40 R \\
60\end{array}$ & 3 & & $\begin{array}{l}\mathrm{ZL} \\
\mathrm{O}\end{array}$ & 1 & & & & $\begin{array}{l}\text { gunflint } \\
\text { flake }\end{array}$ & & & & & \\
\hline $\begin{array}{l}23 S G \\
272- \\
167\end{array}$ & $\begin{array}{l}40 R \\
60\end{array}$ & 3 & & $\begin{array}{l}\mathrm{ZL} \\
\mathrm{O}\end{array}$ & 7 & & & & & & & & & \\
\hline $23 S G$ & $40 R$ & 3 & & ZL & 90 & & & & & & & & & \\
\hline
\end{tabular}




\begin{tabular}{|c|c|c|c|c|c|c|c|c|c|c|c|c|c|c|}
\hline \multicolumn{15}{|c|}{ Archaeological Analysis } \\
\hline $\begin{array}{c}\text { Cata } \\
\text { ogue } \\
\#\end{array}$ & $\begin{array}{c}\text { Uni } \\
t\end{array}$ & $\begin{array}{c}\text { Q } \\
\text { ua } \\
\text { d }\end{array}$ & \begin{tabular}{c||} 
Le \\
ve \\
I
\end{tabular} & $\begin{array}{c}\mathrm{Ti} \\
\mathrm{me} \\
\mathrm{per} \\
\mathrm{iod}\end{array}$ & $\mid \begin{array}{c}\text { Am } \\
\text { oun } \\
t\end{array}$ & \begin{tabular}{|l} 
Hous \\
ehold \\
Furnis \\
hings
\end{tabular} & Clothing & $\mid \begin{array}{c}\text { Perso } \\
\text { nal } \\
\text { Items }\end{array}$ & $\begin{array}{l}\text { Househ } \\
\text { old } \\
\text { product } \\
\text { ion } \\
\text { activity }\end{array}$ & \begin{tabular}{|} 
Househ \\
old \\
produc \\
tion-for \\
sale
\end{tabular} & $\begin{array}{l}\text { Pharma } \\
\text { ceutical }\end{array}$ & $\begin{array}{l}\text { Food } \\
\text { stuffs }\end{array}$ & $\begin{array}{c}\text { Alc } \\
\text { oho } \\
\text { I }\end{array}$ & $\begin{array}{l}\text { Ot } \\
\text { her }\end{array}$ \\
\hline $\begin{array}{l}272- \\
168\end{array}$ & 60 & & & 0 & & & & & & & & & & \\
\hline $\begin{array}{l}23 S G \\
272- \\
168\end{array}$ & $\begin{array}{l}40 R \\
60\end{array}$ & 3 & & $\begin{array}{l}\mathrm{ZL} \\
\mathrm{O}\end{array}$ & 1 & & & & & & & & & \\
\hline \begin{tabular}{|l}
$23 S G$ \\
$272-$ \\
168
\end{tabular} & $\begin{array}{l}40 \mathrm{R} \\
60\end{array}$ & 3 & & $\begin{array}{l}Z L \\
O\end{array}$ & 1 & & & & & & & & & \\
\hline \begin{tabular}{|l}
$23 S G$ \\
$272-$ \\
168
\end{tabular} & $\begin{array}{l}40 R \\
60\end{array}$ & 3 & & $\begin{array}{l}Z L \\
O\end{array}$ & 2 & & & & & & & & & \\
\hline $\begin{array}{l}23 S G \\
272- \\
168\end{array}$ & $\begin{array}{l}40 R \\
60\end{array}$ & 3 & & $\begin{array}{l}Z L \\
O\end{array}$ & 8 & & & & & & & & & \\
\hline $\begin{array}{l}23 S G \\
272- \\
168\end{array}$ & $\begin{array}{l}40 R \\
60\end{array}$ & 3 & & $\begin{array}{l}Z L \\
O\end{array}$ & 2 & & & & & & & & & \\
\hline \begin{tabular}{|l}
$23 S G$ \\
$272-$ \\
168
\end{tabular} & $\begin{array}{l}40 R \\
60\end{array}$ & 3 & & $\begin{array}{l}\mathrm{ZL} \\
\mathrm{O}\end{array}$ & 2 & & & & & & & & & \\
\hline $\begin{array}{l}23 S G \\
272- \\
168\end{array}$ & $\begin{array}{l}40 R \\
60\end{array}$ & 3 & & $\begin{array}{l}Z L \\
O\end{array}$ & 1 & & & & & & & & & \\
\hline $\begin{array}{l}23 S G \\
272- \\
168\end{array}$ & $\begin{array}{l}40 \mathrm{R} \\
60\end{array}$ & 3 & & $\begin{array}{l}Z \mathrm{~L} \\
\mathrm{O}\end{array}$ & 1 & & & & & & & & & \\
\hline $\begin{array}{l}23 S G \\
272- \\
168\end{array}$ & $\begin{array}{l}40 \mathrm{R} \\
60\end{array}$ & 3 & & $\begin{array}{l}Z L \\
O\end{array}$ & 9 & & & & & & & & & \\
\hline $\begin{array}{l}23 S G \\
272- \\
168\end{array}$ & $\begin{array}{l}40 R \\
60\end{array}$ & 3 & & $\begin{array}{l}Z L \\
O\end{array}$ & 2 & & & & & & & & & \\
\hline \begin{tabular}{|l}
$23 S G$ \\
$272-$ \\
168
\end{tabular} & $\begin{array}{l}40 \mathrm{R} \\
60\end{array}$ & 3 & & $\begin{array}{l}\mathrm{ZL} \\
\mathrm{O}\end{array}$ & 6 & & & & & & & & & \\
\hline $\begin{array}{l}23 S G \\
272-\end{array}$ & $\begin{array}{l}40 R \\
60\end{array}$ & 3 & & $\begin{array}{l}\mathrm{ZL} \\
\mathrm{O}\end{array}$ & 5 & & & & & & & & & \\
\hline
\end{tabular}




\begin{tabular}{|c|c|c|c|c|c|c|c|c|c|c|c|c|c|c|}
\hline \multicolumn{15}{|c|}{ Archaeological Analysis } \\
\hline $\begin{array}{c}\text { Catal } \\
\text { ogue } \\
\#\end{array}$ & $\begin{array}{c}\text { Uni } \\
t\end{array}$ & $\begin{array}{c}Q \\
\text { ua } \\
d\end{array}$ & \begin{tabular}{c||l} 
Le & \\
ve & r \\
I & i
\end{tabular} & $\begin{array}{l}\text { Ti } \\
\text { me } \\
\text { per } \\
\text { iod }\end{array}$ & $\mid \begin{array}{c}\text { Am } \\
\text { oun } \\
t\end{array}$ & \begin{tabular}{|l} 
Hous \\
ehold \\
Furnis \\
hings
\end{tabular} & Clothing & $\mid \begin{array}{c}\text { Perso } \\
\text { nal } \\
\text { Items }\end{array}$ & \begin{tabular}{|} 
Househ \\
old \\
product \\
ion \\
activity
\end{tabular} & \begin{tabular}{||c||} 
Househ \\
old \\
produc \\
tion-for \\
sale
\end{tabular} & $\begin{array}{l}\text { Pharma } \\
\text { ceutical }\end{array}$ & $\mid \begin{array}{l}\text { Food } \\
\text { stuffs }\end{array}$ & $\mid \begin{array}{c}\text { Alc } \\
\text { oho } \\
\text { I }\end{array}$ & $\begin{array}{c}\text { Ot } \\
\text { her }\end{array}$ \\
\hline \multicolumn{15}{|l|}{168} \\
\hline \begin{tabular}{|l|}
$23 S G$ \\
$272-$ \\
168 \\
\end{tabular} & $\begin{array}{l}40 R \\
60\end{array}$ & 3 & & $\begin{array}{l}Z L \\
O\end{array}$ & 5 & & & & & & & & & \\
\hline \begin{tabular}{|l|}
$23 S G$ \\
$272-$ \\
168
\end{tabular} & $\begin{array}{l}40 \mathrm{R} \\
60\end{array}$ & 3 & & $\begin{array}{l}Z L \\
O\end{array}$ & 12 & & & & & & & & & \\
\hline $\begin{array}{l}23 S G \\
272- \\
168\end{array}$ & $\begin{array}{l}40 R \\
60\end{array}$ & 3 & & $\begin{array}{l}\text { ZL } \\
O\end{array}$ & 1 & & & & & & & & & \\
\hline \begin{tabular}{|l|}
$23 S G$ \\
$272-$ \\
168 \\
\end{tabular} & $\begin{array}{l}40 \mathrm{R} \\
60\end{array}$ & 3 & & $\begin{array}{l}Z \mathrm{~L} \\
\mathrm{O}\end{array}$ & 1 & & & & & & & & & \\
\hline \begin{tabular}{|l|}
$23 S G$ \\
$272-$ \\
168 \\
\end{tabular} & $\begin{array}{l}40 R \\
60\end{array}$ & 3 & & $\begin{array}{l}Z L \\
O\end{array}$ & 1 & & & & & & & & & \\
\hline \begin{tabular}{|l|}
$23 S G$ \\
$272-$ \\
168 \\
\end{tabular} & $\begin{array}{l}40 R \\
60\end{array}$ & 3 & & $\begin{array}{l}Z L \\
O\end{array}$ & 1 & & & & & & & & & \\
\hline \begin{tabular}{|l|}
$23 S G$ \\
$272-$ \\
168
\end{tabular} & $\begin{array}{l}40 R \\
60\end{array}$ & 3 & & $\begin{array}{l}\mathrm{ZL} \\
\mathrm{O}\end{array}$ & 1 & & & & & & & & & \\
\hline \begin{tabular}{|l|}
$23 S G$ \\
$272-$ \\
168 \\
\end{tabular} & $\begin{array}{l}40 R \\
60\end{array}$ & 3 & & $\begin{array}{l}Z L \\
O\end{array}$ & 5 & & & & & & & & & \\
\hline \begin{tabular}{|l|}
$23 S G$ \\
$272-$ \\
168
\end{tabular} & $\begin{array}{l}40 R \\
60\end{array}$ & 3 & & $\begin{array}{l}Z L \\
O\end{array}$ & 4 & & & \begin{tabular}{|l|} 
frags, \\
clear, \\
thick \\
glass, \\
possib \\
le \\
jewelr \\
y \\
settin \\
g
\end{tabular} & & & & & & \\
\hline \begin{tabular}{|l|}
$23 S G$ \\
$272-$ \\
168
\end{tabular} & $\begin{array}{l}40 R \\
60\end{array}$ & 3 & & $\begin{array}{l}Z \mathrm{~L} \\
\mathrm{O}\end{array}$ & 1 & & & $\begin{array}{l}\text { opaqu } \\
\text { e red } \\
\text { facete }\end{array}$ & & & & & & \\
\hline
\end{tabular}




\begin{tabular}{|c|c|c|c|c|c|c|c|c|c|c|c|c|c|c|}
\hline \multicolumn{15}{|c|}{ Archaeological Analysis } \\
\hline $\begin{array}{c}\text { Catal } \\
\text { ogue } \\
\#\end{array}$ & $\begin{array}{c}\text { Uni } \\
t\end{array}$ & $\begin{array}{c}\text { Q } \\
\text { ua } \\
d\end{array}$ & \begin{tabular}{c||} 
Le \\
ve \\
I
\end{tabular} & $\begin{array}{l}\text { Ti } \\
\text { me } \\
\text { per } \\
\text { iod }\end{array}$ & $\mid \begin{array}{c}\text { Am } \\
\text { oun } \\
t\end{array}$ & \begin{tabular}{|l} 
Hous \\
ehold \\
Furnis \\
hings
\end{tabular} & Clothing & $\begin{array}{c}\text { Perso } \\
\text { nal } \\
\text { Items }\end{array}$ & \begin{tabular}{|} 
Househ \\
old \\
product \\
ion \\
activity
\end{tabular} & \begin{tabular}{||c||} 
Househ \\
old \\
produc \\
tion-for \\
sale
\end{tabular} & $\begin{array}{l}\text { Pharma } \\
\text { ceutical }\end{array}$ & $\mid \begin{array}{l}\text { Food } \\
\text { stuffs }\end{array}$ & $\mid \begin{array}{c}\text { Alc } \\
\text { oho } \\
\text { I }\end{array}$ & $\begin{array}{c}\text { Ot } \\
\text { her }\end{array}$ \\
\hline & & & & & & & & $\begin{array}{l}\text { d } \\
\text { neckl } \\
\text { ace } \\
\text { bead; } \\
\text { bright } \\
\text { red }\end{array}$ & & & & & & \\
\hline \begin{tabular}{|l|}
$23 S G$ \\
$272-$ \\
168
\end{tabular} & $\begin{array}{l}40 R \\
60\end{array}$ & 3 & & $\begin{array}{l}Z L \\
O\end{array}$ & 1 & & & \begin{tabular}{|l} 
clear \\
facete \\
d \\
neckl \\
ace \\
bead
\end{tabular} & & & & & & \\
\hline \begin{tabular}{|l|}
$23 S G$ \\
$272-$ \\
168
\end{tabular} & $\begin{array}{l}40 R \\
60\end{array}$ & 3 & & $\begin{array}{l}Z \mathrm{~L} \\
\mathrm{O}\end{array}$ & 1 & & & \begin{tabular}{|l} 
clear \\
facete \\
d \\
neckl \\
ace \\
bead
\end{tabular} & & & & & & \\
\hline \begin{tabular}{|l|}
$23 S G$ \\
$272-$ \\
168
\end{tabular} & $\begin{array}{l}40 R \\
60\end{array}$ & 3 & & $\begin{array}{l}\text { ZL } \\
O\end{array}$ & 1 & & & $\begin{array}{l}\text { seed } \\
\text { bead, } \\
\text { turqu } \\
\text { oise }\end{array}$ & & & & & & \\
\hline \begin{tabular}{|l|}
$23 S G$ \\
$272-$ \\
168
\end{tabular} & $\begin{array}{l}40 R \\
60\end{array}$ & 3 & & $\begin{array}{l}\text { ZL } \\
\mathrm{O}\end{array}$ & 8 & & & $\begin{array}{l}\text { seed } \\
\text { beads } \\
\text {, dark } \\
\text { purpl } \\
\text { e, }\end{array}$ & & & & & & \\
\hline \begin{tabular}{|l|}
$23 S G$ \\
$272-$ \\
168
\end{tabular} & $\begin{array}{l}40 R \\
60\end{array}$ & 3 & & $\begin{array}{l}Z L \\
O\end{array}$ & 1 & & & & & \begin{tabular}{|l|}
$.22 "$ \\
caliber \\
cartridg \\
e shell, \\
large X \\
with \\
"SUPER \\
"across \\
end
\end{tabular} & & & & \\
\hline \begin{tabular}{|l|}
$23 S G$ \\
$272-$ \\
168 \\
\end{tabular} & $\begin{array}{l}40 R \\
60\end{array}$ & 3 & & $\begin{array}{l}Z \mathrm{~L} \\
\mathrm{O}\end{array}$ & 1 & & & & & $\begin{array}{l}.22 " \\
\text { caliber } \\
\text { lead }\end{array}$ & & & & \\
\hline
\end{tabular}




\begin{tabular}{|c|c|c|c|c|c|c|c|c|c|c|c|c|c|c|}
\hline \multicolumn{15}{|c|}{ Archaeological Analysis } \\
\hline $\begin{array}{c}\text { Catal } \\
\text { ogue } \\
\#\end{array}$ & $\begin{array}{c}\text { Uni } \\
t\end{array}$ & $\begin{array}{c}\text { Q } \\
\text { ua } \\
d\end{array}$ & 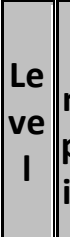 & $\begin{array}{l}\mathrm{Ti} \\
\mathrm{me} \\
\mathrm{per} \\
\mathrm{iod}\end{array}$ & $\mid \begin{array}{c}\text { Am } \\
\text { oun } \\
t\end{array}$ & \begin{tabular}{|l} 
Hous \\
ehold \\
Furnis \\
hings
\end{tabular} & Clothing & \begin{tabular}{|c} 
Perso \\
nal \\
Items
\end{tabular} & \begin{tabular}{|c|} 
Househ \\
old \\
product \\
ion \\
activity
\end{tabular} & \begin{tabular}{||c||} 
Househ \\
old \\
produc \\
tion-for \\
sale
\end{tabular} & 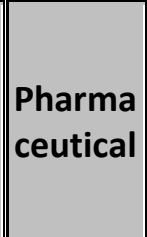 & 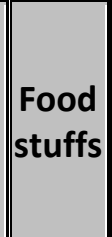 & $\mid \begin{array}{c}\text { Alc } \\
\text { oho } \\
\text { I }\end{array}$ & $\begin{array}{c}\text { Ot } \\
\text { her }\end{array}$ \\
\hline & & & & & & & & & & shot & & & & \\
\hline \begin{tabular}{|l|}
$23 S G$ \\
$272-$ \\
168
\end{tabular} & $\begin{array}{l}40 R \\
60\end{array}$ & 3 & & $\begin{array}{l}\text { ZL } \\
\text { O }\end{array}$ & 4 & & & & & $\begin{array}{l}\text {.19" } \\
\text { caliber } \\
\text { lead } \\
\text { shot }\end{array}$ & & & & \\
\hline \begin{tabular}{|l|}
$23 S G$ \\
$272-$ \\
168
\end{tabular} & $\begin{array}{l}40 R \\
60\end{array}$ & 3 & & $\begin{array}{l}Z \mathrm{~L} \\
\mathrm{O}\end{array}$ & 3 & & & & & $\begin{array}{l}.18 " \\
\text { caliber } \\
\text { lead } \\
\text { shot }\end{array}$ & & & & \\
\hline \begin{tabular}{|l|}
$23 S G$ \\
$272-$ \\
168
\end{tabular} & $\begin{array}{l}40 R \\
60\end{array}$ & 3 & & $\begin{array}{l}\text { ZL } \\
O\end{array}$ & 5 & & & & & $\begin{array}{l}.16 " \\
\text { caliber } \\
\text { lead } \\
\text { shot }\end{array}$ & & & & \\
\hline \begin{tabular}{|l|}
$23 S G$ \\
$272-$ \\
168
\end{tabular} & $\begin{array}{l}40 R \\
60\end{array}$ & 3 & & $\begin{array}{l}\text { ZL } \\
O\end{array}$ & 6 & & & & & $\begin{array}{l}.15 " \\
\text { caliber } \\
\text { lead } \\
\text { shot }\end{array}$ & & & & \\
\hline $\begin{array}{l}23 S G \\
272- \\
168\end{array}$ & $\begin{array}{l}40 R \\
60\end{array}$ & 3 & & $\begin{array}{l}Z \mathrm{~L} \\
\mathrm{O}\end{array}$ & 3 & & & & & $\begin{array}{l}.14 " \\
\text { caliber } \\
\text { lead } \\
\text { shot }\end{array}$ & & & & \\
\hline \begin{tabular}{|l|}
$23 S G$ \\
$272-$ \\
168
\end{tabular} & $\begin{array}{l}40 R \\
60\end{array}$ & 3 & & $\begin{array}{l}Z L \\
O\end{array}$ & 2 & & & & & $\begin{array}{l}.13 " \\
\text { caliber } \\
\text { lead } \\
\text { shot }\end{array}$ & & & & \\
\hline \begin{tabular}{|l|}
$23 S G$ \\
$272-$ \\
168
\end{tabular} & $\begin{array}{l}40 R \\
60\end{array}$ & 3 & & $\begin{array}{l}\text { ZL } \\
O\end{array}$ & 1 & & & & & $\begin{array}{l}.12 " \\
\text { caliber } \\
\text { lead } \\
\text { shot }\end{array}$ & & & & \\
\hline \begin{tabular}{|l|}
$23 S G$ \\
$272-$ \\
168
\end{tabular} & $\begin{array}{l}40 R \\
60\end{array}$ & 3 & & $\begin{array}{l}Z \mathrm{~L} \\
\mathrm{O}\end{array}$ & 3 & & & & & $\begin{array}{l}.11 " \\
\text { caliber } \\
\text { lead } \\
\text { shot }\end{array}$ & & & & \\
\hline \begin{tabular}{|l}
$23 S G$ \\
$272-$ \\
168
\end{tabular} & $\begin{array}{l}40 R \\
60\end{array}$ & 3 & & $\begin{array}{l}Z \mathrm{~L} \\
\mathrm{O}\end{array}$ & 1 & & & & & $\begin{array}{l}.10 " \\
\text { caliber } \\
\text { lead } \\
\text { shot }\end{array}$ & & & & \\
\hline 23SG & $40 R$ & 3 & & ZL & 1 & & & & & $.07 "$ & & & & \\
\hline
\end{tabular}




\begin{tabular}{|c|c|c|c|c|c|c|c|c|c|c|c|c|c|c|}
\hline \multicolumn{15}{|c|}{ Archaeological Analysis } \\
\hline $\mid \begin{array}{c}\text { Catal } \\
\text { ogue } \\
\#\end{array}$ & Uni & $\mid \begin{array}{c}Q \\
\text { ua } \\
\text { d }\end{array}$ & \begin{tabular}{c|} 
Le \\
ve \\
I
\end{tabular} & $\begin{array}{c}\mathrm{Ti} \\
\mathrm{me} \\
\mathrm{per} \\
\mathrm{iod}\end{array}$ & $\mid \begin{array}{c}\text { Am } \\
\text { oun } \\
t\end{array}$ & $\mid \begin{array}{l}\text { Hous } \\
\text { ehold } \\
\text { Furnis } \\
\text { hings }\end{array}$ & Clothing & $\begin{array}{c}\text { Perso } \\
\text { nal } \\
\text { Items }\end{array}$ & \begin{tabular}{|} 
Househ \\
old \\
product \\
ion \\
activity
\end{tabular} & \begin{tabular}{|c} 
Househ \\
old \\
produc \\
tion-for \\
sale
\end{tabular} & \begin{tabular}{|l} 
Pharma \\
ceutical
\end{tabular} & $\begin{array}{l}\text { Food } \\
\text { stuffs }\end{array}$ & $\begin{array}{c}\text { Alc } \\
\text { oho } \\
\text { I }\end{array}$ & $\begin{array}{l}\text { Ot } \\
\text { her }\end{array}$ \\
\hline $\begin{array}{l}272- \\
168\end{array}$ & 60 & & & 0 & & & & & & $\begin{array}{l}\text { caliber } \\
\text { lead } \\
\text { shot }\end{array}$ & & & & \\
\hline \begin{tabular}{|l|}
$23 S G$ \\
$272-$ \\
168
\end{tabular} & \begin{tabular}{|l|}
$40 R$ \\
60
\end{tabular} & 3 & & $\begin{array}{l}\mathrm{ZL} \\
\mathrm{O}\end{array}$ & 1 & & & & & $\begin{array}{l}\text { flattene } \\
\text { d shot, } \\
\text { approxi } \\
\text { mately } \\
.11 " \\
\text { caliber }\end{array}$ & & & & \\
\hline \begin{tabular}{|l|}
$23 S G$ \\
$272-$ \\
168
\end{tabular} & \begin{tabular}{|l|}
$40 R$ \\
60
\end{tabular} & 3 & & $\begin{array}{l}\mathrm{ZL} \\
\mathrm{O}\end{array}$ & 2 & & & & & $\begin{array}{l}\text { flattene } \\
\text { d shot, } \\
\text { approxi } \\
\text { mately } \\
.14 " \\
\text { caliber }\end{array}$ & & & & \\
\hline \begin{tabular}{|l|}
$23 S G$ \\
$272-$ \\
168
\end{tabular} & $\begin{array}{l}40 R \\
60\end{array}$ & 3 & & $\begin{array}{l}\mathrm{ZL} \\
\mathrm{O}\end{array}$ & 1 & & & & & $\begin{array}{l}\text { lead } \\
\text { shot, } \\
\text { approxi } \\
\text { mately } \\
.14 " \\
\text { caliber }\end{array}$ & & & & \\
\hline $\begin{array}{l}23 S G \\
272- \\
168\end{array}$ & $\begin{array}{l}40 R \\
60\end{array}$ & 3 & & $\begin{array}{l}\text { ZL } \\
0\end{array}$ & 2 & & & & & $\begin{array}{l}\text { frags, } \\
\text { brass } \\
\text { percuss } \\
\text { ion cap }\end{array}$ & & & & \\
\hline \begin{tabular}{|l|}
$23 S G$ \\
$272-$ \\
168
\end{tabular} & $\begin{array}{l}40 R \\
60\end{array}$ & 3 & & $\begin{array}{l}\mathrm{ZL} \\
\mathrm{O}\end{array}$ & 1 & & & & \begin{tabular}{|l|} 
spall \\
gunflint \\
, pistol \\
sized
\end{tabular} & & & & & \\
\hline \begin{tabular}{|l|}
$23 S G$ \\
$272-$ \\
168
\end{tabular} & \begin{tabular}{|l|}
$40 R$ \\
60
\end{tabular} & 3 & & $\begin{array}{l}\text { ZL } \\
\text { O }\end{array}$ & 3 & & & & \begin{tabular}{|l|} 
pieces \\
of \\
honey- \\
colored \\
gunflint
\end{tabular} & & & & & \\
\hline \begin{tabular}{|l|}
$23 S G$ \\
$272-$ \\
168
\end{tabular} & $\begin{array}{l}40 R \\
60\end{array}$ & 3 & & $\begin{array}{l}\mathrm{ZL} \\
\mathrm{O}\end{array}$ & 2 & & & & \begin{tabular}{|l|} 
pieces \\
of \\
gunflint \\
, gray-
\end{tabular} & & & & & \\
\hline
\end{tabular}




\begin{tabular}{|c|c|c|c|c|c|c|c|c|c|c|c|c|c|c|}
\hline \multicolumn{15}{|c|}{ Archaeological Analysis } \\
\hline $\begin{array}{c}\text { Catal } \\
\text { ogue } \\
\#\end{array}$ & Uni & $\begin{array}{c}Q \\
\text { ua } \\
d\end{array}$ & $\begin{array}{c}\text { Le } \\
\text { ve } \\
\text { I }\end{array}$ & $\begin{array}{l}\text { Ti } \\
\text { me } \\
\text { per } \\
\text { iod }\end{array}$ & $\begin{array}{c}\text { Am } \\
\text { oun } \\
t\end{array}$ & $\mid \begin{array}{l}\text { Hous } \\
\text { ehold } \\
\text { Furnis } \\
\text { hings }\end{array}$ & Clothing & \begin{tabular}{|c} 
Perso \\
nal \\
Items
\end{tabular} & $\begin{array}{c}\text { Househ } \\
\text { old } \\
\text { product } \\
\text { ion } \\
\text { activity }\end{array}$ & \begin{tabular}{|c} 
Househ \\
old \\
produc \\
tion-for \\
sale
\end{tabular} & \begin{tabular}{|l} 
Pharma \\
ceutical
\end{tabular} & \begin{tabular}{||l} 
Food \\
stuffs
\end{tabular} & $\begin{array}{c}\text { Alc } \\
\text { oho } \\
\text { I }\end{array}$ & $\begin{array}{l}\text { Ot } \\
\text { her }\end{array}$ \\
\hline & & & & & & & & & $\tan$ & & & & & \\
\hline $\begin{array}{l}23 S G \\
272- \\
168\end{array}$ & $\begin{array}{l}40 R \\
60\end{array}$ & 3 & & & 1 & & & & $\begin{array}{l}\text { gunflint } \\
\text { flake, } \\
\text { gray- } \\
\text { tan }\end{array}$ & & & & & \\
\hline \begin{tabular}{|l|}
$23 S G$ \\
$272-$ \\
168
\end{tabular} & $\begin{array}{l}40 R \\
60\end{array}$ & 3 & & $\begin{array}{l}Z L \\
O\end{array}$ & 6 & & & & $\begin{array}{l}\text { gunflint } \\
\text { flakes, } \\
\text { honey- } \\
\text { colored }\end{array}$ & & & & & \\
\hline \begin{tabular}{|l|}
$23 S G$ \\
$272-$ \\
168 \\
\end{tabular} & \begin{tabular}{|l|}
$40 R$ \\
60
\end{tabular} & 3 & & $\begin{array}{l}Z L \\
O\end{array}$ & 1 & & $\begin{array}{l}\text { brass } \\
\text { button, } \\
\text { flat, plain }\end{array}$ & & & & & & & \\
\hline $\begin{array}{l}23 S G \\
272- \\
168\end{array}$ & $\begin{array}{l}40 R \\
60\end{array}$ & 3 & & & 1 & & $\begin{array}{l}\text { multi- } \\
\text { componen } \\
\text { t brass } \\
\text { button }\end{array}$ & & & & & & & \\
\hline \begin{tabular}{|l|}
$23 S G$ \\
$272-$ \\
168
\end{tabular} & \begin{tabular}{|l|}
$40 R$ \\
60
\end{tabular} & 3 & & $\begin{array}{l}Z L \\
O\end{array}$ & 1 & & $\begin{array}{l}\text { sheet } \\
\text { brass } \\
\text { button } \\
\text { blank frag } \\
\text { ( } 2 \text { circular } \\
\text { buttons } \\
\text { having } \\
\text { been cut } \\
\text { or } \\
\text { stamped } \\
\text { out) }\end{array}$ & & & & & & & \\
\hline \begin{tabular}{|l|}
$23 S G$ \\
$272-$ \\
168 \\
\end{tabular} & $\begin{array}{l}40 R \\
60\end{array}$ & 3 & & $\begin{array}{l}Z L \\
O\end{array}$ & 1 & & & & & & & & & \\
\hline $\begin{array}{l}23 S G \\
272- \\
168\end{array}$ & $\begin{array}{l}40 R \\
60\end{array}$ & 3 & & $\begin{array}{l}Z \mathrm{~L} \\
\mathrm{O}\end{array}$ & 1 & & $\begin{array}{l}\text { brass- } \\
\text { plated iron } \\
\text { hook from } \\
\text { a hook- } \\
\text { and-eye }\end{array}$ & & & & & & & \\
\hline \begin{tabular}{|l|}
$23 S G$ \\
$272-$ \\
168
\end{tabular} & \begin{tabular}{|l|}
$40 R$ \\
60
\end{tabular} & 3 & & $\begin{array}{l}Z L \\
O\end{array}$ & 1 & & & $\begin{array}{l}\text { piece } \\
\text { of } \\
\text { brass- }\end{array}$ & & & & & & \\
\hline
\end{tabular}




\begin{tabular}{|c|c|c|c|c|c|c|c|c|c|c|c|c|c|c|}
\hline \multicolumn{15}{|c|}{ Archaeological Analysis } \\
\hline $\mid \begin{array}{c}\text { Catal } \\
\text { ogue } \\
\#\end{array}$ & Uni & $\mid \begin{array}{c}Q \\
\text { ua } \\
\text { d }\end{array}$ & \begin{tabular}{c|} 
Le \\
ve \\
I
\end{tabular} & $\begin{array}{l}\mathrm{Ti} \\
\mathrm{me} \\
\mathrm{per} \\
\mathrm{iod}\end{array}$ & $\mid \begin{array}{c}\text { Am } \\
\text { oun } \\
t\end{array}$ & $\mid \begin{array}{l}\text { Hous } \\
\text { ehold } \\
\text { Furnis } \\
\text { hings }\end{array}$ & Clothing & \begin{tabular}{|c} 
Perso \\
nal \\
Items
\end{tabular} & $\begin{array}{l}\text { Househ } \\
\text { old } \\
\text { product } \\
\text { ion } \\
\text { activity }\end{array}$ & \begin{tabular}{|c} 
Househ \\
old \\
produc \\
tion-for \\
sale
\end{tabular} & \begin{tabular}{|l} 
Pharma \\
ceutical
\end{tabular} & $\begin{array}{l}\text { Food } \\
\text { stuffs }\end{array}$ & $\begin{array}{c}\text { Alc } \\
\text { oho } \\
\text { I }\end{array}$ & $\begin{array}{l}\text { Ot } \\
\text { her }\end{array}$ \\
\hline & & & & & & & & $\begin{array}{l}\text { plated } \\
\text { iron, } \\
\text { possib } \\
\text { le link } \\
\text { for } \\
\text { jewelr } \\
\text { y }\end{array}$ & & & & & & \\
\hline $\begin{array}{l}23 S G \\
272- \\
168\end{array}$ & $\begin{array}{l}40 R \\
60\end{array}$ & 3 & & $\begin{array}{l}\mathrm{ZL} \\
\mathrm{O}\end{array}$ & 1 & & & & & & & & & \\
\hline \begin{tabular}{|l|}
$23 S G$ \\
$272-$ \\
168
\end{tabular} & $\begin{array}{l}40 R \\
60\end{array}$ & 3 & & $\begin{array}{l}\mathrm{ZL} \\
\mathrm{O}\end{array}$ & 1 & & & & & $\begin{array}{l}\text { brass } \\
\text { cartridg } \\
\text { e shell } \\
\text { frag or } \\
\text { cylinder } \\
\text { of } \\
\text { some } \\
\text { kind }\end{array}$ & & & & \\
\hline \begin{tabular}{|l|}
$23 S G$ \\
$272-$ \\
168
\end{tabular} & $\begin{array}{l}40 R \\
60\end{array}$ & 3 & & $\begin{array}{l}\mathrm{ZL} \\
\mathrm{O}\end{array}$ & 4 & & $\begin{array}{l}\text { frags, } \\
\text { brass- } \\
\text { plated iron } \\
\text { straight } \\
\text { pin }\end{array}$ & & & & & & & \\
\hline $\begin{array}{l}23 S G \\
272- \\
168\end{array}$ & $\begin{array}{l}40 R \\
60\end{array}$ & 3 & & $\begin{array}{l}\text { ZL } \\
\text { O }\end{array}$ & 20 & & & & & $\begin{array}{l}\text { frags, } \\
\text { sheet } \\
\text { lead } \\
\text { scrap }\end{array}$ & & & & \\
\hline \begin{tabular}{|l|}
$23 S G$ \\
$272-$ \\
168 \\
\end{tabular} & $\begin{array}{l}40 R \\
60\end{array}$ & 3 & & $\begin{array}{l}\mathrm{ZL} \\
\mathrm{O}\end{array}$ & 1 & & & & & & & & & \\
\hline \begin{tabular}{|l|}
$23 S G$ \\
$272-$ \\
168 \\
\end{tabular} & $\begin{array}{l}40 \mathrm{R} \\
60\end{array}$ & 3 & & $\begin{array}{l}\mathrm{ZL} \\
\mathrm{O}\end{array}$ & 1 & & & & & & & & & \\
\hline \begin{tabular}{|l|}
$23 S G$ \\
$272-$ \\
168
\end{tabular} & $\begin{array}{l}40 \mathrm{R} \\
60\end{array}$ & 3 & & $\begin{array}{l}\mathrm{ZL} \\
\mathrm{O}\end{array}$ & 5 & & & & & & & & & \\
\hline \begin{tabular}{|l}
$23 S G$ \\
$272-$
\end{tabular} & \begin{tabular}{|l|}
$40 R$ \\
60
\end{tabular} & 3 & & $\begin{array}{l}\mathrm{ZL} \\
\mathrm{O}\end{array}$ & 5 & & & & & & & & & \\
\hline
\end{tabular}




\begin{tabular}{|c|c|c|c|c|c|c|c|c|c|c|c|c|c|c|}
\hline \multicolumn{15}{|c|}{ Archaeological Analysis } \\
\hline $\begin{array}{c}\text { Catal } \\
\text { ogue } \\
\#\end{array}$ & Uni & 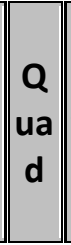 & $\begin{array}{c}\text { Le } \\
\text { ve } \\
\text { I }\end{array}$ & $\begin{array}{l}\text { Ti } \\
\text { me } \\
\text { per } \\
\text { iod }\end{array}$ & $\mid \begin{array}{c}\text { Am } \\
\text { oun } \\
t\end{array}$ & \begin{tabular}{|l} 
Hous \\
ehold \\
Furnis \\
hings
\end{tabular} & Clothing & $\mid \begin{array}{c}\text { Perso } \\
\text { nal } \\
\text { Items }\end{array}$ & \begin{tabular}{|c|} 
Househ \\
old \\
product \\
ion \\
activity
\end{tabular} & \begin{tabular}{|c} 
Househ \\
old \\
produc \\
tion-for \\
sale
\end{tabular} & 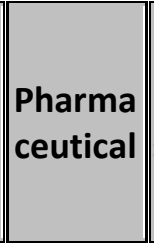 & 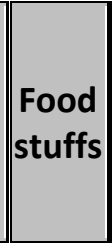 & $\mid \begin{array}{c}\text { Alc } \\
\text { oho } \\
\text { I }\end{array}$ & Ot \\
\hline \multicolumn{15}{|l|}{168} \\
\hline $\begin{array}{l}23 S G \\
272- \\
168\end{array}$ & $\begin{array}{l}40 R \\
60\end{array}$ & 3 & & $\begin{array}{l}Z \mathrm{ZL} \\
\mathrm{O}\end{array}$ & 103 & & & & & & & & & \\
\hline \begin{tabular}{|l|}
$23 S G$ \\
$272-$ \\
168 \\
\end{tabular} & $\begin{array}{l}40 R \\
60\end{array}$ & 3 & & $\begin{array}{l}Z L \\
O\end{array}$ & 10 & & & & & & & & & \\
\hline \begin{tabular}{|l|}
$23 S G$ \\
$272-$ \\
168 \\
\end{tabular} & $\begin{array}{l}40 R \\
60\end{array}$ & 3 & & $\begin{array}{l}Z L \\
O\end{array}$ & 1 & & & & & & & & & \\
\hline \begin{tabular}{|l|}
$23 S G$ \\
$272-$ \\
168 \\
\end{tabular} & $\begin{array}{l}40 R \\
60\end{array}$ & 3 & & $\begin{array}{l}Z \mathrm{ZL} \\
\mathrm{O}\end{array}$ & 1 & & & & $\begin{array}{l}\text { "tumble } \\
\text { d" } \\
\text { stone }\end{array}$ & & & & & \\
\hline \begin{tabular}{|l|}
$23 S G$ \\
$272-$ \\
168
\end{tabular} & $\begin{array}{l}40 R \\
60\end{array}$ & 3 & & $\begin{array}{l}Z L \\
O\end{array}$ & 1 & & & & $\begin{array}{l}\text { "tumble } \\
\text { d" chert } \\
\text { flake }\end{array}$ & & & & & \\
\hline \begin{tabular}{|l|}
$23 S G$ \\
$272-$ \\
168
\end{tabular} & $\begin{array}{l}40 R \\
60\end{array}$ & 3 & & $\begin{array}{l}Z \mathrm{ZL} \\
\mathrm{O}\end{array}$ & 2 & & & & $\begin{array}{l}\text { "tumble } \\
\text { d" glass } \\
\text { frags }\end{array}$ & & & & & \\
\hline \begin{tabular}{|l|}
$23 S G$ \\
$272-$ \\
169 \\
\end{tabular} & $\begin{array}{l}40 R \\
60\end{array}$ & 3 & & $\begin{array}{l}Z L \\
O\end{array}$ & 1 & & & & & & & & & \\
\hline \begin{tabular}{|l|}
$23 S G$ \\
$272-$ \\
169
\end{tabular} & $\begin{array}{l}40 R \\
60\end{array}$ & 3 & & $\begin{array}{l}Z L \\
O\end{array}$ & 1 & & & & & $\begin{array}{l}0.18 " \\
\text { caliber } \\
\text { lead } \\
\text { shot }\end{array}$ & & & & \\
\hline \begin{tabular}{|l|}
$23 S G$ \\
$272-$ \\
170 \\
\end{tabular} & $\begin{array}{l}40 R \\
60\end{array}$ & 3 & & $\begin{array}{l}Z L \\
O\end{array}$ & 2 & & & & & & & & & \\
\hline \begin{tabular}{|l|}
$23 S G$ \\
$272-$ \\
170 \\
\end{tabular} & $\begin{array}{l}40 R \\
60\end{array}$ & 3 & & $\begin{array}{l}Z \mathrm{ZL} \\
\mathrm{O}\end{array}$ & 1 & & & & & & & & & \\
\hline \begin{tabular}{|l|}
$23 S G$ \\
$272-$ \\
170 \\
\end{tabular} & $\begin{array}{l}40 R \\
60\end{array}$ & 3 & & $\begin{array}{l}Z \mathrm{Z} \\
\mathrm{O}\end{array}$ & 2 & & & & & & & & & \\
\hline \begin{tabular}{|l}
$23 S G$ \\
$272-$
\end{tabular} & $\begin{array}{l}40 R \\
60\end{array}$ & 3 & & $\begin{array}{l}Z \mathrm{Z} \\
\mathrm{O}\end{array}$ & 3 & & & & & & & & & \\
\hline
\end{tabular}




\begin{tabular}{|c|c|c|c|c|c|c|c|c|c|c|c|c|c|c|}
\hline \multicolumn{15}{|c|}{ Archaeological Analysis } \\
\hline $\begin{array}{c}\text { Catal } \\
\text { ogue } \\
\#\end{array}$ & $\begin{array}{c}\text { Uni } \\
t\end{array}$ & $\begin{array}{c}\text { Q } \\
\text { ua } \\
d\end{array}$ & \begin{tabular}{c||} 
Le \\
ve \\
I
\end{tabular} & $\begin{array}{l}\text { Ti } \\
\text { me } \\
\text { per } \\
\text { iod }\end{array}$ & $\mid \begin{array}{c}\text { Am } \\
\text { oun } \\
t\end{array}$ & \begin{tabular}{|l} 
Hous \\
ehold \\
Furnis \\
hings
\end{tabular} & Clothing & \begin{tabular}{|c} 
Perso \\
nal \\
Items
\end{tabular} & \begin{tabular}{|c|}
$\begin{array}{c}\text { Househ } \\
\text { old } \\
\text { product } \\
\text { ion } \\
\text { activity }\end{array}$ \\
\end{tabular} & \begin{tabular}{||c|}
$\begin{array}{c}\text { Househ } \\
\text { old } \\
\text { produc } \\
\text { tion-for } \\
\text { sale }\end{array}$ \\
\end{tabular} & $\begin{array}{l}\text { Pharma } \\
\text { ceutical }\end{array}$ & Food & $\mid \begin{array}{c}\text { Alc } \\
\text { oho } \\
\text { I }\end{array}$ & $\begin{array}{c}\text { Ot } \\
\text { her }\end{array}$ \\
\hline \multicolumn{15}{|l|}{170} \\
\hline $\begin{array}{l}23 S G \\
272- \\
170\end{array}$ & $\begin{array}{l}40 R \\
60\end{array}$ & 3 & & $\begin{array}{l}\mathrm{ZL} \\
\mathrm{O}\end{array}$ & 2 & & & & & & & & & \\
\hline \begin{tabular}{|l|}
$23 S G$ \\
$272-$ \\
170
\end{tabular} & $\begin{array}{l}40 R \\
60\end{array}$ & 3 & & $\begin{array}{l}Z L \\
O\end{array}$ & 1 & & & & & & & & & \\
\hline \begin{tabular}{|l|}
$23 S G$ \\
$272-$ \\
170
\end{tabular} & $\begin{array}{l}40 R \\
60\end{array}$ & 3 & & $\begin{array}{l}\mathrm{ZL} \\
\mathrm{O}\end{array}$ & 4 & & & & & & & & & \\
\hline \begin{tabular}{|l|}
$23 S G$ \\
$272-$ \\
170
\end{tabular} & $\begin{array}{l}40 R \\
60\end{array}$ & 3 & & $\begin{array}{l}\text { ZL } \\
\mathrm{O}\end{array}$ & 6 & & & & & & & & & \\
\hline \begin{tabular}{|l|}
$23 S G$ \\
$272-$ \\
170 \\
\end{tabular} & $\begin{array}{l}40 R \\
60\end{array}$ & 3 & & $\begin{array}{l}Z L \\
O\end{array}$ & 1 & & & & & & & & & \\
\hline \begin{tabular}{|l|}
$23 S G$ \\
$272-$ \\
170 \\
\end{tabular} & $\begin{array}{l}40 R \\
60\end{array}$ & 3 & & $\begin{array}{l}Z \mathrm{~L} \\
\mathrm{O}\end{array}$ & 1 & & & & & & & & & \\
\hline \begin{tabular}{|l|}
$23 S G$ \\
$272-$ \\
170 \\
\end{tabular} & $\begin{array}{l}40 R \\
60\end{array}$ & 3 & & $\begin{array}{l}Z L \\
O\end{array}$ & 3 & & & & & & & & & \\
\hline \begin{tabular}{|l|}
$23 S G$ \\
$272-$ \\
170
\end{tabular} & $\begin{array}{l}40 R \\
60\end{array}$ & 3 & & $\begin{array}{l}\mathrm{ZL} \\
\mathrm{O}\end{array}$ & 2 & & & & & & & & & \\
\hline \begin{tabular}{|l}
$23 S G$ \\
$272-$ \\
170
\end{tabular} & $\begin{array}{l}40 R \\
60\end{array}$ & 3 & & $\begin{array}{l}Z \mathrm{~L} \\
\mathrm{O}\end{array}$ & 28 & & & & & & & & & \\
\hline \begin{tabular}{|l|}
$23 S G$ \\
$272-$ \\
170 \\
\end{tabular} & $\begin{array}{l}40 R \\
60\end{array}$ & 3 & & $\begin{array}{l}Z L \\
O\end{array}$ & 2 & & & & & & & & & \\
\hline \begin{tabular}{|l|}
$23 S G$ \\
$272-$ \\
170
\end{tabular} & $\begin{array}{l}40 R \\
60\end{array}$ & 3 & & $\begin{array}{l}Z \mathrm{~L} \\
\mathrm{O}\end{array}$ & 1 & & & $\begin{array}{l}\text { frag, } \\
\text { quart } \\
\text { z } \\
\text { crysta } \\
\text { l, part } \\
\text { of } \\
\text { jewelr }\end{array}$ & & & & & & \\
\hline
\end{tabular}




\begin{tabular}{|c|c|c|c|c|c|c|c|c|c|c|c|c|c|c|}
\hline \multicolumn{15}{|c|}{ Archaeological Analysis } \\
\hline $\mid \begin{array}{c}\text { Catal } \\
\text { ogue } \\
\#\end{array}$ & Uni & $\begin{array}{c}\text { Q } \\
\text { ua } \\
\text { d }\end{array}$ & $\begin{array}{c}\text { Le } \\
\text { ve } \\
\text { I }\end{array}$ & $\begin{array}{l}\text { Ti } \\
\text { me } \\
\text { per } \\
\text { iod }\end{array}$ & $\begin{array}{c}\text { Am } \\
\text { oun } \\
t\end{array}$ & $\mid \begin{array}{l}\text { Hous } \\
\text { ehold } \\
\text { Furnis } \\
\text { hings }\end{array}$ & Clothing & \begin{tabular}{|c} 
Perso \\
nal \\
Items
\end{tabular} & $\begin{array}{c}\text { Househ } \\
\text { old } \\
\text { product } \\
\text { ion } \\
\text { activity }\end{array}$ & \begin{tabular}{|c|}
$\begin{array}{c}\text { Househ } \\
\text { old } \\
\text { produc } \\
\text { tion-for } \\
\text { sale }\end{array}$ \\
\end{tabular} & \begin{tabular}{|l} 
Pharma \\
ceutical
\end{tabular} & \begin{tabular}{||l} 
Food \\
stuffs
\end{tabular} & $\begin{array}{c}\text { Alc } \\
\text { oho } \\
\text { I }\end{array}$ & $\begin{array}{l}\text { Ot } \\
\text { her }\end{array}$ \\
\hline & & & & & & & & $y$ & & & & & & \\
\hline \begin{tabular}{|l|}
$23 S G$ \\
$272-$ \\
170
\end{tabular} & \begin{tabular}{|l|}
$40 R$ \\
60
\end{tabular} & 3 & & $\begin{array}{l}Z L \\
O\end{array}$ & 1 & & & \begin{tabular}{|l} 
neckl \\
ace \\
bead, \\
transl \\
ucent \\
dark \\
purpl \\
e
\end{tabular} & & & & & & \\
\hline \begin{tabular}{|l|}
$23 S G$ \\
$272-$ \\
170
\end{tabular} & $\begin{array}{l}40 R \\
60\end{array}$ & 3 & & $\begin{array}{l}Z L \\
O\end{array}$ & 1 & & & $\begin{array}{l}\text { neckl } \\
\text { ace } \\
\text { bead, } \\
\text { opaqu } \\
\text { e red }\end{array}$ & & & & & & \\
\hline \begin{tabular}{|l|}
$23 S G$ \\
$272-$ \\
170
\end{tabular} & $\begin{array}{l}40 R \\
60\end{array}$ & 3 & & $\begin{array}{l}Z \mathrm{~L} \\
\mathrm{O}\end{array}$ & 1 & & & & & $\begin{array}{l}0.41 " \\
\text { caliber } \\
\text { lead } \\
\text { shot }\end{array}$ & & & & \\
\hline \begin{tabular}{|l|}
$23 S G$ \\
$272-$ \\
170
\end{tabular} & $\begin{array}{l}40 R \\
60\end{array}$ & 3 & & $\begin{array}{l}Z \mathrm{~L} \\
\mathrm{O}\end{array}$ & 1 & & & & & $\begin{array}{l}0.16 " \\
\text { caliber } \\
\text { lead } \\
\text { shot }\end{array}$ & & & & \\
\hline \begin{tabular}{|l|}
$23 S G$ \\
$272-$ \\
170
\end{tabular} & \begin{tabular}{|l|}
$40 R$ \\
60
\end{tabular} & 3 & & $\begin{array}{l}Z L \\
O\end{array}$ & 1 & & & & & $\begin{array}{l}0.15 " \\
\text { caliber } \\
\text { lead } \\
\text { shot }\end{array}$ & & & & \\
\hline \begin{tabular}{|l|}
$23 S G$ \\
$272-$ \\
170
\end{tabular} & $\begin{array}{l}40 R \\
60\end{array}$ & 3 & & $\begin{array}{l}Z L \\
O\end{array}$ & 1 & & & & & $\begin{array}{l}0.14 " \\
\text { caliber } \\
\text { lead } \\
\text { shot }\end{array}$ & & & & \\
\hline $\begin{array}{l}23 S G \\
272- \\
170\end{array}$ & $\begin{array}{l}40 R \\
60\end{array}$ & 3 & & $\begin{array}{l}Z \mathrm{~L} \\
\mathrm{O}\end{array}$ & 3 & & & & & $\begin{array}{l}0.13 " \\
\text { caliber } \\
\text { lead } \\
\text { shot }\end{array}$ & & & & \\
\hline \begin{tabular}{|l|}
$23 S G$ \\
$272-$ \\
170
\end{tabular} & $\begin{array}{l}40 R \\
60\end{array}$ & 3 & & $\begin{array}{l}Z \mathrm{~L} \\
\mathrm{O}\end{array}$ & 3 & & & & & $\begin{array}{l}0.12 " \\
\text { caliber } \\
\text { lead } \\
\text { shot }\end{array}$ & & & & \\
\hline
\end{tabular}




\begin{tabular}{|c|c|c|c|c|c|c|c|c|c|c|c|c|c|c|}
\hline \multicolumn{15}{|c|}{ Archaeological Analysis } \\
\hline $\begin{array}{c}\text { Catal } \\
\text { ogue } \\
\#\end{array}$ & Uni & $\mid \begin{array}{c}Q \\
\text { ua } \\
\text { d }\end{array}$ & \begin{tabular}{|c||l} 
Le & $r$ \\
ve & $r$ \\
I & i
\end{tabular} & $\begin{array}{c}\mathrm{Ti} \\
\mathrm{me} \\
\mathrm{per} \\
\mathrm{iod}\end{array}$ & $\mid \begin{array}{c}\text { Am } \\
\text { oun } \\
t\end{array}$ & $\mid \begin{array}{l}\text { Hous } \\
\text { ehold } \\
\text { Furnis } \\
\text { hings }\end{array}$ & Clothing & $\mid \begin{array}{c}\text { Perso } \\
\text { nal } \\
\text { Items }\end{array}$ & $\begin{array}{l}\text { Househ } \\
\text { old } \\
\text { product } \\
\text { ion } \\
\text { activity }\end{array}$ & \begin{tabular}{|c} 
Househ \\
old \\
produc \\
tion-for \\
sale
\end{tabular} & $\begin{array}{l}\text { Pharma } \\
\text { ceutical }\end{array}$ & \begin{tabular}{|l||} 
Food \\
stuffs
\end{tabular} & $\begin{array}{c}\text { Alc } \\
\text { oho } \\
\text { I }\end{array}$ & $\begin{array}{l}\text { Ot } \\
\text { her }\end{array}$ \\
\hline \begin{tabular}{|l|}
$23 S G$ \\
$272-$ \\
170
\end{tabular} & $\begin{array}{l}40 R \\
60\end{array}$ & 3 & & $\begin{array}{l}Z L \\
O\end{array}$ & 1 & & & & & $\begin{array}{l}0.11 " \\
\text { caliber } \\
\text { lead } \\
\text { shot }\end{array}$ & & & & \\
\hline \begin{tabular}{|l|}
$23 S G$ \\
$272-$ \\
170
\end{tabular} & \begin{tabular}{|l|}
$40 \mathrm{R}$ \\
60
\end{tabular} & 3 & & $\begin{array}{l}Z \mathrm{Z} \\
\mathrm{O}\end{array}$ & 4 & & & & & $\begin{array}{l}0.10 " \\
\text { caliber } \\
\text { lead } \\
\text { shot }\end{array}$ & & & & \\
\hline \begin{tabular}{|l|}
$23 S G$ \\
$272-$ \\
170
\end{tabular} & \begin{tabular}{|l|}
$40 R$ \\
60
\end{tabular} & 3 & & $\begin{array}{l}Z L \\
O\end{array}$ & 1 & & & & & $\begin{array}{l}\text { deform } \\
\text { ed } \\
\text { shot, } \\
\text { approxi } \\
\text { mately } \\
0.12 " \\
\text { caliber }\end{array}$ & & & & \\
\hline $\begin{array}{l}23 S G \\
272- \\
170\end{array}$ & $\begin{array}{l}40 R \\
60\end{array}$ & 3 & & $\begin{array}{l}\mathrm{ZL} \\
\mathrm{O}\end{array}$ & 1 & & & & & $\begin{array}{l}\text { deform } \\
\text { ed } \\
\text { shot, } \\
\text { approxi } \\
\text { mately } \\
0.12 " \\
\text { caliber }\end{array}$ & & & & \\
\hline $\begin{array}{l}23 S G \\
272- \\
170\end{array}$ & $\begin{array}{l}40 R \\
60\end{array}$ & 3 & & $\begin{array}{l}Z \mathrm{~L} \\
\mathrm{O}\end{array}$ & 1 & & & & & \begin{tabular}{|l} 
shot, \\
approxi \\
mately \\
$0.16 "$ \\
caliber
\end{tabular} & & & & \\
\hline $\begin{array}{l}23 S G \\
272- \\
170\end{array}$ & $\begin{array}{l}40 R \\
60\end{array}$ & 3 & & $\begin{array}{l}Z \mathrm{~L} \\
\mathrm{O}\end{array}$ & 1 & & & & & $\begin{array}{l}0.22 " \\
\text { caliber } \\
\text { lead } \\
\text { bullet }\end{array}$ & & & & \\
\hline \begin{tabular}{|l|}
$23 S G$ \\
$272-$ \\
170
\end{tabular} & \begin{tabular}{|l|}
$40 R$ \\
60
\end{tabular} & 3 & & $\begin{array}{l}\text { ZL } \\
O\end{array}$ & 1 & & & & $\begin{array}{l}\text { frag. Of } \\
\text { honey- } \\
\text { colored } \\
\text { gunflint }\end{array}$ & & & & & \\
\hline \begin{tabular}{|l|}
$23 S G$ \\
$272-$ \\
170 \\
\end{tabular} & $\begin{array}{l}40 R \\
60\end{array}$ & 3 & & $\begin{array}{l}\mathrm{ZL} \\
\mathrm{O}\end{array}$ & 3 & & & & $\begin{array}{l}\text { gunflint } \\
\text { flakes, } \\
\text { honey- }\end{array}$ & & & & & \\
\hline
\end{tabular}




\begin{tabular}{|c|c|c|c|c|c|c|c|c|c|c|c|c|c|c|}
\hline \multicolumn{15}{|c|}{ Archaeological Analysis } \\
\hline $\begin{array}{c}\text { Catal } \\
\text { ogue } \\
\#\end{array}$ & $\left(\begin{array}{c}\text { Uni } \\
t\end{array}\right.$ & $\mid \begin{array}{c}Q \\
\text { ua } \\
\text { d }\end{array}$ & \begin{tabular}{c|} 
Le \\
ve \\
I
\end{tabular} & $\begin{array}{c}\mathrm{Ti} \\
\mathrm{me} \\
\mathrm{per} \\
\mathrm{iod}\end{array}$ & $\mid \begin{array}{c}\text { Am } \\
\text { oun } \\
t\end{array}$ & $\mid \begin{array}{l}\text { Hous } \\
\text { ehold } \\
\text { Furnis } \\
\text { hings }\end{array}$ & Clothing & \begin{tabular}{|c} 
Perso \\
nal \\
Items
\end{tabular} & $\begin{array}{l}\text { Househ } \\
\text { old } \\
\text { product } \\
\text { ion } \\
\text { activity }\end{array}$ & \begin{tabular}{|c} 
Househ \\
old \\
produc \\
tion-for \\
sale
\end{tabular} & $\begin{array}{l}\text { Pharma } \\
\text { ceutical }\end{array}$ & $\begin{array}{l}\text { Food } \\
\text { stuffs }\end{array}$ & $\begin{array}{c}\text { Alc } \\
\text { oho } \\
\text { I }\end{array}$ & $\begin{array}{l}\text { Ot } \\
\text { her }\end{array}$ \\
\hline & & & & & & & & & colored & & & & & \\
\hline $\begin{array}{l}23 S G \\
272- \\
170\end{array}$ & $\begin{array}{l}40 R \\
60\end{array}$ & 3 & & $\begin{array}{l}\text { ZL } \\
\text { O }\end{array}$ & 1 & & & & $\begin{array}{l}\text { gunflint } \\
\text { flakes, } \\
\text { gray, } \\
\text { possibly } \\
\text { from } \\
\text { British } \\
\text { gunflint }\end{array}$ & & & & & \\
\hline $\begin{array}{l}23 S G \\
272- \\
170\end{array}$ & $\begin{array}{l}40 R \\
60\end{array}$ & 3 & & $\begin{array}{l}\mathrm{ZL} \\
\mathrm{O}\end{array}$ & 1 & & & & & $\begin{array}{l}\text { frag, } \\
\text { sheet } \\
\text { lead } \\
\text { scrap }\end{array}$ & & & & \\
\hline \begin{tabular}{|l|}
$23 S G$ \\
$272-$ \\
170 \\
\end{tabular} & $\begin{array}{l}40 R \\
60\end{array}$ & 3 & & $\begin{array}{l}\text { ZL } \\
\text { O }\end{array}$ & 1 & & & & & & & & & \\
\hline \begin{tabular}{|l|}
$23 S G$ \\
$272-$ \\
170
\end{tabular} & $\begin{array}{l}40 R \\
60\end{array}$ & 3 & & $\begin{array}{l}\text { ZL } \\
\text { O }\end{array}$ & 1 & & $\begin{array}{l}\text { brass- } \\
\text { plated iron } \\
\text { straight } \\
\text { pin frag }\end{array}$ & & & & & & & \\
\hline $\begin{array}{l}23 S G \\
272- \\
170\end{array}$ & $\begin{array}{l}40 R \\
60\end{array}$ & 3 & & $\begin{array}{l}\mathrm{ZL} \\
\mathrm{O}\end{array}$ & 1 & & $\begin{array}{l}\text { brass- } \\
\text { plated iron } \\
\text { frag } \\
\text { (appears } \\
\text { to be part } \\
\text { of the } \\
\text { base of an } \\
\text { eye for a } \\
\text { hook-and- } \\
\text { eye }\end{array}$ & & & & & & & \\
\hline \begin{tabular}{|l|}
$23 S G$ \\
$272-$ \\
170 \\
\end{tabular} & $\begin{array}{l}40 \mathrm{R} \\
60\end{array}$ & 3 & & $\begin{array}{l}\mathrm{ZL} \\
\mathrm{O}\end{array}$ & 4 & & & & & & & & & \\
\hline \begin{tabular}{|l|}
$23 S G$ \\
$272-$ \\
170 \\
\end{tabular} & $\begin{array}{l}40 R \\
60\end{array}$ & 3 & & $\begin{array}{l}\text { ZL } \\
0\end{array}$ & 10 & & & & & & & & & \\
\hline $\begin{array}{l}23 S G \\
272- \\
170\end{array}$ & $\begin{array}{l}40 R \\
60\end{array}$ & 3 & & $\begin{array}{l}\text { ZL } \\
\text { O }\end{array}$ & 1 & & & & & & & & & \\
\hline
\end{tabular}




\begin{tabular}{|c|c|c|c|c|c|c|c|c|c|c|c|c|c|c|}
\hline \multicolumn{15}{|c|}{ Archaeological Analysis } \\
\hline $\begin{array}{c}\text { Catal } \\
\text { ogue } \\
\#\end{array}$ & $\begin{array}{c}\text { Uni } \\
t\end{array}$ & $\begin{array}{c}Q \\
\text { ua } \\
d\end{array}$ & \begin{tabular}{c||l} 
Le & \\
ve & i \\
I & $i$
\end{tabular} & $\begin{array}{l}\text { Ti } \\
\text { me } \\
\text { per } \\
\text { iod }\end{array}$ & $\mid \begin{array}{c}\text { Am } \\
\text { oun } \\
t\end{array}$ & \begin{tabular}{|l} 
Hous \\
ehold \\
Furnis \\
hings
\end{tabular} & Clothing & $\mid \begin{array}{c}\text { Perso } \\
\text { nal } \\
\text { Items }\end{array}$ & \begin{tabular}{|} 
Househ \\
old \\
product \\
ion \\
activity
\end{tabular} & \begin{tabular}{|l} 
Househ \\
old \\
produc \\
tion-for \\
sale
\end{tabular} & 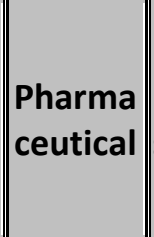 & Food & $\mid \begin{array}{c}\text { Alc } \\
\text { oho } \\
\text { I }\end{array}$ & $\begin{array}{c}\text { Ot } \\
\text { her }\end{array}$ \\
\hline \begin{tabular}{|l|}
$23 S G$ \\
$272-$ \\
171 \\
\end{tabular} & $\begin{array}{l}40 R \\
60\end{array}$ & 3 & & $\begin{array}{l}\mathrm{ZL} \\
\mathrm{O}\end{array}$ & 4 & & & & & & & & & \\
\hline \begin{tabular}{|l|}
$23 S G$ \\
$272-$ \\
171
\end{tabular} & $\begin{array}{l}40 R \\
60\end{array}$ & 3 & & $\begin{array}{l}\text { ZL } \\
O\end{array}$ & 1 & & & & & & & & & \\
\hline \begin{tabular}{|l|}
$23 S G$ \\
$272-$ \\
171
\end{tabular} & $\begin{array}{l}40 R \\
60\end{array}$ & 3 & & $\begin{array}{l}\mathrm{ZL} \\
\mathrm{O}\end{array}$ & 2 & & & & & & & & & \\
\hline \begin{tabular}{|l|}
$23 S G$ \\
$272-$ \\
171
\end{tabular} & $\begin{array}{l}40 R \\
60\end{array}$ & 3 & & $\begin{array}{l}Z \mathrm{~L} \\
\mathrm{O}\end{array}$ & 5 & & & & & & & & & \\
\hline $\begin{array}{l}23 S G \\
272- \\
171\end{array}$ & $\begin{array}{l}40 R \\
60\end{array}$ & 3 & & $\begin{array}{l}\text { ZL } \\
0\end{array}$ & 1 & & & $\begin{array}{l}\text { longit } \\
\text { udinal } \\
\text { frags } \\
\text { of } \\
\text { quart } \\
\text { z } \\
\text { crysta } \\
\text { I }\end{array}$ & & & & & & \\
\hline \begin{tabular}{|l|}
$23 S G$ \\
$272-$ \\
171
\end{tabular} & $\begin{array}{l}40 R \\
60\end{array}$ & 3 & & $\begin{array}{l}Z L \\
O\end{array}$ & 1 & & & & \begin{tabular}{|l|} 
gunflint \\
frag, \\
honey- \\
colored
\end{tabular} & & & & & \\
\hline $\begin{array}{l}23 S G \\
272- \\
171\end{array}$ & $\begin{array}{l}40 R \\
60\end{array}$ & 3 & & $\begin{array}{l}\mathrm{ZL} \\
\mathrm{O}\end{array}$ & 1 & & & & & $\begin{array}{l}\text { frag., } \\
\text { sheet } \\
\text { lead } \\
\text { scrap }\end{array}$ & & & & \\
\hline \begin{tabular}{|l|}
$23 S G$ \\
$272-$ \\
171 \\
\end{tabular} & $\begin{array}{l}40 R \\
60\end{array}$ & 3 & & $\begin{array}{l}\mathrm{ZL} \\
\mathrm{O}\end{array}$ & 1 & & & & & & & & & \\
\hline \begin{tabular}{|l|}
$23 S G$ \\
$272-$ \\
171 \\
\end{tabular} & $\begin{array}{l}40 R \\
60\end{array}$ & 3 & & $\begin{array}{l}Z L \\
O\end{array}$ & 1 & & & & & & & & & \\
\hline \begin{tabular}{|l}
$23 S G$ \\
$272-$ \\
171
\end{tabular} & $\begin{array}{l}40 R \\
60\end{array}$ & 3 & & $\begin{array}{l}\mathrm{ZL} \\
\mathrm{O}\end{array}$ & 9 & & & & & & & & & \\
\hline 23SG & $40 R$ & 3 & 10 & ZO & 4 & & & & & & & & & \\
\hline
\end{tabular}




\begin{tabular}{|c|c|c|c|c|c|c|c|c|c|c|c|c|c|c|}
\hline \multicolumn{15}{|c|}{ Archaeological Analysis } \\
\hline $\begin{array}{c}\text { Catal } \\
\text { ogue } \\
\#\end{array}$ & Uni & $\mid \begin{array}{c}Q \\
\text { ua } \\
\text { d }\end{array}$ & \begin{tabular}{c||} 
Le \\
ve \\
I
\end{tabular} & $\begin{array}{l}\text { Ti } \\
\text { me } \\
\text { per } \\
\text { iod }\end{array}$ & $\mid \begin{array}{c}\text { Am } \\
\text { oun } \\
t\end{array}$ & $\mid \begin{array}{l}\text { Hous } \\
\text { ehold } \\
\text { Furnis } \\
\text { hings }\end{array}$ & Clothing & $\mid \begin{array}{c}\text { Perso } \\
\text { nal } \\
\text { Items }\end{array}$ & $\begin{array}{c}\text { Househ } \\
\text { old } \\
\text { product } \\
\text { ion } \\
\text { activity }\end{array}$ & $\begin{array}{c}\text { Househ } \\
\text { old } \\
\text { produc } \\
\text { tion-for } \\
\text { sale }\end{array}$ & $\begin{array}{l}\text { Pharma } \\
\text { ceutical }\end{array}$ & Food & $\mid \begin{array}{c}\text { Alc } \\
\text { oho } \\
\text { I }\end{array}$ & Ot \\
\hline $\begin{array}{l}272- \\
172\end{array}$ & 60 & & & & & & & & & & & & & \\
\hline $\begin{array}{l}23 S G \\
272- \\
172\end{array}$ & $\begin{array}{l}40 R \\
60\end{array}$ & 3 & 102 & ZO & 1 & & & & & & & & & \\
\hline \begin{tabular}{|l|}
$23 S G$ \\
$272-$ \\
172
\end{tabular} & $\begin{array}{l}40 R \\
60\end{array}$ & 3 & 10 & ZO & 1 & & & & & & & & & \\
\hline \begin{tabular}{|l|}
$23 S G$ \\
$272-$ \\
172
\end{tabular} & $\begin{array}{l}40 R \\
60\end{array}$ & 3 & 10 & ZO & 2 & & & & & $\begin{array}{l}0.18 " \\
\text { caliber } \\
\text { lead } \\
\text { shot }\end{array}$ & & & & \\
\hline \begin{tabular}{|l|}
$23 S G$ \\
$272-$ \\
172
\end{tabular} & $\begin{array}{l}40 R \\
60\end{array}$ & 3 & 10 & ZO & 1 & & & & & $\begin{array}{l}0.17 " \\
\text { caliber } \\
\text { lead } \\
\text { shot }\end{array}$ & & & & \\
\hline \begin{tabular}{|l|}
$23 S G$ \\
$272-$ \\
172
\end{tabular} & $\begin{array}{l}40 R \\
60\end{array}$ & 3 & 10 & ZO & 1 & & & & & $\begin{array}{l}0.15 " \\
\text { caliber } \\
\text { lead } \\
\text { shot }\end{array}$ & & & & \\
\hline \begin{tabular}{|l|}
$23 S G$ \\
$272-$ \\
172
\end{tabular} & $\begin{array}{l}40 R \\
60\end{array}$ & 3 & 10 & $\mathrm{ZO}$ & 1 & & & & & $\begin{array}{l}0.14 " \\
\text { caliber } \\
\text { lead } \\
\text { shot }\end{array}$ & & & & \\
\hline \begin{tabular}{|l|}
$23 S G$ \\
$272-$ \\
172
\end{tabular} & $\begin{array}{l}40 R \\
60\end{array}$ & 3 & 10 & ZO & 2 & & & & & $\begin{array}{l}0.13 " \\
\text { caliber } \\
\text { lead } \\
\text { shot }\end{array}$ & & & & \\
\hline $\begin{array}{l}23 S G \\
272- \\
172\end{array}$ & $\begin{array}{l}40 R \\
60\end{array}$ & 3 & 10 & ZO & 2 & & & & & $\begin{array}{l}0.12 " \\
\text { caliber } \\
\text { lead } \\
\text { shot }\end{array}$ & & & & \\
\hline \begin{tabular}{|l|}
$23 S G$ \\
$272-$ \\
172
\end{tabular} & $\begin{array}{l}40 R \\
60\end{array}$ & 3 & 10 & ZO & 2 & & & & & $\begin{array}{l}0.11 " \\
\text { caliber } \\
\text { lead } \\
\text { shot }\end{array}$ & & & & \\
\hline $\begin{array}{l}23 S G \\
272-\end{array}$ & $\begin{array}{l}40 R \\
60\end{array}$ & 3 & 10 & ZO & 3 & & & & & $\begin{array}{l}0.10 " \\
\text { caliber }\end{array}$ & & & & \\
\hline
\end{tabular}




\begin{tabular}{|c|c|c|c|c|c|c|c|c|c|c|c|c|c|c|}
\hline \multicolumn{15}{|c|}{ Archaeological Analysis } \\
\hline $\begin{array}{c}\text { Catal } \\
\text { ogue } \\
\#\end{array}$ & $\begin{array}{c}\text { Uni } \\
t\end{array}$ & $\begin{array}{c}\text { Q } \\
\text { ua } \\
d\end{array}$ & \begin{tabular}{c||} 
Le \\
ve \\
I
\end{tabular} & $\begin{array}{l}\text { Ti } \\
\text { me } \\
\text { per } \\
\text { iod }\end{array}$ & $\mid \begin{array}{c}\text { Am } \\
\text { oun } \\
t\end{array}$ & \begin{tabular}{|l} 
Hous \\
ehold \\
Furnis \\
hings
\end{tabular} & Clothing & $\begin{array}{c}\text { Perso } \\
\text { nal } \\
\text { Items }\end{array}$ & \begin{tabular}{|} 
Househ \\
old \\
product \\
ion \\
activity
\end{tabular} & \begin{tabular}{||c||} 
Househ \\
old \\
produc \\
tion-for \\
sale
\end{tabular} & $\begin{array}{l}\text { Pharma } \\
\text { ceutical }\end{array}$ & $\mid \begin{array}{l}\text { Food } \\
\text { stuffs }\end{array}$ & $\mid \begin{array}{c}\text { Alc } \\
\text { oho } \\
\text { I }\end{array}$ & $\begin{array}{c}\text { Ot } \\
\text { her }\end{array}$ \\
\hline 172 & & & & & & & & & & $\begin{array}{l}\text { lead } \\
\text { shot }\end{array}$ & & & & \\
\hline $\begin{array}{l}23 S G \\
272- \\
172\end{array}$ & $\begin{array}{l}40 R \\
60\end{array}$ & 3 & 10 & ZO & 1 & & & & & $\begin{array}{l}0.08 " \\
\text { caliber } \\
\text { lead } \\
\text { shot }\end{array}$ & & & & \\
\hline \begin{tabular}{|l|}
$23 S G$ \\
$272-$ \\
172
\end{tabular} & $\begin{array}{l}40 R \\
60\end{array}$ & 3 & 10 & ZO & 1 & & & & & $\begin{array}{l}0.07 " \\
\text { caliber } \\
\text { lead } \\
\text { shot }\end{array}$ & & & & \\
\hline \begin{tabular}{|l|}
$23 S G$ \\
$272-$ \\
172
\end{tabular} & $\begin{array}{l}40 R \\
60\end{array}$ & 3 & 10 & ZO & 1 & & & & & \begin{tabular}{|l|} 
lead \\
shot, \\
flattene \\
d on \\
several \\
sides; \\
appro. \\
$0.13 "$ \\
caliber
\end{tabular} & & & & \\
\hline $\begin{array}{l}23 S G \\
272- \\
172\end{array}$ & $\begin{array}{l}40 R \\
60\end{array}$ & 3 & 10 & ZO & 1 & & & & & $\begin{array}{l}\text { lead } \\
\text { shot, } \\
\text { flattene } \\
\text { d on } \\
\text { several } \\
\text { sides; } \\
\text { appro. } \\
0.12 " \\
\text { caliber }\end{array}$ & & & & \\
\hline \begin{tabular}{|l|}
$23 S G$ \\
$272-$ \\
172
\end{tabular} & $\begin{array}{l}40 R \\
60\end{array}$ & 3 & 10 & ZO & 1 & & & & & \begin{tabular}{|l|} 
lead \\
shot, \\
flattene \\
d; \\
appro. \\
$0.13 "$ \\
caliber
\end{tabular} & & & & \\
\hline \begin{tabular}{|l|}
$23 S G$ \\
$272-$ \\
172
\end{tabular} & $\begin{array}{l}40 R \\
60\end{array}$ & 3 & 10 & ZO & 1 & & & $\begin{array}{l}\text { brass- } \\
\text { plated } \\
\text { iron } \\
\text { jewelr }\end{array}$ & & & & & & \\
\hline
\end{tabular}




\begin{tabular}{|c|c|c|c|c|c|c|c|c|c|c|c|c|c|c|}
\hline \multicolumn{15}{|c|}{ Archaeological Analysis } \\
\hline $\begin{array}{c}\text { Cata } \\
\text { ogue } \\
\#\end{array}$ & $\begin{array}{c}\text { Uni } \\
t\end{array}$ & $\begin{array}{c}\text { Q } \\
\text { ua } \\
\text { d }\end{array}$ & $\begin{array}{c}\text { Le } \\
\text { ve } \\
\text { I }\end{array}$ & \begin{tabular}{|l}
$\mathrm{Ti}$ \\
$\mathrm{me}$ \\
per \\
iod
\end{tabular} & $\mid \begin{array}{c}\text { Am } \\
\text { oun } \\
t\end{array}$ & $\mid \begin{array}{l}\text { Hous } \\
\text { ehold } \\
\text { Furnis } \\
\text { hings }\end{array}$ & Clothing & \begin{tabular}{|c} 
Perso \\
nal \\
Items
\end{tabular} & $\begin{array}{l}\text { Househ } \\
\text { old } \\
\text { product } \\
\text { ion } \\
\text { activity }\end{array}$ & \begin{tabular}{|c} 
Househ \\
old \\
produc \\
tion-for \\
sale
\end{tabular} & \begin{tabular}{|l} 
Pharma \\
ceutical
\end{tabular} & \begin{tabular}{|l} 
Food \\
stuffs
\end{tabular} & $\begin{array}{c}\text { Alc } \\
\text { oho } \\
\text { I }\end{array}$ & $\begin{array}{l}\text { Ot } \\
\text { her }\end{array}$ \\
\hline & & & & & & & & $\begin{array}{l}\text { y } \\
\text { frag.; } \\
\text { thin } \\
\text { wire } \\
\text { with } \\
\text { circul } \\
\text { ar } \\
\text { loop } \\
\text { in one } \\
\text { end }\end{array}$ & & & & & & \\
\hline $\begin{array}{l}23 S G \\
272- \\
172\end{array}$ & $\begin{array}{l}40 R \\
60\end{array}$ & 3 & 10 & 20 & 4 & & & & & & & & & \\
\hline \begin{tabular}{|l}
$23 S G$ \\
$272-$ \\
173
\end{tabular} & $\begin{array}{l}40 R \\
60\end{array}$ & 3 & 11 & 20 & 3 & & & & & & & & & \\
\hline $\begin{array}{l}23 S G \\
272- \\
173\end{array}$ & $\begin{array}{l}40 R \\
60\end{array}$ & 3 & 11 & 20 & 2 & & & & & & & & & \\
\hline $\begin{array}{l}23 S G \\
272- \\
173\end{array}$ & $\begin{array}{l}40 R \\
60\end{array}$ & 3 & 11 & 20 & 1 & & & & & & $\begin{array}{l}\text { frags, } \\
\text { thin } \\
\text { light } \\
\text { blue } \\
\text { pharma } \\
\text { ceutical } \\
\text { bottle } \\
\text { glass }\end{array}$ & & & \\
\hline $\begin{array}{l}23 S G \\
272- \\
173\end{array}$ & $\begin{array}{l}40 R \\
60\end{array}$ & 3 & 11 & ZO & 2 & & & & & & & & & \\
\hline $\begin{array}{l}23 S G \\
272- \\
173\end{array}$ & $\begin{array}{l}40 R \\
60\end{array}$ & 3 & 11 & ZO & 7 & & & & & & & & & \\
\hline \begin{tabular}{|l}
$23 S G$ \\
$272-$ \\
173
\end{tabular} & $\begin{array}{l}40 R \\
60\end{array}$ & 3 & 11 & 20 & 2 & & & & & & & & & \\
\hline $\begin{array}{l}\text { 23SG } \\
272-\end{array}$ & $\begin{array}{l}40 R \\
60\end{array}$ & 3 & 11 & 20 & 1 & & & & & & & & & \\
\hline
\end{tabular}




\begin{tabular}{|c|c|c|c|c|c|c|c|c|c|c|c|c|c|c|}
\hline \multicolumn{15}{|c|}{ Archaeological Analysis } \\
\hline $\begin{array}{c}\text { Catal } \\
\text { ogue } \\
\#\end{array}$ & $\begin{array}{c}\text { Uni } \\
t\end{array}$ & $\begin{array}{c}\text { Q } \\
\text { ua } \\
\text { d }\end{array}$ & \begin{tabular}{c||} 
Le \\
ve \\
I
\end{tabular} & $\begin{array}{c}\mathrm{Ti} \\
\mathrm{me} \\
\mathrm{per} \\
\mathrm{iod}\end{array}$ & $\mid \begin{array}{c}\text { Am } \\
\text { oun } \\
t\end{array}$ & $\mid \begin{array}{l}\text { Hous } \\
\text { ehold } \\
\text { Furnis } \\
\text { hings }\end{array}$ & Clothing & \begin{tabular}{|c} 
Perso \\
nal \\
Items
\end{tabular} & $\begin{array}{c}\text { Househ } \\
\text { old } \\
\text { product } \\
\text { ion } \\
\text { activity }\end{array}$ & \begin{tabular}{|c|}
$\begin{array}{c}\text { Househ } \\
\text { old } \\
\text { produc } \\
\text { tion-for } \\
\text { sale }\end{array}$ \\
\end{tabular} & \begin{tabular}{|l} 
Pharma \\
ceutical
\end{tabular} & $\begin{array}{l}\text { Food } \\
\text { stuffs }\end{array}$ & $\begin{array}{c}\text { Alc } \\
\text { oho } \\
\text { I }\end{array}$ & $\begin{array}{l}\text { Ot } \\
\text { her }\end{array}$ \\
\hline \multicolumn{15}{|l|}{173} \\
\hline \begin{tabular}{|l|}
$23 S G$ \\
$272-$ \\
173
\end{tabular} & $\begin{array}{l}40 R \\
60\end{array}$ & 3 & 11 & 20 & 1 & & & $\begin{array}{l}\text { black } \\
\text { stone, } \\
\text { settin } \\
\text { g for a } \\
\text { piece } \\
\text { of } \\
\text { jewelr } \\
\text { y }\end{array}$ & & & & & & \\
\hline \begin{tabular}{|l|}
$23 S G$ \\
$272-$ \\
173
\end{tabular} & \begin{tabular}{|l|}
$40 R$ \\
60
\end{tabular} & 3 & 112 & ZO & 1 & & & & & $\begin{array}{l}\text { lead } \\
\text { shot, } \\
\text { flattene } \\
\text { d, } \\
\text { widest } \\
\text { diamet } \\
\text { er is } \\
0.19^{\prime \prime}\end{array}$ & & & & \\
\hline \begin{tabular}{|l|}
$23 S G$ \\
$272-$ \\
173
\end{tabular} & $\begin{array}{l}40 R \\
60\end{array}$ & 3 & 11 & ZO & 1 & & & & & $\begin{array}{l}0.17 " \\
\text { caliber } \\
\text { lead } \\
\text { shot }\end{array}$ & & & & \\
\hline \begin{tabular}{|l|}
$23 S G$ \\
$272-$ \\
173
\end{tabular} & $\begin{array}{l}40 R \\
60\end{array}$ & 3 & 11 & 20 & 1 & & & & & $\begin{array}{l}0.14 " \\
\text { caliber } \\
\text { lead } \\
\text { shot }\end{array}$ & & & & \\
\hline \begin{tabular}{|l|}
$23 S G$ \\
$272-$ \\
173
\end{tabular} & $\begin{array}{l}40 R \\
60\end{array}$ & 3 & 11 & 20 & 3 & & & & & $\begin{array}{l}0.13 " \\
\text { caliber } \\
\text { lead } \\
\text { shot }\end{array}$ & & & & \\
\hline $\begin{array}{l}23 S G \\
272- \\
173\end{array}$ & $\begin{array}{l}40 R \\
60\end{array}$ & 3 & 11 & ZO & 2 & & & & & $\begin{array}{l}0.12 " \\
\text { caliber } \\
\text { lead } \\
\text { shot }\end{array}$ & & & & \\
\hline $\begin{array}{l}23 S G \\
272- \\
173\end{array}$ & $\begin{array}{l}40 R \\
60\end{array}$ & 3 & 11 & ZO & 2 & & & & & $\begin{array}{l}0.11 " \\
\text { caliber } \\
\text { lead } \\
\text { shot }\end{array}$ & & & & \\
\hline $23 S G$ & $40 R$ & 3 & 11 & ZO & 2 & & & & & $0.10^{\prime \prime}$ & & & & \\
\hline
\end{tabular}




\begin{tabular}{|c|c|c|c|c|c|c|c|c|c|c|c|c|c|c|}
\hline \multicolumn{15}{|c|}{ Archaeological Analysis } \\
\hline $\begin{array}{c}\text { Catal } \\
\text { ogue } \\
\#\end{array}$ & $\begin{array}{c}\text { Uni } \\
t\end{array}$ & $\begin{array}{c}\text { Q } \\
\text { ua } \\
d\end{array}$ & \begin{tabular}{c||} 
Le \\
ve \\
I
\end{tabular} & $\begin{array}{c}\mathrm{Ti} \\
\mathrm{me} \\
\mathrm{per} \\
\mathrm{iod}\end{array}$ & $\mid \begin{array}{c}\text { Am } \\
\text { oun } \\
t\end{array}$ & $\mid \begin{array}{l}\text { Hous } \\
\text { ehold } \\
\text { Furnis } \\
\text { hings }\end{array}$ & Clothing & $\mid \begin{array}{c}\text { Perso } \\
\text { nal } \\
\text { Items }\end{array}$ & \begin{tabular}{|} 
Househ \\
old \\
product \\
ion \\
activity
\end{tabular} & \begin{tabular}{|} 
Househ \\
old \\
produc \\
tion-for \\
sale
\end{tabular} & $\begin{array}{l}\text { Pharma } \\
\text { ceutical }\end{array}$ & \begin{tabular}{|l||} 
Food \\
stuffs
\end{tabular} & $\begin{array}{c}\text { Alc } \\
\text { oho } \\
\text { I }\end{array}$ & $\begin{array}{l}\text { Ot } \\
\text { her }\end{array}$ \\
\hline $\begin{array}{l}272- \\
173\end{array}$ & 60 & & & & & & & & & $\begin{array}{l}\text { caliber } \\
\text { lead } \\
\text { shot }\end{array}$ & & & & \\
\hline \begin{tabular}{|l}
$23 S G$ \\
$272-$ \\
173
\end{tabular} & $\begin{array}{l}40 R \\
60\end{array}$ & 3 & 112 & ZO & 1 & & & & & $\begin{array}{l}0.07 " \\
\text { caliber } \\
\text { lead } \\
\text { shot }\end{array}$ & & & & \\
\hline \begin{tabular}{|l}
$23 S G$ \\
$272-$ \\
173
\end{tabular} & $\begin{array}{l}40 R \\
60\end{array}$ & 3 & 112 & ZO & 1 & & & & & $\begin{array}{l}0.06 " \\
\text { caliber } \\
\text { lead } \\
\text { shot }\end{array}$ & & & & \\
\hline $\begin{array}{l}23 S G \\
272- \\
173\end{array}$ & $\begin{array}{l}40 R \\
60\end{array}$ & 3 & 11 & ZO & 1 & & & & & $\begin{array}{l}\text { lead } \\
\text { shot, } \\
\text { flattene } \\
\text { d; } 0.15 " \\
\text { caliber } \\
\text { at } \\
\text { widest }\end{array}$ & & & & \\
\hline \begin{tabular}{|l|}
$23 S G$ \\
$272-$ \\
173
\end{tabular} & $\begin{array}{l}40 R \\
60\end{array}$ & 3 & 112 & $\mathrm{ZO}$ & 1 & & & & & $\begin{array}{l}\text { lead } \\
\text { shot, } \\
\text { flattene } \\
\text { d, } 0.07 " \\
\text { in } \\
\text { diamet } \\
\text { er }\end{array}$ & & & & \\
\hline \begin{tabular}{|l|}
$23 S G$ \\
$272-$ \\
173
\end{tabular} & $\begin{array}{l}40 R \\
60\end{array}$ & 3 & 11 & 20 & 1 & & $\begin{array}{l}\text { brass- } \\
\text { plated iron } \\
\text { straight } \\
\text { pin frag }\end{array}$ & & & & & & & \\
\hline \begin{tabular}{|l|}
$23 S G$ \\
$272-$ \\
173
\end{tabular} & $\begin{array}{l}40 R \\
60\end{array}$ & 3 & 11 & 20 & 1 & & $\begin{array}{l}\text { brass- } \\
\text { plated iron } \\
\text { bangle/tin } \\
\text { king cone } \\
\text { frag }\end{array}$ & & & & & & & \\
\hline \begin{tabular}{|l|}
$23 S G$ \\
$272-$ \\
173
\end{tabular} & $\begin{array}{l}40 R \\
60\end{array}$ & 3 & 112 & ZO & 2 & & $\begin{array}{l}\text { frags } \\
\text { brass- } \\
\text { plated iron } \\
\text { eyelet }\end{array}$ & & & & & & & \\
\hline
\end{tabular}




\begin{tabular}{|c|c|c|c|c|c|c|c|c|c|c|c|c|c|c|}
\hline \multicolumn{15}{|c|}{ Archaeological Analysis } \\
\hline $\begin{array}{c}\text { Catal } \\
\text { ogue } \\
\#\end{array}$ & Uni & $\mid \begin{array}{c}Q \\
\text { ua } \\
\text { d }\end{array}$ & \begin{tabular}{c||} 
Le \\
ve \\
I
\end{tabular} & $\begin{array}{l}\mathrm{Ti} \\
\mathrm{me} \\
\mathrm{per} \\
\text { iod }\end{array}$ & $\mid \begin{array}{c}\text { Am } \\
\text { oun } \\
t\end{array}$ & $\begin{array}{l}\text { Hous } \\
\text { ehold } \\
\text { Furnis } \\
\text { hings }\end{array}$ & Clothing & $\mid \begin{array}{c}\text { Perso } \\
\text { nal } \\
\text { Items }\end{array}$ & \begin{tabular}{|c|} 
Househ \\
old \\
product \\
ion \\
activity
\end{tabular} & \begin{tabular}{|c} 
Househ \\
old \\
produc \\
tion-for \\
sale
\end{tabular} & $\begin{array}{l}\text { Pharma } \\
\text { ceutical }\end{array}$ & Food & $\mid \begin{array}{c}\text { Alc } \\
\text { oho } \\
\text { I }\end{array}$ & $\begin{array}{c}\text { Ot } \\
\text { her }\end{array}$ \\
\hline & & & & & & & from shoe & & & & & & & \\
\hline \begin{tabular}{|l|}
$23 S G$ \\
$272-$ \\
173
\end{tabular} & $\begin{array}{l}40 R \\
60\end{array}$ & 3 & 11 & ZO & 2 & & & & & & & & & \\
\hline \begin{tabular}{|l|}
$23 S G$ \\
$272-$ \\
173
\end{tabular} & $\begin{array}{l}40 R \\
60\end{array}$ & 3 & 11 & 20 & 2 & & & & & \begin{tabular}{|l} 
frags \\
molten \\
lead \\
waste
\end{tabular} & & & & \\
\hline \begin{tabular}{|l|}
$23 S G$ \\
$272-$ \\
173
\end{tabular} & $\begin{array}{l}40 R \\
60\end{array}$ & 3 & 11 & ZO & 1 & & & & & $\begin{array}{l}\text { lead } \\
\text { trailer } \\
\text { from } \\
\text { shot } \\
\text { manufa } \\
\text { cture }\end{array}$ & & & & \\
\hline \begin{tabular}{|l|}
$23 S G$ \\
$272-$ \\
173
\end{tabular} & $\begin{array}{l}40 R \\
60\end{array}$ & 3 & 11 & ZO & 29 & & & & & $\begin{array}{l}\text { frags } \\
\text { sheet } \\
\text { lead } \\
\text { scrap }\end{array}$ & & & & \\
\hline $\begin{array}{l}23 S G \\
272- \\
173\end{array}$ & $\begin{array}{l}40 R \\
60\end{array}$ & 3 & 11 & ZO & 3 & & & & & & & & & \\
\hline \begin{tabular}{|l|}
$23 S G$ \\
$272-$ \\
173
\end{tabular} & $\begin{array}{l}40 R \\
60\end{array}$ & 3 & 11 & ZO & 1 & & & & \begin{tabular}{|l|} 
frag \\
tumble \\
d glass, \\
light \\
blue- \\
green \\
window \\
glass
\end{tabular} & & & & & \\
\hline $\begin{array}{l}23 S G \\
272- \\
173\end{array}$ & $\begin{array}{l}40 R \\
60\end{array}$ & 3 & 11 & ZO & 2 & & & & $\begin{array}{l}\text { tumble } \\
\text { d } \\
\text { stones }\end{array}$ & & & & & \\
\hline \begin{tabular}{|l|}
$23 S G$ \\
$272-$ \\
174
\end{tabular} & $\begin{array}{l}40 R \\
60\end{array}$ & 3 & 12 & 20 & 1 & & & & & & & & & \\
\hline $\begin{array}{l}23 S G \\
272- \\
174\end{array}$ & $\begin{array}{l}40 R \\
60\end{array}$ & 3 & 12 & ZO & 7 & & & & & & & & & \\
\hline
\end{tabular}




\begin{tabular}{|c|c|c|c|c|c|c|c|c|c|c|c|c|c|c|}
\hline \multicolumn{15}{|c|}{ Archaeological Analysis } \\
\hline $\begin{array}{c}\text { Catal } \\
\text { ogue } \\
\#\end{array}$ & $\begin{array}{c}\text { Uni } \\
t\end{array}$ & $\begin{array}{c}Q \\
\text { ua } \\
d\end{array}$ & \begin{tabular}{c||} 
Le \\
ve \\
I
\end{tabular} & $\begin{array}{l}\text { Ti } \\
\text { me } \\
\text { per } \\
\text { iod }\end{array}$ & $\mid \begin{array}{c}\text { Am } \\
\text { oun } \\
t\end{array}$ & $\begin{array}{l}\text { Hous } \\
\text { ehold } \\
\text { Furnis } \\
\text { hings }\end{array}$ & Clothing & $\mid \begin{array}{c}\text { Perso } \\
\text { nal } \\
\text { Items }\end{array}$ & \begin{tabular}{|} 
Househ \\
old \\
product \\
ion \\
activity
\end{tabular} & \begin{tabular}{||c||} 
Househ \\
old \\
produc \\
tion-for \\
sale
\end{tabular} & \begin{tabular}{|l||} 
Pharma \\
ceutical
\end{tabular} & $\left(\begin{array}{l}\text { Food } \\
\text { stuffs }\end{array}\right.$ & $\mid \begin{array}{c}\text { Alc } \\
\text { oho } \\
\text { I }\end{array}$ & $\begin{array}{c}\text { Ot } \\
\text { her }\end{array}$ \\
\hline \begin{tabular}{|l|}
$23 S G$ \\
$272-$ \\
174
\end{tabular} & $\begin{array}{l}40 R \\
60\end{array}$ & 3 & 12 & $\mathrm{ZO}$ & 1 & & & & & \begin{tabular}{|l|} 
brass \\
percuss \\
ion cap, \\
fired
\end{tabular} & & & & \\
\hline \begin{tabular}{|l|}
$23 S G$ \\
$272-$ \\
174
\end{tabular} & $\begin{array}{l}40 R \\
60\end{array}$ & 3 & 12 & ZO & 1 & & & & & $\begin{array}{l}0.22 " \\
\text { caliber } \\
\text { lead } \\
\text { shot }\end{array}$ & & & & \\
\hline \begin{tabular}{|l|}
$23 S G$ \\
$272-$ \\
174
\end{tabular} & $\begin{array}{l}40 R \\
60\end{array}$ & 3 & 12 & ZO & 1 & & & & & \begin{tabular}{|l|}
$0.17 " c a$ \\
liber \\
lead \\
shot
\end{tabular} & & & & \\
\hline $\begin{array}{l}23 S G \\
272- \\
174\end{array}$ & $\begin{array}{l}40 R \\
60\end{array}$ & 3 & 12 & ZO & 1 & & & & & $\begin{array}{l}0.16 " c a \\
\text { liber } \\
\text { lead } \\
\text { shot }\end{array}$ & & & & \\
\hline \begin{tabular}{|l|}
$23 S G$ \\
$272-$ \\
174
\end{tabular} & $\begin{array}{l}40 R \\
60\end{array}$ & 3 & 12 & $\mathrm{ZO}$ & 4 & & & & & $\begin{array}{l}0.14 " \\
\text { caliber } \\
\text { lead } \\
\text { shot }\end{array}$ & & & & \\
\hline $\begin{array}{l}23 S G \\
272- \\
174\end{array}$ & $\begin{array}{l}40 R \\
60\end{array}$ & 3 & 12 & ZO & 1 & & & & & $\begin{array}{l}0.13 " \\
\text { caliber } \\
\text { lead } \\
\text { shot }\end{array}$ & & & & \\
\hline \begin{tabular}{|l|}
$23 S G$ \\
$272-$ \\
174
\end{tabular} & $\begin{array}{l}40 R \\
60\end{array}$ & 3 & 12 & ZO & 3 & & & & & $\begin{array}{l}0.12 " \\
\text { caliber } \\
\text { lead } \\
\text { shot }\end{array}$ & & & & \\
\hline \begin{tabular}{|l|}
$23 S G$ \\
$272-$ \\
174
\end{tabular} & $\begin{array}{l}40 R \\
60\end{array}$ & 3 & 12 & $\mathrm{ZO}$ & 1 & & & & & $\begin{array}{l}0.11 " \\
\text { caliber } \\
\text { lead } \\
\text { shot }\end{array}$ & & & & \\
\hline \begin{tabular}{|l|}
$23 S G$ \\
$272-$ \\
174
\end{tabular} & $\begin{array}{l}40 R \\
60\end{array}$ & 3 & 12 & ZO & 2 & & & & & $\begin{array}{l}0.10 " \\
\text { caliber } \\
\text { lead } \\
\text { shot }\end{array}$ & & & & \\
\hline \begin{tabular}{|l|}
$23 S S G$ \\
$272-$
\end{tabular} & $\begin{array}{l}40 R \\
60\end{array}$ & 3 & 12 & ZO & 1 & & & & & $\begin{array}{l}0.09 " \\
\text { caliber }\end{array}$ & & & & \\
\hline
\end{tabular}




\begin{tabular}{|c|c|c|c|c|c|c|c|c|c|c|c|c|c|c|}
\hline \multicolumn{15}{|c|}{ Archaeological Analysis } \\
\hline $\mid \begin{array}{c}\text { Catal } \\
\text { ogue } \\
\#\end{array}$ & $\begin{array}{c}\text { Uni } \\
t\end{array}$ & $\begin{array}{c}\text { Q } \\
\text { ua } \\
\text { d }\end{array}$ & $\begin{array}{c}\text { Le } \\
\text { ve } \\
\text { I }\end{array}$ & $\begin{array}{l}\text { Ti } \\
\text { me } \\
\text { per } \\
\text { iod }\end{array}$ & $\mid \begin{array}{c}\text { Am } \\
\text { oun } \\
t\end{array}$ & $\mid \begin{array}{l}\text { Hous } \\
\text { ehold } \\
\text { Furnis } \\
\text { hings }\end{array}$ & Clothing & $\begin{array}{c}\text { Perso } \\
\text { nal } \\
\text { Items }\end{array}$ & $\begin{array}{l}\text { Househ } \\
\text { old } \\
\text { product } \\
\text { ion } \\
\text { activity }\end{array}$ & \begin{tabular}{|c} 
Househ \\
old \\
produc \\
tion-for \\
sale
\end{tabular} & \begin{tabular}{|l} 
Pharma \\
ceutical
\end{tabular} & $\begin{array}{l}\text { Food } \\
\text { stuffs }\end{array}$ & $\begin{array}{c}\text { Alc } \\
\text { oho } \\
\text { I }\end{array}$ & $\begin{array}{l}\text { Ot } \\
\text { her }\end{array}$ \\
\hline 174 & & & & & & & & & & $\begin{array}{l}\text { lead } \\
\text { shot }\end{array}$ & & & & \\
\hline $\begin{array}{l}23 S G \\
272- \\
174\end{array}$ & $\begin{array}{l}40 R \\
60\end{array}$ & 3 & 12 & 20 & 1 & & & & & $\begin{array}{l}\text { lead } \\
\text { shot, } \\
\text { flattene } \\
\text { d on } \\
\text { bottom } \\
\text { 0.12" } \\
\text { at } \\
\text { widest }\end{array}$ & & & & \\
\hline \begin{tabular}{|l|}
$23 S G$ \\
$272-$ \\
174 \\
\end{tabular} & $\begin{array}{l}40 R \\
60\end{array}$ & 3 & 12 & 20 & 2 & & & & $\begin{array}{l}\text { gunflint } \\
\text { flakes }\end{array}$ & & & & & \\
\hline \begin{tabular}{|l|}
$23 S G$ \\
$272-$ \\
174 \\
\end{tabular} & $\begin{array}{l}40 R \\
60\end{array}$ & 3 & 12 & 20 & 1 & & & & & & & & & \\
\hline \begin{tabular}{|l|}
$23 S G$ \\
$272-$ \\
174 \\
\end{tabular} & $\begin{array}{l}40 R \\
60\end{array}$ & 3 & 12 & 20 & 1 & & & & & $\begin{array}{l}\text { frag } \\
\text { lead } \\
\text { waste }\end{array}$ & & & & \\
\hline \begin{tabular}{|l|}
$23 S G$ \\
$272-$ \\
174 \\
\end{tabular} & $\begin{array}{l}40 R \\
60\end{array}$ & 3 & 12 & 20 & 1 & & & & $\begin{array}{l}\text { "tumble } \\
\text { d" } \\
\text { stone }\end{array}$ & & & & & \\
\hline \begin{tabular}{|l|}
$23 S G$ \\
$272-$ \\
175
\end{tabular} & $\begin{array}{l}40 R \\
60\end{array}$ & 3 & 13 & 20 & 1 & & & & & & & & & \\
\hline $\begin{array}{l}23 S G \\
272- \\
175\end{array}$ & $\begin{array}{l}40 R \\
60\end{array}$ & 3 & 13 & 20 & 1 & & & & & & & & & \\
\hline \begin{tabular}{|l|}
$23 S G$ \\
$272-$ \\
175
\end{tabular} & $\begin{array}{l}40 R \\
60\end{array}$ & 3 & 13 & 20 & 5 & & & & & & & & & \\
\hline \begin{tabular}{|l|}
$23 S G$ \\
$272-$ \\
175
\end{tabular} & $\begin{array}{l}40 R \\
60\end{array}$ & 3 & 13 & 20 & 1 & & & & & $\begin{array}{l}0.18 " \\
\text { caliber } \\
\text { lead } \\
\text { shot }\end{array}$ & & & & \\
\hline \begin{tabular}{|l|}
$23 S G$ \\
$272-$ \\
175
\end{tabular} & $\begin{array}{l}40 R \\
60\end{array}$ & 3 & 13 & 20 & 3 & & & & & $\begin{array}{l}0.13 " \\
\text { caliber } \\
\text { lead }\end{array}$ & & & & \\
\hline
\end{tabular}




\begin{tabular}{|c|c|c|c|c|c|c|c|c|c|c|c|c|c|c|}
\hline \multicolumn{15}{|c|}{ Archaeological Analysis } \\
\hline $\mid \begin{array}{c}\text { Catal } \\
\text { ogue } \\
\#\end{array}$ & Uni & $\mid \begin{array}{c}Q \\
\text { ua } \\
\text { d }\end{array}$ & $\mid$\begin{tabular}{c||} 
Le \\
ve \\
I
\end{tabular} & $\begin{array}{c}\mathrm{Ti} \\
\mathrm{me} \\
\mathrm{per} \\
\mathrm{iod}\end{array}$ & $\mid \begin{array}{c}\text { Am } \\
\text { oun } \\
t\end{array}$ & $\mid \begin{array}{l}\text { Hous } \\
\text { ehold } \\
\text { Furnis } \\
\text { hings }\end{array}$ & Clothing & $\begin{array}{c}\text { Perso } \\
\text { nal } \\
\text { Items }\end{array}$ & \begin{tabular}{|} 
Househ \\
old \\
product \\
ion \\
activity
\end{tabular} & \begin{tabular}{|} 
Househ \\
old \\
produc \\
tion-for \\
sale
\end{tabular} & \begin{tabular}{|l} 
Pharma \\
ceutical
\end{tabular} & $\begin{array}{l}\text { Food } \\
\text { stuffs }\end{array}$ & $\begin{array}{c}\text { Alc } \\
\text { oho } \\
\text { I }\end{array}$ & $\begin{array}{l}\text { Ot } \\
\text { her }\end{array}$ \\
\hline & & & & & & & & & & shot & & & & \\
\hline \begin{tabular}{|l|}
$23 S G$ \\
$272-$ \\
175
\end{tabular} & $\begin{array}{l}40 R \\
60\end{array}$ & 3 & 13 & $\mathrm{ZO}$ & 1 & & & & & $\begin{array}{l}0.12 " \\
\text { caliber } \\
\text { lead } \\
\text { shot }\end{array}$ & & & & \\
\hline $\begin{array}{l}23 S G \\
272- \\
175\end{array}$ & $\begin{array}{l}40 R \\
60\end{array}$ & 3 & 13 & ZO & 1 & & & & \begin{tabular}{|l|} 
gunflint \\
flake; \\
honey- \\
colored
\end{tabular} & & & & & \\
\hline \begin{tabular}{|l|}
$23 S G$ \\
$272-$ \\
175
\end{tabular} & $\begin{array}{l}40 R \\
60\end{array}$ & 3 & 13 & 20 & 1 & & $\begin{array}{l}\text { brass- } \\
\text { plated iron } \\
\text { straight } \\
\text { pin frag }\end{array}$ & & & & & & & \\
\hline \begin{tabular}{|l|}
$23 S G$ \\
$272-$ \\
175 \\
\end{tabular} & $\begin{array}{l}40 R \\
60\end{array}$ & 3 & 13 & ZO & 2 & & & & & & & & & \\
\hline \begin{tabular}{|l|}
$23 S G$ \\
$272-$ \\
175
\end{tabular} & $\begin{array}{l}40 R \\
60\end{array}$ & 3 & 13 & 20 & 3 & & & & & $\begin{array}{l}\text { frags } \\
\text { sheet } \\
\text { lead } \\
\text { waste/s } \\
\text { crap }\end{array}$ & & & & \\
\hline \begin{tabular}{|l|}
$23 S G$ \\
$272-$ \\
175
\end{tabular} & $\begin{array}{l}40 R \\
60\end{array}$ & 3 & 13 & ZO & 1 & & & & & $\begin{array}{l}\text { lead } \\
\text { object, } \\
\text { possibl } \\
\text { y } \\
\text { related } \\
\text { to a } \\
\text { cap- } \\
\text { and- } \\
\text { ball gun }\end{array}$ & & & & \\
\hline \begin{tabular}{|l|}
$23 S G$ \\
$272-$ \\
176 \\
\end{tabular} & $\begin{array}{l}40 \mathrm{R} \\
60\end{array}$ & 3 & 14 & 20 & 1 & & & & & & & & & \\
\hline \begin{tabular}{|l|}
$23 S G$ \\
$272-$ \\
176 \\
\end{tabular} & $\begin{array}{l}40 \mathrm{R} \\
60\end{array}$ & 3 & 14 & 20 & 1 & & & & & & & & & \\
\hline $\begin{array}{l}23 S G \\
272-\end{array}$ & $\begin{array}{l}40 \mathrm{R} \\
60\end{array}$ & 3 & 14 & ZO & 1 & & & & & & & & & \\
\hline
\end{tabular}




\begin{tabular}{|c|c|c|c|c|c|c|c|c|c|c|c|c|c|c|}
\hline \multicolumn{15}{|c|}{ Archaeological Analysis } \\
\hline $\begin{array}{c}\text { Cata } \\
\text { ogue } \\
\#\end{array}$ & Uni & $\begin{array}{c}\text { Q } \\
\text { ua } \\
\text { d }\end{array}$ & $\begin{array}{c}\text { Le } \\
\text { ve } \\
\text { I }\end{array}$ & $\begin{array}{l}\text { Ti } \\
\text { me } \\
\text { per } \\
\text { iod }\end{array}$ & $\mid \begin{array}{c}\text { Am } \\
\text { oun } \\
t\end{array}$ & $\mid \begin{array}{l}\text { Hous } \\
\text { ehold } \\
\text { Furnis } \\
\text { hings }\end{array}$ & Clothing & $\mid \begin{array}{c}\text { Perso } \\
\text { nal } \\
\text { Items }\end{array}$ & $\begin{array}{c}\text { Househ } \\
\text { old } \\
\text { product } \\
\text { ion } \\
\text { activity }\end{array}$ & \begin{tabular}{|c|}
$\begin{array}{c}\text { Househ } \\
\text { old } \\
\text { produc } \\
\text { tion-for } \\
\text { sale }\end{array}$ \\
\end{tabular} & \begin{tabular}{|l} 
Pharma \\
ceutical
\end{tabular} & \begin{tabular}{||l} 
Food \\
stuffs
\end{tabular} & $\begin{array}{c}\text { Alc } \\
\text { oho } \\
\text { I }\end{array}$ & $\begin{array}{l}\text { Ot } \\
\text { her }\end{array}$ \\
\hline 176 & & & & & & & & & & & & & & \\
\hline \begin{tabular}{|l}
$23 S G$ \\
$272-$ \\
176
\end{tabular} & \begin{tabular}{|l|}
$40 R$ \\
60
\end{tabular} & 3 & 14 & 20 & 3 & & & & & & & & & \\
\hline $\begin{array}{l}23 S G \\
272- \\
176\end{array}$ & $\begin{array}{l}40 R \\
60\end{array}$ & 3 & 14 & 20 & 1 & & & & & & & & & \\
\hline \begin{tabular}{|l}
$23 S G$ \\
$272-$ \\
176
\end{tabular} & \begin{tabular}{|l|}
$40 R$ \\
60
\end{tabular} & 3 & 14 & 20 & 12 & & & & & & & & & \\
\hline \begin{tabular}{|l}
$23 S G$ \\
$272-$ \\
176
\end{tabular} & $\begin{array}{l}40 R \\
60\end{array}$ & 3 & 14 & 20 & 2 & & & & & & & & & \\
\hline $\begin{array}{l}23 S G \\
272- \\
176\end{array}$ & $\begin{array}{l}40 \mathrm{R} \\
60\end{array}$ & 3 & 14 & 20 & 1 & & & $\begin{array}{l}\text { jewelr } \\
\text { y frag, } \\
\text { mediu } \\
\text { m } \\
\text { blue } \\
\text { glass } \\
\text { frag, } \\
\text { possib } \\
\text { ly a } \\
\text { ring } \\
\text { or } \\
\text { earrin } \\
\text { g } \\
\text { settin } \\
\text { g }\end{array}$ & & & & & & \\
\hline $\begin{array}{l}23 S G \\
272- \\
176\end{array}$ & $\begin{array}{l}40 \mathrm{R} \\
60\end{array}$ & 3 & 14 & 20 & 1 & & & $\begin{array}{l}\text { black } \\
\text { opaqu } \\
\text { e } \\
\text { bead }\end{array}$ & & & & & & \\
\hline \begin{tabular}{|l}
$23 S G$ \\
$272-$ \\
176
\end{tabular} & \begin{tabular}{|l|}
$40 R$ \\
60
\end{tabular} & 3 & 14 & 20 & 1 & & & $\begin{array}{l}\text { seed } \\
\text { bead, } \\
\text { turqu } \\
\text { oise }\end{array}$ & & & & & & \\
\hline $\begin{array}{l}23 S G \\
272-\end{array}$ & \begin{tabular}{|l|}
$40 R$ \\
60
\end{tabular} & 3 & 14 & 20 & 1 & & & & & $\begin{array}{l}0.29 " \\
\text { caliber }\end{array}$ & & & & \\
\hline
\end{tabular}




\begin{tabular}{|c|c|c|c|c|c|c|c|c|c|c|c|c|c|c|}
\hline \multicolumn{15}{|c|}{ Archaeological Analysis } \\
\hline $\begin{array}{c}\text { Cata } \\
\text { ogue } \\
\#\end{array}$ & Uni & $\begin{array}{c}\text { Q } \\
\text { ua } \\
\text { d }\end{array}$ & $\begin{array}{c}\text { Le } \\
\text { ve } \\
\text { I }\end{array}$ & $\begin{array}{l}\text { Ti } \\
\text { me } \\
\text { per } \\
\text { iod }\end{array}$ & $\mid \begin{array}{c}\text { Am } \\
\text { oun } \\
t\end{array}$ & $\mid \begin{array}{l}\text { Hous } \\
\text { ehold } \\
\text { Furnis } \\
\text { hings }\end{array}$ & Clothing & \begin{tabular}{|c} 
Perso \\
nal \\
Items
\end{tabular} & $\begin{array}{l}\text { Househ } \\
\text { old } \\
\text { product } \\
\text { ion } \\
\text { activity }\end{array}$ & \begin{tabular}{|c} 
Househ \\
old \\
produc \\
tion-for \\
sale
\end{tabular} & \begin{tabular}{|l} 
Pharma \\
ceutical
\end{tabular} & \begin{tabular}{||l} 
Food \\
stuffs
\end{tabular} & $\begin{array}{c}\text { Alc } \\
\text { oho } \\
\text { I }\end{array}$ & $\begin{array}{l}\text { Ot } \\
\text { her }\end{array}$ \\
\hline 176 & & & & & & & & & & $\begin{array}{l}\text { lead } \\
\text { shot }\end{array}$ & & & & \\
\hline $\begin{array}{l}23 S G \\
272- \\
176\end{array}$ & \begin{tabular}{|l|}
$40 R$ \\
60
\end{tabular} & 3 & 14 & 20 & 1 & & & & & $\begin{array}{l}0.13 " \\
\text { caliber } \\
\text { lead } \\
\text { shot }\end{array}$ & & & & \\
\hline $\begin{array}{l}23 S G \\
272- \\
176\end{array}$ & \begin{tabular}{|l|}
$40 R$ \\
60
\end{tabular} & 3 & 14 & 20 & 1 & & & & & $\begin{array}{l}0.11 " \\
\text { caliber } \\
\text { lead } \\
\text { shot }\end{array}$ & & & & \\
\hline $\begin{array}{l}23 S G \\
272- \\
176\end{array}$ & \begin{tabular}{|l|}
$40 R$ \\
60
\end{tabular} & 3 & 14 & 20 & 1 & & & & & $\begin{array}{l}0.10 " \\
\text { caliber } \\
\text { lead } \\
\text { shot }\end{array}$ & & & & \\
\hline $\begin{array}{l}23 S G \\
272- \\
176\end{array}$ & \begin{tabular}{|l|}
$40 R$ \\
60
\end{tabular} & 3 & 14 & 20 & 1 & & & & $\begin{array}{l}\text { gunflint } \\
\text { flake, } \\
\text { honey- } \\
\text { colored }\end{array}$ & & & & & \\
\hline $\begin{array}{l}23 S G \\
272- \\
176\end{array}$ & $\begin{array}{l}40 R \\
60\end{array}$ & 3 & 14 & 20 & 1 & & $\begin{array}{l}\text { brass } \\
\text { straight } \\
\text { pin }\end{array}$ & & & & & & & \\
\hline $\begin{array}{l}23 S G \\
272- \\
176\end{array}$ & $\begin{array}{l}40 R \\
60\end{array}$ & 3 & 14 & 20 & 1 & & $\begin{array}{l}\text { frag, } \\
\text { brass- } \\
\text { plated } \\
\text { iron, looks } \\
\text { like base } \\
\text { of eye } \\
\text { from a } \\
\text { hook-and- } \\
\text { eye }\end{array}$ & & & & & & & \\
\hline $\begin{array}{l}23 S G \\
272- \\
176\end{array}$ & \begin{tabular}{|l|}
$40 R$ \\
60
\end{tabular} & 3 & 14 & 20 & 1 & & & & & & & & & \\
\hline $\begin{array}{l}23 S G \\
272- \\
176\end{array}$ & $\begin{array}{l}40 R \\
60\end{array}$ & 3 & 14 & 20 & 1 & & & & & $\begin{array}{l}\text { frag, } \\
\text { lead } \\
\text { trailer, } \\
\text { from } \\
\text { shot }\end{array}$ & & & & \\
\hline
\end{tabular}




\begin{tabular}{|c|c|c|c|c|c|c|c|c|c|c|c|c|c|c|}
\hline \multicolumn{15}{|c|}{ Archaeological Analysis } \\
\hline $\begin{array}{c}\text { Catal } \\
\text { ogue } \\
\#\end{array}$ & Uni & $\begin{array}{c}\text { Q } \\
\text { ua } \\
\text { d }\end{array}$ & \begin{tabular}{c||} 
Le \\
ve \\
I
\end{tabular} & $\begin{array}{l}\mathrm{Ti} \\
\mathrm{me} \\
\mathrm{per} \\
\mathrm{iod}\end{array}$ & $\mid \begin{array}{c}\text { Am } \\
\text { oun } \\
t\end{array}$ & \begin{tabular}{|l} 
Hous \\
ehold \\
Furnis \\
hings
\end{tabular} & Clothing & \begin{tabular}{|c} 
Perso \\
nal \\
Items
\end{tabular} & \begin{tabular}{|c|}
$\begin{array}{c}\text { Househ } \\
\text { old } \\
\text { product } \\
\text { ion } \\
\text { activity }\end{array}$ \\
\end{tabular} & \begin{tabular}{|c} 
Househ \\
old \\
produc \\
tion-for \\
sale
\end{tabular} & \begin{tabular}{|l} 
Pharma \\
ceutical
\end{tabular} & $\begin{array}{l}\text { Food } \\
\text { stuffs }\end{array}$ & $\mid \begin{array}{c}\text { Alc } \\
\text { oho } \\
\text { I }\end{array}$ & $\begin{array}{l}\text { Ot } \\
\text { her }\end{array}$ \\
\hline & & & & & & & & & & $\begin{array}{l}\text { manufa } \\
\text { cture }\end{array}$ & & & & \\
\hline $\begin{array}{l}23 S G \\
272- \\
176\end{array}$ & $\begin{array}{l}40 R \\
60\end{array}$ & 3 & 14 & ZO & 3 & & & & & $\begin{array}{l}\text { frags, } \\
\text { sheet } \\
\text { lead } \\
\text { waste }\end{array}$ & & & & \\
\hline \begin{tabular}{|l|}
$23 S G$ \\
$272-$ \\
176
\end{tabular} & $\begin{array}{l}40 R \\
60\end{array}$ & 3 & 14 & 20 & 2 & & & & & $\begin{array}{l}\text { frags, } \\
\text { lead } \\
\text { waste }\end{array}$ & & & & \\
\hline \begin{tabular}{|l|}
$23 S G$ \\
$272-$ \\
178 \\
\end{tabular} & $\begin{array}{l}40 \mathrm{R} \\
60\end{array}$ & 3 & 15 & ZO & 2 & & & & & & & & & \\
\hline $\begin{array}{l}23 S G \\
272- \\
179\end{array}$ & $\begin{array}{l}40 R \\
60\end{array}$ & 3 & 15 & ZO & 1 & & & & & & & & & \\
\hline \begin{tabular}{|l|}
$23 S G$ \\
$272-$ \\
179 \\
\end{tabular} & $\begin{array}{l}40 R \\
60\end{array}$ & 3 & 15 & 20 & 2 & & & & & & & & & \\
\hline \begin{tabular}{|l|}
$23 S G$ \\
$272-$ \\
179 \\
\end{tabular} & $\begin{array}{l}40 R \\
60\end{array}$ & 3 & 15 & ZO & 1 & & & & & & & & & \\
\hline \begin{tabular}{|l|}
$23 S G$ \\
$272-$ \\
179 \\
\end{tabular} & $\begin{array}{l}40 R \\
60\end{array}$ & 3 & 15 & ZO & 6 & & & & & & & & & \\
\hline \begin{tabular}{|l|}
$23 S G$ \\
$272-$ \\
179
\end{tabular} & $\begin{array}{l}40 R \\
60\end{array}$ & 3 & 15 & 20 & 1 & & & & & $\begin{array}{l}0.18 " \\
\text { caliber } \\
\text { lead } \\
\text { shot }\end{array}$ & & & & \\
\hline $\begin{array}{l}23 S G \\
272- \\
179\end{array}$ & $\begin{array}{l}40 R \\
60\end{array}$ & 3 & 15 & ZO & 1 & & & & & $\begin{array}{l}0.14 " \\
\text { caliber } \\
\text { lead } \\
\text { shot }\end{array}$ & & & & \\
\hline $\begin{array}{l}23 S G \\
272- \\
179\end{array}$ & $\begin{array}{l}40 R \\
60\end{array}$ & 3 & 15 & ZO & 1 & & & & & $\begin{array}{l}0.12 " \\
\text { caliber } \\
\text { lead } \\
\text { shot }\end{array}$ & & & & \\
\hline $\begin{array}{l}23 S G \\
272-\end{array}$ & $\begin{array}{l}40 R \\
60\end{array}$ & 3 & 15 & ZO & 1 & & & & & $\begin{array}{l}0.09 " \\
\text { caliber }\end{array}$ & & & & \\
\hline
\end{tabular}




\begin{tabular}{|c|c|c|c|c|c|c|c|c|c|c|c|c|c|c|}
\hline \multicolumn{15}{|c|}{ Archaeological Analysis } \\
\hline $\mid \begin{array}{c}\text { Catal } \\
\text { ogue } \\
\#\end{array}$ & Uni & $\mid \begin{array}{c}Q \\
\text { ua } \\
\text { d }\end{array}$ & $\mid$\begin{tabular}{c||} 
Le \\
ve \\
I
\end{tabular} & $\begin{array}{c}\mathrm{Ti} \\
\mathrm{me} \\
\mathrm{per} \\
\mathrm{iod}\end{array}$ & $\mid \begin{array}{c}\text { Am } \\
\text { oun } \\
t\end{array}$ & $\mid \begin{array}{l}\text { Hous } \\
\text { ehold } \\
\text { Furnis } \\
\text { hings }\end{array}$ & Clothing & \begin{tabular}{|c} 
Perso \\
nal \\
Items
\end{tabular} & \begin{tabular}{|} 
Househ \\
old \\
product \\
ion \\
activity
\end{tabular} & \begin{tabular}{|c} 
Househ \\
old \\
produc \\
tion-for \\
sale
\end{tabular} & \begin{tabular}{|l} 
Pharma \\
ceutical
\end{tabular} & $\begin{array}{l}\text { Food } \\
\text { stuffs }\end{array}$ & $\begin{array}{c}\text { Alc } \\
\text { oho } \\
\text { I }\end{array}$ & $\begin{array}{l}\text { Ot } \\
\text { her }\end{array}$ \\
\hline 179 & & & & & & & & & & $\begin{array}{l}\text { lead } \\
\text { shot }\end{array}$ & & & & \\
\hline $\begin{array}{l}23 S G \\
272- \\
179\end{array}$ & $\begin{array}{l}40 R \\
60\end{array}$ & 3 & 15 & ZO & 1 & & & & & $\begin{array}{l}\text { lead } \\
\text { shot, } \\
\text { flattene } \\
\text { d; } 0.11 " \\
\text { at } \\
\text { widest }\end{array}$ & & & & \\
\hline $\begin{array}{l}23 S G \\
272- \\
179\end{array}$ & $\begin{array}{l}40 R \\
60\end{array}$ & 3 & 15 & ZO & 1 & & & & $\begin{array}{l}\text { gunflint } \\
\text { flake; } \\
\text { honey- } \\
\text { colored }\end{array}$ & & & & & \\
\hline $\begin{array}{l}23 S G \\
272- \\
179\end{array}$ & $\begin{array}{l}40 R \\
60\end{array}$ & 3 & 15 & ZO & 1 & & $\begin{array}{l}\text { brass- } \\
\text { plated iron } \\
\text { straight } \\
\text { pin }\end{array}$ & & & & & & & \\
\hline \begin{tabular}{|l|}
$23 S G$ \\
$272-$ \\
179
\end{tabular} & \begin{tabular}{|l|}
$40 R$ \\
60
\end{tabular} & 3 & 15 & ZO & 1 & & $\begin{array}{l}\text { brass- } \\
\text { plated iron } \\
\text { bangle/tub } \\
\text { e/tinkling } \\
\text { cone }\end{array}$ & & & & & & & \\
\hline $\begin{array}{l}23 S G \\
272- \\
179\end{array}$ & $\begin{array}{l}40 R \\
60\end{array}$ & 3 & 15 & ZO & 1 & & & & & $\begin{array}{l}\text { brass } \\
\text { frag, } \\
\text { possibl } \\
\text { y part } \\
\text { of } \\
\text { percuss } \\
\text { ion cap }\end{array}$ & & & & \\
\hline $\begin{array}{l}23 S G \\
272- \\
179\end{array}$ & $\begin{array}{l}40 R \\
60\end{array}$ & 3 & 15 & ZO & 1 & & & $\begin{array}{l}\text { iron } \\
\text { buckl } \\
\text { e; } \\
\text { proba } \\
\text { bly } \\
\text { harne } \\
\text { ss } \\
\text { buckl } \\
\text { e }\end{array}$ & & & & & & \\
\hline $23 S G$ & $40 R$ & 3 & 15 & & 1 & & & & & & & & & \\
\hline
\end{tabular}




\begin{tabular}{|c|c|c|c|c|c|c|c|c|c|c|c|c|c|}
\hline \multicolumn{14}{|c|}{ Archaeological Analysis } \\
\hline $\begin{array}{c}\text { Catal } \\
\text { ogue } \\
\#\end{array}$ & $\begin{array}{c}\text { Uni } \\
t\end{array}$ & $\begin{array}{c}\text { Q } \\
\text { ua } \\
\text { d }\end{array}$ & \begin{tabular}{|c||c} 
Le & Ti \\
ve & me \\
I & per \\
& iod
\end{tabular} & $\left(\begin{array}{c}A m \\
\text { oun } \\
t\end{array}\right.$ & \begin{tabular}{|l|} 
Hous \\
ehold \\
Furnis \\
hings
\end{tabular} & Clothing & $\begin{array}{c}\text { Perso } \\
\text { nal } \\
\text { Items }\end{array}$ & $\begin{array}{c}\text { Househ } \\
\text { old } \\
\text { product } \\
\text { ion } \\
\text { activity }\end{array}$ & \begin{tabular}{|c|} 
Househ \\
old \\
produc \\
tion-for \\
sale
\end{tabular} & \begin{tabular}{|l} 
Pharma \\
ceutical
\end{tabular} & Food & $\mid \begin{array}{c}\text { Alc } \\
\text { oho } \\
\text { I }\end{array}$ & $\begin{array}{l}\text { Ot } \\
\text { her }\end{array}$ \\
\hline \begin{tabular}{|l|}
$272-$ \\
180
\end{tabular} & 60 & & & & & & & & & & & & \\
\hline \begin{tabular}{|l|}
$23 S G$ \\
$272-$ \\
13 \\
\end{tabular} & $\begin{array}{l}110 \\
\text { R30 }\end{array}$ & 2 & \begin{tabular}{l|l}
1 & $Z L$ \\
$O$
\end{tabular} & 1 & & & & & & & & & \\
\hline \begin{tabular}{|l|}
$23 S G$ \\
$272-$ \\
13
\end{tabular} & $\begin{array}{l}110 \\
\text { R30 }\end{array}$ & 2 & \begin{tabular}{l|l}
1 & $Z L$ \\
$O$
\end{tabular} & 1 & & & & & & & & & \\
\hline \begin{tabular}{|l|}
$23 S G$ \\
$272-$ \\
13
\end{tabular} & $\begin{array}{l}110 \\
\text { R30 }\end{array}$ & 2 & \begin{tabular}{l|l}
1 & $Z L$ \\
$O$ &
\end{tabular} & 1 & & & & $\begin{array}{l}\text { brass } \\
\text { eyelet, } \\
\text { possibly } \\
\text { from a } \\
\text { shoe }\end{array}$ & & & & & \\
\hline \begin{tabular}{|l|}
$23 S G$ \\
$272-$ \\
13
\end{tabular} & $\begin{array}{l}110 \\
\text { R30 }\end{array}$ & 2 & \begin{tabular}{l|l}
1 & $\mathrm{ZL}$ \\
$\mathrm{O}$
\end{tabular} & 1 & & & & & $\begin{array}{l}\text { lead } \\
\text { splatter } \\
\text { /sprue, } \\
\text { from } \\
\text { making } \\
\text { shot }\end{array}$ & & & & \\
\hline \begin{tabular}{|l|}
$23 S G$ \\
$272-$ \\
13
\end{tabular} & $\begin{array}{l}110 \\
\text { R30 }\end{array}$ & 2 & \begin{tabular}{l|l}
1 & $Z L$ \\
0
\end{tabular} & 1 & & & & $\begin{array}{l}\text { lead } \\
\text { seal or } \\
\text { button } \\
\text { frag }\end{array}$ & & & & & \\
\hline \multirow[t]{2}{*}{\begin{tabular}{|l|}
$23 S G$ \\
$272-$ \\
13 \\
\end{tabular}} & $\begin{array}{l}110 \\
\text { R30 }\end{array}$ & 2 & \begin{tabular}{l|l}
1 & $\mathrm{ZL}$ \\
$\mathrm{O}$
\end{tabular} & 17 & & & & & & & & & \\
\hline & & & & $\begin{array}{l}232 \\
0\end{array}$ & & & & & & & & & \\
\hline
\end{tabular}

\section{Ceramic analysis}

\begin{tabular}{|c|c|c|c|c|c|c|c|c|c|}
\hline 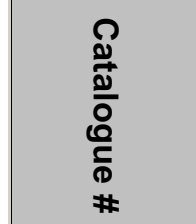 & 돌 & $\begin{array}{l}0 \\
\frac{0}{\Perp 0} \\
0\end{array}$ & $\left|\begin{array}{l}\mathbb{D} \\
\mathbb{D} \\
\mathbb{\Phi}\end{array}\right|$ & $\begin{array}{l}\not 1 \\
\overline{\overline{3}} \\
0 \\
0 \\
0 \\
\frac{0}{0} \\
\overline{0}\end{array}$ & 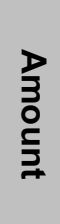 & 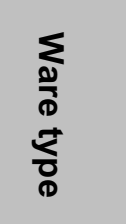 & $\begin{array}{l}\frac{0}{\phi 0} \\
\stackrel{0}{0} \\
\frac{0}{3} \\
\frac{3}{0} \\
\frac{0}{3}\end{array}$ & $\begin{array}{l}\overrightarrow{0} \\
\frac{7}{3} \\
\text { ¿্ঠ }\end{array}$ & 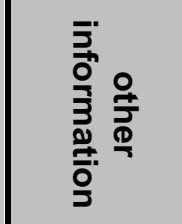 \\
\hline 23SG272-6 & $\begin{array}{l}150 \\
\text { R0 }\end{array}$ & 1 & & $\overline{\mathrm{ZO}}$ & \multicolumn{2}{|c|}{ 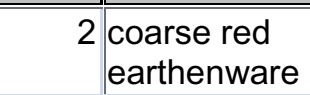 } & & & $\begin{array}{l}\text { caramel- } \\
\text { brown lead }\end{array}$ \\
\hline
\end{tabular}




\begin{tabular}{|c|c|c|c|c|c|c|c|c|c|}
\hline $\begin{array}{l}\text { O } \\
\stackrel{0}{0} \\
\frac{0}{0} \\
\stackrel{0}{0} \\
\frac{1}{0} \\
\# \\
\#\end{array}$ & 돌 & 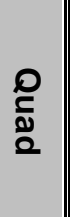 & $\underset{\Phi}{\stackrel{\Phi}{\infty}}$ & \begin{tabular}{l}
-1 \\
$\overline{3}$ \\
$\overline{0}$ \\
0 \\
$\mathbb{0}$ \\
$\frac{0}{0}$ \\
\hdashline
\end{tabular} & 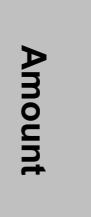 & 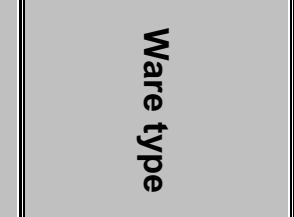 & $\begin{array}{l}\frac{0}{0} \\
\frac{0}{0} \\
\frac{0}{*} \\
\frac{0}{3}\end{array}$ & 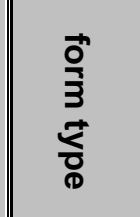 & 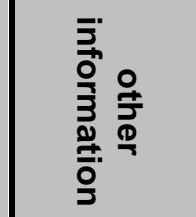 \\
\hline & & & & & & & & & glaze \\
\hline 23SG272-6 & $\begin{array}{l}150 \\
\text { R0 }\end{array}$ & 1 & & $\mathrm{ZO}$ & & 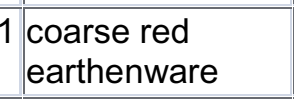 & & & $\begin{array}{l}\text { brown } \\
\text { ?lead glaze }\end{array}$ \\
\hline 23SG272-6 & $\begin{array}{l}150 \\
\text { Ro }\end{array}$ & 1 & & $\mathrm{ZO}$ & & 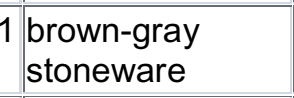 & & & $\begin{array}{l}\text { brown-gray } \\
\text { glaze }\end{array}$ \\
\hline 23SG272-6 & $\begin{array}{l}150 \\
\text { R0 }\end{array}$ & 1 & & $\mathrm{ZO}$ & & 1 pearlware & & $\begin{array}{l}\text { plate/sa } \\
\text { ucer/bo } \\
\text { wl }\end{array}$ & \\
\hline 23SG272-6 & $\begin{array}{l}150 \\
\text { R0 }\end{array}$ & 1 & & $\mathrm{ZO}$ & & $\begin{array}{l}1 \text { pearlware/cream } \\
\text { ware }\end{array}$ & & & \\
\hline 23SG272-6 & $\begin{array}{l}150 \\
\text { R0 }\end{array}$ & 1 & & $\mathrm{ZO}$ & & 1 whiteware & & bowl & \\
\hline 23SG272-6 & $\begin{array}{l}150 \\
\text { R0 }\end{array}$ & 1 & & 20 & & 1 whiteware & & & \\
\hline 23SG272-6 & $\begin{array}{l}150 \\
\text { R0 }\end{array}$ & 1 & & 20 & & 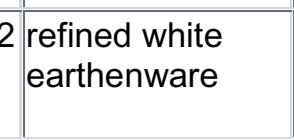 & $\begin{array}{l}\text { blue- } \\
\text { decorated }\end{array}$ & & $\begin{array}{l}\text { maybe } \\
\text { have been } \\
\text { "tumbled" }\end{array}$ \\
\hline 23SG272-6 & $\begin{array}{l}150 \\
\text { R0 }\end{array}$ & 1 & & 20 & & 1 yellow ware & & bowl & \\
\hline 23SG272-7 & $\begin{array}{l}150 \\
\text { Ro }\end{array}$ & 1 & 6 & 20 & & 1 gray stoneware & & $\begin{array}{l}\text { ?large } \\
\text { crock }\end{array}$ & \\
\hline 23SG272-7 & $\begin{array}{l}150 \\
\text { R0 }\end{array}$ & 1 & & 20 & & 1 pearlware & & & "tumbled" \\
\hline 23SG272-7 & $\begin{array}{l}150 \\
\text { RO }\end{array}$ & 1 & 6 & 20 & & 1 whiteware & $\begin{array}{l}\text { blue- } \\
\text { decorated } \\
\text { transfer- } \\
\text { print or } \\
\text { hand- } \\
\text { painted }\end{array}$ & & \\
\hline 23SG272-7 & $\begin{array}{l}150 \\
\text { R0 }\end{array}$ & 1 & 6 & $\mathrm{ZO}$ & & 2 whiteware & & & \\
\hline 23SG272-7 & $\begin{array}{l}150 \\
\text { Ro }\end{array}$ & 1 & 6 & $\mathrm{ZO}$ & & 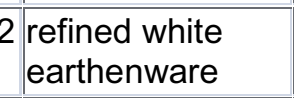 & & & \\
\hline 23SG272-7 & $\begin{array}{l}150 \\
\text { Ro }\end{array}$ & 1 & 6 & $\mathrm{ZO}$ & & 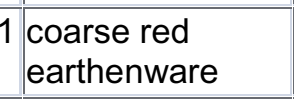 & & & $\begin{array}{l}\text { dark brown } \\
\text { lead glaze }\end{array}$ \\
\hline 23SG272-7 & $\begin{array}{l}150 \\
\text { R0 }\end{array}$ & 1 & 6 & $\mathrm{ZO}$ & & 1 yellow ware & $\begin{array}{l}\text { white/crea } \\
\text { m-colored } \\
\text { annular } \\
\text { rings }\end{array}$ & & \\
\hline 23SG272-7 & $\begin{array}{l}150 \\
\text { R0 }\end{array}$ & 1 & 6 & 20 & & 1 yellow ware & & & \\
\hline 23SG272-8 & $\begin{array}{l}150 \\
\text { R0 }\end{array}$ & 1 & 7 & 20 & & 1 whiteware & $\begin{array}{l}\text { dark blue } \\
\text { decoration, }\end{array}$ & & \\
\hline
\end{tabular}




\begin{tabular}{|c|c|c|c|c|c|c|c|c|c|}
\hline 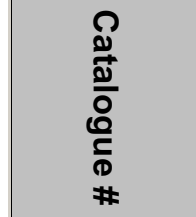 & $\underset{F}{\stackrel{c}{\rightleftarrows}}$ & 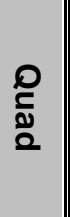 & 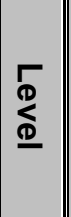 & 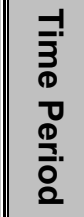 & $\begin{array}{l}\frac{1}{3} \\
\stackrel{0}{0} \\
\frac{1}{2}\end{array}$ & 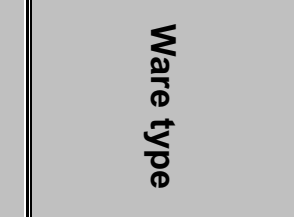 & $\begin{array}{l}\frac{0}{0} \\
\frac{0}{a} \\
\frac{0}{0} \\
\frac{0}{2}\end{array}$ & $\begin{array}{l}\frac{0}{0} \\
3 \\
\frac{1}{2} \\
\frac{0}{0}\end{array}$ & $\begin{array}{l}\overline{5} \\
\overline{0} \\
\frac{7}{3} \\
\frac{0}{5} \\
\frac{0}{0} \\
\frac{0}{0}\end{array}$ \\
\hline & & & & & & & $\begin{array}{l}\text { probably } \\
\text { flow-blue } \\
\text { transfer } \\
\text { print }\end{array}$ & & \\
\hline 23SG272-8 & $\begin{array}{l}150 \\
\text { RO }\end{array}$ & 1 & & $\mathrm{ZO}$ & & 1 whiteware & $\begin{array}{l}\text { "fern" of } \\
\text { mocha } \\
\text { design }\end{array}$ & & \\
\hline 23SG272-8 & $\begin{array}{l}150 \\
\text { RO }\end{array}$ & 1 & & $\mathrm{ZO}$ & & 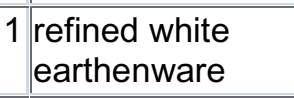 & & & \\
\hline 23SG272-8 & $\begin{array}{l}150 \\
\text { R0 }\end{array}$ & 1 & & $\mathrm{ZO}$ & & 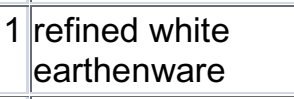 & & & "tumbled" \\
\hline 23SG272-9 & $\begin{array}{l}150 \\
\text { R0 }\end{array}$ & 1 & & $\mathrm{ZO}$ & & 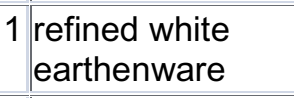 & & & $\begin{array}{l}\text { possibly } \\
\text { "tumbled" }\end{array}$ \\
\hline \begin{tabular}{|l}
$23 S G 272-$ \\
10
\end{tabular} & $\begin{array}{l}150 \\
\text { RO }\end{array}$ & 1 & & $\mathrm{ZO}$ & & 1 pearlware & & & \\
\hline $\begin{array}{l}\text { 23SG272- } \\
10\end{array}$ & $\begin{array}{l}150 \\
\text { RO }\end{array}$ & 1 & & $\mathrm{ZO}$ & & 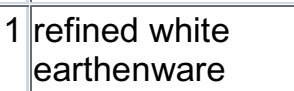 & & & \\
\hline $\begin{array}{l}\text { 23SG272- } \\
10\end{array}$ & $\begin{array}{l}150 \\
\text { R0 }\end{array}$ & 1 & & $\mathrm{ZO}$ & & 1 white glaze chip & & & \\
\hline $\begin{array}{l}\text { 23SG272- } \\
163\end{array}$ & $\begin{array}{l}40 \mathrm{R} \\
60\end{array}$ & 3 & & ZLO & & $1 \begin{array}{l}\text { whiteware or } \\
\text { ironstone }\end{array}$ & & & $\begin{array}{l}\text { highly } \\
\text { exfoliated } \\
\text { and rust- } \\
\text { stained }\end{array}$ \\
\hline $\begin{array}{l}\text { 23SG272- } \\
163\end{array}$ & $\begin{array}{l}40 \mathrm{R} \\
60\end{array}$ & 3 & 8 & ZLO & & 1 porcelain & & figurine & \\
\hline $\begin{array}{l}\text { 23SG272- } \\
164\end{array}$ & $\begin{array}{l}40 R \\
60\end{array}$ & 3 & 8 & ZLO & & $\begin{array}{l}1 \text { refined white } \\
\text { earthenware/cre } \\
\text { amware }\end{array}$ & & & \\
\hline $\begin{array}{l}\text { 23SG272- } \\
164\end{array}$ & $\begin{array}{l}40 R \\
60\end{array}$ & 3 & & ZLO & & $1 \begin{array}{l}\text { plain white } \\
\text { pearlware }\end{array}$ & & & \\
\hline $\begin{array}{l}\text { 23SG272- } \\
164\end{array}$ & $\begin{array}{l}40 \mathrm{R} \\
60\end{array}$ & 3 & & ZLO & & 1 whiteware & $\begin{array}{l}\text { red } \\
\text { transfer- } \\
\text { print, red } \\
\text { floral } \\
\text { design }\end{array}$ & $\begin{array}{l}\text { plate/pl } \\
\text { atter }\end{array}$ & \\
\hline $\begin{array}{l}\text { 23SG272- } \\
164\end{array}$ & $\begin{array}{l}40 \mathrm{R} \\
60\end{array}$ & 3 & & ZLO & & 1 whiteware & & & $\begin{array}{l}\text { Slightly } \\
\text { curved }\end{array}$ \\
\hline \begin{tabular}{|l} 
23SG272- \\
164
\end{tabular} & $\begin{array}{l}40 R \\
60\end{array}$ & 3 & & ZLO & & 1 whiteware & & & \\
\hline $\begin{array}{l}\text { 23SG272- } \\
164\end{array}$ & $\begin{array}{l}40 R \\
60\end{array}$ & 3 & & ZLO & & 2 whiteware & $\begin{array}{l}\text { handpainte } \\
\text { d or } \\
\text { stamped, } \\
\text { dark green } \\
\text { design on }\end{array}$ & & $\begin{array}{l}\text { similar to } \\
\text { one in } \\
23 \text { SG272- } \\
166\end{array}$ \\
\hline
\end{tabular}




\begin{tabular}{|c|c|c|c|c|c|c|c|c|c|}
\hline $\begin{array}{l}\text { 尺 } \\
\stackrel{0}{0} \\
\frac{0}{0} \\
\stackrel{0}{0} \\
\frac{1}{0} \\
\# \\
\#\end{array}$ & $\underset{ }{c}$ & 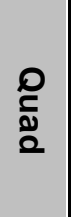 & 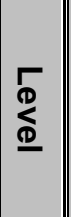 & $\begin{array}{l}-1 \\
\overline{3} \\
\overline{0} \\
0 \\
\mathbb{0} \\
\frac{0}{0} \\
0\end{array}$ & $\begin{array}{l}2 \\
3 \\
0 \\
\\
\end{array}$ & 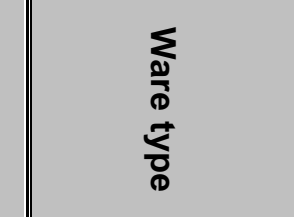 & $\begin{array}{l}\frac{0}{0} \\
\frac{0}{0} \\
\frac{0}{3} \\
\frac{0}{3}\end{array}$ & 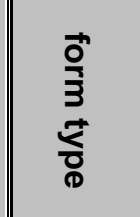 & 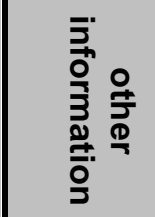 \\
\hline & & & & & & & $\begin{array}{l}\text { white } \\
\text { backgroun } \\
\text { d }\end{array}$ & & \\
\hline $\begin{array}{l}\text { 23SG272- } \\
164\end{array}$ & $\begin{array}{l}40 \mathrm{R} \\
60\end{array}$ & 3 & 8 & ZLO & & $1 \begin{array}{l}\text { gray salt-glazed } \\
\text { stoneware }\end{array}$ & & & \\
\hline $\begin{array}{l}\text { 23SG272- } \\
164\end{array}$ & $\begin{array}{l}40 \mathrm{R} \\
60\end{array}$ & 3 & 8 & ZLO & & $\begin{array}{l}1 \text { gray salt-glazed } \\
\text { stoneware }\end{array}$ & & $\begin{array}{l}\text { large } \\
\text { crock or } \\
\text { jar }\end{array}$ & \\
\hline 23SG272-3 & $\begin{array}{l}150 \\
\text { RO }\end{array}$ & 1 & 2 & ZLO & & 1 kaoline & & $\begin{array}{l}\text { pipe } \\
\text { stem of } \\
\text { smokin } \\
\text { g pipe }\end{array}$ & \\
\hline 23SG272-3 & $\begin{array}{l}150 \\
\text { RO }\end{array}$ & 1 & 2 & ZLO & & 1 white stoneware & & $\begin{array}{l}\text { small- } \\
\text { medium } \\
\text { cosmeti } \\
\text { c jar or } \\
\text { canister }\end{array}$ & \\
\hline 23SG272-3 & $\begin{array}{l}150 \\
\text { R0 }\end{array}$ & 1 & 2 & ZLO & & 2 creamware & & $\begin{array}{l}\text { plate/bo } \\
\text { wl }\end{array}$ & \\
\hline 23SG272-3 & $\begin{array}{l}150 \\
\text { R0 }\end{array}$ & 1 & & ZLO & & 1 pearlware & & & \\
\hline 23SG272-3 & $\begin{array}{l}150 \\
\text { R0 }\end{array}$ & 1 & 2 & ZLO & & 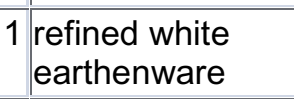 & & $\begin{array}{l}\text { plate/bo } \\
\text { wl }\end{array}$ & \\
\hline 23SG272-3 & $\begin{array}{l}150 \\
\text { Ro }\end{array}$ & 1 & 2 & ZLO & & 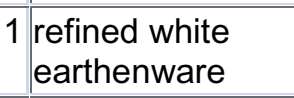 & & & \\
\hline 23SG272-3 & $\begin{array}{l}150 \\
\text { R0 }\end{array}$ & 1 & 2 & ZLO & & 1 whiteware & & $\begin{array}{l}\text { med-lg } \\
\text { vessel }\end{array}$ & \\
\hline 23SG272-3 & $\begin{array}{l}150 \\
\text { Ro }\end{array}$ & 1 & & ZLO & & 1 whiteware & & $\begin{array}{l}\text { plate/bo } \\
\text { wl }\end{array}$ & \\
\hline 23SG272-3 & $\begin{array}{l}150 \\
\text { RO }\end{array}$ & 1 & 2 & ZLO & & 1 whiteware & & & \\
\hline 23SG272-3 & $\begin{array}{l}150 \\
\text { R0 }\end{array}$ & 1 & & ZLO & & 1 whiteware & & $\begin{array}{l}\text { med. } \\
\text { Bowl? }\end{array}$ & \\
\hline 23SG272-3 & $\begin{array}{l}150 \\
\text { R0 }\end{array}$ & 1 & 2 & ZLO & & 1 whiteware & & $\begin{array}{l}\text { plate/bo } \\
\text { wl }\end{array}$ & \\
\hline 23SG272-3 & $\begin{array}{l}150 \\
\text { RO }\end{array}$ & 1 & 2 & ZLO & & $\begin{array}{l}1 \text { pearlware/white } \\
\text { ware }\end{array}$ & $\begin{array}{l}\text { blue } \\
\text { decoration, } \\
\text { possibly } \\
\text { transfer } \\
\text { print }\end{array}$ & & \\
\hline 23SG272-3 & $\begin{array}{l}150 \\
\text { Ro }\end{array}$ & 1 & & ZLO & & $\begin{array}{l}1 \text { pearlware/white } \\
\text { ware }\end{array}$ & & & \\
\hline 23SG272-3 & $\begin{array}{l}150 \\
\text { RO }\end{array}$ & 1 & & ZLO & & 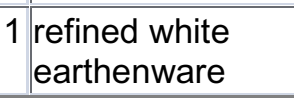 & $\begin{array}{l}\text { green } \\
\text { decoration }\end{array}$ & & $\begin{array}{l}\text { has been } \\
\text { "tumbled" }\end{array}$ \\
\hline
\end{tabular}




\begin{tabular}{|c|c|c|c|c|c|c|c|c|c|}
\hline 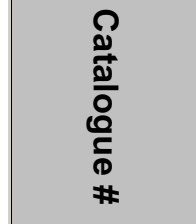 & 돌 & $\begin{array}{l}0 \\
\frac{0}{2} \\
\text { ¿ }\end{array}$ & 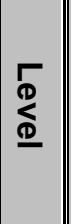 & 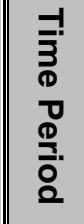 & $\begin{array}{l}\frac{1}{3} \\
0 \\
\frac{0}{2} \\
2\end{array}$ & 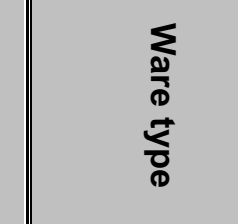 & 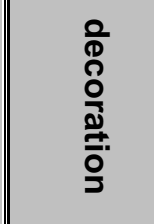 & 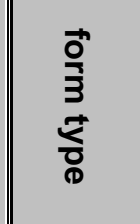 & 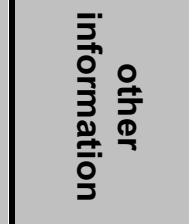 \\
\hline 23SG272-3 & $\begin{array}{l}150 \\
\text { RO }\end{array}$ & $\overline{1}$ & & ZLO & & 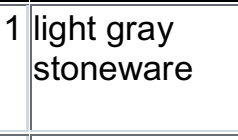 & & $\begin{array}{l}\text { large } \\
\text { bowl or } \\
\text { crock }\end{array}$ & \\
\hline 23SG272-3 & $\begin{array}{l}150 \\
\text { R0 }\end{array}$ & 1 & & ZLO & & $\begin{array}{l}2 \text { olive-brown } \\
\text { stoneware }\end{array}$ & & $\begin{array}{l}\text { large } \\
\text { crock }\end{array}$ & \\
\hline 23SG272-3 & $\begin{array}{l}150 \\
\text { R0 }\end{array}$ & 1 & & ZLO & & 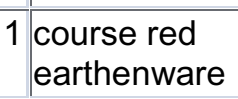 & & & $\begin{array}{l}\text { dark brown } \\
\text { lead glaze }\end{array}$ \\
\hline 23SG272-3 & $\begin{array}{l}150 \\
\text { R0 }\end{array}$ & 1 & & ZLO & & 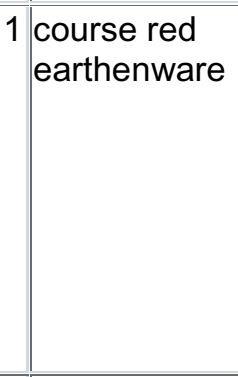 & & & $\begin{array}{l}\text { variegated } \\
\text { caramel- } \\
\text { colored } \\
\text { lead glaze, } \\
\text { (might be } \\
\text { considered } \\
\text { refined red } \\
\text { earthenwar } \\
\text { e }\end{array}$ \\
\hline 23SG272-3 & $\begin{array}{l}150 \\
\text { R0 }\end{array}$ & 1 & & ZLO & & 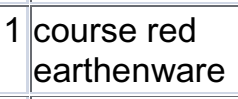 & & & \\
\hline 23SG272-4 & $\begin{array}{l}150 \\
\text { R0 }\end{array}$ & 1 & & ZLO & & 1 creamware & & $\begin{array}{l}\text { plate/sa } \\
\text { ucer/bo } \\
\text { wl }\end{array}$ & \\
\hline 23SG272-4 & $\begin{array}{l}150 \\
\text { R0 }\end{array}$ & 1 & & ZLO & & 1 whiteware & & $\begin{array}{l}\text { ?mediu } \\
\text { m bowl }\end{array}$ & \\
\hline 23SG272-4 & $\begin{array}{l}150 \\
\text { R0 }\end{array}$ & 1 & & ZLO & & 1 whiteware & & \begin{tabular}{|l} 
small- \\
medium \\
bowl
\end{tabular} & \\
\hline 23SG272-4 & $\begin{array}{l}150 \\
\text { RO }\end{array}$ & 1 & & ZLO & & 2 whiteware & & & \\
\hline 23SG272-4 & $\begin{array}{l}150 \\
\text { R0 }\end{array}$ & 1 & & ZLO & & 1 whiteware & $\begin{array}{l}\text { purple } \\
\text { transfer- } \\
\text { print }\end{array}$ & & \\
\hline 23SG272-4 & $\begin{array}{l}150 \\
\text { R0 }\end{array}$ & 1 & & ZLO & & 1 whiteware & $\begin{array}{l}\text { interior } \\
\text { green } \\
\text { transfer- } \\
\text { print }\end{array}$ & $\begin{array}{l}\text { small- } \\
\text { medium } \\
\text { bowl }\end{array}$ & \\
\hline 23SG272-4 & $\begin{array}{l}150 \\
\text { R0 }\end{array}$ & 1 & & ZLO & & 1 pearlware & $\begin{array}{l}\text { interior } \\
\text { blue } \\
\text { transfer- } \\
\text { print }\end{array}$ & $\begin{array}{l}\text { med- } \\
\text { large } \\
\text { bowl }\end{array}$ & $\begin{array}{l}\text { pssibly a } \\
\text { chinoiserie } \\
\text { design }\end{array}$ \\
\hline 23SG272-4 & $\begin{array}{l}150 \\
\text { R0 }\end{array}$ & 1 & 3 & ZLO & & 3 yellow ware & & $\begin{array}{l}\text { med-lg } \\
\text { bowl or } \\
\text { pitcher }\end{array}$ & \\
\hline 23SG272-4 & $\begin{array}{l}150 \\
\text { R0 }\end{array}$ & 1 & & ZLO & & 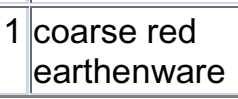 & & & \\
\hline
\end{tabular}




\begin{tabular}{|c|c|c|c|c|c|c|c|c|c|}
\hline 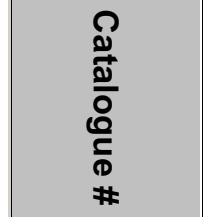 & $\stackrel{c}{\mathrm{C}}$ & 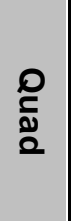 & 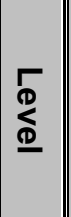 & $\begin{array}{l}-1 \\
\overline{3} \\
0 \\
0 \\
\Phi \\
\frac{0}{0} \\
0\end{array}$ & $\begin{array}{l}\frac{1}{3} \\
\stackrel{0}{0} \\
\frac{2}{2}\end{array}$ & 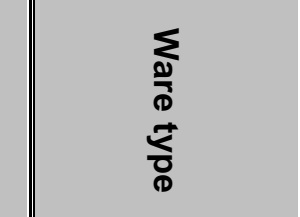 & $\begin{array}{l}\frac{0}{0} \\
\frac{0}{0} \\
\frac{0}{*} \\
\frac{0}{3}\end{array}$ & 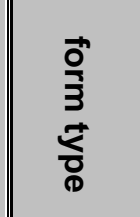 & 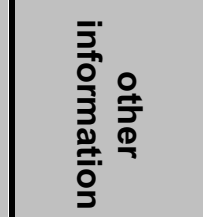 \\
\hline 23SG272-4 & $\begin{array}{l}150 \\
\text { Ro }\end{array}$ & $\overline{1}$ & & ZLO & & 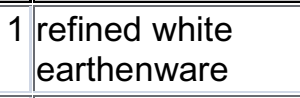 & & & \\
\hline 23SG272-4 & $\begin{array}{l}150 \\
\text { RO }\end{array}$ & 1 & & ZLO & & 2 gray stoneware & & $\begin{array}{l}\text { large } \\
\text { crock }\end{array}$ & $\begin{array}{l}\text { light gray } \\
\text { exterior, } \\
\text { dark grey } \\
\text { interior }\end{array}$ \\
\hline 23SG272-4 & $\begin{array}{l}150 \\
\text { R0 }\end{array}$ & 1 & & ZLO & & $\begin{array}{l}1 \text { white kaolin } \\
\text { pipes }\end{array}$ & & $\begin{array}{l}\text { pipe } \\
\text { stem }\end{array}$ & \\
\hline 23SG272-5 & $\begin{array}{l}150 \\
\text { Ro }\end{array}$ & 1 & & ZLO & & 1 white kaolin pipe & & $\begin{array}{l}\text { pipeste } \\
\mathrm{m}\end{array}$ & \\
\hline 23SG272-5 & $\begin{array}{l}150 \\
\text { R0 }\end{array}$ & 1 & & ZLO & & 2 creamware & & bowl? & \\
\hline 23SG272-5 & $\begin{array}{l}150 \\
\text { RO }\end{array}$ & 1 & & ZLO & & 1 whiteware & $\begin{array}{l}\text { brown } \\
\text { transfer- } \\
\text { print }\end{array}$ & $\begin{array}{l}\text { med-lg } \\
\text { bowl/se } \\
\text { rving } \\
\text { dish }\end{array}$ & \\
\hline 23SG272-5 & $\begin{array}{l}150 \\
\text { RO }\end{array}$ & 1 & & ZLO & & 1 whiteware & \begin{tabular}{|l|} 
black \\
transfer- \\
print
\end{tabular} & & \\
\hline 23SG272-5 & $\begin{array}{l}150 \\
\text { RO }\end{array}$ & 1 & & ZLO & & 1 whiteware & $\begin{array}{l}\text { green } \\
\text { transfer- } \\
\text { print }\end{array}$ & $\begin{array}{l}\text { med-lg } \\
\text { bowl }\end{array}$ & \\
\hline $\begin{array}{l}\text { 23SG272- } \\
14\end{array}$ & $\begin{array}{l}110 \\
\text { R30 }\end{array}$ & 2 & 2 & ZLO & & 1 stoneware & & & $\begin{array}{l}\text { gray-white } \\
\text { interior and } \\
\text { gray-tan } \\
\text { exterior } \\
\text { glaze }\end{array}$ \\
\hline $\begin{array}{l}23 \text { SG272- } \\
14\end{array}$ & $\begin{array}{l}110 \\
\text { R30 }\end{array}$ & 2 & & ZLO & & 1 stoneware & & & $\begin{array}{l}\text { innter } \\
\text { portion is } \\
\text { tan and } \\
\text { both interior } \\
\text { and exterior } \\
\text { portions of } \\
\text { the paste } \\
\text { are gray }\end{array}$ \\
\hline $\begin{array}{l}23 S G 272- \\
14\end{array}$ & $\begin{array}{l}110 \\
\text { R30 }\end{array}$ & 2 & 2 & ZLO & & 1 red earthenware & & $\begin{array}{l}\text { possibly } \\
\text { flower } \\
\text { pot }\end{array}$ & \\
\hline $\begin{array}{l}\text { 23SG272- } \\
14\end{array}$ & $\begin{array}{l}110 \\
\text { R30 }\end{array}$ & 2 & & ZLO & & 1 red earthenware & & $\begin{array}{l}\text { possibly } \\
\text { flower } \\
\text { pot }\end{array}$ & \\
\hline $\begin{array}{l}23 S G 272- \\
14\end{array}$ & $\begin{array}{l}110 \\
\text { R30 }\end{array}$ & 2 & 2 & ZLO & & 4 red earthenware & $\begin{array}{l}\text { light- } \\
\text { colored } \\
\text { orange- } \\
\text { pink }\end{array}$ & & $\begin{array}{l}\text { circular hole } \\
\text { in middle }\end{array}$ \\
\hline
\end{tabular}




\begin{tabular}{|c|c|c|c|c|c|c|c|c|c|}
\hline 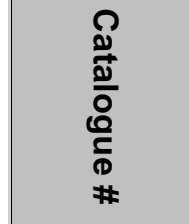 & $\stackrel{c}{s}$ & $\begin{array}{l}0 \\
\stackrel{0}{2} \\
\stackrel{2}{2}\end{array}$ & 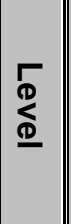 & 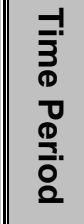 & 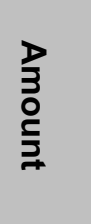 & 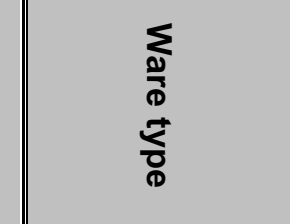 & 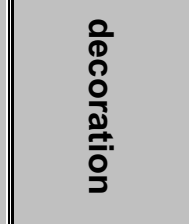 & 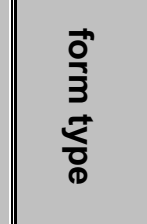 & 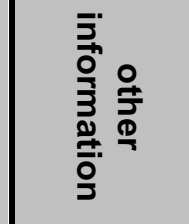 \\
\hline $\begin{array}{l}23 S G 272- \\
14\end{array}$ & $\begin{array}{l}110 \\
\text { R30 }\end{array}$ & 2 & & ZLO & & 1 pearlware & \begin{tabular}{|l} 
blue \\
transfer- \\
print
\end{tabular} & bowl/jar & \\
\hline \begin{tabular}{|l}
$23 S G 272-$ \\
14
\end{tabular} & $\begin{array}{l}110 \\
\text { R30 }\end{array}$ & 2 & & ZLO & & 2 pearlware & \begin{tabular}{|l} 
blue \\
transfer- \\
print
\end{tabular} & bowl & \\
\hline \begin{tabular}{|l}
$23 S G 272-$ \\
14
\end{tabular} & $\begin{array}{l}110 \\
\text { R30 }\end{array}$ & 2 & & ZLO & & 4 whiteware & $\begin{array}{l}\text { black/dark } \\
\text { brown } \\
\text { transfer- } \\
\text { printed }\end{array}$ & & \\
\hline $\begin{array}{l}23 S G 272- \\
14\end{array}$ & $\begin{array}{l}110 \\
\text { R30 }\end{array}$ & 2 & 2 & ZLO & 15 & 5 Creamware & $\begin{array}{l}\text { indetermin } \\
\text { ate } \\
\text { embossed } \\
\text { design }\end{array}$ & $\begin{array}{l}\text { bowl/pl } \\
\text { ate }\end{array}$ & $\begin{array}{l}3 \text { have } \\
\text { heavy } \\
\text { stains }\end{array}$ \\
\hline $\begin{array}{l}\text { 23SG272- } \\
14\end{array}$ & $\begin{array}{l}110 \\
\text { R30 }\end{array}$ & 2 & & ZLO & & $\begin{array}{l}1 \text { pearlware/white } \\
\text { ware }\end{array}$ & & & $\begin{array}{l}\text { blue shell- } \\
\text { edged }\end{array}$ \\
\hline $\begin{array}{l}23 S G 272- \\
14\end{array}$ & $\begin{array}{l}110 \\
\text { R30 }\end{array}$ & 2 & & ZLO & & 7 pearlware & & & \\
\hline $\begin{array}{l}23 S G 272- \\
14\end{array}$ & $\begin{array}{l}110 \\
\text { R30 }\end{array}$ & 2 & & ZLO & & $\begin{array}{l}3 \text { pearlware/white } \\
\text { ware }\end{array}$ & & & \\
\hline $\begin{array}{l}23 S G 272- \\
14\end{array}$ & $\begin{array}{l}\text { 110 } \\
\text { R30 }\end{array}$ & 2 & & ZLO & & 1 pearlware & & & $\begin{array}{l}\text { plain white } \\
\text { interior and } \\
\text { orange-tan } \\
\text { exterior }\end{array}$ \\
\hline $\begin{array}{l}23 S G 272- \\
14\end{array}$ & $\begin{array}{l}110 \\
\text { R30 }\end{array}$ & 2 & & ZLO & & 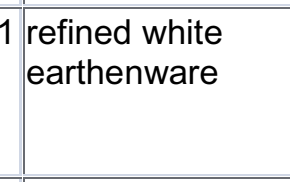 & $\begin{array}{l}\text { yellow } \\
\text { ?floral } \\
\text { (?petals) } \\
\text { design }\end{array}$ & & \\
\hline \begin{tabular}{|l}
$23 S G 272-$ \\
14
\end{tabular} & $\begin{array}{l}110 \\
\text { R30 }\end{array}$ & 2 & & ZLO & & 1 red stoneware & & & $\begin{array}{l}\text { olive- } \\
\text { green/brow } \\
\text { n glaze }\end{array}$ \\
\hline \begin{tabular}{|l}
$23 S G 272-$ \\
14 \\
\end{tabular} & $\begin{array}{l}110 \\
\text { R30 }\end{array}$ & 2 & 2 & ZLO & & $\begin{array}{l}2 \text { coarse red } \\
\text { earthenware }\end{array}$ & & & \\
\hline $\begin{array}{l}23 S G 272- \\
14\end{array}$ & $\begin{array}{l}110 \\
\text { R30 }\end{array}$ & 2 & 2 & ZLO & & 1 porcelain & & & $\begin{array}{l}\text { probably } \\
\text { Chinese } \\
\text { export }\end{array}$ \\
\hline $\begin{array}{l}23 S G 272- \\
15\end{array}$ & $\begin{array}{l}110 \\
\text { R30 }\end{array}$ & 2 & & ZLO & & 3 white kaolin clay & & & \\
\hline $\begin{array}{l}23 S G 272- \\
15\end{array}$ & $\begin{array}{l}110 \\
\text { R30 }\end{array}$ & 2 & & ZLO & & 3 red earthenware & & $\begin{array}{l}\text { possibly } \\
\text { flower } \\
\text { pot }\end{array}$ & \\
\hline $\begin{array}{l}23 S G 272- \\
15\end{array}$ & $\begin{array}{l}110 \\
\text { R30 }\end{array}$ & 2 & & ZLO & & 1 red earthenware & & & $\begin{array}{l}\text { small bit of } \\
\text { dark brown } \\
\text { lead glaze }\end{array}$ \\
\hline
\end{tabular}




\begin{tabular}{|c|c|c|c|c|c|c|c|c|c|}
\hline 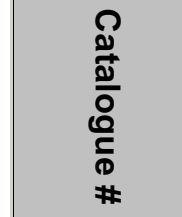 & $\stackrel{c}{\stackrel{c}{丂}}$ & $\begin{array}{l}0 \\
\stackrel{0}{0} \\
\stackrel{0}{2}\end{array}$ & $\underset{\Phi}{\stackrel{\Phi}{\Phi}}$ & 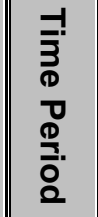 & 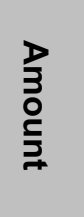 & 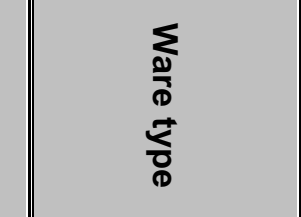 & $\begin{array}{l}\frac{0}{0} \\
0 \\
\frac{0}{3} \\
\frac{0}{0} \\
\frac{0}{2}\end{array}$ & 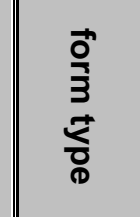 & 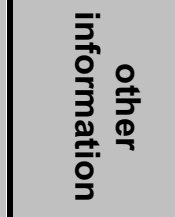 \\
\hline & & & & & & & & & on exterior \\
\hline $\begin{array}{l}23 S G 272- \\
15\end{array}$ & $\begin{array}{l}110 \\
\text { R30 }\end{array}$ & 2 & 3 & ZLO & & 1 gray stoneware & $\begin{array}{l}\text { molded } \\
\text { bands and } \\
\text { red- } \\
\text { colored } \\
\text { slip }\end{array}$ & & \\
\hline $\begin{array}{l}23 S G 272- \\
15\end{array}$ & $\begin{array}{l}110 \\
\text { R30 }\end{array}$ & 2 & 3 & ZLO & & 1 pearlware & $\begin{array}{l}\text { dark blue } \\
\text { transfer- } \\
\text { print }\end{array}$ & bowl & \\
\hline $\begin{array}{l}23 S G 272- \\
15\end{array}$ & $\begin{array}{l}110 \\
\text { R30 }\end{array}$ & 2 & 3 & ZLO & & $\begin{array}{l}1 \text { pearlware/white } \\
\text { ware }\end{array}$ & $\begin{array}{l}\text { dark blue } \\
\text { transfer- } \\
\text { printed }\end{array}$ & & \\
\hline $\begin{array}{l}\text { 23SG272- } \\
15\end{array}$ & $\begin{array}{l}110 \\
\text { R30 }\end{array}$ & 2 & 3 & ZLO & & 1 pearlware & $\begin{array}{l}\text { light blue } \\
\text { transfer- } \\
\text { print }\end{array}$ & $\begin{array}{l}\text { bowl/pl } \\
\text { ate }\end{array}$ & \\
\hline $\begin{array}{l}23 S G 272- \\
15\end{array}$ & $\begin{array}{l}110 \\
\text { R30 }\end{array}$ & 2 & 3 & ZLO & & 1 whiteware? & $\begin{array}{l}\text { light blue } \\
\text { transfer- } \\
\text { print }\end{array}$ & & \\
\hline $\begin{array}{l}\text { 23SG272- } \\
15\end{array}$ & $\begin{array}{l}110 \\
\text { R30 }\end{array}$ & 2 & 3 & ZLO & & 1 whiteware & \begin{tabular}{|l} 
red \\
transfer- \\
print
\end{tabular} & & \\
\hline $\begin{array}{l}\text { 23SG272- } \\
15\end{array}$ & $\begin{array}{l}110 \\
\text { R30 }\end{array}$ & 2 & 3 & ZLO & & 1 pearlware & $\begin{array}{l}\text { hand- } \\
\text { painted } \\
\text { polychrom } \\
\text { e, olive- } \\
\text { green } \\
\text { design on } \\
\text { interior }\end{array}$ & $\begin{array}{l}\text { bowl/pl } \\
\text { ate }\end{array}$ & \\
\hline $\begin{array}{l}\text { 23SG272- } \\
15\end{array}$ & $\begin{array}{l}110 \\
\text { R30 }\end{array}$ & 2 & 3 & ZLO & & 2 whiteware & $\begin{array}{l}\text { hand- } \\
\text { painted } \\
\text { light blue } \\
\text { decoration }\end{array}$ & & \\
\hline $\begin{array}{l}\text { 23SG272- } \\
15\end{array}$ & $\begin{array}{l}110 \\
\text { R30 }\end{array}$ & 2 & 3 & ZLO & & 1 pearlware & $\begin{array}{l}\text { green } \\
\text { shell- } \\
\text { edged }\end{array}$ & & \\
\hline $\begin{array}{l}\text { 23SG272- } \\
15\end{array}$ & $\begin{array}{l}110 \\
\text { R30 }\end{array}$ & 2 & 3 & ZLO & & 3 pearlware & & \begin{tabular}{|l} 
bowl/pl \\
ate
\end{tabular} & \\
\hline $\begin{array}{l}\text { 23SG272- } \\
15\end{array}$ & $\begin{array}{l}110 \\
\text { R30 }\end{array}$ & 2 & 3 & ZLO & & 1 whiteware & & & \\
\hline $\begin{array}{l}\text { 23SG272- } \\
15\end{array}$ & $\begin{array}{l}110 \\
\text { R30 }\end{array}$ & 2 & 3 & ZLO & & $\begin{array}{l}1 \text { pearlware/white } \\
\text { ware }\end{array}$ & & & \\
\hline $\begin{array}{l}23 S G 272- \\
15\end{array}$ & $\begin{array}{l}110 \\
\text { R30 }\end{array}$ & 2 & 3 & ZLO & & $\begin{array}{l}1 \text { whiteware/ironst } \\
\text { one }\end{array}$ & & & \\
\hline 23SG272- & 110 & 2 & 3 & ZLO & & 1 whiteware/ironst & & bowl? & \\
\hline
\end{tabular}




\begin{tabular}{|c|c|c|c|c|c|c|c|c|c|}
\hline 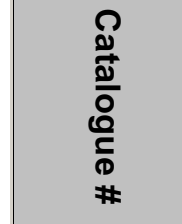 & $\stackrel{c}{\mathrm{c}}$ & 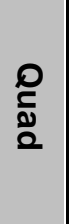 & \begin{tabular}{|l|}
$\Gamma$ \\
$\mathbb{D}$ \\
$\mathbb{\Phi}$ \\
\end{tabular} & 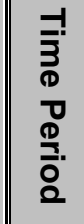 & $\begin{array}{l}\stackrel{D}{3} \\
\stackrel{0}{0} \\
\frac{0}{J}\end{array}$ & 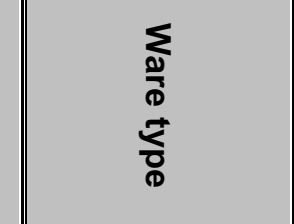 & $\begin{array}{l}\frac{0}{0} \\
\stackrel{0}{0} \\
0 \\
\frac{0}{0} \\
\frac{0}{0}\end{array}$ & $\begin{array}{l}\frac{0}{0} \\
3 \\
\text { ¿ } \\
\text { ¿ }\end{array}$ & 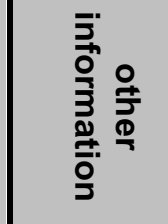 \\
\hline 15 & R30 & & & & & one & & & \\
\hline $\begin{array}{l}23 S G 272- \\
15\end{array}$ & $\begin{array}{l}110 \\
\text { R30 }\end{array}$ & 2 & 3 & ZLO & & $\begin{array}{l}1 \text { refined white } \\
\text { earthenware/iro } \\
\text { nstone }\end{array}$ & $\begin{array}{l}\text { interior } \\
\text { cream- } \\
\text { colored or } \\
\text { yellow- } \\
\text { colored } \\
\text { with } \\
\text { machine } \\
\text { bands } \\
\text { evident }\end{array}$ & & \\
\hline $\begin{array}{l}23 \text { SG272- } \\
16\end{array}$ & $\begin{array}{l}110 \\
\text { R30 }\end{array}$ & 2 & & ZLO & & 4 white kaolin clay & & & \\
\hline $\begin{array}{l}23 S G 272- \\
16\end{array}$ & $\begin{array}{l}110 \\
\text { R30 }\end{array}$ & 2 & & ZLO & & 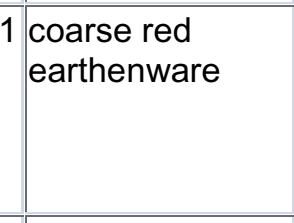 & & & $\begin{array}{l}\text { clear lead } \\
\text { glaze } \\
\text { (appears } \\
\text { caramel } \\
\text { colored) }\end{array}$ \\
\hline $\begin{array}{l}\text { 23SG272- } \\
16\end{array}$ & $\begin{array}{l}110 \\
\text { R30 }\end{array}$ & 2 & & ZLO & & 2 creamware & & & \\
\hline $\begin{array}{l}23 \text { SG272- } \\
16\end{array}$ & $\begin{array}{l}110 \\
\text { R30 }\end{array}$ & 2 & & ZLO & & $\begin{array}{l}4 \text { pearlware/white } \\
\text { ware }\end{array}$ & & & \\
\hline $\begin{array}{l}\text { 23SG272- } \\
16\end{array}$ & $\begin{array}{l}110 \\
\text { R30 }\end{array}$ & 2 & & ZLO & & 1 whiteware & & bowl & \\
\hline $\begin{array}{l}23 S G 272- \\
16\end{array}$ & $\begin{array}{l}110 \\
\text { R30 }\end{array}$ & 2 & & ZLO & & 1 whiteware & & $\begin{array}{l}\text { plate/bo } \\
\text { wl }\end{array}$ & \\
\hline $\begin{array}{l}23 \text { SG272- } \\
16\end{array}$ & $\begin{array}{l}110 \\
\text { R30 }\end{array}$ & 2 & & ZLO & & 5 whiteware & & plate & \\
\hline $\begin{array}{l}\text { 23SG272- } \\
16\end{array}$ & $\begin{array}{l}110 \\
\text { R30 }\end{array}$ & 2 & & ZLO & & 1 whiteware & & $\begin{array}{l}\text { plate/lg } \\
\text { bowl }\end{array}$ & \\
\hline $\begin{array}{l}23 \text { SG272- } \\
16\end{array}$ & $\begin{array}{l}110 \\
\text { R30 }\end{array}$ & 2 & & ZLO & & 2 whiteware & & $\begin{array}{l}\text { plate/bo } \\
\text { wl }\end{array}$ & \\
\hline $\begin{array}{l}23 S G 272- \\
16\end{array}$ & $\begin{array}{l}110 \\
\text { R30 }\end{array}$ & 2 & & ZLO & & 5 whiteware & & & \\
\hline $\begin{array}{l}\text { 23SG272- } \\
16\end{array}$ & $\begin{array}{l}110 \\
\text { R30 }\end{array}$ & 2 & & ZLO & & 3 whiteware & $\begin{array}{l}\text { dark blue } \\
\text { transfer- } \\
\text { print }\end{array}$ & & \\
\hline $\begin{array}{l}\text { 23SG272- } \\
16\end{array}$ & $\begin{array}{l}110 \\
\text { R30 }\end{array}$ & 2 & & ZLO & & 1 pearlware & \begin{tabular}{|l} 
dark blue \\
transfer- \\
print
\end{tabular} & $\begin{array}{l}\text { plate/bo } \\
\text { wl }\end{array}$ & \\
\hline $\begin{array}{l}\text { 23SG272- } \\
16\end{array}$ & $\begin{array}{l}110 \\
\text { R30 }\end{array}$ & 2 & 4 & ZLO & & 3 whiteware & $\begin{array}{l}\text { red } \\
\text { transfer- } \\
\text { print }\end{array}$ & & \\
\hline $\begin{array}{l}\text { 23SG272- } \\
16\end{array}$ & $\begin{array}{l}110 \\
\text { R30 }\end{array}$ & 2 & & ZLO & & 1 whiteware & $\begin{array}{l}\text { purple } \\
\text { transfer- }\end{array}$ & & \\
\hline
\end{tabular}




\begin{tabular}{|c|c|c|c|c|c|c|c|c|c|}
\hline $\begin{array}{l}\text { O } \\
\stackrel{0}{ \pm} \\
\stackrel{0}{0} \\
\stackrel{0}{0} \\
\stackrel{D}{0} \\
\#\end{array}$ & $\stackrel{c}{\stackrel{c}{丂}}$ & $\begin{array}{l}0 \\
\stackrel{0}{ \pm} \\
\stackrel{0}{2}\end{array}$ & \begin{tabular}{|l|}
$\Gamma$ \\
$\mathbb{D}$ \\
$\Phi$ \\
$\Phi$
\end{tabular} & 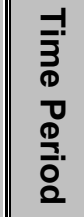 & 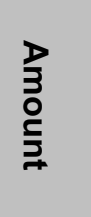 & 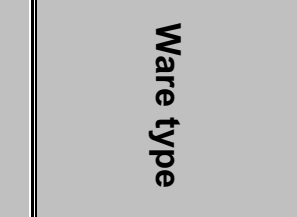 & $\begin{array}{l}\frac{0}{0} \\
0 \\
\frac{0}{3} \\
\frac{0}{0} \\
\frac{0}{2}\end{array}$ & 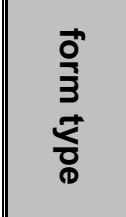 & 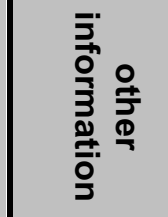 \\
\hline & & & & & & & print & & \\
\hline $\begin{array}{l}\text { 23SG272- } \\
16\end{array}$ & $\begin{array}{l}110 \\
\text { R30 }\end{array}$ & 2 & & ZLO & & $\begin{array}{l}1 \text { pearlware/white } \\
\text { ware }\end{array}$ & $\begin{array}{l}\text { black } \\
\text { transfer- } \\
\text { print }\end{array}$ & $\begin{array}{l}\text { mug or } \\
\text { bowl }\end{array}$ & \\
\hline $\begin{array}{l}23 S G 272- \\
16\end{array}$ & $\begin{array}{l}110 \\
\text { R30 }\end{array}$ & 2 & & ZLO & & 12 pearlware & \begin{tabular}{|l} 
blue \\
transfer- \\
print
\end{tabular} & $\begin{array}{l}\text { cup or } \\
\text { tea } \\
\text { bowl }\end{array}$ & \\
\hline $\begin{array}{l}\text { 23SG272- } \\
16\end{array}$ & $\begin{array}{l}110 \\
\text { R30 }\end{array}$ & 2 & & ZLO & & 1 pearlware & \begin{tabular}{|l|} 
banded \\
(light blue) \\
or shell- \\
edged \\
(blue)
\end{tabular} & & $\begin{array}{l}\text { possibily } \\
\text { "tumbled" }\end{array}$ \\
\hline $\begin{array}{l}\text { 23SG272- } \\
16\end{array}$ & $\begin{array}{l}\text { 110 } \\
\text { R30 }\end{array}$ & 2 & 4 & ZLO & & 1 whiteware & $\begin{array}{l}\text { hand- } \\
\text { painted } \\
\text { green } \\
\text { design }\end{array}$ & & \\
\hline $\begin{array}{l}\text { 23SG272- } \\
16\end{array}$ & $\begin{array}{l}110 \\
\text { R30 }\end{array}$ & 2 & & ZLO & & 1 pearlware & $\begin{array}{l}\text { blue } \\
\text { decoration, } \\
\text { probably } \\
\text { transfer } \\
\text { print }\end{array}$ & & \\
\hline $\begin{array}{l}\text { 23SG272- } \\
16\end{array}$ & $\begin{array}{l}110 \\
\text { R30 }\end{array}$ & 2 & & ZLO & & 1 pearlware & & teacup & \\
\hline $\begin{array}{l}23 S G 272- \\
16\end{array}$ & $\begin{array}{l}110 \\
\text { R30 }\end{array}$ & 2 & & ZLO & & 1 pearlware & & $\begin{array}{l}\text { cup/bo } \\
\text { wl }\end{array}$ & \\
\hline $\begin{array}{l}\text { 23SG272- } \\
16\end{array}$ & $\begin{array}{l}110 \\
\text { R30 }\end{array}$ & 2 & & ZLO & & 1 pearlware & & $\begin{array}{l}\text { ring } \\
\text { from } \\
\text { some } \\
\text { kind of } \\
\text { lid } \\
\end{array}$ & \\
\hline $\begin{array}{l}\text { 23SG272- } \\
16\end{array}$ & $\begin{array}{l}110 \\
\text { R30 }\end{array}$ & 2 & & ZLO & & 6 pearlware & & & \\
\hline $\begin{array}{l}\text { 23SG272- } \\
16\end{array}$ & $\begin{array}{l}110 \\
\text { R30 }\end{array}$ & 2 & & ZLO & & 1 yellow ware & & $\begin{array}{l}\text { large } \\
\text { bowl }\end{array}$ & $\begin{array}{l}\text { yellow } \\
\text { banded }\end{array}$ \\
\hline $\begin{array}{l}\text { 23SG272- } \\
16\end{array}$ & $\begin{array}{l}110 \\
\text { R30 }\end{array}$ & 2 & & ZLO & & 1 gray stoneware & & & \\
\hline $\begin{array}{l}\text { 23SG272- } \\
16\end{array}$ & $\begin{array}{l}110 \\
\text { R30 }\end{array}$ & 2 & & ZLO & & $\begin{array}{l}3 \text { refined white } \\
\text { earthenware/iro } \\
\text { nstone }\end{array}$ & & & \\
\hline $\begin{array}{l}\text { 23SG272- } \\
16\end{array}$ & $\begin{array}{l}110 \\
\text { R30 }\end{array}$ & 2 & & ZLO & & $\begin{array}{l}3 \text { refined white } \\
\text { earthenware/iro } \\
\text { nstone }\end{array}$ & & & \\
\hline $\begin{array}{l}\text { 23SG272- } \\
16\end{array}$ & $\begin{array}{l}110 \\
\text { R30 }\end{array}$ & 2 & & ZLO & & 1 dark blue frag. & & & \\
\hline
\end{tabular}




\begin{tabular}{|c|c|c|c|c|c|c|c|c|c|}
\hline 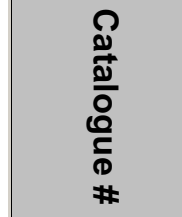 & 돌 & 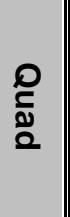 & 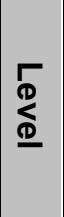 & 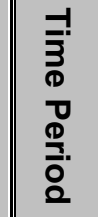 & $\begin{array}{l}\frac{1}{3} \\
\text { o } \\
\frac{1}{2}\end{array}$ & 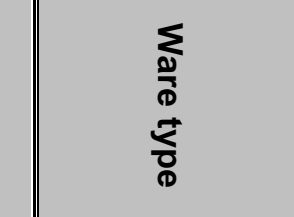 & 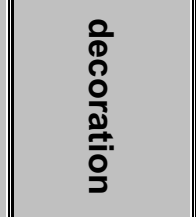 & 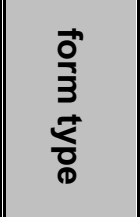 & 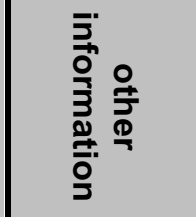 \\
\hline $\begin{array}{l}23 S G 272- \\
17\end{array}$ & $\begin{array}{l}110 \\
\text { R30 }\end{array}$ & 2 & & 20 & & $\begin{array}{l}1 \text { pearlware/white } \\
\text { ware }\end{array}$ & $\begin{array}{l}\text { dark brown } \\
\text { annular } \\
\text { band on } \\
\text { interior }\end{array}$ & $\begin{array}{l}\text { bowl or } \\
\text { cup }\end{array}$ & \\
\hline $\begin{array}{l}23 \text { SG272- } \\
17\end{array}$ & $\begin{array}{l}110 \\
\text { R30 }\end{array}$ & 2 & & $\mathrm{ZO}$ & & 7 pearlware & \begin{tabular}{|l} 
blue \\
transfer- \\
print
\end{tabular} & & \\
\hline $\begin{array}{l}23 \text { SG272- } \\
17\end{array}$ & $\begin{array}{l}110 \\
\text { R30 }\end{array}$ & 2 & & $Z O$ & & 1 pearlware & \begin{tabular}{|l} 
blue \\
transfer- \\
print
\end{tabular} & bowl & \\
\hline $\begin{array}{l}23 S G 272- \\
17\end{array}$ & $\begin{array}{l}110 \\
\text { R30 }\end{array}$ & 2 & & 20 & & 1 pearlware & $\begin{array}{l}\text { scalloped/ } \\
\text { embossed } \\
\text { blue- } \\
\text { decorated }\end{array}$ & plate & \\
\hline \begin{tabular}{|l}
$23 S G 272-$ \\
17
\end{tabular} & $\begin{array}{l}110 \\
\text { R30 }\end{array}$ & 2 & & $\mathrm{ZO}$ & & 1 pearlware & $\begin{array}{l}\text { blue hand- } \\
\text { painted }\end{array}$ & & \\
\hline $\begin{array}{l}\text { 23SG272- } \\
17\end{array}$ & $\begin{array}{l}110 \\
\text { R30 }\end{array}$ & 2 & & $\mathrm{ZO}$ & & 1 pearlware & & $\begin{array}{l}\text { plate/sa } \\
\text { ucer }\end{array}$ & \\
\hline $\begin{array}{l}23 \text { SG272- } \\
17\end{array}$ & $\begin{array}{l}110 \\
\text { R30 }\end{array}$ & 2 & & $\mathrm{ZO}$ & & 3 pearlware & & & \\
\hline $\begin{array}{l}3 \text { SG272- } \\
65\end{array}$ & $\begin{array}{l}40 R \\
60\end{array}$ & 3 & & ZLO & & 1 gray stoneware & & $\begin{array}{l}\text { large } \\
\text { crock or } \\
\text { pot }\end{array}$ & $\begin{array}{l}\text { dark brown } \\
\text { glossy } \\
\text { glaze }\end{array}$ \\
\hline $\begin{array}{l}\text { 23SG272- } \\
165\end{array}$ & $\begin{array}{l}40 R \\
60\end{array}$ & 3 & & ZLO & & 1 yellow ware & & $\begin{array}{l}\text { bowl or } \\
\text { pitcher }\end{array}$ & \\
\hline $\begin{array}{l}\text { 23SG272- } \\
165\end{array}$ & $\begin{array}{l}40 R \\
60\end{array}$ & 3 & & ZLO & & 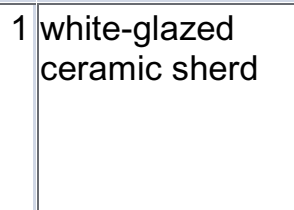 & & & $\begin{array}{l}\text { iron } \\
\text { corrosion } \\
\text { adhereing } \\
\text { to one nail } \\
\text { shank }\end{array}$ \\
\hline $\begin{array}{l}\text { 23SG272- } \\
166\end{array}$ & $\begin{array}{l}40 R \\
60\end{array}$ & 3 & 8 & ZLO & & 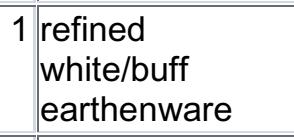 & & $\begin{array}{l}\text { possibly } \\
\text { a crock }\end{array}$ & $\begin{array}{l}\text { mottled } \\
\text { chocolate } \\
\text { brown glaze }\end{array}$ \\
\hline \begin{tabular}{|l} 
23SG272- \\
166
\end{tabular} & $\begin{array}{l}40 \mathrm{R} \\
60\end{array}$ & 3 & 8 & ZLO & & 2 yellow ware & & & \\
\hline $\begin{array}{l}\text { 23SG272- } \\
166\end{array}$ & $\begin{array}{l}40 \mathrm{R} \\
60\end{array}$ & 3 & 8 & ZLO & & 1 whiteware & $\begin{array}{l}\text { handpainte } \\
\text { d or } \\
\text { stamped, } \\
\text { small } \\
\text { green } \\
\text { design on } \\
\text { exterior }\end{array}$ & & \\
\hline $\begin{array}{l}\text { 23SG272- } \\
166\end{array}$ & $\begin{array}{l}40 R \\
60\end{array}$ & 3 & 8 & ZLO & & 1 whiteware & \begin{tabular}{|l|} 
black \\
transfer-
\end{tabular} & & \\
\hline
\end{tabular}




\begin{tabular}{|c|c|c|c|c|c|c|c|c|c|}
\hline 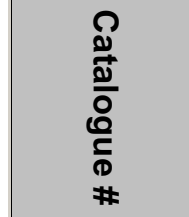 & $\underset{\rightleftharpoons}{\stackrel{c}{\rightleftharpoons}}$ & 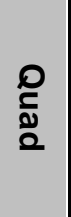 & 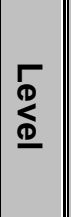 & 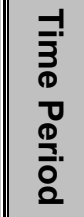 & $\begin{array}{l}\frac{D}{3} \\
0 \\
\\
\end{array}$ & 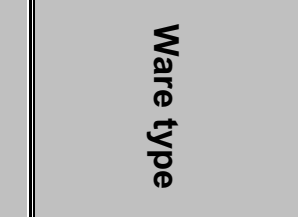 & $\begin{array}{l}\frac{0}{0} \\
0 \\
\frac{0}{a} \\
\frac{0}{*} \\
\frac{0}{2}\end{array}$ & 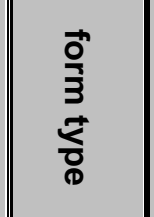 & $\begin{array}{l}\overline{5} \\
\overline{0} \\
\frac{7}{3} \\
\frac{0}{5} \\
\frac{0}{0} \\
\frac{0}{0}\end{array}$ \\
\hline & & & & & & & $\begin{array}{l}\text { print, } \\
\text { possibly } \\
\text { floral } \\
\text { design }\end{array}$ & & \\
\hline \begin{tabular}{|l|}
$23 S G 272-$ \\
166
\end{tabular} & $\begin{array}{l}40 \mathrm{R} \\
60\end{array}$ & 3 & 8 & ZLO & & 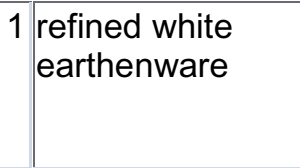 & & & $\begin{array}{l}\text { dark blue } \\
\text { glaze, } \\
\text { possibly } \\
\text { pearlware }\end{array}$ \\
\hline \begin{tabular}{|l|}
$23 S G 272-$ \\
168
\end{tabular} & $\begin{array}{l}40 \mathrm{R} \\
60\end{array}$ & 3 & 9 & ZLO & & 1 stoneware & & $\begin{array}{l}\text { crock/ju } \\
\mathrm{g}\end{array}$ & $\begin{array}{l}\text { some dark } \\
\text { brown glaze }\end{array}$ \\
\hline $\begin{array}{l}\text { 23SG272- } \\
168\end{array}$ & $\begin{array}{l}40 \mathrm{R} \\
60\end{array}$ & 3 & 9 & ZLO & & 1 faience & & & $\begin{array}{l}\text { plain white } \\
\text { tin-glaze, } \\
\text { "waterworn" } \\
\text { looking, } \\
\text { possible } \\
\text { gastrolith }\end{array}$ \\
\hline \begin{tabular}{|l}
$23 S G 272-$ \\
168
\end{tabular} & $\begin{array}{l}40 R \\
60\end{array}$ & 3 & 9 & ZLO & & 1 plain creamware & & $\begin{array}{l}\text { possibl } \\
\text { e } \\
\text { saucer }\end{array}$ & \\
\hline \begin{tabular}{|l|}
$23 S G 272-$ \\
168
\end{tabular} & $\begin{array}{l}40 \mathrm{R} \\
60\end{array}$ & 3 & 9 & ZLO & & 2 plain creamware & & $\begin{array}{l}\text { possibl } \\
\text { e } \\
\text { saucer }\end{array}$ & \\
\hline $\begin{array}{l}\text { 23SG272- } \\
168\end{array}$ & $\begin{array}{l}40 R \\
60\end{array}$ & 3 & 9 & ZLO & & $\begin{array}{l}\text { Chinese export } \\
\text { porcelain }\end{array}$ & $\begin{array}{l}\text { blue } \\
\text { decoration } \\
\text { on interior }\end{array}$ & bowl & \\
\hline \begin{tabular}{|l|}
$23 S G 272-$ \\
168
\end{tabular} & $\begin{array}{l}40 \mathrm{R} \\
60\end{array}$ & 3 & 9 & ZLO & & 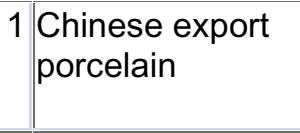 & $\begin{array}{l}\text { light brown } \\
\text { decoration } \\
\text { on exterior }\end{array}$ & bowl & \\
\hline $\begin{array}{l}\text { 23SG272- } \\
168\end{array}$ & $\begin{array}{l}40 \mathrm{R} \\
60\end{array}$ & 3 & 9 & ZLO & & 1 plain pearlware & & \begin{tabular}{|l|} 
small- \\
medium \\
bowl
\end{tabular} & \\
\hline $\begin{array}{l}\text { 23SG272- } \\
168\end{array}$ & $\begin{array}{l}40 \mathrm{R} \\
60\end{array}$ & 3 & 9 & ZLO & & 1 pearlware & $\begin{array}{l}\text { handpainte } \\
\text { d orange } \\
\text { floral } \\
\text { design on } \\
\text { exterior }\end{array}$ & \begin{tabular}{|l} 
small- \\
medium \\
bowl
\end{tabular} & \\
\hline \begin{tabular}{|l}
$23 S G 272-$ \\
168 \\
\end{tabular} & $\begin{array}{l}40 \mathrm{R} \\
60\end{array}$ & 3 & 9 & ZLO & & 1 pearlware & & & \\
\hline \begin{tabular}{|l}
$23 S G 272-$ \\
168 \\
\end{tabular} & $\begin{array}{l}40 \mathrm{R} \\
60\end{array}$ & 3 & 9 & ZLO & & 1 plain whiteware & & $\begin{array}{l}\text { plate or } \\
\text { bowl }\end{array}$ & \\
\hline \begin{tabular}{|l|}
$23 S G 272-$ \\
168
\end{tabular} & $\begin{array}{l}40 \mathrm{R} \\
60\end{array}$ & 3 & & ZLO & & 1 plain whiteware & & \begin{tabular}{|l|} 
medium \\
-large \\
bowl or \\
pitcher
\end{tabular} & \\
\hline
\end{tabular}




\begin{tabular}{|c|c|c|c|c|c|c|c|c|c|}
\hline 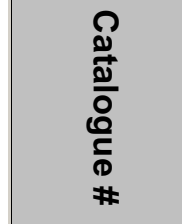 & 돌 & 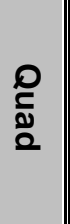 & $\underset{\mathbb{D}}{\Gamma}$ & 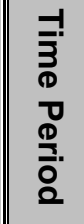 & $\begin{array}{l}\frac{1}{3} \\
\text { o } \\
\frac{1}{3} \\
\end{array}$ & 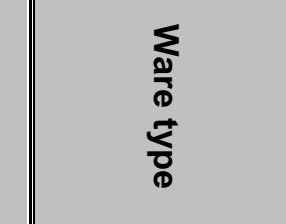 & $\begin{array}{l}\frac{0}{0} \\
\stackrel{0}{0} \\
\frac{0}{20} \\
\frac{0}{0} \\
\frac{0}{2}\end{array}$ & 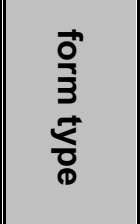 & 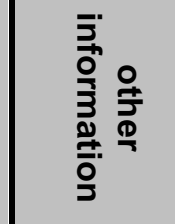 \\
\hline $\begin{array}{l}\text { 23SG272- } \\
168\end{array}$ & $\begin{array}{l}40 \mathrm{R} \\
60\end{array}$ & 3 & & ZLO & & 1 plain whiteware & & $\begin{array}{l}\text { indeter } \\
\text { minate }\end{array}$ & \\
\hline $\begin{array}{l}\text { 23SG272- } \\
168\end{array}$ & $\begin{array}{l}40 R \\
60\end{array}$ & 3 & & ZLO & & 1 whiteware & $\begin{array}{l}\text { blue } \\
\text { transfer } \\
\text { print, rows } \\
\text { of thin } \\
\text { lines }\end{array}$ & $\begin{array}{l}\text { medium } \\
\text { bown }\end{array}$ & \\
\hline $\begin{array}{l}\text { 23SG272- } \\
168\end{array}$ & $\begin{array}{l}40 R \\
60\end{array}$ & 3 & & ZLO & & 1 whiteware & $\begin{array}{l}\text { blue } \\
\text { transfer } \\
\text { print, rows } \\
\text { of thin } \\
\text { lines }\end{array}$ & $\begin{array}{l}\text { indeter } \\
\text { minate }\end{array}$ & \\
\hline $\begin{array}{l}\text { 23SG272- } \\
168\end{array}$ & $\begin{array}{l}40 \mathrm{R} \\
60\end{array}$ & 3 & 9 & ZLO & & 1 whiteware & $\begin{array}{l}\text { blue } \\
\text { transfer } \\
\text { print }\end{array}$ & & \\
\hline $\begin{array}{l}\text { 23SG272- } \\
168\end{array}$ & $\begin{array}{l}40 R \\
60\end{array}$ & 3 & 9 & ZLO & & 1 whiteware & $\begin{array}{l}\text { blue-black } \\
\text { transfer } \\
\text { rpint, floral } \\
\text { design }\end{array}$ & \begin{tabular}{|l|} 
outflarin \\
g bowl \\
or plate
\end{tabular} & \\
\hline $\begin{array}{l}\text { 23SG272- } \\
168\end{array}$ & $\begin{array}{l}40 \mathrm{R} \\
60\end{array}$ & 3 & 9 & ZLO & & 1 whiteware & $\begin{array}{l}\text { brown } \\
\text { transfer } \\
\text { print, floral } \\
\text { design on } \\
\text { interior and } \\
\text { plain white } \\
\text { exterior }\end{array}$ & \begin{tabular}{|l|} 
outflarin \\
g bowl \\
or plate
\end{tabular} & \\
\hline $\begin{array}{l}\text { 23SG272- } \\
168\end{array}$ & $\begin{array}{l}40 \mathrm{R} \\
60\end{array}$ & 3 & & ZLO & & 1 whiteware & \begin{tabular}{|l} 
brown \\
transfer \\
print, \\
geometric \\
design on \\
one side
\end{tabular} & & \\
\hline $\begin{array}{l}\text { 23SG272- } \\
168\end{array}$ & $\begin{array}{l}40 R \\
60\end{array}$ & 3 & & ZLO & & 1 whiteware & $\begin{array}{l}\text { green } \\
\text { handpainte } \\
\text { d or } \\
\text { spongewar } \\
\text { e/stamped } \\
\text { floral } \\
\text { design }\end{array}$ & & \\
\hline $\begin{array}{l}\text { 23SG272- } \\
168\end{array}$ & $\begin{array}{l}40 R \\
60\end{array}$ & 3 & 9 & ZLO & & 1 white 1 earthenware & & $\begin{array}{l}\text { plate or } \\
\text { platter }\end{array}$ & $\begin{array}{l}\text { blue shell- } \\
\text { edged }\end{array}$ \\
\hline $\begin{array}{l}\text { 23SG272- } \\
168\end{array}$ & $\begin{array}{l}40 R \\
60\end{array}$ & 3 & 9 & ZLO & & 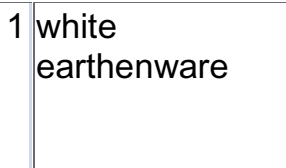 & $\begin{array}{l}\text { black } \\
\text { annular } \\
\text { decoration } \\
\text { on exterior }\end{array}$ & bowl & \\
\hline
\end{tabular}




\begin{tabular}{|c|c|c|c|c|c|c|c|c|c|}
\hline 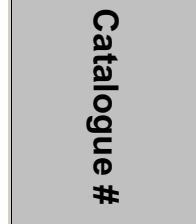 & $\underset{\rightleftharpoons}{\stackrel{c}{\supset}}$ & $\begin{array}{l}0 \\
\stackrel{0}{2} \\
\stackrel{2}{2}\end{array}$ & 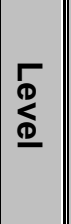 & 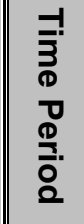 & $\begin{array}{l}\frac{1}{3} \\
\text { o } \\
\frac{2}{2} \\
\end{array}$ & 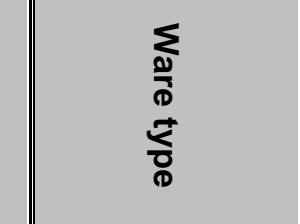 & $\begin{array}{l}\frac{0}{0} \\
\stackrel{0}{0} \\
\frac{0}{2} \\
\frac{0}{0} \\
\frac{0}{2}\end{array}$ & 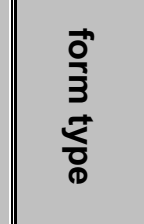 & 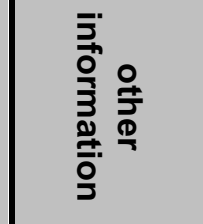 \\
\hline \begin{tabular}{|l} 
23SG272- \\
168
\end{tabular} & $\begin{array}{l}40 \mathrm{R} \\
60\end{array}$ & 3 & & ZLO & & 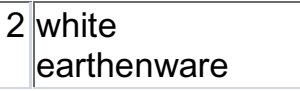 & $\begin{array}{l}\text { light blue } \\
\text { decoration }\end{array}$ & & \\
\hline $\begin{array}{l}\text { 23SG272- } \\
168\end{array}$ & $\begin{array}{l}40 R \\
60\end{array}$ & 3 & & ZLO & & 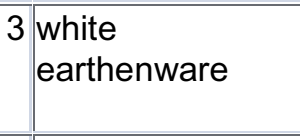 & $\begin{array}{l}\text { blue } \\
\text { transfer- } \\
\text { print }\end{array}$ & & \\
\hline $\begin{array}{l}\text { 23SG272- } \\
168\end{array}$ & $\begin{array}{l}40 \mathrm{R} \\
60\end{array}$ & 3 & & ZLO & & $\begin{array}{l}9 \text { plain white } \\
\text { refined } \\
\text { earthenware }\end{array}$ & & & \\
\hline $23 S G 272-5$ & $\begin{array}{l}150 \\
\text { RO }\end{array}$ & 1 & & ZLO & & 1 whiteware & $\begin{array}{l}\text { purple } \\
\text { transfer- } \\
\text { print }\end{array}$ & $\begin{array}{l}\text { med-lg } \\
\text { bowl }\end{array}$ & \\
\hline 23SG272-5 & $\begin{array}{l}150 \\
\text { RO }\end{array}$ & 1 & 4 & ZLO & & 1 whiteware & $\begin{array}{l}\text { purple } \\
\text { transfer } \\
\text { print }\end{array}$ & bowl & \\
\hline 23SG272-5 & $\begin{array}{l}150 \\
\mathrm{R} 0\end{array}$ & 1 & 4 & ZLO & & 3 whiteware & & $\begin{array}{l}\text { large } \\
\text { bowl }\end{array}$ & \\
\hline $23 S G 272-5$ & $\begin{array}{l}150 \\
\text { RO }\end{array}$ & 1 & 4 & ZLO & & $\begin{array}{l}3 \text { whiteware/ironst } \\
\text { one }\end{array}$ & & & \\
\hline 23SG272-5 & $\begin{array}{l}150 \\
\text { RO }\end{array}$ & 1 & 4 & ZLO & & $1 \begin{array}{l}\text { white } \\
\text { earthenware/ } \\
\text { pearlware }\end{array}$ & $\begin{array}{l}\text { blue } \\
\text { decorated }\end{array}$ & $\begin{array}{l}\text { plate or } \\
\text { large } \\
\text { bowl }\end{array}$ & \\
\hline 23SG272-5 & $\begin{array}{l}150 \\
\text { R0 }\end{array}$ & 1 & 4 & ZLO & & 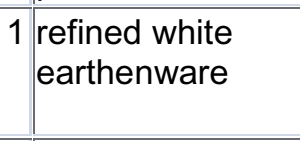 & $\begin{array}{l}\text { green } \\
\text { glaze } \\
\text { design }\end{array}$ & & "tumbled" \\
\hline 23SG272-5 & $\begin{array}{l}150 \\
\text { Ro }\end{array}$ & 1 & 4 & ZLO & & 1 yellow ware & & & \\
\hline 23SG272-5 & $\begin{array}{l}150 \\
\text { RO }\end{array}$ & 1 & 4 & ZLO & & 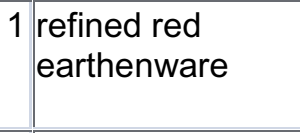 & & $\begin{array}{l}\text { med- } \\
\text { large } \\
\text { bowl }\end{array}$ & $\begin{array}{l}\text { possibly } \\
\text { early } \\
\text { Albany slig }\end{array}$ \\
\hline 23SG272-5 & $\begin{array}{l}150 \\
\text { RO }\end{array}$ & 1 & 4 & ZLO & & $\begin{array}{l}5 \text { coarse red } \\
\text { earthenware }\end{array}$ & & $\begin{array}{l}\text { possibil } \\
\text { y large } \\
\text { flower } \\
\text { pot }\end{array}$ & \\
\hline 23SG272-5 & $\begin{array}{l}150 \\
\text { RO }\end{array}$ & 1 & 4 & ZLO & & 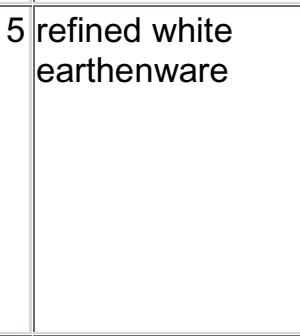 & & & $\begin{array}{l}1 \text { has } \\
\text { slightly blue } \\
\text { cast, which } \\
\text { could be } \\
\text { decoration } \\
\text { or a } \\
\text { pearlware } \\
\text { background }\end{array}$ \\
\hline 23SG272-6 & $\begin{array}{l}150 \\
\text { RO }\end{array}$ & 1 & 5 & $\mathrm{ZO}$ & & 1 white Kaolin & & pipe & \\
\hline 23SG272-6 & 150 & 1 & 5 & $\mathrm{ZO}$ & & 1 coarse red & & & \\
\hline
\end{tabular}




\begin{tabular}{|c|c|c|c|c|c|c|c|c|c|}
\hline $\begin{array}{l}\text { 尺 } \\
\stackrel{0}{0} \\
\frac{0}{0} \\
\stackrel{0}{0} \\
\frac{1}{0} \\
\#\end{array}$ & $\stackrel{c}{\mathrm{C}}$ & 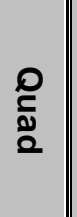 & \begin{tabular}{|l|}
$\mathbf{D}$ \\
$\stackrel{\Phi}{\Phi}$ \\
\end{tabular} & \begin{tabular}{l} 
검 \\
$\overline{3}$ \\
0 \\
0 \\
0 \\
$\frac{0}{0}$ \\
$\vdots$ \\
\hdashline
\end{tabular} & 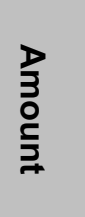 & 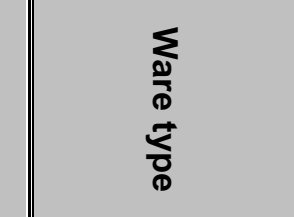 & $\begin{array}{l}\frac{0}{0} \\
\frac{0}{0} \\
\frac{0}{3} \\
\frac{0}{3}\end{array}$ & $\begin{array}{l}\underset{0}{0} \\
3 \\
3 \\
\frac{1}{3}\end{array}$ & 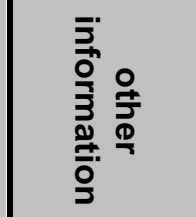 \\
\hline & Ro & & & & & earthenware & & & \\
\hline $\begin{array}{l}\text { 23SG272- } \\
168\end{array}$ & $\begin{array}{l}40 \mathrm{R} \\
60\end{array}$ & 3 & 9 & ZLO & & $\begin{array}{l}1 \text { plain white } \\
\text { refined } \\
\text { earthenware }\end{array}$ & & $\begin{array}{l}\text { bowl or } \\
\text { cup }\end{array}$ & \\
\hline $\begin{array}{l}\text { 23SG272- } \\
168\end{array}$ & $\begin{array}{l}40 \mathrm{R} \\
60\end{array}$ & 3 & 9 & ZLO & & $\begin{array}{l}1 \text { plain yellow } \\
\text { ware }\end{array}$ & & $\begin{array}{l}\text { bowl/pit } \\
\text { cher }\end{array}$ & \\
\hline \begin{tabular}{|l} 
23SG272- \\
168
\end{tabular} & $\begin{array}{l}40 \mathrm{R} \\
60\end{array}$ & 3 & 9 & ZLO & & 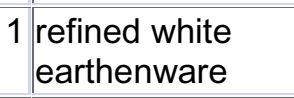 & & & $\begin{array}{l}\text { chocolate } \\
\text { brown glaze }\end{array}$ \\
\hline $\begin{array}{l}\text { 23SG272- } \\
168\end{array}$ & $\begin{array}{l}40 \mathrm{R} \\
60\end{array}$ & 3 & 9 & ZLO & & 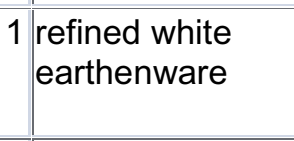 & $\begin{array}{l}\text { light green } \\
\text { handpainte } \\
\text { d design }\end{array}$ & & \\
\hline $\begin{array}{l}\text { 23SG272- } \\
170\end{array}$ & $\begin{array}{l}40 \mathrm{R} \\
60\end{array}$ & 3 & 9 & ZLO & & 1 gray stoneware & & & salt-glazed \\
\hline $\begin{array}{l}\text { 23SG272- } \\
170\end{array}$ & $\begin{array}{l}40 \mathrm{R} \\
60\end{array}$ & 3 & 9 & ZLO & & 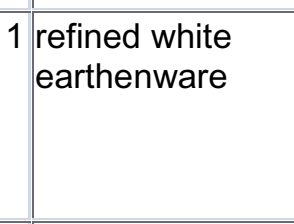 & & $\begin{array}{l}\text { large } \\
\text { crock or } \\
\text { large } \\
\text { mixing } \\
\text { bowl }\end{array}$ & $\begin{array}{l}\text { mottled } \\
\text { brown lead } \\
\text { glaze }\end{array}$ \\
\hline $\begin{array}{l}\text { 23SG272- } \\
170\end{array}$ & $\begin{array}{l}40 \mathrm{R} \\
60\end{array}$ & 3 & 9 & ZLO & & 1 yellow ware & $\begin{array}{l}\text { cream- } \\
\text { colored } \\
\text { ridges on } \\
\text { exterior }\end{array}$ & $\begin{array}{l}\text { bowl or } \\
\text { pitcher }\end{array}$ & \\
\hline $\begin{array}{l}\text { 23SG272- } \\
170\end{array}$ & $\begin{array}{l}40 \mathrm{R} \\
60\end{array}$ & 3 & 9 & ZLO & & 1 whiteware & $\begin{array}{l}\text { green } \\
\text { transfer- } \\
\text { printed }\end{array}$ & & \\
\hline $\begin{array}{l}\text { 23SG272- } \\
170\end{array}$ & $\begin{array}{l}40 \mathrm{R} \\
60\end{array}$ & 3 & 9 & ZLO & & $\begin{array}{l}2 \text { pearlware/white } \\
\text { ware }\end{array}$ & $\begin{array}{l}\text { dark blue } \\
\text { transfer- } \\
\text { printed }\end{array}$ & & $\begin{array}{l}\text { plain white } \\
\text { glaze on } \\
\text { exterior }\end{array}$ \\
\hline $\begin{array}{l}\text { 23SG272- } \\
170\end{array}$ & $\begin{array}{l}40 \mathrm{R} \\
60\end{array}$ & 3 & 9 & ZLO & & $\begin{array}{l}1 \text { pearlware/white } \\
\text { ware }\end{array}$ & & & \\
\hline $\begin{array}{l}\text { 23SG272- } \\
170\end{array}$ & $\begin{array}{l}40 \mathrm{R} \\
60\end{array}$ & 3 & 9 & ZLO & & 1 whiteware & & $\begin{array}{l}\text { plate or } \\
\text { bowl }\end{array}$ & \\
\hline $\begin{array}{l}\text { 23SG272- } \\
170\end{array}$ & $\begin{array}{l}40 \mathrm{R} \\
60\end{array}$ & 3 & 9 & ZLO & & 9 whiteware & & \begin{tabular}{|l|} 
plate or \\
bowl
\end{tabular} & \\
\hline $\begin{array}{l}\text { 23SG272- } \\
170\end{array}$ & $\begin{array}{l}40 \mathrm{R} \\
60\end{array}$ & 3 & 9 & ZLO & & 1 ironstone & & $\begin{array}{l}\text { bowl or } \\
\text { pitcher }\end{array}$ & \\
\hline $\begin{array}{l}\text { 23SG272- } \\
171\end{array}$ & $\begin{array}{l}40 \mathrm{R} \\
60\end{array}$ & 3 & 9 & ZLO & & $\begin{array}{l}1 \text { whiteware/ironst } \\
\text { one }\end{array}$ & & \begin{tabular}{|l|} 
medium \\
-large \\
bowl
\end{tabular} & \\
\hline $\begin{array}{l}\text { 23SG272- } \\
171\end{array}$ & $\begin{array}{l}40 \mathrm{R} \\
60\end{array}$ & 3 & 9 & ZLO & & 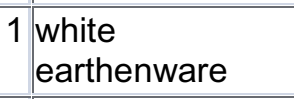 & & & \\
\hline $\begin{array}{l}23 S G 272- \\
171\end{array}$ & $\begin{array}{l}40 \mathrm{R} \\
60\end{array}$ & 3 & 9 & ZLO & & 1 stoneware & & & $\begin{array}{l}\text { gray body } \\
\text { with } \\
\text { medium }\end{array}$ \\
\hline
\end{tabular}




\begin{tabular}{|c|c|c|c|c|c|c|c|c|c|}
\hline 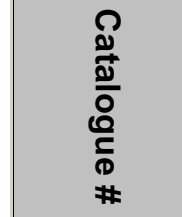 & 돌 & 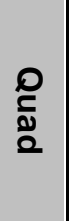 & $\underset{\Phi}{\stackrel{\Phi}{\Phi}}$ & $\begin{array}{l}\text { 검 } \\
\overline{3} \\
0 \\
0 \\
0 \\
\frac{0}{0} \\
\vdots \\
0\end{array}$ & $\begin{array}{l}\frac{1}{3} \\
\text { o } \\
\frac{2}{2} \\
2\end{array}$ & 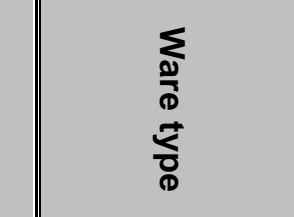 & 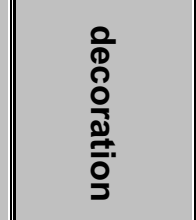 & $\begin{array}{l}\text { o } \\
\frac{3}{3} \\
\text { ¿ } \\
\text { ¿ }\end{array}$ & 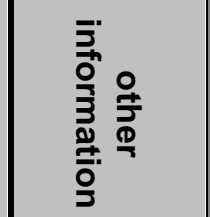 \\
\hline & & & & & & & & & brown glaze \\
\hline $\begin{array}{l}\text { 23SG272- } \\
172\end{array}$ & $\begin{array}{l}40 \mathrm{R} \\
60\end{array}$ & 3 & 9 & ZLO & & 1 pearlware & & & \\
\hline $\begin{array}{l}\text { 23SG272- } \\
172\end{array}$ & $\begin{array}{l}40 \mathrm{R} \\
60\end{array}$ & 3 & 9 & ZLO & & 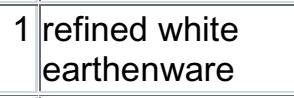 & & & \\
\hline $\begin{array}{l}\text { 23SG272- } \\
172\end{array}$ & $\begin{array}{l}40 \mathrm{R} \\
60\end{array}$ & 3 & 9 & ZLO & & 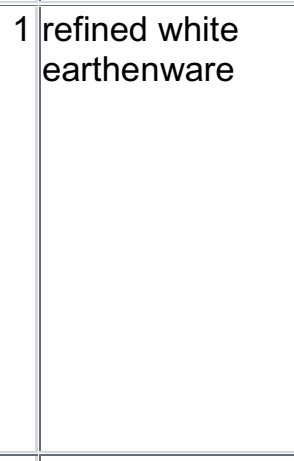 & $\begin{array}{l}\text { possibly } \\
\text { blue } \\
\text { transfer- } \\
\text { printed, } \\
\text { some red } \\
\text { present } \\
\text { (possibly } \\
\text { blue and } \\
\text { red } \\
\text { handpainte } \\
\text { d) } \\
\end{array}$ & & \\
\hline $\begin{array}{l}\text { 23SG272- } \\
173\end{array}$ & $\begin{array}{l}40 \mathrm{R} \\
60\end{array}$ & 3 & 11. & $\mathrm{ZO}$ & & 1 faience & & & \\
\hline $\begin{array}{l}\text { 23SG272- } \\
173\end{array}$ & $\begin{array}{l}40 \mathrm{R} \\
60\end{array}$ & 3 & 11 . & 20 & & 1 late creamware & & $\begin{array}{l}\text { med- } \\
\text { large } \\
\text { bowl }\end{array}$ & \\
\hline $\begin{array}{l}23 \text { SG272- } \\
17\end{array}$ & $\begin{array}{l}110 \\
\text { R30 }\end{array}$ & 2 & 5 & $\mathrm{ZO}$ & & $\begin{array}{l}1 \text { white and blue } \\
\text { pearlware }\end{array}$ & $\begin{array}{l}\text { possibily } \\
\text { transfer- } \\
\text { print }\end{array}$ & & \\
\hline $\begin{array}{l}\text { 23SG272- } \\
17\end{array}$ & $\begin{array}{l}110 \\
\text { R30 }\end{array}$ & 2 & & $\mathrm{ZO}$ & & 1 whiteware & \begin{tabular}{|l} 
blue \\
transfer- \\
print
\end{tabular} & bowl & \\
\hline $\begin{array}{l}23 \text { SG272- } \\
17\end{array}$ & $\begin{array}{l}110 \\
\text { R30 }\end{array}$ & 2 & & $\mathrm{ZO}$ & & 2 whiteware & $\begin{array}{l}\text { brown } \\
\text { transfer- } \\
\text { print }\end{array}$ & $\begin{array}{l}\text { plate/lar } \\
\text { ge bowl }\end{array}$ & \\
\hline $\begin{array}{l}23 S G 272- \\
17\end{array}$ & $\begin{array}{l}110 \\
\text { R30 }\end{array}$ & 2 & 5 & $\mathrm{ZO}$ & & 1 whiteware & $\begin{array}{l}\text { red } \\
\text { transfer- } \\
\text { print }\end{array}$ & \begin{tabular}{|l} 
bowl/pl \\
ate
\end{tabular} & \\
\hline $\begin{array}{l}\text { 23SG272- } \\
17\end{array}$ & $\begin{array}{l}110 \\
\text { R30 }\end{array}$ & 2 & & $\mathrm{ZO}$ & & 2 whiteware & \begin{tabular}{|l} 
red \\
transfer- \\
print
\end{tabular} & & \\
\hline $\begin{array}{l}23 S G 272- \\
17\end{array}$ & $\begin{array}{l}110 \\
\text { R30 }\end{array}$ & 2 & & $\mathrm{ZO}$ & & 1 whiteware & \begin{tabular}{|l} 
purple \\
transfer- \\
print
\end{tabular} & $\begin{array}{l}\text { bowl/pl } \\
\text { ate }\end{array}$ & \\
\hline $\begin{array}{l}23 \text { SG272- } \\
17\end{array}$ & $\begin{array}{l}110 \\
\text { R30 }\end{array}$ & 2 & 5 & $\mathrm{ZO}$ & & 2 whiteware & $\begin{array}{l}\text { black } \\
\text { transfer- } \\
\text { print }\end{array}$ & & \\
\hline $\begin{array}{l}\text { 23SG272- } \\
17\end{array}$ & $\begin{array}{l}110 \\
\text { R30 }\end{array}$ & 2 & & $\mathrm{ZO}$ & & 1 whiteware & $\begin{array}{l}\text { green } \\
\text { transfer- }\end{array}$ & & \\
\hline
\end{tabular}




\begin{tabular}{|c|c|c|c|c|c|c|c|c|c|}
\hline 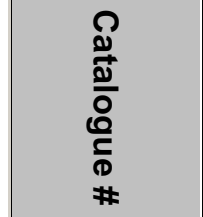 & 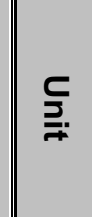 & 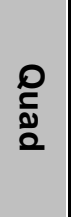 & 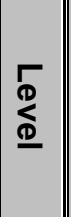 & 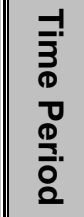 & 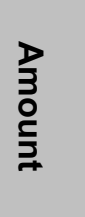 & 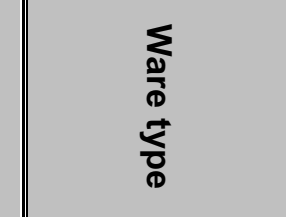 & 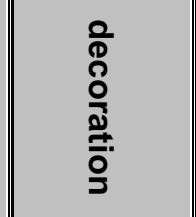 & 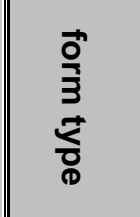 & $\begin{array}{l}\overline{5} \\
\overline{0} \\
\frac{7}{3} \\
\frac{0}{5} \\
\frac{0}{0} \\
\frac{0}{0}\end{array}$ \\
\hline & & & & & & & $\begin{array}{l}\text { print, fleur- } \\
\text { de-lys } \\
\text { design }\end{array}$ & & \\
\hline $\begin{array}{l}23 \text { SG272- } \\
17\end{array}$ & $\begin{array}{l}110 \\
\text { R30 }\end{array}$ & 2 & & $\mathrm{ZO}$ & & 1 whiteware & $\begin{array}{l}\text { green } \\
\text { hand- } \\
\text { painted or } \\
\text { annular } \\
\text { decoration }\end{array}$ & bowl & \\
\hline $\begin{array}{l}\text { 23SG272- } \\
17\end{array}$ & $\begin{array}{l}110 \\
\text { R30 }\end{array}$ & 2 & & $\mathrm{ZO}$ & & 1 whiteware & & plate & \\
\hline $\begin{array}{l}\text { 23SG272- } \\
17\end{array}$ & $\begin{array}{l}110 \\
\text { R30 }\end{array}$ & 2 & & $\mathrm{ZO}$ & & 1 whiteware & & $\begin{array}{l}\text { plate/lar } \\
\text { ge bowl }\end{array}$ & \\
\hline $\begin{array}{l}\text { 23SG272- } \\
17\end{array}$ & $\begin{array}{l}110 \\
\text { R30 }\end{array}$ & 2 & & $\mathrm{ZO}$ & & 7 whiteware & & & \\
\hline $\begin{array}{l}23 \text { SG272- } \\
17\end{array}$ & $\begin{array}{l}110 \\
\text { R30 }\end{array}$ & 2 & & $\mathrm{ZO}$ & & $\begin{array}{l}1 \text { pearlware/white } \\
\text { ware }\end{array}$ & $\begin{array}{l}\text { brown thin } \\
\text { band } \\
\text { w/yellow } \\
\text { band } \\
\text { below, } \\
\text { orange } \\
\text { arch-like } \\
\text { ?floral } \\
\text { design } \\
\text { above } \\
\text { bands }\end{array}$ & & \\
\hline $\begin{array}{l}\text { 23SG272- } \\
17\end{array}$ & $\begin{array}{l}110 \\
\text { R30 }\end{array}$ & 2 & & $\mathrm{ZO}$ & & 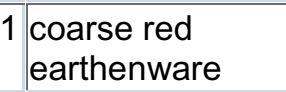 & & & $\begin{array}{l}\text { dark brown } \\
\text { lead glaze }\end{array}$ \\
\hline \begin{tabular}{|l|}
$23 S G 272-$ \\
18
\end{tabular} & $\begin{array}{l}110 \\
\text { R30 }\end{array}$ & 2 & & $\mathrm{ZO}$ & & $\begin{array}{l}1 \text { pearlware/white } \\
\text { ware }\end{array}$ & $\begin{array}{l}\text { darkbrown, } \\
\text { thin, yellow } \\
\text { bands }\end{array}$ & \begin{tabular}{|l} 
tea \\
bowl or \\
cup
\end{tabular} & \\
\hline $\begin{array}{l}\text { 23SG272- } \\
18\end{array}$ & $\begin{array}{l}110 \\
\text { R30 }\end{array}$ & 2 & & $\mathrm{ZO}$ & & $\begin{array}{l}1 \text { pearlware/white } \\
\text { ware }\end{array}$ & & & \\
\hline 23SG272-2 & $\begin{array}{l}150 \\
\text { RO }\end{array}$ & 1 & & ZLO & & 1 whiteware & & $\begin{array}{l}\text { probabl } \\
\text { y } \\
\text { medium } \\
\text { bowl }\end{array}$ & \\
\hline $\begin{array}{l}\text { 23SG272- } \\
173\end{array}$ & $\begin{array}{l}40 \mathrm{R} \\
60\end{array}$ & 3 & 11 & $\mathrm{ZO}$ & & 1 late creamware & & $\begin{array}{l}\text { med- } \\
\text { large } \\
\text { bowl }\end{array}$ & \\
\hline $\begin{array}{l}\text { 23SG272- } \\
173\end{array}$ & $\begin{array}{l}40 \mathrm{R} \\
60\end{array}$ & 3 & 11 & $\mathrm{ZO}$ & & 1 pearlware & & & \\
\hline $\begin{array}{l}\text { 23SG272- } \\
173\end{array}$ & $\begin{array}{l}40 \mathrm{R} \\
60\end{array}$ & 3 & 11 & $\mathrm{ZO}$ & & 1 whiteware & \begin{tabular}{|l} 
blue \\
transfer- \\
printed, \\
floral
\end{tabular} & $\begin{array}{l}\text { small } \\
\text { bowl }\end{array}$ & \\
\hline
\end{tabular}




\begin{tabular}{|c|c|c|c|c|c|c|c|c|c|}
\hline 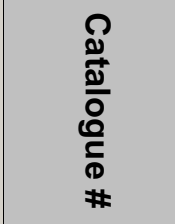 & $\stackrel{c}{ㄱ}$ & 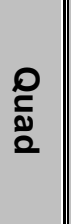 & $\underset{\substack{\Phi \\
\Phi}}{\stackrel{\Phi}{\Phi}}$ & 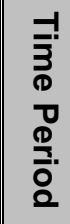 & $\begin{array}{l}\frac{1}{3} \\
\text { 0 } \\
\frac{2}{2} \\
\end{array}$ & 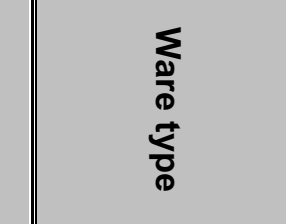 & $\begin{array}{l}\frac{0}{0} \\
\stackrel{8}{0} \\
\frac{0}{30} \\
\frac{0}{0} \\
\frac{0}{2}\end{array}$ & 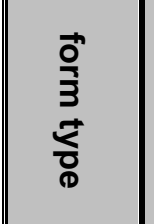 & 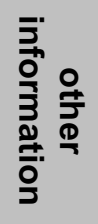 \\
\hline & & & & & & & $\begin{array}{l}\text { design on } \\
\text { interior and } \\
\text { exterior }\end{array}$ & & \\
\hline $\begin{array}{l}\text { 23SG272- } \\
173\end{array}$ & $\begin{array}{l}40 \mathrm{R} \\
60\end{array}$ & 3 & 11 & $\mathrm{ZO}$ & & 1 whiteware & $\begin{array}{l}\text { dark brown } \\
\text { transfer- } \\
\text { prin on } \\
\text { interior }\end{array}$ & $\begin{array}{l}\text { medium } \\
\text { bowl/sa } \\
\text { ucer }\end{array}$ & \\
\hline $\begin{array}{l}\text { 23SG272- } \\
173\end{array}$ & $\begin{array}{l}40 \mathrm{R} \\
60\end{array}$ & 3 & 11. & $\mathrm{ZO}$ & & 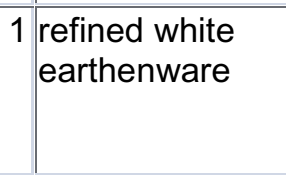 & $\begin{array}{l}\text { handpainte } \\
\text { d green } \\
\text { design on } \\
\text { exterior }\end{array}$ & & \\
\hline $\begin{array}{l}\text { 23SG272- } \\
173\end{array}$ & $\begin{array}{l}40 \mathrm{R} \\
60\end{array}$ & 3 & 11. & $\mathrm{ZO}$ & & 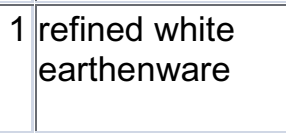 & \begin{tabular}{|l|} 
blue- \\
decoreated \\
design
\end{tabular} & & \\
\hline $\begin{array}{l}\text { 23SG272- } \\
173\end{array}$ & $\begin{array}{l}40 \mathrm{R} \\
60\end{array}$ & 3 & 11. & ZO & & 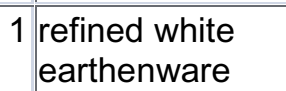 & & & \\
\hline $\begin{array}{l}\text { 23SG272- } \\
174\end{array}$ & $\begin{array}{l}40 \mathrm{R} \\
60\end{array}$ & 3 & 12 & ZO & & 1 whiteware & $\begin{array}{l}\text { blue } \\
\text { transfer } \\
\text { print }\end{array}$ & $\begin{array}{l}\text { med- } \\
\text { large } \\
\text { bowl }\end{array}$ & \\
\hline $\begin{array}{l}\text { 23SG272- } \\
174\end{array}$ & $\begin{array}{l}40 \mathrm{R} \\
60\end{array}$ & 3 & 12 & $\mathrm{ZO}$ & & 1 whiteware & \begin{tabular}{|l} 
blue \\
transfer \\
print
\end{tabular} & $\begin{array}{l}\text { med- } \\
\text { large } \\
\text { bowl }\end{array}$ & \\
\hline $\begin{array}{l}\text { 23SG272- } \\
174\end{array}$ & $\begin{array}{l}40 \mathrm{R} \\
60\end{array}$ & 3 & 12 & $\mathrm{ZO}$ & & 1 pearlware & & \begin{tabular}{|l} 
med- \\
large \\
vessel, \\
possibly \\
plate/bo \\
wl
\end{tabular} & \\
\hline $\begin{array}{l}\text { 23SG272- } \\
174\end{array}$ & $\begin{array}{l}40 \mathrm{R} \\
60\end{array}$ & 3 & 12 & $\mathrm{ZO}$ & & 1 late creamware & & & \\
\hline $\begin{array}{l}\text { 23SG272- } \\
174\end{array}$ & $\begin{array}{l}40 \mathrm{R} \\
60\end{array}$ & 3 & 12 & ZO & & 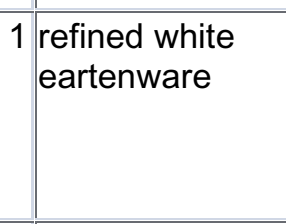 & $\begin{array}{l}\text { handpainte } \\
\text { d yellow \& } \\
\text { brown } \\
\text { design on } \\
\text { exterior }\end{array}$ & $\begin{array}{l}\text { med- } \\
\text { large } \\
\text { bowl }\end{array}$ & \\
\hline $\begin{array}{l}\text { 23SG272- } \\
174\end{array}$ & $\begin{array}{l}40 \mathrm{R} \\
60\end{array}$ & 3 & 12 & $\mathrm{ZO}$ & & $\begin{array}{l}9 \text { refined white } \\
\text { earthenware }\end{array}$ & & & \\
\hline $\begin{array}{l}\text { 23SG272- } \\
175\end{array}$ & $\begin{array}{l}40 \mathrm{R} \\
60\end{array}$ & 3 & 13 & ZO & & 1 pearlware & $\begin{array}{l}\text { handpainte } \\
\text { d blue } \\
\text { design on } \\
\text { light blue } \\
\text { backgroun } \\
\text { d on } \\
\text { interior }\end{array}$ & $\begin{array}{l}\text { plate/bo } \\
\text { wl }\end{array}$ & \\
\hline 23SG272- & 40R & 3 & 13 & ZO & & 1 pearlware & blue-and- & large & \\
\hline
\end{tabular}




\begin{tabular}{|c|c|c|c|c|c|c|c|c|c|}
\hline 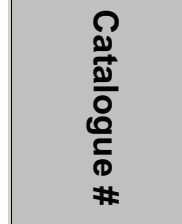 & 돌 & 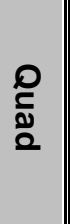 & $\underset{\mathbb{\Phi}}{\Gamma}$ & 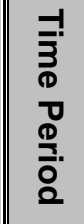 & 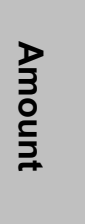 & 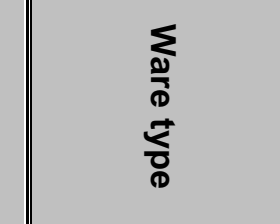 & $\begin{array}{l}\frac{0}{0} \\
\frac{2}{0} \\
0 \\
\frac{0}{2} \\
\frac{0}{2}\end{array}$ & 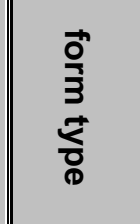 & 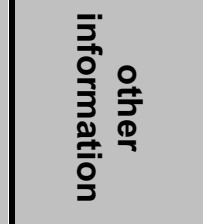 \\
\hline 175 & 60 & & & & & & $\begin{array}{l}\text { green } \\
\text { handpainte } \\
\text { d on } \\
\text { exterior }\end{array}$ & $\begin{array}{l}\text { holloww } \\
\text { are } \\
\text { vessel, } \\
\text { pitcher/l } \\
\text { arge } \\
\text { bowl/se } \\
\text { rving } \\
\text { dish or } \\
\text { lid? }\end{array}$ & \\
\hline $\begin{array}{l}\text { 23SG272- } \\
175\end{array}$ & $\begin{array}{l}40 R \\
60\end{array}$ & 3 & 13 & $\mathrm{ZO}$ & & 1 pearlware & $\begin{array}{l}\text { blue shell- } \\
\text { edge }\end{array}$ & plate & \\
\hline $\begin{array}{l}\text { 23SG272- } \\
175\end{array}$ & $\begin{array}{l}40 \mathrm{R} \\
60\end{array}$ & 3 & 13 & $\mathrm{ZO}$ & & 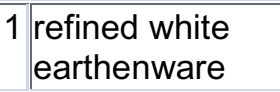 & $\begin{array}{l}\text { green } \\
\text { shell-edge }\end{array}$ & & \\
\hline $\begin{array}{l}\text { 23SG272- } \\
175\end{array}$ & $\begin{array}{l}40 R \\
60\end{array}$ & 3 & 13 & $\mathrm{ZO}$ & & 1 whiteware & & $\begin{array}{l}\text { holloww } \\
\text { are }\end{array}$ & \\
\hline $\begin{array}{l}\text { 23SG272- } \\
175\end{array}$ & $\begin{array}{l}40 \mathrm{R} \\
60\end{array}$ & 3 & 13 & $\mathrm{ZO}$ & & 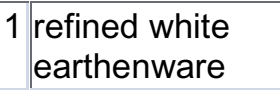 & & & \\
\hline $\begin{array}{l}\text { 23SG272- } \\
176\end{array}$ & $\begin{array}{l}40 R \\
60\end{array}$ & 3 & 14 & $\mathrm{ZO}$ & & 1 stoneware & & $\begin{array}{l}\text { jug or } \\
\text { crock }\end{array}$ & $\begin{array}{l}\text { dark } \\
\text { reddish- } \\
\text { brown } \\
\text { paste/ } \\
\text { chocolate } \\
\text { brown glaze } \\
\text { on interior; } \\
\text { brown \& } \\
\text { green glaze } \\
\text { on exterior }\end{array}$ \\
\hline $\begin{array}{l}\text { 23SG272- } \\
176\end{array}$ & $\begin{array}{l}40 R \\
60\end{array}$ & 3 & 14 & $\mathrm{ZO}$ & & 1 late creamware & & $\begin{array}{l}\text { holloww } \\
\text { are }\end{array}$ & \\
\hline $\begin{array}{l}\text { 23SG272- } \\
176\end{array}$ & $\begin{array}{l}40 R \\
60\end{array}$ & 3 & 14 & $\mathrm{ZO}$ & & 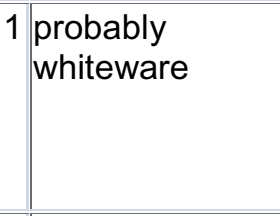 & $\begin{array}{l}\text { black or } \\
\text { dark brown } \\
\text { transfer- } \\
\text { print on } \\
\text { interior }\end{array}$ & $\begin{array}{l}\text { large } \\
\text { shallow } \\
\text { bowl }\end{array}$ & \\
\hline $\begin{array}{l}\text { 23SG272- } \\
176\end{array}$ & $\begin{array}{l}40 R \\
60\end{array}$ & 3 & 14 & $\mathrm{ZO}$ & & 2 whiteware & & & \\
\hline \begin{tabular}{|l} 
23SG272- \\
176
\end{tabular} & $\begin{array}{l}40 \mathrm{R} \\
60\end{array}$ & 3 & 14 & $\mathrm{ZO}$ & & 2 pearlware & & & \\
\hline $\begin{array}{l}\text { 23SG272- } \\
176\end{array}$ & $\begin{array}{l}40 R \\
60\end{array}$ & 3 & 14 & $\mathrm{ZO}$ & & 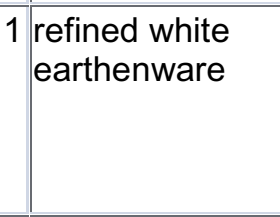 & $\begin{array}{l}\text { green } \\
\text { hand- } \\
\text { painted } \\
\text { design on } \\
\text { exterior }\end{array}$ & & \\
\hline $\begin{array}{l}\text { 23SG272- } \\
176\end{array}$ & $\begin{array}{l}40 \mathrm{R} \\
60\end{array}$ & 3 & 14 & ZO & & 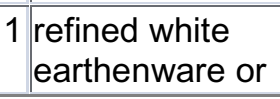 & & & \\
\hline
\end{tabular}




\begin{tabular}{|c|c|c|c|c|c|c|c|c|c|}
\hline 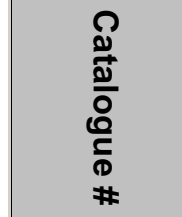 & 돌 & 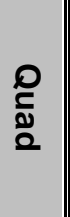 & $\underset{\mathbb{D}}{\Gamma}$ & 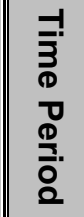 & 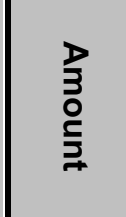 & 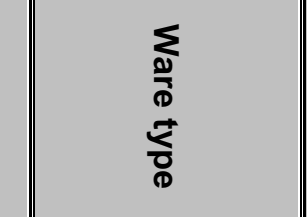 & 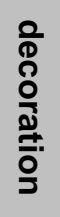 & 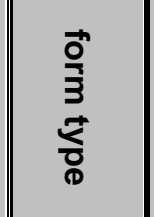 & 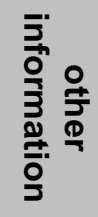 \\
\hline & & & & & \multicolumn{2}{|r|}{ ironstone } & & & \\
\hline $\begin{array}{l}\text { 23SG272- } \\
176\end{array}$ & $\begin{array}{l}40 R \\
60\end{array}$ & 3 & 14 & $\mathrm{ZO}$ & \multicolumn{2}{|r|}{1 frag, white? } & & & \\
\hline $\begin{array}{l}\text { 23SG272- } \\
179\end{array}$ & $\begin{array}{l}40 R \\
60\end{array}$ & 3 & 15 & $\mathrm{ZO}$ & \multicolumn{2}{|r|}{1 whiteware } & & $\begin{array}{l}\text { medium } \\
\text {-large } \\
\text { bowl }\end{array}$ & \\
\hline $\begin{array}{l}\text { 23SG272- } \\
179\end{array}$ & $\begin{array}{l}40 R \\
60\end{array}$ & 3 & 15 & $\mathrm{ZO}$ & \multicolumn{2}{|r|}{2 late creamware } & & & \\
\hline $\begin{array}{l}\text { 23SG272- } \\
179\end{array}$ & $\begin{array}{l}40 R \\
60\end{array}$ & 3 & 15 & $\mathrm{ZO}$ & \multicolumn{2}{|r|}{ 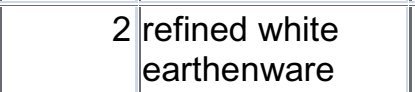 } & & & \\
\hline $\begin{array}{l}23 S G 272- \\
13\end{array}$ & $\begin{array}{l}110 \\
\text { R30 }\end{array}$ & 2 & & ZLO & \multicolumn{2}{|r|}{ 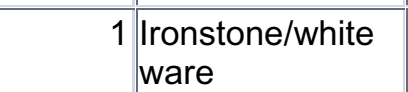 } & & $\begin{array}{l}\text { plate/pl } \\
\text { atter }\end{array}$ & \\
\hline $\begin{array}{l}23 S G 272- \\
13\end{array}$ & $\begin{array}{l}110 \\
\text { R30 }\end{array}$ & 2 & & ZLO & \multicolumn{2}{|r|}{1 red earthenware } & & $\begin{array}{l}\text { possibly } \\
\text { flowerp } \\
\text { ot }\end{array}$ & \\
\hline $\begin{array}{l}\text { 23SG272- } \\
14\end{array}$ & $\begin{array}{l}110 \\
\text { R30 }\end{array}$ & 2 & & ZLO & \multicolumn{2}{|r|}{$\begin{array}{l}18 \begin{array}{l}\text { frags. Kaolin } \\
\text { clay }\end{array} \\
\end{array}$} & & & \\
\hline & & & & & 425 & & & & \\
\hline
\end{tabular}


APPENDIX C

INVENTORY ANALYSIS 


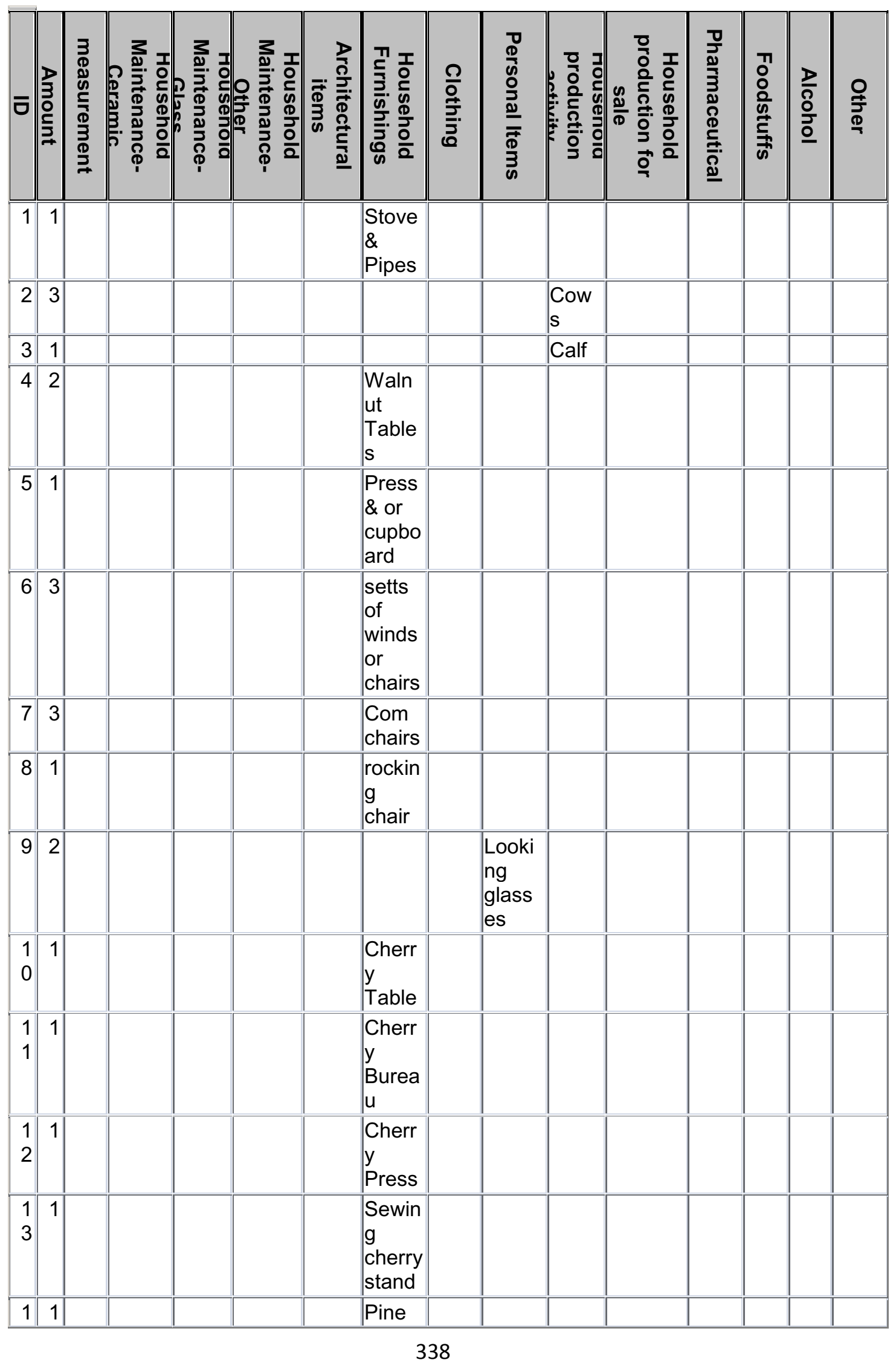




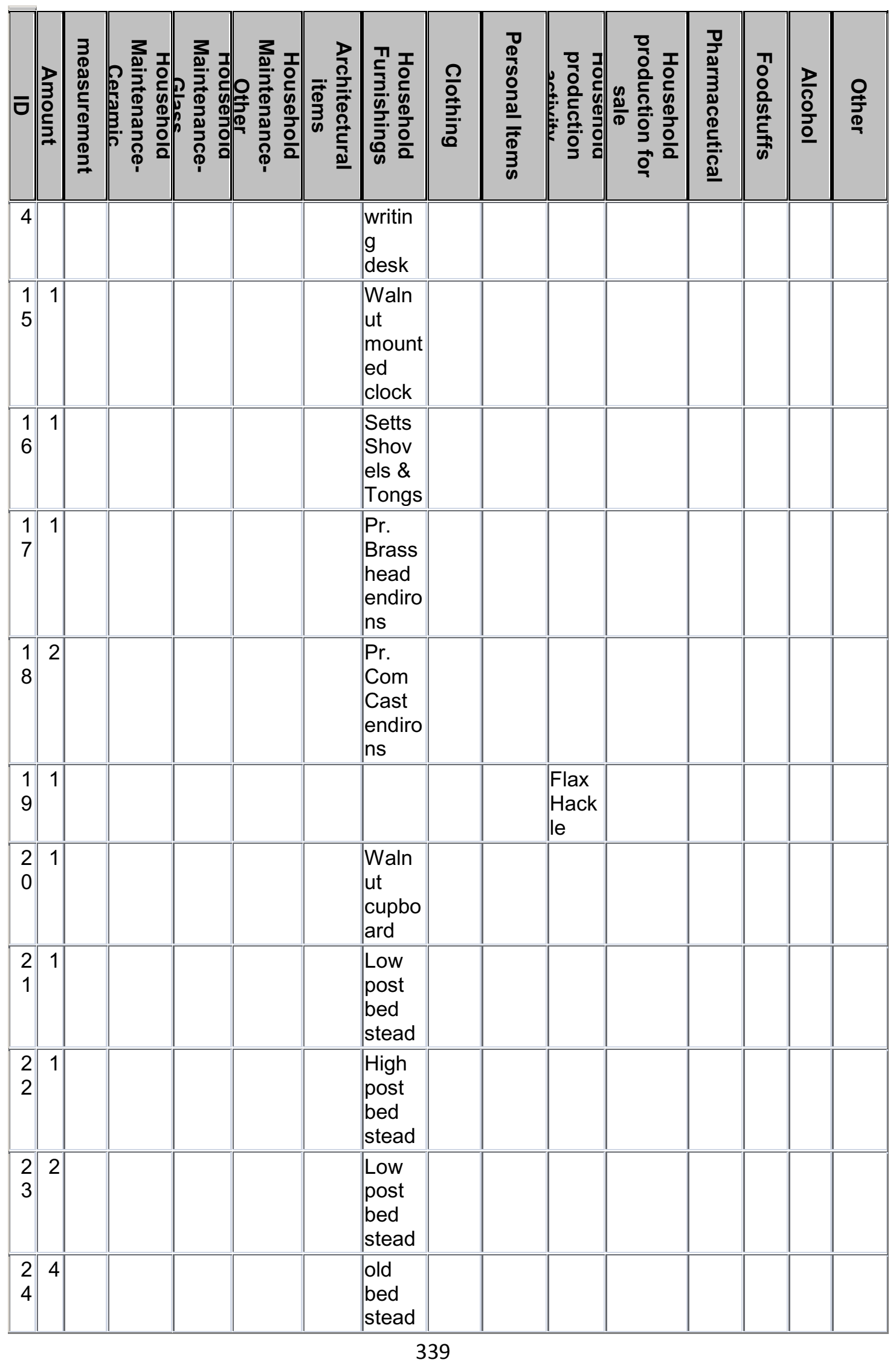




\begin{tabular}{|c|c|c|c|c|c|c|c|c|c|c|c|c|c|c|c|}
\hline б & 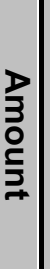 & $\begin{array}{l}3 \\
3 \\
0 \\
0 \\
0 \\
0 \\
\overline{2} \\
0 \\
3 \\
0 \\
0 \\
2 \\
2\end{array}$ & 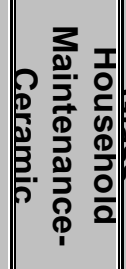 & 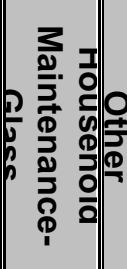 & 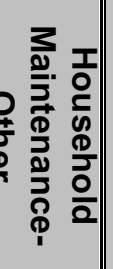 & 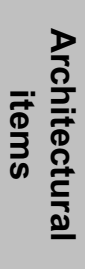 & 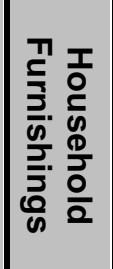 & $\begin{array}{l}\frac{\Omega}{0} \\
\frac{+}{5} \\
\frac{5}{0}\end{array}$ & 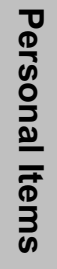 & 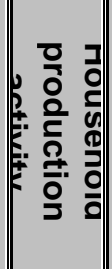 & 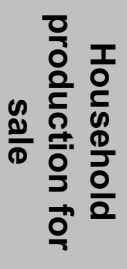 & 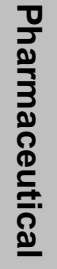 & 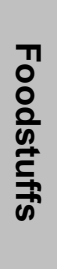 & $\begin{array}{l}\frac{D}{\delta} \\
\frac{0}{\sigma} \\
\text { o }\end{array}$ & $\begin{array}{l}\text { 옴 } \\
\text { Ф્ }\end{array}$ \\
\hline & & & & & & & $\mathrm{s}$ & & & & & & & & \\
\hline $\begin{array}{l}2 \\
5\end{array}$ & 4 & & & & & & $\begin{array}{l}\text { feathe } \\
r \text { beds }\end{array}$ & & & & & & & & \\
\hline $\begin{array}{l}2 \\
6\end{array}$ & 4 & & & & & & $\begin{array}{l}\text { feathe } \\
r \text { beds } \\
\text { small } \\
\text { er }\end{array}$ & & & & & & & & \\
\hline $\begin{array}{l}2 \\
7\end{array}$ & 2 & & & & & & $\begin{array}{l}\text { Mattr } \\
\text { as }\end{array}$ & & & & & & & & \\
\hline $\begin{array}{l}2 \\
8 \\
\end{array}$ & 9 & & & & & & $\begin{array}{l}\text { Straw } \\
\text { beds }\end{array}$ & & & & & & & & \\
\hline $\begin{array}{l}2 \\
9\end{array}$ & $\begin{array}{l}1 \\
4\end{array}$ & & & & & & $\begin{array}{l}\text { bed } \\
\text { quilts }\end{array}$ & & & & & & & & \\
\hline $\begin{array}{l}3 \\
0\end{array}$ & $\begin{array}{l}1 \\
2\end{array}$ & & & & & & $\begin{array}{l}\text { bed } \\
\text { blank } \\
\text { ets }\end{array}$ & & & & & & & & \\
\hline $\begin{array}{l}3 \\
1\end{array}$ & $\begin{array}{l}2 \\
4\end{array}$ & & & & & & $\begin{array}{l}\text { Cotto } \\
n \\
\text { sheet } \\
\text { s }\end{array}$ & & & & & & & & \\
\hline $\begin{array}{l}3 \\
2\end{array}$ & 2 & & & & & & $\begin{array}{l}\text { Linen } \\
\text { sheet } \\
\text { s }\end{array}$ & & & & & & & & \\
\hline $\begin{array}{l}3 \\
3\end{array}$ & 6 & & & & & & $\begin{array}{l}\text { Cotto } \\
\text { n } \\
\text { sheet } \\
\text { s }\end{array}$ & & & & & & & & \\
\hline $\begin{array}{l}3 \\
4 \\
\end{array}$ & 4 & & & & & & $\begin{array}{l}\text { bed } \\
\text { sprea } \\
\text { ds }\end{array}$ & & & & & & & & \\
\hline $\begin{array}{l}3 \\
5\end{array}$ & 1 & & & & & & $\begin{array}{l}\text { bed } \\
\text { sprea } \\
\text { ds } \\
\text { Small } \\
\text { er }\end{array}$ & & & & & & & & \\
\hline $\begin{array}{l}3 \\
6\end{array}$ & 3 & & & & & & $\begin{array}{l}\text { Table } \\
\text { cloths }\end{array}$ & & & & & & & & \\
\hline $\begin{array}{l}3 \\
7\end{array}$ & 1 & & & & & & $\begin{array}{l}\text { table } \\
\text { cloth }\end{array}$ & & & & & & & & \\
\hline $\begin{array}{l}3 \\
8\end{array}$ & 7 & & & & & & $\begin{array}{l}\text { Table } \\
\text { cloths } \\
\text { of } \\
\text { Linen }\end{array}$ & & & & & & & & \\
\hline
\end{tabular}




\begin{tabular}{|c|c|c|c|c|c|c|c|c|c|c|c|c|c|c|c|}
\hline б & 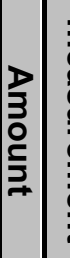 & 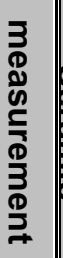 & 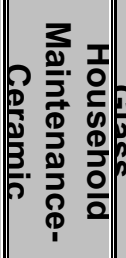 & 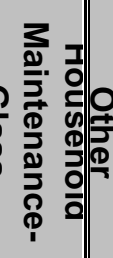 & 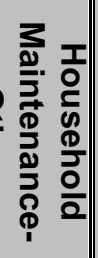 & 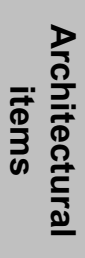 & 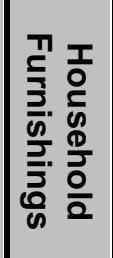 & $\begin{array}{l}\frac{\Omega}{0} \\
\frac{0}{5} \\
\frac{5}{3} \\
0\end{array}$ & 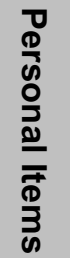 & 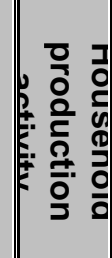 & 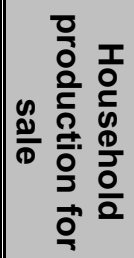 & 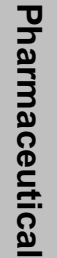 & 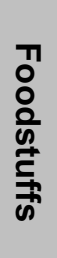 & $\begin{array}{l}\frac{8}{8} \\
\frac{0}{2} \\
\text { o }\end{array}$ & 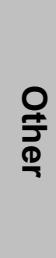 \\
\hline \begin{tabular}{|l|}
3 \\
9
\end{tabular} & \begin{tabular}{|l|}
4 \\
3
\end{tabular} & & & & & & $\begin{array}{l}\text { Linen } \\
\text { Towel } \\
\text { s }\end{array}$ & & & & & & & & \\
\hline \begin{tabular}{|l|}
4 \\
0
\end{tabular} & \begin{tabular}{|l|}
1 \\
1
\end{tabular} & & & & & & $\begin{array}{l}\text { Long } \\
\text { pillow } \\
\text { cases }\end{array}$ & & & & & & & & \\
\hline \begin{tabular}{|l|}
4 \\
1
\end{tabular} & \begin{tabular}{|l|}
2 \\
4 \\
\end{tabular} & & & & & & $\begin{array}{l}\text { short } \\
\text { pillow } \\
\text { cases }\end{array}$ & & & & & & & & \\
\hline \begin{tabular}{|l|}
4 \\
2 \\
\end{tabular} & 2 & & & & & & $\begin{array}{l}\text { Ticks } \\
\text { for } \\
\text { feathe } \\
r \text { beds }\end{array}$ & & & & & & & & \\
\hline \begin{tabular}{|l|}
4 \\
3
\end{tabular} & \begin{tabular}{|l|}
1 \\
\end{tabular} & & & & & & $\begin{array}{l}\text { Musq } \\
\text { uitoe } \\
\text { bars }\end{array}$ & & & & & & & & \\
\hline \begin{tabular}{|l|}
4 \\
4 \\
\end{tabular} & 1 & & & & & & & $\begin{array}{l}\text { cotto } \\
n\end{array}$ & & & & & & & \\
\hline \begin{tabular}{|l|}
4 \\
5
\end{tabular} & $\mid 1$ & & & & & & & $\begin{array}{l}\text { cotto } \\
n\end{array}$ & & & & & & & \\
\hline \begin{tabular}{|l|}
4 \\
6
\end{tabular} & \begin{tabular}{|ll}
1 & $y$ \\
2 &
\end{tabular} & & & & & & & $\begin{array}{l}\text { blea } \\
\text { chd. } \\
\text { Cott } \\
\text { on }\end{array}$ & & & & & & & \\
\hline \begin{tabular}{|l|}
4 \\
7
\end{tabular} & $\begin{array}{ll}2 & y \\
2 & \end{array}$ & & & & & & & $\begin{array}{l}\text { blea } \\
\text { chd. } \\
\text { Cott } \\
\text { on }\end{array}$ & & & & & & & \\
\hline \begin{tabular}{|l|}
4 \\
8 \\
\end{tabular} & \begin{tabular}{|l|l|l}
1 & $y$ \\
0 &.
\end{tabular} & & & & & & & $\begin{array}{l}\text { blea } \\
\text { chd. } \\
\text { Cott } \\
\text { on }\end{array}$ & & & & & & & \\
\hline \begin{tabular}{|l|}
4 \\
9
\end{tabular} & \begin{tabular}{|l|l|l}
2 & $y$ \\
4 &
\end{tabular} & & & & & & & $\begin{array}{l}\text { fine } \\
\text { brow } \\
\text { n } \\
\text { Cott } \\
\text { on }\end{array}$ & & & & & & & \\
\hline \begin{tabular}{|l|}
5 \\
0
\end{tabular} & $\begin{array}{lll}1 & y \\
2 & \end{array}$ & & & & & & & $\begin{array}{l}\text { Ger } \\
\text { man } \\
\text { Line } \\
n\end{array}$ & & & & & & & \\
\hline $\begin{array}{l}5 \\
1\end{array}$ & \begin{tabular}{|l|l|}
1 & $y$ \\
0 &
\end{tabular} & & & & & & & $\begin{array}{l}\text { Ger } \\
\text { man }\end{array}$ & & & & & & & \\
\hline
\end{tabular}




\begin{tabular}{|c|c|c|c|c|c|c|c|c|c|c|c|c|c|c|c|}
\hline б & 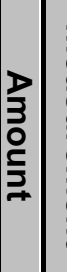 & 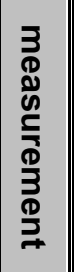 & 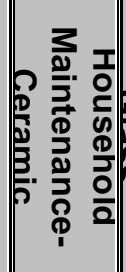 & 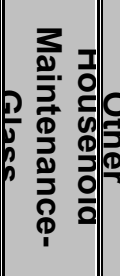 & 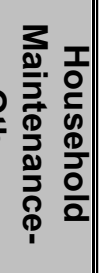 & 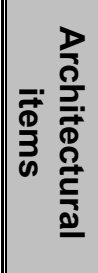 & 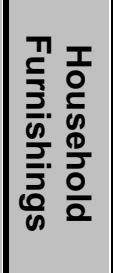 & $\mid \begin{array}{l}\frac{0}{0} \\
\frac{5}{5} \\
\frac{5}{0}\end{array}$ & 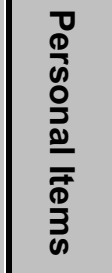 & 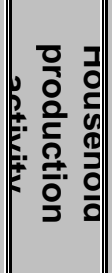 & 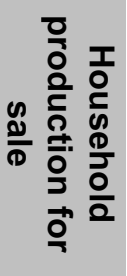 & 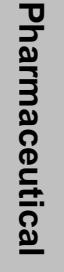 & 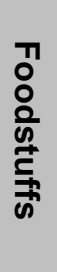 & $\begin{array}{l}\frac{8}{\Omega} \\
\frac{0}{2} \\
\text { 으 }\end{array}$ & 암 \\
\hline & & & & & & & & \begin{tabular}{|l|} 
Line \\
$n$
\end{tabular} & & & & & & & \\
\hline $\begin{array}{l}5 \\
2\end{array}$ & & yds & & & & & & $\begin{array}{l}\text { Ger } \\
\text { man } \\
\text { Line } \\
n\end{array}$ & & & & & & & \\
\hline $\begin{array}{l}5 \\
3\end{array}$ & & yds & & & & & & \begin{tabular}{|l|} 
blue \\
Jean \\
es
\end{tabular} & & & & & & & \\
\hline \begin{tabular}{|l|}
5 \\
4
\end{tabular} & & yds & & & & & & \begin{tabular}{|l} 
Russ \\
ia \\
diap \\
er
\end{tabular} & & & & & & & \\
\hline $\begin{array}{l}5 \\
5\end{array}$ & $\begin{array}{lll}1 & y \\
8 & \end{array}$ & yds & & & & & & \begin{tabular}{|l} 
Russ \\
ia \\
diap \\
er \\
\end{tabular} & & & & & & & \\
\hline $\begin{array}{l}5 \\
6 \\
\end{array}$ & 1 & & & & & & & & $\begin{array}{l}\text { Silver } \\
\text { watch }\end{array}$ & & & & & & \\
\hline $\begin{array}{l}5 \\
7\end{array}$ & 3 & & & & & & $\begin{array}{l}\text { Setts } \\
\text { windo } \\
\text { Curtai } \\
\text { ns }\end{array}$ & & & & & & & & \\
\hline $\begin{array}{l}5 \\
8\end{array}$ & 4 & & & & & & & & $\begin{array}{l}\text { Cotto } \\
n \\
\text { umbr } \\
\text { ellars }\end{array}$ & & & & & & \\
\hline $\begin{array}{l}5 \\
9\end{array}$ & 6 & & & $\begin{array}{l}\text { wine } \\
\text { glass } \\
\text { es }\end{array}$ & & & & & & & & & & & \\
\hline $\begin{array}{l}6 \\
0\end{array}$ & 5 & & & \begin{tabular}{|l|} 
Cutt \\
glass \\
drinki \\
ng \\
glass \\
es \\
\end{tabular} & & & & & & & & & & & \\
\hline $\begin{array}{l}6 \\
1\end{array}$ & 4 & & & \begin{tabular}{|l} 
Lgr \\
plain \\
Tum \\
blers
\end{tabular} & & & & & & & & & & & \\
\hline $\begin{array}{l}6 \\
2\end{array}$ & 1 & & & $\begin{array}{l}\text { Glas } \\
\text { s } \\
\text { pitch }\end{array}$ & & & & & & & & & & & \\
\hline
\end{tabular}




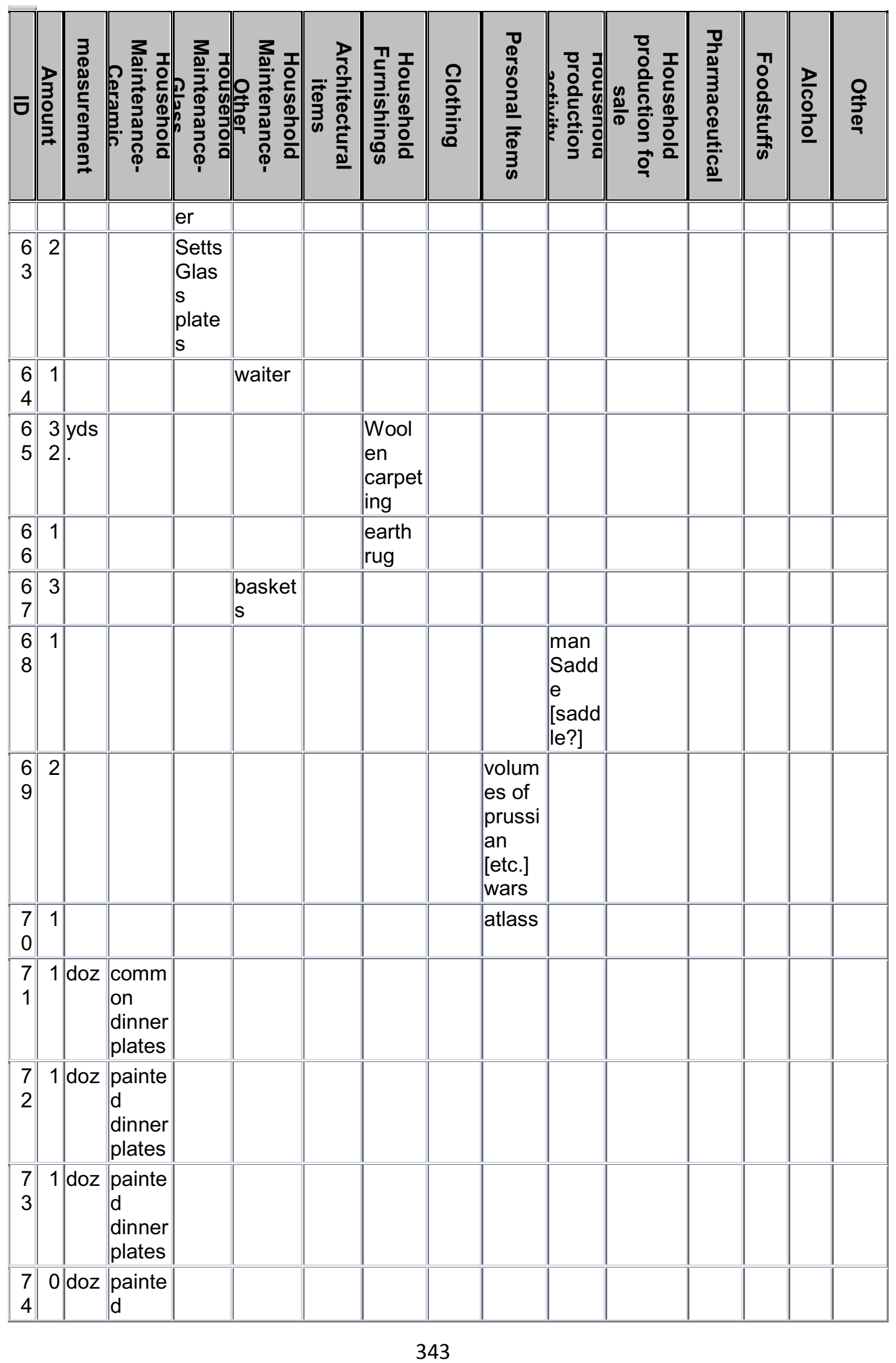




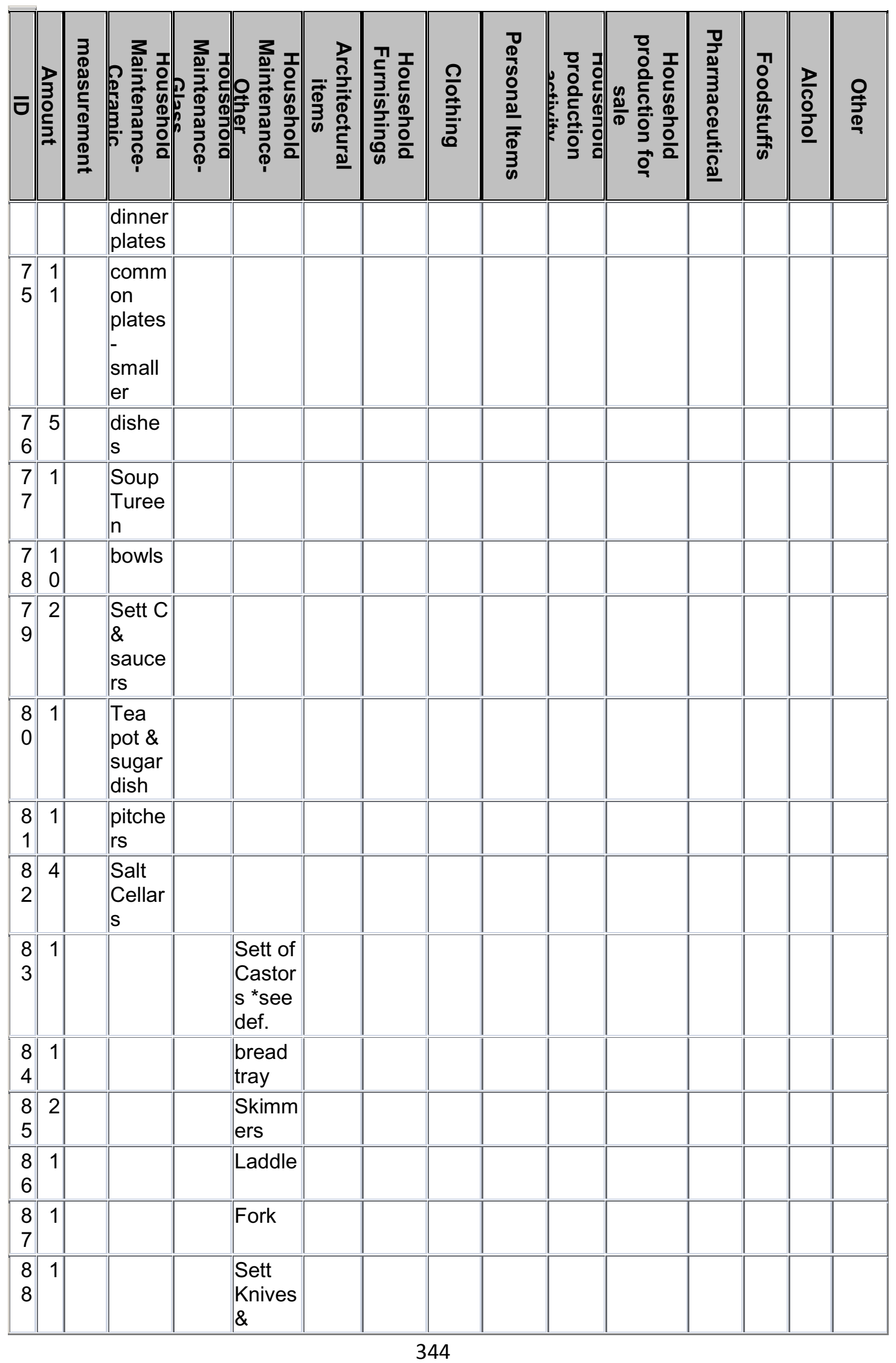




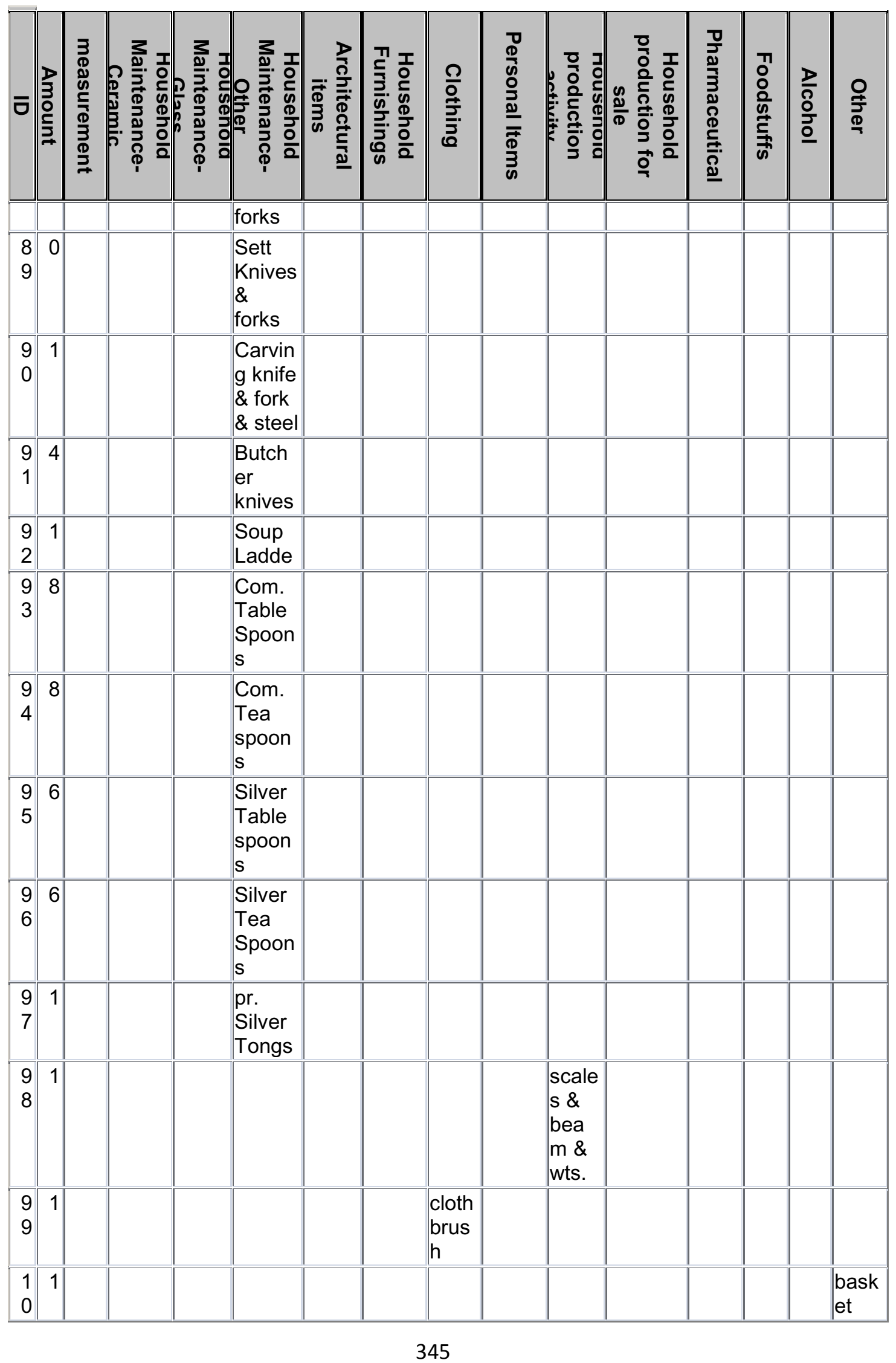




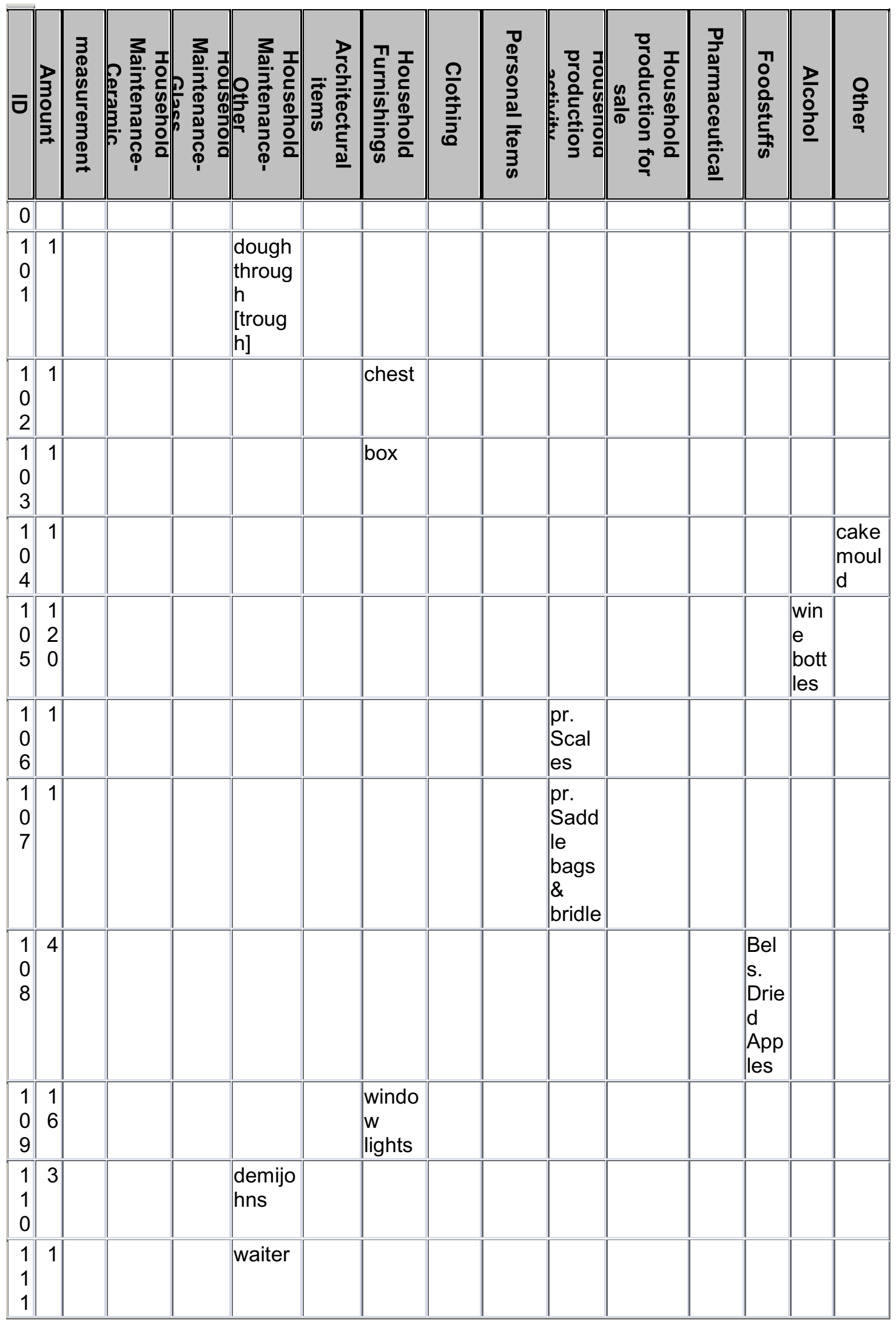




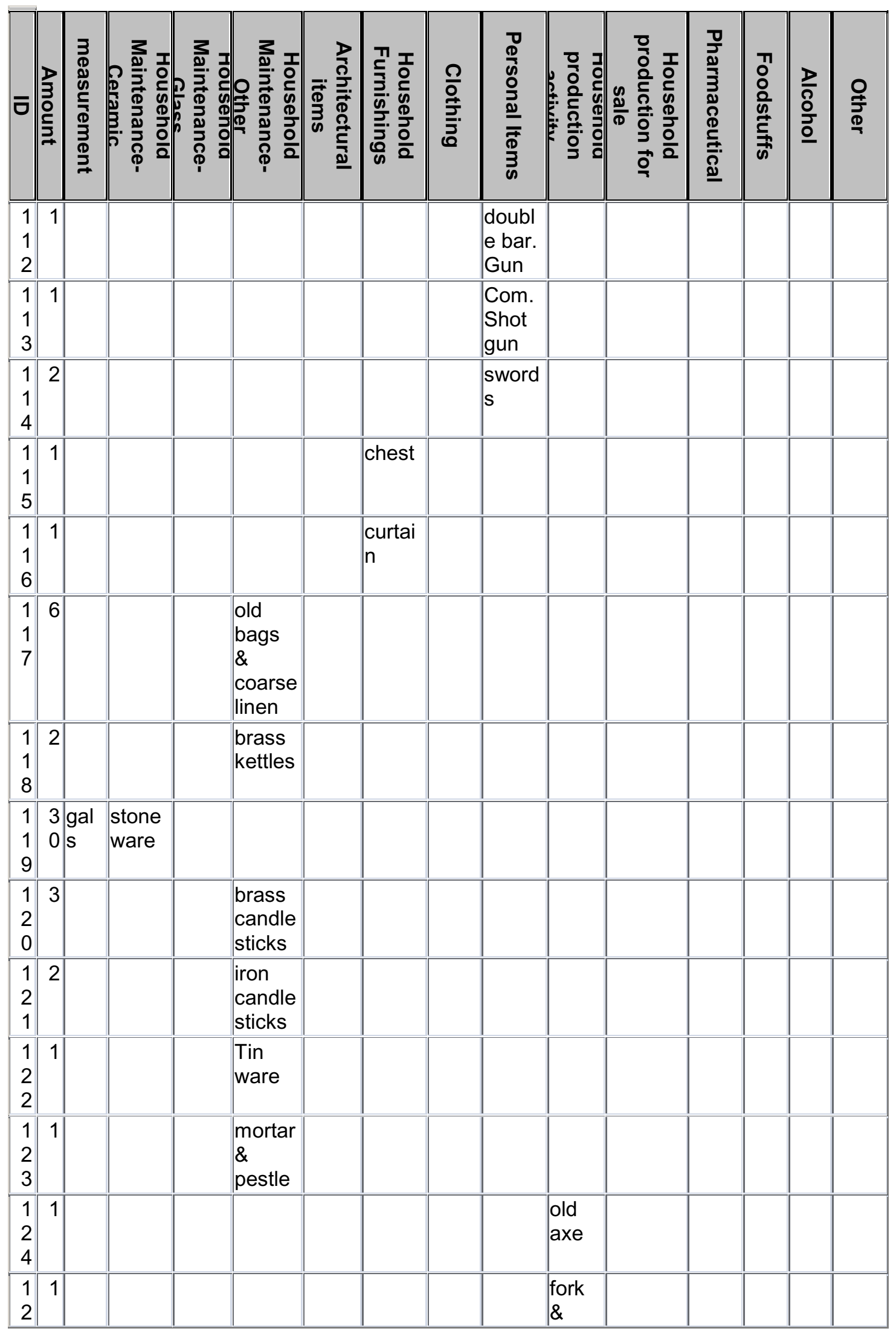




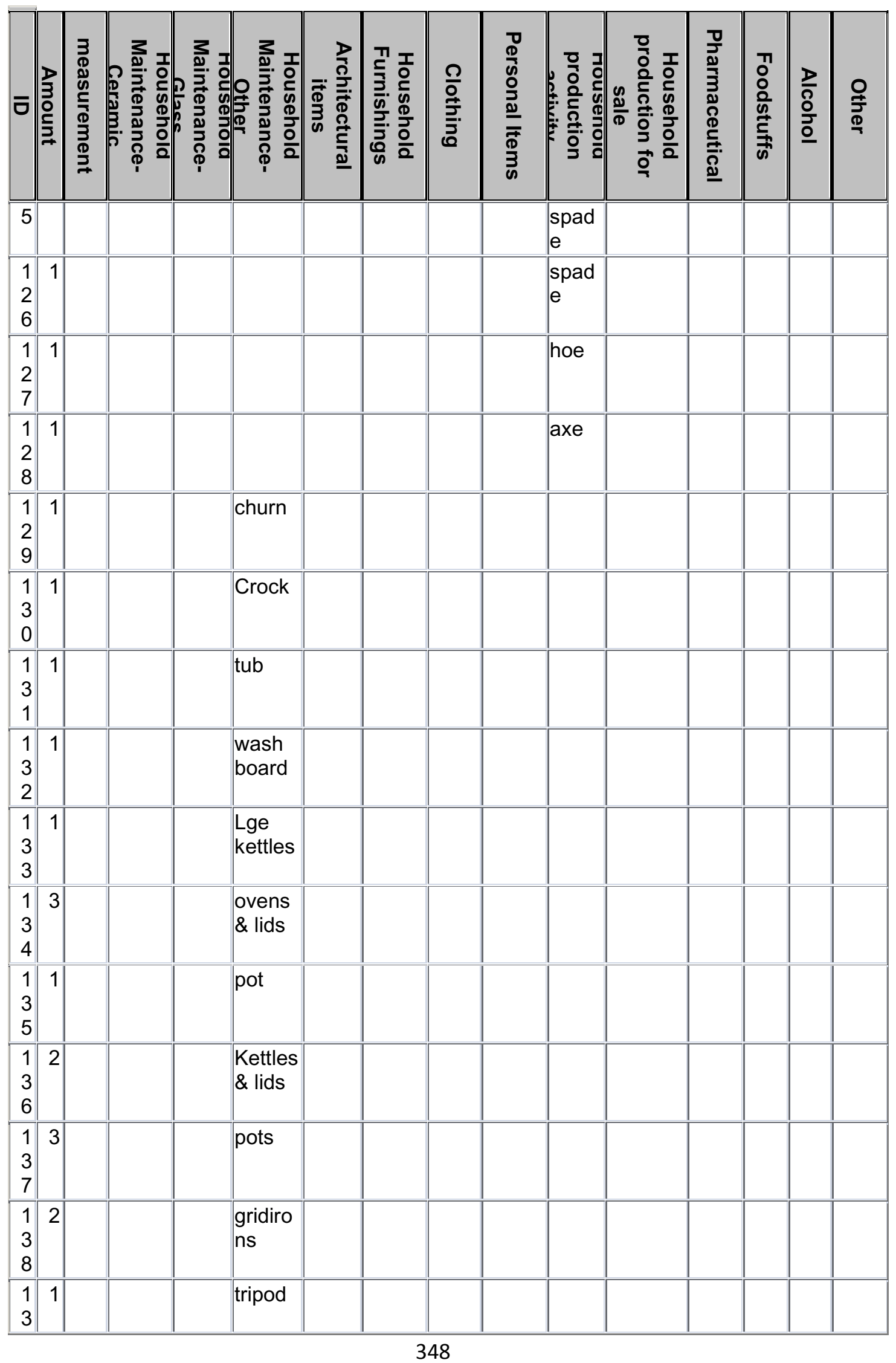




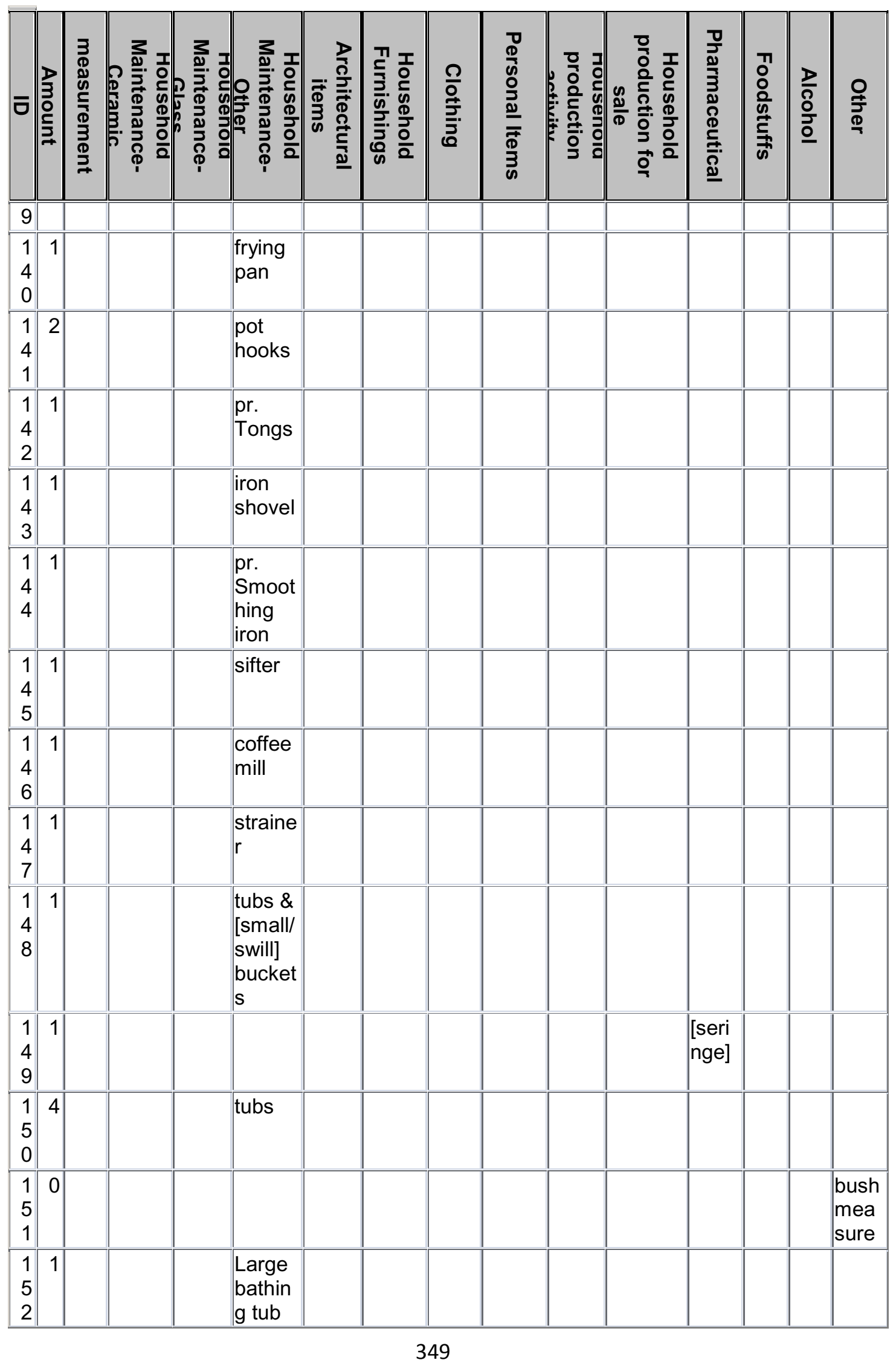




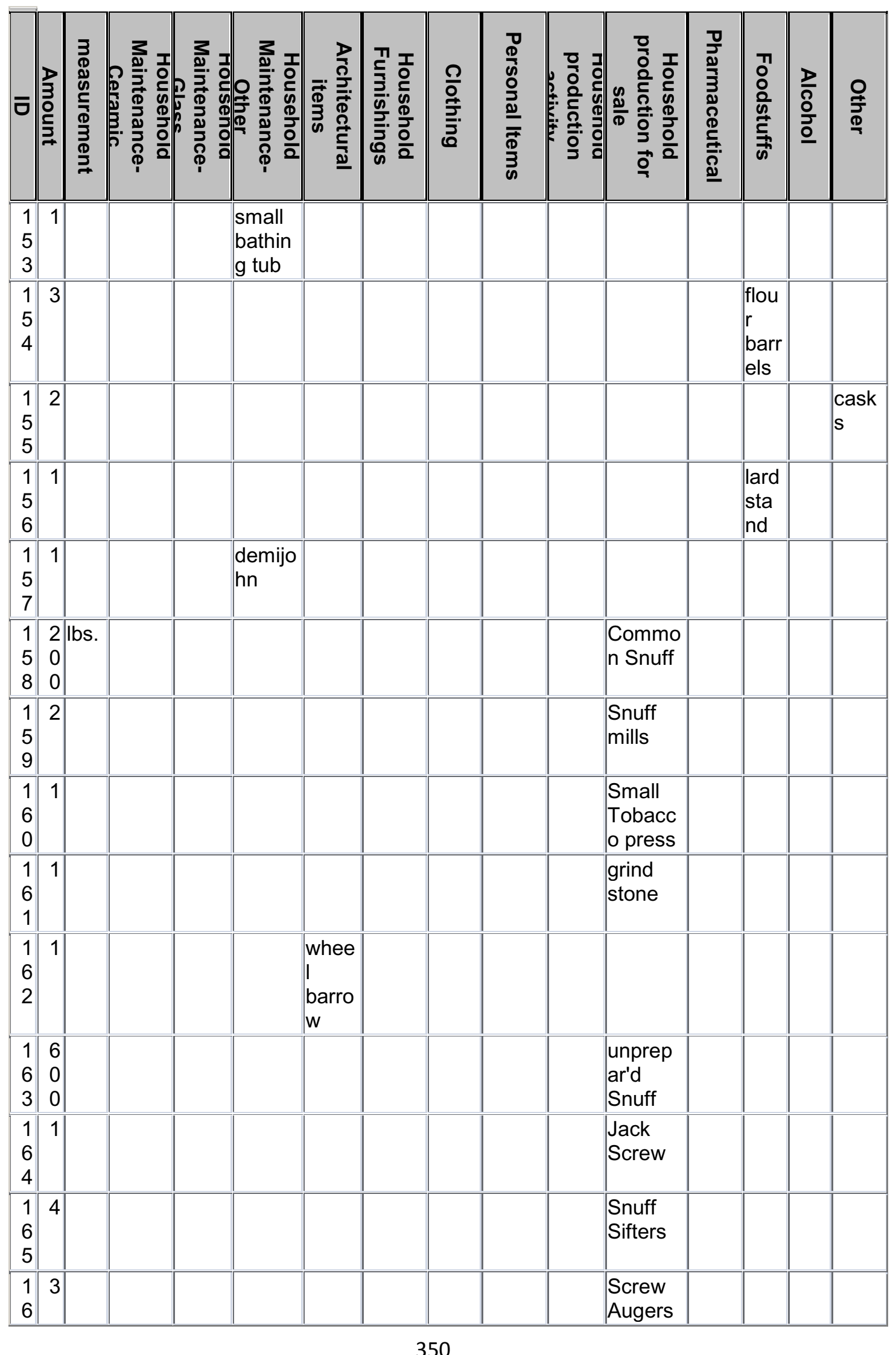




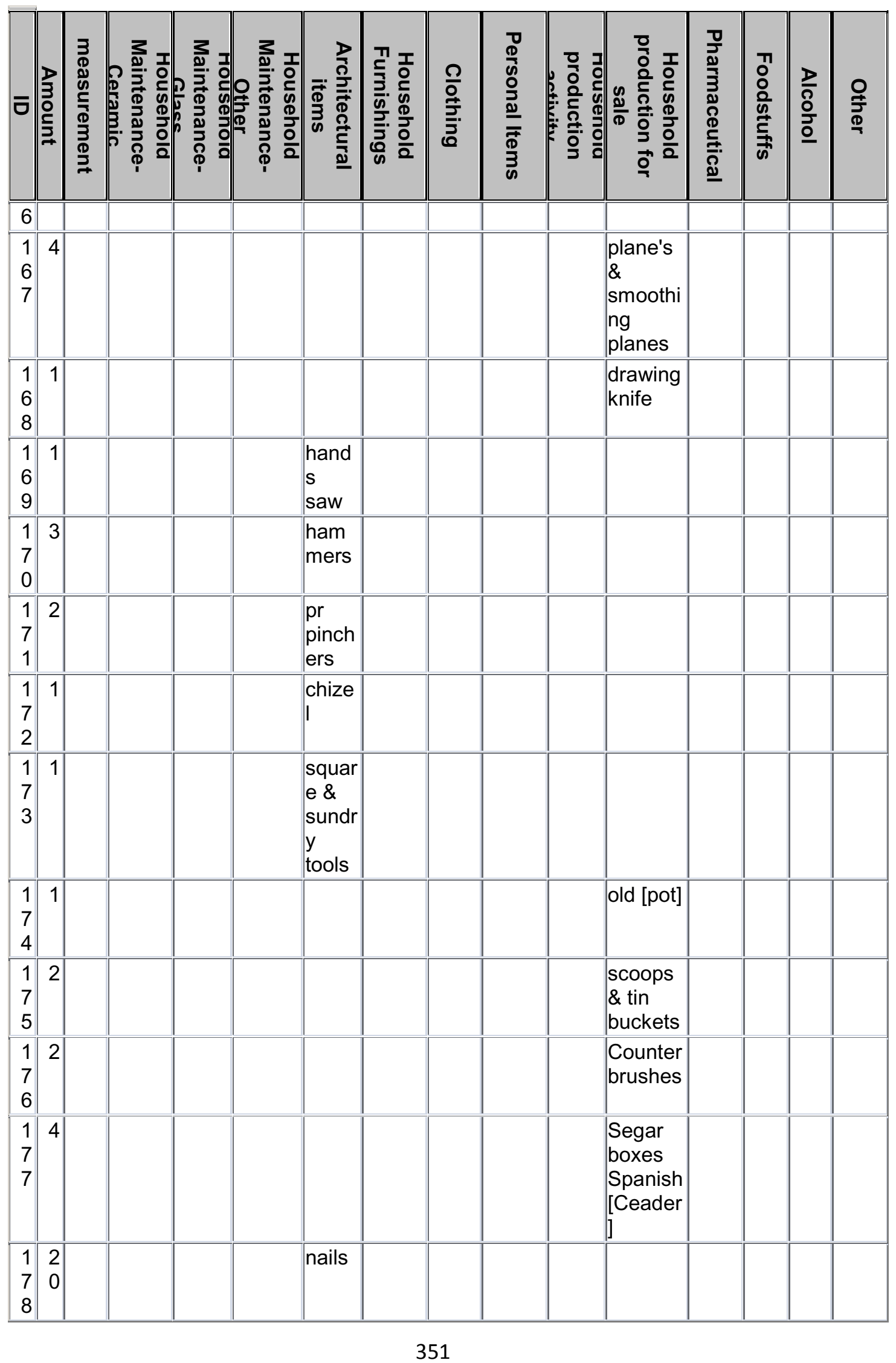




\begin{tabular}{|c|c|c|c|c|c|c|c|c|c|c|c|c|c|c|c|}
\hline б & 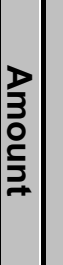 & 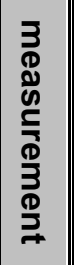 & 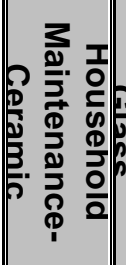 & 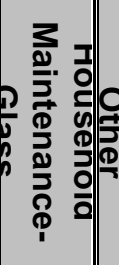 & 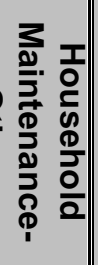 & 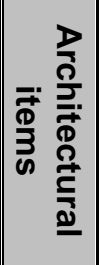 & 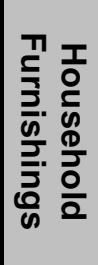 & $\begin{array}{l}\frac{0}{0} \\
\frac{5}{5} \\
\frac{5}{0}\end{array}$ & 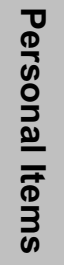 & 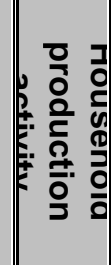 & 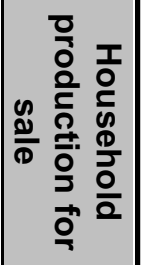 & 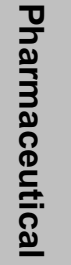 & 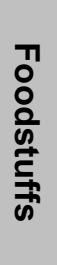 & $\begin{array}{l}\frac{D}{\delta} \\
\frac{0}{\sigma} \\
\text { o }\end{array}$ & 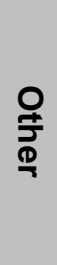 \\
\hline \begin{tabular}{|l|}
1 \\
7 \\
9
\end{tabular} & \begin{tabular}{|l|}
2 \\
7 \\
7 \\
\end{tabular} & & & & & & & & & & $\begin{array}{l}\text { Spanish } \\
\text { or } \\
\text { Havann } \\
\text { a } \\
\text { Tobco. }\end{array}$ & & & & \\
\hline $\begin{array}{l}1 \\
8 \\
0\end{array}$ & \begin{tabular}{|l|}
1 \\
5 \\
7
\end{tabular} & & & & & & & & & & \begin{tabular}{|l|} 
St. \\
Doming \\
o \\
Tobco.
\end{tabular} & & & & \\
\hline $\begin{array}{l}1 \\
8 \\
1\end{array}$ & \begin{tabular}{|l|}
1 \\
4 \\
\end{tabular} & & & & & & & & & & \begin{tabular}{|l|} 
Carrots \\
Natchit \\
oches \\
Tobco.
\end{tabular} & & & & \\
\hline $\begin{array}{l}1 \\
8 \\
2\end{array}$ & \begin{tabular}{|l|}
1 \\
7 \\
5 \\
\end{tabular} & & & & & & & & & & \begin{tabular}{|l|} 
Com. \\
Tobacc \\
\end{tabular} & & & & \\
\hline $\begin{array}{l}1 \\
8 \\
3\end{array}$ & & \begin{tabular}{|l|} 
box \\
es
\end{tabular} & & & & & & & & & $\begin{array}{l}\text { Bro. } \\
\text { Soap }\end{array}$ & & & & \\
\hline \begin{tabular}{l|}
1 \\
8 \\
4
\end{tabular} & & $\begin{array}{l}\text { rea } \\
\mathrm{m}\end{array}$ & & & & & & & & & $\begin{array}{l}\text { wrappin } \\
\text { g paper }\end{array}$ & & & & \\
\hline $\begin{array}{l}1 \\
8 \\
5\end{array}$ & 2 & & & & & & & & & & boxes & & & & \\
\hline $\begin{array}{l}1 \\
8 \\
6\end{array}$ & 1 & & & & & & & & & & $\begin{array}{l}\text { box } \\
\text { mould }\end{array}$ & & & & \\
\hline $\begin{array}{l}1 \\
8 \\
7\end{array}$ & 2 & & & & & & & & & & $\begin{array}{l}\text { strainer } \\
\text { boxes }\end{array}$ & & & & \\
\hline $\begin{array}{l}1 \\
8 \\
8\end{array}$ & & $\begin{array}{l}\text { keg } \\
\text { s }\end{array}$ & & & & & & & & & \begin{tabular}{|l|} 
chewin \\
g \\
Tobacc \\
0
\end{tabular} & & & & \\
\hline $\begin{array}{l}1 \\
8 \\
9\end{array}$ & \begin{tabular}{|l|}
2 \\
5
\end{tabular} & & & & & & & & & & \begin{tabular}{|l|} 
one] \\
half \\
Spanish \\
Segars
\end{tabular} & & & & \\
\hline $\begin{array}{l}1 \\
9 \\
0\end{array}$ & \begin{tabular}{|l|}
1 \\
4
\end{tabular} & & & & & & & & & & $\begin{array}{l}\text { melee } \\
\text { Spanish } \\
\text { Segars }\end{array}$ & & & & \\
\hline 1 & & box & & & & & & & & & half & & & & \\
\hline
\end{tabular}




\begin{tabular}{|c|c|c|c|c|c|c|c|c|c|c|c|c|c|c|c|}
\hline$\sigma \|$ & 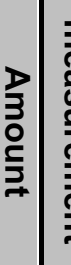 & 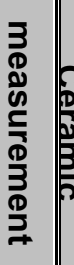 & 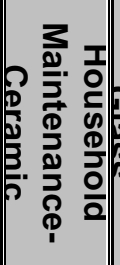 & c & 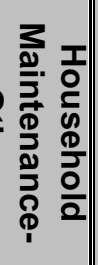 & 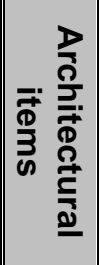 & 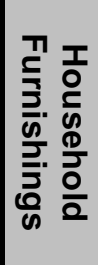 & $\begin{array}{l}\frac{0}{0} \\
\frac{5}{5} \\
\frac{5}{0}\end{array}$ & 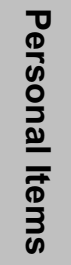 & 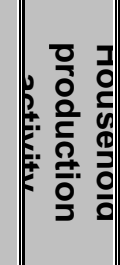 & 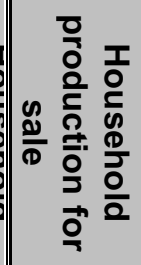 & 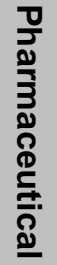 & 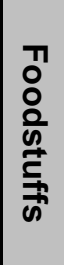 & $\begin{array}{l}\frac{8}{8} \\
\frac{0}{2} \\
\text { 으 }\end{array}$ & 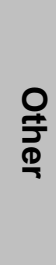 \\
\hline \begin{tabular}{|l|}
9 \\
1
\end{tabular} & $4 \mathrm{es}$ & $\overline{\text { es }}$ & & & & & & & & & $\begin{array}{l}\text { Spanish } \\
\text { Segars }\end{array}$ & & & & \\
\hline \begin{tabular}{|l|}
1 \\
9 \\
2
\end{tabular} & \begin{tabular}{l|l}
6 & $b c$ \\
9 & $e s$
\end{tabular} & $\begin{array}{l}\text { ox } \\
\text { es }\end{array}$ & & & & & & & & & $\begin{array}{l}\text { melee } \\
\text { Spanish } \\
\text { Segars }\end{array}$ & & & & \\
\hline \begin{tabular}{|l|}
1 \\
9 \\
3
\end{tabular} & 3 & & & & & & & & & & $\begin{array}{l}\text { Spanish } \\
\text { Segars }\end{array}$ & & & & \\
\hline \begin{tabular}{|l|}
1 \\
9 \\
4
\end{tabular} & \begin{tabular}{l|}
8 \\
0
\end{tabular} & & & & & & & & & & $\begin{array}{l}\text { Spanish } \\
\text { Cavand } \\
\text { ish }\end{array}$ & & & & \\
\hline \begin{tabular}{|l|}
1 \\
9 \\
5
\end{tabular} & $9 \mid \begin{array}{ll}\mathrm{b} c \\
\mathrm{le}\end{array}$ & $\begin{array}{l}\text { Dott } \\
\text { es }\end{array}$ & & & & & & & & & $\begin{array}{l}\text { macoob } \\
\text { a snuff }\end{array}$ & & & & \\
\hline \begin{tabular}{|l|}
1 \\
9 \\
6
\end{tabular} & \begin{tabular}{l|l}
1 & $\mathrm{bc}$ \\
4 & $\mathrm{le}$
\end{tabular} & $\begin{array}{l}\text { ott } \\
\text { es }\end{array}$ & & & & & & & & & $\begin{array}{l}\text { Natchit } \\
\text { oches } \\
\text { snuff }\end{array}$ & & & & \\
\hline \begin{tabular}{|l|}
1 \\
9 \\
7
\end{tabular} & $6 \begin{array}{l}\mathrm{k} \epsilon \\
\mathrm{s}\end{array}$ & keg & & & & & & & & & snuff & & & & \\
\hline \begin{tabular}{|l|}
1 \\
9 \\
8
\end{tabular} & 1 & & & & & & & & & & $\begin{array}{l}\text { Tobacc } \\
\text { o Cutter }\end{array}$ & & & & \\
\hline \begin{tabular}{|l|}
1 \\
9 \\
9
\end{tabular} & 2 & & & & & & & & & & $\begin{array}{l}\text { pr. } \\
\text { Scales } \\
\& \\
\text { weights }\end{array}$ & & & & \\
\hline \begin{tabular}{|l|}
2 \\
0 \\
0
\end{tabular} & 1 & & & & & & & & & & $\begin{array}{l}\text { Stove \& } \\
\text { pipes }\end{array}$ & & & & \\
\hline \begin{tabular}{|l|}
2 \\
0 \\
1
\end{tabular} & 1 & & & & & & & & & & $\begin{array}{l}\text { Tobacc } \\
\text { o press } \\
\& \text { screw }\end{array}$ & & & & \\
\hline \begin{tabular}{|l|}
2 \\
0 \\
2
\end{tabular} & 4 & & & & & & & & & & $\begin{array}{l}\text { Segar } \\
\text { tables }\end{array}$ & & & & \\
\hline \begin{tabular}{|l|}
2 \\
0 \\
3
\end{tabular} & $\begin{array}{l}2 \\
0\end{array}$ & & & & & & & & & & $\begin{array}{l}\text { Carrots } \\
\text { Com. } \\
\text { Tobacc } \\
\text { o }\end{array}$ & & & & \\
\hline \begin{tabular}{|l|}
2 \\
0 \\
4
\end{tabular} & $\left.\begin{array}{l}9 \\
0 \\
0\end{array}\right]$ & & & & & & & & & & $\begin{array}{l}\text { Tobacc } \\
\text { o [?] }\end{array}$ & & & & \\
\hline
\end{tabular}




\begin{tabular}{|c|c|c|c|c|c|c|c|c|c|c|c|c|c|c|c|}
\hline б & 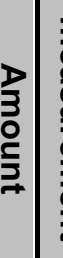 & 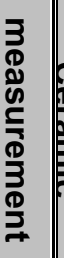 & 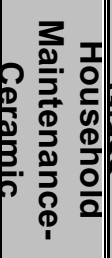 & 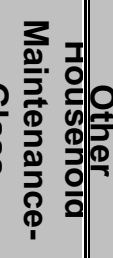 & 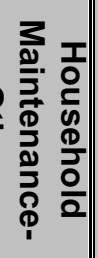 & 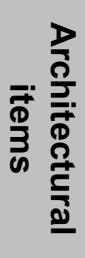 & 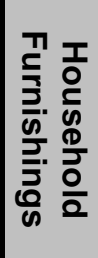 & $\begin{array}{l}\frac{0}{0} \\
\text { 寄 } \\
0\end{array}$ & 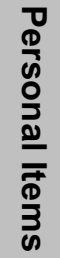 & 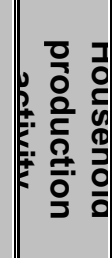 & 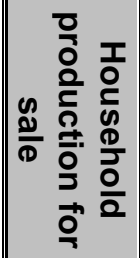 & 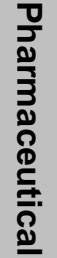 & 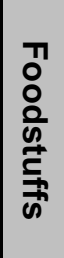 & $\begin{array}{l}\frac{D}{2} \\
\frac{0}{0} \\
\underline{0}\end{array}$ & $\begin{array}{l}\text { 옴 } \\
\text { क्ञ }\end{array}$ \\
\hline \begin{tabular}{|l|}
2 \\
0 \\
5
\end{tabular} & $\begin{array}{l}6 \\
0\end{array}$ & & & & & & & & & $\begin{array}{l}\text { head } \\
\text { s of } \\
\text { gees } \\
\text { e }\end{array}$ & & & & & \\
\hline \begin{tabular}{|l|}
2 \\
0 \\
6
\end{tabular} & $\begin{array}{l}6 \\
0 \\
0\end{array}$ & bs. & & & & & & & & & $\begin{array}{l}\text { Natchiti } \\
\text { choch } \\
\text { snuff }\end{array}$ & & & & \\
\hline $\begin{array}{l}2 \\
0 \\
7\end{array}$ & $\begin{array}{l}1 \\
7\end{array}$ & & & & & & & & & & $\begin{array}{l}\text { Carrot } \\
\text { moulds }\end{array}$ & & & & \\
\hline
\end{tabular}

